\title{
Formulación material y espacial del modelo geométricamente exacto de piezas alargadas
}

\author{
autor \\ Carlos Lázaro Fernández \\ Ingeniero de Caminos, Canales y Puertos
}

Tesis doctoral presentada en el

Departamento de Mecánica de Medios Continuos y Teoría de Estructuras de la Universidad Politécnica de Valencia

director

Prof. Dr. Salvador Monleón Cremades

Valencia, julio de 2005

(c) Carlos Lázaro Fernández, 2005 

A Juliane, Sara y Adrián 



\section{Resumen}

El análisis de la respuesta mecánica de piezas alargadas que experimentan grandes desplazamientos y rotaciones constituye un campo en el que se han producido avances significativos en las tres últimas décadas, tanto desde el punto de vista de la formulación de modelos físicos como de la búsqueda de soluciones numéricas. El ámbito de aplicación de este tipo de modelos se separa quizás del abanico de problemas de los que tradicionalmente se ha ocupado la ingeniería civil, aproximándose más a situaciones propias de otras disciplinas, como la aeronáutica, la robótica o la biomecánica.

Dentro de la variedad de modelos en una dimensión desarrollados para el análisis no lineal de piezas alargadas, el propuesto por Simó como extensión del trabajo de Reissner es capaz de reproducir rotaciones arbitrariamente grandes de las secciones transversales. Su sencillez conceptual y la potencia de las soluciones numéricas basadas en él lo han convertido en referencia obligada y punto de partida de muchas investigaciones recientes. El propio Simó introdujo la denominación de modelo geométricamente exacto de piezas alargadas para referirse a él. No obstante, el modelo de Reissner-Simó no está libre de dificultades. Éstas derivan principalmente del tratamiento exacto de las rotaciones, que exige trabajar en un espacio de configuraciones no lineal ni conmutativo.

Esta tesis examina los fundamentos del modelo geométricamente exacto y su conexión con la teoría no lineal de la elasticidad. La relación entre variables materiales y espaciales a través de la transformación definida por la rotación de cada sección establece el método de análisis. En un primer paso se ha desarrollado completamente la cinemática del modelo y las ecuaciones de campo en sus dos facetas -material y espacial-, lo que ha permitido sistematizar el proceso deductivo y aportar algunos resultados teóricos novedosos. El análisis del problema desde el punto de vista variacional ha puesto de manifiesto las conexiones formales entre las ecuaciones de la estática de las piezas alargadas y las ecuaciones de la dinámica de sólidos rígidos, y ha conducido a actualizar y generalizar la analogía cinética de Kirchhoff empleando el lenguaje moderno de la mecánica clásica.

Como paso previo al desarrollo de soluciones numéricas se ha deducido la expresión espacial del operador tangente a partir de la linealización consistente de la ecuación de trabajos virtuales. Cuando este proceso se lleva a cabo antes de la discretización da lugar a la aparición de nuevos términos adicionales en el operador, aunque su influencia en los resultados numéricos ha resultado ser despreciable. También se ha deducido la forma material del operador, aún no publicada. El hecho de que las expresiones del operador tangente sean independientes de la parametrización escogida para la descripción de las rotaciones constituye un aspecto destacado de la formulación empleada. 
La parte final de la tesis se centra en la solución numérica del modelo. En una primera etapa se ha desarrollado un elemento finito basado en la forma espacial del operador, que es esencialmente el propuesto por Simó y Vu-Quoc con algunas modificaciones introducidas por Ibrahimbegović y Taylor. El análisis de diversos ejemplos muestra la potencia del modelo, pero también pone de manifiesto algunos problemas numéricos. Por último se ha desarrollado un nuevo elemento finito empleando el operador tangente en forma material, que incorpora la interpolación esférica propuesta por Crisfield y Jelenić. Después de procesar distintos casos se concluye que el elemento material proporciona la misma precisión en la solución que el espacial, pero su velocidad de convergencia es considerablemente menor. 


\section{Resum}

L'anàlisi de la resposta mecànica de peces allargades que experimenten grans desplaçaments i rotacions constitueix un camp en què s'han produït avenços significatius en les tres últimes dècades, tant des del punt de vista de la formulació de models físics com de la recerca de solucions numèriques. L'àmbit d'aplicació d'aquest tipus de models se separa potser de la sèrie de problemes de què tradicionalment s'ha ocupat l'enginyeria civil, aproximant-se més a situacions pròpies d'altres disciplines, com l'aeronàutica, la robòtica o la biomecànica.

Dins de la varietat de models en una dimensió desenvolupats per a l'anàlisi no lineal de peces allargades, el proposat per Simó com a extensió del treball de Reissner és capaç de reproduir rotacions arbitràriament grans de les seccions transversals. La senzillesa conceptual del model i la potència de les solucions numèriques basades en ell l'han convertit en referència obligada i punt de partida de moltes investigacions recents. El propi Simó va introduir la denominació de model geomètricament exacte de peces allargades per a referir-se a ell. No obstant això, el model de Reissner-Simó no està lliure de dificultats. Aquestes deriven principalment del tractament exacte de les rotacions, que exigeix treballar en un espai de configuracions no lineal ni commutatiu.

Aquesta tesi examina els fonaments del model geomètricament exacte i la connexió d'aquest amb la teoria no lineal de l'elasticitat. La relació entre variables materials i espacials a través de la transformació definida per la rotació de cada secció estableix el mètode d'anàlisi. En un primer pas s'ha desenvolupat completament la cinemàtica del model i les equacions de camp en les seues dues facetes -material i espacial-, la qual cosa ha permès sistematitzar el procés deductiu i aportar alguns resultats teòrics nous. L'anàlisi del problema des del punt de vista variacional ha posat de manifest les connexions formals entre les equacions de l'estàtica de les peces allargades i les equacions de la dinàmica de sòlids rígids, i ha conduït a actualitzar i generalitzar l'analogia cinètica de Kirchhoff emprant el llenguatge modern de la mecànica clàssica.

Com a pas previ al desenvolupament de solucions numèriques s'ha deduït l'expressió espacial de l'operador tangent a partir de la linealització consistent de l'equació de treballs virtuals. Quan aquest procés es porta a terme abans de la discretització dóna lloc a l'aparició de nous termes addicionals en l'operador, encara que la influència d'aquests en els resultats numèrics ha resultat ser negligible. També s'ha deduit la forma material de l'operador, encara no publicada. El fet que les expressions de l'operador tangent siguen independents de la parametrització triada per a la descripció de les rotacions constitueix un aspecte destacat de la formulació emprada.

La part final de la tesi se centra en la solució numèrica del model. En una primera etapa s'ha desenvolupat un element finit basat en la forma espacial de l'operador, que és essencialment el proposat per Simó i Vu-Quoc amb algunes modificacions introduïdes per Ibrahimbegović i Taylor. L'anàlisi de diversos exemples mostra la potència 
del model, però també posa de manifest alguns problemes numèrics. Finalment s'ha desenvolupat un nou element finit emprant l'operador tangent en forma material, que incorpora la interpolació esfèrica proposada per Crisfield i Jelenić. Després de processar distints casos es conclou que l'element material proporciona la mateixa precisió en la solució que l'espacial, però la velocitat de convergència és considerablement menor. 


\begin{abstract}
The analysis of the mechanical behaviour of rods subject to large displacements and rotations constitutes a research field where significant advances from the viewpoint of both, formulation of physical models and finding of computational solutions have taken place over the last three decades. The realm of applications of this kind of models differs from the scope of problems traditionally treated by civil engineering, approaching other technologies as aeronautics, robotics or biomechanics.

Among the variety of one dimensional models available for non linear analysis of rods, the one proposed by Simó as a extension of the work of Reissner is capable to treat arbitrarily large rotations of the cross-sections. Its conceptual simplicity and the model-based powerful numerical solutions have turned it into a main reference and a starting point for many recent research works. Simó coined the label geometrically exact rod model to refer to his model. Nevertheless the Reissner-Simó model is not free of difficulties. These are mainly caused by the exact treatment of rotations, requiring to work in a non linear, non commutative configuration space.

This dissertation examines the foundations of the geometrically exact model and its connections to the non linear theory of elasticity. The relation between material and spatial variables through the transformation defined by the rotation of the crosssections determines the approach method. As a first step, model kinematics and field equations in both forms -material and spatial- have been fully developed. This allowed to systematize the deductive process and to contribute some new theoretical results. The problem analysis from the variational viewpoint has shown the formal connections between the equations of rod elastostatics and the equations of rigid body dynamics, and has led to the generalization and update of Kirchhoff's kinetic analogy employing the modern language of classical mechanics.

Prior to the development of numerical solutions, the expression of the spatial tangent operator has been derived from a consistent linearization of the virtual work equation. When linearization is carried on before discretization, new additional terms in the operator appear; their influence on the numerical results has been shown to be negligible. The material form of the operator (still unpublished) has also been obtained. The fact that the expressions of the tangent operator are independent of the selected parameterization of rotations is a remarkable feature of the formulation.

The final part of the dissertation focuses on the model numerical solution. In a first stage a finite element based on the spatial form of the operator has been developed, which is essentially the one proposed by Simó and $\mathrm{Vu}-\mathrm{Quoc}$ with some changes introduced by Ibrahimbegović and Taylor. The analysis of several examples shows the power of this model, but also poses some numerical problems. As a last contribution, a new finite element using the material tangent operator and the spherical interpolation proposed by Crisfield and Jelenić was developed. Processing of several examples
\end{abstract}


has led to the conclusion that solutions obtained using the material element reach the same precision as with the spatial one, but the convergence rate is much slower. 


\section{Agradecimientos}

Esta tesis doctoral ha sido el fruto de varios años de trabajo durante los cuales he recibido el apoyo de muchas personas. Quisiera agradecer en primer lugar a mi director de tesis, el Dr. Salvador Monleón, la confianza que ha depositado en mí, la libertad que me ha concedido para buscar nuevos caminos, así como su guía inestimable y su apoyo constante para recorrerlos. Algunos han resultado ser difíciles o equivocados; otros han conducido, finalmente, a resultados y conclusiones que espero sean interesantes. Esta libertad es la que más satisfacciones me ha proporcionado en el curso de la investigación.

A Juliane, y por supuesto a Sara y Adrián, les debo el cariño con el que me han apoyado y la paciencia con la que han soportado mis ausencias. Espero estar aún a tiempo de recuperar el tiempo perdido. A mis amigos, y especialmente a Conchi, les agradezco el ánimo que me han infundido en los momentos más difíciles. Debo nombrar también a mis padres que siempre me alentaron en esta empresa.

Al Dr. Alberto Domingo tengo que agradecerle la inestimable ayuda que me ha prestado, el tiempo que me ha dedicado durante toda esta etapa, y la seguridad de que el resultado merece la pena. Sin su impulso y apoyo difícilmente hubiera podido llegar hasta aquí.

Agradezco también la ayuda recibida de mis compañeros de la unidad docente, y quiero mencionar especialmente al Dr. José Casanova y al Dr. Antonio Agüero, que han estado siempre dispuestos a escuchar el relato de mis dificultades y a ofrecer sus interesantes sugerencias.

Finalmente quiero mencionar a José Chamorro, Juanjo Ferrer, Marina Selma, Enrique Moreno, Francisco Palacios, Luis Claramonte y todos los demás miembros del área de ingeniería de $\mathrm{CM}$, que también han contribuido al resultado de este trabajo. 



\section{Índice general}

Índice general IX

Índice de figuras XII

1 Introducción $\quad 1$

1.1. El modelo geométricamente exacto de piezas alargadas . . . . . . . . . 1

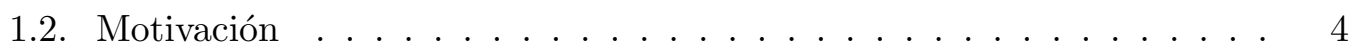

1.3. Objetivos de la tesis $\ldots \ldots \ldots \ldots \ldots$

1.4. Contenido . . . . . . . . . . . . . . . . . 6

1.5. Notación . . . . . . . . . . . . . . . . . . . . . . . 8

2 Estado del Arte $\quad 13$

2.1. Contexto histórico . . . . . . . . . . . . . . . . 13

2.2. El modelo de Reissner-Simó . . . . . . . . . . . . . . . . . . . . . . . 19

2.3. Soluciones numéricas del modelo de Reissner-Simó . . . . . . . . . . . 21

2.3.1. El tratamiento de las rotaciones . . . . . . . . . . . . . 21

2.3.2. La solución numérica de Simó y Vu-Quoc . . . . . . . . . . . . 24

2.3.3. Clasificación de las soluciones numéricas del modelo geométri-

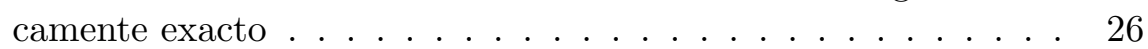

2.3.4. Extensión de las soluciones numéricas a otros tipos de problemas 29

3 Cinemática del modelo RS $\quad 31$

3.1. Configuración inicial y configuración de referencia . . . . . . . . . . . 33

3.2. Geometría de la configuración inicial . . . . . . . . . . . . . 36

3.2.1. Tensor de curvaturas iniciales . . . . . . . . . . . . . 36

3.2.2. Métrica de la sección transversal . . . . . . . . . . . . . . . . . . . . . . . . 38

3.3. Hipótesis cinemática . . . . . . . . . . . . . . . . . . . . . 39

3.4. Interpretación geométrica del tensor $\boldsymbol{\Lambda} \ldots \ldots \ldots$. . . . . . . . 41

3.5. Geometría de la configuración actual . . . . . . . . . . . . . . 45

3.5.1. Cambio de orientación de la sección . . . . . . . . . . . 45

3.5.2. Gradiente de la deformación. Deformaciones generalizadas . . . 46

3.5.3. Objetividad de las medidas de la deformación . . . . . . . . . . 49 
4 Ecuaciones de campo $\quad 53$

4.1. Construcción de la ecuación de trabajos virtuales . . . . . . . . . . . 53

4.1.1. Variación de la configuración . . . . . . . . . . . . . . 54

4.1.2. Definición de la variación corrotacional . . . . . . . . . . . 55

4.1.3. Variación del gradiente de la deformación . . . . . . . . . . . 57

4.1.4. Ecuación de trabajos virtuales del modelo RS . . . . . . . . . . 58

4.2. Cálculo de la variación de las deformaciones generalizadas . . . . . . . 62

4.3. Ecuaciones de equilibrio . . . . . . . . . . . . . . . . . . . . 67

4.4. Ecuaciones constitutivas . . . . . . . . . . . . . . . 70

$\begin{array}{llr}5 & \text { La analogía dinámica } & \mathbf{7 7}\end{array}$

5.1. Las ecuaciones de Euler-Poincaré . . . . . . . . . . . . . . . . . . . 84

5.2. Las ecuaciones de Lie-Poisson . . . . . . . . . . . . . . . . . 87

$\begin{array}{lll}6 & \text { El operador tangente en el modelo RS } & 95\end{array}$

6.1. Resultados preliminares . . . . . . . . . . . . . . . . 97

6.1.1. Propiedades elementales de la variación y la variación corrotacional ......................... 98

6.1.2. Segunda variación de la configuración . . . . . . . . . . . . . 99

6.2. Forma espacial del equilibrio tangente . . . . . . . . . . . . . . . 101

6.2.1. Linealización del trabajo virtual de las fuerzas internas. . . . . 101

6.2.2. Linealización del trabajo virtual de las fuerzas exteriores . . . . 107

6.2.3. Equilibrio tangente . . . . . . . . . . . . . . . . 110

6.2.4. Análisis de los términos adicionales al operador de Simó . . . . 112

6.3. Forma material del equilibrio tangente . . . . . . . . . . . . . . 116

6.3.1. Linealización del trabajo virtual de las fuerzas internas. . . . . 116

6.3.2. Linealización del trabajo virtual de las fuerzas exteriores . . . . 122

6.3.3. Ecuación de equilibrio tangente . . . . . . . . . . . . . 123

$\begin{array}{llr}7 & \text { Solución numérica basada en la forma espacial } & 127\end{array}$

7.1. Discretización en elementos finitos . . . . . . . . . . . . . . . . 128

7.2. Solución incremental pura . . . . . . . . . . . . . . . . . . . 129

7.2.1. Construcción de la ecuación incremental . . . . . . . . . . . . 130

7.2.2. Actualización de la configuración . . . . . . . . . . . . . . . . 134

7.2.3. Relación entre la interpolación y la objetividad de la solución . 137

7.3. Solución incremental-iterativa . . . . . . . . . . . . . . . . 142

7.3.1. Expresión del residuo . . . . . . . . . . . . . . . . . . 142

7.3.2. Linealización del residuo . . . . . . . . . . . . . . . . . . . . 144

7.3.3. Proceso iterativo . . . . . . . . . . . . . . . . 148

7.4. Flujo del programa . . . . . . . . . . . . . . . . . . . . . 152

7.4.1. Datos iniciales . . . . . . . . . . . . . . . . . . . . . . . . . . . . . . . . . . . . . . . . . . . .

7.4.2. Proceso incremental . . . . . . . . . . . . . . . 153

7.4.3. Proceso incremental - iterativo . . . . . . . . . . . . 155

7.5. Resultados . . . . . . . . . . . . . . . . . . 157 
7.5.1. Análisis de la solución de Simó y Vu-Quoc al problema de la

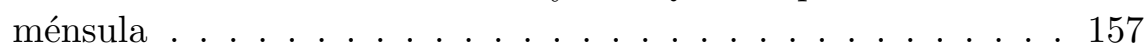

7.5.2. Solución incremental pura de la ménsula de Simó y Vu-Quoc . 163

7.5.3. Evaluación del efecto de la parte antisimétrica del operador . . 166

7.5.4. Ejemplos . . . . . . . . . . . . . 176

8 Solución numérica basada en la forma material 203

8.1. Formulación del elemento . . . . . . . . . . . . . . . . . 204

8.1.1. Interpolación . . . . . . . . . . . . . . . . . 204

8.1.2. Predicción . . . . . . . . . . . . . . . . . 205

8.1.3. Corrección . . . . . . . . . . . . . . . . . 209

8.1.4. Actualización de la configuración . . . . . . . . . . . . . . . 214

8.2. Flujo del programa . . . . . . . . . . . . . . . . . . 217

8.2.1. Datos iniciales . . . . . . . . . . . . . . . . . . 217

8.2.2. Predicción . . . . . . . . . . . . . . . . . . . 218

8.2.3. Corrección . . . . . . . . . . . . . . . 221

8.3. Resultados . . . . . . . . . . . . . . . . . . . . . . 224

9 Conclusiones 233

9.1. Resumen del trabajo realizado . . . . . . . . . . . . . . 233

9.2. Conclusiones . . . . . . . . . . . . . . . . . . . . . 234

9.3. Aportaciones originales . . . . . . . . . . . . . . . . . 237

9.4. Líneas de investigación propuestas . . . . . . . . . . . . . . 239

\begin{tabular}{|ll}
\hline A Algunas propiedades de la variación corrotacional & 241
\end{tabular}

\begin{tabular}{|ll}
\hline B Cálculo del operador T & 243
\end{tabular}

$\begin{array}{lr}\text { Bibliografía } & 247\end{array}$ 


\section{Índice de figuras}

2.1. Reproducción de la tabla 4 de Euler . . . . . . . . . . . . . . . . . . 15

2.2. Analogía cinética de Kirchhoff (figura 47 de Love) . . . . . . . . . . . . . . . 17

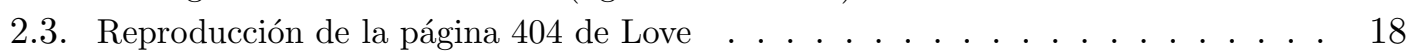

3.1. Configuraciones y sistemas de referencia . . . . . . . . . . . . . . . . 32

3.2. Configuración inicial y configuración de referencia . . . . . . . . . . . . . . . 34

3.3. Cambio de configuración . . . . . . . . . . . . . . . . . . . . . . 39

3.4. Objetos materiales y espaciales . . . . . . . . . . . . . . . . . 43

3.5. Alargamiento y distorsión . . . . . . . . . . . . . . . . . . 50

3.6. Cambio de orientación del sistema de referencia . . . . . . . . . . . . . . . 51

4.1. Variación de la rotación. Forma espacial y material . . . . . . . . . . . . . 61

7.1. Velocidad incremental . . . . . . . . . . . . . . . . . . . . . 132

7.2. Interpolación esférica . . . . . . . . . . . . . . . . . . . . . 139

7.3. Control de carga . . . . . . . . . . . . . . . . . . . . . . . . . . . 149

7.4. Control de desplazamiento . . . . . . . . . . . . . . . . . . . . . . . . . 149

7.5. Control de arco . . . . . . . . . . . . . . . . . . . . . 150

7.6. Trayectoria del extremo de la ménsula de Simó y Vu-Quoc. Solución exacta . . . 158

7.7. Trayectoria del extremo de la estructura A $(E / G=2,5)$. Soluciones incrementales con $n$ variable . . . . . . . . . . . . . . . . . . . . . . 164

7.8. Trayectoria del extremo de la estructura $\mathrm{C} 2(E / G=2,5)$. Soluciones incrementales con $n$ variable. . . . . . . . . . . . . . . . . . . . 165

7.9. Estructura $1 \ldots \ldots \ldots$. . . . . . . . . . . . . . . 167

7.10. Estructura 1. Acción de un momento de eje fijo de módulo $4 \pi$. Solución iterativa 168

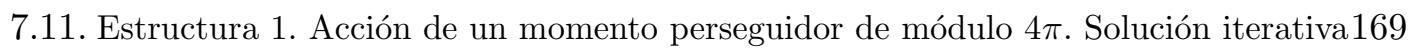

7.12. Estructura 1 con un momento de eje fijo en el extremo. Error relativo en los desplazamientos nodales en la solución incremental pura en 20 pasos . . . . . . 170

7.13. Estructura 1 con un momento perseguidor en el extremo. Error relativo en los desplazamientos nodales en la solución incremental pura en 20 pasos . . . . . . 171

7.14. Estructura 2 . . . . . . . . . . . . . . . . . . . . . 172

7.15. Estructura 2. Acción de una fuerza de eje fijo $F_{z}=1$. Solución iterativa . . . . . 173

7.16. Estructura 2. Acción de una fuerza perseguidora de módulo 1. Solución iterativa 173

7.17. Estructura 2 con una fuerza de eje fijo en el extremo. Error relativo en los desplazamientos nodales en la solución incremental pura en 20 pasos . . . . . . . . 174 
7.18. Estructura 2 con una fuerza perseguidora en el extremo. Error relativo en los desplazamientos nodales en la solución incremental pura en 20 pasos . . . . . . 175

7.19. Figuras 1.8 y 1.9 de Brush et al. . . . . . . . . . . . . . . . . . . . . 176

7.20. Figura 1.10 de Brush et al. . . . . . . . . . . . . . . . . . . . . . . . 177

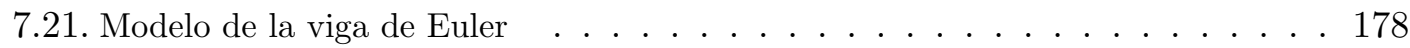

7.22. Comparación de trayectorias de equilibrio para la pieza comprimida con una perturbación inicial. . . . . . . . . . . . . . . . . . 178

7.23. Viga de Euler. Trayectoria de equilibrio del nodo 6 . . . . . . . . . . . . . . . 179

7.24. Proceso de deformación de la viga de Euler (módulo de la fuerza en $\mathrm{kN}$, coordenadas en $\mathrm{m}$ ) . . . . . . . . . . . . . . . . . . 180

7.25. Esfuerzos correspondientes a $F=3599,4 k N$. . . . . . . . . . . . . . . . . . . 180

7.26. Trabajo y energía durante la deformación . . . . . . . . . . . . . . . . . . . . 181

7.27. Densidad de energía de deformación, esfuerzo axil y valor de $\mathcal{H}$ en cada elemento 182

7.28. Valores del primer $(\mathcal{H})$ y tercer invariante $\left(C_{(1)}\right)$ en cada elemento . . . . . . . 183

7.29. Superficies invariantes y esfuerzos . . . . . . . . . . . . . . . 183

7.30. Voladizo de Bathe y Bolourchi . . . . . . . . . . . . . . . . . . . . . . . . 184

7.31. Proceso de deformación del voladizo de Bathe. Fuerza perseguidora en el extremo (módulo de la fuerza en lb, coordenadas en in) . . . . . . . . . . . . . . . . 186

7.32. Voladizo de Bathe. Trayectoria de equilibrio del nodo 9 . . . . . . . . . . . . . 187

7.33. Esfuerzos en el voladizo de Bathe. $F=3000 \mathrm{lb}$, perseguidora . . . . . . . . . . 187

7.34. Densidad de energía de deformación, esfuerzo axil y valor de $\mathcal{H}$ en cada elemento 188

7.35. Modelo del pórtico de Argyris (figura 6.3.1. de Argyris et al.) . . . . . . . . . . 189

7.36. Pórtico de Argyris. Trayectorias de equilibrio del desplazamiento horizontal para la fuerza espacial / perseguidora . . . . . . . . . . . . . . . . . 192

7.37. Pórtico de Argyris. Trayectorias de equilibrio del desplazamiento vertical para la fuerza espacial / perseguidora . . . . . . . . . . . . . . . 192

7.38. Proceso de deformación del pórtico bajo la acción de una fuerza de eje fijo (módulo de la fuerza en $\mathrm{kN}$, coordenadas en $\mathrm{cm}$ ) . . . . . . . . . . . . . 193

7.39. Proceso de deformación del pórtico bajo la acción de una fuerza perseguidora (módulo de la fuerza en $\mathrm{kN}$, coordenadas en $\mathrm{cm}$ ) . . . . . . . . . . . . 193

7.40. Malla geodésica . . . . . . . . . . . . . . . . . . . . . . . 194

7.41. Malla geodésica. Trayectoria de equilibrio (desplazamiento vertical de la cúspide) 196

7.42. Malla geodésica. Energía de deformación . . . . . . . . . . . . . . . . . . . . . 196

7.43. Situación inicial . . . . . . . . . . . . . . . . . . . . . . . . . 197

7.44. Paso 21. . . . . . . . . . . . . . . . . . . . . . . . 198

7.45. Paso 33. . . . . . . . . . . . . . . . . . . . . 198

7.46. Paso 61. . . . . . . . . . . . . . . . . . . . . . 199

7.47. Paso 106 . . . . . . . . . . . . . . . . . . . . . . . 199

7.48. Paso 134 . . . . . . . . . . . . . . . . . . . . . . 200

7.49. Paso 178 ........................... . . . 200

7.50. Paso 200 . . . . . . . . . . . . . . . . . . . 201

8.1. Transformaciones en el elemento material . . . . . . . . . . . . . . . . . . . 207

8.2. Rotación de referencia . . . . . . . . . . . . . . . . . . . . . 215

8.3. Rotaciones locales . . . . . . . . . . . . . . . . . . . . 215 
8.4. Trayectoria del extremo de la estructura A $(E / G=2,5)$. Solución incremental pura en 50 incrementos. Comparación de la forma espacial y material . . . . . . 224

8.5. Error relativo en la posición del extremo de la estructura A $(E / G=2,5)$. Solución incremental pura en 50 incrementos. Comparación de la forma espacial y material 225

8.6. Estructura A $(E / G=2,5)$ con un momento $2 \pi$ aplicado en el extremo. Aproximación a la solución exacta en función del número de elementos . . . . . . . . . 226

8.7. Estructura $\mathrm{A}(E / G=2,5)$ con un momento $2 \pi$ aplicado en el extremo. Geometría de la solución en función del número de elementos . . . . . . . . . . . . . . . . 226

8.8. Estructura A $(E / G=2,5)$ con un momento $2 \pi$ aplicado en el extremo. Número de iteraciones para la convergencia en función del número de elementos . . . . . 227

8.9. Estructura $\mathrm{C} 2$ con $M=2 \pi \quad 10^{-2}$. Solución material. Número de iteraciones por paso necesarias para la convergencia . . . . . . . . . . . . . . . 228

8.10. Esfuerzos en la ménsula curva sometida a una fuerza de eje fijo . . . . . . . . . 230

8.11. Esfuerzos en la ménsula curva sometida a una fuerza perseguidora . . . . . . . 230

8.12. Distancia entre las posiciones nodales obtenidas con las soluciones espacial y material en el problema de la ménsula curva sometida a una fuerza perseguidora en el extremo . . . . . . . . . . . . . . . . . . . . . 231 


\section{Capítulo 1}

\section{Introducción}

En las últimas décadas se han producido avances significativos en el análisis teórico y el desarrollo de modelos numéricos de comportamiento mecánico de piezas alargadas que experimentan grandes desplazamientos y rotaciones. Esta clase de modelos es de aplicación en el ámbito de tecnologías en las que se emplean sistemas estructurales o mecanismos formados por piezas muy flexibles, como en la aeronáutica o la robótica. Incluso en disciplinas como la biología existen procesos biomecánicos que se pueden simular mediante modelos de piezas alargadas flexibles. Las aplicaciones en ingeniería civil son más escasas, pero también aparecen problemas, como el del tendido de conducciones submarinas, que pueden ser analizados con este tipo de técnicas.

Dentro del amplio grupo de modelos unidimensionales desarrollados para el análisis no lineal de piezas alargadas, el formulado por Simó en 1985 [58] -denominado modelo geométricamente exacto de piezas alargadas - es capaz de reproducir rotaciones arbitrariamente grandes de las secciones transversales. Su sencillez conceptual y la potencia de las soluciones numéricas basadas en él lo han convertido en referencia obligada y punto de partida de numerosas investigaciones recientes. El estudio del modelo geométricamente exacto, el análisis de sus fundamentos teóricos y la búsqueda de nuevas soluciones constituye el objeto de esta tesis.

\subsection{El modelo geométricamente exacto de piezas alargadas}

Dentro del campo de la mecánica de sólidos deformables, el análisis de la flexión de piezas alargadas que experimentan grandes desplazamientos y rotaciones de la tangente a la directriz, o problema de la elástica, ha sido el primer problema geométricamente no lineal resuelto con rigor matemático. La elástica de Euler (1744) [18] es la forma que adopta una pieza alargada plana sometida a fuerzas opuestas en sus extremos, admitiendo que no sufre deformaciones de extensión ni corte, pero sin restricciones en cuanto a la magnitud de las curvaturas. El procedimiento empleado por Euler es puramente variacional, de hecho, el problema de la elástica aparece como 
ejemplo de aplicación del cálculo de variaciones, que él mismo había descubierto ${ }^{1}$.

La elástica de Euler, cuyo único fundamento mecánico es la hipótesis de proporcionalidad entre el momento flector y la curvatura, es un caso particular del problema general de la deformación de un sólido alargado. Para que se produjeran avances significativos en el análisis de este tipo de sólidos fue necesario disponer de un fundamento físico riguroso -la mecánica del medio continuo- que no se desarrolló hasta finales del siglo XIX y comienzos de XX. En ese contexto diversos investigadores comprendieron que una teoría de piezas alargadas se puede construir $(a)$ como caso particular de la mecánica de sólidos (tridimensional), introduciendo ciertas restricciones cinemáticas derivadas de la forma de la pieza alargada, lo que da lugar a una teoría basada en el continuo tridimensional, o bien $(b)$ modelando el sólido como un continuo unidimensional o curva de Cosserat cuyos puntos llevan asociado un conjunto de vectores directores que definen su cinemática, a partir de los cuales se puede desarrollar una teoría directa.

La primera alternativa ha resultado ser fuente de diversas teorías de vigas derivadas de la teoría lineal de la elasticidad (Navier, Timoshenko, Oumansky-Vlassov,...) usadas habitualmente en ingeniería civil [45]. La restricción o hipótesis cinemática que caracteriza una cierta teoría se concreta en una ecuación que relaciona las componentes del desplazamiento (o de la posición final) de los puntos del sólido con un conjunto de funciones - los desplazamientos generalizados- cuya variable independiente es la posición inicial de los puntos de la directriz. Sin embargo, el desarrollo de modelos de piezas alargadas en el ámbito de la teoría cinemáticamente no lineal conduce a formulaciones complejas, ya que después de seleccionar el tensor de deformaciones apropiado entre los distintos disponibles en la teoría no lineal, es necesario escribir sus componentes en función de los desplazamientos generalizados. El resultado de esta operación proporciona las deformaciones generalizadas, pero su expresión no es sencilla debido a la no linealidad de las componentes de la deformación. La complejidad de las ecuaciones constitutivas en la teoría no lineal, unida a las inconsistencias que aparecen como consecuencia de las restricciones cinemáticas complica aún más la situación. Generalmente es necesario introducir ciertas simplificaciones en el proceso de deducción de las ecuaciones para alcanzar resultados manejables.

Las formulación de una teoría directa es más sencilla: las deformaciones generalizadas en cada punto de la curva de Cosserat (la directriz) se definen a partir de los vectores directores; los esfuerzos -o resultantes de tensiones- se introducen a priori y las ecuaciones constitutivas que relacionan las deformaciones generalizadas con los esfuerzos se postulan directamente. Esto permite eludir las complicaciones derivadas del empleo de una ecuación constitutiva tridimensional, pero a cambio limita la información proporcionada por el modelo al nivel del conjunto de la sección, ya que -como señala Naghdi [47]- el modelo no establece una relación entre los puntos de la curva de Cosserat y los puntos materiales del sólido.

Aunque las dos clases de teorías señaladas parten de puntos opuestos, es evidente que debe existir una conexión estrecha entre ellas, y es precisamente en este sentido

\footnotetext{
${ }^{1}$ Con ello no sólo aporta la solución al problema de la elástica sino que sienta las bases de la mecánica del medio continuo computacional.
} 
en el que se orienta el trabajo de Antman [3], que interpreta la hipótesis cinemática como una función no lineal de los vectores directores y establece un nexo entre la teoría directa y las variables de la mecánica de sólidos tridimensional.

En este contexto, Simó [58] propone un modelo que ofrece la sencillez conceptual de las hipótesis cinemáticas tradicionales, pero a la vez permite emplear la potencia de la teoría directa para su aplicación a la solución de problemas no lineales. La cinemática propuesta por Simó es simple: la sección transversal de la pieza, que se supone indeformable en su plano, puede experimentar una rotación finita, independiente de la orientación de la tangente a la directriz deformada. Es una generalización de la cinemática de Love-Kirchhoff (que considera únicamente flexión y extensión) permitiendo también deformaciones de corte, sin introducir limitaciones en la magnitud de las deformaciones. La auténtica aportación de Simó consiste en emplear la matriz de rotación de la sección como variable generalizada del modelo. La configuración de la pieza queda definida por (a) el vector posición de cada punto de la directriz, y (b) la rotación de la sección transversal en cada punto. Simó sustituye los vectores directores de la teoría directa por una matriz ortogonal. Esta elección posibilita la descripción exacta de las rotaciones de las secciones transversales en problemas de grandes desplazamientos, pero a cambio introduce una dificultad importante en la formulación, por el hecho de que las matrices ortogonales son elementos del grupo no conmutativo de las rotaciones en $\mathbb{R}^{3}$. El propio Simó se refiere a su modelo como modelo geométricamente exacto de piezas alargadas en [31, y esta denominación ha sido adoptada con cierta generalidad en la literatura. El modelo se conoce también como teoría de Reissner-Simó (que en ocasiones abreviaremos como teoría RS), pues Reissner puede ser considerado como su precursor.

En el análisis de problemas geométricamente no lineales es usual hacer referencia a la forma material y espacial de las ecuaciones y variables del modelo. En el caso del modelo geométricamente exacto la rotación $\boldsymbol{\Lambda}$ actúa como operador que transforma objetos materiales en espaciales, sustituyendo al gradiente de la deformación $\mathbf{F}^{*}$ que tiene el mismo papel en la teoría tridimensional. Con ello se logra que la transformación material $\leftrightarrow$ espacial se pueda interpretar también como un cambio de sistema de referencia.

En el desarrollo original del modelo [58], después de definir la hipótesis cinemática, Simó formula la expresión tridimensional de la potencia, y a partir de ella deduce las ecuaciones de equilibrio dinámico de la pieza. El procedimiento es riguroso pero no aclara suficientemente el paso del sólido tridimensional al modelo unidimensional. En la siguiente contribución [62] dedicada al desarrollo de un elemento finito basado en su teoría, las ecuaciones de la estática del modelo constituyen el punto de partida desde el que se deduce la ecuación de trabajos virtuales en forma espacial con la que se formulará el elemento. Otras versiones posteriores del modelo geométricamente exacto (Ibrahimbegović [25], Cardona et al. [11]) siguen el mismo procedimiento, con lo que tampoco se añade nada acerca de la relación entre la teoría tridimensional y el modelo.

Otra cuestión especialmente importante para el desarrollo de herramientas numéricas es el cálculo del operador tangente del modelo, que Simó y Vu-Quoc [62] abordan con un planteamiento riguroso basado en la linealización consistente de la ecuación 
de trabajos virutales. Sin embargo, la parte más delicada de la linealización, aquella que tiene que ver con la parte geométrica del operador, es la menos desarrollada en su artículo. Esta cuestión tampoco es tratada con más detalle en aportaciones posteriores; únicamente Kapania y Li [34] desarrollan con detalle este cálculo, aunque prescinden de la segunda variación de las variables de la configuración. Todas las referencias consultadas fundamentan la discretización en la forma espacial del operador. La forma material, que según creemos aún no ha sido publicada, podría ser el origen de nuevas formulaciones numéricas.

Por último, resaltaremos que uno de los aspectos más importantes y menos valorados del trabajo de Simó en su vertiente computacional es el de la elección de las variables incrementales relacionadas con las rotaciones nodales. Simó emplea como variables incrementales las denominadas rotaciones incrementales (spins). Éstas aparecen de forma natural en el cálculo de la variación de las deformaciones generalizadas. Desde el punto de vista físico son las variables conjugadas de las fuerzas generalizadas con unidades de momento (momentos distribuidos), y desde el punto de vista de la geometría analítica se pueden interpretar como vectores tangentes al espacio de las rotaciones, lo que las convierte en parámetros locales del grupo de las rotaciones; éstos parámetros solamente se usan para actualizar las rotaciones, pero no para almacenar su estado. Se puede decir que la formulación del elemento de Simó es independiente de la parametrización escogida para almacenar las rotaciones. Esto lo contrapone a otras formulaciones numéricas del modelo geométricamente exacto (Cardona y Geradin [11], Ibrahimbegović, Frey y Kozar [27], Ritto-Corrêa y Camotim [54]) basadas en parametrizaciones totales de la rotación -empleando generalmente las coordenadas del vector rotación--. La principal ventaja de las formulaciones totales estriba en la simetría de la matriz de rigidez tangente del elemento, si bien su expresión es muy complicada. Por otra parte la parametrización local conduce a una matriz tangente cuya parte geométrica es asimétrica, sin embargo su expresión es mucho más simple que la obtenida con la parametrización total, ya que la rotación incremental es una variable intrínseca al modelo.

\subsection{Motivación}

El origen de esta tesis se sitúa en el texto de Monleón [45]. En él se desarrolla un procedimiento sistemático para la construcción de teorías lineales de piezas alargadas y láminas partiendo de cualquier hipótesis cinemática lineal físicamente razonable. El proceso deductivo toma como punto de partida el teorema de la mínima energía potencial: la minimización de la energía de deformación del sólido y del potencial de las fuerzas exteriores conduce, empleando el cálculo de variaciones, a las ecuaciones de Euler-Lagrange, que son, precisamente, las ecuaciones de equilibrio del modelo. Esta aproximación pone de manifiesto que los sistemas de ecuaciones diferenciales que rigen las teorías lineales de piezas alargadas, cuyas funciones incógnita -los desplazamientos generalizados y los esfuerzos- dependen de la longitud de arco sobre la pieza, son formalmente análogos a las ecuaciones que describen el movimiento de puntos o sistemas mecánicos sencillos mediante las coordenadas generalizadas y los momenta, 
que son funciones dependientes de la variable tiempo.

Uno de los aspectos más relevantes del trabajo de Monleón ha sido el sistematizar el desarrollo de las ecuaciones de cualquier teoría lineal de piezas alargadas, expresándolas como sistema lineal de ecuaciones diferenciales ordinarias en los desplazamientos generalizados y los esfuerzos. La integración de este tipo de sistemas responde también a una metodología precisa; sus soluciones se pueden obtener de forma explícita si el sistema es de coeficientes constantes (piezas de sección y curvatura constantes). Como extensión de su formulación, Monleón también aplica su método en el ámbito de teorías no lineales, introduciendo algunas simplificaciones en la cinemática necesarias para poder manipular las ecuaciones con técnicas análogas a las propuestas en el caso lineal. En este caso los sistemas de ecuaciones diferenciales deducidos no son lineales y no es posible sistematizar su solución.

La idea original de la tesis consistió en la búsqueda de soluciones a problemas no lineales de piezas alargadas basada en dos premisas: (a) la extensión de la analogía entre problemas de estática de vigas y problemas dinámicos al caso no lineal, y $(b)$ el empleo de soluciones desarrolladas en el ámbito de la mecánica clásica para sistemas dinámicos como base para la construcción de soluciones del problema de la pieza alargada.

En el campo de la solución numérica de sistemas dinámicos no lineales se han producido avances recientes en la construcción de integradores numéricos que respetan la geometría del espacio de las configuraciones y otras magnitudes características de estos sistemas, como por ejemplo los basados en las expansiones de Magnus y Fer (Iserles [29], Iserles y Nørsett [30]), o los integradores discretos (Marsden, Pekarsky y Shkoller [42, Wendlandt y Marsden [65]). El libro de Hairer, Lubich y Wanner [23] constituye una revisión actual de esta clase de métodos, y presta una atención especial a sistemas de ecuaciones diferenciales en grupos de Lie, como el grupo de las rotaciones en $\mathbb{R}^{3}$, ya que éste es el espacio de las configuraciones de una clase amplia de problemas dinámicos no lineales.

En este contexto, optamos por situar el modelo geométricamente exacto de piezas alargadas en el centro de la investigación, ya que, de entre todos los modelos útiles para tratar problemas de grandes desplazamientos, es el que emplea la descripción cinemática más próxima a la de los problemas no lineales en dinámica. Por tanto, nos sitúa en la mejor posición para extender la analogía lineal al ámbito de los desplazamientos finitos. Aunque inicialmente experimentamos soluciones numéricas aplicables a sistemas dinámicos mediante integradores basados en la forma discreta de la función lagrangiana [42], pronto abandonamos esta línea porque los problemas de piezas alargadas son problemas de contorno, lo que conduce naturalmente a técnicas como el método de elementos finitos. Sin embargo, las técnicas aplicables a problemas dinámicos se han desarrollado específicamente para la solución de problemas de valores iniciales, y no son versátiles ni resultan apropiadas para resolver los problemas de la estática de piezas alargadas. 


\subsection{Objetivos de la tesis}

Dentro del marco del análisis del equilibrio estático de piezas alargadas mediante la teoría de Reissner-Simó, con un modelo constitutivo hiperelástico, se plantean en esta tesis los siguientes objetivos encaminados a arrojar luz sobre algunos aspectos poco estudiados de la teoría y vincular cuestiones que han sido tratadas de forma dispersa en distintas fuentes:

1. Establecer una conexión rigurosa entre el modelo geométricamente exacto y la cinemática no lineal del sólido deformable, definiendo las expresiones que relacionan las deformaciones generalizadas del modelo con las medidas no lineales de la deformación del sólido.

2. Construir la ecuación de trabajos virtuales del modelo a partir de la ecuación correspondiente al sólido tridimensional, y deducir las ecuaciones de equilibrio de la pieza alargada obteniendo la forma material y espacial de las mismas.

3. Desarrollar la expresión formal de las ecuaciones constitutivas del modelo admitiendo como única hipótesis que el sólido tridimensional es hiperelástico.

4. Explorar el paralelismo existente entre las ecuaciones de equilibrio de la teoría de Reissner-Simó y las ecuaciones del movimiento de un sólido rígido, tratando de generalizar la analogía cinética de Kirchhoff.

5. Examinar la deducción de la expresión espacial del operador tangente mediante la linealización consistente de la ecuación de trabajos virtuales previa a la discretización. Obtener la forma material del mismo y comprobar la concordancia entre las dos formulaciones.

6. Revisar la formulación del elemento finito propuesto por Simó y Vu-Quoc (basado en el operador tangente espacial) a la luz de los resultados anteriores, y explorar la posibilidad de formular un elemento partiendo de la forma material del operador.

\subsection{Contenido}

Esta tesis doctoral consta de nueve capítulos. En el primero de ellos se introduce el problema tratado, explicando las causas que motivan su análisis y los objetivos que se pretende cubrir en la investigación.

El segundo capítulo contiene una revisión del estado del Arte acerca de los modelos geométricamente exactos de piezas alargadas. Después de repasar las distintas contribuciones a lo largo de la historia que encuadran la teoría de Reissner-Simo, se presenta el trabajo precursor de Reissner, y los fundamentos y claves del modelo. Posteriormente se repasan las contribuciones actuales al análisis de piezas con grandes rotaciones y se ofrece una perspectiva de los principales modelos numéricos para su análisis. Por último se establece una clasificación de las soluciones numéricas construidas directamente a partir del modelo geométricamente exacto, así como una 
relación de trabajos que amplían su campo de aplicación a, por ejemplo, problemas dinámicos.

El capítulo tercero desarrolla el estudio de la cinemática del modelo tomando como punto de partida la restricción que la hipótesis de Reissner introduce en los desplazamientos de los puntos del sólido. También incluye un análisis de la rotación como operador que permite transformar variables materiales en espaciales. El capítulo se cierra con la definición de las deformaciones generalizadas en forma material y espacial, y su expresión en función del gradiente de la deformación del sólido.

El cuarto capítulo está dedicado a la deducción de las ecuaciones de campo. El punto de partida es la ecuación de trabajos virtuales en el sólido tridimensional. Introduciendo en ella las restricciones cinemáticas definidas en el capítulo anterior, se alcanza la expresión (material y espacial) de la ecuación de trabajos virtuales característica del modelo en función de las variaciones de la configuración y de las deformaciones generalizadas. Su integración por partes proporciona las conocidas ecuaciones de equilibrio del modelo; para llevarla a cabo es necesario obtener previamente las variaciones de las deformaciones generalizadas en función de las variaciones de la configuración. A continuación se establece la equivalencia entre la definición energética y la estática de los esfuerzos, que es la expresión formal de las ecuaciones constitutivas, y se incluyen las ecuaciones constitutivas correspondientes al material elástico lineal.

En el capítulo quinto se desarrolla la analogía dinámica como extensión de la analogía lineal de Monleón [45] y de la analogía cinética de Kirchhoff. Las ecuaciones de equilibrio se pueden escribir en forma compacta, bien en función de las deformaciones generalizadas, o en función de los esfuerzos. Esto conduce a dos estructuras conocidas en la mecánica clásica: las ecuaciones de Euler-Poincaré y las ecuaciones de Lie-Poisson. Empleando esta última es posible $(a)$ definir la función de Hamilton del problema homogéneo de equilibrio de la pieza alargada, y (b) introducir el corchete de la pieza alargada RS como caso particular del corchete de Lie-Poisson, lo que completa la analogía.

La deducción del operador tangente mediante la linealización consistente de la ecuación de trabajos virtuales, antes de la discretización, se lleva a cabo en el sexto capítulo. Como paso previo se calcula la segunda variación de la configuración demostrando que tiene componentes no nulas, que no habían sido consideradas hasta el momento en el cálculo del operador. El resultado, en comparación con el operador de Simó, contiene nuevos términos antisimétricos. El análisis de los mismos muestra que se anulan en configuraciones de equilibrio. La última parte del capítulo se dedica a la obtención de la forma material del operador tangente, que aún no ha sido publicada, y a demostrar su correspondencia con la forma espacial.

El capítulo séptimo contiene la formulación y el desarrollo del elemento finito basado en la forma espacial del operador tangente, empleando la interpolación de las rotaciones incrementales, es decir, la parametrización local de las rotaciones propuesta por Simó. Después de deducir las expresiones necesarias para las operaciones de predicción (incrementales) y de corrección (iterativas), y de mostrar el diagrama de flujo del programa de cálculo, se ha valorado la importancia de los nuevos términos adicionales del operador estudiando dos casos con el operador de Simó y el deducido en la tesis. Finalmente se ha procesado diversos ejemplos comparando los resultados 
obtenidos con los de la literatura para validar el programa.

En el capítulo octavo se propone un nuevo elemento finito basado en la forma material del operador tangente. La idea para su formulación consiste en calcular la rigidez local del elemento mediante la expresión material del operador, y luego transformarla a la forma espacial empleando las rotaciones nodales; de este modo se puede realizar el ensamblaje y aprovechar los procesos desarrollados en el capítulo anterior. Después de desarrollar las expresiones necesarias y describir el flujo del programa se ha comparado la respuesta del nuevo elemento material con la del elemento espacial.

Por último, el capítulo noveno recoge las conclusiones de la tesis, resume las aportaciones originales y plantea nuevas líneas de investigación.

\subsection{Notación}

Aunque en el capítulo tercero volveremos sobre la cuestión de la notación, indicamos a continuación los criterios básicos empleados. Las variables físicas se denotan, en general, mediante el tipo romans, o mediante letras griegas. Los objetos espaciales se denotan mediante minúsculas y los materiales con mayúsculas. Por otro lado, para referirnos a vectores emplearemos negrita cursiva, y para denotar matrices o tensores utilizaremos negrita recta. Las matrices antisimétricas se identifican además mediante el acento $\widehat{~}$. Por ejemplo: $\boldsymbol{m}$ es la forma espacial del vector momento y $\boldsymbol{M}$ la forma material del mismo; $\delta \boldsymbol{\omega}$ es la variación de la rotación (vector en forma espacial) y $\delta \widehat{\boldsymbol{\omega}}$ es el tensor antisimétrico asociado (también en la forma espacial); la forma material de estos entes es $\delta \boldsymbol{\Omega}$ (vector) y $\delta \widehat{\boldsymbol{\Omega}}$ (tensor antisimétrico).

El tensor de rotación $\boldsymbol{\Lambda}$ es una excepción, pues transforma objetos materiales en espaciales, y se representa por claridad como letra mayúscula. También lo son el tensor gradiente de la deformación $\mathbf{F}^{*}$ y el primer tensor de tensiones de Piola $\mathbf{P}^{*}$. El vector posición de los puntos del sólido o de los puntos de la directriz tampoco es un objeto material o espacial. Se representará con minúscula $\boldsymbol{x}$ cuando denote la posición de puntos en la configuración actual y con mayúscula $\boldsymbol{X}$ cuando indique puntos en la configuración de referencia. Otra excepción la constituyen las matrices y vectores que resultan de la discretización y se emplean en la solución mediante elementos finitos. En este caso se emplea letra sans serif mayúscula para las matrices y minúscula para los vectores. Por ejemplo $\mathbf{K}$ es la matriz de rigidez (la mayúscula no se asocia ya al carácter material) y $\mathbf{f}$ es el vector de fuerzas (que ya no se denota en cursiva).

En los cuadros siguientes reflejamos la notación empleada para los distintos objetos que aparecen en el texto:

\begin{tabular}{lccc} 
& c. referencia & c. inicial & c. actual \\
\hline Sistema de referencia de la sección & $\left\{\boldsymbol{A}_{1} \boldsymbol{A}_{2} \boldsymbol{A}_{3}\right\}$ & $\left\{\boldsymbol{a}_{01} \boldsymbol{a}_{02} \boldsymbol{a}_{03}\right\}$ & $\left\{\boldsymbol{a}_{1} \boldsymbol{a}_{2} \boldsymbol{a}_{3}\right\}$ \\
Posición de los puntos del sólido & $\boldsymbol{X}^{*}$ & $\boldsymbol{x}_{0}^{*}$ & $\boldsymbol{x}^{*}$ \\
Posición relativa de los puntos de la sección & $\boldsymbol{R}^{*}$ & $\boldsymbol{r}_{0}^{*}$ & $\boldsymbol{r}^{*}$ \\
Posición de los puntos de la directriz & $\boldsymbol{X}$ & $\boldsymbol{x}_{0}$ & $\boldsymbol{x}$ \\
\hline
\end{tabular}

Cuadro 1.1: Variables del sólido y de la configuración 


\begin{tabular}{lc}
\hline Rotación inicial & $\boldsymbol{\Lambda}_{0}$ \\
Rotación deformacional & $\boldsymbol{\Lambda}_{d}$ \\
Rotación total & $\boldsymbol{\Lambda}$ \\
\hline
\end{tabular}

Cuadro 1.2: Rotaciones

\begin{tabular}{lccc} 
& material & & espacial \\
\hline Gradiente de la deformación & & $\mathbf{F}^{*}$ & \\
Vectores del gradiente $(K=1,2,3)$ & $\boldsymbol{F}_{K}^{*}$ & & $\boldsymbol{f}_{K}^{*}$ \\
\hline $\begin{array}{l}\text { Primer tensor de tensiones de Piola-Kirchhoff } \\
\text { Vectores tensión de Piola-Kirchhoff }(K=1,2,3)\end{array}$ & $\boldsymbol{P}^{* K}$ & & $\boldsymbol{P}^{* K}$ \\
\hline Fuerzas de volumen & & & $\boldsymbol{b}^{*}$ \\
Fuerzas de superficie & & $\overline{\boldsymbol{t}}^{*}$ \\
\hline
\end{tabular}

Cuadro 1.3: Otras variables asociadas al sólido

\begin{tabular}{llc} 
& material & espacial \\
\hline Tangente a la directriz & $\boldsymbol{\Gamma}$ & $\boldsymbol{\gamma}$ \\
Cambio de orientación del sistema de referencia (vector) & $\boldsymbol{K}$ & $\boldsymbol{\kappa}$ \\
Tensores antisimétricos asociados & $\widehat{\boldsymbol{\Gamma}}$ & $\widehat{\boldsymbol{\gamma}}$ \\
& $\widehat{\mathbf{K}}$ & $\widehat{\boldsymbol{\kappa}}$ \\
\hline Tangente a la directriz inicial & $\boldsymbol{\Gamma}_{0}$ & $\boldsymbol{\gamma}_{0}$ \\
Curvatura inicial de la directriz (vector) & $\boldsymbol{K}_{0}$ & $\boldsymbol{\kappa}_{0}$ \\
\hline Extensión y distorsión & $\boldsymbol{\Gamma}_{d}$ & $\boldsymbol{\gamma}_{d}$ \\
Cambio de orientación deformacional (vector) & $\boldsymbol{K}_{d}$ & $\boldsymbol{\kappa}_{d}$ \\
\hline
\end{tabular}

Cuadro 1.4: Deformaciones generalizadas

\begin{tabular}{lcc} 
& material & espacial \\
\hline Esfuerzos con unidades de fuerza & $\boldsymbol{N}$ & $\boldsymbol{n}$ \\
Esfuerzos con unidades de momento & $\boldsymbol{M}$ & $\boldsymbol{m}$ \\
Tensores antisimétricos asociados & $\widehat{\mathbf{N}}$ & $\widehat{\mathbf{n}}$ \\
& $\widehat{\mathbf{M}}$ & $\widehat{\mathbf{m}}$ \\
\hline Fuerzas generalizadas & $\boldsymbol{Q}_{n}$ & $\boldsymbol{q}_{n}$ \\
Momentos generalizados & $\boldsymbol{Q}_{m}$ & $\boldsymbol{q}_{m}$ \\
Tensores antisimétricos asociados & $\widehat{\mathbf{Q}}_{n}$ & $\widehat{\mathbf{q}}_{n}$ \\
& $\widehat{\mathbf{Q}}_{m}$ & $\widehat{\mathbf{q}}_{m}$ \\
\hline Fuerzas en los extremos de la pieza $(a=1,2)$ & $\boldsymbol{N}_{a}$ & $\boldsymbol{n}_{a}$ \\
Momentos en los extremos de la pieza $(a=1,2)$ & $\boldsymbol{M}_{a}$ & $\boldsymbol{m}_{a}$ \\
Tensores antisimétricos asociados & $\widehat{\mathbf{N}}_{a}$ & $\widehat{\mathbf{n}}_{a}$ \\
& $\widehat{\mathbf{M}}_{a}$ & $\widehat{\mathbf{m}}_{a}$ \\
\hline
\end{tabular}

Cuadro 1.5: Variables estáticas asociadas al modelo 
material

$\left[\begin{array}{ll}\mathbf{C}_{\Gamma \Gamma} & \mathbf{C}_{\Gamma K} \\ \mathbf{C}_{K \Gamma} & \mathbf{C}_{K K}\end{array}\right]$ espacial

$\left[\begin{array}{ll}\mathbf{c}_{\gamma \gamma} & \mathbf{c}_{\gamma \kappa} \\ \mathbf{c}_{\kappa \gamma} & \mathbf{c}_{\kappa \kappa}\end{array}\right]$

Cuadro 1.6: Matriz constitutiva

\begin{tabular}{lcc} 
& material & espacial \\
\hline Variación de la posición & $\delta \chi$ & $\delta \boldsymbol{x}$ \\
Variación de la rotación & $\delta \boldsymbol{\Omega}$ & $\delta \boldsymbol{\omega}$ \\
Tensores antisimétricos asociados & $-\overline{\boldsymbol{\Omega}}$ & $-\overline{\boldsymbol{\omega}}$ \\
\hline
\end{tabular}

Cuadro 1.7: Variaciones de la configuración

\begin{tabular}{lcc} 
& material & espacial \\
\hline Variaciones de la configuración & $\delta \boldsymbol{\Phi}$ & $\delta \boldsymbol{\phi}$ \\
Vector de deformaciones generalizadas & $\boldsymbol{E}$ & $\boldsymbol{\epsilon}$ \\
Vector de esfuerzos & $\boldsymbol{F}$ & $\boldsymbol{f}$ \\
Vector de fuerzas generalizadas & $\boldsymbol{Q}$ & $\boldsymbol{q}$ \\
Vector de fuerzas en los extremos & $\boldsymbol{F}_{a}$ & $\boldsymbol{f}_{a}$ \\
Bloques $(6 \times 6)$ del núcleo del operador tangente $(r, s=0,1)$ & $\mathbf{D}_{r s}$ & $\mathbf{d}_{r s}$ \\
Bloque $(6 \times 6)$ de fuerzas en extremos $(a=1,2)$ & $\mathbf{F}_{a}$ & $\mathbf{f}_{a}$ \\
\hline
\end{tabular}

Cuadro 1.8: Variables en notación compacta (seis componentes)

\begin{tabular}{lcc} 
& material & espacial \\
\hline Incremento de la posición & $\Delta \boldsymbol{\chi}_{i}$ & $\Delta \boldsymbol{x}_{i}$ \\
Rotación incremental & $\Delta \boldsymbol{\Omega}_{i}$ & $\Delta \boldsymbol{\omega}_{i}$ \\
Incrementos de la configuración $(6 \times 1)$ & $\Delta \boldsymbol{\Phi}_{i}$ & $\Delta \boldsymbol{\phi}_{i}$ \\
\hline Fuerzas aplicadas en los nodos & $\boldsymbol{N}_{i}$ & $\boldsymbol{n}_{i}$ \\
Momentos aplicados en los nodos & $\boldsymbol{M}_{i}$ & $\boldsymbol{m}_{i}$ \\
Fuerzas y momentos aplicados en nodos $(6 \times 1)$ & $\boldsymbol{F}_{i}$ & $\boldsymbol{f}_{i}$ \\
\hline
\end{tabular}

Cuadro 1.9: Variables de la solución numérica asociadas al nodo $i$ 


\begin{tabular}{lcc} 
& material & espacial \\
\hline Incrementos nodales de la configuración & $\Delta \boldsymbol{\Phi}^{e}$ & $\Delta \boldsymbol{\phi}^{e}$ \\
Vector de fuerzas internas & $\mathbf{p}_{\text {mat }}^{e}$ & $\mathbf{p}^{e}$ \\
Vector de fuerzas nodales iniciales & $\mathbf{f}_{\text {mat }}^{0 e}$ & $\mathbf{f}^{0 e}$ \\
Matriz de rigidez tangente & $\mathbf{K}_{m a t}^{e}$ & $\mathbf{K}^{e}$ \\
Matriz de rigidez tangente para la corrección & $\widetilde{\mathbf{K}}_{\text {mat }}^{e^{e}}$ & $\widetilde{\mathbf{K}}^{e}$ \\
\hline Rotación en puntos de Gauss & & $\boldsymbol{\Lambda}^{e}$ \\
Tangente a la directriz en puntos de Gauss & $\boldsymbol{\Gamma}^{e}$ & $\gamma^{e}$ \\
Cambio de orientación en puntos de Gauss & $\boldsymbol{K}^{e}$ & $\boldsymbol{\kappa}^{e}$ \\
Esfuerzos en puntos de Gauss (fuerzas) & $\boldsymbol{N}^{e}$ & $\boldsymbol{n}^{e}$ \\
Esfuerzos en puntos de Gauss (momentos) & $\boldsymbol{M}^{e}$ & $\boldsymbol{m}^{e}$ \\
\hline
\end{tabular}

Cuadro 1.10: Variables de la solución numérica asociadas al elemento $e$

\begin{tabular}{lcc} 
& material & espacial \\
\hline Incrementos de la configuración & - & $\Delta \boldsymbol{\Phi}$ \\
Vector de fuerzas internas & - & $\mathbf{p}$ \\
Vector de fuerzas exteriores & - & $\mathbf{f}$ \\
Residuo & - & $\mathbf{r}$ \\
Matriz de rigidez tangente & $\mathbf{K}$ \\
Matriz de rigidez tangente para la corrección & - & $\widetilde{\mathbf{K}}$ \\
Velocidad incremental & - & $\mathbf{v}$ \\
\hline
\end{tabular}

Cuadro 1.11: Variables globales de la solución numérica (estructura completa) 



\section{Capítulo 2}

\section{Estado del Arte}

\subsection{Contexto histórico}

En 1744 Leonhard Euler publicó Methodus Inveniendi Lineas Curvas Maximi Minimivi Proprietate Gaudentes [18. Su anexo Additamentum I. De Curvis Elasticis se puede considerar origen de la teoría sobre la que trata este trabajo. En él se presenta la solución al problema no lineal de la deformación plana de una pieza alargada sometida a flexión partiendo únicamente del principio según el cual la energía de deformación (fuerza potencial según Euler) acumulada en el cuerpo debe ser mínima en la curva elástica solución del problema. El proceso de deducción de la ecuación que determina la deformada de la pieza se lleva a cabo como aplicación del método de máximos y mínimos (es decir, del cálculo de variaciones) ideado y desarrollado por el matemático en el cuerpo de esta obra ${ }^{1}$. El trabajo de Euler contiene la primera aplicación de un método variacional a la solución de un problema elástico, y al mismo tiempo constituye el primer estudio sistemático de la elástica, es decir, de la solución en deformaciones del problema plano de la pieza flectada sin restricción alguna a la magnitud de los desplazamientos o de las deformaciones (curvaturas). El problema resuelto por Euler considera únicamente las deformaciones por flexión, pero no las debidas a la extensión ni al corte. Euler toma como punto de partida la expresión de la fuerza potencial de un fleje sometido a flexión ${ }^{2}$

$$
\int \frac{d S}{R^{2}}
$$

Teniendo en cuenta la proporcionalidad momento - curvatura, esta expresión es equivalente a

$$
\int \frac{M^{2}}{(E I)^{2}} d S
$$

\footnotetext{
${ }^{1}$ Según Dou 17: "La misma obra euleriana tiene un inmenso valor para la física teórica de los medios continuos. Principalmente porque muchas leyes físicas se enuncian en forma de principios variacionales (...) En particular el primer aditamento constituye el primer tratado de elasticidad".

${ }^{2}$ Comunicada a Euler por Daniel Bernoulli en carta fechada el 20 de octubre de 1742 (Dou [17]).
} 
que es precisamente la energía de deformación (flexión) dividida por la rigidez a flexión $E I$. El problema de la determinación de la deformada de una pieza alargada sometida a flexión se puede formular en los siguientes términos:

Hallar entre todas las curvas de igual longitud que pasan por los puntos $A$ y $B$ y son tangentes en esos puntos a rectas dadas, aquella en la que la expresión (2.1) sea mínima.

A continuación exponemos el razonamiento de Euler en forma sintética: si consideramos unos ejes $x$ e $y$ con origen en el punto A, el diferencial de arco se puede expresar en función de la derivada de $y$ como

$$
d S=d x \sqrt{1+\left(y^{\prime}\right)^{2}},
$$

y la curvatura

$$
\frac{1}{R}=\frac{y^{\prime \prime}}{\left(1+\left(y^{\prime}\right)^{2}\right)^{3 / 2}} .
$$

Se trata pues de minimizar

$$
\int \frac{d S}{R^{2}}=\int \frac{\left(y^{\prime \prime}\right)^{2}}{\left(1+\left(y^{\prime}\right)^{2}\right)^{5 / 2}} d x
$$

con la restricción $\int d S=l$, es decir

$$
\int \sqrt{1+\left(y^{\prime}\right)^{2}} d x-l=0 .
$$

Si denominamos $L\left(y^{\prime}, y^{\prime \prime}\right)=\frac{\left(y^{\prime \prime}\right)^{2}}{\left(1+\left(y^{\prime}\right)^{2}\right)^{5 / 2}}$ al integrando de la función a minimizar y $f\left(y^{\prime}\right)=\sqrt{1+\left(y^{\prime}\right)^{2}}$ al integrando de la restricción geométrica, el problema es equivalente (Lanczos [37, ec. (214.6)]) al de minimización del funcional

$$
\int F\left(y^{\prime}, y^{\prime \prime}\right) d x
$$

con

$$
F\left(y^{\prime}, y^{\prime \prime}\right)=L\left(y^{\prime}, y^{\prime \prime}\right)+\alpha f\left(y^{\prime}\right),
$$

donde $\alpha$ es un multiplicador de Lagrange. Las ecuaciones de Euler-Lagrange que permiten obtener la curva solución del problema en este caso son (Lanczos [37, ec. $(210.11)])$

$$
-\frac{d}{d x} \frac{\partial F}{\partial y^{\prime}}+\frac{d^{2}}{d x^{2}} \frac{\partial F}{\partial y^{\prime \prime}}=0 .
$$

Desarrollando e integrando estas ecuaciones Euler define la elástica a partir de la siguiente ecuación diferencial de primer orden

$$
\frac{d y}{d x}=\frac{\alpha+\beta x+\gamma x^{2}}{\sqrt{a^{4}-\left(\alpha+\beta x+\gamma x^{2}\right)^{2}}}
$$




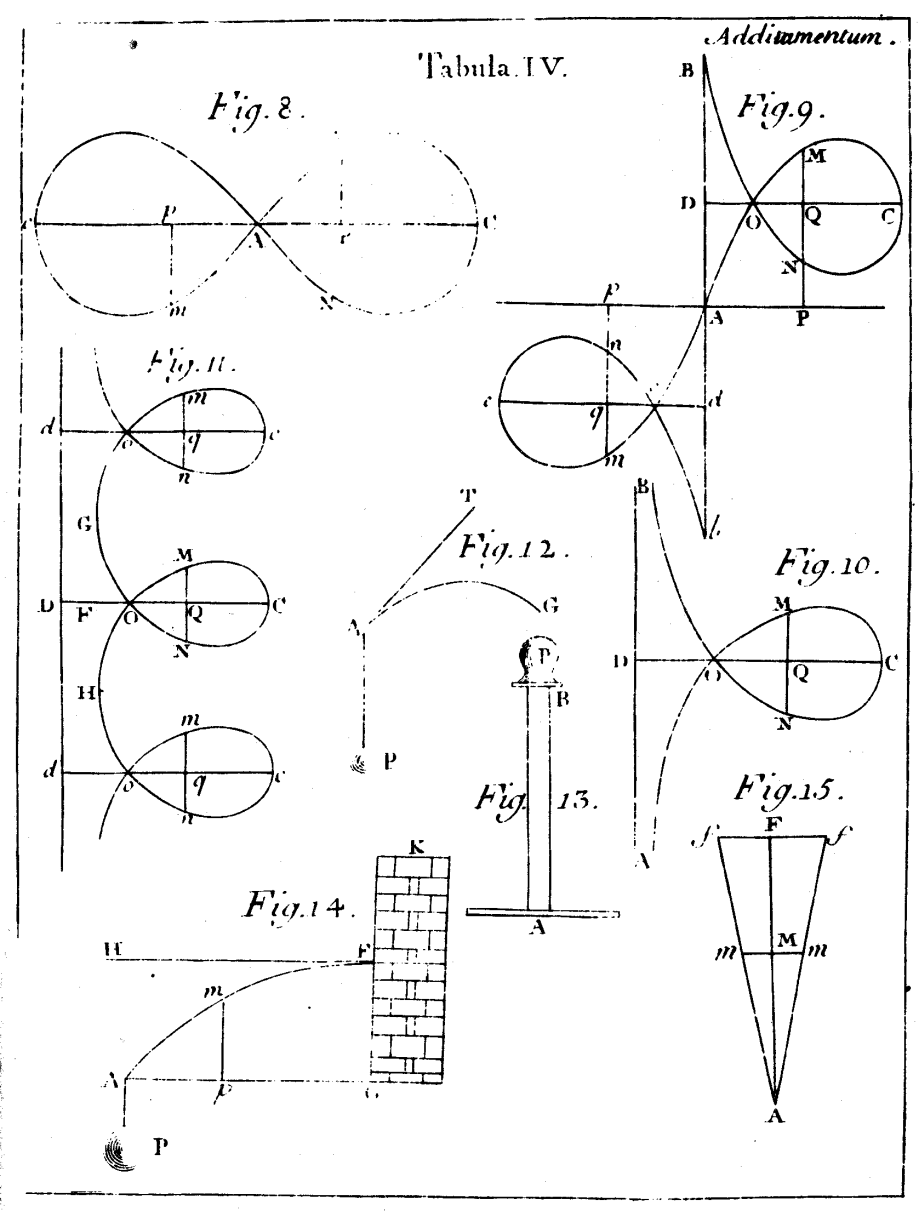

Figura 2.1: Reproducción de la tabla 4 de Euler

en la que $\alpha, \beta$ y $\gamma$ dependen de las condiciones de contorno, y a es un parámetro introducido por conveniencia. Esta expresión coincide con la que se obtiene estableciendo a priori el equilibrio de una pieza de rigidez $E I$ sometida a un momento flector producida por una fuerza $P$ aplicada a una distancia $c$ del extremo A [18, §251]

$$
\frac{d y}{d x}=\frac{-P\left((1 / 2) x^{2}+c x+f\right)}{\sqrt{(E I)^{2}-P^{2}\left((1 / 2) x^{2}+c x+f\right)^{2}}} .
$$

A partir del resultado (2.10) Euler ofrece una clasificación de las soluciones de la pieza sometida a fuerzas de compresión en sus extremos en diferentes categorías en función de la relación entre la fuerza y la rigidez a flexión (figura 2.1).

Empleando una terminología actual podríamos decir que el modelo de Euler es un modelo directo en el sentido de que se basa en la existencia a priori de una relación constitutiva entre las variables (generalizadas) del modelo -en este caso la relación 
momento - curvatura-, sin establecer conexiones con las variables ligadas a los puntos del sólido (tensiones o deformaciones) propias de la teoría de la elasticidad, que en la época del matemático aún no había sido suficientemente desarrollada.

Un siglo después Kirchhoff [35] extendió el trabajo de Euler al caso tridimensional (sus conclusiones fueron recogidas y ampliadas en el tratado de Love [40]). Para ello definió sobre cada uno de los puntos de la directriz deformada un sistema de referencia "móvil" que resulta de la deformación del sistema inicial sobre la sección transversal. Por hipótesis, las secciones transversales siguen siendo perpendiculares a la directriz después de la deformación, y el sistema de referencia en la configuración deformada es el resultado de una rotación del sistema inicial, permaneciendo uno de sus ejes tangente a la directriz. La rotación del sistema móvil se define mediante ángulos de Euler, y su incorporación a la formulación indica que Kirchhoff y Love identifican la rotación como uno de los aspectos esenciales de la teoría, que no había aparecido en la obra de Euler.

Si se imagina que el sistema de referencia móvil se desplaza sobre la directriz con velocidad unitaria entonces su velocidad angular expresada en componentes del sistema móvil define un vector $\boldsymbol{K}=\left\{K_{1} K_{2} K_{3}\right\}^{\top}$ cuyas componentes son precisamente la curvatura y la torsión (en el sentido geométrico) de la directriz deformada. Volviendo a emplear un concepto actual, podríamos decir que las componentes de $\boldsymbol{K}$ son las deformaciones generalizadas asociadas al modelo. Como conclusión al estudio de la cinemática Love incluye en [40, §253] las ecuaciones que relacionan las deformaciones generalizadas con las derivadas de los ángulos de Euler.

Kirchhoff y Love abandonan el método variacional de Euler, y recurren al planteamiento del equilibrio de la rebanada para la deducción de las ecuaciones de equilibrio interno, asumiendo la hipótesis de proporcionalidad entre momentos (flectores y torsores, entendidos como variables estáticas generalizadas) y componentes de $\boldsymbol{K}$

$$
M_{1}=G J K_{1} \quad M_{2}=E I_{2} K_{2} \quad M_{3}=E I_{3} K_{3} .
$$

Las componentes de $\boldsymbol{K}$ aparecen, junto con los momentos, el axil y los cortantes en las ecuaciones de equilibrio de la pieza -véase ecs. (5.9) en un capítulo posterior, y ecs. (10) y (11) en [40, §254]-.

Otro aspecto tratado por Kirchhoff y desarrollado por Love [40] (que lo generaliza al caso en que se permite la extensión de la directriz) es la conexión del modelo con la teoría de la elasticidad: las expresiones simplificadas de las componentes de la deformación se deducen suponiendo que éstas son pequeñas, y que también lo son las dimensiones de la sección transversal en relación con la longitud de la pieza. El cálculo de las tensiones a partir de las deformaciones conduce a la conclusión de que la hipótesis de proporcionalidad entre los momentos (entendidos ahora como resultantes de tensiones) y las componentes de $\boldsymbol{K}$ es suficientemente correcta.

El aspecto más interesante del trabajo de Kirchhoff desde el punto de vista de esta tesis lo constituye la denominada analogía cinética: Kirchoff demuestra que la expresión

$$
\frac{1}{2}\left(G J\left(K_{1}\right)^{2}+E I_{2}\left(K_{2}\right)^{2}+E I_{3}\left(K_{3}\right)^{2}\right)+N
$$




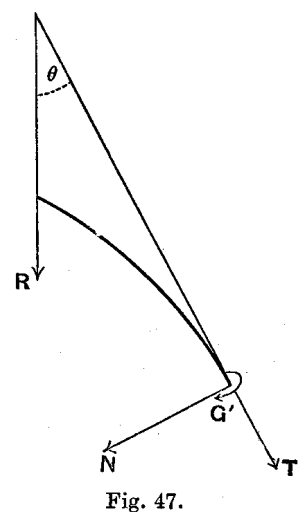

Figura 2.2: Analogía cinética de Kirchhoff (figura 47 de Love)

es constante a lo largo de la directriz en un problema en el que sólo actúen cargas en los extremos, y constituye por tanto un invariante del sistema, que además es formalmente idéntico a la expresión de la energía total de un sólido que gira en un campo gravitatorio con un punto fijo en el espacio (una peonza). Es posible establecer una analogía entre los dos problemas equiparando la longitud de arco al tiempo, los momentos sobre la sección a las componentes del momento angular, el vector curvatura a la velocidad angular, y el axil a la energía potencial del sólido. En el caso plano, la analogía se puede establecer entre la deformada de la pieza comprimida por una fuerza $F$ y la oscilación libre de un péndulo rígido de peso $F$; se puede observar en la figura $\left.{ }^{3} 2.2\right)$. En este caso el invariante es

$$
\frac{1}{2} E I(K)^{2}+N=\text { constante. }
$$

Teniendo en cuenta que $K=d \theta / d S$, siendo $\theta$ el giro de la tangente a la directriz deformada, y expresando $N$ en función de $F$

$$
\frac{1}{2} E I\left(\frac{d \theta}{d S}\right)^{2}+F\left(\cos \theta-\cos \theta_{1}\right)=0 .
$$

Las soluciones a esta ecuación diferencial pueden expresarse en forma de integrales elípticas de Jacobi (Love [40, §263]) y conducen a la misma clasificación de formas de la elástica establecida por Euler con el método expuesto antes (figura 2.3).

A mediados de los años 70 Antman [1] y Green, Naghdi y Wenner [21], 22] habían establecido las bases rigurosas para la formulación de teorías de piezas alargadas. Éstas pueden construirse $(a)$ como restricción a una dimensión de las ecuaciones de campo del sólido tridimensional (teorías basadas en el continuo tridimensional), o bien (b) modelando el sólido como continuo unidimensional con un conjunto de vectores directores asociados en cada uno de sus puntos (teorías directas). La última categoría

\footnotetext{
${ }^{3}$ La notación de los esfuerzos es diferente de la empleada en este trabajo.
} 

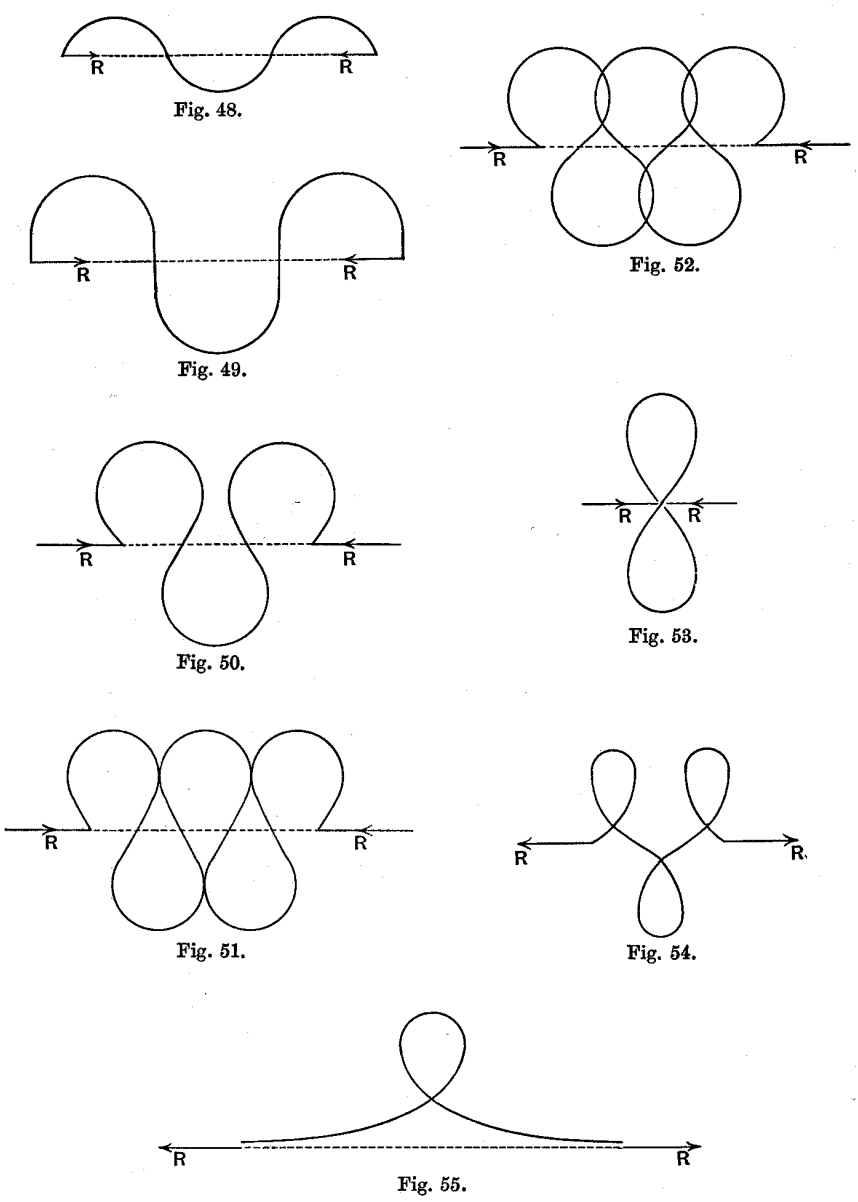

Figura 2.3: Reproducción de la página 404 de Love 
nace con el trabajo de los hermanos Cosserat [12]. La referencia [3] de Antman pone el punto final a esta introducción histórica. En ella se establece la conexión estrecha entre los dos grupos de teorías mencionados, al interpretar la hipótesis cinemática como una función no lineal de los vectores directores, estableciendo un nexo entre la teoría directa y las variables de la mecánica de sólidos tridimensional.

\subsection{El modelo de Reissner-Simó}

Reissner se puede considerar como el precursor del modelo geométricamente exacto. En las referencias (1973) [51] y (1981) [52] estudia el problema clásico de grandes desplazamientos de vigas esbeltas de directriz curva como generalización de la formulación de Kirchhoff y Love. Los trabajos de Reissner aportan la consideración explícita de las deformaciones debidas al axil y al cortante en el marco de una teoría no lineal en la que no se restringe la magnitud de estas deformaciones ni de los desplazamientos asociados.

A partir de la cinemática propuesta por Reissner y de las bases rigurosas de las teorías de piezas alargadas establecidas por Antman y Naghdi, Simó (1985) [58] desarrolla una teoría de vigas con deformaciones finitas (a finite strain beam formulation). En palabras de Simó

"The approach discussed here (...) should be regarded as a convenient parametrization of a three-dimensional extension of the classical KirchhoffLove rod model due to Antman."

La hipótesis cinemática admite que las secciones transversales permanece planas, pero pueden experimentar una rotación finita. La hipótesis permite deformaciones finitas de extensión y corte de la directriz (en contraste con el modelo de Kirchhoff-Love). Las configuraciones de la viga quedan completamente definidas especificando la evolución del vector posición de la curva de centros geométricos de las secciones transversales, así como de la matriz ortogonal que define la rotación de cada sección transversal. El modelo resultante es conocido como teoría de piezas alargadas de ReissnerSimó, o modelo geométricamente exacto de piezas alargadas. Este término fue acuñado por el propio Simó en [31] (a geometrically exact rod model) haciendo referencia al caracter exacto de la descripción de la cinemática del movimiento de la sección transversal.

Reissner toma como base las ecuaciones diferenciales de equilibrio de fuerzas y momentos de un elemento diferencial de directriz (deducidas a partir del principio de acción y reacción) expresadas en un sistema de referencia global

$$
\begin{aligned}
n^{\prime}+q_{n} & =0 \\
\boldsymbol{m}^{\prime}+\boldsymbol{x}^{\prime} \times \boldsymbol{n}+\boldsymbol{q}_{\boldsymbol{m}} & =\mathbf{0},
\end{aligned}
$$

donde $\boldsymbol{n}$ y $\boldsymbol{m}$ son los vectores que recogen las componentes de fuerza y momento de las fuerzas internas (o esfuerzos), $\boldsymbol{q}_{\boldsymbol{n}}$ y $\boldsymbol{q}_{\boldsymbol{m}}$ son las fuerzas y momentos generalizados, y $\boldsymbol{x}^{\prime}$ es la tangente a la directriz deformada. 
A continuación introduce $\stackrel{\nabla}{\delta} \boldsymbol{\gamma}$ y $\stackrel{\nabla}{\delta} \boldsymbol{\kappa}$, que constituyen una definición especial de las variaciones de las deformaciones generalizadas, conjugadas de los esfuerzos $\boldsymbol{n}$ y $\boldsymbol{m}$, y obtiene su expresión en función de la variación de las posiciones de la directriz $\delta \boldsymbol{x}$, y de las variaciones de la rotación de la sección transversal $\delta \boldsymbol{\omega}$, conjugadas de las fuerzas y momentos generalizados $\boldsymbol{q}_{\boldsymbol{n}}$ y $\boldsymbol{q}_{\boldsymbol{m}}$. Para ello parte de (2.16) y de una ecuación de trabajos virtuales

$$
\begin{aligned}
& \int_{\Gamma}(\boldsymbol{n} \cdot \stackrel{\nabla}{\delta} \boldsymbol{\gamma}+\boldsymbol{m} \cdot \stackrel{\nabla}{\delta} \boldsymbol{\kappa}) d S= \\
& \qquad \int_{\Gamma}\left(\boldsymbol{q}_{\boldsymbol{n}} \cdot \delta \boldsymbol{x}+\boldsymbol{q}_{\boldsymbol{m}} \cdot \delta \boldsymbol{\omega}\right) d S+[\boldsymbol{n} \cdot \delta \boldsymbol{x}]_{S_{1}}^{S_{2}}+[\boldsymbol{m} \cdot \delta \boldsymbol{\omega}]_{S_{1}}^{S_{2}}
\end{aligned}
$$

que postula a priori. El resultado es

$$
\begin{aligned}
& \stackrel{\nabla}{\delta} \boldsymbol{\gamma}=(\delta \boldsymbol{x})^{\prime}+\boldsymbol{x}^{\prime} \times \delta \boldsymbol{\omega} \\
& \nabla \\
& \delta \boldsymbol{\kappa}=(\delta \boldsymbol{\omega})^{\prime} .
\end{aligned}
$$

Esta es, precisamente, la definición de lo que se conoce como variación corrotacional de las deformaciones generalizadas, expresión que fue introducida por Cardona y Geradin (1988) [11, y que reproduce exactamente el carácter de esta operación. Reissner llama la atención sobre el hecho de que, así como $(\delta \boldsymbol{x})^{\prime}=\delta\left(\boldsymbol{x}^{\prime}\right)$, no es posible establecer una relación de este tipo para $\delta \boldsymbol{\omega}$. De hecho, no existe ninguna función $\boldsymbol{\omega}$ de la que derive $\delta \boldsymbol{\omega}[52$, p. 735].

El autor elabora la expresión escalar de las seis ecuaciones de equilibrio con todas las variables expresadas en el sistema actual; en ellas aparecen las componentes de los esfuerzos y de las deformaciones en ese sistema (ecuaciones 23 y 24, [51]). Estas ecuaciones junto con un sistema de seis ecuaciones constitutivas constituyen lo que Reissner denomina ecuaciones intrínsecas de la teoría de vigas, pues forman un sistema completo de ecuaciones diferenciales sin referencia directa a las variables cinemáticas.

Por otra parte, Simó [58] introduce el sistema de referencia $\left\{\boldsymbol{a}_{1}, \boldsymbol{a}_{2}, \boldsymbol{a}_{3}\right\}$ móvil o intrínseco, unido a la sección transversal en su rotación. Su teoría se desarrolla a partir de la hipótesis cinemática

$$
\boldsymbol{x}^{*}=\boldsymbol{x}+\boldsymbol{r}=\boldsymbol{x}+\left[\begin{array}{lll}
\boldsymbol{a}_{1} & \boldsymbol{a}_{2} & \boldsymbol{a}_{3}
\end{array}\right] \boldsymbol{R}=\boldsymbol{x}+\boldsymbol{\Lambda} \boldsymbol{R},
$$

donde $\boldsymbol{R}$ es el vector posición (relativa al origen de la sección) de un punto material cualquiera de la sección transversal en la configuración de referencia, $\boldsymbol{\Lambda}$ es la matriz ortogonal que define la rotación de la sección transversal después de la deformación, y $\boldsymbol{r}$ es la posición (relativa al origen de la sección) del punto material de la sección en la configuración deformada. La elección de Simó supone una novedad

En la sección 3, Simó define los esfuerzos $\boldsymbol{n}$ y $\boldsymbol{m}$ como resultantes de fuerzas y momentos, respectivamente, de las fuerzas por unidad de superficie sobre la sección transversal, y empleando las ecuaciones de conservación del momento lineal y angular de la teoría tridimensional deduce las expresiones de equilibrio dinámico de fuerzas y 
momentos de la teoría de vigas, que coinciden con las ecuaciones (2.16) salvo por los términos correspondientes a las fuerzas de inercia.

En la sección 4, obtiene la expresión de la potencia de las tensiones (stress power) del modelo de viga a partir de la teoría tridimensional, lo que permite la interpretación de las componentes de la deformación $\gamma$ y $\kappa$ en términos de las variables cinemáticas $\boldsymbol{x}$ y $\boldsymbol{\Lambda}$, con $\boldsymbol{\gamma}=\boldsymbol{x}^{\prime}$ y $\boldsymbol{\kappa}$ igual al vector axial correspondiente a la matriz antisimétrica $\widehat{\kappa}=\Lambda^{\prime} \Lambda^{\top}$.

Las aportaciones fundamentales de Simó [58] son:

1. La formulación matemática de la hipótesis cinemática para el caso general tridimensional en términos del vector posición de los puntos de la directriz, y de la rotación de la sección transversal (con independencia de la parametrización escogida para representarla).

2. El establecimiento de la relación entre la deformación conjugada a los momentos y la variable cinemática asociada, que es la matriz ortogonal que define la rotación.

3. La escritura de las ecuaciones en el caso dinámico general, partiendo de la expresión de la potencia del sólido tridimensional en términos del primer tensor de Piola-Kirchhoff y de la derivada en el tiempo del gradiente de la deformación.

4. La adopción de una terminología basada en la consideración de la matriz de rotación como operador que permite el paso de la configuración material a la espacial.

5. El desarrollo de una formulación que permitió la solución del problema por elementos finitos.

\subsection{Soluciones numéricas del modelo de Reissner-Simó}

\subsubsection{El tratamiento de las rotaciones}

Desde el punto de vista computacional pronto se reconoció que una de las principales dificultades para el desarrollo de soluciones numéricas la constituye el tratamiento preciso de la rotación de las secciones transversales. El primer autor en enfrentarse a esta dificultad en el ámbito del análisis no lineal de piezas alargadas fue Argyris, que junto con Dunne y Scharpf [5] propuso en 1978 una nueva definición conmutativa (aproximada) de las rotaciones, denominada rotación semitangencial, que empleó para formular un elemento finito apropiado para el análisis no lineal de estructuras. Su artículo de 1982 [4] constituye también una referencia obligada en el estudio de del problema de las rotaciones.

Estrictamente hablando, una rotación en el espacio es un elemento de una variedad denominada grupo especial ortogonal $\mathrm{SO}(3)$ [43]. Los elementos del grupo ortogonal son matrices $\boldsymbol{\Lambda}$ de orden 3 que cumplen la restricción $\boldsymbol{\Lambda}^{\top} \boldsymbol{\Lambda}=\mathbf{1}$ y cuyo determinante es igual a 1. El teorema de Euler [43, teorema 9.2.1] demuestra la equivalencia entre la representación matricial de la rotación y su representación vectorial $\boldsymbol{\theta}$ como rotación 
alrededor de un eje: el módulo del vector es el ángulo de rotación $\theta$, y su orientación determina el eje de rotación.

La fórmula de Rodrigues (1840) [55] establece la relación entre la matriz $\boldsymbol{\Lambda}$ y el vector $\boldsymbol{\theta}$ a través de la matriz antisimétrica $\widehat{\boldsymbol{\theta}}$ cuyo vector axial es $\boldsymbol{\theta}$ :

$$
\boldsymbol{\Lambda}=\mathbf{1}+\frac{\operatorname{sen} \theta}{\theta} \widehat{\boldsymbol{\theta}}+\frac{1-\cos \theta}{\theta^{2}} \widehat{\boldsymbol{\theta}}^{2}
$$

o la expresión alternativa

$$
\boldsymbol{\Lambda}=\cos \theta \mathbf{1}+\frac{\operatorname{sen} \theta}{\theta} \widehat{\boldsymbol{\theta}}+\frac{1-\cos \theta}{\theta^{2}} \boldsymbol{\theta} \otimes \boldsymbol{\theta} .
$$

La fórmula de Rodrigues define la denominada parametrización vectorial o vectorlike parametrization, que ha sido la más frecuentemente empleada en las soluciones numéricas del modelo geométricamente exacto. Crisfield [15] denomina a $\boldsymbol{\theta}$ pseudovector para recordar que la composición de dos rotaciones no se obtiene como suma de los vectores que las representan.

Ritto-Corrêa y Camotim [53] han señalado las siguientes ventajas de la parametrización vectorial: $(a)$ su significado físico directo, $(b)$ el empleo de un conjunto mínimo de tres parámetros, $(c)$ la correspondencia uno a uno entre el tensor ortogonal y la parametrización hasta valores muy grandes de la rotación $(\theta=\pi)$, pero también los siguientes inconvenientes: (a) requiere el uso de funciones trigonométricas, y $(b)$ no es diferenciable en $\theta=2 k \pi$. Cardona y Geradin (1988) [11, Ibrahimbegović, Frey y Kozar (1995) [27] y Ritto-Corrêa y Camotim [53] han trabajado con esta parametrización.

De hecho, es posible construir innumerables parametrizaciones pseudo-vectoriales de la rotación a partir de cualquier función biunívoca de $\theta$ definida para un cierto rango de valores del vector [54, eq. (17)]

$$
a=a(\theta) .
$$

Mencionaremos tres, que aunque han sido referidas en la literatura, han tenido un uso muy limitado:

1. Los parámetros de Rodrigues (citados por Crisfield [15] y Morán [46])

$$
\boldsymbol{a}_{\omega}=\frac{2 \tan (\theta / 2)}{\theta} \boldsymbol{\theta}
$$

2. Los parámetros pseudo-rotacionales (citados por Ritto-Corrêa y Camotim [54])

$$
\boldsymbol{a}_{q}=\frac{2 \operatorname{sen} \theta}{\theta(1+\cos \theta)} \boldsymbol{\theta}
$$

3. Los parámetros semi-tangenciales (citados por Ritto-Corrêa y Camotim [54])

$$
\boldsymbol{a}_{c}=\frac{\operatorname{sen} \theta}{\theta} \boldsymbol{\theta}
$$


Por otra parte Argyris [4] muestra que el cálculo de la exponencial de la matriz antisimétrica $\widehat{\theta}$ conduce de nuevo a la expresión $(2.20)$

$$
\exp (\widehat{\boldsymbol{\theta}})=\sum_{1}^{n} \frac{1}{n !} \widehat{\boldsymbol{\theta}}^{n}=\mathbf{1}+\frac{\operatorname{sen} \theta}{\theta} \widehat{\boldsymbol{\theta}}+\frac{1-\cos \theta}{\theta^{2}} \widehat{\boldsymbol{\theta}}^{2}
$$

Esta fórmula tiene una interpretación geométrica en términos de grupos de Lie (Hairer, Lubich y Wanner [23, lema 6.4]): la exponencial de una matriz es una aplicación que transforma elementos del álgebra $\mathfrak{g}$ (espacio tangente al grupo en el elemento neutro) en elementos del grupo $G$. En el caso del grupo $\mathrm{SO}(3)$ el álgebra $\mathfrak{s o}(3)$ es el conjunto de las matrices antisimétricas, por eso la aplicación exponencial produce una matriz ortogonal. Esta propiedad permite obtener una rotación $\boldsymbol{\Lambda}_{\epsilon}$ próxima a una dada, $\boldsymbol{\Lambda}$, mediante la siguiente operación

$$
\boldsymbol{\Lambda}_{\varepsilon}=\exp (\varepsilon \delta \widehat{\boldsymbol{\omega}}) \boldsymbol{\Lambda},
$$

donde $\delta \widehat{\boldsymbol{\omega}}$ es tangente a $\mathrm{SO}(3)$ en $\boldsymbol{\Lambda}$ (ver la figura 4.1). El vector axial $\delta \boldsymbol{\omega}$ asociado a $\delta \widehat{\boldsymbol{\omega}}$ es la denominada variación de la rotación y se puede interpretar como una parametrización local de las rotaciones en el entorno de $\boldsymbol{\Lambda}$ (la parametrización natural). La expresión anterior será la pieza clave de la formulación numérica de Simó. La variación de la rotación también ha sido denominada spin por Ritto-Corrêa y Camotim (2002) [53.

Otras parametrizaciones con un número mínimo de parámetros, como los ángulos de Euler o los de Cardan (Goldstein [20]) apenas han encontrado aplicación en el análisis de piezas alargadas.

Es evidente que en las parametrizaciones mencionadas los tres parámetros son independientes. No sucede lo mismo con otras alternativas basadas en más de 3 parámetros: en este caso el carácter de variedad del grupo de las rotaciones introduce necesariamente relaciones de dependencia entre ellos. Entre los sistemas con cuatro parámetros mencionaremos los parámetros de Cayley-Klein (Goldstein [20]) y los cuaterniones unitarios o parámetros de Euler-Rodrigues (Marsden y Ratiu [43]). Stuelpnagel 64] propuso un sistema con cinco parámetros cuya aplicación es limitada. La ventaja de los sistemas con más de tres parámetros es la de no poseer singularidades, lo que aporta ventajas importantes en el cálculo. A cambio presentan el inconveniente de su dependencia, y su significado físico es menos evidente. Su uso como parametrización del modelo geométricamente exacto se limita a la tesis de Morán [46] que lleva a cabo un estudio comparativo de los resultados del modelo empleando distintas parametrizaciones. Sin embargo, no podemos pasar por alto la ventaja excepcional que supone trabajar con cuaterniones unitarios para almacenar la información de las rotaciones nodales o en los puntos de Gauss [62, 28] porque, frente al empleo de la matriz completa, reducen drásticamente las necesidades de almacenamiento y facilitan el cálculo del producto (composición) de rotaciones. Incluiremos por tanto la expresión de la parametrización mediante cuaterniones unitarios.

Ésta se basa en cuatro números $\left(q_{0}, q_{1}, q_{2}, q_{3}\right)$ cuya suma de cuadrados es igual a 1. Los tres últimos se interpretan como componentes de un vector, de modo que 
el cuaternión también se pueden expresar así: $\left(q_{0}, \mathbf{q}\right)$. La aplicación que define la parametrización es

$$
\left(q_{0}, \mathbf{q}\right) \mapsto \boldsymbol{\Lambda}=\left(q_{0}^{2}-1\right) \mathbf{1}+2 q_{0} \hat{\mathbf{q}}+2 \mathbf{q} \otimes \mathbf{q} .
$$

El producto de cuaterniones -que define la composición de rotaciones- está determinado por la expresión

$$
\left(a_{0}, \mathbf{a}\right) \star\left(b_{0}, \mathbf{b}\right)=\left(a_{0} b_{0}-\mathbf{a} \cdot \mathbf{b}, a_{0} \mathbf{b}+b_{0} \mathbf{a}+\mathbf{a} \times \mathbf{b}\right) .
$$

Además, la interpretación geométrica de la rotación por medio de la relación con el vector rotación es sencilla

$$
\left(q_{0}, \mathbf{q}\right)=\left(\cos \frac{\theta}{2}, \sin \frac{\theta}{2} \boldsymbol{n}\right)
$$

$\operatorname{con} \boldsymbol{n}=\boldsymbol{\theta} / \theta$.

\subsubsection{La solución numérica de Simó y Vu-Quoc}

Tras la publicación de los fundamentos de su teoría, Simó aborda junto con VuQuoc 62] la solución numérica de problemas en el marco de la misma. La dificultad principal para la formulación de métodos aproximados de solución estriba en el carácter no aditivo de las variables que describen la configuración, por la presencia de la rotación finita de la sección transversal. La expresión (2.17) de la ecuación de trabajos virtuales lleva a Simó a emplear el vector desplazamiento de la directriz, $\Delta \boldsymbol{x}$, y el vector rotación incremental (o simplemente rotación incremental), $\Delta \omega$, como variables incrementales incógnita. Esta última es la versión incremental de la variación de la rotación ${ }^{4}, \delta \boldsymbol{\omega}$, conjugada de los momentos generalizados $\boldsymbol{q}_{\boldsymbol{m}}$. Empleando $\Delta \boldsymbol{\omega}$, que es el vector axial de la matriz antisimétrica $\Delta \hat{\boldsymbol{\omega}}$, las rotaciones se pueden actualizar mediante la expresión -compárese con (2.27)-

$$
\boldsymbol{\Lambda}_{(n+1)}=\exp \left(\Delta \hat{\mathbf{w}}_{(n)}\right) \boldsymbol{\Lambda}_{(n)} .
$$

La actualización de las deformaciones generalizadas (especialmente del cambio de orientación $\boldsymbol{\kappa}$ ) a partir de los desplazamientos y las rotaciones incrementales no es directa y requiere el uso de expresiones específicas en las que aparece el operador $\mathbf{T}$ del que hablaremos en la siguiente sección.

A partir de la elección de las variables incrementales Simó y Vu-Quoc linealizan la ecuación de trabajos virtuales apoyándose en la idea de derivada direccional 62 , sec. 4.1.]. El resultado es un operador tangente con una parte constitutiva simétrica y una parte geométrica no simétrica con la siguiente estructura [62, ecuación 4.14]

$$
\int_{\Gamma}\left\{\delta \boldsymbol{x}^{\top} \delta \boldsymbol{\omega}^{\top} \delta \boldsymbol{x}^{\prime \top} \delta \boldsymbol{\omega}^{\prime \top}\right\}\left[\begin{array}{cccc}
\mathbf{0} & \mathbf{0} & \mathbf{0} & \mathbf{0} \\
\mathbf{0} & \widehat{\gamma} \widehat{\mathbf{n}} & \widehat{\mathbf{n}} & \mathbf{0} \\
\mathbf{0} & -\widehat{\mathbf{n}} & \mathbf{0} & \mathbf{0} \\
\mathbf{0} & -\widehat{\mathbf{m}} & \mathbf{0} & \mathbf{0}
\end{array}\right]\left\{\begin{array}{c}
\Delta \boldsymbol{x} \\
\Delta \boldsymbol{\omega} \\
\Delta \boldsymbol{x}^{\prime} \\
\Delta \boldsymbol{\omega}^{\prime}
\end{array}\right\} d S .
$$

\footnotetext{
${ }^{4} \delta \boldsymbol{\omega}$ es el vector axial asociado a la matriz antisimétrica $\delta \hat{\boldsymbol{\omega}}=\delta \boldsymbol{\Lambda} \boldsymbol{\Lambda}^{\top}$; esta matriz es tangente al grupo de las rotaciones en $\boldsymbol{\Lambda}$.
} 
Los autores analizan esta parte del operador y llegan a la conclusión de que la simetría se recupera una vez alcanzada la trayectoria de equilibrio en problemas con fuerzas exteriores conservativas, y que el carácter asimétrico del operador fuera de la trayectoria de equilibrio es una consecuencia del hecho de que el espacio de las configuraciones es una variedad y no un espacio vectorial aditivo.

Simó (1992) [59] vuelve a ocuparse tras un tiempo del problema de la simetría del operador, esta vez desde una perspectiva matemática, y llega a la conclusión de que si en lugar de linealizar la ecuación de trabajos virtuales operando con la derivada direccional, se emplea la derivada covariante, entonces se obtiene un operador que es siempre simétrico fuera de la trayectoria de equilibrio. En su análisis acerca de la estructura geométrica del operador, parte de la expresión del trabajo virtual de las fuerzas internas y externas, que en un problema conservativo se puede expresar como derivada de la energía potencial $V$ en la dirección de la variación, y simbólicamente se puede escribir del siguiente modo

$$
\delta V=d V \cdot \delta \phi=0
$$

El operador se define a partir de la variación de la expresión anterior, es decir, de su derivada direccional en la dirección de una nueva variación $\Delta \phi$

$$
\Delta \delta V=d(d V \cdot \delta \phi) \cdot \Delta \phi .
$$

Simó deriva formalmente la expresión del trabajo virtual empleando la noción de derivada covariante $\nabla(\cdot)$ (op. cit. ecuación 2.19)

$$
\Delta \delta V=(\nabla d V \cdot \delta \phi) \cdot \Delta \phi+d V \cdot(\nabla \delta \phi \cdot \Delta \phi),
$$

y llega a la conclusión de que el primer sumando de esta expresión proporciona un operador simétrico, y que la elección de una parametrización adecuada lleva precisamente a que éste último coincida con la parte simétrica del operador calculado con la derivada direccional. Finalmente propone su empleo como operador tangente, en sustitución del asimétrico. Tras la contribución de Simó, varios autores han examinado las posibles ventajas computacionales derivadas de emplear la parte simétrica del operador tangente [26], [53], llegando a la conclusión de que su empleo no está justificado pues supone un deterioro importante de la velocidad de convergencia.

Una cuestión crucial en el desarrollo de Simó y Vu-Quoc es que todas las operaciones que conducen a la expresión del operador tangente son independientes de la parametrización escogida para describir la rotación total de la sección. La parametrización sólo interviene en el proceso numérico de almacenamiento y actualización de la configuración. De hecho, estos autores proponen operar (parcialmente) con cuaterniones unitarios o parámetros de Euler para su almacenamiento, transformando los cuaterniones en matrices de rotación mediante la expresión (2.28) a fin de calcular la actualización de las rotaciones con la fórmula (2.31), y finalmente extraer los nuevos cuaterniones correspondientes a las rotaciones actualizadas utilizando el algoritmo de Spurrier 63. Por estas razones, el procedimiento propuesto por Simó y Vu-Quoc ha sido denominado por otros autores parametrización- $\boldsymbol{\Lambda}$ [27], si bien esta calificación 
resulta confusa ${ }^{5}$ puesto que $\boldsymbol{\Lambda}$ denota un elemento del grupo de las rotaciones, independientemente de cuál sea la parametrización escogida para representarlo. Kapania y Li (2003) [34] han empleado también la expresión parametrización no-vectorial.

En este sentido, nos parece más acertado el análisis que efectúan Ritto-Corrêa y Camotim (2003) [53], que indica que el procedimiento de Simó comprende dos niveles de parametrización:

- la parametrización global, que sirve para registrar la rotación $\boldsymbol{\Lambda}$ (entendida como variable estado) de una cierta sección (sea en un nodo, o en un punto de integración) en una determinada etapa del proceso de cálculo (al final de un incremento, o de una iteración) -Simó y Vu-Quoc escogen cuaterniones como parámetros globales, aunque sería posible emplear otros, como los ángulos de Euler-. Ésta no interviene en el proceso incremental.

- la parametrización local, que sirve para describir el cambio en la variable estado $\boldsymbol{\Lambda}$ en cada una de las etapas del cálculo; dicho cambio, que es a su vez un elemento del grupo de las rotaciones, actúa sobre $\boldsymbol{\Lambda}$ como una rotación incremental. Simó y Vu-Quoc la describen mediante las coordenadas locales $\Delta \boldsymbol{\omega}$, cuya relación con la rotación incremental viene definida por la aplicación exponencial ${ }^{6}$ [23, lema 6.4] (exponential map), $\exp (\Delta \hat{\boldsymbol{\omega}})$. Esta relación justifica la expresión (2.31) introducida para la actualización de las rotaciones. Las componentes de $\Delta \boldsymbol{\omega}$ actúan como parámetros (o coordenadas) locales del la rotación incremental en el entorno de la rotación total $\boldsymbol{\Lambda}$. La matriz antisimétrica $\Delta \hat{\boldsymbol{\omega}}$, cuyo vector axial es $\Delta \boldsymbol{\omega}$, es un elemento del espacio tangente al grupo de las rotaciones en el elemento $\Lambda$; en este sentido se puede decir que las componentes de $\Delta \boldsymbol{\omega}$ son las coordenadas naturales del grupo en $\boldsymbol{\Lambda}$. Por ello emplearemos la denominación parametrización local (o natural) para describir la metodología desarrollada por Simó y Vu-Quoc.

\subsubsection{Clasificación de las soluciones numéricas del modelo geométricamente exacto}

Ritto-Corrêa y Camotim [54 han dividido las soluciones numéricas del modelo geométricamente exacto en dos categorías en función de la técnica empleada para la actualización de las rotaciones. Cada categoría está estrechamente relacionada con un tipo de parametrización:

1. Modelos numéricos con actualización aditiva. Si $\boldsymbol{a}$ es una cierta parametrización vectorial de las rotaciones entonces el paso desde el estado $k$ hasta el $k+1$ tiene lugar del siguiente modo

$$
\boldsymbol{\Lambda}^{(k)}=\boldsymbol{\Lambda}\left(\boldsymbol{a}^{(k)}\right) \rightarrow \boldsymbol{\Lambda}^{(k+1)}=\boldsymbol{\Lambda}\left(\boldsymbol{a}^{(k+1)}\right)=\boldsymbol{\Lambda}\left(\boldsymbol{a}^{(k)}+\Delta \boldsymbol{a}\right) .
$$

\footnotetext{
${ }^{5}$ Como indican Ritto-Corrêa y Camotim 53].

${ }^{6} \mathrm{Su}$ expresión desarrollada se conoce también como fórmula de Rodrigues (1840) [43, proposición 9.2.5.].
} 
La expresión $\boldsymbol{\Lambda}(\boldsymbol{a})$ es la aplicación que construye la matriz de rotación a partir de los parámetros -la exponencial en el caso de $\boldsymbol{a}=\boldsymbol{\theta}$-. Las variables incrementales en este tipo de modelos son los incrementos de las componentes de los parámetros $\boldsymbol{\Delta} \boldsymbol{a}$. Como las variables incrementales en el operador tangente son las rotaciones incrementales $\Delta \omega$ es necesario disponer de una expresión que las relacione con $\Delta \boldsymbol{a}$. Cuando se emplea la parametrización vectorial natural $\boldsymbol{a}=\boldsymbol{\theta}$ la relación viene definida por la siguiente expresión (Cardona [11], Ibrahimbegović et al. [27], y la interesante demostración de Ritto-Corrêa et al. $[53)^{7}$

$$
\Delta \omega=\mathbf{T}(\theta) \Delta \theta
$$

con

$$
\mathbf{T}(\boldsymbol{\theta})=\frac{\sin \theta}{\theta} \mathbf{1}+\frac{1-\cos \theta}{\theta^{2}} \widehat{\boldsymbol{\theta}}+\frac{\theta-\sin \theta}{\theta^{3}} \boldsymbol{\theta} \otimes \boldsymbol{\theta} .
$$

Evidentemente la actualización aditiva está ligada al empleo de una parametrización vectorial de las rotaciones. La actualización aditiva tiene, a su vez, dos versiones:

a) Aquella en la que el vector rotación representa las rotaciones totales (desde el inicio del proceso de deformación). Esta perspectiva ha sido la adoptada en los modelos numéricos mencionados de Cardona (1988) [11], Ibrahimbegović et al. (1995) [27] y Ritto-Corrêa (2002) [53].

b) Una variante propuesta por Cardona y Geradin en la misma referencia [1] en la que el vector rotación representa las rotaciones incrementales desde la última configuración de equilibrio.

2. Modelos numéricos con actualización multiplicativa. Considerando una cierta parametrización local de las rotaciones $\Delta \mathbf{a}$ el paso $k \rightarrow k+1$ se produce del siguiente modo

$$
\mathbf{\Lambda}^{(k+1)}=\boldsymbol{\Lambda}(\Delta \mathbf{a}) \boldsymbol{\Lambda}^{(k)} .
$$

Si se emplea la parametrización local natural $\Delta \mathbf{a}=\Delta \boldsymbol{\omega}$ entonces $\boldsymbol{\Lambda}(\Delta \mathbf{a})=$ $\exp (\Delta \widehat{\boldsymbol{\omega}})$. Este es, evidentemente, el camino seguido por Simó y Vu-Quoc.

El empleo de la actualización aditiva, o lo que es lo mismo, de la parametrización vectorial, complica considerablemente las expresiones del operador tangente debido a que es necesario introducir la expresión (2.33) en el núcleo del operador (que originalmente depende de $\Delta \boldsymbol{\omega}$ ). Esto llevó a Cardona y Geradin [11] a eliminar algunos términos. La primera linealización completa de la parametrización vectorial se encuentra en Ibrahimbegović et al. (2005) [27].

Como contrapartida a su complicación, este tipo de parametrizaciones ofrecen tres ventajas:

\footnotetext{
${ }^{7}$ Ninguna de las dos primeras referencias ofrece una demostración completa de la expresión del operador, únicamente indican el camino seguido. Por ello, hemos incluido la demostración completa en el apéndice B. Ritto-Corrêa sí ofrece una prueba empleando una vía alternativa.
} 
1. La matriz geométrica es simétrica, debido a que el espacio de los parámetros es lineal. Curiosamente, las expresiones del operador obtenidas en [27] no mostraban esa simetría, debido a su complejidad. Ritto-Corrêa y Camotim 53] revisan desde el origen el proceso de linealización y llegan finalmente a expresiones simétricas.

2. La actualización de las deformaciones generalizadas se lleva a cabo directamente a partir de los valores nodales de la rotación. Por tanto no se requiere una fórmula específica de actualización como en el caso de las actualización multiplicativa.

3. El uso de parametrizaciones totales conduce, en problemas hiperelásticos, a soluciones independientes del camino seguido (path-independent) [16]. No sucede lo mismo con parametrizaciones incrementales (variante $b$ ) o locales.

Por otro lado existen numerosas referencias en las que se emplea la actualización multiplicativa propuesta por Simó. Citaremos las siguientes: Ibrahimbegović (1995) [25] desarrolla un elemento curvo de 2 y 3 nodos basándose en la parametrización local. El mismo autor (1997) compara en [26] los dos tipos de parametrización y extiende la formulación a problemas dinámicos. Más adelante, junto con Taylor (2002) [28] trabaja con la parametrización local y la versión simétrica del operador tangente de Simó, basándose en la justificación recogida en [59]; en su artículo proponen un interesante procedimiento de actualización de las rotaciones empleando exclusivamente cuaterniones que emplearemos en nuestro desarrollo. Kapania y Li (2003) [34] también emplean la metodología de Simó para desarrollar un elemento curvo de 4 nodos.

Crisfield y Jelenić (1999) [16] tratan la cuestión de la objetividad (invarianza del modelo numérico frente a cambios del sistema de referencia) llegando a la conclusión de que ninguna de las discretizaciones propuestas garantiza la objetividad de los resultados. Romero, que también estudia este problema analizando distintos tipos de parametrización, explica esta situación (2004) [56, p. 125] en el contexto de la actualización multiplicativa ${ }^{8}$. Crisfield y Jelenić (1999) [32], manteniendo la actualización multiplicativa, proponen un nuevo elemento basado en $(a)$ el uso de rotaciones locales mediante las que se pueden calcular las deformaciones generalizadas de forma objetiva y $(b)$ unas funciones de forma modificadas que garantizan la objetividad de la interpolación. Con él resuelven problemas estáticos y dinámicos.

Por último, y también en el marco de las formulaciones basadas en la actualización multiplicativa, mencionamos el elemento de Jelenić y Saje (1995) [33] que, mediante el uso de un principio variacional generalizado, elimina de las incógnitas el campo de desplazamientos y mantiene únicamente las rotaciones incrementales, así como

\footnotetext{
8 “... debido a la no conmutatividad de la actualización de las rotaciones, los valores de las rotaciones en cada punto de integración no dependen solamente de las rotaciones nodales, sino también de la historia de deformaciones ocurrida en cada punto de integración. De esta forma, dos deformaciones cuyas variables nodales difieran sólo en un movimiento de sólido rígido pueden corresponder a dos campos de rotaciones, que en los puntos de integración no están relacionados por una única rotación."
} 
el elemento propuesto por Zupan y Saje (2003) [66], 67] que se fundamenta en la interpolación de las deformaciones generalizadas y mantiene, por tanto, la objetividad.

\subsubsection{Extensión de las soluciones numéricas a otros tipos de problemas}

La incorporación del alabeo al modelo geométricamente exacto y su implementación numérica fue llevada a cabo por Simó y Vu-Quoc en 1991 [31].

La solución de problemas de láminas mediante técnicas basadas en un planteamiento análogo al del modelo geométricamente exacto fue abordada por Simó y Fox y Rifai en una serie de cuatro artículos que comienza en 1989, de los cuales incluimos la referencia del primero [60]. Sólo añadiremos que Betsch, Menzel y Stein [9] se ocupan también del problema de la parametrización en el contexto de las láminas geométricamente exactas, y que el modelo de Simó ha dado lugar a una línea de investigación muy fructífera en este terreno.

El tratamiento de los problemas dinámicos es otro campo en el que se han producido aportaciones interesantes basadas en el modelo geométricamente exacto. Ya hemos mencionado que distintos autores han extendido la formulación a este tipo de problemas [26], [32]. Añadimos la referencia de Simó, Tarnow y Doblaré (1995) [61] que tiene un interés especial en el contexto de la tesis, puesto que incorpora al análisis dinámico del modelo geométricamente exacto una técnica específica de integración geométrica que conserva los invariantes del sistema mecánico: el método energía-momento. También tiene interés la contribución reciente de Romero y Armero (2002) [57] que emplea el mismo tipo de técnica de integración temporal e incorpora una nueva solución numérica del modelo de Reissner-Simó basada en la interpolación directa de los incrementos de los vectores directores.

La incorporación de comportamientos inelásticos es un aspecto poco estudiado en el modelo exacto. Terminamos la revisión de literatura citando la interesante contribución de Nukala y White (2004) [48] que emplea un elemento mixto basado en el principio variacional de Hellinger-Reissner con interpolación lineal de los esfuerzos para el análisis plástico de piezas alargadas. 



\section{Capítulo 3}

\section{Cinemática del modelo de Reissner-Simó}

En esta capítulo se estudia la descripción del cambio de forma de una pieza alargada $\mathcal{B}$ en el marco de la teoría de Reissner-Simó (RS). Se considerará el caso general de la pieza de directriz inicial curva. La hipótesis cinemática se basa en la indeformabilidad de la sección transversal, y en admitir que ésta puede experimentar una rotación finita relativa a su posición original.

Es conveniente introducir tres configuraciones útiles para una adecuada clasificación de las variables del problema. Supondremos que la pieza se encuentra inicialmente libre de tensiones y la geometría de su directriz es curva; esta situación define la denominada configuración inicial. La importancia de la configuración inicial reside en los siguientes hechos: (a) las fuerzas de volumen que actúan a lo largo del proceso de deformación se definen con referencia al diferencial de volumen de la geometría inicial, (b) dado que esta configuración constituye el origen de tensiones, el cambio de forma se evalúa a partir de ella, y (c) las fuerzas internas que actúan en el sólido deformado se referirán también al diferencial de área en la geometría inicial.

Después de la actuación de las cargas la pieza se deforma hasta su configuración actual. Es en esta configuración en la que se formula el principio de trabajos virtuales y las ecuaciones de equilibrio. Los vectores que describen las fuerzas generalizadas sobre la pieza, así como las fuerzas internas o esfuerzos, pertenecen a ella. También las deformaciones generalizadas son características de la configuración actual.

Además, es útil definir una situación ideal en la que (a) la curva formada por la directriz deformada se endereza primero hasta su forma inicial y luego hasta transformarse en parte de una recta, y (b) las secciones transversales recuperan primero la rotación sufrida en el proceso de deformación y después la rotación inicial debida a la curvatura original de la pieza; esta será la denominada configuración de referencia. Cuando las variables asociadas a la configuración actual se retrotraen idealmente junto con la directriz y las secciones transversales hasta la configuración de referencia, entonces adquieren el aspecto (intrínseco) que tendrían para un observador que avanza sobre la directriz deformada. Por eso esta configuración permite la definición de los valores intrínsecos de los esfuerzos y las deformaciones generalizadas, así como 
la formulación más sencilla de las ecuaciones constitutivas. Cuando el principio de trabajos virtuales y las ecuaciones de equilibrio se escriben en la configuración de referencia diremos que están expresados en su forma material.

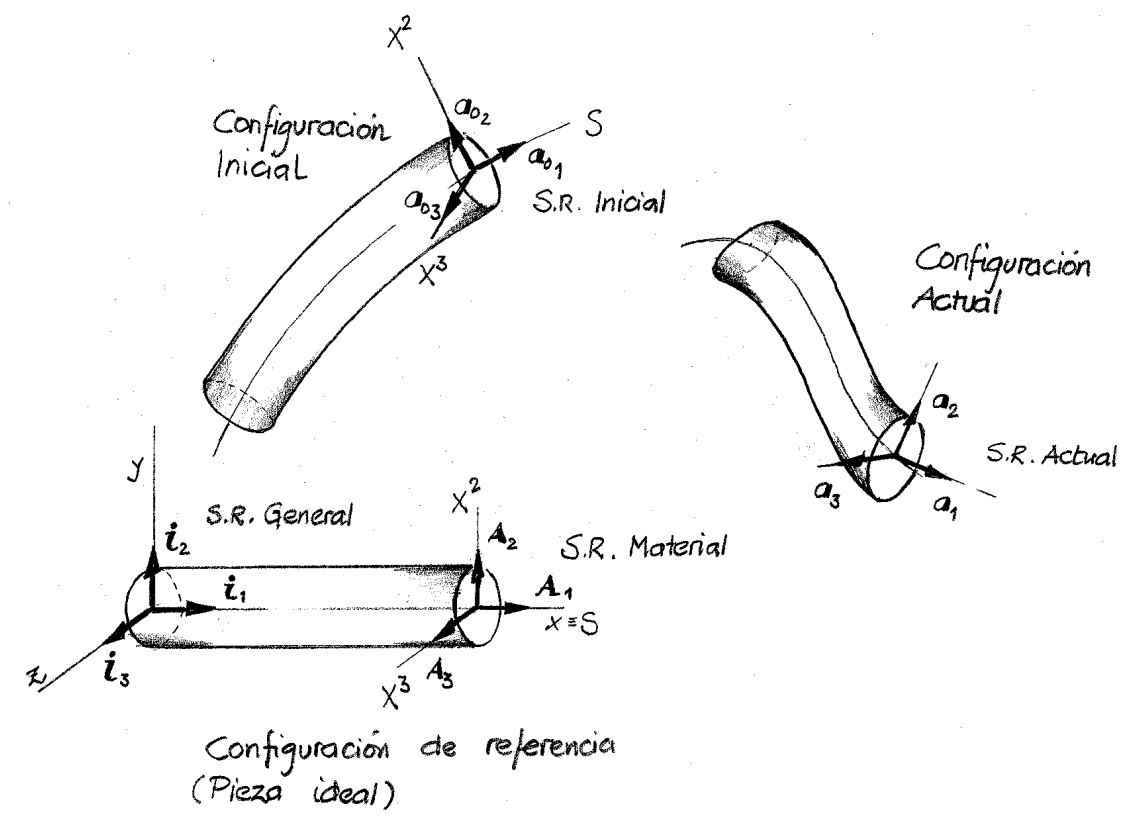

Figura 3.1: Configuraciones y sistemas de referencia

El espacio está dotado de un sistema de coordenadas cartesianas $(x, y, z)$ que permite definir en cada punto una base ortonormal $\left\{\begin{array}{lll}\boldsymbol{i}_{1} & \boldsymbol{i}_{2} & \boldsymbol{i}_{3}\end{array}\right\}$ que denominamos sistema de referencia del espacio o sistema de referencia general. Cuando empleemos notación de índices utilizaremos para este sistema los índices $a, b, c \ldots$ Por otra parte, cada una de las configuraciones descritas lleva asociado un sistema de referencia:

- El sistema de referencia material $\left\{\boldsymbol{A}_{1} \boldsymbol{A}_{2} \boldsymbol{A}_{3}\right\}$ es el asociado a la configuración de referencia. Coincide con el sistema general, y por ello también se empleará los índices $a, b, c \ldots$ Las coordenadas correspondientes a este sistema son $\left(S, X^{2}, X^{3}\right)$, idénticas por definición a las cartesianas $(x, y, z)$. Los puntos materiales del sólido se etiquetan por medio de sus coordenadas $\left(S, X^{2}, X^{3}\right)$ en la configuración de referencia, que son las variables independientes del problema. En este sentido, toda la formulación del problema es lagrangiana.

- El sistema de referencia inicial $\left\{\begin{array}{lll}\boldsymbol{a}_{01} & \boldsymbol{a}_{02} & \boldsymbol{a}_{03}\end{array}\right\}$, se define a partir del triedro de Frênet-Serret de la curva que describe la geometría inicial de la directriz. Dado que el triedro de Frênet define una base ortonormal del espacio en cada punto de la directriz, el sistema inicial se puede obtener por medio de una transformación ortogonal de la base material, definida por el tensor $\boldsymbol{\Lambda}_{0}$. El 
triedro de Frênet es la base natural (en los puntos de la directriz inicial) del sistema de coordenadas curvilíneas $\left(S, X^{2}, X^{3}\right)$. Este sistema es el resultado de curvar las rectas coordenadas del sistema de referencia material hasta la forma inicial de la pieza. Los índices asociados a este sistema serán las mayúsculas $I, J, K \ldots$

- El sistema de referencia actual $\left\{\begin{array}{lll}\boldsymbol{a}_{1} & \boldsymbol{a}_{2} & \boldsymbol{a}_{3}\end{array}\right\}$, define la orientación de la sección transversal después de la actuación de las cargas. También es el resultado de una transformación ortogonal del sistema material definida por el tensor $\boldsymbol{\Lambda}$. En notación indicial se empleará las minúsculas $i, j, k \ldots$

\subsection{Configuración inicial y configuración de referencia}

La descripción geométrica de la pieza en su configuración inicial se realiza a partir de la curva $\Gamma_{0}$ que define la directriz $\boldsymbol{x}_{0}(S):\left[S_{1}, S_{2}\right] \subset \mathbb{R} \rightarrow \mathbb{R}^{3}$. El parámetro $S$ es la longitud de arco medida sobre la directriz. La intersección de $\mathcal{B}$ con el plano perpendicular a la directriz define la sección transversal $A_{s}$, cuyo contorno denotaremos $\partial A_{s}$. Denominaremos $A_{1}$ y $A_{2}$ a las secciones de los extremos de la pieza. El contorno $\partial \mathcal{B}$ del sólido está formado por la unión de $A_{1}, A_{2}$ y $\partial \overline{\mathcal{B}}$; este último término representa el contorno lateral de la pieza.

La curva $\Gamma_{0}$ posee en cada uno de sus puntos una base asociada denominada triedro de Frênet-Serret. El triedro queda determinado por tres vectores unitarios y perpendiculares entre si (tangente, normal y binormal), que se definen de la siguiente forma

$$
\begin{aligned}
\boldsymbol{a}_{01} & =\frac{d \boldsymbol{x}_{0}}{d S} \\
\boldsymbol{a}_{02} & =\frac{1}{\chi} \frac{d \boldsymbol{a}_{01}}{d S} \\
\boldsymbol{a}_{03} & =\frac{1}{\tau}\left(\frac{d \boldsymbol{a}_{02}}{d S}+\chi \boldsymbol{a}_{01}\right),
\end{aligned}
$$

donde $\chi$ es el módulo de $d \boldsymbol{a}_{01} / d S$ y mide la curvatura de $\Gamma_{0}$, y $\tau$ mide la torsión de $\Gamma_{0}$. Los vectores $\left\{\boldsymbol{a}_{01}, \boldsymbol{a}_{02}, \boldsymbol{a}_{03}\right\}$ constituyen, en cada punto de la directriz, la base ortonormal asociada a la sección transversal en la configuración inicial, es decir, el sistema de referencia inicial.

En la descripción geométrica de la configuración inicial se adopta el sistema de coordenadas curvilíneas $\left\{S, X^{2}, X^{3}\right\}$, en el que la coordenada $S$ tiene el significado descrito en el párrafo anterior, y las coordenadas $X^{2}$ y $X^{3}$ describen la distancia de los puntos del sólido a la directriz, medida en la dirección de los vectores unitarios $\boldsymbol{a}_{02}$ (normal) y $\boldsymbol{a}_{03}$ (binormal), contenidos en el plano de la sección transversal.

La posición de un punto cualquiera del sólido en la configuración inicial $\boldsymbol{x}_{0}^{*}$ se puede expresar mediante la siguiente ecuación vectorial

$$
\boldsymbol{x}_{0}^{*}=\boldsymbol{x}_{0}+\boldsymbol{r}_{0}^{*},
$$




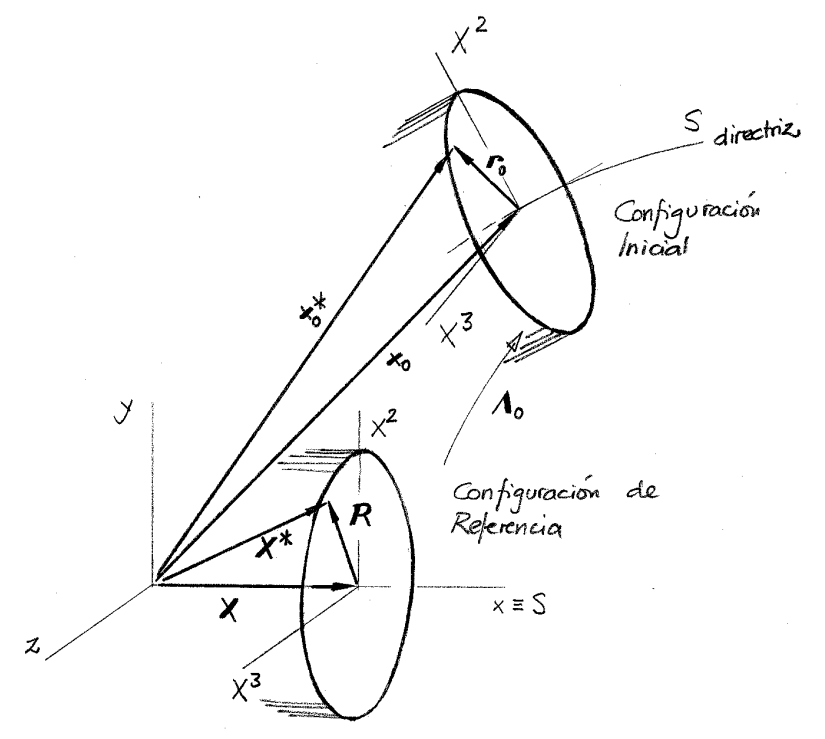

Figura 3.2: Configuración inicial y configuración de referencia

donde $\boldsymbol{r}_{0}^{*}$ expresa la posición del punto material relativa al origen de la sección (intersección con la directriz). Las componentes de $\boldsymbol{r}_{0}^{*}$ en el sistema de referencia inicial, que denotaremos $\left(r_{0}^{*}\right)^{I}$, valen $\left\{0, X^{2}, X^{3}\right\}^{\top}$ ya que

$$
\boldsymbol{r}_{0}^{*}=\boldsymbol{a}_{0 I}\left(r_{0}^{*}\right)^{I}=\boldsymbol{a}_{02} X^{2}+\boldsymbol{a}_{03} X^{3}=\left[\begin{array}{lll}
\boldsymbol{a}_{01} & \boldsymbol{a}_{02} & \boldsymbol{a}_{03}
\end{array}\right]\left\{\begin{array}{c}
0 \\
X^{2} \\
X^{3}
\end{array}\right\} .
$$

Introducimos ahora la configuración de referencia en la que la pieza curva se endereza hasta transformarse en una pieza ideal, con la misma sección transversal que la original, de directriz recta coincidente con el eje $x$ del sistema de referencia del espacio. Esta configuración está dotada de un sistema de coordenadas cartesianas $\left\{S, X^{2}, X^{3}\right\}$ que coinciden con las coordenadas $\{x, y, z\}$ del espacio. La base natural correspondiente a las coordenadas $\left\{S, X^{2}, X^{3}\right\}$, que denominamos sistema de referencia material, está formada por los vectores $\left\{\begin{array}{lll}\boldsymbol{A}_{1} & \boldsymbol{A}_{2} & \boldsymbol{A}_{3}\end{array}\right\}$, que coinciden con los vectores ortonormales $\left\{\begin{array}{lll}\boldsymbol{i}_{1} & \boldsymbol{i}_{2} & \boldsymbol{i}_{3}\end{array}\right\}$ del sistema de referencia general. La posición de un punto material del sólido en la configuración de referencia $\boldsymbol{X}^{*}$ se puede expresar mediante la siguiente ecuación vectorial

$$
\boldsymbol{X}^{*}=\boldsymbol{X}+\boldsymbol{R}^{*}
$$

donde $\boldsymbol{R}^{*}$ expresa la posición del punto relativa al origen de la sección (intersección con la directriz). Las componentes de $\boldsymbol{R}^{*}$ en el sistema de referencia material, $\left(R^{*}\right)^{a}$, 
valen $\left\{0, X^{2}, X^{3}\right\}^{\top}$, puesto que

$$
\boldsymbol{R}^{*}=\boldsymbol{A}_{a}\left(R^{*}\right)^{a}=\boldsymbol{A}_{2} X^{2}+\boldsymbol{A}_{3} X^{3}=\left[\begin{array}{lll}
\boldsymbol{A}_{1} & \boldsymbol{A}_{2} & \boldsymbol{A}_{3}
\end{array}\right]\left\{\begin{array}{c}
0 \\
X^{2} \\
X^{3}
\end{array}\right\} .
$$

Comparando esta expresión con (3.3) se observa la coincidencia de las componentes de $\boldsymbol{r}_{0}^{*}$ en la base inicial con las de $\boldsymbol{R}^{*}$ en la base material, $\left(R^{*}\right)^{a}=\left(r_{0}^{*}\right)^{I}$. En todo el texto emplearemos el asterisco $\left(^{*}\right)$ para denotar objetos inherentes a cada uno de los puntos materiales del sólido tridimensional. Por el contrario, los vectores sin asterisco serán, en general, los pertenecientes al modelo unidimensional de la pieza alargada.

El sistema inicial $\left\{\boldsymbol{a}_{01}, \boldsymbol{a}_{02}, \boldsymbol{a}_{03}\right\}$ se puede obtener a partir de una rotación finita inicial del sistema de referencia material $\left\{\boldsymbol{A}_{1}, \boldsymbol{A}_{2}, \boldsymbol{A}_{3}\right\}$, definida por el tensor ortogonal $\boldsymbol{\Lambda}_{0}(S)$. Dado que el sistema material coincide con el sistema de referencia del espacio (sistema general) se tiene:

$$
\boldsymbol{a}_{0 I}=\boldsymbol{A}_{a}\left(\Lambda_{0}\right)^{a}{ }_{I}=\boldsymbol{i}_{a}\left(\Lambda_{0}\right)^{a}{ }_{I} .
$$

Por tanto, las componentes del tensor de rotación inicial en el sistema general coinciden con las de los vectores $\boldsymbol{a}_{0 K}$ en ese mismo sistema, escritas en columna

$$
\boldsymbol{\Lambda}_{0}=\left[\begin{array}{lll}
\boldsymbol{a}_{01} & \boldsymbol{a}_{02} & \boldsymbol{a}_{03}
\end{array}\right]
$$

y en forma abreviada

$$
\left(\Lambda_{0}\right)^{a}{ }_{I}=\left(a_{0 I}\right)^{a} .
$$

Retornando ahora a la ecuación (3.3), ésta se puede interpretar como cambio de base del vector $\boldsymbol{r}_{0}^{*}$ como consecuencia de (3.7). Las componentes del vector en el sistema del espacio se obtienen a partir de las componentes en el sistema inicial mediante la siguiente relación

$$
\left(r_{0}^{*}\right)^{a}=\left(\Lambda_{0}\right)^{a}{ }_{I}\left(r_{0}^{*}\right)^{I} .
$$

Por tanto, la expresión de (3.2) en componentes del sistema general es

$$
\left(x_{0}^{*}\right)^{a}=\left(x_{0}\right)^{a}+\left(\Lambda_{0}\right)^{a}{ }_{I}\left(r_{0}^{*}\right)^{I} .
$$

Desde otro punto de vista, si se tiene en cuenta que en la ecuación (3.3) la matriz de los vectores de la base inicial es, precisamente, el tensor de rotación inicial, y el vector columna esta formado por las componentes de $\boldsymbol{R}^{*}$ en la base del espacio, entonces el vector $\boldsymbol{r}_{0}^{*}$ se puede interpretar como el resultado de la rotación de $\boldsymbol{R}^{*}$ mediante el tensor $\boldsymbol{\Lambda}_{0}$

$$
\boldsymbol{r}_{0}^{*}=\boldsymbol{\Lambda}_{0} \boldsymbol{R}^{*} .
$$

En componentes del sistema general

$$
\left(r_{0}^{*}\right)^{a}=\left(\Lambda_{0}\right)^{a}{ }_{b}\left(R^{*}\right)^{b} .
$$

Esta expresión confirma que la forma inicial de la pieza es el resultado de la actuación de la rotación $\boldsymbol{\Lambda}_{0}(S)$ sobre cada una de las secciones de una pieza ideal con la misma sección transversal, de directriz recta coincidente con el eje $x$ del sistema general de referencia, además de un desplazamiento inicial de la directriz definido por el vector $\boldsymbol{x}_{0}$. 


\subsection{Geometría de la configuración inicial}

\subsubsection{Tensor de curvaturas iniciales}

La relación entre los vectores de la base inicial y sus derivadas viene dada por las ecuaciones (3.1), y se puede expresar matricialmente de la siguiente forma

$$
\left\{d \boldsymbol{a}_{01} / d S \quad d \boldsymbol{a}_{02} / d S \quad d \boldsymbol{a}_{03} / d S\right\}=\left\{\begin{array}{lll}
\boldsymbol{a}_{01} & \boldsymbol{a}_{02} & \boldsymbol{a}_{03}
\end{array}\right\}\left[\begin{array}{ccc}
0 & -\chi & 0 \\
\chi & 0 & -\tau \\
0 & \tau & 0
\end{array}\right] .
$$

Escribiremos esta última relación en forma abreviada

$$
\frac{d}{d S} \boldsymbol{a}_{0 K}=\boldsymbol{a}_{0 I}\left(\widehat{\mathrm{k}}_{0}\right)_{K}^{I}
$$

Denominaremos tensor de curvaturas iniciales al tensor $\widehat{\mathbf{k}}_{0}$. La notación responde a su carácter antisimétrico.

Derivando la ecuación (3.6) y empleando la ecuación (3.14) se obtiene fácilmente la expresión del tensor de curvaturas iniciales en función del tensor de rotación inicial, en componentes del sistema de referencia inicial de la sección,

$$
\frac{d}{d S} \boldsymbol{a}_{0 K}=\boldsymbol{i}_{a}\left(\frac{d}{d S} \Lambda_{0}\right)^{a}{ }_{K}=\boldsymbol{a}_{0 I}\left(\Lambda_{0}^{\boldsymbol{\top}}\right)^{I}{ }_{a}\left(\frac{d}{d S} \Lambda_{0}\right)_{K}^{a}=\boldsymbol{a}_{0 I}\left(\widehat{\mathrm{k}}_{0}\right)_{K}^{I} .
$$

Comparando este resultado con (3.14) se deduce que el valor de las componentes de $\widehat{\mathbf{k}}_{0}$ en sistema inicial $\left(\widehat{\mathrm{k}}_{0}\right)_{K}^{I}$ es el indicado en la expresión (3.13) obtenida antes. Denotaremos estas componentes mediante el superíndice I. Por tanto

$$
\stackrel{\mathrm{I}}{\widehat{\mathbf{k}}_{0}}=\boldsymbol{\Lambda}_{0}^{\top} \frac{d}{d S} \boldsymbol{\Lambda}_{0}
$$

Por otra parte, si consideramos de nuevo la definición (3.7) de la matriz de rotación inicial, es sencillo comprobar que las componentes de cada uno de los vectores $\boldsymbol{a}_{0 K}$ de la base inicial en cualquier sistema del espacio se pueden obtener multiplicando la matriz de rotación por las componentes de los vectores del sistema general $\boldsymbol{i}_{K}$ en ese mismo sistema

$$
\boldsymbol{a}_{0 K}=\boldsymbol{\Lambda}_{0} \boldsymbol{i}_{K} .
$$

Derivando, se obtiene el siguiente resultado

$$
\frac{d}{d S} \boldsymbol{a}_{0 K}=\frac{d}{d S} \boldsymbol{\Lambda}_{0} \boldsymbol{i}_{K}=\frac{d}{d S} \boldsymbol{\Lambda}_{0} \boldsymbol{\Lambda}_{0}^{\top} \boldsymbol{a}_{0 K},
$$

que permite definir una nueva expresión del tensor de curvaturas iniciales válida en el sistema de referencia del espacio

$$
\widehat{\mathbf{k}}_{0}=\frac{d}{d S} \boldsymbol{\Lambda}_{0} \boldsymbol{\Lambda}_{0}^{\top} .
$$

El tensor $\widehat{\mathbf{k}}_{0}$ es un objeto perteneciente a la configuración inicial. 
Volviendo a la fórmula (3.18) de las derivadas de los vectores del sistema inicial, su expresión en el sistema general es

$$
\left(\frac{d}{d S} a_{0 K}\right)^{a}=\left(\frac{d}{d S} \Lambda_{0}\right)_{I}^{a}\left(\Lambda_{0}^{\top}\right)_{b}^{I}\left(\boldsymbol{a}_{0 K}\right)^{b} .
$$

Las componentes de $\widehat{\mathbf{k}}_{0}$ en el sistema general son, por tanto,

$$
\left(\widehat{\mathrm{k}}_{0}\right)_{b}^{a}=\left(\frac{d}{d S} \Lambda_{0}\right)^{a}{ }_{I}\left(\Lambda_{0}^{\top}\right)_{b}^{I} .
$$

Más adelante será útil emplear esta expresión en notación compacta, en la que denotaremos mediante el superíndice s (spatial, en inglés) las componentes del tensor en el sistema general

$$
\widehat{\mathbf{k}}_{0}^{\mathrm{S}}=\frac{d}{d S} \boldsymbol{\Lambda}_{0} \boldsymbol{\Lambda}_{0}^{\top} .
$$

Es sencillo comprobar que la relación entre las componentes del tensor de curvaturas en los sistemas inicial y general, empleando $\boldsymbol{\Lambda}_{0}$ como matriz de cambio de base, es

$$
\left(\widehat{\mathrm{k}}_{0}\right)_{K}^{I}=\left(\Lambda_{0}^{\top}\right)^{I}{ }_{a}\left(\widehat{\mathrm{k}}_{0}\right)_{b}^{a}\left(\Lambda_{0}\right)_{K}^{b},
$$

por lo tanto, en notación compacta,

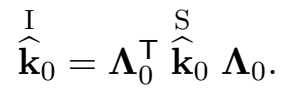

La expresión (3.18) se puede escribir así

$$
\frac{d}{d S} \stackrel{\mathrm{S}}{\boldsymbol{a}_{0 K}}=\stackrel{\widehat{\mathbf{k}}_{0}}{\stackrel{\boldsymbol{a}}{\mathbf{a}}_{0 K}}
$$

La expresión de las derivadas es la misma en componentes del sistema inicial, por tanto

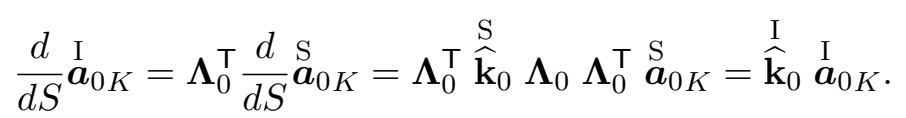

Generalmente expresaremos todos los objetos en el sistema de referencia del espacio (sistema general), prescindiendo por ello del superíndice s.

De la misma forma que al vector posición $\boldsymbol{r}_{0}^{*}$, perteneciente a la configuración inicial, corresponde el vector $\boldsymbol{R}^{*}$ de la configuración de referencia, es posible introducir un nuevo tensor $\widehat{\mathbf{K}}_{0}$ perteneciente a la pieza ideal, definido de forma que la actuación de la rotación $\boldsymbol{\Lambda}_{0}(S)$ sobre él diese como resultado el tensor de curvaturas iniciales $\widehat{\mathbf{k}}_{0}$

$$
\widehat{\mathbf{k}}_{0}=\boldsymbol{\Lambda}_{0} \widehat{\mathbf{K}}_{0} \boldsymbol{\Lambda}_{0}^{\top}
$$

La relación inversa es

$$
\widehat{\mathbf{K}}_{0}=\boldsymbol{\Lambda}_{0}^{\top} \widehat{\mathbf{k}}_{0} \boldsymbol{\Lambda}_{0} .
$$

Si se compara esta última ecuación con la expresión (3.22) es inmediato concluir que las componentes de $\widehat{\mathbf{k}}_{0}$ en el sistema inicial coinciden con las componentes de $\widehat{\mathbf{K}}_{0}$ en el sistema general

$$
\widehat{\mathrm{k}}_{0}=\stackrel{\mathrm{S}}{\widehat{\mathbf{K}}_{0}}
$$




\subsubsection{Métrica de la sección transversal}

La geometría de la sección en la configuración inicial se basa en el triedro de Frênet $\left\{\boldsymbol{a}_{01}, \boldsymbol{a}_{02}, \boldsymbol{a}_{03}\right\}$ y se define a partir del vector posición de un punto de la sección (3.2), (3.3):

$$
\boldsymbol{x}_{0}^{*}\left(S, X^{2}, X^{3}\right)=\boldsymbol{x}_{0}(S)+X^{2} \boldsymbol{a}_{02}(S)+X^{3} \boldsymbol{a}_{03}(S) .
$$

Los vectores de la base natural en cada punto de la sección se obtienen derivando el vector posición respecto de las coordenadas del sistema inicial

$$
\begin{aligned}
& \boldsymbol{g}_{01}^{*}=\frac{\partial \boldsymbol{x}_{0}^{*}}{\partial S}=\frac{d}{d S} \boldsymbol{x}_{0}+X^{2} \frac{d}{d S} \boldsymbol{a}_{02}+X^{3} \frac{d}{d S} \boldsymbol{a}_{03} \\
& \boldsymbol{g}_{02}^{*}=\frac{\partial \boldsymbol{x}_{0}^{*}}{\partial X^{2}}=\boldsymbol{a}_{02} \\
& \boldsymbol{g}_{03}^{*}=\frac{\partial \boldsymbol{x}_{0}^{*}}{\partial X^{3}}=\boldsymbol{a}_{03} .
\end{aligned}
$$

Empleando la ecuación (3.13) y operando, resulta

$$
\begin{aligned}
& \boldsymbol{g}_{01}^{*}=\left(1-\chi X^{2}\right) \boldsymbol{a}_{01}-\tau X^{3} \boldsymbol{a}_{02}+\tau X^{2} \boldsymbol{a}_{03} \\
& \boldsymbol{g}_{02}^{*}=\boldsymbol{a}_{02} \\
& \boldsymbol{g}_{03}^{*}=\boldsymbol{a}_{03} .
\end{aligned}
$$

Las componentes covariantes del tensor métrico $\left(\mathrm{g}_{0}^{*}\right)_{I J}=\boldsymbol{g}_{0 I}^{*} \cdot \boldsymbol{g}_{0 J}^{*}$ son

$$
\left[\begin{array}{ccc}
\left(1-\chi X^{2}\right)^{2}+\tau^{2}\left(\left(X^{2}\right)^{2}+\left(X^{3}\right)^{2}\right) & -\tau X^{3} & \tau X^{2} \\
-\tau X^{3} & 1 & 0 \\
\tau X^{2} & 0 & 1
\end{array}\right]
$$

Se puede observar que la base natural sólo coincide con el sistema de referencia inicial de la sección en la directriz $\left(X^{2}=X^{3}=0\right)$. Además, el sistema, en general no es ortogonal. El diferencial de volumen en la geometría inicial es

$$
d V_{0}^{*}=\sqrt{\mathrm{g}_{0}^{*}} d S d X^{2} d X^{3}
$$

donde $\mathrm{g}_{0}^{*}$ denota el determinante del tensor métrico $\mathbf{g}_{0}^{*}$, y su raíz cuadrada es

$$
\sqrt{\mathrm{g}_{0}^{*}}=1-\chi X^{2} \text {. }
$$

El diferencial de superficie sobre los puntos de la sección transversal es

$$
d A_{0}^{*}=\sqrt{\mathrm{g}_{0}^{*}\left(\mathrm{~g}_{0}^{*}\right)^{11}} d X^{2} d X^{3}
$$

y como

$$
\left(\mathrm{g}_{0}^{*}\right)^{11}=\frac{1}{\mathrm{~g}_{0}^{*}} \operatorname{Adj}\left(\mathrm{g}_{0}^{*}\right)_{11}=\frac{1}{\mathrm{~g}_{0}^{*}} .
$$

Luego $d A_{0}^{*}=d X^{2} d X^{3}$. Las expresiones del diferencial de volumen y de superficie de la sección transversal quedan, finalmente,

$$
\begin{aligned}
& d V_{0}^{*}=\left(1-\chi X^{2}\right) d S d X^{2} d X^{3} \\
& d A_{0}^{*}=d X^{2} d X^{3}
\end{aligned}
$$


Si la directriz está contenida en un plano, entonces la torsión de la directriz es nula $\tau=0$, y la base natural será ortogonal en todos los puntos de la sección. La expresión del tensor métrico se simplifica:

$$
\left[\begin{array}{ccc}
\left(1-\chi X^{2}\right)^{2} & 0 & 0 \\
0 & 1 & 0 \\
0 & 0 & 1
\end{array}\right]
$$

\subsection{Hipótesis cinemática}

La posición de un punto material después de la deformación $\boldsymbol{x}^{*}$ se expresa en función de la posición deformada de la directriz, $\boldsymbol{x}$, y de la posición relativa del punto respecto del origen de la sección, $\boldsymbol{r}^{*}$, mediante la ecuación vectorial

$$
\boldsymbol{x}^{*}=\boldsymbol{x}+\boldsymbol{r}^{*}
$$

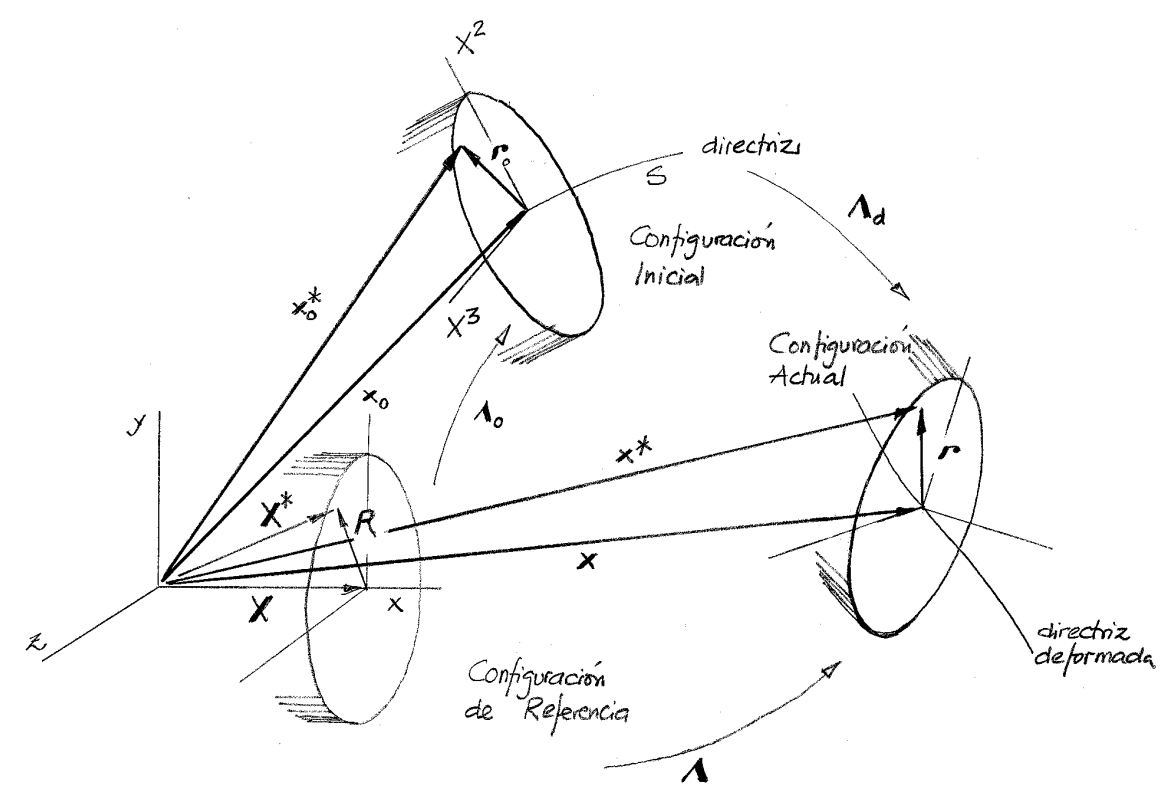

Figura 3.3: Cambio de configuración

La hipótesis cinemática de Reissner-Simó considera que, tras la deformación, las secciones transversales de la pieza experimentan una rotación finita, permaneciendo indeformables en su plano. La posibilidad de que se produzcan deformaciones transversales se traduce en que la normal a la sección deformada no tiene por qué ser tangente a la directriz deformada. El triedro $\left\{\begin{array}{lll}\boldsymbol{a}_{1} & \boldsymbol{a}_{2} & \boldsymbol{a}_{3}\end{array}\right\}$ es el resultado de la rotación de la sección, es decir de la rotación del triedro inicial $\left\{\begin{array}{llll}\boldsymbol{a}_{01} & \boldsymbol{a}_{02} & \boldsymbol{a}_{03}\end{array}\right\}$, y caracteriza la sección deformada, pues $\boldsymbol{a}_{1}$ define su normal, y $\boldsymbol{a}_{2}, \boldsymbol{a}_{3}$ son vectores contenidos en 
ella. Las componentes del nuevo triedro en el sistema de referencia inicial, escritas en columna, $\left[\begin{array}{lll}\boldsymbol{a}_{1} & \boldsymbol{a}_{2} & \boldsymbol{a}_{3}\end{array}\right]$ definen un tensor ortogonal que denotamos $\boldsymbol{\Lambda}_{\mathrm{d}}$ y caracteriza la rotación desde la configuración inicial hasta la actual ${ }^{1}$. Denominaremos rotación deformacional a $\boldsymbol{\Lambda}_{\mathrm{d}}$. Entonces

$$
\boldsymbol{r}^{*}=X^{2} \boldsymbol{a}_{2}+X^{3} \boldsymbol{a}_{3}=\left[\begin{array}{lll}
\boldsymbol{a}_{1} & \boldsymbol{a}_{2} & \boldsymbol{a}_{3}
\end{array}\right]\left\{\begin{array}{c}
0 \\
X^{2} \\
X^{3}
\end{array}\right\}=\boldsymbol{\Lambda}_{\mathrm{d}} \boldsymbol{r}_{0}^{*} .
$$

El vector posición en la sección después de la deformación se obtiene, por tanto, mediante de la actuación de $\boldsymbol{\Lambda}_{\mathrm{d}}$ sobre el vector posición antes de la deformación. La ecuación vectorial anterior, obtenida en el sistema de referencia inicial, es cierta en cualquier sistema de referencia del espacio, y en particular lo es cuando todos sus términos se expresan en el sistema de referencia general:

$$
\boldsymbol{r}^{*}=\boldsymbol{\Lambda}_{\mathrm{d}} \boldsymbol{r}_{0}^{*}
$$

Entonces, empleando la expresión (3.11) de $\boldsymbol{r}_{0}^{*}$ como resultado de la rotación inicial del vector posición sobre la sección de la pieza ideal $\boldsymbol{R}^{*}$,

$$
\boldsymbol{r}^{*}=\boldsymbol{\Lambda}_{\mathrm{d}} \boldsymbol{r}_{0}^{*}=\boldsymbol{\Lambda}_{\mathrm{d}} \boldsymbol{\Lambda}_{0} \boldsymbol{R}^{*}
$$

Podemos escribir esta expresión como

$$
\boldsymbol{r}^{*}=\boldsymbol{\Lambda} \boldsymbol{R}^{*}
$$

introduciendo el tensor de rotación $\boldsymbol{\Lambda}=\boldsymbol{\Lambda}_{\mathrm{d}} \boldsymbol{\Lambda}_{0}$ que transforma vectores de la configuración de referencia en vectores de la configuración actual. Entonces la posición de un punto material después de la deformación es

$$
\begin{aligned}
\boldsymbol{x}^{*}\left(S, X^{2}, X^{3}\right) & =\boldsymbol{x}(S)+\boldsymbol{\Lambda}_{\mathrm{d}}(S) \boldsymbol{\Lambda}_{0}(S) \boldsymbol{R}^{*}\left(X^{2}, X^{3}\right) \\
& =\boldsymbol{x}(S)+\boldsymbol{\Lambda}(S) \boldsymbol{R}^{*}\left(X^{2}, X^{3}\right) .
\end{aligned}
$$

Esta ecuación vectorial, que constituye la hipótesis cinemática de la teoría de Reissner-Simó, válida en cualquier sistema de referencia fijo del espacio, lo es en particular en el sistema general de referencia. Se observa que el tensor $\boldsymbol{\Lambda}_{0}(S)$ que define el cambio de coordenadas entre el sistema general y el inicial es un dato. Además, las componentes de $\boldsymbol{R}^{*}$ en el sistema general sólo dependen de las coordenadas $X^{2}$ y $X^{3}$. Las variables $\boldsymbol{x}(S) \in \mathbb{R}^{3}$ y $\boldsymbol{\Lambda}(S) \in \mathrm{SO}(3)$, dependientes únicamente de la coordenada $S$, constituyen las variables generalizadas que definen la configuración, que son las incógnitas de la teoría RS. El conjunto $\mathbb{R}^{3} \times \mathrm{SO}(3)$ define el correspondiente espacio de las configuraciones, que es una variedad diferenciable, y no un espacio vectorial lineal. Es interesante destacar que el espacio de las configuraciones coincide con el espacio de los movimientos euclídeos en $\mathbb{R}^{3}$ (traslaciones y rotaciones), y que esta coincidencia permite establecer una analogía entre la respuesta del modelo RS y cierta clase de problemas dinámicos, que desarrollaremos posteriormente.

\footnotetext{
${ }^{1}$ El empleo del subíndice d hace referencia a que esta rotación es la que produce la deformación.
} 


\subsection{Interpretación geométrica del tensor $\Lambda$}

La ecuación (3.36) muestra como el vector $\boldsymbol{r}^{*}$ es el resultado de la transformación de $\boldsymbol{R}^{*}$ por medio del tensor ortogonal $\boldsymbol{\Lambda}$. En este sentido, Simó [58] considera $\boldsymbol{\Lambda}$ como una aplicación tangente o mapa de derivadas (derivative map o tangent map), mediante el que se puede llevar a cabo el push-forward de objetos (vectores o tensores) asociados a la configuración de referencia - materiales-, convirtiéndolos en objetos espaciales que residen en la configuración actual. La operación inversa, o pullback, permite convertir objetos espaciales en materiales. Es interesante advertir cómo Simó toma esta terminología de la geometría diferencial, y en concreto de su aplicación a la mecánica del continuo recogida en Marsden y Hughes [41], modificándola para el caso particular de rotaciones finitas: a diferencia de [41, que consideran la transformación definida por el cambio de configuración, y su operador tangente $\mathbf{F}^{*}$ (el gradiente de la deformación), Simó restringe la transformación a la rotación y no emplea el gradiente de la deformación como operador tangente. Esta restricción presenta una ventaja formal: la transformación entre la configuración espacial y la material es formalmente idéntica a un cambio de coordenadas entre el sistema de referencia general y el actual. En adelante utilizaremos la palabra acción para referirnos al push-forward, y retroacción para el pull-back. La terminología de Simó ha sido adoptada por la mayoría de los autores que han tratado este problema. A continuación analizamos con mayor detalle esta cuestión.

El operador $\boldsymbol{\Lambda}$ transforma los vectores de la base material $\boldsymbol{A}^{a}$ en los vectores de la actual $\boldsymbol{a}_{i}$, uno a uno. Su expresión tensorial es, por tanto

$$
\boldsymbol{\Lambda}=\delta_{c}^{k} \boldsymbol{a}_{k} \otimes \boldsymbol{A}^{c}
$$

y su expresión en componentes en la base material (o general) es

$$
\Lambda=\Lambda_{c}^{b} \boldsymbol{A}_{b} \otimes \boldsymbol{A}^{c} .
$$

Igualando ambas se deduce que

$$
\boldsymbol{a}_{k}=\boldsymbol{A}_{b} \Lambda^{b}{ }_{k}=\boldsymbol{A}_{b}\left(a_{k}\right)^{b}
$$

expresión que muestra que las componentes de $\boldsymbol{\Lambda}$ en la base material (o general) se obtienen ordenando en columnas las componentes en esta misma base de los vectores de la base actual. De esta forma queda justificada la expresión (3.33).

Desde otro punto de vista, las componentes de $\boldsymbol{A}_{k}$ en esta misma base son $\left(A_{k}\right)^{a}=$ $\delta^{a}{ }_{k}$. Si se hace actuar ahora el operador $\boldsymbol{\Lambda}$ sobre $\boldsymbol{A}_{k}$ se obtiene, por definición, el vector $\boldsymbol{a}_{k}$ de la base actual

$$
\boldsymbol{a}_{k}=\Lambda \boldsymbol{A}_{k}
$$

escribiendo esta expresión en componentes y operando

$$
\boldsymbol{A}_{b}\left(a_{k}\right)^{b}=\boldsymbol{A}_{b} \Lambda^{b}{ }_{a}\left(A_{k}\right)^{a}=\boldsymbol{A}_{b} \Lambda^{b}{ }_{a} \delta^{a}{ }_{k}=\boldsymbol{A}_{b} \Lambda^{b}{ }_{k} .
$$

Este resultado es idéntico al formulado en (3.40). El tensor $\boldsymbol{\Lambda}$ es, por definición, ortogonal. Por tanto $\boldsymbol{\Lambda}^{-1}=\boldsymbol{\Lambda}^{\top}$. A continuación se analiza el efecto de la transformación sobre un vector $\boldsymbol{W}$. 
Acción. El operador $\boldsymbol{\Lambda}$ actúa sobre un vector tangente $\boldsymbol{W}=\boldsymbol{A}_{b} W^{b}$ en la configuración de referencia (objeto material)

$$
\boldsymbol{w}=\boldsymbol{\Lambda} \boldsymbol{W}=\boldsymbol{\Lambda} \boldsymbol{A}_{b} W^{b}=\boldsymbol{a}_{b} W^{b} .
$$

El resultado es un vector tangente $\boldsymbol{w}$ en la configuración actual (objeto espacial). Sus componentes en la base actual son iguales a las de $\boldsymbol{W}$ en la base material. Por tanto, la acción de $\boldsymbol{\Lambda}$ sobre $\boldsymbol{W}$ (o simplemente acción sobre $\boldsymbol{W}$ ) es una rotación con las mismas características que la de la sección transversal. Las componentes del vector espacial $\boldsymbol{w}$ en la base material son

$$
w^{a}=\Lambda_{b}^{a} W^{b},
$$

puesto que

$$
\boldsymbol{w}=\boldsymbol{\Lambda} \boldsymbol{W}=\boldsymbol{A}_{a} \Lambda_{b}^{a} W^{b}=\boldsymbol{A}_{a} w^{a} .
$$

Retroacción. Es la operación inversa. La retroacción de $\boldsymbol{w}$ transforma este objeto espacial en el objeto material $\boldsymbol{W}$, deshaciendo la rotación.

$$
\boldsymbol{W}=\boldsymbol{\Lambda}^{-1} \boldsymbol{w}=\boldsymbol{\Lambda}^{\top} \boldsymbol{w}
$$

Luego la relación entre las componentes de $\boldsymbol{W}$ y $\boldsymbol{w}$ en la base material es

$$
W^{b}=\left(\Lambda^{\top}\right)^{b}{ }_{a} w^{a} .
$$

Cambio de coordenadas. Considérese la expresión en componentes del vector espacial $\boldsymbol{w}$ respecto de la base material, $\boldsymbol{w}=\boldsymbol{A}_{b} w^{b}$. Para expresar este vector en componentes intrínsecas (referidas a la base actual) se puede hacer uso de la expresión inversa a (3.40)

$$
\boldsymbol{A}_{b}=\boldsymbol{a}_{k}\left(\Lambda^{\top}\right)^{k}{ }_{b}
$$

Entonces

$$
\boldsymbol{w}=\boldsymbol{a}_{k}\left(\Lambda^{\top}\right)_{b}^{k} w^{b}=\boldsymbol{a}_{k} w^{k},
$$

luego las componentes de $\boldsymbol{w}$ respecto de la base actual son

$$
w^{k}=\left(\Lambda^{\top}\right)^{k}{ }_{b} w^{b} .
$$

Esta expresión es formalmente idéntica a la ecuación (3.47). Por lo tanto se puede afirmar que las componentes intrínsecas (respecto del sistema de referencia actual) de un vector espacial $\boldsymbol{w}$ son iguales a las componentes respecto del sistema general del vector material correspondiente $\boldsymbol{W}$ (la retroacción de $\boldsymbol{w}$ ).

Denotando con el superíndice $\mathrm{M}$ las componentes de un vector en el sistema material, y con el superíndice A las componentes en el sistema actual (intrínsecas), las relaciones anteriores se pueden resumir así:

$$
\begin{array}{ll}
\stackrel{M}{\boldsymbol{w}}=\boldsymbol{\Lambda} \stackrel{M}{\boldsymbol{W}} \text { (acción) } & \stackrel{M}{\boldsymbol{W}}=\boldsymbol{\Lambda}^{\top} \stackrel{M}{\boldsymbol{w}} \text { (retroacción) } \\
\stackrel{M}{\boldsymbol{w}}=\boldsymbol{\Lambda} \stackrel{A}{\boldsymbol{w}} & \stackrel{A}{\boldsymbol{w}}=\boldsymbol{\Lambda}^{\top} \stackrel{M}{\boldsymbol{w}} \text { (cambio de coordenadas) }
\end{array}
$$



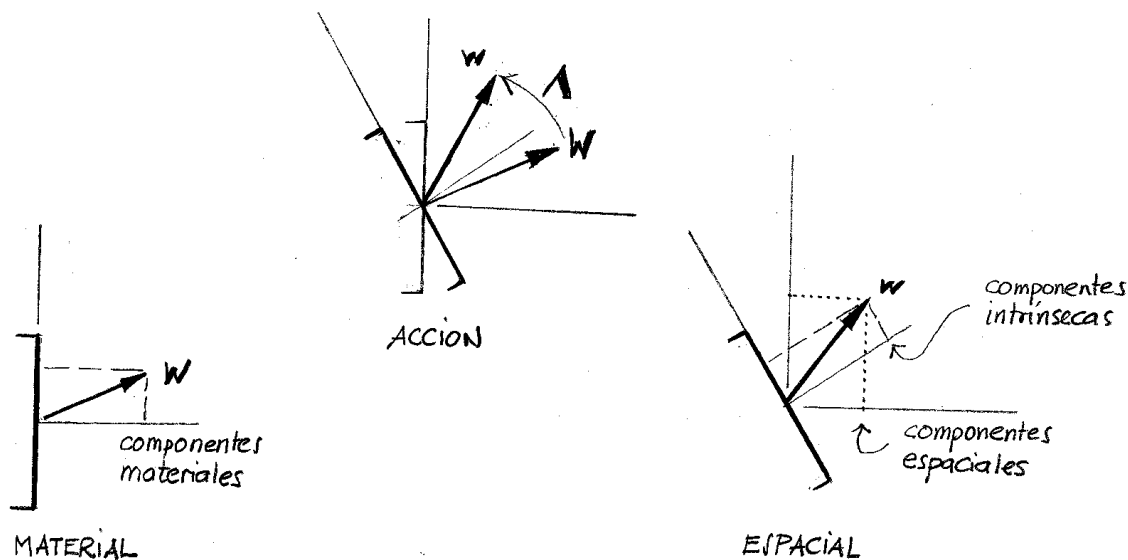

ESPACIAL

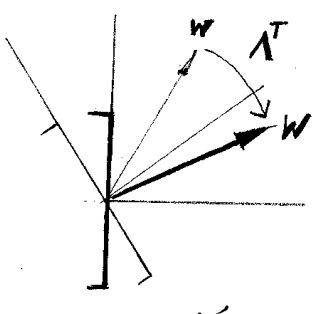

RETROACCIÓN

Figura 3.4: Objetos materiales y espaciales

Teniendo en cuenta todas estas consideraciones estamos en condiciones de fijar un criterio para la notación. En general emplearemos mayúsculas para denotar objetos materiales (como el vector posición de un punto material relativa al origen de la sección en la configuración de referencia $\boldsymbol{R}^{*}$ ) y la forma material de objetos espaciales (como la retroacción del tensor de cambio de orientación de la sección $\widehat{\mathbf{K}}$ ), y minúsculas para los objetos espaciales (como el tensor de cambio de orientación $\widehat{\kappa}$ ), o la forma espacial de objetos materiales (como el vector posición de un punto material relativa al origen de la sección en la configuración actual $\boldsymbol{r}^{*}$ ). Existen algunos tensores que escapan a este criterio: el tensor rotación $\boldsymbol{\Lambda}$, el tensor gradiente de la deformación $\mathbf{F}^{*}$, y el primer tensor de tensiones de Piola-Kirchhoff $\mathbf{P}^{*}$ son ejemplos. Se trata de tensores de dos puntos [41, que actúan transformando objetos materiales en objetos espaciales, y no se pueden catalogar como pertenecientes a una de estas dos configuraciones. Se emplearán mayúsculas para representarlos, manteniendo el criterio predominante en la literatura. Mención especial merecen los vectores posición de la configuración de referencia $\boldsymbol{X}^{*}, \boldsymbol{X}$ y de la configuración deformada $\boldsymbol{x}^{*}, \boldsymbol{x}$, cuyo origen es el del sistema de referencia general. No se trata de objetos materiales (ni espaciales), pues en sentido estricto sólo se puede calificar de esta forma a objetos cuyo origen se encuentre en los puntos de la configuración de referencia (o de la configuración actual). Los vectores (tensores) materiales son, en términos de geometría diferencial, 


\begin{tabular}{cccccc} 
& \multicolumn{3}{c}{ acción } & \multicolumn{3}{c}{ retroacción } \\
\hline vector & $\boldsymbol{W}$ & $\boldsymbol{\Lambda} \boldsymbol{W} \rightarrow$ & $\boldsymbol{w}$ & $\boldsymbol{\Lambda}^{\top} \boldsymbol{w} \rightarrow$ & $\boldsymbol{W}$ \\
tensor & $\mathbf{H}$ & $\boldsymbol{\Lambda} \mathbf{H} \boldsymbol{\Lambda}^{\top} \rightarrow$ & $\mathbf{h}$ & $\boldsymbol{\Lambda}^{\top} \mathbf{h} \boldsymbol{\Lambda} \rightarrow$ & $\mathbf{H}$ \\
\hline & material & & espacial & & material
\end{tabular}

Cuadro 3.1: Acción y Retroacción

vectores (tensores) tangentes a la variedad definida por la configuración de referencia, y los vectores (tensores) espaciales lo son a la variedad definida por la configuración actual.

Las operaciones de acción y retroacción pueden generalizarse a tensores de segundo orden. Si $\mathbf{H}$ es un tensor material de segundo orden $\mathbf{H}=H_{b}^{a} \boldsymbol{A}_{a} \otimes \boldsymbol{A}^{b}$, la acción de $\boldsymbol{\Lambda}$ lo transforma en un objeto espacial $\mathbf{h}$ a través de la operación

$$
\mathbf{h}=\mathbf{\Lambda} \mathbf{H} \Lambda^{\top}
$$

cuyas coordenadas en el sistema general son

$$
h_{d}^{c}=\Lambda_{a}^{c} H_{b}^{a}\left(\Lambda^{\top}\right)_{d}^{b} .
$$

De la misma forma se define la retroacción del tensor espacial $\mathbf{h}$

$$
\mathbf{H}=\boldsymbol{\Lambda}^{\top} \mathbf{h} \boldsymbol{\Lambda}
$$

con coordenadas en el sistema material

$$
H_{b}^{a}=\left(\Lambda^{\top}\right)^{a}{ }_{c} h_{d}^{c} \Lambda_{b}^{d} .
$$

Al igual que en el caso vectorial esta última expresión coincide con la expresión del cambio de coordenadas que proporciona las componentes de $\mathbf{h}$ en la base actual (componentes intrínsecas). Por tanto, las componentes intrínsecas de $\mathbf{h}$ son iguales a las componentes de $\mathbf{H}$ en el sistema general. Todos los resultados anteriores se resumen en el cuadro 3.1 .

Por otra parte conviene notar que las relaciones establecidas en la descripción de la geometría inicial de la pieza pueden interpretarse de forma análoga. Las expresiones (3.11) y (3.24) que reproducimos por conveniencia

$$
\begin{aligned}
\boldsymbol{r}_{0}^{*} & =\boldsymbol{\Lambda}_{0} \boldsymbol{R}^{*} \\
\widehat{\mathbf{k}}_{0} & =\boldsymbol{\Lambda}_{0} \widehat{\mathbf{K}}_{0} \boldsymbol{\Lambda}_{0}^{\top},
\end{aligned}
$$

muestran la acción de la rotación inicial $\boldsymbol{\Lambda}_{0}$ sobre el vector posición $\boldsymbol{R}^{*}$ y sobre el tensor de curvatura inicial $\widehat{\mathbf{K}}_{0}$, que son objetos materiales (asociados a la pieza ideal). Los objetos resultantes pertenecen en este caso a la configuración inicial. De modo análogo se establece la equivalencia entre las coordenadas en el sistema de referencia general de los objetos materiales, y las coordenadas intrínsecas (en el sistema inicial) de los objetos asociados a la configuración inicial. 


\subsection{Geometría de la configuración actual}

La deducción de las ecuaciones de equilibrio por procedimientos energéticos, que se abordará en el próximo capítulo, requiere el cálculo del tensor gradiente de la deformación. Previamente conviene introducir una medida del cambio de orientación de la sección. Para ello se evaluarán las derivadas del sistema de referencia actual.

\subsubsection{Cambio de orientación de la sección}

La expresión del gradiente de la deformación involucra las derivadas de los vectores del sistema actual, que se calculan a continuación a partir de la expresión (3.41), y de la retroacción de los vectores de la base actual:

$$
\frac{d}{d S} \boldsymbol{a}_{K}=\frac{d}{d S}\left(\boldsymbol{\Lambda} \boldsymbol{A}_{K}\right)=\left(\frac{d}{d S} \boldsymbol{\Lambda}\right) \boldsymbol{A}_{K}+\boldsymbol{\Lambda} \frac{d}{d S} \boldsymbol{A}_{K}
$$

El segundo sumando es nulo, puesto que el sistema material es constante a lo largo de la directriz. El primer sumando se puede expresar así

$$
\left(\frac{d}{d S} \boldsymbol{\Lambda}\right) \boldsymbol{A}_{K}=\left(\frac{d}{d S} \boldsymbol{\Lambda}\right) \boldsymbol{\Lambda}^{\top} \boldsymbol{a}_{K}
$$

El factor de $\boldsymbol{a}_{K}$ es un tensor antisimétrico que denominaremos $\widehat{\boldsymbol{\kappa}} \in \mathfrak{s o}(3)$,

$$
\widehat{\kappa}=\Lambda^{\prime} \Lambda^{\top} .
$$

La antisimetría de $\widehat{\kappa}$ se puede comprobar fácilmente teniendo en cuenta que $\boldsymbol{\Lambda}$ es ortogonal y cumple $\boldsymbol{\Lambda}^{\top} \boldsymbol{\Lambda}=\mathbf{1}$. Por tanto, $d\left(\boldsymbol{\Lambda}^{\top} \boldsymbol{\Lambda}\right) / d S=\mathbf{0}$, y basta derivar el producto para obtener el resultado deseado. El tensor $\widehat{\mathbf{K}}$ describe la rotación infinitesimal que experimenta el sistema de referencia actual al avanzar $d S$ sobre la directriz, y es, por definición, un objeto espacial. Si un observador ideal avanzase por la directriz deformada, el tensor $\widehat{\boldsymbol{\kappa}}$ representaría la velocidad angular de la base actual. En la literatura [53, 62 se ha denominado curvatura de forma imprecisa, ya que $\widehat{\kappa}$ únicamente coincide con el tensor de curvatura de la directriz deformada cuando no se permite la distorsión de la sección (hipótesis de Love-Kirchhoff). En este trabajo lo llamaremos tensor de cambio de orientación de la sección. Las derivadas de los vectores de la base actual se pueden expresar, por tanto, en función de los propios vectores de la siguiente forma

$$
\frac{d}{d S} \boldsymbol{a}_{K}=\widehat{\kappa} \boldsymbol{a}_{K}
$$

Por otra parte, la retroacción de $\widehat{\boldsymbol{\kappa}}$, empleando la expresión (3.53), produce el objeto material $\widehat{\mathbf{K}}$

$$
\widehat{\mathbf{K}}=\boldsymbol{\Lambda}^{\top} \widehat{\kappa} \boldsymbol{\Lambda}=\boldsymbol{\Lambda}^{\top} \boldsymbol{\Lambda}^{\prime} \boldsymbol{\Lambda}^{\top} \boldsymbol{\Lambda}=\boldsymbol{\Lambda}^{\top} \boldsymbol{\Lambda}^{\prime}
$$

Las componentes de $\widehat{\mathbf{K}}$ en el sistema inicial coinciden con las componentes de $\widehat{\boldsymbol{K}}$ en la base actual.

Es importante señalar que $\widehat{\boldsymbol{K}}$ mide el cambio de orientación final en la configuración actual, sin tener en cuenta la forma original de la pieza, que cuando es curva ya 
lleva asociado un cambio de orientación inicial de la sección transversal. Es interesante cuantificar qué parte del cambio de orientación está directamente relacionada con el cambio de forma de la pieza. Para ello se puede volver a trabajar con el primer sumando teniendo en cuenta que la rotación total se puede descomponer como producto de la inicial y la deformacional. Entonces

$$
\left(\frac{d}{d S} \boldsymbol{\Lambda}\right) \boldsymbol{A}_{K}=\frac{d}{d S}\left(\boldsymbol{\Lambda}_{\mathrm{d}} \boldsymbol{\Lambda}_{0}\right) \boldsymbol{A}_{K}=\boldsymbol{\Lambda}_{\mathrm{d}}^{\prime} \boldsymbol{\Lambda}_{0} \boldsymbol{A}_{K}+\boldsymbol{\Lambda}_{\mathrm{d}} \boldsymbol{\Lambda}_{0}^{\prime} \boldsymbol{A}_{K}
$$

El primer sumando, a su vez, se puede expresar así

$$
\boldsymbol{\Lambda}_{\mathrm{d}}^{\prime} \boldsymbol{\Lambda}_{0} \boldsymbol{A}_{K}=\boldsymbol{\Lambda}_{\mathrm{d}}^{\prime} \boldsymbol{\Lambda}_{\mathrm{d}}^{\top} \boldsymbol{\Lambda}_{\mathrm{d}} \boldsymbol{\Lambda}_{0} \boldsymbol{A}_{K}=\widehat{\boldsymbol{\kappa}}_{\mathrm{d}} \boldsymbol{a}_{K}
$$

Hemos introducido el tensor antisimétrico $\widehat{\mathbf{k}}_{\mathrm{d}}=\boldsymbol{\Lambda}_{\mathrm{d}}^{\prime} \boldsymbol{\Lambda}_{\mathrm{d}}^{\top}$ que mide el cambio de orientación desde la configuración inicial a la actual. Ésta es precisamente la parte del cambio de orientación que produce deformación. El segundo sumando se puede desarrollar así

$$
\boldsymbol{\Lambda}_{\mathrm{d}} \boldsymbol{\Lambda}_{0}^{\prime} \boldsymbol{A}_{K}=\boldsymbol{\Lambda}_{\mathrm{d}} \boldsymbol{\Lambda}_{0}^{\prime} \boldsymbol{\Lambda}_{0}^{\top} \boldsymbol{\Lambda}_{0} \boldsymbol{A}_{K}=\boldsymbol{\Lambda}_{\mathrm{d}} \widehat{\mathbf{k}}_{0} \boldsymbol{\Lambda}_{\mathrm{d}}^{\top} \boldsymbol{\Lambda}_{\mathrm{d}} \boldsymbol{\Lambda}_{0} \boldsymbol{A}_{K}
$$

En él aparece el tensor de curvaturas iniciales $\widehat{\mathbf{k}}_{0}=\boldsymbol{\Lambda}_{0}^{\prime} \boldsymbol{\Lambda}_{0}^{\top}$, introducido anteriormente, que es un objeto perteneciente a la configuración inicial. La acción de la rotación $\boldsymbol{\Lambda}_{\mathrm{d}}$ sobre este objeto proporciona el objeto espacial $\widehat{\boldsymbol{\kappa}}_{0}$

$$
\widehat{\mathbf{k}}_{0}=\boldsymbol{\Lambda}_{\mathrm{d}} \widehat{\mathbf{k}}_{0} \boldsymbol{\Lambda}_{\mathrm{d}}^{\top} \text {. }
$$

Por tanto, el segundo sumando puede escribirse así

$$
\boldsymbol{\Lambda}_{\mathrm{d}} \boldsymbol{\Lambda}_{0}^{\prime} \boldsymbol{A}_{K}=\widehat{\boldsymbol{\kappa}}_{0} \boldsymbol{a}_{K}
$$

De estas expresiones se deduce que el cambio de orientación total se puede descomponer como suma de la curvatura inicial empujada a la configuración actual más el cambio de orientación desde la configuración inicial a la actual que es el asociado a la deformación del sólido.

$$
\widehat{\mathbf{\kappa}}=\widehat{\boldsymbol{\kappa}}_{0}+\widehat{\mathbf{k}}_{\mathrm{d}}
$$

Por tanto las derivadas de los vectores de la base se pueden expresar así

$$
\frac{d}{d S} \boldsymbol{a}_{K}=\widehat{\boldsymbol{\kappa}} \boldsymbol{a}_{K}=\left(\widehat{\boldsymbol{\kappa}}_{0}+\widehat{\boldsymbol{\kappa}}_{\mathrm{d}}\right) \boldsymbol{a}_{K}
$$

\subsubsection{Gradiente de la deformación. Deformaciones generalizadas}

El tensor gradiente de la deformación $\mathbf{F}^{*}$ describe la transformación de los vectores $\boldsymbol{g}_{0}^{* K}$ de la base natural en la configuración inicial en tres vectores $\boldsymbol{f}_{K}^{*}$ asociados a la configuración actual:

$$
\mathbf{F}^{*}=\boldsymbol{f}_{K}^{*} \otimes \boldsymbol{g}_{0}^{* K} .
$$

Expresando los vectores $\boldsymbol{f}_{K}^{*}$ en componentes de la base del espacio, $\boldsymbol{f}_{K}^{*}=\boldsymbol{i}_{a} F_{K}^{a}$ resulta

$$
\mathbf{F}^{*}=\left(\mathrm{F}^{*}\right)^{a}{ }_{K} \boldsymbol{i}_{a} \otimes \boldsymbol{g}_{0}^{* K} .
$$




$$
\boldsymbol{f}_{K}^{*} \otimes \boldsymbol{g}_{0}^{* K}=\frac{\partial \boldsymbol{x}^{*}}{\partial S} \otimes \boldsymbol{g}_{0}^{* 1}+\frac{\partial \boldsymbol{x}^{*}}{\partial X^{2}} \otimes \boldsymbol{g}_{0}^{* 2}+\frac{\partial \boldsymbol{x}^{*}}{\partial X^{3}} \otimes \boldsymbol{g}_{0}^{* 3} .
$$

Teniendo en cuenta (3.37) se tiene

$$
\boldsymbol{f}_{1}^{*}=\frac{\partial \boldsymbol{x}^{*}}{\partial S}=\frac{d}{d S}\left(\boldsymbol{x}+\boldsymbol{\Lambda} \boldsymbol{R}^{*}\right)=\boldsymbol{x}^{\prime}+\boldsymbol{\Lambda}^{\prime} \boldsymbol{R}^{*}
$$

ya que las componentes de $\boldsymbol{R}^{*}$ son independientes de $S$. El segundo término se puede reelaborar de forma que

$$
f_{1}^{*}=x^{\prime}+\Lambda^{\prime} \Lambda^{\top} \Lambda R^{*}=x^{\prime}+\widehat{\kappa} r^{*}
$$

El factor de $\boldsymbol{r}^{*}$ es precisamente el tensor de cambio de orientación total $\widehat{\kappa}$ introducido en la ecuación (3.55). A partir de la expresión del primer vector del gradiente es posible introducir la definición de las deformaciones generalizadas ${ }^{2} \gamma$ y $\widehat{\kappa}$, que son variables espaciales dependientes de $S$

$$
\begin{aligned}
& \gamma(S)=\boldsymbol{x}^{\prime} \\
& \widehat{\boldsymbol{\kappa}}(S)=\boldsymbol{\Lambda}^{\prime} \boldsymbol{\Lambda}^{\top} .
\end{aligned}
$$

La primera variable es el vector tangente a la directriz deformada, y admite una descomposición teniendo en cuenta que sólo una parte está directamente relacionada con la deformación del sólido. El vector tangente a la directriz en la configuración inicial es $\boldsymbol{a}_{01}$; después de la deformación la sección experimenta la rotación deformacional $\boldsymbol{\Lambda}_{\mathrm{d}}$ que transforma $\boldsymbol{a}_{01}$ en el vector normal a la sección transversal deformada $\boldsymbol{a}_{1}$. Si denominamos $\gamma_{0}$ a este objeto espacial, entonces la diferencia entre $\boldsymbol{\gamma}$ y $\gamma_{0}$ es una medida de la distorsión y el alargamiento de la pieza que denominaremos $\gamma_{\mathrm{d}}$. Podemos pues escribir

$$
\boldsymbol{\gamma}=\boldsymbol{\Lambda}_{\mathrm{d}} \boldsymbol{x}_{0}^{\prime}+\gamma_{\mathrm{d}}=\boldsymbol{a}_{1}+\gamma_{\mathrm{d}}=\gamma_{0}+\gamma_{\mathrm{d}} .
$$

Esta expresión es formalmente análoga a la descomposición (3.59) del cambio de orientación del triedro.

La segunda variable es el tensor antisimétrico que mide el cambio en el triedro que define la orientación de la sección desde la configuración de referencia a la actual al avanzar por la directriz, introducido en la sección anterior. Teniendo en cuenta la antisimetría de $\widehat{\boldsymbol{\kappa}}$ se puede introducir el vector $\boldsymbol{\kappa} \in \mathbb{R}^{3}$-vector axial de $\widehat{\boldsymbol{\kappa}}-$, que cumple

$$
\widehat{\kappa} \boldsymbol{v}=\boldsymbol{\kappa} \times \boldsymbol{v} \quad \forall \boldsymbol{v} \in \mathbb{R}^{3} .
$$

En la sección anterior se mostró que $\widehat{\mathbf{k}}$ se puede descomponer en el tensor de curvatura inicial en su forma espacial más el cambio de orientación causante de la deformación $(3.59)$

$$
\widehat{\boldsymbol{\kappa}}=\widehat{\boldsymbol{\kappa}}_{0}+\widehat{\boldsymbol{\kappa}}_{\mathrm{d}}
$$

\footnotetext{
${ }^{2}$ La definición aquí recogida de la deformación generalizada $\gamma$-el vector tangente a la directriz deformada $\boldsymbol{x}^{\prime}$-, que coincide con la empleada por Ritto-Corrêa et al. [53], se diferencia de la definición habitual -utilizada por Simó [58 - heredada de la teoría lineal $\boldsymbol{x}^{\prime}-\boldsymbol{a}_{1}$, que coincide con la variable que luego denominamos $\gamma_{\mathrm{d}}$.
} 
Dado que los tensores $\widehat{\boldsymbol{K}}_{0} \mathrm{y} \widehat{\boldsymbol{\kappa}}_{\mathrm{d}}$ también son antisimétricos es posible definir los vectores axiales $\boldsymbol{\kappa}_{0}$ y $\boldsymbol{\kappa}_{\mathrm{d}}$ correspondientes, que cumplen

$$
\begin{array}{ll}
\widehat{\boldsymbol{\kappa}}_{0} \boldsymbol{v} & =\boldsymbol{\kappa}_{0} \times \boldsymbol{v} \\
\widehat{\boldsymbol{\kappa}}_{\mathrm{d}} \boldsymbol{v} & =\boldsymbol{\kappa}_{\mathrm{d}} \times \boldsymbol{v} \quad \forall \boldsymbol{v} \in \mathbb{R}^{3},
\end{array}
$$

entonces

$$
\kappa=\kappa_{0}+\kappa_{\mathrm{d}} .
$$

Por tanto, la forma espacial del primer vector del gradiente es

$$
\boldsymbol{f}_{1}^{*}=\boldsymbol{\gamma}+\boldsymbol{\kappa} \times \boldsymbol{r}^{*}=\left(\gamma_{0}+\gamma_{\mathrm{d}}\right)+\left(\boldsymbol{\kappa}_{0}+\boldsymbol{\kappa}_{\mathrm{d}}\right) \times \boldsymbol{r}^{*} .
$$

Es interesante notar cómo el segundo sumando de esta expresión tiene la misma estructura que las derivadas de los vectores de la base actual (3.60). Por otra parte, teniendo en cuenta la expresión (3.33) se observa que el segundo y el tercer vector del gradiente coinciden con los vectores respectivos de la base actual

$$
\begin{aligned}
& \boldsymbol{f}_{2}^{*}=\frac{\partial \boldsymbol{x}^{*}}{\partial X^{2}}=\boldsymbol{a}_{2} \\
& \boldsymbol{f}_{3}^{*}=\frac{\partial \boldsymbol{x}^{*}}{\partial X^{3}}=\boldsymbol{a}_{3} .
\end{aligned}
$$

La forma material de $\boldsymbol{f}_{1}^{*}$ se calcula a partir de su retroacción

$$
\begin{aligned}
\boldsymbol{F}_{1}^{*} & =\boldsymbol{\Lambda}^{\top} \boldsymbol{f}_{1}^{*} \\
& =\boldsymbol{\Lambda}^{\top} \boldsymbol{x}^{\prime}+\boldsymbol{\Lambda}^{\top} \widehat{\boldsymbol{K}} \boldsymbol{r}^{*} \\
& =\boldsymbol{\Lambda}^{\top} \boldsymbol{x}^{\prime}+\boldsymbol{\Lambda}^{\top} \widehat{\boldsymbol{\kappa}} \boldsymbol{\Lambda} \boldsymbol{\Lambda}^{\top} \boldsymbol{r}^{*} \\
& =\boldsymbol{\Lambda}^{\top} \boldsymbol{x}^{\prime}+\boldsymbol{\Lambda}^{\top} \widehat{\boldsymbol{K}} \boldsymbol{\Lambda} \boldsymbol{R}^{*} .
\end{aligned}
$$

En esta expresión es posible identificar la forma material de las deformaciones generalizadas, que es efectivamente

$$
\begin{aligned}
\boldsymbol{\Gamma}(S) & =\boldsymbol{\Lambda}^{\top} \boldsymbol{\gamma}=\boldsymbol{\Lambda}^{\top} \boldsymbol{x}^{\prime} \\
\widehat{\mathbf{K}}(S) & =\boldsymbol{\Lambda}^{\top} \widehat{\boldsymbol{K}} \boldsymbol{\Lambda}=\boldsymbol{\Lambda}^{\top} \boldsymbol{\Lambda}^{\prime} .
\end{aligned}
$$

De la misma forma que para la forma espacial, también es posible aquí introducir el vector axial $\boldsymbol{K}$ asociado a $\widehat{\mathbf{K}}$, así como la descomposición de las deformaciones generalizadas en una fracción inicial ${ }^{3}$

$$
\begin{aligned}
\boldsymbol{K}_{0} & =\boldsymbol{\Lambda}^{\top} \boldsymbol{\kappa}_{0}=\boldsymbol{\Lambda}_{0}^{\top} \boldsymbol{k}_{0} \\
\boldsymbol{\Gamma}_{0} & =\boldsymbol{\Lambda}^{\top} \gamma_{0}=\boldsymbol{\Lambda}_{0}^{\top} \boldsymbol{a}_{01}=\boldsymbol{A}_{1},
\end{aligned}
$$

\footnotetext{
${ }^{3}$ Nótese (a) que $\boldsymbol{k}_{0}$ es el vector axial asociado al tensor de curvaturas iniciales $\widehat{\mathbf{k}}_{0}$, así como (b) la coincidencia de la forma material $\boldsymbol{\Gamma}_{0}$ con el primer vector de la base material.
} 
y otra deformacional

$$
\begin{aligned}
\boldsymbol{K}_{\mathrm{d}} & =\boldsymbol{\Lambda}^{\top} \boldsymbol{\kappa}_{\mathrm{d}} \\
\boldsymbol{\Gamma}_{\mathrm{d}} & =\boldsymbol{\Lambda}^{\top} \gamma_{\mathrm{d}} .
\end{aligned}
$$

Entonces, la forma material del primer tensor del gradiente es

$$
\boldsymbol{F}_{1}^{*}=\boldsymbol{\Gamma}+\boldsymbol{K} \times \boldsymbol{R}^{*}=\left(\boldsymbol{\Gamma}_{0}+\boldsymbol{\Gamma}_{\mathrm{d}}\right)+\left(\boldsymbol{K}_{0}+\boldsymbol{K}_{\mathrm{d}}\right) \times \boldsymbol{R}^{*} .
$$

Siguiendo el mismo procedimiento es posible obtener la versión material de los otros dos vectores

$$
\begin{aligned}
& \boldsymbol{F}_{2}^{*}=\boldsymbol{\Lambda}^{\top} \boldsymbol{f}_{2}=\boldsymbol{A}_{2} \\
& \boldsymbol{F}_{3}^{*}=\boldsymbol{\Lambda}^{\top} \boldsymbol{f}_{3}=\boldsymbol{A}_{3} .
\end{aligned}
$$

Recordamos que la expresión de los vectores $\boldsymbol{F}_{K}^{*}$ en componentes del sistema general, coincide con la de los vectores $\boldsymbol{f}_{K}^{*}$ en componentes del sistema actual.

En las figuras 3.5 y 3.6 se puede apreciar el significado físico de las variables introducidas, así como su descomposición en la fracción inicial y la deformacional.

\subsubsection{Objetividad de las medidas de la deformación}

A continuación analizamos una propiedad esencial de las medidas de la deformación introducidas en la sección anterior. Se trata de la objetividad o invarianza respecto a los movimientos de sólido rígido, es decir, la propiedad por la cual el cambio de orientación, y la medida de la distorsión y el alargamiento, en su forma material, no se ven afectadas por la superposición de un movimiento de sólido rígido. En efecto, consideremos la nueva configuración

$$
(\underline{\boldsymbol{x}}, \underline{\boldsymbol{\Lambda}})=(\mathbf{Q} \boldsymbol{x}+\boldsymbol{r}, \mathbf{Q} \boldsymbol{\Lambda}),
$$

que es el resultado de superponer a $(\boldsymbol{x}, \boldsymbol{\Lambda})$ un movimiento de sólido rígido definido por una traslación $\boldsymbol{r}$ y una rotación $\mathbf{Q}$. Si calculamos las deformaciones generalizadas correspondientes a la nueva configuración

$$
\begin{aligned}
& \underline{\boldsymbol{\Gamma}}=\underline{\boldsymbol{\Lambda}}^{\top} \underline{\boldsymbol{x}}^{\prime}=\boldsymbol{\Lambda}^{\top} \mathbf{Q}^{\top} \mathbf{Q}^{\prime} \boldsymbol{x}+\boldsymbol{\Lambda}^{\top} \mathrm{Q}^{\top} \mathrm{Q} \boldsymbol{x}^{\prime}=\boldsymbol{\Gamma} \\
& \underline{\widehat{\mathbf{K}}}=\underline{\boldsymbol{\Lambda}}^{\top} \underline{\boldsymbol{\Lambda}}^{\prime}=\boldsymbol{\Lambda}^{\top} \mathbf{Q}^{\top} \mathbf{Q}^{\prime} \boldsymbol{\Lambda}+\boldsymbol{\Lambda}^{\top} \mathbf{Q}^{\top} \mathbf{Q} \boldsymbol{\Lambda}^{\prime}=\widehat{\mathbf{K}},
\end{aligned}
$$

comprobamos que son idénticas a las de la configuración original. Por ello diremos que las deformaciones generalizadas del modelo geométricamente exacto son objetivas. Este resultado ha sido reflejado por diversos autores como Crisfield y Jelenić [16, Proposicion 3.1] y Ritto-Corrêa et al. [53].

La cuestión de la objetividad volverá a aparecer en el contexto de la solución numérica del problema y la interpolación de las variables de la configuración. 

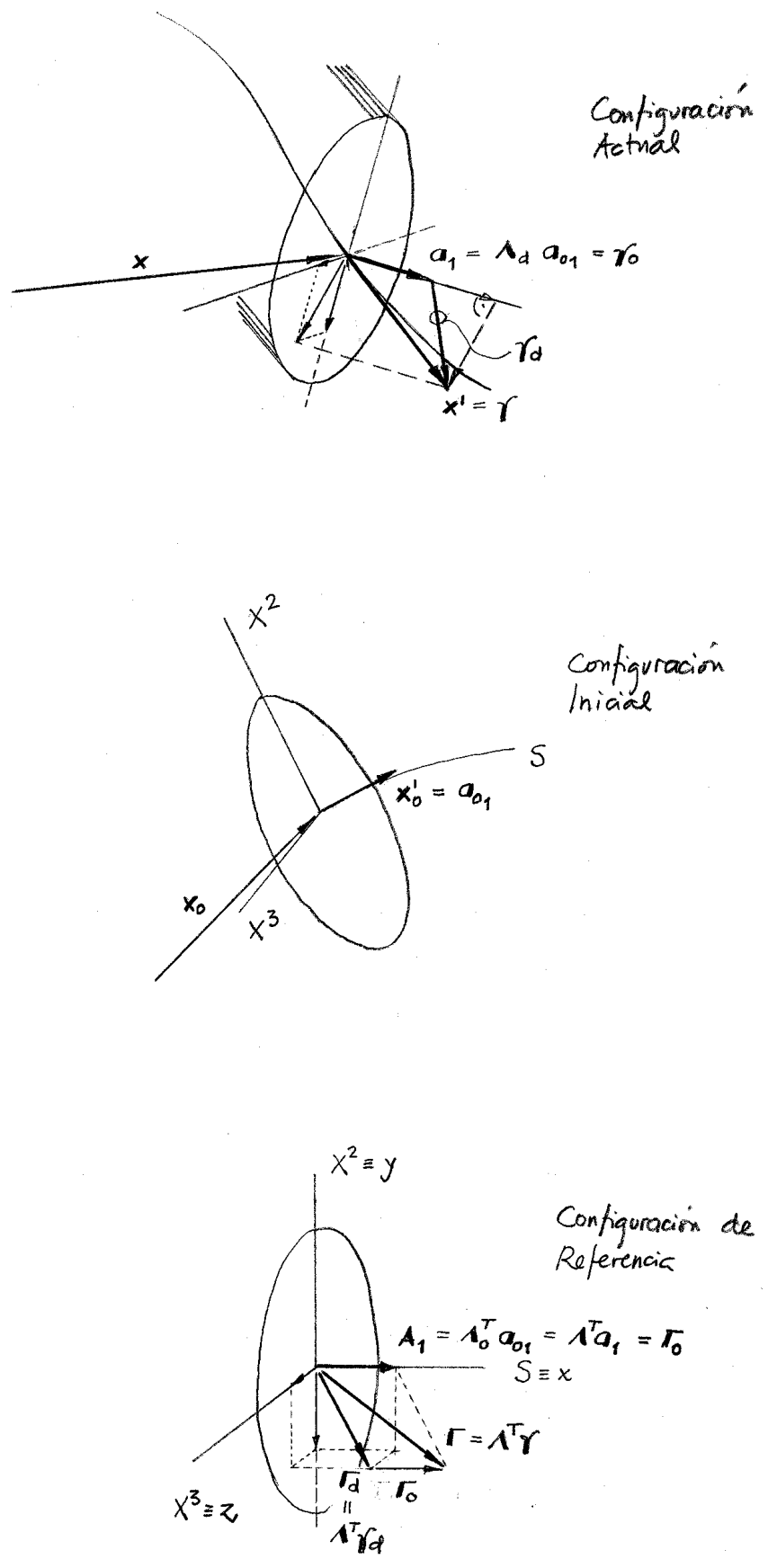

Figura 3.5: Alargamiento y distorsión 

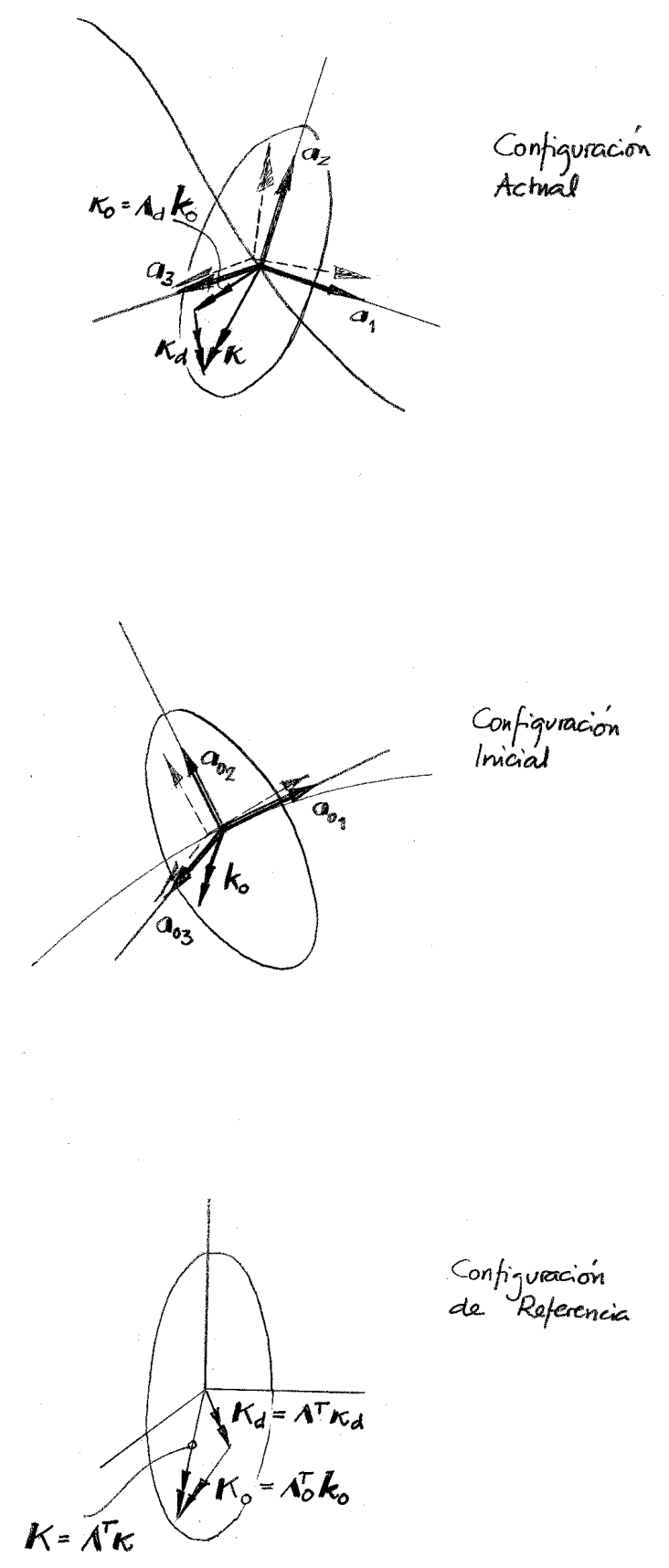

Figura 3.6: Cambio de orientación del sistema de referencia 



\section{Capítulo 4}

\section{Ecuaciones de campo}

El análisis de las condiciones de equilibrio de la pieza alargada con la hipótesis de Reissner-Simó se aborda en este capítulo desde un perspectiva variacional, a partir de la ecuación de trabajos virtuales correspondiente al modelo 1D de la pieza alargada. Su expresión se deduce a partir de la ecuación de trabajos virtuales (ETV) correspondiente al sólido tridimensional. En la formulación tridimensional de la ecuación se ha optado por expresar el trabajo virtual de las fuerzas internas en función del producto contraído del primer tensor de tensiones de Piola-Kirchhoff $\mathbf{P}^{*}$ y la variación del tensor gradiente de la deformación $\delta \mathbf{F}^{*}$. La elección de esta definición de las tensiones responde a su carácter conjugado del gradiente de la deformación [41, cap.3, teorema 2.4], calculado en el capítulo anterior para la cinemática RS. El gradiente se expresa en función de las deformaciones generalizadas, que son las variables del modelo que describen el cambio de forma del sólido. Una de las cuestiones cruciales en este capítulo se plantea al transformar el término del trabajo virtual de las fuerzas internas con el fin de expresarlo en función de las variables del modelo, para lo que será necesario introducir una nueva definición de variación de objetos tangentes a la configuración actual que se aplicará al cálculo de la variación de las deformaciones generalizadas. Una vez formulada la versión 1D del principio de trabajos virtuales, el cálculo de variaciones permite deducir las ecuaciones de equilibrio del modelo RS en su forma espacial y material. La expresión de las ecuaciones constitutivas del modelo completa el conjunto de ecuaciones que rigen la estática de la pieza alargada.

\subsection{Construcción de la ecuación de trabajos virtuales}

El punto de partida del desarrollo es la ecuación de trabajos virtuales en el sólido. Ésta indica que $\boldsymbol{x}^{*}$ es una configuración de equilibrio si, y sólo si,

$$
\int_{V_{0}} \mathbf{P}^{*}: \delta \mathbf{F}^{*} d V_{0}^{*}=\int_{V_{0}} \boldsymbol{b}^{*} \cdot \delta \boldsymbol{x}^{*} d V_{0}^{*}+\int_{\partial V_{0}} \overline{\boldsymbol{t}}^{*} \cdot \delta \boldsymbol{x}^{*} d A_{0}^{*}
$$

para cualquier variación cinemáticamente admisible $\delta \boldsymbol{x}^{*}$ de la configuración. $V_{0}$ y $\partial V_{0}$ son, respectivamente, el volumen y el contorno de la pieza en la configuración inicial. $\boldsymbol{b}^{*}\left(S, X^{2}, X^{3}\right)$ es el campo de fuerzas de volumen, $\overline{\boldsymbol{t}}^{*}\left(S, X^{2}, X^{3}\right)$ es el campo 
de fuerzas de superficie, y $\delta \mathbf{F}^{*}=\partial \delta \boldsymbol{x}^{*} / \partial \boldsymbol{X}$ es la variación del gradiente de la deformación. Es necesario remarcar que, si bien las variables estáticas y cinemáticas residen en la configuración actual, se entienden como funciones de las variables independientes $S, X^{2}, X^{3}$ asociadas a la geometría de la configuración inicial, puesto que la formulación del problema es lagrangiana.

\subsubsection{Variación de la configuración}

En primer lugar se calcula la variación de la posición de los puntos materiales de la pieza, que denominaremos variación de la configuración. La hipótesis cinemática viene dada por la expresión (3.37) $\boldsymbol{x}^{*}=\boldsymbol{x}+\boldsymbol{r}^{*}$, escrita en componentes del sistema de referencia general. La variación de $\boldsymbol{x}^{*}$ se deduce a partir de la cinemática, y de la definición de acción de $\boldsymbol{\Lambda}$ sobre $\boldsymbol{R}^{*}$, ya que $\boldsymbol{r}^{*}=\boldsymbol{\Lambda} \boldsymbol{R}^{*}$. Además es necesario tener en cuenta que sólo las variables $\boldsymbol{x}$ y $\boldsymbol{\Lambda}$ son objeto de variación; la variación $\delta \boldsymbol{R}^{*}$ es nula, puesto que $\boldsymbol{R}^{*}$ pertenece a la pieza ideal

$$
\begin{aligned}
\delta \boldsymbol{x}^{*} & =\delta \boldsymbol{x}+\delta\left(\boldsymbol{\Lambda} \boldsymbol{R}^{*}\right) \\
& =\delta \boldsymbol{x}+\delta \boldsymbol{\Lambda} \boldsymbol{\Lambda}^{\top} \boldsymbol{\Lambda} \boldsymbol{R}^{*} \\
& =\delta \boldsymbol{x}+\delta \widehat{\boldsymbol{\omega}} \boldsymbol{r}^{*} .
\end{aligned}
$$

En esta expresión se introduce la nueva variable espacial $\delta \widehat{\boldsymbol{\omega}} \in \mathfrak{s o}(3)$

$$
\delta \widehat{\boldsymbol{\omega}}=\delta \boldsymbol{\Lambda}^{\top},
$$

denominada en la literatura rotación virtual ${ }^{1}$ o spin [53], que es una matriz antisimétrica cuyo vector axial $\delta \boldsymbol{\omega}$ queda definido por la relación

$$
\delta \widehat{\boldsymbol{\omega}} \boldsymbol{v}=\delta \boldsymbol{\omega} \times \boldsymbol{v} \quad \forall \boldsymbol{v} \in \mathbb{R}^{3} .
$$

Por lo tanto, la variación de $\boldsymbol{x}^{*}$ se puede escribir así

$$
\delta \boldsymbol{x}^{*}=\delta \boldsymbol{x}+\delta \boldsymbol{\omega} \times \boldsymbol{r}^{*} .
$$

Es fundamental señalar, como se indica en [52, p.735] que, a pesar de la notación empleada, no existe una función $\boldsymbol{\omega}$ asociada a $\delta \boldsymbol{\omega}$. La rotación virtual también se puede

\footnotetext{
${ }^{1}$ Por otra parte, volviendo al cálculo de la variación de la configuración, el segundo sumando se puede desarrollar a partir de la descomposición de la rotación total en la parte inicial y la deformacional

$$
\delta\left(\boldsymbol{\Lambda} \boldsymbol{R}^{*}\right)=\delta\left(\boldsymbol{\Lambda}_{\mathrm{d}} \boldsymbol{\Lambda}_{0} \boldsymbol{R}^{*}\right)
$$

Dado que la rotación inicial $\boldsymbol{\Lambda}_{0}$ es un dato, no está sujeta a variación. Desarrollando la expresión anterior

$$
\begin{aligned}
\delta\left(\boldsymbol{\Lambda} \boldsymbol{R}^{*}\right) & =\delta \boldsymbol{\Lambda}_{\mathrm{d}} \boldsymbol{\Lambda}_{0} \boldsymbol{R}^{*} \\
& =\delta \boldsymbol{\Lambda}_{\mathrm{d}} \boldsymbol{\Lambda}_{\mathrm{d}}^{\top} \boldsymbol{\Lambda}_{\mathrm{d}} \boldsymbol{\Lambda}_{0} \boldsymbol{R}^{*} \\
& =\delta \widehat{\boldsymbol{\omega}}_{\mathrm{d}} \boldsymbol{r}^{*} .
\end{aligned}
$$

Por lo tanto, comparando con la expresión obtenida antes para el segundo sumando se deduce que la variación de la rotación deformacional, $\delta \widehat{\boldsymbol{\omega}}_{\mathrm{d}}=\delta \boldsymbol{\Lambda}_{\mathrm{d}} \boldsymbol{\Lambda}_{\mathrm{d}}^{\top}$, es igual a la variación de la rotación total, $\delta \widehat{\boldsymbol{\omega}}$.
} 
introducir calculando directamente la variación de la rotación $\boldsymbol{\Lambda}$ en la dirección del vector $\delta \widehat{\boldsymbol{\omega}}$ (elemento de $\mathfrak{s o}(3))$ tangente al grupo de las rotaciones $\mathrm{SO}(3)$ ([58], [27])

$$
\begin{aligned}
\delta \boldsymbol{\Lambda} & =\left.\frac{d}{d \varepsilon} \boldsymbol{\Lambda}_{\varepsilon}\right|_{\varepsilon=0} \\
& =\left.\frac{d}{d \varepsilon}[\exp (\varepsilon \delta \widehat{\boldsymbol{\omega}}) \boldsymbol{\Lambda}]\right|_{\varepsilon=0} \\
& =\delta \widehat{\boldsymbol{\omega}} \boldsymbol{\Lambda} .
\end{aligned}
$$

\subsubsection{Definición de la variación corrotacional}

Cuando en la sección siguiente estudiemos las ecuaciones constitutivas, veremos cómo la expresión natural de las mismas se obtiene empleando la forma material de las deformaciones generalizadas y de los esfuerzos (fuerzas internas del modelo); ambas se relacionan directamente con la forma material de los vectores $\boldsymbol{F}_{K}^{*}$ del gradiente de la deformación y $\boldsymbol{P}^{* K}$ del primer tensor de tensiones de Piola-Kirchhoff. Esta situación aconseja, para analizar el integrando de la expresión del trabajo virtual de las fuerzas internas $\mathbf{P}^{*}: \delta \mathbf{F}^{*}$, emplear la forma material de la descomposición de los tensores $\mathbf{P}^{*}$ y $\mathbf{F}^{*}$ de forma que

$$
\begin{aligned}
\mathbf{P}^{*} & =\boldsymbol{P}^{* K} \otimes \boldsymbol{g}_{0 K}^{*} \\
\delta \mathbf{F}^{*} & =\delta \boldsymbol{F}_{K}^{*} \otimes \boldsymbol{g}_{0}^{* K} .
\end{aligned}
$$

En la variación del gradiente se ha tenido en cuenta que los vectores de base natural inicial no están sujetos a variación ya que no dependen de las variables de la configuración. Con esta descomposición, el producto contraído del integrando se convierte en la suma de tres productos escalares

$$
\mathbf{P}^{*}: \delta \mathbf{F}^{*}=\boldsymbol{P}^{* K} \cdot \delta \boldsymbol{F}_{K}^{*}
$$

Consideremos ahora una expresión genérica con la misma estructura que uno de los sumandos de la expresión anterior

$$
\boldsymbol{W} \cdot \delta \boldsymbol{V}
$$

Se trata del producto escalar de dos vectores en su forma material. El resultado del producto debe ser independiente de la forma en la que expresemos los vectores (material o espacial), ya que la transformación entre una y otra equivale a un cambio de base. Podemos pues operar en la expresión anterior para obtener su forma espacial

$$
\boldsymbol{W} \cdot \delta \boldsymbol{V}=\boldsymbol{W}^{\top} \delta \boldsymbol{V}=\boldsymbol{w}^{\top} \boldsymbol{\Lambda} \delta\left(\boldsymbol{\Lambda}^{\top} \boldsymbol{v}\right)=\boldsymbol{w} \cdot \boldsymbol{\Lambda} \delta\left(\boldsymbol{\Lambda}^{\top} \boldsymbol{v}\right)
$$

Este resultado sugiere la introducción de una nueva definición de variación que actuará sobre objetos espaciales. Si $\boldsymbol{v}$ es un vector tangente definido en la configuración deformada - un objeto espacial-, entonces la variación corrotacional de $\boldsymbol{v}$ se define del siguiente modo

$$
\stackrel{\nabla}{\delta} \boldsymbol{v}=\boldsymbol{\Lambda} \delta\left(\boldsymbol{\Lambda}^{\top} \boldsymbol{v}\right)=\boldsymbol{\Lambda} \delta \boldsymbol{V}
$$


en esta definición se observa que, antes de calcular la variación, se retrotrae $\boldsymbol{v}$ a la configuración inicial, obteniendo el objeto material $\boldsymbol{V}$. El resultado de la variación del objeto material, se empuja de nuevo a la configuración espacial evaluando la acción de $\boldsymbol{\Lambda}$ sobre $\delta \boldsymbol{V}$. Con esta definición la expresión anterior resulta ${ }^{2}$

$$
\boldsymbol{W} \cdot \delta \boldsymbol{V}=\boldsymbol{w} \cdot \stackrel{\nabla}{\delta} \boldsymbol{v}
$$

A partir del resultado anterior es inmediato deducir que

$$
\mathbf{P}^{*}: \delta \mathbf{F}^{*}=\boldsymbol{p}^{* K} \cdot \stackrel{\nabla}{\delta} \boldsymbol{f}_{K}^{*}
$$

Por otra parte, la variación de un tensor de segundo orden $\mathbf{h}$ tangente a la configuración actual se calcula por el mismo procedimiento

$$
\stackrel{\nabla}{\delta} \mathbf{h}=\boldsymbol{\Lambda} \delta\left(\boldsymbol{\Lambda}^{\top} \mathbf{h} \boldsymbol{\Lambda}\right) \boldsymbol{\Lambda}^{\top}=\boldsymbol{\Lambda} \delta \mathbf{H} \boldsymbol{\Lambda}^{\top}
$$

Por la forma de definirlo $\stackrel{\nabla}{\delta}(\cdot)$ es un objeto espacial igual a la variación de $(\cdot)$ desde el punto de vista de un observador que se mueve junto con la base actual. El nombre de variación corrotacional (co-rotational variation) fue introducido por Cardona y Geradin [11, p.2417]; Ritto-Corrêa et al. [53, p.1015] la denominan co-rotated virtual rate. Podemos evaluar la variación corrotacional a partir de su definición (los detalles técnicos del desarrollo siguiente se pueden encontrar en la proposición 4.2.1)

$$
\stackrel{\nabla}{\delta} \boldsymbol{v}=\boldsymbol{\Lambda} \delta\left(\boldsymbol{\Lambda}^{\top} \boldsymbol{v}\right)=\delta \boldsymbol{v}-\delta \boldsymbol{\Lambda} \boldsymbol{\Lambda}^{\top} \boldsymbol{v}=\delta \boldsymbol{v}-\delta \boldsymbol{\omega} \times \boldsymbol{v}
$$

El resultado tiene dos sumandos: el primero es la variación de $\boldsymbol{v}$ desde el punto de vista de un observador situado en el sistema de referencia fijo del espacio. El segundo es la corrección debida al movimiento de la base actual a medida que el observador avanza por la directriz de la pieza deformada. La variación corrotacional de $\boldsymbol{v}$ es, por tanto, la variación desde el punto de vista de un observador situado en el sistema de referencia de la sección.

En este sentido se obtiene una expresión que es formalmente equivalente a la denominada derivada corrotacional de un vector (derivada desde el punto de vista del observador que se encuentra en el sistema de referencia móvil). Simó et al. [62] emplean esta definición (corrotated rate) en el contexto dinámico. En el marco de la estática resultará ilustrativo analizar la composición de la derivada de un vector tangente a la configuración actual respecto de la variable $S$.

$$
\begin{aligned}
\frac{d}{d S} \boldsymbol{v}=\frac{d}{d S}(\boldsymbol{\Lambda} \boldsymbol{V}) & =\frac{d}{d S} \boldsymbol{\Lambda} \boldsymbol{V}+\boldsymbol{\Lambda} \frac{d}{d S} \boldsymbol{V} \\
& =\frac{d}{d S} \boldsymbol{\Lambda} \boldsymbol{\Lambda}^{\top} \boldsymbol{\Lambda} \boldsymbol{V}+\boldsymbol{\Lambda} \frac{d}{d S} \boldsymbol{V} \\
& =\boldsymbol{\kappa} \times \boldsymbol{v}+\boldsymbol{\Lambda} \frac{d}{d S}\left(\boldsymbol{\Lambda}^{\top} \boldsymbol{v}\right) .
\end{aligned}
$$

\footnotetext{
${ }^{2}$ Es necesario remarcar que, en contra de lo que se concluiría con un simple cambio de notación $\boldsymbol{W} \cdot \delta \boldsymbol{V} \neq \boldsymbol{w} \cdot \delta \boldsymbol{v}$.
} 
El segundo sumando es precisamente la derivada corrotacional de $\boldsymbol{v}$, es decir la derivada desde el punto de vista de un observador que avanza por la directriz montado en el sistema de referencia de la sección. El primer sumando es la corrección por la rotación de la base actual, que se expresa en función del cambio de orientación de ésta $(\boldsymbol{\kappa})$. Esta descomposición resultará útil más adelante para entender la relación entre la forma espacial y material de las ecuaciones de equilibrio del modelo ${ }^{3}$. De forma abreviada, la expresión de la derivada corrotacional es

$$
\stackrel{\nabla}{v}=v^{\prime}-\kappa \times v
$$

\subsubsection{Variación del gradiente de la deformación}

A continuación se evalúa la variación del gradiente de la deformación a partir de su expresión $\mathbf{F}^{*}=\boldsymbol{f}_{K}^{*} \otimes \boldsymbol{g}_{0}^{* K}$, con

$$
\begin{aligned}
& \boldsymbol{f}_{1}^{*}=\boldsymbol{\gamma}+\widehat{\boldsymbol{\kappa}} \boldsymbol{r}^{*} \\
& \boldsymbol{f}_{2}^{*}=\boldsymbol{a}_{2} \\
& \boldsymbol{f}_{3}^{*}=\boldsymbol{a}_{3}
\end{aligned}
$$

dados por las ecuaciones (3.70) y (3.71). Con la definición de variación corrotacional introducida en la sección anterior la variación del tensor gradiente de la deformación se puede expresar así a partir de la ecuación (3.63)

$$
\delta \mathbf{F}^{*}=\stackrel{\nabla}{\delta} \boldsymbol{f}_{K}^{*} \otimes \boldsymbol{g}_{0}^{* K} .
$$

La variación corrotacional de $\boldsymbol{f}_{1}^{*}$ es

$$
\stackrel{\nabla}{\delta} \boldsymbol{f}_{1}^{*}=\stackrel{\nabla}{\delta} \boldsymbol{\gamma}+\stackrel{\nabla}{\delta} \widehat{\boldsymbol{\kappa}} \boldsymbol{r}^{*}+\widehat{\boldsymbol{\kappa}} \stackrel{\nabla}{\delta} \boldsymbol{r}^{*}
$$

La variación $\stackrel{\nabla}{\delta} \boldsymbol{r}^{*}$ es nula, puesto que $\stackrel{\nabla}{\delta} \boldsymbol{r}=\boldsymbol{\Lambda} \delta \boldsymbol{R}^{*}$, y $\delta \boldsymbol{R}^{*}=\mathbf{0}$. Entonces

$$
\stackrel{\nabla}{\delta} \boldsymbol{f}_{1}^{*}=\stackrel{\nabla}{\delta} \boldsymbol{\gamma}+\stackrel{\nabla}{\delta} \widehat{\boldsymbol{\kappa}} \boldsymbol{r}^{*}=\stackrel{\nabla}{\delta} \boldsymbol{\gamma}+\stackrel{\nabla}{\delta} \boldsymbol{\kappa} \times \boldsymbol{r}^{*}
$$

donde se ha introducido el vector axial $\stackrel{\nabla}{\delta} \boldsymbol{\kappa}$ correspondiente a $\stackrel{\nabla}{\delta} \widehat{\boldsymbol{\kappa}} \in \mathfrak{s o}(3)$ por conveniencia. Por otra parte, las variaciones espaciales del segundo y el tercer vector son nulas puesto que

$$
\begin{aligned}
& \stackrel{\nabla}{\delta} \boldsymbol{f}_{2}^{*}=\boldsymbol{\Lambda} \delta \boldsymbol{A}_{2}=\mathbf{0} \\
& \stackrel{\nabla}{\delta} \boldsymbol{f}_{3}^{*}=\boldsymbol{\Lambda} \delta \boldsymbol{A}_{3}=\mathbf{0} .
\end{aligned}
$$

\footnotetext{
${ }^{3}$ La estructura de las ecuaciones es idéntica en ambos casos, si en la forma espacial se sustituye la derivada de los esfuerzos $\boldsymbol{n}$ y $\boldsymbol{m}$ por su descomposición como suma de la derivada corrotacional y el término corrector, como podrá comprobarse más adelante
} 


\subsubsection{Ecuación de trabajos virtuales del modelo RS}

\section{Forma espacial}

Introduciendo las expresiones de las variaciones (4.5), (4.15) y (4.17) en la ecuación de trabajos virtuales del sólido se puede expresar cada uno de sus términos en función de las variables generalizadas asociadas a la cinemática RS. El término de fuerzas de volumen resulta

$$
\begin{aligned}
\int_{V_{0}} \boldsymbol{b}^{*} \cdot \delta \boldsymbol{x}^{*} d V_{0}^{*} & =\int_{\Gamma_{0}} \int_{A_{s}} \boldsymbol{b}^{*} \cdot \delta \boldsymbol{x} \sqrt{\mathrm{g}_{0}^{*}} d X^{2} d X^{3} d S \\
& +\int_{\Gamma_{0}} \int_{A_{s}} \boldsymbol{b}^{*} \cdot\left(\delta \boldsymbol{\omega} \times \boldsymbol{r}^{*}\right) \sqrt{\mathrm{g}_{0}^{*}} d X^{2} d X^{3} d S \\
& =\int_{\Gamma_{0}}\left(\int_{A_{s}} \boldsymbol{b}^{*} \sqrt{\mathrm{g}_{0}^{*}} d X^{2} d X^{3}\right) \cdot \delta \boldsymbol{x} d S \\
& +\int_{\Gamma_{0}}\left(\int_{A_{s}} \boldsymbol{r}^{*} \times \boldsymbol{b}^{*} \sqrt{\mathrm{g}_{0}^{*}} d X^{2} d X^{3}\right) \cdot \delta \boldsymbol{\omega} d S
\end{aligned}
$$

El término correspondiente a las fuerzas de superficie se descompone en una integral sobre el contorno lateral y dos integrales sobre las secciones extremas

$$
\int_{\partial V_{0}} \overline{\boldsymbol{t}}^{*} \cdot \delta \boldsymbol{x}^{*} d A_{0}^{*}=\int_{\partial \bar{V}_{0}} \overline{\boldsymbol{t}}^{*} \cdot \delta \boldsymbol{x}^{*} \bar{\mu} d \bar{S} d S+\int_{A_{1}} \overline{\boldsymbol{t}}^{*} \cdot \delta \boldsymbol{x}^{*} d A_{0}^{*}+\int_{A_{2}} \overline{\boldsymbol{t}}^{*} \cdot \delta \boldsymbol{x}^{*} d A_{0}^{*} .
$$

A su vez,

$$
\begin{aligned}
\int_{\partial \bar{V}_{0}} \overline{\boldsymbol{t}}^{*} \cdot \delta \boldsymbol{x}^{*} \bar{\mu} d \bar{S} d S & =\int_{\Gamma_{0}} \int_{\partial A_{s}} \overline{\boldsymbol{t}}^{*} \cdot \delta \boldsymbol{x} \bar{\mu} d \bar{S} d S \\
& +\int_{\Gamma_{0}} \int_{\partial A_{s}} \overline{\boldsymbol{t}}^{*} \cdot\left(\delta \boldsymbol{\omega} \times \boldsymbol{r}^{*}\right) \bar{\mu} d \bar{S} d S \\
& =\int_{\Gamma_{0}}\left(\int_{\partial A_{s}} \overline{\boldsymbol{t}}^{*} \bar{\mu} d \bar{S}\right) \cdot \delta \boldsymbol{x} d S \\
& +\int_{\Gamma_{0}}\left(\int_{\partial A_{s}} \boldsymbol{r}^{*} \times \overline{\boldsymbol{t}}^{*} \bar{\mu} d \bar{S}\right) \cdot \delta \boldsymbol{\omega} d S
\end{aligned}
$$

por tanto

$$
\begin{aligned}
\int_{\partial V_{0}} \overline{\boldsymbol{t}}^{*} \cdot \delta \boldsymbol{x}^{*} d A_{0}^{*} & =\int_{\Gamma_{0}}\left(\int_{\partial A_{s}} \overline{\boldsymbol{t}}^{*} \bar{\mu} d \bar{S}\right) \cdot \delta \boldsymbol{x} d S \\
& +\int_{\Gamma_{0}}\left(\int_{\partial A_{s}} \boldsymbol{r}^{*} \times \overline{\boldsymbol{t}}^{*} \bar{\mu} d \bar{S}\right) \cdot \delta \boldsymbol{\omega} d S \\
& +\int_{A_{1}} \overline{\boldsymbol{t}}^{*} d A_{0}^{*} \cdot \delta \boldsymbol{x}\left(S_{1}\right)+\int_{A_{1}} \boldsymbol{r}^{*} \times \overline{\boldsymbol{t}}^{*} d A_{0}^{*} \cdot \delta \boldsymbol{\omega}\left(S_{1}\right) \\
& +\int_{A_{2}} \overline{\boldsymbol{t}}^{*} d A_{0}^{*} \cdot \delta \boldsymbol{x}\left(S_{2}\right)+\int_{A_{2}} \boldsymbol{r}^{*} \times \overline{\boldsymbol{t}}^{*} d A_{0}^{*} \cdot \delta \boldsymbol{\omega}\left(S_{2}\right) .
\end{aligned}
$$


Por último, teniendo en cuenta que el primer tensor de Piola-Kirchhoff puede expresarse como $\mathbf{P}^{*}=\boldsymbol{p}^{* K} \otimes \boldsymbol{g}_{0 K}^{*}$, y el resultado (4.9), el término correspondiente al trabajo virtual de las tensiones es

$$
\begin{aligned}
\int_{V_{0}} \mathbf{P}^{*}: \delta \mathbf{F}^{*} d V & =\int_{\Gamma_{0}} \int_{A_{s}} \boldsymbol{p}^{* 1} \cdot \stackrel{\nabla}{\delta} \boldsymbol{f}_{1}^{*} \sqrt{\mathrm{g}_{0}^{*}} d X^{2} d X^{3} d S \\
& =\int_{\Gamma_{0}}\left(\int_{A_{s}} \boldsymbol{p}^{* 1} \sqrt{\mathrm{g}_{0}^{*}} d X^{2} d X^{3}\right) \cdot \stackrel{\nabla}{\delta} \boldsymbol{\gamma} d S \\
& +\int_{\Gamma_{0}}\left(\int_{A_{s}} \boldsymbol{r}^{*} \times \boldsymbol{p}^{* 1} \sqrt{\mathrm{g}_{0}^{*}} d X^{2} d X^{3}\right) \cdot \stackrel{\nabla}{\delta} \boldsymbol{\kappa} d S .
\end{aligned}
$$

Finalmente resulta la siguiente expresión:

\section{Forma espacial de la ETV para el modelo de RS}

La configuración $(\boldsymbol{x}, \boldsymbol{\Lambda})$ es una configuración de equilibrio si, y sólo si,

$$
\begin{aligned}
\int_{\Gamma_{0}}(\boldsymbol{n} \cdot \stackrel{\nabla}{\delta} \gamma & +\boldsymbol{m} \cdot \stackrel{\nabla}{\delta} \boldsymbol{\kappa}) d S \\
=\int_{\Gamma_{0}}\left(\boldsymbol{q}_{n} \cdot \delta \boldsymbol{x}+\boldsymbol{q}_{m} \cdot \delta \boldsymbol{\omega}\right) d S & +\boldsymbol{n}_{1} \cdot \delta \boldsymbol{x}\left(S_{1}\right)+\boldsymbol{n}_{2} \cdot \delta \boldsymbol{x}\left(S_{2}\right) \\
& +\boldsymbol{m}_{1} \cdot \delta \boldsymbol{\omega}\left(S_{1}\right)+\boldsymbol{m}_{2} \cdot \delta \boldsymbol{\omega}\left(S_{2}\right),
\end{aligned}
$$

para cualquier variación cinemáticamente admisible $(\delta \boldsymbol{x}, \delta \boldsymbol{\omega})$.

Las variables estáticas que intervienen en el principio 1D se interpretan a continuación:

- Esfuerzos. Las resultantes de los vectores tensión que actúan sobre la sección en la configuración deformada ponderadas mediante la hipótesis cinemática se denominan $\boldsymbol{n}$ y $\boldsymbol{m}$, y coinciden con la definición clásica de los esfuerzos. En efecto, si denotamos $\boldsymbol{t}^{* 1}$ al vector tensión, medido por unidad de superficie inicial, que actúa en cada punto de la sección transversal deformada, y recordamos que $\boldsymbol{a}_{0}{ }^{1}$ es el vector unitario normal en cualquier punto de la sección inicial, entonces

$$
\boldsymbol{t}^{* 1} d A_{0}^{*}=\mathbf{P}^{*} \boldsymbol{a}_{0}{ }^{1} d A_{0}^{*}=\mathbf{P}^{*} \boldsymbol{g}_{0}^{* 1} \frac{d A_{0}^{*}}{\sqrt{\left(\mathrm{g}_{0}^{*}\right)^{11}}}
$$

es la fuerza que se ejerce sobre cada diferencial de superficie deformado que inicialmente pertenece a una sección transversal de la pieza. Por lo tanto, se puede escribir

$$
\boldsymbol{t}^{* 1} d A_{0}^{*}=\left(\boldsymbol{p}^{* K} \otimes \boldsymbol{g}_{0 K}^{*}\right) \boldsymbol{g}_{0}^{* 1} \frac{d A_{0}^{*}}{\sqrt{\left(\mathrm{g}_{0}^{*}\right)^{11}}}=\boldsymbol{p}^{* 1} \frac{d A_{0}^{*}}{\sqrt{\left(\mathrm{g}_{0}^{*}\right)^{11}}} .
$$

En el capítulo anterior dedujimos que $\sqrt{\left(\mathrm{g}_{0}^{*}\right)^{11}}=1 / \sqrt{\mathrm{g}_{0}^{*}}$, con lo que finalmente se obtiene

$$
p^{* 1} \sqrt{\mathrm{g}_{0}^{*}}=\boldsymbol{t}^{* 1} \text {. }
$$


Teniendo en cuenta que el diferencial de superficie sobre la sección transversal es

$$
d A_{0}^{*}=d X^{2} d X^{3}
$$

se puede escribir

$$
\begin{aligned}
\boldsymbol{n} & =\int_{A_{s}} \boldsymbol{p}^{* 1} \sqrt{\mathrm{g}_{0}^{*}} d X^{2} d X^{3}=\int_{A_{s}} \boldsymbol{t}^{* 1} d A_{0}^{*} \\
\boldsymbol{m} & =\int_{A_{s}} \boldsymbol{r}^{*} \times \boldsymbol{p}^{* 1} \sqrt{\mathrm{g}_{0}^{*}} d X^{2} d X^{3}=\int_{A_{s}} \boldsymbol{r}^{*} \times \boldsymbol{t}^{* 1} d A_{0}^{*} .
\end{aligned}
$$

- Fuerzas y momentos sobre las secciones extremas. Las expresiones anteriores permiten definir de forma análoga las resultantes de las fuerzas de superficie sobre las secciones extremas de la pieza (fuerzas y momentos)

$$
\begin{aligned}
\boldsymbol{n}_{1} & =\int_{A_{1}} \overline{\boldsymbol{t}} d A_{0}^{*} & \boldsymbol{n}_{2} & =\int_{A_{2}} \overline{\boldsymbol{t}} d A_{0}^{*} \\
\boldsymbol{m}_{1} & =\int_{A_{1}} \boldsymbol{r}^{*} \times \overline{\boldsymbol{t}}^{*} d A_{0}^{*} & \boldsymbol{m}_{2} & =\int_{A_{2}} \boldsymbol{r}^{*} \times \overline{\boldsymbol{t}}^{*} d A_{0}^{*} .
\end{aligned}
$$

- Fuerzas generalizadas. Por último, se definen las fuerzas y momentos por unidad de longitud de la directriz originadas por las fuerzas de volumen y las fuerzas de superficie sobre el contorno lateral de la pieza, a partir de las expresiones restantes

$$
\begin{aligned}
\boldsymbol{q}_{n} & =\int_{A_{s}} \boldsymbol{b}^{*} \sqrt{\mathrm{g}_{0}^{*}} d X^{2} d X^{3}+\int_{\partial A_{s}} \overline{\boldsymbol{t}}^{*} \bar{\mu} d \bar{S} \\
\boldsymbol{q}_{m} & =\int_{A_{s}} \boldsymbol{r}^{*} \times \boldsymbol{b}^{*} \sqrt{\mathrm{g}_{0}^{*}} d X^{2} d X^{3}+\int_{\partial A_{s}} \boldsymbol{r}^{*} \times \overline{\boldsymbol{t}}^{*} \bar{\mu} d \bar{S}
\end{aligned}
$$

\section{Forma material}

La forma material del PTV del modelo se obtiene haciendo uso de la retroacción de las variables que intervienen en la forma espacial del principio. Comenzamos definiendo la retroacción de las variaciones de la configuración, $\delta \boldsymbol{x}$ y $\delta \widehat{\boldsymbol{\omega}}$.

$$
\begin{aligned}
\delta \chi & =\boldsymbol{\Lambda}^{\top} \delta \boldsymbol{x} \\
\delta \widehat{\boldsymbol{\Omega}} & =\boldsymbol{\Lambda}^{\top} \delta \widehat{\boldsymbol{\omega}} \boldsymbol{\Lambda}=\boldsymbol{\Lambda}^{\top} \delta \boldsymbol{\Lambda} .
\end{aligned}
$$

La última definición constituye la forma material de la variación de la rotación. De manera análoga a la forma espacial (4.6), la forma material de la rotación virtual se puede definir como vector tangente al grupo $\mathrm{SO}(3)$ en el elemento neutro del grupo. Así, para calcular la derivada de $\boldsymbol{\Lambda}$ en la dirección $\delta \widehat{\boldsymbol{\Omega}}$, la exponencial de esta última actúa en primer lugar

$$
\begin{aligned}
\delta \boldsymbol{\Lambda} & =\left.\frac{d}{d \varepsilon} \boldsymbol{\Lambda}_{\varepsilon}\right|_{\varepsilon=0} \\
& =\left.\frac{d}{d \varepsilon}[\boldsymbol{\Lambda} \exp (\varepsilon \delta \widehat{\boldsymbol{\Omega}})]\right|_{\varepsilon=0} \\
& =\boldsymbol{\Lambda} \delta \widehat{\boldsymbol{\Omega}} .
\end{aligned}
$$




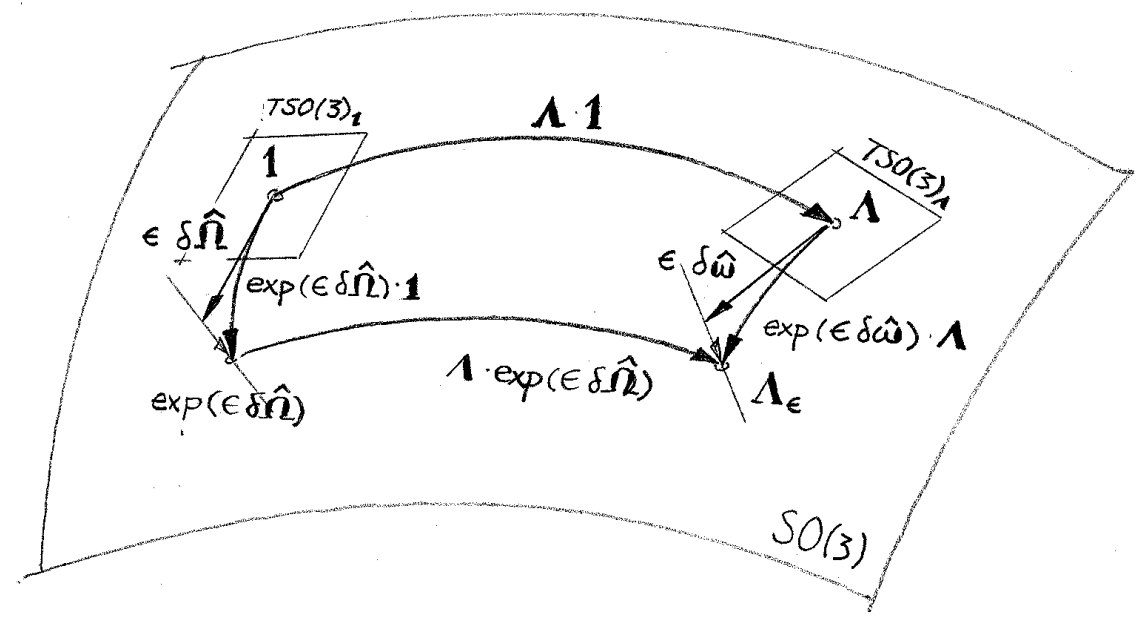

Figura 4.1: Variación de la rotación. Forma espacial y material

El vector axial correspondiente a $\delta \widehat{\boldsymbol{\Omega}}$ es $\delta \boldsymbol{\Omega}$ y su relación con $\delta \boldsymbol{\omega}$ es

$$
\delta \boldsymbol{\Omega}=\boldsymbol{\Lambda}^{\top} \delta \boldsymbol{\omega} .
$$

A continuación evaluamos la retroacción de la variación de las deformaciones generalizadas empleando las definiciones $(3.72)$ de la forma material de las deformaciones generalizadas

$$
\begin{gathered}
\boldsymbol{\Lambda}^{\top} \stackrel{\nabla}{\delta} \boldsymbol{\gamma}=\boldsymbol{\Lambda}^{\top} \boldsymbol{\Lambda} \delta\left(\boldsymbol{\Lambda}^{\top} \boldsymbol{\gamma}\right)=\delta \boldsymbol{\Gamma} \\
\boldsymbol{\Lambda}^{\top} \stackrel{\nabla}{\delta} \widehat{\boldsymbol{\kappa}} \boldsymbol{\Lambda}=\boldsymbol{\Lambda}^{\top} \boldsymbol{\Lambda} \delta\left(\boldsymbol{\Lambda}^{\top} \widehat{\boldsymbol{\kappa}} \boldsymbol{\Lambda}\right) \boldsymbol{\Lambda}^{\top} \boldsymbol{\Lambda}=\delta \widehat{\mathbf{K}}
\end{gathered}
$$

que, como cabe esperar, proporciona la variación de la versión material de éstas. El vector axial correspondiente a $\delta \widehat{\mathbf{K}}$ es $\delta \boldsymbol{K}$, y su relación con la variación corrotacional de $\boldsymbol{\kappa}$ es

$$
\delta \boldsymbol{K}=\boldsymbol{\Lambda}^{\top} \stackrel{\nabla}{\delta} \boldsymbol{\kappa} .
$$

Por lo que respecta a las variables estáticas, la forma material de los esfuerzos y las fuerzas generalizadas es

$$
\begin{array}{ll}
\boldsymbol{N}=\boldsymbol{\Lambda}^{\top} \boldsymbol{n} & \boldsymbol{Q}_{n}=\boldsymbol{\Lambda}^{\top} \boldsymbol{q}_{n} \\
\boldsymbol{M}=\boldsymbol{\Lambda}^{\top} \boldsymbol{m} & \boldsymbol{Q}_{m}=\boldsymbol{\Lambda}^{\top} \boldsymbol{q}_{m}
\end{array}
$$

Estas relaciones muestran cómo el uso de la retroacción permite trabajar siempre en el sistema de referencia inicial (que en el análisis de la pieza recta hacemos coincidir con el espacial), ya que las componentes en este sistema de la forma material de los 
vectores coinciden con las componentes intrínsecas (en el sistema actual) de su forma espacial.

La forma espacial de la ecuación de trabajos virtuales se obtiene expresando los productos escalares $\boldsymbol{v} \cdot \boldsymbol{w}$ como operación matricial, y multiplicando por $\boldsymbol{\Lambda} \boldsymbol{\Lambda}^{\top}$

$$
\boldsymbol{v} \cdot \boldsymbol{w}=\boldsymbol{v}^{\top} \boldsymbol{w}=\boldsymbol{v}^{\top} \boldsymbol{\Lambda} \boldsymbol{\Lambda}^{\top} \boldsymbol{w}=\left(\boldsymbol{\Lambda}^{\top} \boldsymbol{v}\right) \cdot\left(\boldsymbol{\Lambda}^{\top} \boldsymbol{w}\right)=\boldsymbol{V} \cdot \boldsymbol{W} .
$$

El resultado, teniendo en cuenta las expresiones anteriores, es el siguiente:

\section{Forma material de la ETV para la teoría RS}

La configuración $(\boldsymbol{x}, \boldsymbol{\Lambda})$ es una configuración de equilibrio si, y sólo si,

$$
\begin{aligned}
\int_{\Gamma_{0}}(\boldsymbol{N} \cdot \delta \boldsymbol{\Gamma}+\boldsymbol{M} \cdot \delta \boldsymbol{K}) d S & \\
=\int_{\Gamma_{0}}\left(\boldsymbol{Q}_{n} \cdot \delta \boldsymbol{\chi}+\boldsymbol{Q}_{m} \cdot \delta \boldsymbol{\Omega}\right) d S & +\boldsymbol{N}_{1} \cdot \delta \boldsymbol{\chi}\left(S_{1}\right)+\boldsymbol{N}_{2} \cdot \delta \boldsymbol{\chi}\left(S_{2}\right) \\
& +\boldsymbol{M}_{1} \cdot \delta \boldsymbol{\Omega}\left(S_{1}\right)+\boldsymbol{M}_{2} \cdot \delta \boldsymbol{\Omega}\left(S_{2}\right)
\end{aligned}
$$

para cualquier variación cinemáticamente admisible $(\delta \boldsymbol{\chi}, \delta \boldsymbol{\Omega})$.

\subsection{Cálculo de la variación de las deformaciones generalizadas}

En esta sección se evalúa la variación de las deformaciones generalizadas en función de las variaciones de la configuración.

\section{Forma espacial}

La siguiente proposición recoge el cálculo de la variación de $\gamma$ y $\kappa$ en función de $\delta \boldsymbol{x}$ у $\delta \boldsymbol{\omega}$.

Proposición 4.2.1. La variación de la forma espacial de las deformaciones generalizadas de la viga con la cinemática de Reissner-Simó admite la siguiente expresión en función de las variaciones de la configuración

$$
\begin{aligned}
& \nabla \\
& \delta \boldsymbol{\gamma}=\delta \boldsymbol{x}^{\prime}+\boldsymbol{\gamma} \times \delta \boldsymbol{\omega} \\
& \nabla \\
& \delta \boldsymbol{\kappa}=(\delta \boldsymbol{\omega})^{\prime} .
\end{aligned}
$$

Demostración. En primer lugar se calcula la variación de $\gamma$. Aplicando la definición de variación de objetos espaciales (4.8) a $\gamma$ se tiene

$$
\stackrel{\nabla}{\delta} \gamma=\boldsymbol{\Lambda} \delta\left(\boldsymbol{\Lambda}^{\top} \gamma\right)
$$


Sustituyendo $\gamma$ por su definición (3.64),

$$
\begin{aligned}
\stackrel{\nabla}{\delta} \gamma & =\boldsymbol{\Lambda} \delta\left(\boldsymbol{\Lambda}^{\top} \boldsymbol{x}^{\prime}\right) \\
& =\boldsymbol{\Lambda} \delta\left(\boldsymbol{\Lambda}^{\top}\right) \boldsymbol{x}^{\prime}+\boldsymbol{\Lambda} \boldsymbol{\Lambda}^{\top} \delta\left(\boldsymbol{x}^{\prime}\right) .
\end{aligned}
$$

El término $\delta\left(\boldsymbol{\Lambda}^{\top}\right)$ se obtiene a partir de la siguiente relación

$$
0=\delta\left(\boldsymbol{\Lambda}^{\top} \boldsymbol{\Lambda}\right)=\delta\left(\boldsymbol{\Lambda}^{\top}\right) \boldsymbol{\Lambda}+\boldsymbol{\Lambda}^{\top} \delta \boldsymbol{\Lambda}
$$

luego

$$
\delta\left(\boldsymbol{\Lambda}^{\top}\right)=-\boldsymbol{\Lambda}^{\top} \delta \boldsymbol{\Lambda} \boldsymbol{\Lambda}^{\top} .
$$

Entonces, teniendo en cuenta que $\delta\left(\boldsymbol{x}^{\prime}\right)=\delta \boldsymbol{x}^{\prime}$

$$
\begin{aligned}
\stackrel{\nabla}{\delta} \boldsymbol{\gamma} & =\delta \boldsymbol{x}^{\prime}-\delta \boldsymbol{\Lambda} \boldsymbol{\Lambda}^{\top} \boldsymbol{x}^{\prime} \\
& =\delta \boldsymbol{x}^{\prime}-\delta \widehat{\boldsymbol{\omega}} \boldsymbol{x}^{\prime},
\end{aligned}
$$

que expresada en función del vector axial conduce a la expresión (4.30a) del enunciado.

A continuación se evalúa la variación de $\boldsymbol{\kappa}$ como función de las variaciones de la configuración. Partiendo de (4.10)

$$
\stackrel{\nabla}{\delta \widehat{\mathbf{K}}}=\boldsymbol{\Lambda} \delta\left(\boldsymbol{\Lambda}^{\top} \widehat{\boldsymbol{\kappa}} \boldsymbol{\Lambda}\right) \boldsymbol{\Lambda}^{\top}
$$

Sustituyendo $\widehat{\kappa}$ por su definición (3.64),

$$
\begin{aligned}
\stackrel{\nabla}{\delta} \widehat{\boldsymbol{\kappa}} & =\boldsymbol{\Lambda} \delta\left(\boldsymbol{\Lambda}^{\top} \boldsymbol{\Lambda}^{\prime} \boldsymbol{\Lambda}^{\top} \boldsymbol{\Lambda}\right) \boldsymbol{\Lambda}^{\top} \\
& =\boldsymbol{\Lambda} \delta\left(\boldsymbol{\Lambda}^{\top} \boldsymbol{\Lambda}^{\prime}\right) \boldsymbol{\Lambda}^{\top} .
\end{aligned}
$$

El termino central se puede escribir como

$$
\delta\left(\boldsymbol{\Lambda}^{\top} \boldsymbol{\Lambda}^{\prime}\right)=-\boldsymbol{\Lambda}^{\top} \delta \boldsymbol{\Lambda} \boldsymbol{\Lambda}^{\top} \boldsymbol{\Lambda}^{\prime}+\boldsymbol{\Lambda}^{\top} \delta \boldsymbol{\Lambda}^{\prime} .
$$

Sustituyendo en la expresión anterior

$$
\begin{aligned}
\stackrel{\nabla}{\delta} \widehat{\boldsymbol{\kappa}} & =-\boldsymbol{\Lambda} \boldsymbol{\Lambda}^{\top} \delta \boldsymbol{\Lambda} \boldsymbol{\Lambda}^{\top} \boldsymbol{\Lambda}^{\prime} \boldsymbol{\Lambda}^{\top}+\boldsymbol{\Lambda} \boldsymbol{\Lambda}^{\top} \delta \boldsymbol{\Lambda}^{\prime} \boldsymbol{\Lambda}^{\top} \\
& =-\delta \boldsymbol{\Lambda} \boldsymbol{\Lambda}^{\top} \boldsymbol{\Lambda}^{\prime} \boldsymbol{\Lambda}^{\top}+\delta \boldsymbol{\Lambda}^{\prime} \boldsymbol{\Lambda}^{\top} .
\end{aligned}
$$

El último término se puede analizar a partir de la derivada de $\delta \widehat{\boldsymbol{\omega}}$

$$
\begin{aligned}
(\delta \widehat{\boldsymbol{\omega}})^{\prime} & =\left(\delta \boldsymbol{\Lambda} \boldsymbol{\Lambda}^{\top}\right)^{\prime} \\
& =\delta \boldsymbol{\Lambda}^{\prime} \boldsymbol{\Lambda}^{\top}+\delta \boldsymbol{\Lambda}\left(\boldsymbol{\Lambda}^{\top}\right)^{\prime} \\
& =\delta \boldsymbol{\Lambda}^{\prime} \boldsymbol{\Lambda}^{\top}-\delta \boldsymbol{\Lambda} \boldsymbol{\Lambda}^{\top} \boldsymbol{\Lambda}^{\prime} \boldsymbol{\Lambda}^{\top} .
\end{aligned}
$$

Despejando el primer término a la derecha de la igualdad e introduciéndolo en la expresión anterior se obtiene un resultado final drásticamente simplificado

$$
\stackrel{\nabla}{\delta}=(\delta \widehat{\boldsymbol{\omega}})^{\prime},
$$

que empleando vectores axiales proporciona la expresión (4.30b) del enunciado. 
Comentario 4.2.2. La expresión (4.30a) de la variación corrotacional de $\boldsymbol{\gamma}$ se puede obtener directamente a partir de la definición (4.11) de la variación corrotacional. Lo mismo sucede para la variación corrotacional de $\boldsymbol{\kappa}$, para la que se puede deducir la siguiente expresión alternativa a la ecuación (4.30b):

$$
\stackrel{\nabla}{\delta}=\delta \boldsymbol{\kappa}-\delta \boldsymbol{\omega} \times \boldsymbol{\kappa} .
$$

Este razonamiento permite una interpretación correcta de la expresión (2.10) de Ibrahimbegović [25].

El resultado de la proposición anterior es útil para definir un operador diferencial

$$
\overline{\mathbf{b}}=\left[\begin{array}{cc}
\mathbf{1} \frac{d}{d S} & \widehat{\gamma} \\
\mathbf{0} & \mathbf{1} \frac{d}{d S}
\end{array}\right]
$$

que relaciona la variación de las deformaciones generalizadas con la variación de las variables de la configuración

$$
\left\{\begin{array}{l}
\nabla \\
\delta \boldsymbol{\gamma} \\
\nabla \\
\delta \boldsymbol{\kappa}
\end{array}\right\}=\left[\begin{array}{cc}
\mathbf{1} \frac{d}{d S} & \widehat{\boldsymbol{\gamma}} \\
\mathbf{0} & \mathbf{1} \frac{d}{d S}
\end{array}\right]\left\{\begin{array}{l}
\delta \boldsymbol{x} \\
\delta \boldsymbol{\omega}
\end{array}\right\} .
$$

Introduciendo la variables

$$
\begin{aligned}
& \delta \boldsymbol{\phi}=\left\{\begin{array}{ll}
\delta \boldsymbol{x} & \delta \boldsymbol{\omega}
\end{array}\right\}^{\top} \\
& \stackrel{\nabla}{\nabla}=\left\{\begin{array}{ll}
\nabla & \nabla \\
\delta \boldsymbol{\epsilon} & \delta \boldsymbol{\kappa}
\end{array}\right\}^{\top},
\end{aligned}
$$

se puede escribir de forma abreviada

$$
\stackrel{\nabla}{\delta \boldsymbol{\epsilon}}=\overline{\mathbf{b}} \delta \phi
$$

con

$$
\overline{\mathbf{b}}=\mathbf{\epsilon}+\mathbf{I} \frac{d}{d S}, \quad \boldsymbol{\epsilon}=\left[\begin{array}{ll}
\mathbf{0} & \widehat{\gamma} \\
\mathbf{0} & \mathbf{0}
\end{array}\right], \quad \mathbf{I} \frac{d}{d S}=\left[\begin{array}{cc}
\mathbf{1} \frac{d}{d S} & \mathbf{0} \\
\mathbf{0} & \mathbf{1} \frac{d}{d S}
\end{array}\right]
$$

Resulta útil utilizar la siguiente expresión alternativa, función explícita de las variaciones de la configuración y sus derivadas

$$
\stackrel{\nabla}{\delta \boldsymbol{\epsilon}}=\left[\begin{array}{ll}
\boldsymbol{\epsilon} & \mathbf{I}
\end{array}\right]\left\{\begin{array}{l}
\delta \phi \\
\delta \phi^{\prime}
\end{array}\right\}
$$

en la que aparece la matriz

$$
\mathbf{b}=\left[\begin{array}{ll}
\boldsymbol{\epsilon} & \mathbf{I}
\end{array}\right]=\left[\begin{array}{llll}
\mathbf{0} & \widehat{\gamma} & \mathbf{1} & \mathbf{0} \\
\mathbf{0} & \mathbf{0} & \mathbf{0} & \mathbf{1}
\end{array}\right] .
$$




\section{Forma material}

Desarrollamos ahora la versión material de la proposición anterior.

Proposición 4.2.3. La variación de la forma material de las deformaciones generalizadas de la viga con la cinemática de Reissner-Simó admite la siguiente expresión en función de las variaciones de la configuración

$$
\begin{aligned}
\delta \boldsymbol{\Gamma} & =(\delta \boldsymbol{\chi})^{\prime}+\boldsymbol{K} \times \delta \boldsymbol{\chi}+\boldsymbol{\Gamma} \times \delta \boldsymbol{\Omega} \\
\delta \boldsymbol{K} & =(\delta \boldsymbol{\Omega})^{\prime}+\boldsymbol{K} \times \delta \boldsymbol{\Omega} .
\end{aligned}
$$

Demostración. La variación de $\boldsymbol{\Gamma}$ es

$$
\begin{aligned}
\delta \boldsymbol{\Gamma} & =\delta\left(\boldsymbol{\Lambda}^{\top} \boldsymbol{x}^{\prime}\right) \\
& =\delta\left(\boldsymbol{\Lambda}^{\top}\right) \boldsymbol{x}^{\prime}+\boldsymbol{\Lambda}^{\top} \delta\left(\boldsymbol{x}^{\prime}\right) \\
& =-\boldsymbol{\Lambda}^{\top} \delta \boldsymbol{\Lambda} \boldsymbol{\Lambda}^{\top} \boldsymbol{x}^{\prime}+\boldsymbol{\Lambda}^{\top} \delta\left(\boldsymbol{x}^{\prime}\right) .
\end{aligned}
$$

El último término se puede analizar a partir de la derivada de $\delta \boldsymbol{\chi}$, empleando las definiciones (3.72)

$$
\begin{aligned}
(\delta \boldsymbol{\chi})^{\prime} & =\left(\boldsymbol{\Lambda}^{\top} \delta \boldsymbol{x}\right)^{\prime} \\
& =\left(\boldsymbol{\Lambda}^{\top}\right)^{\prime} \delta \boldsymbol{x}+\boldsymbol{\Lambda}^{\top}(\delta \boldsymbol{x})^{\prime} \\
& =-\boldsymbol{\Lambda}^{\top} \boldsymbol{\Lambda}^{\prime} \boldsymbol{\Lambda}^{\top} \delta \boldsymbol{x}+\boldsymbol{\Lambda}^{\top} \delta \boldsymbol{x}^{\prime} \\
& =-\widehat{\mathbf{K}} \delta \boldsymbol{\chi}+\boldsymbol{\Lambda}^{\top} \delta \boldsymbol{x}^{\prime} .
\end{aligned}
$$

Despejando el segundo término, sustituyendo en la expresión anterior y empleando las definiciones (4.22)

$$
\delta \boldsymbol{\Gamma}=(\delta \boldsymbol{\chi})^{\prime}+\widehat{\mathbf{K}} \delta \boldsymbol{\chi}-\delta \widehat{\boldsymbol{\Omega}} \boldsymbol{\Gamma} .
$$

Empleando vectores axiales resulta la expresión (4.43a) del enunciado, que es similar a la de la variación espacial, salvo por el término adicional $\boldsymbol{K} \times \delta \boldsymbol{\chi}$. Para el cálculo de la variación de $\boldsymbol{K}$ se puede hacer uso de la expresión (4.34)

$$
\begin{aligned}
\delta \widehat{\mathbf{K}} & =\delta\left(\boldsymbol{\Lambda}^{\top} \boldsymbol{\Lambda}^{\prime}\right) \\
& =-\boldsymbol{\Lambda}^{\top} \delta \boldsymbol{\Lambda} \boldsymbol{\Lambda}^{\top} \boldsymbol{\Lambda}^{\prime}+\boldsymbol{\Lambda}^{\top} \delta \boldsymbol{\Lambda}^{\prime}
\end{aligned}
$$

El último término se despeja a partir de la derivada de $\widehat{\Omega}$

$$
\begin{aligned}
(\delta \widehat{\boldsymbol{\Omega}})^{\prime} & =\left(\boldsymbol{\Lambda}^{\top} \delta \boldsymbol{\Lambda}\right)^{\prime} \\
& =-\boldsymbol{\Lambda}^{\top} \boldsymbol{\Lambda}^{\prime} \boldsymbol{\Lambda}^{\top} \delta \boldsymbol{\Lambda}+\boldsymbol{\Lambda}^{\top} \delta \boldsymbol{\Lambda}^{\prime} .
\end{aligned}
$$

Sustituyendo en la expresión anterior y empleando las definiciones (3.72) y (4.22) se tiene

$$
\delta \widehat{\mathbf{K}}=(\delta \widehat{\boldsymbol{\Omega}})^{\prime}+\widehat{\mathbf{K}} \delta \widehat{\boldsymbol{\Omega}}-\delta \widehat{\boldsymbol{\Omega}} \widehat{\mathbf{K}}=(\delta \widehat{\boldsymbol{\Omega}})^{\prime}+[\widehat{\mathbf{K}}, \delta \widehat{\boldsymbol{\Omega}}] .
$$


El operador $[\cdot, \cdot]$ se denomina conmutador, y cumple, al igual que el producto vectorial, la identidad de Jacobi [49, Definición 1.45], [23, Lema 6.2]. Empleando el vector axial $\delta \boldsymbol{\Omega}$ introducido en la sección anterior

$$
\delta \widehat{\boldsymbol{\Omega}} \boldsymbol{w}=\delta \boldsymbol{\Omega} \times \boldsymbol{w}, \quad \forall \boldsymbol{w} \in \mathbb{R}^{3},
$$

se cumple

$$
[\widehat{\mathbf{K}}, \delta \widehat{\boldsymbol{\Omega}}] \boldsymbol{w}=(\boldsymbol{K} \times \delta \boldsymbol{\Omega}) \times \boldsymbol{w}, \quad \forall \boldsymbol{w} \in \mathbb{R}^{3}
$$

La variación de $\boldsymbol{K}$ en función del vector axial $\delta \boldsymbol{\Omega}$ es la expresión (4.43b) del enunciado.

Al igual que en la versión espacial, es posible definir un operador diferencial $\overline{\mathbf{B}}$ que relaciona variaciones de las deformaciones con variaciones de la configuración

$$
\left\{\begin{array}{l}
\delta \boldsymbol{\Gamma} \\
\delta \boldsymbol{K}
\end{array}\right\}=\left[\begin{array}{cc}
\frac{d}{d S} \mathbf{1}+\widehat{\mathbf{K}} & \widehat{\boldsymbol{\Gamma}} \\
\mathbf{0} & \frac{d}{d S} \mathbf{1}+\widehat{\mathbf{K}}
\end{array}\right]\left\{\begin{array}{l}
\delta \chi \\
\delta \boldsymbol{\Omega}
\end{array}\right\}
$$

Introduciendo la variables

$$
\begin{aligned}
\delta \boldsymbol{\Phi} & =\left\{\begin{array}{ll}
\delta \boldsymbol{\chi} & \delta \boldsymbol{\Omega}
\end{array}\right\}^{\top} \\
\delta \boldsymbol{E} & =\left\{\begin{array}{ll}
\delta \boldsymbol{\Gamma} & \delta \boldsymbol{K}
\end{array}\right\}^{\top}
\end{aligned}
$$

se puede escribir de forma abreviada

$$
\delta \boldsymbol{E}=\overline{\mathbf{B}} \delta \boldsymbol{\Phi}
$$

con

$$
\overline{\mathbf{B}}=\mathbf{E}+\mathbf{I} \frac{d}{d S}, \quad \mathbf{E}=\left[\begin{array}{cc}
\widehat{\mathbf{K}} & \widehat{\boldsymbol{\Gamma}} \\
\mathbf{0} & \widehat{\mathbf{K}}
\end{array}\right], \quad \mathbf{I} \frac{d}{d S}=\left[\begin{array}{cc}
\frac{d}{d S} \mathbf{1} & \mathbf{0} \\
\mathbf{0} & \frac{d}{d S} \mathbf{1}
\end{array}\right]
$$

También se utilizará la siguiente expresión alternativa, función explícita de las variaciones de la configuración y sus derivadas

$$
\delta \boldsymbol{E}=\left[\begin{array}{ll}
\mathbf{E} & \mathbf{I}
\end{array}\right]\left\{\begin{array}{l}
\delta \boldsymbol{\Phi} \\
\delta \boldsymbol{\Phi}^{\prime}
\end{array}\right\}
$$

en la que se ha introducido la matriz

$$
\mathbf{B}=\left[\begin{array}{ll}
\mathbf{E} & \mathbf{I}
\end{array}\right]=\left[\begin{array}{cccc}
\widehat{\mathbf{K}} & \widehat{\Gamma} & \mathbf{1} & \mathbf{0} \\
\mathbf{0} & \widehat{\mathbf{K}} & \mathbf{0} & \mathbf{1}
\end{array}\right]
$$




\subsection{Ecuaciones de equilibrio}

\section{Forma espacial}

Introduciendo las expresiones (4.30a) y (4.30b) en el término correspondiente al trabajo virtual de los esfuerzos en su forma espacial,

$$
\begin{aligned}
\int_{\Gamma_{0}}(\boldsymbol{n} \cdot \stackrel{\nabla}{\delta} \boldsymbol{\gamma}+\boldsymbol{m} \cdot \stackrel{\nabla}{\delta} \boldsymbol{\kappa}) d S & =\int_{\Gamma_{0}} \boldsymbol{n} \cdot\left(\delta \boldsymbol{x}^{\prime}+\boldsymbol{\gamma} \times \delta \boldsymbol{\omega}\right) d S+\int_{\Gamma_{0}} \boldsymbol{m} \cdot(\delta \boldsymbol{\omega})^{\prime} d S \\
& =\int_{\Gamma_{0}}\left(\boldsymbol{n} \cdot \delta \boldsymbol{x}^{\prime}+(\boldsymbol{n} \times \gamma) \cdot \delta \boldsymbol{\omega}\right) d S+\int_{\Gamma_{0}} \boldsymbol{m} \cdot(\delta \boldsymbol{\omega})^{\prime} d S
\end{aligned}
$$

Integrando por partes y reagrupando términos

$$
\begin{aligned}
\int_{\Gamma_{0}}(\boldsymbol{n} \cdot \stackrel{\nabla}{\delta} \boldsymbol{\gamma}+\boldsymbol{m} \cdot \stackrel{\nabla}{\delta} \boldsymbol{\kappa}) d S & =\int_{\Gamma_{0}}\left(-\boldsymbol{n}^{\prime}\right) \cdot \delta \boldsymbol{x} d S+\int_{\Gamma_{0}}\left(-\boldsymbol{m}^{\prime}+\boldsymbol{n} \times \gamma\right) \cdot \delta \boldsymbol{\omega} d S \\
& +[\boldsymbol{n} \cdot \delta \boldsymbol{x}]_{S_{1}}^{S_{2}}+[\boldsymbol{m} \cdot \delta \boldsymbol{\omega}]_{S_{1}}^{S_{2}} .
\end{aligned}
$$

Igualando este resultado al trabajo virtual de las fuerzas externas, y considerando que las integrales deben anularse para cualquier variación cinemáticamente admisible, se obtienen las siguientes expresiones

Forma espacial de las ecuaciones de equilibrio RS

$$
\begin{array}{r}
\boldsymbol{n}^{\prime}+\boldsymbol{q}_{n}=\mathbf{0} \\
\boldsymbol{m}^{\prime}+\gamma \times \boldsymbol{n}+\boldsymbol{q}_{m}=\mathbf{0}
\end{array}
$$

Condiciones de contorno en el extremo inicial

$$
\begin{aligned}
{\left[\boldsymbol{n}\left(S_{1}\right)+\boldsymbol{n}_{1}\right] \cdot \delta \boldsymbol{x}\left(S_{1}\right) } & =0 \\
{\left[\boldsymbol{m}\left(S_{1}\right)+\boldsymbol{m}_{1}\right] \cdot \delta \boldsymbol{\omega}\left(S_{1}\right) } & =0,
\end{aligned}
$$

Condiciones de contorno en el extremo final

$$
\begin{aligned}
{\left[\boldsymbol{n}\left(S_{2}\right)-\boldsymbol{n}_{2}\right] \cdot \delta \boldsymbol{x}\left(S_{2}\right) } & =0 \\
{\left[\boldsymbol{m}\left(S_{2}\right)-\boldsymbol{m}_{2}\right] \cdot \delta \boldsymbol{\omega}\left(S_{2}\right) } & =0
\end{aligned}
$$

La forma matricial de las ecuaciones de equilibrio es

$$
\left\{\begin{array}{c}
\boldsymbol{n}^{\prime} \\
\boldsymbol{m}^{\prime}
\end{array}\right\}=\left[\begin{array}{cc}
\mathbf{0} & 0 \\
-\widehat{\gamma} & 0
\end{array}\right]\left\{\begin{array}{c}
\boldsymbol{n} \\
\boldsymbol{m}
\end{array}\right\}-\left\{\begin{array}{c}
\boldsymbol{q}_{n} \\
\boldsymbol{q}_{m}
\end{array}\right\} .
$$


Empleando la notación ${ }^{4}$

$$
\begin{aligned}
& \boldsymbol{f}=\left\{\begin{array}{ll}
\boldsymbol{n} & \boldsymbol{m}
\end{array}\right\}^{\top} \\
& \boldsymbol{q}=\left\{\begin{array}{ll}
\boldsymbol{q}_{n} & \boldsymbol{q}_{m}
\end{array}\right\}^{\top},
\end{aligned}
$$

las ecuaciones se pueden expresar en forma abreviada

$$
f^{\prime}=\epsilon^{\top} f-q
$$

La matriz del sistema $\boldsymbol{\epsilon}^{\top}$ es precisamente el primer bloque traspuesto del operador $\mathbf{b}$ que relaciona la variación de las deformaciones generalizadas con la variación de las variables de la configuración y sus derivadas. La matriz $\boldsymbol{\epsilon}$ aparecerá posteriormente en la linealización del principio de trabajos virtuales.

\section{Forma Material}

Introduciendo las expresiones (4.43a) y (4.43b) en el término correspondiente al trabajo virtual de los esfuerzos en su forma material,

$$
\begin{aligned}
\int_{\Gamma_{0}}(\boldsymbol{N} \cdot \delta \boldsymbol{\Gamma}+\boldsymbol{M} \cdot \delta \boldsymbol{K}) d S & =\int_{\Gamma_{0}} \boldsymbol{N} \cdot\left((\delta \boldsymbol{\chi})^{\prime}+\boldsymbol{\Gamma} \times \delta \boldsymbol{\Omega}+\boldsymbol{K} \times \delta \boldsymbol{\chi}\right) d S \\
& +\int_{\Gamma_{0}} \boldsymbol{M} \cdot\left((\delta \boldsymbol{\Omega})^{\prime}+\boldsymbol{K} \times \delta \boldsymbol{\Omega}\right) d S \\
& =\int_{\Gamma_{0}}\left(\boldsymbol{N} \cdot \delta(\boldsymbol{\chi})^{\prime}+(\boldsymbol{N} \times \boldsymbol{K}) \cdot \delta \boldsymbol{\chi}+(\boldsymbol{N} \times \boldsymbol{\Gamma}) \cdot \delta \boldsymbol{\Omega}\right) d S \\
& +\int_{\Gamma_{0}}\left(\boldsymbol{M} \cdot(\delta \boldsymbol{\Omega})^{\prime}+(\boldsymbol{M} \times \boldsymbol{K}) \cdot \delta \boldsymbol{\Omega}\right) d S .
\end{aligned}
$$

Integrando por partes y reagrupando términos

$$
\begin{aligned}
\int_{\Gamma_{0}}(\boldsymbol{N} \cdot \delta \boldsymbol{\Gamma}+\boldsymbol{M} \cdot \delta \boldsymbol{K}) d S & =\int_{\Gamma_{0}}\left(-\boldsymbol{N}^{\prime}+\boldsymbol{N} \times \boldsymbol{K}\right) \cdot \delta \boldsymbol{\chi} d S \\
& +\int_{\Gamma_{0}}\left(-\boldsymbol{M}^{\prime}+\boldsymbol{M} \times \boldsymbol{K}+\boldsymbol{N} \times \boldsymbol{\Gamma}\right) \cdot \delta \boldsymbol{\Omega} d S \\
& +[\boldsymbol{N} \cdot \delta \boldsymbol{\chi}]_{S_{1}}^{S_{2}}+[\boldsymbol{M} \cdot \delta \boldsymbol{\Omega}]_{S_{1}}^{S_{2}} .
\end{aligned}
$$

Igualando este resultado al trabajo virtual de las fuerzas externas, y considerando que las integrales deben anularse para cualquier variación cinemáticamente admisible, se obtienen las siguientes expresiones

\footnotetext{
${ }^{4}$ La nueva variable $\boldsymbol{f}$, que agrupa las componentes de los esfuerzos, no debe confundirse con los vectores $\boldsymbol{f}_{1}^{*}, \boldsymbol{f}_{2}^{*}, \boldsymbol{f}_{3}^{*}$ que componen la forma espacial del gradiente de la deformación, introducidos en la sección 3.5 .
} 
Forma material de las ecuaciones de equilibrio RS

$$
\begin{array}{r}
\boldsymbol{N}^{\prime}+\boldsymbol{K} \times \boldsymbol{N}+\boldsymbol{Q}_{n}=\mathbf{0} \\
\boldsymbol{M}^{\prime}+\boldsymbol{K} \times \boldsymbol{M}+\boldsymbol{\Gamma} \times \boldsymbol{N}+\boldsymbol{Q}_{m}=\mathbf{0}
\end{array}
$$

Condiciones de contorno en el extremo inicial

$$
\begin{aligned}
{\left[\boldsymbol{N}\left(S_{1}\right)+\boldsymbol{N}_{1}\right] \cdot \delta \boldsymbol{\chi}\left(S_{1}\right) } & =0 \\
{\left[\boldsymbol{M}\left(S_{1}\right)+\boldsymbol{M}_{1}\right] \cdot \delta \boldsymbol{\Omega}\left(S_{1}\right) } & =0
\end{aligned}
$$

Condiciones de contorno en el extremo final

$$
\begin{aligned}
{\left[\boldsymbol{N}\left(S_{2}\right)-\boldsymbol{N}_{2}\right] \cdot \delta \boldsymbol{\chi}\left(S_{1}\right) } & =0 \\
{\left[\boldsymbol{M}\left(S_{2}\right)-\boldsymbol{M}_{2}\right] \cdot \delta \boldsymbol{\Omega}\left(S_{2}\right) } & =0
\end{aligned}
$$

Comentario 4.3.1. Denotando mediante el símbolo $\stackrel{\nabla}{\boldsymbol{v}}$ a la derivada corrotacional del vector $\boldsymbol{v}$, definida en la sección anterior

$$
\stackrel{\nabla}{\boldsymbol{v}}=\boldsymbol{\Lambda} d / d S\left(\boldsymbol{\Lambda}^{\top} \boldsymbol{v}\right)
$$

habiamos probado que la derivada de $\boldsymbol{v}$ respecto de la variable $S$ admite la siguiente descomposición

$$
\boldsymbol{v}^{\prime}=\stackrel{\nabla}{\boldsymbol{v}}+\kappa \times \boldsymbol{v}
$$

Si sustituimos las derivadas de los esfuerzos en la expresión de la forma espacial de las ecuaciones de equilibrio del modelo por su descomposición en función de la derivada corrotacional, resultan las siguientes ecuaciones

$$
\begin{array}{r}
\stackrel{\nabla}{n}+\boldsymbol{\kappa} \times \boldsymbol{n}+\boldsymbol{q}_{n}=\mathbf{0} \\
\stackrel{\nabla}{\boldsymbol{m}}+\boldsymbol{\kappa} \times \boldsymbol{m}+\boldsymbol{\gamma} \times \boldsymbol{n}+\boldsymbol{q}_{m}=\mathbf{0},
\end{array}
$$

que poseen la misma estructura que la forma material que se acaba de deducir. Este resultado demuestra que la forma espacial de las ecuaciones de equilibrio es igual que la material cuando la derivada convencional se sustituye por la corrotacional. Esta propiedad se puede extender a las expresiones espaciales (4.30a) y (4.30b) de las variaciones de las deformaciones generalizadas que resultan ser idénticas a las materiales si se sustituyen las derivadas convencionales por las corrotacionales.

Es posible escribir las ecuaciones intrínsecas de equilibrio en forma matricial

$$
\left\{\begin{array}{l}
N^{\prime} \\
M^{\prime}
\end{array}\right\}=\left[\begin{array}{cc}
-\widehat{\mathbf{K}} & 0 \\
-\widehat{\Gamma} & -\widehat{\mathbf{K}}
\end{array}\right]\left\{\begin{array}{l}
\boldsymbol{N} \\
\boldsymbol{M}
\end{array}\right\}-\left\{\begin{array}{l}
\boldsymbol{Q}_{n} \\
\boldsymbol{Q}_{m}
\end{array}\right\},
$$


Empleando la notación ${ }^{5}$

$$
\begin{aligned}
& \boldsymbol{F}=\left\{\begin{array}{ll}
\boldsymbol{N} & \boldsymbol{M}
\end{array}\right\}^{\top} \\
& \boldsymbol{Q}=\left\{\begin{array}{ll}
\boldsymbol{Q}_{n} & \boldsymbol{Q}_{m}
\end{array}\right\}^{\top},
\end{aligned}
$$

las ecuaciones se pueden expresar en forma abreviada

$$
\boldsymbol{F}^{\prime}=\mathbf{E}^{\top} \boldsymbol{F}-\boldsymbol{Q}
$$

La matriz del sistema $\mathbf{E}^{\top}$ es, como en el caso espacial, el primer bloque traspuesto del operador $\mathbf{B}$ que relaciona la variación de las deformaciones generalizadas con la variación de las variables de la configuración y sus derivadas. $\mathbf{E}$ aparecerá posteriormente en la linealización del principio de trabajos virtuales.

\subsection{Ecuaciones constitutivas}

Las conclusiones obtenidas a partir del principio de los trabajos virtuales son aplicables con independencia de la relación constitutiva que define la respuesta del material. En particular serán ciertas para un material hiperelástico caracterizado por su densidad de energía de deformación $\rho_{0}^{*} \mathcal{W}^{*}\left(\mathbf{F}^{*}\right)$, que depende del gradiente de la deformación [41]. La energía de deformación de la pieza alargada en la configuración $\boldsymbol{x}^{*}$ vendrá definida por

$$
U\left(\boldsymbol{x}^{*}\right)=\int_{V_{0}} \mathcal{W}^{*}\left(\mathbf{F}^{*}\right) \rho_{0}^{*} d V
$$

La siguiente proposición muestra cómo la energía de deformación de la pieza alargada admite una expresión en función de las deformaciones generalizadas.

Proposición 4.4.1. La energía de deformación de la pieza alargada con la cinemática de Reissner-Simó admite la siguiente expresión en función de la forma material de las deformaciones generalizadas $\boldsymbol{\Gamma}, \boldsymbol{K}$

$$
U(\boldsymbol{x}, \boldsymbol{\Lambda})=\int_{\Gamma_{0}} \rho_{0} \mathcal{W}(\boldsymbol{\Gamma}, \boldsymbol{K}) d S,
$$

donde $\rho_{0}$ es la masa por unidad de longitud de la pieza alargada en la configuración de referencia, y $\rho_{0} \mathcal{W}(\boldsymbol{\Gamma}, \boldsymbol{K})$ es la densidad de energía de deformación por unidad de longitud.

Demostración. La integral de volumen de la energía de deformación de la pieza se puede dividir en una integral sobre la sección transversal y una integral sobre la longitud de la pieza

$$
U\left(\boldsymbol{x}^{*}\right)=\int_{\Gamma_{0}} \int_{A_{s}} \mathcal{W}^{*}\left(\mathbf{F}^{*}\right) \rho_{0}^{*} \sqrt{\mathrm{g}_{0}^{*}} d X^{2} d X^{3} d S
$$

\footnotetext{
${ }^{5}$ Cabe el mismo comentario que en el caso espacial: la nueva variable $\boldsymbol{F}$ agrupa las componentes de los esfuerzos, y no debe ser confundida con los vectores $\boldsymbol{F}_{1}^{*}, \boldsymbol{F}_{2}^{*}, \boldsymbol{F}_{3}^{*}$ que componen la forma espacial del gradiente de la deformación.
} 
A su vez, el gradiente de la deformación únicamente es función de las deformaciones generalizadas y de las variables $X^{2}$ y $X^{3}$, ya que el primer vector (3.75) es

$$
\boldsymbol{F}_{1}^{*}=\boldsymbol{\Gamma}+\boldsymbol{K} \times \boldsymbol{R}^{*},
$$

las componentes de $\boldsymbol{R}^{*}$ en el sistema de referencia material son $\left\{0, X^{2}, X^{3}\right\}^{\top}$ y los vectores $\boldsymbol{F}_{2}^{*}$ y $\boldsymbol{F}_{3}^{*}$ son constantes en ese sistema (3.76). Teniendo en cuenta la descomposición de $\boldsymbol{F}_{1}^{*}$ se puede definir la densidad de energía de deformación por unidad de longitud de la siguiente forma

$$
\rho_{0} \mathcal{W}(\boldsymbol{\Gamma}, \boldsymbol{K})=\int_{A_{s}} \mathcal{W}^{*}\left(\mathbf{F}^{*}\left(\boldsymbol{\Gamma}, \boldsymbol{K}, X^{2}, X^{3}\right)\right) \rho_{0}^{*} \sqrt{\mathrm{g}_{0}^{*}} d X^{2} d X^{3} .
$$

En esta expresión $\rho_{0}$ es la masa por unidad de longitud de la pieza alargada en la configuración de referencia, y se define así

$$
\rho_{0}=\int_{A_{s}} \rho_{0}^{*} \sqrt{\mathrm{g}_{0}^{*}} d X^{2} d X^{3}
$$

Teniendo en cuenta (4.66), (4.67) y (4.68) se obtiene el resultado buscado.

La expresión de la energía de deformación en función de la forma material de las deformaciones generalizadas permite demostrar su independencia del sistema de referencia espacial, es decir, su invarianza por la izquierda frente a rotaciones. Esta propiedad, como adelantamos en el capítulo anterior, se denomina objetividad:

Proposición 4.4.2. La densidad lineal de energía de deformación en la pieza alargada con la cinemática de Reissner-Simó es invariante frente a rotaciones por la izquierda (cambios del sistema de referencia).

Demostración. En la proposición anterior se ha demostrado que la densidad lineal de energía de deformación puede ser expresada en función de la forma material de las deformaciones generalizadas, $\boldsymbol{\Gamma}$ y $\boldsymbol{K}$. Sea $\mathbf{Q}$ una matriz ortogonal que actúa por la izquierda sobre la configuración $\boldsymbol{x}, \boldsymbol{\Lambda}$ produciendo las siguientes transformaciones

$$
\begin{aligned}
\boldsymbol{\Lambda} & \rightarrow \mathrm{Q} \boldsymbol{\Lambda} \\
\boldsymbol{x}^{\prime} & \rightarrow \mathrm{Q} \boldsymbol{x}^{\prime} \\
\boldsymbol{\Lambda}^{\prime} & \rightarrow \mathrm{Q} \boldsymbol{\Lambda}^{\prime} .
\end{aligned}
$$

La forma material de las deformaciones generalizadas resulta ser invariante frente a este tipo de transformación

$$
\begin{aligned}
\boldsymbol{\Gamma} & =\boldsymbol{\Lambda}^{\top} \boldsymbol{x}^{\prime} \rightarrow \boldsymbol{\Lambda}^{\top} \mathbf{Q}^{\top} \mathbf{Q} \boldsymbol{x}^{\prime}=\boldsymbol{\Gamma} \\
\widehat{\mathbf{K}} & =\boldsymbol{\Lambda}^{\top} \boldsymbol{\Lambda}^{\prime} \rightarrow \boldsymbol{\Lambda}^{\top} \mathbf{Q}^{\top} \mathbf{Q} \boldsymbol{\Lambda}^{\prime}=\widehat{\mathbf{K}},
\end{aligned}
$$

y teniendo en cuenta la dependencia de la densidad lineal de energía de deformación de $\boldsymbol{\Gamma}$ y $\boldsymbol{K}$, queda demostrado el enunciado. 
La actuación por la izquierda del tensor $\mathbf{Q}$ sobre las variables de la configuración es equivalente al efecto de un cambio del sistema de referencia espacial, por lo que también queda probada como corolario la independencia de $\rho_{0} \mathcal{W}(\boldsymbol{\Gamma}, \boldsymbol{K})$ respecto del sistema de referencia espacial.

Por otra parte, la definición energética

$$
\mathbf{P}^{*}=\rho_{0}^{*} \frac{\partial \mathcal{W}^{*}}{\partial \mathbf{F}^{*}}
$$

del primer tensor de Piola-Kirchhoff es, a su vez, la ecuación constitutiva que relaciona variables estáticas y cinemáticas en el sólido hiperelástico. En el modelo de la pieza alargada hiperelástica, definido por la densidad lineal de energía de deformación, la relación constitutiva debe establecerse a partir de sus derivadas respecto de las variables $\boldsymbol{\Gamma}$ y $\boldsymbol{K}$. La siguiente proposición demuestra que estas derivadas coinciden con la forma material de los esfuerzos, definidos como resultantes de tensiones, es decir, como la forma material de las ecuaciones (4.19). En la demostración se vuelve a hacer uso de la expresión material (3.75) del primer vector del gradiente de la deformación $\boldsymbol{F}_{1}^{*}$ cuya expresión en componentes es

$$
\left(F_{1}^{*}\right)^{a}=\Gamma^{a}+e^{a}{ }_{b c} K^{b}\left(R^{*}\right)^{c} .
$$

Proposición 4.4.3. Las derivadas parciales de la densidad de energía de deformación por unidad de longitud respecto de la forma material de las deformaciones generalizadas $\boldsymbol{\Gamma}$ y $\boldsymbol{K}$ son las resultantes de los vectores tensión que actúan sobre cada sección transversal en la configuración deformada en su forma material, que coinciden con la definición clásica de los esfuerzos.

$$
\begin{aligned}
& \boldsymbol{N}=\rho_{0} \frac{\partial \mathcal{W}}{\partial \boldsymbol{\Gamma}}=\int_{A_{s}} \boldsymbol{T}^{* 1} d A_{0}^{*} \\
& \boldsymbol{M}=\rho_{0} \frac{\partial \mathcal{W}}{\partial \boldsymbol{K}}=\int_{A_{s}} \boldsymbol{R}^{*} \times \boldsymbol{T}^{* 1} d A_{0}^{*} .
\end{aligned}
$$

Demostración. Introduciendo en la definición (4.67) de la densidad lineal de energía de deformación la definición energética del primer tensor de tensiones de PiolaKirchhoff, $\mathbf{P}^{*}=\rho_{0}^{*} \partial \mathcal{W}^{*} / \partial \mathbf{F}^{*}$, y derivando

$$
\rho_{0} \frac{\partial \mathcal{W}}{\partial \boldsymbol{\Gamma}}=\int_{A_{s}} \rho_{0}^{*} \frac{\partial \mathcal{W}^{*}}{\partial \mathbf{F}^{*}}: \frac{\partial \mathbf{F}^{*}}{\partial \boldsymbol{\Gamma}} \sqrt{\mathrm{g}_{0}^{*}} d X^{2} d X^{3}=\int_{A_{s}} \mathbf{P}^{*}: \frac{\partial \mathbf{F}^{*}}{\partial \boldsymbol{\Gamma}} \sqrt{\mathrm{g}_{0}^{*}} d X^{2} d X^{3}
$$

Expresando $\mathbf{F}^{*}$ como $\boldsymbol{F}_{K}^{*} \otimes \boldsymbol{g}_{0}^{* K}$ y teniendo en cuenta que sólo $\boldsymbol{F}_{1}^{*}$ depende de $\boldsymbol{\Gamma}$ $(3.75)$,

$$
\frac{\partial\left(\mathrm{F}^{*}\right)^{a} K}{\partial \Gamma^{b}}=\frac{\partial\left(F_{1}^{*}\right)^{a}}{\partial \Gamma^{b}}=\delta_{b}^{a}
$$

luego

$$
\frac{\partial \mathbf{F}^{*}}{\partial \boldsymbol{\Gamma}}=\mathbf{1} \otimes \boldsymbol{g}_{0}^{* 1} .
$$


El primer tensor de Piola-Kirchhoff se puede descomponer de la siguiente forma: $\mathbf{P}^{*}=\boldsymbol{P}^{* K} \otimes \boldsymbol{g}_{0 K}^{*}$. Entonces

$$
\left(\mathrm{P}^{*}\right)_{a}{ }^{K} \frac{\partial\left(\mathrm{F}^{*}\right)_{K}^{a}}{\partial \Gamma^{b}}=\left(\mathrm{P}^{*}\right)_{a}{ }^{1} \frac{\partial\left(\mathrm{F}^{*}\right)_{1}^{a}}{\partial \Gamma^{b}}=\left(\mathrm{P}^{*}\right)_{a}{ }^{1} \delta_{b}^{a}=\left(\mathrm{P}^{*}\right)_{b}{ }^{1}=\left(P^{* 1}\right)_{b},
$$

es decir

$$
\mathbf{P}^{*}: \frac{\partial \mathbf{F}^{*}}{\partial \boldsymbol{\Gamma}}=\boldsymbol{P}^{* 1}
$$

Teniendo en cuenta la relación $\boldsymbol{P}^{* 1} \sqrt{\mathrm{g}_{0}^{*}}=\boldsymbol{T}^{* 1}$, versión material de la obtenida en la sección anterior, y la fórmula del diferencial de superficie sobre la sección transversal $d A_{0}^{*}=d X^{2} d X^{3}$, resulta la expresión (4.74a).

Por otro lado

$$
\rho_{0} \frac{\partial \mathcal{W}}{\partial \boldsymbol{K}}=\int_{A_{s}} \rho_{0}^{*} \frac{\partial \mathcal{W}^{*}}{\partial \mathbf{F}^{*}}: \frac{\partial \mathbf{F}^{*}}{\partial \boldsymbol{K}} \sqrt{\mathrm{g}_{0}^{*}} d X^{2} d X^{3}=\int_{A_{s}} \mathbf{P}^{*}: \frac{\partial \mathbf{F}^{*}}{\partial \boldsymbol{K}} \sqrt{\mathrm{g}_{0}^{*}} d X^{2} d X^{3}
$$

Dado que sólo $\boldsymbol{F}_{1}^{*}$ depende de $\boldsymbol{K}(\overline{3.70})$,

$$
\frac{\partial\left(\mathrm{F}^{*}\right)_{K}^{a}}{\partial K^{b}}=\frac{\partial\left(F_{1}^{*}\right)^{a}}{\partial K^{b}}=e^{a}{ }_{d c} \delta_{b}^{d}\left(R^{*}\right)^{c}=e_{b c}^{a}\left(R^{*}\right)^{c},
$$

luego

$$
\frac{\partial \mathbf{F}^{*}}{\partial \boldsymbol{K}}=\frac{\partial \boldsymbol{F}_{1}^{*}}{\partial \boldsymbol{K}} \otimes \boldsymbol{g}_{0}^{* 1}=\cdot \times \boldsymbol{R}^{*} \otimes \boldsymbol{g}_{0}^{* 1}
$$

Desarrollando el integrando en componentes

$$
\begin{aligned}
\left(\mathrm{P}^{*}\right)_{a}{ }^{K} \frac{\partial\left(\mathrm{F}^{*}\right)^{a} K}{\partial K^{b}}=\left(\mathrm{P}^{*}\right)_{a}{ }^{1} \frac{\partial\left(\mathrm{F}^{*}\right)^{a}{ }_{1}}{\partial K^{b}}=\left(P^{* 1}\right)_{a} e_{b c}^{a}\left(R^{*}\right)^{c}=e_{b c}{ }^{a}\left(R^{*}\right)^{c}\left(P^{* 1}\right)_{a} \\
\mathbf{P}^{*}: \frac{\partial \mathbf{F}^{*}}{\partial \boldsymbol{K}}=\boldsymbol{R}^{*} \times \boldsymbol{P}^{* 1} .
\end{aligned}
$$

considerando, como antes, que $\boldsymbol{P}^{* 1} \sqrt{\mathrm{g}_{0}^{*}}=\boldsymbol{T}^{* 1}$, y que el diferencial de superficie sobre la sección transversal es $d A_{0}^{*}=d X^{2} d X^{3}$, resulta la expresión (4.74b).

Las ecuaciones (4.74) son la forma material de las ecuaciones constitutivas del modelo RS, y relacionan las variables estáticas $\boldsymbol{N}$ y $\boldsymbol{M}$ con las deformaciones generalizadas $\boldsymbol{\Gamma}$ y $\boldsymbol{K}$. Es frecuente emplear la versión incremental de estas ecuaciones que relaciona incrementos de esfuerzos e incrementos de deformaciones generalizadas

$$
\left\{\begin{array}{c}
\Delta \boldsymbol{N} \\
\Delta \boldsymbol{M}
\end{array}\right\}=\left[\begin{array}{ll}
\mathbf{C}_{\Gamma \Gamma} & \mathbf{C}_{\Gamma K} \\
\mathbf{C}_{K \Gamma} & \mathbf{C}_{K K}
\end{array}\right]\left\{\begin{array}{c}
\Delta \boldsymbol{\Gamma} \\
\Delta \boldsymbol{K}
\end{array}\right\}
$$

con

$$
\begin{aligned}
\mathbf{C}_{\Gamma \Gamma} & =\rho_{0} \frac{\partial^{2} \mathcal{W}}{\partial \boldsymbol{\Gamma} \partial \boldsymbol{\Gamma}} & \mathbf{C}_{\Gamma K} & =\rho_{0} \frac{\partial^{2} \mathcal{W}}{\partial \boldsymbol{\Gamma} \partial \boldsymbol{K}} \\
\mathbf{C}_{K \Gamma} & =\rho_{0} \frac{\partial^{2} \mathcal{W}}{\partial \boldsymbol{K} \partial \boldsymbol{\Gamma}} & \mathbf{C}_{K K} & =\rho_{0} \frac{\partial^{2} \mathcal{W}}{\partial \boldsymbol{K} \partial \boldsymbol{K}},
\end{aligned}
$$


y por la igualdad de derivadas cruzadas

$$
\mathbf{C}_{\Gamma \Gamma}=\mathbf{C}_{\Gamma \Gamma}^{\top} \quad \mathbf{C}_{\Gamma K}=\mathbf{C}_{K \Gamma}^{\top} \quad \mathbf{C}_{K K}=\mathbf{C}_{K K}^{\top},
$$

con lo que la matriz constitutiva incremental es, en conjunto, simétrica.

La deducción de la densidad lineal $\rho_{0} \mathcal{W}$ partiendo de la densidad de energía en el sólido $\mathcal{W}^{*}$ mediante la expresión (4.67) no es una tarea sencilla, porque las restricciones en el movimiento de la sección transversal que introduce la hipótesis cinemática distorsionan la respuesta tridimensional del material. Por esta razón es usual postular directamente la densidad lineal de energía de deformación para construir una teoría directa, no derivada de la teoría tridimensional. En este caso las ecuaciones constitutivas aparecen de manera natural en su forma material o intrinseca, ya que ello permite independizar la respuesta del material de la rotación $\boldsymbol{\Lambda}$.

En el caso particular del material elástico lineal e isótropo se puede postular una expresión para la energía de deformación que tenga la misma estructura que la utilizada en el problema lineal, y dependa de la parte de las deformaciones generalizadas asociada al cambio de forma desde la configuración inicial a la actual

$$
\rho_{0} \mathcal{W}(\boldsymbol{\Gamma}, \boldsymbol{K})=\frac{1}{2}\left\{\boldsymbol{\Gamma}-\boldsymbol{\Gamma}_{0}, \boldsymbol{K}-\boldsymbol{K}_{0}\right\}\left[\begin{array}{cc}
\mathbf{C}_{\Gamma} & \mathbf{0} \\
\mathbf{0} & \mathbf{C}_{K}
\end{array}\right]\left\{\begin{array}{c}
\boldsymbol{\Gamma}-\boldsymbol{\Gamma}_{0} \\
\boldsymbol{K}-\boldsymbol{K}_{0}
\end{array}\right\}
$$

con

$$
\mathbf{C}_{\Gamma}=\left[\begin{array}{ccc}
E A & 0 & 0 \\
0 & G A_{Q 2} & 0 \\
0 & 0 & G A_{Q 3}
\end{array}\right] \quad \mathbf{C}_{K}=\left[\begin{array}{ccc}
G J & 0 & 0 \\
0 & E I_{2} & 0 \\
0 & 0 & E I_{3}
\end{array}\right]
$$

Esta expresión es una forma cuadrática de lo que denominaremos deformaciones físicas del modelo (en su forma material)

$$
\begin{aligned}
\boldsymbol{\Gamma}_{\mathrm{d}} & =\boldsymbol{\Gamma}-\boldsymbol{\Gamma}_{0} \\
\boldsymbol{K}_{\mathrm{d}} & =\boldsymbol{K}-\boldsymbol{K}_{0},
\end{aligned}
$$

introducidas ya en la sección anterior. En la matriz de la forma cuadrática aparecen las rigideces frente al esfuerzo axil $(E A)$, los esfuerzos cortantes $\left(G A_{Q 2}, G A_{Q 3}\right)$, el momento torsor $(G J)$ y los momentos flectores $\left(E I_{2}, E I_{3}\right)$ características de la teoría lineal.

Por otra parte, en la sección anterior ya se hizo notar que $\boldsymbol{\Gamma}_{0}$ coincide con la forma material de la tangente a la directriz inicial, es decir, con el vector $\boldsymbol{A}_{1}$ de la base material de componentes $\{1,0,0\}^{\top}$ en el sistema general. Por tanto

$$
\boldsymbol{\Gamma}_{\mathrm{d}}=\boldsymbol{\Gamma}-\boldsymbol{A}_{1}=\left\{\Gamma_{1}-1, \Gamma_{2}, \Gamma_{3}\right\}^{\top} .
$$

La primera componente es una medida del alargamiento del elemento diferencial de directriz $^{6}$; es el factor de la rigidez frente al esfuerzo axil, y es la variable que se

\footnotetext{
${ }^{6}$ Es importante señalar que esta medida del alargamiento no coincide con el alargamiento del diferencial de longitud inicial $d S$, que se obtendría como diferencia entre el diferencial de longitud deformado y el inicial.
} 
corresponde con la derivada del desplazamiento en la dirección de la directriz, $u^{\prime}$, en la teoría lineal. Las dos componentes restantes son las distorsiones en las direcciones de los ejes de la sección.

También es interesante notar que, cuando la pieza es inicialmente recta, entonces la forma material del vector de curvaturas iniciales es nula ${ }^{7}, \boldsymbol{K}_{0}=\mathbf{0}$, y la deformación física $\boldsymbol{K}_{\mathrm{d}}$ coincide con la generalizada $\boldsymbol{K}$.

Aplicando el resultado (4.74) se obtiene la forma material de las ecuaciones constitutivas correspondientes al material isótropo lineal.

$$
\left\{\begin{array}{l}
\boldsymbol{N} \\
\boldsymbol{M}
\end{array}\right\}=\left[\begin{array}{cc}
\mathbf{C}_{\Gamma} & \mathbf{0} \\
\mathbf{0} & \mathbf{C}_{K}
\end{array}\right]\left\{\begin{array}{l}
\boldsymbol{\Gamma}_{\mathrm{d}} \\
\boldsymbol{K}_{\mathrm{d}}
\end{array}\right\}
$$

Si se calcula ahora la versión incremental de las ecuaciones constitutivas es inmediato comprobar que, en el caso del material de Hooke

$$
\mathbf{C}_{\Gamma \Gamma}=\mathbf{C}_{\Gamma} \quad \mathbf{C}_{\Gamma K}=\mathbf{C}_{K \Gamma}=\mathbf{0} \quad \mathbf{C}_{K K}=\mathbf{C}_{K} .
$$

La forma espacial de las ecuaciones constitutivas para el material isótropo lineal se obtiene a partir de la retroacción de las variables materiales

$$
\left\{\begin{array}{c}
\boldsymbol{n} \\
\boldsymbol{m}
\end{array}\right\}=\left[\begin{array}{cc}
\boldsymbol{\Lambda} & \mathbf{0} \\
\mathbf{0} & \boldsymbol{\Lambda}
\end{array}\right]\left[\begin{array}{cc}
\mathbf{C}_{\Gamma} & \mathbf{0} \\
\mathbf{0} & \mathbf{C}_{K}
\end{array}\right]\left[\begin{array}{cc}
\boldsymbol{\Lambda}^{\top} & \mathbf{0} \\
\mathbf{0} & \boldsymbol{\Lambda}^{\top}
\end{array}\right]\left\{\begin{array}{l}
\gamma_{\mathrm{d}} \\
\boldsymbol{\kappa}_{\mathrm{d}}
\end{array}\right\} .
$$

Por lo tanto

$$
\left\{\begin{array}{c}
\boldsymbol{n} \\
\boldsymbol{m}
\end{array}\right\}=\left[\begin{array}{cc}
\mathbf{c}_{\gamma} & \mathbf{0} \\
\mathbf{0} & \mathbf{c}_{\kappa}
\end{array}\right]\left\{\begin{array}{c}
\gamma_{\mathrm{d}} \\
\boldsymbol{\kappa}_{\mathrm{d}}
\end{array}\right\}
$$

con

$$
\begin{aligned}
& \mathbf{c}_{\gamma}=\boldsymbol{\Lambda} \mathbf{C}_{\Gamma} \boldsymbol{\Lambda}^{\top} \\
& \mathbf{c}_{\kappa}=\boldsymbol{\Lambda} \mathbf{C}_{K} \boldsymbol{\Lambda}^{\top} .
\end{aligned}
$$

\footnotetext{
${ }^{7}$ Para comprobarlo basta recordar que las componentes del tensor de curvaturas iniciales $\widehat{\mathbf{k}}_{0}(3.13)$ se anulan si la directriz inicial es recta.
} 



\section{Capítulo 5}

\section{La analogía dinámica}

El análisis desarrollado en el capítulo anterior sugiere la posibilidad de observar la estructura de las ecuaciones del problema homogéneo de equilibrio de la pieza recta con la cinemática de Reissner-Simó bajo el prisma de los principios variacionales, en particular del principio de mínima energía potencial. Este punto de vista, esencialmente lagrangiano, pone de manifiesto un notable paralelismo entre la estructura de los problemas de equilibrio de piezas alargadas, y la de los problemas que aborda la mecánica clásica analizados a partir del principio de mínima acción, lo que permite establecer diversas analogías. La referencia clásica en este sentido es la llamada analogía cinética de Kirchhoff (1859) [35], sobre la que volveremos en este capítulo. La equivalencia formal entre los problemas de estática y los sistemas dinámicos no se circunscribe al problema de Kirchhoff; se puede extender a problemas lineales [45], [39], e incluso a problemas no lineales [38]. En este capítulo desarrollamos la analogía para el caso particular del modelo cinemático RS, haciendo hincapié en sus aspectos más formales que hacen uso de las técnicas matemáticas más recientes [43]. Comenzamos repasando las analogías citadas antes de entrar con más profundidad en el problema de Reissner-Simó.

\section{La analogía lineal}

Monleón [45] ha mostrado cómo las ecuaciones que rigen los problemas lineales de equilibrio de piezas alargadas elásticas pueden ser deducidas por un procedimiento lagrangiano, con independencia de la hipótesis cinemática considerada, siempre que esta cumpla los requisitos de linealidad. La energía potencial $V$ del sistema, suma de la energía de deformación y el potencial de las fuerzas exteriores, se puede expresar en función de los desplazamientos generalizados $\mathbf{u}$ y sus derivadas

$$
V\left(\mathbf{u}, \mathbf{u}^{\prime}\right)=\int_{\Gamma} L\left(S, \mathbf{u}, \mathbf{u}^{\prime}\right) d S-\left(\mathbf{f}_{1} \cdot \mathbf{u}_{1}+\mathbf{f}_{2} \cdot \mathbf{u}_{2}\right)
$$

y actúa como funcional de acción del problema de equilibrio. La función $L$ es la lagrangiana del problema de equilibrio y es la suma de la densidad lineal de energía de deformación y del potencial por unidad de longitud de las fuerzas exteriores 45 , 
ecs. (3.19) y (3.20)]

$$
L\left(S, \mathbf{u}, \mathbf{u}^{\prime}\right)=\frac{1}{2}\left\{\mathbf{u}^{\top} \mathbf{u}^{\prime}\right\}\left[\begin{array}{ll}
\mathbf{D}_{00} & \mathbf{D}_{01} \\
\mathbf{D}_{10} & \mathbf{D}_{11}
\end{array}\right]\left\{\begin{array}{l}
\mathbf{u} \\
\mathbf{u}^{\prime}
\end{array}\right\}-\mathbf{q}^{\top} \cdot \mathbf{u}
$$

donde $\mathbf{q}$ es el vector de fuerzas generalizadas y los bloques $\mathbf{D}_{r s}$ dependen de la hipótesis cinemática y la matriz de constantes elásticas. El equilibrio del sistema se da cuando la energía potencial es estacionaria para cualquier variación cinemáticamente admisible de la configuración

$$
\delta V\left(\mathbf{u}, \mathbf{u}^{\prime}\right)=0 .
$$

El cálculo de variaciones asegura que esta condición se satisface si se cumplen las ecuaciones de Euler-Lagrange en todos los puntos de la directriz

$$
\frac{d}{d S} \frac{\partial L}{\partial \mathbf{u}^{\prime}}=\frac{\partial L}{\partial \mathbf{u}}
$$

Introduciendo las variables energéticas $\mathbf{p}(S)=\partial L / \partial \mathbf{u}^{\prime}$ y derivando en la ecuación (5.2) se tiene

$$
\mathbf{p}=\mathbf{D}_{10} \mathbf{u}+\mathbf{D}_{11} \mathbf{u}^{\prime}
$$

Monleón [45, ecuación (3.28)] demuestra que estas variables coinciden con la resultante de las tensiones que actúan sobre la sección transversal ponderadas por medio de la matriz de la hipótesis cinemática; son, por tanto, los esfuerzos que actúan en cada punto de la pieza. Introduciendo p en las ecuaciones de Euler-Lagrange resulta $\mathbf{p}^{\prime}=\partial L / \partial \mathbf{u}$. Derivando $L$ resulta

$$
\mathbf{p}^{\prime}=\mathbf{D}_{00} \mathbf{u}+\mathbf{D}_{01} \mathbf{u}^{\prime}-\mathbf{q}
$$

Despejando las derivadas de los desplazamientos generalizados y de los esfuerzos de las dos ecuaciones anteriores [45, ecuaciones (4.3) a (4.5)] resultan las ecuaciones de equilibrio en forma canónica

$$
\left\{\begin{array}{l}
\mathbf{u}^{\prime} \\
\mathbf{p}^{\prime}
\end{array}\right\}=\left[\begin{array}{cc}
-\mathbf{D}_{11}^{-1} \mathbf{D}_{10} & \mathbf{D}_{11}^{-1} \\
\mathbf{D}_{00}-\mathbf{D}_{01} \mathbf{D}_{11}^{-1} \mathbf{D}_{10} & \mathbf{D}_{01} \mathbf{D}_{11}^{-1}
\end{array}\right]\left\{\begin{array}{l}
\mathbf{u} \\
\mathbf{p}
\end{array}\right\}-\left\{\begin{array}{l}
\mathbf{0} \\
\mathbf{q}
\end{array}\right\} .
$$

Todas estas consideraciones, y en especial la ecuación (5.4), conducen al establecimiento de una analogía formal entre las ecuaciones de equilibrio estático de una pieza alargada elástica con una hipótesis cinemática lineal con $n$ desplazamientos generalizados, y las ecuaciones de equilibrio dinámico de un sistema mecánico con $n$ grados de libertad, cuya energía total venga dada por la función $H=\mathbf{p} \cdot \mathbf{u}^{\prime}-L$. La longitud de $\operatorname{arco} S$ en la viga es análoga a la variable tiempo en el sistema dinámico, los desplazamientos generalizados de la viga son análogos a los grados de libertad del sistema, y los esfuerzos lo son a los momenta. El autor ha desarrollado esta analogía lineal en [39]. Como generalización de esta idea el autor también ha ampliado la analogía a problemas de equilibrio no lineal de piezas alargadas dotadas de una hipótesis cinemática general definida como función de vectores directores -las variables generalizadas- pertenecientes a un espacio lineal [38. 


\section{La analogía cinética de Kirchhoff}

Ya en el marco de los problemas no lineales, Kirchhoff desarrolló en 1859 [35] una teoría de flexión de piezas alargadas inicialmente rectas considerando rotaciones finitas de la sección transversal, como extensión del trabajo original de Euler [18]. La teoría de Kirchhoff se basa en las siguientes hipótesis ( $c f$. Antman [2]): (a) el vector momento depende linealmente de las curvaturas y la torsión de la directriz, (b) la directriz de la pieza es inextensible, (c) la pieza no presenta deformación por cortante, y (d) la sección transversal es indeformable en su plano. Kirchhoff muestra cómo con estas hipótesis, las ecuaciones de equilibrio de la pieza son formalmente análogas a las ecuaciones del movimiento de un sólido con un punto fijo en un campo gravitatorio (una peonza pesada).

Es muy sencillo comprobar la analogía de Kirchhoff introduciendo algunas restricciones en las ecuaciones obtenidas en el capítulo anterior, como se muestra a continuación. La ausencia de deformación debida al esfuerzo axil y a los esfuerzos cortantes implica: (a) que la sección transversal permanece perpendicular a la tangente a la directriz deformada, y (b) que la tangente a la directriz es un vector unitario, ya que no hay cambio en la longitud de arco, es decir $d s=d S$. Estas conclusiones se resumen en la siguiente ecuación expresada en forma espacial

$$
\boldsymbol{x}^{\prime}=\boldsymbol{a}_{1},
$$

que se puede retrotraer a la configuración material

$$
\Lambda^{\top} \boldsymbol{x}^{\prime}=\boldsymbol{\Lambda}^{\top} \boldsymbol{a}_{1} .
$$

Por lo tanto

$$
\Gamma=\boldsymbol{A}_{1}
$$

Sustituyendo este resultado en las ecuaciones de equilibrio (4.58) cuando no hay fuerzas aplicadas sobre la directriz, se tiene

$$
\begin{aligned}
& \boldsymbol{N}^{\prime}=\boldsymbol{N} \times \boldsymbol{K} \\
& \boldsymbol{M}^{\prime}=\boldsymbol{M} \times \boldsymbol{K}+\boldsymbol{N} \times \boldsymbol{A}_{1} .
\end{aligned}
$$

Las ecuaciones son formalmente idénticas a las de la peonza de Lagrange, recogidas por ejemplo en [43, ecs. (15.73) y (15.74)]. La analogía se establece, por tanto, en los siguientes términos:

1. La longitud de arco sobre la directriz $S$ equivale al tiempo.

2. La deformación $\boldsymbol{K}$ es análoga a la velocidad angular de la peonza expresada en su propio sistema de referencia.

3. Los esfuerzos $\boldsymbol{M}$ se corresponden con el momento angular expresado también en el su sistema de referencia. 
4. Los esfuerzos $\boldsymbol{N}$ se corresponden con el producto del vector que describe la dirección de la gravedad observada desde el sistema de referencia de la peonza, por su masa, la aceleración de la gravedad y la distancia entre el punto de apoyo y el centro de gravedad.

5. El tensor de rigideces $\mathbf{C}_{K}$ de la pieza es análogo al tensor de inercia del sólido, pues establece la relación entre el momento angular $\boldsymbol{M}$ y la velocidad angular K.

Desarrollando estas ecuaciones para $\boldsymbol{A}_{1}=\{1,0,0\}^{\top}$ resulta

$$
\begin{aligned}
\frac{d}{d S} N & =Q_{2} K_{3}-Q_{3} K_{2} \\
\frac{d}{d S} Q_{2} & =Q_{3} K_{1}-N K_{3} \\
\frac{d}{d S} Q_{3} & =N K_{2}-Q_{2} K_{1} \\
\frac{d}{d S} M_{1} & =M_{2} K_{3}-M_{3} K_{2} \\
\frac{d}{d S} M_{2} & =M_{3} K_{1}-M_{1} K_{3}+Q_{3} \\
\frac{d}{d S} M_{3} & =M_{1} K_{2}-M_{2} K_{1}-Q_{2}
\end{aligned}
$$

que es precisamente la expresión de las ecuaciones de equilibrio incluida con otra notación en Love [40, §254, ecs. (10), (11)]. En el epígrafe 260 de la referencia citada se desarrolla la analogía, obteniendo finalmente la expresión equivalente a la energía total de la peonza -ec. (3)-

$$
\frac{1}{2}\left(G J\left(K_{1}\right)^{2}+E I_{2}\left(K_{2}\right)^{2}+E I_{3}\left(K_{3}\right)^{2}\right)+N=\text { const } .
$$

que expresada en función de las variables estáticas es igual a

$$
\frac{1}{2}\left(\frac{\left(M_{1}\right)^{2}}{G J}+\frac{\left(M_{2}\right)^{2}}{E I_{2}}+\frac{\left(M_{3}\right)^{2}}{E I_{3}}\right)+N=\text { const. }
$$

Esta función invariante es la función de Hamilton de la analogía de Kirchhoff.

\section{Introducción a la analogía dinámica para el modelo RS}

Retornamos ahora al problema de la pieza alargada con la cinemática de ReissnerSimó, formada por un material hiperelástico para el que es posible definir una densidad de energía de deformación por unidad de longitud $\rho_{0} \mathcal{W}(\boldsymbol{\Gamma}, \boldsymbol{K})$ función de las deformaciones generalizadas, que -al menos a nivel teórico- se deduce de la densidad de energía de deformación que caracteriza a este tipo de materiales. En este capítulo mostraremos cómo establecer también una analogía dinámica para este problema, generalizando la analogía de Kirchhoff. 
La proposición 4.4 .3 muestra cómo las resultantes de tensiones (en fuerzas y momentos) sobre la sección transversal se obtienen como derivadas de la densidad de energía de deformación. Se establece así en el modelo de Reissner-Simó una definición constitutiva de estas resultantes de tensiones, que coinciden con la definición clásica de los esfuerzos y se obtienen como derivadas de $\rho_{0} \mathcal{W}(\boldsymbol{\Gamma}, \boldsymbol{K})$ respecto de las variables cinemáticas $\boldsymbol{\Gamma}$ y $\boldsymbol{K}$. La densidad de energía de deformación actúa pues como función potencial de los esfuerzos. Por comodidad introducimos la notación

$$
\mathcal{L}(\boldsymbol{\Gamma}, \boldsymbol{K})=\rho_{0} \mathcal{W}(\boldsymbol{\Gamma}, \boldsymbol{K}) .
$$

La función $\mathcal{L}$ es la función lagrangiana del problema de rotaciones finitas cuando no actúan fuerzas generalizadas sobre la directriz (problema homogéneo). Con todos estos supuestos es posible reescribir las ecuaciones intrínsecas de equilibrio (4.58) para el problema homogéneo de la pieza recta de sección constante de la siguiente forma

$$
\begin{aligned}
\frac{d}{d S} \frac{\partial \mathcal{L}}{\partial \boldsymbol{\Gamma}} & =\frac{\partial \mathcal{L}}{\partial \boldsymbol{\Gamma}} \times \boldsymbol{K} \\
\frac{d}{d S} \frac{\partial \mathcal{L}}{\partial \boldsymbol{K}} & =\frac{\partial \mathcal{L}}{\partial \boldsymbol{\Gamma}} \times \boldsymbol{\Gamma}+\frac{\partial \mathcal{L}}{\partial \boldsymbol{K}} \times \boldsymbol{K}
\end{aligned}
$$

Estas ecuaciones se pueden organizar en forma matricial

$$
\frac{d}{d S}\left\{\begin{array}{l}
\partial \mathcal{L} / \partial \boldsymbol{\Gamma} \\
\partial \mathcal{L} / \partial \boldsymbol{K}
\end{array}\right\}=\left[\begin{array}{cc}
\mathbf{0} & \partial \mathcal{L} \widehat{\mathcal{L}} \boldsymbol{\Gamma} \\
\partial \mathcal{L} \widehat{/ \partial} \boldsymbol{\Gamma} & \partial \mathcal{L} \widehat{\mathcal{L}} \boldsymbol{K}
\end{array}\right]\left\{\begin{array}{l}
\boldsymbol{\Gamma} \\
\boldsymbol{K}
\end{array}\right\} .
$$

En este caso, a diferencia de la expresión matricial alternativa (4.61) de las ecuaciones de equilibrio, la matriz del sistema sí es antisimétrica. Las expresiones (5.13) y (5.14) recuerdan la estructura de las ecuaciones de Euler del movimiento de un sólido libre -véase, por ejemplo, Arnold [7, sección 29], o Marsden y Ratiu [43, ec.(1.24)]-

$$
\frac{d}{d t} \frac{\partial L}{\partial \boldsymbol{\Omega}}=\frac{\partial L}{\partial \boldsymbol{\Omega}} \times \boldsymbol{\Omega}
$$

o en forma matricial

$$
\frac{d}{d t} \frac{\partial L}{\partial \boldsymbol{\Omega}}=\frac{\widehat{\partial L}}{\partial \boldsymbol{\Omega}} \boldsymbol{\Omega}
$$

en las que $\boldsymbol{\Omega}$ es la velocidad angular del sólido expresada en el sistema de referencia intrínseco, y $L$ es la función lagrangiana del problema. En ambos casos el vector de las derivadas de las variables mecánicas -el vector de esfuerzos $\{\partial \mathcal{L} / \partial \boldsymbol{\Gamma}, \partial \mathcal{L} / \partial \boldsymbol{K}\}^{\top}$ en el problema de la viga, y el vector momento angular $\partial L / \partial \boldsymbol{\Omega}$ en el del sólido librese iguala al producto de una matriz antisimétrica formada por las componentes de las propias variables mecánicas que multiplica al vector de las variables cinemáticas -las deformaciones generalizadas $\{\boldsymbol{\Gamma}, \boldsymbol{K}\}^{\top}$ en el problema de la viga y la velocidad angular $\boldsymbol{\Omega}$ en el problema del sólido-. Parece claro que existe una estrecha relación entre ambos sistemas de ecuaciones diferenciales, cuyo origen se encuentra en la estructura del espacio de las configuraciones, que en los dos casos es un grupo de Lie: el grupo 
$\mathrm{SO}(3)$ en el problema del sólido y el grupo SE(3) - de los movimientos euclídeos- en el de la viga ${ }^{1}$.

Las ecuaciones de Euler expresadas en la forma (5.15) se denominan ecuaciones de Euler-Poincaré [43]. Estas ecuaciones aparecen con esta forma en el capítulo 2 de la Mécanique Analytique de Lagrange [36], Poincaré [50] las generaliza para cualquier grupo de Lie -véase [43, sección 1.2]-. Marsden y Ratiu recogen esta generalización en el teorema 13.5.3. de la obra citada, que se reproduce a continuación:

Teorema (Marsden y Ratiu). Sea $G$ un grupo de Lie, $L: T G \rightarrow \mathbb{R}$ una función lagrangiana invariante por la izquierda y $l: \mathfrak{g} \rightarrow \mathbb{R}$ su restricción al elemento neutro. Dada la trayectoria $g(t) \in G$, sea $\xi(t)=g(t)^{-1} \cdot \dot{g}(t) \in \mathfrak{g}$. Entonces, los siguientes enunciados son equivalentes:

1. $g(t)$ satisface las ecuaciones de Euler-Lagrange para $L$ en $G$.

2. El principio variacional

$$
\delta \int L(g(t), \dot{g}(t)) d t=0
$$

se satisface para cualquier variación que se anule en los extremos del dominio det.

3. Se cumplen las ecuaciones de Euler-Poincaré para l en $\mathfrak{g}$

$$
\frac{d}{d t} \frac{\partial l}{\partial \xi}=\operatorname{ad}_{\xi}^{*} \frac{\partial l}{\partial \xi}
$$

\section{El principio variacional reducido}

$$
\delta \int l(\xi(t)) d t=0
$$

se satisface en $\mathfrak{g}$ para variaciones de la forma

$$
\delta \xi=\dot{\delta} \dot{\omega}+[\xi, \delta \omega]
$$

que se anulan en los extremos del dominio de $t$.

Este teorema establece un puente entre las ecuaciones de Euler-Lagrange que se cumplen para los elementos del grupo $G$, y las ecuaciones de Euler-Poincaré, que rigen el mismo sistema físico, pero relacionan elementos del álgebra $\mathfrak{g}$-el espacio tangente a $G$ en el elemento neutro-. Marsden y Ratiu denominan reducción a la traducción del principio variacional original al principio variacional en $\mathfrak{g}$. Cuando el grupo $G$ es el ortogonal SO(3) las ecuaciones de Euler-Poincaré (5.18) coinciden con las ecuaciones de Euler (5.15) del movimiento del sólido rígido libre: los elementos del álgebra son los vectores de velocidad angular $\boldsymbol{\Omega} \in \mathbb{R}^{3}$, el vector momento angular

\footnotetext{
${ }^{1}$ Marsden y Ratiu [43] contiene un análisis de la estructura del grupo SE(3).
} 
$\partial L / \partial \boldsymbol{\Omega}$ es un elemento del espacio dual $\mathfrak{g}^{*}$, el operador ad $\mathrm{d}_{\xi}^{*}$ es el producto vectorial entre los elementos del álgebra y su dual, y el conmutador $[\cdot, \cdot \cdot]$ es el producto vectorial entre elementos del álgebra.

En un problema de valores iniciales en el que $\xi(0)$ sea conocido es posible, disponiendo de un método numérico de solución de (5.18), avanzar la solución $\xi(t)$ en el álgebra $\mathfrak{g}$ hasta el valor de $t$ deseado. Una vez conocida la solución en el álgebra es necesario reconstruir la solución en el grupo. Para ello se emplea la siguiente ecuación de reconstrucción en sistemas invariantes por la izquierda

$$
\dot{g}(t)=g(t) \xi(t)
$$

La reconstrucción se puede llevar a cabo conociendo el valor inicial $g(0)$ y disponiendo de otro método numérico para avanzar la solución en $G$.

El esquema reducción - reconstrucción es importante porque permite dividir y simplificar el proceso de solución de un problema de valores iniciales en dos fases, la primera de las cuales se desarrolla en el álgebra, y es independiente de los elementos del grupo, y por lo tanto de su parametrización. La parametrización del grupo es precisamente el aspecto que más complejidad introduce en los procesos de solución, empezando por la elección de la más adecuada, elección que no es obvia, y de la que se tratará en el capítulo correspondiente. La escritura de las ecuaciones en la forma de Euler-Lagrange requiere, sin embargo, la elección previa de una parametrización del grupo de las soluciones, para poder derivar la función lagrangiana respecto de cada parámetro.

En el problema de equilibrio de piezas alargadas que nos ocupa, las ventajas del binomio reducción-reconstrucción son algo diferentes. En primer lugar, se trata de un problema de contorno, y por lo tanto no es viable un proceso de solución como el descrito para el de valores iniciales. Sin embargo es posible proporcionar una interpretación física directa para las ecuaciones reducidas y para las de reconstrucción:

- Las ecuaciones de Euler-Poincaré (ecuaciones reducidas) se identifican con las ecuaciones de equilibrio del problema físico, y éstas pueden formularse íntegramente en términos de elementos del álgebra -las deformaciones generalizadas-, y de su espacio dual -los esfuerzos-, prescindiendo totalmente de la parametrización escogida para el grupo.

- Las ecuaciones de reconstrucción se identifican con las ecuaciones cinemáticas, que relacionan las deformaciones generalizadas con los elementos del grupo -el espacio de las configuraciones- $\mathrm{y}$ sus derivadas.

En la sección siguiente particularizamos el teorema de Marsden y Ratiu al problema homogéneo de la pieza alargada, en el que el espacio de las configuraciones es el grupo especial euclídeo SE(3), demostrando que las expresiones (5.13) coinciden con las ecuaciones de Euler-Poincaré en $\mathfrak{s e}(3)$, que es el espacio de las deformaciones generalizadas. Este resultado, que Holms, Marsden y Ratiu [24, pág. 27] han demostrado a partir del teorema anterior empleando herramientas de cálculo en grupos de Lie, 
se deduce a continuación de forma directa para nuestro problema, y permite relacionar el principio variacional reducido con la forma material del principio de trabajos virtuales empleada en la sección anterior.

Finalmente, la última sección de este capítulo plantea el problema en términos de los esfuerzos, que son las variables duales pertenecientes al álgebra $\mathfrak{s e}(3)^{*}$, dual de $\mathfrak{s e}(3)$; en ella se muestra que las ecuaciones escritas en función de los esfuerzos tienen la denominada estructura de Lie-Poisson, y se establecen paralelismos con los problemas de dinámica.

\subsection{Las ecuaciones de Euler-Poincaré}

El estudio del problema de equilibrio de la pieza alargada desde un punto de vista lagrangiano se basa en admitir que existe una función que define su energía potencial, y que las ecuaciones de equilibrio se deducen a partir de la condición de estacionariedad de esta función. Es evidente que esta definición pone de manifiesto la necesidad de que las fuerzas que actúan sobre el sólido tengan un carácter conservativo para que la definición de potencial tenga sentido. Esta condición puede ser excesivamente restrictiva frente a un planteamiento general del problema. En particular, cuando se admite que las rotaciones de la sección son finitas es sencillo imaginar fuerzas no conservativas: por ejemplo aquellas que acompañan a la sección transversal en su rotación (que posteriormente definiremos como intrínsecas), o los momentos de eje fijo en el espacio [4], [54].

En esta sección estamos interesados en investigar la estructura y el origen de las ecuaciones que gobiernan el problema; por ello vamos a simplificarlo al máximo suponiendo que la pieza es recta, prescindiendo de las fuerzas generalizadas, y admitiendo únicamente la existencia de valores prescritos de las deformaciones generalizadas $\boldsymbol{\Gamma}, \boldsymbol{K}$ en los extremos de la pieza. Este tipo de condición de contorno, que es equivalente a imponer el valor de los esfuerzos en los extremos de la pieza, no tiene una utilidad práctica directa, pero es muy conveniente para demostrar que las ecuaciones de equilibrio tienen la estructura de Euler-Poincaré. Denominaremos problema homogéneo al problema de equilibrio formulado en estos términos.

El desarrollo de la estática de la pieza alargada desde el punto de vista lagrangiano se basa en el siguiente principio:

La configuración $(\boldsymbol{x}, \boldsymbol{\Lambda})$ es solución del problema de equilibrio de la pieza alargada si la energía potencial $V$ asociada a dicha configuración es estacionaria.

La condición de estacionariedad de $V$ es equivalente a la condición de nulidad de la derivada direccional del potencial en cualquier dirección tangente a la configuración de equilibrio - entendida la configuración como una curva definida en la variedad de las soluciones, que en el problema que nos ocupa es el grupo $\mathrm{SE}(3)-$. Una formulación alternativa del principio anterior indica que la configuración $(\boldsymbol{x}, \boldsymbol{\Lambda})$ es una configuración de equilibrio cuando la variación de la energía potencial es nula para cualquier 
variación cinemáticamente admisible de la configuración, es decir

$$
\delta V=0 \quad \forall \delta \boldsymbol{x}, \delta \widehat{\boldsymbol{\omega}} \in T \mathrm{SE}(3) .
$$

En esta expresión $\delta V$ es la derivada direccional del potencial $V$, que se calcula de la siguiente forma

$$
\delta V=\left.\frac{d}{d \varepsilon} V(\boldsymbol{x}+\varepsilon \delta \boldsymbol{x}, \exp (\varepsilon \delta \widehat{\boldsymbol{\omega}}) \boldsymbol{\Lambda})\right|_{\varepsilon=0}
$$

En este punto conviene llamar la atención sobre la diferencia entre la forma de derivar el vector posición $\boldsymbol{x}$ y el tensor de rotación $\boldsymbol{\Lambda}$, debida a que este último es un elemento del grupo $\mathrm{SO}(3)$. La derivada se calcula en la dirección $(\delta \boldsymbol{x}, \delta \widehat{\boldsymbol{\omega}})$ tangente a $\mathrm{SE}(3)$ en $(\boldsymbol{x}, \boldsymbol{\Lambda})$. El vector tangente $(\delta \boldsymbol{x}, \delta \widehat{\boldsymbol{\omega}})$ es, por lo tanto, un objeto espacial (véase la figura 4.1). Dado que las variables cinemáticas son conocidas en los extremos de la pieza, entonces el vector de las variaciones será nulo en ambos puntos para que las variaciones sean cinemáticamente admisibles.

Sin embargo, para evaluar de forma correcta la variación del potencial es necesario recordar que hemos demostrado la dependencia de la densidad lineal de energía de deformación respecto de las variables $\boldsymbol{\Gamma}$ y $\boldsymbol{K}$, que son variables materiales. Si, como hemos supuesto, no actúan fuerzas sobre la directriz, y en los extremos se prescribe el valor de las deformaciones generalizadas, entonces la energía potencial se compone de un único término debido a la energía de deformación

$$
V(\boldsymbol{x}, \boldsymbol{\Lambda})=\int_{\Gamma_{0}} \mathcal{L}(\boldsymbol{\Gamma}, \boldsymbol{K}) d S
$$

con la notación introducida antes

$$
\mathcal{L}(\boldsymbol{\Gamma}, \boldsymbol{K})=\rho_{0} \mathcal{W}(\boldsymbol{\Gamma}, \boldsymbol{K}),
$$

Por lo tanto, dado que las variables de las que depende el potencial son, en última instancia, variables materiales, la derivada direccional debe calcularse en la configuración material o intrínseca, derivando en la dirección $(\delta \boldsymbol{\chi}, \delta \widehat{\boldsymbol{\Omega}})$, que es tangente al grupo en el elemento neutro $\Lambda^{\top} \boldsymbol{\Lambda}$, y es por tanto un elemento del álgebra $\mathfrak{s e}(3)$. Entonces

$$
\begin{aligned}
\delta V & =\delta \int_{\Gamma_{0}} \mathcal{L}(\boldsymbol{\Gamma}, \boldsymbol{K}) d S \\
& =\int_{\Gamma_{0}}\left(\frac{\partial \mathcal{L}}{\partial \boldsymbol{\Gamma}} \cdot \delta \boldsymbol{\Gamma}+\frac{\partial \mathcal{L}}{\partial \boldsymbol{K}} \cdot \delta \boldsymbol{K}\right) d S=0 .
\end{aligned}
$$

En esta expresión $\delta \boldsymbol{\Gamma}$ y $\delta \boldsymbol{K}$ representan las derivadas direccionales de las deformaciones generalizadas en la dirección $(\delta \boldsymbol{\chi}, \delta \widehat{\boldsymbol{\Omega}})$. El cálculo de estas derivadas -variacionesya se ha llevado a cabo en la proposición 4.2.3. El resultado se recoge en las expresiones (4.43a) y (4.43b) que repetimos aquí por conveniencia ( $\delta \boldsymbol{\Omega}$ es el vector axial correspondiente a $\delta \widehat{\mathbf{\Omega}})$ :

$$
\begin{aligned}
\delta \boldsymbol{\Gamma} & =(\delta \boldsymbol{\chi})^{\prime}+\boldsymbol{K} \times \delta \boldsymbol{\chi}+\boldsymbol{\Gamma} \times \delta \boldsymbol{\Omega} \\
\delta \boldsymbol{K} & =(\delta \boldsymbol{\Omega})^{\prime}+\boldsymbol{K} \times \delta \boldsymbol{\Omega} .
\end{aligned}
$$


Dado que, por hipótesis, el valor de las deformaciones generalizadas en los extremos de la pieza es un dato, entonces su variación en los extremos debe ser nula para que la variación de la configuración sea cinemáticamente admisible (es decir, para que sea tal que se respeten las condiciones de contorno).

Las ecuaciones de equilibrio se obtienen integrando por partes; este proceso ya se ha desarrollado en la sección 4.3. El resultado son las ecuaciones (5.13) que coinciden con las ecuaciones (4.58) salvo por los términos debidos a las fuerzas generalizadas, que por hipótesis no aparecen en este caso.

Si retornamos a la última integral de la igualdad anterior e identificamos las derivadas de la densidad de energía de deformación con la forma material de los esfuerzos en virtud de la proposición 4.4.3, es inmediato reconocer el término correspondiente al trabajo virtual de las fuerzas internas de la versión material del principio de los trabajos virtuales formulado en la expresión (4.29)

$$
\int_{\Gamma_{0}}(\boldsymbol{N} \cdot \delta \boldsymbol{\Gamma}+\boldsymbol{M} \cdot \delta \boldsymbol{K}) d S
$$

Con las hipótesis consideradas (ausencia de fuerzas generalizadas a lo largo de la directriz y deformaciones generalizadas prescritas en los extremos de la pieza), el trabajo virtual de las fuerzas exteriores es nulo: el producido por las fuerzas generalizadas evidentemente lo es, y el trabajo virtual que se desarrolla en los extremos de la pieza también es nulo ya que la variación de las deformaciones generalizadas en los extremos es nula. Por lo tanto, la única contribución restante al trabajo virtual es la debida a las fuerzas internas y esta debe ser, a su vez, nula.

Todos los razonamientos anteriores se pueden concretar en la siguiente proposición:

Proposición 5.1.1. Consideremos una pieza alargada recta de sección constante con la cinemática de Reissner-Simó, sobre la que no actúan fuerzas exteriores y únicamente se prescribe el valor de las deformaciones generalizadas en sus extremos (problema homogéneo). La energía potencial de la pieza coincide en estas condiciones con su energía de deformación, que es función de la forma material de las deformaciones generalizadas y es independiente del sistema de referencia (invariante frente a rotaciones por la izquierda). Con estas condiciones los siguientes enunciados son equivalentes:

1. La configuración $(\boldsymbol{x}(S), \boldsymbol{\Lambda}(S))$ es solución del problema de equilibrio.

2. El principio variacional (reducido)

$$
\delta \int_{\Gamma_{0}} \rho_{0} \mathcal{W}(\boldsymbol{\Gamma}, \boldsymbol{K}) d S=0
$$

se satisface en $\mathfrak{s e}(3)$ para variaciones $\delta \boldsymbol{\Gamma}$ y $\delta \boldsymbol{K}$ de la forma

$$
\begin{aligned}
\delta \boldsymbol{\Gamma} & =(\delta \boldsymbol{\chi})^{\prime}+\boldsymbol{K} \times \delta \boldsymbol{\chi}+\boldsymbol{\Gamma} \times \delta \boldsymbol{\Omega} \\
\delta \boldsymbol{K} & =(\delta \boldsymbol{\Omega})^{\prime}+\boldsymbol{K} \times \delta \boldsymbol{\Omega},
\end{aligned}
$$

que se anulan en los extremos de la pieza. 
3. Las ecuaciones de equilibrio del sólido tienen la estructura de Euler-Poincaré en el álgebra $\mathfrak{s e}(3)$ del grupo especial euclídeo.

$$
\begin{aligned}
\frac{d}{d S} \frac{\partial \mathcal{L}}{\partial \boldsymbol{\Gamma}} & =\frac{\partial \mathcal{L}}{\partial \boldsymbol{\Gamma}} \times \boldsymbol{K} \\
\frac{d}{d S} \frac{\partial \mathcal{L}}{\partial \boldsymbol{K}} & =\frac{\partial \mathcal{L}}{\partial \boldsymbol{\Gamma}} \times \boldsymbol{\Gamma}+\frac{\partial \mathcal{L}}{\partial \boldsymbol{K}} \times \boldsymbol{K},
\end{aligned}
$$

$\operatorname{con} \mathcal{L}(\boldsymbol{\Gamma}, \boldsymbol{K})=\rho_{0} \mathcal{W}(\boldsymbol{\Gamma}, \boldsymbol{K})$.

4. El trabajo virtual de las fuerzas internas es nulo para cualquier variación $(\delta \boldsymbol{\chi}, \delta \boldsymbol{\Omega})$ cinemáticamente admisible de la configuración.

Los razonamientos que conducen a la proposición anterior proporcionan una explicación a la definición de variación corrotacional introducida en la sección 4.3 para formular el principio de trabajos virtuales en su versión espacial. El análisis lagrangiano del problema se fundamenta en el cálculo de la derivada (la variación) del funcional definido por la densidad de energía de deformación por unidad de longitud. Son precisamente las características de la densidad de energía de deformación las que condicionan el problema: se trata de una función independiente del sistema de referencia espacial, lo que implica que es invariante frente a rotaciones por la izquierda. Este tipo de invarianza implica su dependencia de la forma material de las deformaciones generalizadas. Por ello la derivada de esta función debe evaluarse en la configuración material, en direcciones tangentes a la retroacción de las variables cinemáticas a esta configuración. Cuando se define el principio de trabajos virtuales en su forma espacial es necesario introducir una definición de variación de las variables cinemáticas espaciales que sea compatible con la variación definida para la forma material. Esto justifica las definiciones de la variación corrotacional (4.8) y (4.10) en las que el objeto espacial se retrotrae a la configuración material, se evalúa su derivada en esta configuración y el resultado se empuja de nuevo a la configuración espacial.

El alcance de la proposición se puede ampliar parcialmente suponiendo que actúan fuerzas exteriores originadas por un potencial. No desarrollamos este caso porque el análisis del problema homogéneo basta, como se ha visto, para demostrar la estructura matemática de las ecuaciones de campo. La situación más general, en la que pueden actuar fuerzas exteriores cuyo carácter no sea conservativo, y el material no tiene una respuesta hiperelástica, queda cubierta por el principio de trabajos virtuales (4.29), que puede ser interpretado como generalización de la ecuación (5.24).

\subsection{Las ecuaciones de Lie-Poisson}

Consideremos a continuación la transformación de Legendre ${ }^{2}$ de la función de densidad de energía de deformación $\mathcal{L}(\boldsymbol{\Gamma}, \boldsymbol{K})$ actuando sobre las deformaciones generalizadas $\boldsymbol{E}=\{\boldsymbol{\Gamma}, \boldsymbol{K}\}^{\top}$ e introduciendo la variable conjugada $\boldsymbol{F}$, de forma que

$$
\mathcal{H}(\boldsymbol{F})=\boldsymbol{F} \cdot \boldsymbol{E}-\mathcal{L}(\boldsymbol{E}),
$$

\footnotetext{
${ }^{2}$ [7, pág. 61 y ss.] contiene una definición de este tipo de transformaciones.
} 
expresión en la que $\boldsymbol{E}=\boldsymbol{E}(\boldsymbol{F})$, y la variable conjugada $\boldsymbol{F}$ se define como

$$
\boldsymbol{F}=\frac{\partial \mathcal{L}}{\partial \boldsymbol{E}}=\{\partial \mathcal{L} / \partial \boldsymbol{\Gamma}, \partial \mathcal{L} / \partial \boldsymbol{K}\}^{\top},
$$

y pertenece a $\mathfrak{s e}^{*}(3)$, el álgebra dual de $\mathfrak{s e}(3)$. Es inmediato comprobar que las componentes de $\boldsymbol{F}$ son precisamente los esfuerzos $\{\boldsymbol{N}, \boldsymbol{M}\}^{\top}$ definidos en la proposición 4.4.3. Para que la transformación (5.27) tenga sentido, es necesario que la definición (5.28) sea invertible, pues en caso contrario no es posible expresar $\boldsymbol{E}$ en función de $\boldsymbol{F}$.

Denominamos función de Hamilton del problema homogéneo de rotaciones finitas a la función $\mathcal{H}$, ya que tiene el mismo papel que la función homónima en la Dinámica. Si calculamos ahora la derivada de la nueva función en la dirección de $\boldsymbol{F}$ se tiene

$$
\frac{\partial \mathcal{H}}{\partial \boldsymbol{F}}=\boldsymbol{E}+\boldsymbol{F} \cdot \frac{\partial \boldsymbol{E}}{\partial \boldsymbol{F}}-\frac{\partial \mathcal{L}}{\partial \boldsymbol{E}} \cdot \frac{\partial \boldsymbol{E}}{\partial \boldsymbol{F}}=\boldsymbol{E}
$$

Desarrollando este resultado se tiene

$$
\begin{aligned}
\frac{\partial \mathcal{H}}{\partial \boldsymbol{N}} & =\boldsymbol{\Gamma} \\
\frac{\partial \mathcal{H}}{\partial \boldsymbol{M}} & =\boldsymbol{K}
\end{aligned}
$$

que es la versión dual de las ecuaciones constitutivas.

Haciendo uso ahora de esta nueva definición constitutiva, así como de la definición (5.28) de las variables conjugadas, las ecuaciones de Euler-Poincaré (las ecuaciones de equilibrio del problema homogéneo), admiten también una escritura dual

$$
\begin{aligned}
\boldsymbol{N}^{\prime} & =\boldsymbol{N} \times \frac{\partial \mathcal{H}}{\partial \boldsymbol{M}} \\
\boldsymbol{M}^{\prime} & =\boldsymbol{N} \times \frac{\partial \mathcal{H}}{\partial \boldsymbol{N}}+\boldsymbol{M} \times \frac{\partial \mathcal{H}}{\partial \boldsymbol{M}},
\end{aligned}
$$

en la que sólo intervienen los esfuerzos, que son elementos del álgebra dual $\mathfrak{s e}^{*}(3)$. La estructura de las ecuaciones de equilibrio expresadas en función de los elementos de $\mathfrak{s e}^{*}(3)$ recibe el nombre de Lie-Poisson [43]. En forma matricial se puede escribir

$$
\left\{\begin{array}{l}
\boldsymbol{N}^{\prime} \\
\boldsymbol{M}^{\prime}
\end{array}\right\}=\left[\begin{array}{cc}
\mathbf{0} & \widehat{\mathbf{N}} \\
\widehat{\mathbf{N}} & \widehat{\mathbf{M}}
\end{array}\right]\left\{\begin{array}{l}
\partial \mathcal{H} / \partial \boldsymbol{N} \\
\partial \mathcal{H} / \partial \boldsymbol{M}
\end{array}\right\}
$$

y de forma abreviada

$$
\boldsymbol{F}^{\prime}=\widehat{\mathbf{F}} \nabla \mathcal{H}(\boldsymbol{F})
$$

expresión en la que se ha introducido la matriz antisimétrica

$$
\widehat{\mathbf{F}}=\left[\begin{array}{cc}
\mathbf{0} & \widehat{\mathbf{N}} \\
\widehat{\mathbf{N}} & \widehat{\mathbf{M}}
\end{array}\right],
$$


que depende linealmente de las variables duales $\boldsymbol{N}, \boldsymbol{M}$ y define la estructura de LiePoisson $^{3}$. $\widehat{\mathbf{F}}$ aparecerá posteriormente al linealizar el principio de trabajos virtuales, y no debe ser confundida con la expresión material del tensor de deformaciones $\mathbf{F}$. Mielke y Holmes [44] han sido los primeros en detectar la estructura hamiltoniana -no canónica- del problema, válida para cualquier función de Hamilton definida por medio de la transformación de Legendre (5.27).

El resultado que se prueba a continuación es equivalente al teorema de conservación de la energía en Mecánica Clásica. La demostración para funciones de Hamilton definidas en espacios lineales es de sobra conocida y se encuentra, por ejemplo, en Lanczos [37]:

Proposición 5.2.1. Sea $\mathcal{H}=\mathcal{H}(\mathbf{F})$ la función de Hamilton del problema homogéneo de equilibrio de la pieza alargada con la cinemática de Reissner-Simó, que es independiente de la variable $S$. Entonces el valor de $\mathcal{H}$ es constante en el dominio de $S y$ es un invariante del sistema.

Demostración. Derivando $\mathcal{H}$ respecto de la variable $S$

$$
\frac{d \mathcal{H}}{d S}=\frac{\partial \mathcal{H}}{\partial \boldsymbol{F}} \cdot \frac{d \boldsymbol{F}}{d S}=\frac{\partial \mathcal{H}}{\partial \boldsymbol{N}} \cdot \boldsymbol{N}^{\prime}+\frac{\partial \mathcal{H}}{\partial \boldsymbol{M}} \cdot \boldsymbol{M}^{\prime}
$$

Haciendo uso de las ecuaciones de Lie-Poisson (5.31)

$$
\frac{d \mathcal{H}}{d S}=\frac{\partial \mathcal{H}}{\partial \boldsymbol{N}} \cdot\left(\boldsymbol{N} \times \frac{\partial \mathcal{H}}{\partial \boldsymbol{M}}\right)+\frac{\partial \mathcal{H}}{\partial \boldsymbol{M}} \cdot\left(\boldsymbol{N} \times \frac{\partial \mathcal{H}}{\partial \boldsymbol{N}}\right)+\frac{\partial \mathcal{H}}{\partial \boldsymbol{M}} \cdot\left(\boldsymbol{M} \times \frac{\partial \mathcal{H}}{\partial \boldsymbol{M}}\right) .
$$

El último término es nulo y el segundo se puede reordenar, de forma que

$$
\frac{d \mathcal{H}}{d S}=\frac{\partial \mathcal{H}}{\partial \boldsymbol{N}} \cdot\left(\boldsymbol{N} \times \frac{\partial \mathcal{H}}{\partial \boldsymbol{M}}\right)-\frac{\partial \mathcal{H}}{\partial \boldsymbol{N}} \cdot\left(\boldsymbol{N} \times \frac{\partial \mathcal{H}}{\partial \boldsymbol{M}}\right)=0 .
$$

La función de Hamilton es, por lo tanto, un invariante del sistema. Su interpretación física para el problema de la pieza alargada no es inmediata. Para establecerla

\footnotetext{
${ }^{3}$ La estructura de Lie-Poisson definida por las ecuaciones (5.33) es precisamente la de un sistema hamiltoniano no canónico, en contraposición a los sistemas hamiltonianos canónicos, definidos por una relación del tipo

$$
\boldsymbol{X}^{\prime}=\mathbf{J} \nabla \mathcal{H}(\boldsymbol{X})
$$

en la que $\mathbf{J}$ es la llamada matriz simpléctica$$
\mathbf{J}=\left[\begin{array}{cc}
0 & 1 \\
-1 & 0
\end{array}\right]
$$

que es, como $\widehat{\mathbf{F}}$, una matriz antisimétrica (las ecuaciones de los modelos lineales de piezas alargadas son de este tipo). La matriz $\mathbf{J}$ permite definir el corchete (canónico) de Poisson

$$
\{F, G\}_{\text {canonical }}=\nabla F(\boldsymbol{X})^{\top} \mathbf{J} \nabla G(\boldsymbol{X}) .
$$

Las referencias [20], 23] y [43] desarrollan la teoría de los sistemas hamiltonianos y de Poisson desde un punto de vista físico, numérico y matemático, respectivamente.
} 
podemos calcular la densidad de energía complementaria de la pieza. Ésta se obtiene, en el problema homogéneo, como diferencia entre el producto escalar del vector de esfuerzos por el vector de deformaciones físicas definidas en (4.78), y la densidad de energía de deformación

$$
\rho_{0} \overline{\mathcal{W}}(\boldsymbol{N}, \boldsymbol{M})=\boldsymbol{N} \cdot\left(\boldsymbol{\Gamma}-\boldsymbol{A}_{1}\right)+\boldsymbol{M} \cdot \boldsymbol{K}-\rho_{0} \mathcal{W}(\boldsymbol{\Gamma}, \boldsymbol{K}) .
$$

Comparando esta expresión con la definición de la función de Hamilton (5.27) resulta

$$
\mathcal{H}(\boldsymbol{N}, \boldsymbol{M})=\rho_{0} \overline{\mathcal{W}}(\boldsymbol{N}, \boldsymbol{M})+\boldsymbol{N} \cdot \boldsymbol{A}_{1} .
$$

Es decir, la función de Hamilton del problema homogéneo es la suma de la energía complementaria de la pieza y la primera componente del vector $\boldsymbol{N}$ (en el sistema de referencia intrínseco), que habitualmente se identifica con el esfuerzo axil $N$. Para el material elástico lineal se tiene, a partir de la definición de la densidad de energía de deformación (4.76)

$$
\begin{aligned}
\mathcal{H}(\boldsymbol{N}, \boldsymbol{M}) & =\boldsymbol{N} \cdot \boldsymbol{\Gamma}+\boldsymbol{M} \cdot \boldsymbol{K} \\
& -\frac{1}{2} \mathbf{C}_{\Gamma}\left(\boldsymbol{\Gamma}-\boldsymbol{A}_{1}\right) \cdot\left(\boldsymbol{\Gamma}-\boldsymbol{A}_{1}\right)-\frac{1}{2} \mathbf{C}_{K} \boldsymbol{K} \cdot \boldsymbol{K} .
\end{aligned}
$$

Sumando y restando el término $\boldsymbol{N} \cdot \boldsymbol{A}_{1}$ y expresando las deformaciones generalizadas en función de los esfuerzos resulta

$$
\begin{aligned}
\mathcal{H}(\boldsymbol{N}, \boldsymbol{M}) & =\boldsymbol{N} \cdot \mathbf{C}_{\Gamma}^{-1} \boldsymbol{N}+\boldsymbol{M} \cdot \mathbf{C}_{K}^{-1} \boldsymbol{M}+\boldsymbol{N} \cdot \boldsymbol{A}_{1} \\
& -\frac{1}{2}\left(\boldsymbol{N} \cdot \mathbf{C}_{\Gamma}^{-1} \boldsymbol{N}+\boldsymbol{M} \cdot \mathbf{C}_{K}^{-1} \boldsymbol{M}\right) \\
& =\frac{1}{2}\left(\boldsymbol{N} \cdot \mathbf{C}_{\Gamma}^{-1} \boldsymbol{N}+\boldsymbol{M} \cdot \mathbf{C}_{K}^{-1} \boldsymbol{M}\right)+\boldsymbol{N} \cdot \boldsymbol{A}_{1}
\end{aligned}
$$

Su expresión en componentes tomando en consideración la composición de las matrices constitutivas en el caso lineal (4.77) es

$$
\begin{aligned}
\mathcal{H}\left(N, Q_{2}, Q_{3},\right. & \left.M_{1}, M_{2}, M_{3}\right) \\
= & \frac{1}{2}\left(\frac{N^{2}}{E A}+\frac{\left(Q_{2}\right)^{2}}{G A_{Q 2}}+\frac{\left(Q_{3}\right)^{2}}{G A_{Q 3}}+\frac{\left(M_{1}\right)^{2}}{G J}+\frac{\left(M_{2}\right)^{2}}{E I_{2}}+\frac{\left(M_{3}\right)^{2}}{E I_{3}}\right)+N
\end{aligned}
$$

Volviendo ahora a la expresión (5.33) de las ecuaciones de Lie-Poisson, consideremos dos funciones escalares de las variables duales, $K(\boldsymbol{F}), G(\boldsymbol{F})$. Entonces, la matriz estructural $\widehat{\mathbf{F}}$ permite definir el siguiente operador, que se denomina corchete de Lie-Poisson [20, ec. 9-95b], [23, sección VII.2.6.], [43, sección 10.1]

$$
\{K, G\}_{\text {Lie-Poisson }}=\nabla K(\boldsymbol{F})^{\top} \widehat{\mathbf{F}} \nabla G(\boldsymbol{F}) .
$$

Por otra parte, si calculamos la derivada de la función $K$ respecto de la variable independiente $S$ se tiene

$$
K^{\prime}=\nabla K(\boldsymbol{F}) \cdot \boldsymbol{F}^{\prime},
$$


y haciendo uso de las ecuaciones (5.33) y de la definición del corchete de Lie-Poisson (5.39) resulta

$$
K^{\prime}=\{K, \mathcal{H}\}_{\text {Lie-Poisson }}
$$

En la literatura se denomina formalismo de Hamilton a esta expresión. El corchete de Lie-Poisson cumple las propiedades de bilinealidad y antisimetría, así como la propiedad de Jacobi [23, sec. VII.2.2, lema 2.3].

Si particularizamos la definición del corchete de Lie-Poisson al problema de equilibrio de la pieza alargada en la teoría de Reissner-Simó desarrollando la expresión (5.39), podemos introducir la siguiente definición:

Definición 5.2.2. Denominamos corchete de la pieza alargada $\mathrm{R} S$ al corchete de Lie-Poisson correspondiente al problema de equilibrio del modelo de Reissner-Simó

$$
\begin{aligned}
& \{K, G\}_{\text {Reissner-Simo }} \\
& =-\boldsymbol{N} \cdot\left(\frac{\partial K}{\partial \boldsymbol{M}} \times \frac{\partial G}{\partial \boldsymbol{N}}-\frac{\partial G}{\partial \boldsymbol{M}} \times \frac{\partial K}{\partial \boldsymbol{N}}\right)-\boldsymbol{M} \cdot\left(\frac{\partial K}{\partial \boldsymbol{M}} \times \frac{\partial G}{\partial \boldsymbol{M}}\right) .
\end{aligned}
$$

Empleando esta definición, y considerando cada una de las componentes de $\boldsymbol{F}$ como funciones definidas en el espacio dual $\mathfrak{g}^{*}$, las ecuaciones de equilibrio del modelo RS se pueden expresar así por medio del formalismo de Hamilton

$$
\boldsymbol{F}^{\prime}=\{\boldsymbol{F}, \mathcal{H}\}_{\text {Reissner-Simo }},
$$

y para cualquier función $K$ de las variables duales $\boldsymbol{N}, \boldsymbol{M}$ se cumple

$$
K^{\prime}=\{K, \mathcal{H}\}_{\text {Reissner-Simo }} .
$$

Esta última característica del corchete de Lie-Poisson (y en particular, de ReissnerSimó) proporciona una elegante versión de la demostración de la proposición 5.2.1. En efecto, si se toma la función $K=\mathcal{H}$, entonces se cumple

$$
\mathcal{H}^{\prime}=\{\mathcal{H}, \mathcal{H}\}_{\text {Reissner-Simo }}=0,
$$

puesto que, observando la definición (5.41) del corchete, este se anula cuando ambas funciones $K$ y $G$ son iguales. Por lo tanto, $\mathcal{H}$ es constante en el dominio de $S$, y es un invariante del sistema.

La definición (5.39) del corchete de Lie-Poisson permite introducir una familia de funciones $C(\boldsymbol{F})$ que cumplen la propiedad

$$
\{C, G\}_{\text {Lie-Poisson }}=0 \quad \forall G .
$$

Sophus Lie denominó funciones distinguidas a esta familia de funciones, y actualmente se denominan funciones de Casimir. A partir de la propiedad (5.40) es inmediato comprobar que $C$ es un invariante del sistema, ya que $C^{\prime}=0$. En el caso particular de la pieza de Reissner-Simó es sencillo comprobar, a partir de la expresión (5.41) que las siguientes funciones son funciones de Casimir

$$
\begin{aligned}
& C_{(1)}=\boldsymbol{N} \cdot \boldsymbol{N} \\
& C_{(2)}=\boldsymbol{N} \cdot \boldsymbol{M},
\end{aligned}
$$


invariantes, por tanto, del problema homogéneo de equilibrio de la pieza alargada. Es sencillo comprobar con un razonamiento mecánico, que el carácter invariante de la primera función $C_{(1)}$ es consecuencia de la ecuación de equilibrio de fuerzas expresadas en el sistema global

$$
\boldsymbol{n}=\boldsymbol{n}_{2},
$$

donde $\boldsymbol{n}_{2}$ es el vector de fuerzas en el extremo final $S=S_{2}$ de la pieza. Multiplicando el vector por si mismo

$$
\boldsymbol{n} \cdot \boldsymbol{n}=\boldsymbol{N}^{\top} \boldsymbol{\Lambda}^{\top} \boldsymbol{\Lambda} \boldsymbol{N}=\boldsymbol{N} \cdot \boldsymbol{N}=\text { const } .
$$

El carácter invariante de la segunda función es el resultado de la ecuación de equilibrio de momentos

$$
\boldsymbol{m}+\boldsymbol{x} \times \boldsymbol{n}=\boldsymbol{m}_{0},
$$

donde $\boldsymbol{m}_{0}$ es el momento resultante en el origen del sistema de referencia espacial. Multiplicando escalarmente por $\boldsymbol{n}$ y teniendo en cuenta que es constante

$$
\boldsymbol{n} \cdot \boldsymbol{m}+\boldsymbol{n} \cdot(\boldsymbol{x} \times \boldsymbol{n})=\boldsymbol{n} \cdot \boldsymbol{m}=\text { const },
$$

y expresando la ecuación en función de las variables materiales

$$
\boldsymbol{n} \cdot \boldsymbol{m}=\boldsymbol{N}^{\top} \boldsymbol{\Lambda}^{\top} \boldsymbol{\Lambda} \boldsymbol{M}=\boldsymbol{N} \cdot \boldsymbol{M}=\text { const } .
$$

La expresión en componentes de las funciones de Casimir en el problema homogéneo de la pieza alargada es

$$
\begin{aligned}
& C_{(1)}=N^{2}+\left(Q_{2}\right)^{2}+\left(Q_{3}\right)^{2} \\
& C_{(2)}=N M_{1}+Q_{2} M_{2}+Q_{3} M_{3} .
\end{aligned}
$$

Comentario 5.2.3. Es inmediato comprobar que las ecuaciones del modelo de Kirchhoff (5.8) se pueden obtener mediante el corchete de Reissner-Simó

$$
\boldsymbol{F}^{\prime}=\{\boldsymbol{F}, \mathcal{H}\}_{\text {Reissner-Simo }},
$$

con la siguiente función de Hamilton

$$
\mathcal{H}(\boldsymbol{N}, \boldsymbol{M})=\frac{1}{2}\left(\boldsymbol{M} \cdot \mathbf{C}_{K}^{-1} \boldsymbol{M}\right)+\boldsymbol{N} \cdot \boldsymbol{A}_{1} .
$$

Esta función se obtiene eliminando el término correspondiente a $\mathbf{C}_{\Gamma}$ de la función de Hamilton para el problema de Reissner-Simó (5.38), y coincide con la expresión (5.11) obtenida por Kirchhoff.

Por otra parte, los invariantes del problema de Reissner-Simó definidos por las funciones $C_{(1)}$ y $C_{(2)}$, también son invariantes en el problema de Kirchhoff, puesto que únicamente dependen de la definición (5.41) del corchete, que es la misma en ambos casos. 
Las proposiciones 5.1.1 y 5.2.1 que demuestran la estructura de Euler-Poincaré de las ecuaciones de equilibrio del modelo de Reissner-Simó y su relación con el Principio de Trabajos Virtuales, junto con la demostración de su estructura de Lie-Poisson, desarrollada en esta sección, permiten llevar a cabo una generalización de la analogía de Kirchhoff [35]. En la analogía generalizada la longitud medida sobre la directriz en la configuración inicial es análoga a la variable tiempo, y la función $\mathcal{H}$ juega el papel de la función de energía total en el sistema dinámico. 



\section{Capítulo 6}

\section{El operador tangente en el modelo de Reissner-Simó}

La linealización consistente de la ecuación de trabajos virtuales en sus formas material y espacial ha sido una de las cuestiones más debatidas en el contexto de las teorías de vigas con rotaciones finitas. El resultado de la linealización proporciona el denominado operador tangente, que constituye el núcleo de los procedimientos de solución incrementales. El operador es, además, necesario para la determinación de la estabilidad del equilibrio, pues está relacionado con la segunda variación de la energía potencial.

Simó y Vu-Quoc (1986) 62] abordan por primera vez la linealización de la ecuación de trabajos virtuales en el marco estricto del modelo RS. Como se ha mencionado en la revisión de literatura, el proceso de linealización de las fuerzas internas les conduce a un operador no simétrico. Según Simó, el operador recupera la simetría una vez se ha retornado a la trayectoria de equilibrio, si las fuerzas actuantes son conservativas. La linealización propuesta en [62 se basa en el cálculo de la derivada direccional de la ecuación de trabajos virtuales en la dirección de la variación de la configuración.

El cálculo del operador tangente es uno de los aspectos cruciales de la tesis doctoral, pues el resultado que obtenemos en este capítulo no coincide con el clásico, contenido en [62, ni con otros recogidos en la literatura como Cardona y Geradin (1988) [11, Ibrahimbegović (1995) [25], o más recientemente Kapania y Li (2003) [34, que reproducen el resultado de Simó y su colaborador. El motivo de la discrepancia se encuentra en el proceso de evaluación de la derivada direccional de la ecuación de trabajos virtuales, y más concretamente del trabajo virtual de las fuerzas internas. El artículo de Kapania y Li [34] ofrece una visión muy clara del cálculo efectuado por Simó, que resumimos a continuación. El trabajo virtual de las fuerzas internas, en su forma espacial

$$
\delta W_{i n t}=\int_{\Gamma_{0}}(\boldsymbol{n} \cdot \stackrel{\nabla}{\delta} \boldsymbol{\gamma}+\boldsymbol{m} \cdot \stackrel{\nabla}{\delta} \boldsymbol{\kappa}) d S
$$

se puede expresar en función de las variaciones de la configuración (sección 4.3) como

$$
\delta W_{i n t}=\int_{\Gamma_{0}}\left(\boldsymbol{n} \cdot \delta \boldsymbol{x}^{\prime}-\widehat{\gamma} \boldsymbol{n} \cdot \delta \boldsymbol{\omega}+\boldsymbol{m} \cdot(\delta \boldsymbol{\omega})^{\prime}\right) d S,
$$


y en forma matricial

$$
\delta W_{i n t}=\int_{\Gamma_{0}}\left\{\delta \boldsymbol{x}^{\top} \delta \boldsymbol{\omega}^{\top} \delta \boldsymbol{x}^{\prime \top} \delta \boldsymbol{\omega}^{\prime \top}\right\}\left\{\begin{array}{c}
\mathbf{0} \\
-\widehat{\gamma} \boldsymbol{n} \\
\boldsymbol{n} \\
\boldsymbol{m}
\end{array}\right\} d S .
$$

Kapania y Li evalúan la derivada direccional de esta expresión calculando la variación $\Delta$ en una dirección independiente de la primera variación $\delta$ [34, ecuación 55]

$$
\Delta \delta W_{\text {int }}=\int_{\Gamma_{0}}\left\{\delta \boldsymbol{x}^{\top} \delta \boldsymbol{\omega}^{\top} \delta \boldsymbol{x}^{\top \top} \delta \boldsymbol{\omega}^{\prime \top}\right\} \Delta\left\{\begin{array}{c}
\mathbf{0} \\
-\widehat{\gamma} \boldsymbol{n} \\
\boldsymbol{n} \\
\boldsymbol{m}
\end{array}\right\} d S
$$

$\mathrm{Al}$ introducir la variación $\Delta$ en la integral, los autores citados consideran que ésta no tiene efecto sobre el vector que contiene las variaciones originales de la configuración y sus derivadas. Es decir

$$
\begin{aligned}
\Delta \delta \boldsymbol{x}=\Delta \delta \boldsymbol{\omega} & =\mathbf{0} \\
\Delta \delta \boldsymbol{x}^{\prime}=\Delta \delta \boldsymbol{\omega}^{\prime} & =\mathbf{0} .
\end{aligned}
$$

Como ellos mismos indican, la segunda variación se evalúa desde el punto de vista de un observador situado en el sistema de referencia fijo, es decir, la variación espacial. Su relación con la variación corrotacional, como se puede comprobar en la ecuación (4.11), es la siguiente

$$
\Delta \boldsymbol{v}=\stackrel{\nabla}{\Delta} \boldsymbol{v}+\Delta \widehat{\boldsymbol{\omega}} \boldsymbol{v}
$$

Mediante esta expresión, y partiendo de la ecuación constitutiva espacial (6.23) que se deduce más adelante, es posible calcular las variaciones de cada uno de los elementos del vector del integrando:

$$
\begin{aligned}
\Delta \boldsymbol{n} & =\mathbf{c}_{\gamma \gamma} \stackrel{\nabla}{\Delta} \boldsymbol{\gamma}+\mathbf{c}_{\gamma \kappa} \stackrel{\nabla}{\Delta} \boldsymbol{\kappa}+\Delta \widehat{\boldsymbol{\omega}} \boldsymbol{n} \\
& =\mathbf{c}_{\gamma \gamma}\left(\Delta \boldsymbol{x}^{\prime}+\widehat{\gamma} \Delta \boldsymbol{\omega}\right)+\mathbf{c}_{\gamma \kappa} \Delta \boldsymbol{\omega}^{\prime}-\widehat{\mathbf{n}} \Delta \boldsymbol{\omega}, \\
\Delta \boldsymbol{m} & =\mathbf{c}_{\kappa \gamma} \stackrel{\nabla}{\Delta} \boldsymbol{\gamma}+\mathbf{c}_{\kappa \kappa} \stackrel{\nabla}{\Delta} \boldsymbol{\kappa}+\Delta \widehat{\boldsymbol{\omega}} \boldsymbol{m} \\
& =\mathbf{c}_{\kappa \gamma}\left(\Delta \boldsymbol{x}^{\prime}+\widehat{\gamma} \Delta \boldsymbol{\omega}\right)+\mathbf{c}_{\kappa \kappa} \Delta \boldsymbol{\omega}^{\prime}-\widehat{\mathbf{m}} \Delta \boldsymbol{\omega},
\end{aligned}
$$

y teniendo en cuenta la variación de los esfuerzos $\boldsymbol{n}$,

$$
\begin{aligned}
\Delta(-\widehat{\gamma} \boldsymbol{n}) & =\widehat{\mathbf{n}} \Delta \gamma-\widehat{\gamma} \Delta \boldsymbol{n} \\
& =\widehat{\mathbf{n}} \Delta \boldsymbol{x}^{\prime}-\widehat{\gamma} \mathbf{c}_{\gamma \gamma}\left(\Delta \boldsymbol{x}^{\prime}+\widehat{\gamma} \Delta \boldsymbol{\omega}\right)-\widehat{\gamma} \mathbf{c}_{\gamma \kappa} \Delta \boldsymbol{\omega}^{\prime}+\widehat{\gamma} \widehat{\mathbf{n}} \Delta \boldsymbol{\omega}
\end{aligned}
$$


Ordenando estos resultados intermedios se llega a la siguiente expresión de la variación del trabajo virtual de las fuerzas internas

$$
\begin{array}{r}
\Delta \delta W_{\text {int }}= \\
\int_{\Gamma_{0}}\left\{\delta \boldsymbol{x}^{\top} \delta \boldsymbol{\omega}^{\top} \delta \boldsymbol{x}^{\prime \top} \delta \boldsymbol{\omega}^{\prime \top}\right\} \\
{\left[\begin{array}{ccccc}
\mathbf{0} & \mathbf{0} & \mathbf{0} & \mathbf{0} \\
\mathbf{0} & -\widehat{\gamma} \mathbf{c}_{\gamma \gamma} \widehat{\gamma} & -\widehat{\gamma} \mathbf{c}_{\gamma \gamma} & -\widehat{\gamma} \mathbf{c}_{\gamma \kappa} \\
\mathbf{0} & \mathbf{c}_{\gamma \gamma} \widehat{\gamma} & \mathbf{c}_{\gamma \gamma} & \mathbf{c}_{\gamma \kappa} \\
\mathbf{0} & \mathbf{c}_{\kappa \gamma} \widehat{\gamma} & \mathbf{c}_{\kappa \gamma} & \mathbf{c}_{\kappa \kappa}
\end{array}\right]\left\{\begin{array}{l}
\Delta \boldsymbol{x} \\
\Delta \boldsymbol{\omega} \\
\Delta \boldsymbol{x}^{\prime} \\
\Delta \boldsymbol{\omega}^{\prime}
\end{array}\right\} d S+} \\
\int_{\Gamma_{0}}\left\{\delta \boldsymbol{x}^{\top} \delta \boldsymbol{\omega}^{\top} \delta \boldsymbol{x}^{\prime \top} \delta \boldsymbol{\omega}^{\prime \top}\right\}\left[\begin{array}{cccc}
\mathbf{0} & \mathbf{0} & \mathbf{0} & \mathbf{0} \\
\mathbf{0} & \widehat{\gamma} \widehat{\mathbf{n}} & \widehat{\mathbf{n}} & \mathbf{0} \\
\mathbf{0} & -\widehat{\mathbf{n}} & \mathbf{0} & \mathbf{0} \\
\mathbf{0} & -\widehat{\mathbf{m}} & \mathbf{0} & \mathbf{0}
\end{array}\right]\left\{\begin{array}{l}
\Delta \boldsymbol{x} \\
\Delta \boldsymbol{\omega} \\
\Delta \boldsymbol{x}^{\prime} \\
\Delta \boldsymbol{\omega}^{\prime}
\end{array}\right\} d S .
\end{array}
$$

El primer sumando se identifica con la parte constitutiva $^{1}$, y el segundo, con la parte geométrica del operador tangente. Estas expresiones, con diferentes notaciones, aparecen en las referencias [62], [25] y [34] entre otras.

En este punto, un análisis detallado de la linealización de la ecuación de trabajos virtuales nos conduce a un resultado que difiere del ofrecido en la literatura. El origen de la discrepancia radica en la inexactitud de las ecuaciones (6.3); en concreto, demostraremos que $\Delta \delta \boldsymbol{\omega} \neq \mathbf{0}$ y $\Delta \delta \boldsymbol{\omega}^{\prime} \neq \mathbf{0}$. A partir de este resultado preliminar, ya no es posible prescindir de los términos correspondientes a la segunda variación de la configuración en la linealización de la ecuación de trabajos virtuales. Esto conduce naturalmente a nuevas expresiones que analizaremos detalladamente.

El capítulo se organiza de la siguiente forma: en primer lugar se deducen las expresiones de las segundas variaciones de las variables de la configuración. A continuación se ofrecen las nuevas expresiones del operador tangente en su forma espacial y se analiza la relevancia de los términos que difieren del resultado de Simó. Por último, se obtiene la forma material del operador tangente y su relación con el operador espacial.

\subsection{Resultados preliminares}

En primer lugar presentaremos unas propiedades básicas de la variación espacial y de la variación corrotacional que serán útiles a la hora de linealizar la ecuación de trabajos virtuales (en el apéndice $\mathrm{A}$ se incluyen algunas propiedades adicionales de la variación corrotacional). Posteriormente evaluaremos la segunda variación de la configuración.

\footnotetext{
${ }^{1}$ Este término se denomina con frecuencia en la literatura parte material. Preferimos utilizar el calificativo constitutiva ya que resulta igualmente preciso y evita la confusión con el significado con el que se dota al adjetivo material en este trabajo.
} 


\subsubsection{Propiedades elementales de la variación y la variación corrotacional}

Consideremos la variación del producto escalar de dos vectores $\boldsymbol{a}$ y $\boldsymbol{b}$ en su forma espacial

$$
\Delta(\boldsymbol{a} \cdot \boldsymbol{b})
$$

Dado que el producto escalar es invariante frente a un cambio de sistema de referencia, si $\boldsymbol{A}=\boldsymbol{\Lambda}^{\top} \boldsymbol{a}$, y $\boldsymbol{B}=\boldsymbol{\Lambda}^{\top} \boldsymbol{b}$ son la forma material de $\boldsymbol{a}$ y $\boldsymbol{b}$, entonces se cumple $\boldsymbol{A} \cdot \boldsymbol{B}=\boldsymbol{a} \cdot \boldsymbol{b}$, y por tanto

$$
\Delta(\boldsymbol{A} \cdot \boldsymbol{B})=\Delta(\boldsymbol{a} \cdot \boldsymbol{b}) .
$$

El término de la izquierda se desarrolla de forma trivial como

$$
\Delta \boldsymbol{A} \cdot \boldsymbol{B}+\boldsymbol{A} \cdot \Delta \boldsymbol{B}
$$

El de la derecha de la igualdad, sin embargo, admite dos desarrollos posibles. Por una parte se puede escribir

$$
\Delta(\boldsymbol{a} \cdot \boldsymbol{b})=\Delta \boldsymbol{a} \cdot \boldsymbol{b}+\boldsymbol{a} \cdot \Delta \boldsymbol{b}
$$

Pero también se cumple

$$
\begin{aligned}
\Delta(\boldsymbol{a} \cdot \boldsymbol{b})=\Delta\left(\boldsymbol{a}^{\top} \boldsymbol{\Lambda} \boldsymbol{\Lambda}^{\top} \boldsymbol{b}\right) & =\Delta\left(\boldsymbol{a}^{\top} \boldsymbol{\Lambda}\right) \boldsymbol{\Lambda}^{\top} \boldsymbol{b}+\boldsymbol{a}^{\top} \boldsymbol{\Lambda} \Delta\left(\boldsymbol{\Lambda}^{\top} \boldsymbol{b}\right) \\
& =\stackrel{\nabla}{ } \boldsymbol{a} \cdot \boldsymbol{b}+\boldsymbol{a} \cdot \stackrel{\nabla}{\Delta} \boldsymbol{b} .
\end{aligned}
$$

La equivalencia entre estas expresiones se puede comprobar a partir de la conocida definición de variación corrotacional

$$
\stackrel{\nabla}{\Delta} \boldsymbol{v}=\Delta v-\Delta \omega \times v
$$

y da lugar a la siguiente proposición

Proposición 6.1.1. La propiedad de Jacobi para el producto de vectores espaciales se aplica indistintamente a la variación espacial o la variación corrotacional

$$
\Delta(\boldsymbol{a} \cdot \boldsymbol{b})=\Delta \boldsymbol{a} \cdot \boldsymbol{b}+\boldsymbol{a} \cdot \Delta \boldsymbol{b}=\stackrel{\nabla}{\Delta} \boldsymbol{a} \cdot \boldsymbol{b}+\boldsymbol{a} \cdot \stackrel{\nabla}{\Delta} \boldsymbol{b}
$$

Demostración. Desarrollando la expresión del producto mediante variaciones corrotacionales

$$
\begin{aligned}
\stackrel{\nabla}{\Delta} \boldsymbol{a} \cdot \boldsymbol{b}+\boldsymbol{a} \cdot \stackrel{\nabla}{\Delta} \boldsymbol{b} & =\Delta \boldsymbol{a} \cdot \boldsymbol{b}-(\Delta \boldsymbol{\omega} \times \boldsymbol{a}) \cdot \boldsymbol{b}+\boldsymbol{a} \cdot \Delta \boldsymbol{b}-\boldsymbol{a} \cdot(\Delta \boldsymbol{\omega} \times \boldsymbol{b}) \\
& =\Delta \boldsymbol{a} \cdot \boldsymbol{b}+\boldsymbol{a} \cdot \Delta \boldsymbol{b}-(\boldsymbol{a} \times \boldsymbol{b}) \cdot \Delta \boldsymbol{\omega}-\Delta \boldsymbol{\omega} \cdot(\boldsymbol{b} \times \boldsymbol{a}),
\end{aligned}
$$

lo que prueba el enunciado, dado que los dos últimos términos se cancelan. 


\subsubsection{Segunda variación de la configuración}

La evaluación de las segunda variación se calcula de modo natural sobre la forma material de las primeras variaciones, y da lugar a la siguiente proposición

Proposición 6.1.2. La expresión de las segundas variaciones de la configuración y de sus derivadas en forma material es la siguiente

$$
\begin{aligned}
\Delta(\delta \chi) & =-\Delta \boldsymbol{\Omega} \times \delta \chi \\
\Delta(\delta \boldsymbol{\Omega}) & =\mathbf{0} \\
\Delta\left(\delta \chi^{\prime}\right) & =-\Delta \boldsymbol{\Omega}^{\prime} \times \delta \chi-\Delta \boldsymbol{\Omega} \times \delta \chi^{\prime} \\
\Delta\left(\delta \boldsymbol{\Omega}^{\prime}\right) & =\mathbf{0} .
\end{aligned}
$$

Demostración. Consideremos en primer lugar el cálculo de la segunda variación de $\delta \widehat{\Omega}$

$$
\Delta(\delta \widehat{\mathbf{\Omega}})=\Delta\left(\boldsymbol{\Lambda}^{\top} \delta \boldsymbol{\Lambda}\right)=-\boldsymbol{\Lambda}^{\top} \Delta \boldsymbol{\Lambda} \boldsymbol{\Lambda}^{\top} \delta \boldsymbol{\Lambda}+\boldsymbol{\Lambda}^{\top} \Delta(\delta \boldsymbol{\Lambda})=-\Delta \widehat{\boldsymbol{\Omega}} \delta \widehat{\boldsymbol{\Omega}}+\boldsymbol{\Lambda}^{\top} \Delta(\delta \boldsymbol{\Lambda}) .
$$

El término $\Delta(\delta \boldsymbol{\Lambda})$ se puede calcular haciendo uso de la definición de derivada direccional

$$
\Delta(\delta \boldsymbol{\Lambda})=\Delta(\boldsymbol{\Lambda} \delta \widehat{\boldsymbol{\Omega}})=\left.\frac{d}{d \varepsilon}(\boldsymbol{\Lambda} \exp (\varepsilon \Delta \widehat{\boldsymbol{\Omega}}) \delta \widehat{\boldsymbol{\Omega}})\right|_{\varepsilon=0}=\boldsymbol{\Lambda} \Delta \widehat{\boldsymbol{\Omega}} \delta \widehat{\boldsymbol{\Omega}} .
$$

Sustituyendo en la expresión anterior se concluye que

$$
\Delta(\delta \widehat{\mathbf{\Omega}})=\mathbf{0} .
$$

A partir de esta expresión, y teniendo en cuenta que la segunda variación conmuta con la derivada respecto de $S$

$$
\Delta\left(\delta \widehat{\boldsymbol{\Omega}}^{\prime}\right)=\frac{d}{d S} \Delta(\delta \widehat{\boldsymbol{\Omega}})=\mathbf{0} .
$$

Por lo que respecta a la variación de $\chi$ se tiene

$$
\Delta(\delta \boldsymbol{\chi})=\Delta\left(\boldsymbol{\Lambda}^{\top} \delta \boldsymbol{x}\right)=\left.\frac{d}{d \varepsilon}\left(\exp (-\varepsilon \Delta \widehat{\boldsymbol{\Omega}}) \boldsymbol{\Lambda}^{\top} \delta \boldsymbol{x}\right)\right|_{\varepsilon=0}=-\Delta \widehat{\boldsymbol{\Omega}} \delta \boldsymbol{\chi} .
$$

El cálculo de la segunda variación de la derivada es sencillo considerando que la derivada conmuta con la variación

$$
\Delta\left(\delta \chi^{\prime}\right)=\frac{d}{d S}(-\Delta \widehat{\boldsymbol{\Omega}} \delta \chi)=-\Delta \widehat{\boldsymbol{\Omega}}^{\prime} \delta \chi-\Delta \widehat{\boldsymbol{\Omega}} \delta \chi^{\prime} .
$$

La forma espacial de las segundas variaciones se obtiene a partir de los resultados de la proposición anterior, empleando la definición (6.8) de variación corrotacional. 
Proposición 6.1.3. La expresión de las segundas variaciones de la configuración y de sus derivadas en forma espacial es la siguiente

$$
\begin{aligned}
\Delta \delta \boldsymbol{x} & =\mathbf{0} \\
\Delta \delta \boldsymbol{\omega} & =\Delta \boldsymbol{\omega} \times \delta \boldsymbol{\omega} \\
\Delta \delta \boldsymbol{x}^{\prime} & =\mathbf{0} \\
\Delta \delta \boldsymbol{\omega}^{\prime} & =\Delta \boldsymbol{\omega}^{\prime} \times \delta \boldsymbol{\omega}+\Delta \boldsymbol{\omega} \times \delta \boldsymbol{\omega}^{\prime} .
\end{aligned}
$$

Demostración. La primera igualdad es inmediata teniendo en cuenta que $\delta \boldsymbol{x}$ pertenece a un espacio lineal. Para demostrar la segunda igualdad basta considerar que, a partir de la definición (6.8) de variación corrotacional y del resultado de la proposición anterior,

$$
\stackrel{\nabla}{\Delta} \delta \boldsymbol{\omega}=\boldsymbol{\Lambda} \Delta\left(\boldsymbol{\Lambda}^{\top} \delta \boldsymbol{\omega}\right)=\mathbf{\Lambda} \Delta(\delta \boldsymbol{\Omega})=\mathbf{0}
$$

luego

$$
\Delta \delta \boldsymbol{\omega}=\stackrel{\nabla}{\Delta} \delta \boldsymbol{\omega}+\Delta \boldsymbol{\omega} \times \delta \boldsymbol{\omega}=\Delta \boldsymbol{\omega} \times \delta \boldsymbol{\omega}
$$

La tercera ecuación se deduce directamente de la primera, y la última se obtiene de forma inmediata derivando la segunda.

Comentario 6.1.4. Es fácil observar cómo existe una dualidad ente la forma espacial y la forma material de las segundas variaciones de la configuración: cuando la forma material se anula, la forma espacial tiene una estructura del tipo $\Delta \boldsymbol{\omega} \times \cdot$ (o su derivada), y viceversa. Este resultado se puede resumir en la siguiente tabla:

\begin{tabular}{c|ccc|c} 
& $\Delta(\cdot)$ & & $\Delta(\cdot)$ & \\
\hline$\delta \boldsymbol{\chi}$ & $-\Delta \boldsymbol{\Omega} \times \delta \boldsymbol{\chi}$ & $\leftrightarrow$ & $\mathbf{0}$ & $\delta \boldsymbol{x}$ \\
\hline$\delta \boldsymbol{\Omega}$ & $\mathbf{0}$ & $\leftrightarrow$ & $\Delta \boldsymbol{\omega} \times \delta \boldsymbol{\omega}$ & $\delta \boldsymbol{\omega}$ \\
\hline$\delta \boldsymbol{\chi}^{\prime}$ & $-\Delta \boldsymbol{\Omega}^{\prime} \times \delta \boldsymbol{\chi}-\Delta \boldsymbol{\Omega} \times \delta \boldsymbol{\chi}^{\prime}$ & $\leftrightarrow$ & $\mathbf{0}$ & $\delta \boldsymbol{x}^{\prime}$ \\
\hline$\delta \boldsymbol{\Omega}^{\prime}$ & $\mathbf{0}$ & $\leftrightarrow$ & $\Delta \boldsymbol{\omega}^{\prime} \times \delta \boldsymbol{\omega}+\Delta \boldsymbol{\omega} \times \delta \boldsymbol{\omega}^{\prime}$ & $\delta \boldsymbol{\omega}^{\prime}$ \\
\hline & forma material & & forma espacial &
\end{tabular}

Cuadro 6.1: Dualidad de las segundas variaciones de la configuración

Por otro lado, a partir de la definición (6.8) se deduce directamente que la segunda variación corrotacional responde al siguiente esquema:

$$
\begin{aligned}
& \stackrel{\nabla}{\Delta} \delta \boldsymbol{x}=-\Delta \boldsymbol{\omega} \times \delta \boldsymbol{x} \\
& \nabla \\
& \Delta \delta \boldsymbol{\omega}=\mathbf{0} \\
& \stackrel{\nabla}{\Delta} \delta \boldsymbol{x}^{\prime}=-\Delta \boldsymbol{\omega} \times \delta \boldsymbol{x}^{\prime} \\
& { }_{\Delta}^{\nabla} \delta \boldsymbol{\omega}^{\prime}=\Delta \boldsymbol{\omega}^{\prime} \times \delta \boldsymbol{\omega} .
\end{aligned}
$$




\subsection{Forma espacial del equilibrio tangente}

\subsubsection{Linealización del trabajo virtual de las fuerzas internas}

Con las consideraciones hechas en la introducción al presente capítulo y en la sección anterior, se puede abordar la linealización de la ecuación de trabajos virtuales que conduce a la expresión del equilibrio tangente. Comenzaremos operando con la forma espacial. En la linealización del trabajo virtual de las fuerzas internas presentamos dos formas alternativas de llevar a cabo el proceso de linealización: (a) se puede linealizar directamente cada uno de los factores originales del integrando (la variación corrotacional de las deformaciones y los esfuerzos), o bien (b) expresando el integrando como producto de las variaciones de la configuración y sus derivadas por un vector que contiene esfuerzos y deformaciones generalizadas (tal como se muestra en la introducción) se puede linealizar el producto. El primer procedimiento tiene la ventaja de producir por separado la parte geométrica y la constitutiva del operador tangente. El procedimiento (b) conduce a expresiones que permiten analizar la diferencia entre el nuevo operador geométrico y el publicado por Simó 62]. Sin embargo, es fácil comprobar que en ambos casos el resultado final es el mismo.

\section{Procedimiento a: linealización directa de los factores del integrando}

El trabajo virtual de las fuerzas internas, expresado en forma espacial, es (4.18), $(3.64)$

$$
\delta W_{i n t}=\int_{\Gamma_{0}}(\boldsymbol{n} \cdot \stackrel{\nabla}{\delta} \boldsymbol{\gamma}+\boldsymbol{m} \cdot \stackrel{\nabla}{\delta} \boldsymbol{\kappa}) d S=\int_{\Gamma_{0}} \stackrel{\nabla}{\delta} \boldsymbol{\epsilon} \cdot \boldsymbol{f} d S
$$

donde empleamos las variables introducidas en la sección 4.3 -ecuaciones (4.38b) y (4.56a) - que reproducimos por conveniencia

$$
\begin{aligned}
& \stackrel{\nabla}{\delta \boldsymbol{\epsilon}}=\left\{\begin{array}{ll}
\nabla & \nabla \\
\delta \boldsymbol{\delta} & \delta \boldsymbol{\kappa}
\end{array}\right\}^{\top} \\
& \boldsymbol{f}=\left\{\begin{array}{ll}
\boldsymbol{n} & \boldsymbol{m}
\end{array}\right\}^{\top} \text {. }
\end{aligned}
$$

Estas variables condensan en símbolos únicos las componentes traslacional y rotacional de la deformación y las componentes de fuerza y de momento de los esfuerzos, respectivamente.

La variación del trabajo virtual de las fuerzas internas se calcula haciendo uso de la propiedad (A.2) de la variación corrotacional, con el fin de preservar la consistencia de la linealización, de la siguiente forma

$$
\Delta \int_{\Gamma_{0}} \stackrel{\nabla}{\delta} \boldsymbol{\epsilon} \cdot \boldsymbol{f} d S=\int_{\Gamma_{0}} \stackrel{\nabla}{\Delta} \underset{\nabla}{\delta} \boldsymbol{\epsilon} \cdot \boldsymbol{f} d S+\int_{\Gamma_{0}} \stackrel{\nabla}{\delta} \boldsymbol{\epsilon} \cdot \stackrel{\nabla}{\Delta} \boldsymbol{f} d S
$$

El primer término corresponde a la linealización de la variación de las deformaciones, y va a dar lugar a la parte geométrica de la matriz de rigidez. Considerando 
la expresión (4.30a),

$$
\begin{aligned}
\stackrel{\nabla}{\Delta}(\stackrel{\nabla}{\delta} \boldsymbol{\gamma}) & =\stackrel{\nabla}{\Delta}\left(\delta \boldsymbol{x}^{\prime}+\boldsymbol{\gamma} \times \delta \boldsymbol{\omega}\right) \\
& =\stackrel{\nabla}{\Delta} \delta \boldsymbol{x}^{\prime}+\stackrel{\nabla}{\Delta}(\boldsymbol{\gamma} \times \delta \boldsymbol{\omega}) .
\end{aligned}
$$

El primer sumando vale, según la ecuación (6.12c),

$$
\stackrel{\nabla}{\Delta} \delta \boldsymbol{x}^{\prime}=-\Delta \widehat{\boldsymbol{\omega}} \delta \boldsymbol{x}^{\prime}
$$

El segundo se analiza teniendo en cuenta la propiedad (A.4) y la forma incremental de la ecuación (4.30a).

$$
\begin{aligned}
\stackrel{\nabla}{\Delta}(\boldsymbol{\gamma} \times \delta \boldsymbol{\omega}) & =\stackrel{\nabla}{\Delta} \boldsymbol{\gamma} \times \delta \boldsymbol{\omega}+\boldsymbol{\gamma} \times \stackrel{\nabla}{\Delta} \delta \boldsymbol{\omega} \\
& =\left(\Delta \boldsymbol{x}^{\prime}+\boldsymbol{\gamma} \times \Delta \boldsymbol{\omega}\right) \times \delta \boldsymbol{\omega} \\
& =\Delta \widehat{\mathbf{x}}^{\prime} \delta \boldsymbol{\omega}+\widehat{\gamma} \Delta \boldsymbol{\omega} \times \delta \boldsymbol{\omega} .
\end{aligned}
$$

Por tanto

$$
\stackrel{\nabla}{\Delta}(\stackrel{\nabla}{\delta})=-\Delta \widehat{\boldsymbol{\omega}} \delta \boldsymbol{x}^{\prime}+\Delta \widehat{\mathbf{x}}^{\prime} \delta \boldsymbol{\omega}+\widehat{\gamma} \Delta \boldsymbol{\omega} \times \delta \boldsymbol{\omega} .
$$

Por otra parte, mediante la ecuación (6.12d) se deduce

$$
\stackrel{\nabla}{\Delta}(\stackrel{\nabla}{\delta} \boldsymbol{\kappa})=\stackrel{\nabla}{\Delta}\left(\delta \boldsymbol{\omega}^{\prime}\right)=\Delta \widehat{\boldsymbol{\omega}}^{\prime} \delta \boldsymbol{\omega}
$$

A partir de los resultados anteriores se obtiene el integrando del primer sumando en función de las variaciones de la configuración

$$
\begin{aligned}
& \stackrel{\nabla}{\Delta}(\stackrel{\nabla}{\delta} \gamma) \cdot \boldsymbol{n}+\stackrel{\nabla}{\Delta}(\stackrel{\nabla}{\delta} \boldsymbol{\kappa}) \cdot \boldsymbol{m} \\
& =-\left(\Delta \widehat{\boldsymbol{\omega}} \delta \boldsymbol{x}^{\prime}\right) \cdot \boldsymbol{n}+\left(\Delta \widehat{\mathbf{x}}^{\prime} \delta \boldsymbol{\omega}\right) \cdot \boldsymbol{n}+(\widehat{\gamma} \Delta \boldsymbol{\omega} \times \delta \boldsymbol{\omega}) \cdot \boldsymbol{n}+\left(\Delta \widehat{\boldsymbol{\omega}}^{\prime} \delta \boldsymbol{\omega}\right) \cdot \boldsymbol{m}
\end{aligned}
$$

Si analizamos el tercer sumando podemos escribir

$$
\begin{aligned}
(\widehat{\gamma} \Delta \boldsymbol{\omega} \times \delta \boldsymbol{\omega}) \cdot \boldsymbol{n} & =((\widehat{\gamma \times \Delta \boldsymbol{\omega}}) \delta \boldsymbol{\omega}) \cdot \boldsymbol{n} \\
& =-\delta \boldsymbol{\omega}^{\top}(\widehat{\gamma \times \Delta \boldsymbol{\omega}}) \boldsymbol{n} \\
& =-\delta \boldsymbol{\omega}^{\top}(\gamma \times \Delta \boldsymbol{\omega}) \times \boldsymbol{n} \\
& =\delta \boldsymbol{\omega}^{\top} \boldsymbol{n} \times(\boldsymbol{\gamma} \times \Delta \boldsymbol{\omega}),
\end{aligned}
$$

lo que permite obtener finalmente la expresión del integrando:

$$
\begin{aligned}
& \delta \boldsymbol{x}^{\prime \top} \Delta \widehat{\boldsymbol{\omega}} \boldsymbol{n}-\delta \boldsymbol{\omega}^{\top} \Delta \widehat{\mathbf{x}}^{\prime} \boldsymbol{n}+\delta \boldsymbol{\omega}^{\top} \boldsymbol{n} \times(\gamma \times \Delta \boldsymbol{\omega})-\delta \boldsymbol{\omega}^{\top} \Delta \widehat{\boldsymbol{\omega}}^{\prime} \boldsymbol{m} \\
& =-\delta \boldsymbol{x}^{\prime} \widehat{\mathbf{n}} \Delta \boldsymbol{\omega}+\delta \boldsymbol{\omega}^{\top} \widehat{\mathbf{n}} \Delta \mathbf{x}^{\prime}+\delta \boldsymbol{\omega}^{\top} \widehat{\mathbf{n}} \widehat{\gamma} \Delta \boldsymbol{\omega}+\delta \boldsymbol{\omega}^{\top} \widehat{\mathbf{m}} \Delta \boldsymbol{\omega}^{\prime} .
\end{aligned}
$$


Agrupando factores el término geométrico del operador tangente en su forma espacial es

$$
\int_{\Gamma_{0}}\left\{\delta \boldsymbol{x}^{\top} \delta \boldsymbol{\omega}^{\top} \delta \boldsymbol{x}^{\prime \top} \delta \boldsymbol{\omega}^{\prime \top}\right\}\left[\begin{array}{cccc}
\mathbf{0} & \mathbf{0} & \mathbf{0} & \mathbf{0} \\
\mathbf{0} & \widehat{\mathbf{n}} \widehat{\boldsymbol{\gamma}} & \widehat{\mathbf{n}} & \widehat{\mathbf{m}} \\
\mathbf{0} & -\widehat{\mathbf{n}} & \mathbf{0} & \mathbf{0} \\
\mathbf{0} & \mathbf{0} & \mathbf{0} & \mathbf{0}
\end{array}\right]\left\{\begin{array}{c}
\Delta \boldsymbol{x} \\
\Delta \boldsymbol{\omega} \\
\Delta \boldsymbol{x}^{\prime} \\
\Delta \boldsymbol{\omega}^{\prime}
\end{array}\right\} d S .
$$

Denominaremos forma espacial del núcleo de la parte geométrica del operador a la matriz del integrando. Si empleamos las variables que condensan las componentes traslacionales y rotacionales de las variaciones de la configuración introducidas en la sección 4.2 -ecuación (4.38a)-

$$
\begin{aligned}
\delta \phi & =\left\{\begin{array}{ll}
\delta \boldsymbol{x} & \delta \boldsymbol{\omega}
\end{array}\right\}^{\top} \\
\Delta \boldsymbol{\phi} & =\left\{\begin{array}{ll}
\Delta \boldsymbol{x} & \Delta \boldsymbol{\omega}
\end{array}\right\}^{\top},
\end{aligned}
$$

la expresión anterior se convierte en

$$
\int_{\Gamma_{0}}\left\{\delta \boldsymbol{\phi}^{\top} \delta \boldsymbol{\phi}^{\prime \top}\right\}\left[\begin{array}{ll}
\mathbf{d}_{00}^{G} & \mathbf{d}_{01}^{G} \\
\mathbf{d}_{10}^{G} & \mathbf{d}_{11}^{G}
\end{array}\right]\left\{\begin{array}{c}
\Delta \phi \\
\Delta \phi^{\prime}
\end{array}\right\} d S
$$

con el siguiente significado de las matrices en el integrando

$$
\begin{aligned}
& \mathbf{d}_{00}^{G}=\left[\begin{array}{cc}
\mathbf{0} & \mathbf{0} \\
\mathbf{0} & \widehat{\mathbf{n}} \widehat{\gamma}
\end{array}\right] \\
& \mathbf{d}_{01}^{G}=\left[\begin{array}{cc}
\mathbf{0} & \mathbf{0} \\
\widehat{\mathbf{n}} & \widehat{\mathbf{m}}
\end{array}\right] \\
& \mathbf{d}_{10}^{G}=\left[\begin{array}{cc}
\mathbf{0} & -\widehat{\mathbf{n}} \\
\mathbf{0} & \mathbf{0}
\end{array}\right] \\
& \mathbf{d}_{11}^{G}=\left[\begin{array}{ll}
\mathbf{0} & \mathbf{0} \\
\mathbf{0} & \mathbf{0}
\end{array}\right] .
\end{aligned}
$$

Los resultados anteriores permiten concluir que la parte geométrica del operador tangente, como sucedía con la obtenida por Simó y Vu-Quoc en 62 no es simétrica. Además, no parece posible lograr una expresión compacta en función del operador de deformaciones $\overline{\mathbf{b}}$ definido en (4.37). La discrepancia entre el núcleo de nuestro operador

$$
\left[\begin{array}{cccc}
0 & 0 & 0 & 0 \\
0 & \widehat{\mathbf{n}} \widehat{\gamma} & \widehat{\mathbf{n}} & \widehat{\mathbf{m}} \\
\mathbf{0} & -\widehat{\mathbf{n}} & \mathbf{0} & \mathbf{0} \\
\mathbf{0} & 0 & \mathbf{0} & \mathbf{0}
\end{array}\right]
$$

y el núcleo del operador de Simó y Vu-Quoc

$$
\left[\begin{array}{cccc}
0 & 0 & 0 & 0 \\
0 & \widehat{\gamma} \widehat{\mathbf{n}} & \widehat{\mathbf{n}} & 0 \\
0 & -\widehat{\mathbf{n}} & \mathbf{0} & 0 \\
0 & -\widehat{\mathbf{m}} & \mathbf{0} & 0
\end{array}\right]
$$


es evidente. Es importante hacer notar que tampoco parece posible lograr una expresión compacta del operador de Simó en función de $\overline{\mathbf{b}}$.

Comentario 6.2.1. El núcleo de la parte geométrica del operador obtenido en la tesis es, precisamente, el resultado de trasponer el núcleo del operador geométrico de Simó y Vu-Quoc.

El segundo término de (6.14) corresponde a la linealización del vector de esfuerzos (fuerzas internas) y da lugar a la parte constitutiva del operador tangente. La versión incremental de las ecuaciones constitutivas (4.74) se reproduce aquí

$$
\left\{\begin{array}{c}
\Delta \boldsymbol{N} \\
\Delta \boldsymbol{M}
\end{array}\right\}=\left[\begin{array}{ll}
\mathbf{C}_{\Gamma \Gamma} & \mathbf{C}_{\Gamma K} \\
\mathbf{C}_{K \Gamma} & \mathbf{C}_{K K}
\end{array}\right]\left\{\begin{array}{c}
\Delta \boldsymbol{\Gamma} \\
\Delta \boldsymbol{K}
\end{array}\right\}
$$

Para calcular la forma espacial de la relación constitutiva incremental se emplea la definición de variación corrotacional

$$
\begin{array}{ll}
\Delta \boldsymbol{\Gamma}=\boldsymbol{\Lambda}^{\top} \stackrel{\nabla}{\Delta} \gamma & \Delta K=\Lambda^{\top} \stackrel{\nabla}{\Delta} \boldsymbol{\kappa} \\
\stackrel{\nabla}{\Delta} \boldsymbol{n}=\boldsymbol{\Lambda} \Delta \boldsymbol{N} & \stackrel{\nabla}{\Delta m}=\boldsymbol{\Lambda} \Delta \boldsymbol{M}
\end{array}
$$

Entonces

$$
\left\{\begin{array}{l}
{ }_{\Delta}^{\Delta} \boldsymbol{n} \\
\nabla \\
\Delta \boldsymbol{m}
\end{array}\right\}=\left[\begin{array}{cc}
\boldsymbol{\Lambda} & \mathbf{0} \\
\mathbf{0} & \boldsymbol{\Lambda}
\end{array}\right]\left[\begin{array}{ll}
\mathbf{C}_{\Gamma \Gamma} & \mathbf{C}_{\Gamma K} \\
\mathbf{C}_{K \Gamma} & \mathbf{C}_{K K}
\end{array}\right]\left[\begin{array}{cc}
\boldsymbol{\Lambda}^{\top} & \mathbf{0} \\
\mathbf{0} & \boldsymbol{\Lambda}^{\top}
\end{array}\right]\left\{\begin{array}{c}
\nabla_{\Delta} \boldsymbol{\gamma} \\
\nabla \\
\Delta \boldsymbol{\kappa}
\end{array}\right\}
$$

que se puede escribir como

$$
\left\{\begin{array}{l}
\nabla \\
\Delta \boldsymbol{n} \\
\nabla \\
\Delta \boldsymbol{m}
\end{array}\right\}=\left[\begin{array}{ll}
\mathbf{c}_{\gamma \gamma} & \mathbf{c}_{\gamma \kappa} \\
\mathbf{c}_{\kappa \gamma} & \mathbf{c}_{\kappa \kappa}
\end{array}\right]\left\{\begin{array}{l}
\nabla \\
\Delta \gamma \\
\nabla \\
\Delta \kappa
\end{array}\right\}
$$

Aplicando los resultados (4.30a) y (4.30b) y operando, el integrando del segundo sumando resulta

$$
\begin{aligned}
\stackrel{\nabla}{\nabla} \cdot \stackrel{\nabla}{\Delta} \boldsymbol{n}+\stackrel{\nabla}{\nabla} \cdot \stackrel{\nabla}{\nabla} \boldsymbol{m} & =\delta \boldsymbol{x}^{\prime \top} \mathbf{c}_{\gamma \gamma} \Delta \boldsymbol{x}^{\prime}+\delta \boldsymbol{x}^{\prime \top} \mathbf{c}_{\gamma \gamma} \widehat{\boldsymbol{\gamma}} \Delta \boldsymbol{\omega}+\delta \boldsymbol{x}^{\prime \top} \mathbf{c}_{\gamma \kappa} \Delta \boldsymbol{\omega}^{\prime} \\
& -\delta \boldsymbol{\omega}^{\top} \widehat{\gamma} \mathbf{c}_{\gamma \gamma} \Delta \boldsymbol{x}^{\prime}-\delta \boldsymbol{\omega}^{\top} \widehat{\gamma} \mathbf{c}_{\gamma \gamma} \widehat{\boldsymbol{\gamma}} \Delta \boldsymbol{\omega}-\delta \boldsymbol{\omega}^{\top} \widehat{\gamma} \mathbf{c}_{\gamma \kappa} \Delta \boldsymbol{\omega}^{\prime} \\
& \delta \boldsymbol{\omega}^{\prime}{ }^{\top} \mathbf{c}_{\kappa \gamma} \Delta \boldsymbol{x}^{\prime}+\delta \boldsymbol{\omega}^{\prime}{ }^{\top} \mathbf{c}_{\kappa \gamma} \widehat{\gamma} \Delta \boldsymbol{\omega}+\delta \boldsymbol{\omega}^{\prime}{ }^{\top} \mathbf{c}_{\kappa \kappa} \Delta \boldsymbol{\omega}^{\prime}
\end{aligned}
$$

El término constitutivo del operador tangente en su forma espacial se obtiene agrupando factores

$$
\int_{\Gamma_{0}}\left\{\delta \boldsymbol{x}^{\top} \delta \boldsymbol{\omega}^{\top} \delta \boldsymbol{x}^{\prime \top} \delta \boldsymbol{\omega}^{\prime \top}\right\}\left[\begin{array}{cccc}
\mathbf{0} & \mathbf{0} & \mathbf{0} & \mathbf{0} \\
\mathbf{0} & -\widehat{\gamma} \mathbf{c}_{\gamma \gamma} \widehat{\gamma} & -\widehat{\gamma} \mathbf{c}_{\gamma \gamma} & -\widehat{\gamma}_{\gamma \kappa} \\
\mathbf{0} & \mathbf{c}_{\gamma \gamma} \widehat{\gamma} & \mathbf{c}_{\gamma \gamma} & \mathbf{c}_{\gamma \kappa} \\
\mathbf{0} & \mathbf{c}_{\kappa \gamma} \widehat{\gamma} & \mathbf{c}_{\kappa \gamma} & \mathbf{c}_{\kappa \kappa}
\end{array}\right]\left\{\begin{array}{c}
\Delta \boldsymbol{x} \\
\Delta \boldsymbol{\omega} \\
\Delta \boldsymbol{x}^{\prime} \\
\Delta \boldsymbol{\omega}^{\prime}
\end{array}\right\} d S,
$$

o también

$$
\int_{\Gamma_{0}}\left\{\delta \phi^{\top} \quad \delta \phi^{\prime \top}\right\}\left[\begin{array}{ll}
\mathbf{d}_{00}^{M} & \mathbf{d}_{01}^{M} \\
\mathbf{d}_{10}^{M} & \mathbf{d}_{11}^{M}
\end{array}\right]\left\{\begin{array}{c}
\Delta \phi \\
\Delta \phi^{\prime}
\end{array}\right\} d S
$$


con

$$
\begin{aligned}
& \mathbf{d}_{00}^{M}=\left[\begin{array}{cc}
\mathbf{0} & \mathbf{0} \\
\mathbf{0} & -\widehat{\gamma} \mathbf{c}_{\gamma \gamma} \widehat{\gamma}
\end{array}\right]=\left[\begin{array}{cc}
\mathbf{0} & \mathbf{0} \\
-\widehat{\boldsymbol{\gamma}} & \mathbf{0}
\end{array}\right]\left[\begin{array}{cc}
\mathbf{c}_{\gamma \gamma} & \mathbf{c}_{\gamma \kappa} \\
\mathbf{c}_{\kappa \gamma} & \mathbf{c}_{\kappa \kappa}
\end{array}\right]\left[\begin{array}{ll}
\mathbf{0} & \widehat{\gamma} \\
\mathbf{0} & \mathbf{0}
\end{array}\right] \\
& \mathbf{d}_{01}^{M}=\left[\begin{array}{cc}
\mathbf{0} & \mathbf{0} \\
-\widehat{\boldsymbol{\gamma}} \mathbf{c}_{\gamma \gamma} & -\widehat{\gamma} \mathbf{c}_{\gamma \kappa}
\end{array}\right]=\left[\begin{array}{cc}
\mathbf{0} & \mathbf{0} \\
-\widehat{\gamma} & \mathbf{0}
\end{array}\right]\left[\begin{array}{ll}
\mathbf{c}_{\gamma \gamma} & \mathbf{c}_{\gamma \kappa} \\
\mathbf{c}_{\kappa \gamma} & \mathbf{c}_{\kappa \kappa}
\end{array}\right] \\
& \mathbf{d}_{10}^{M}=\mathbf{d}_{01}^{M \top} \\
& \mathbf{d}_{11}^{M}=\left[\begin{array}{ll}
\mathbf{c}_{\gamma \gamma} & \mathbf{c}_{\gamma \kappa} \\
\mathbf{c}_{\kappa \gamma} & \mathbf{c}_{\kappa \kappa}
\end{array}\right] \text {. }
\end{aligned}
$$

Es inmediato comprobar que esta parte del operador es simétrica. Conviene además señalar que la parte constitutiva del operador tangente calculada a partir de la variación corrotacional coincide con la parte correspondiente del operador de Simó (incluida en la introducción al capítulo).

El término constitutivo del operador tangente admite una expresión alternativa en la que se hace uso del operador $\overline{\mathbf{b}}$ definido en (4.37).

$$
\int_{\Gamma_{0}}\left\{\delta \boldsymbol{x}^{\top} \quad \delta \boldsymbol{\omega}^{\top}\right\}\left[\begin{array}{cc}
\frac{d}{d S} \mathbf{1} & \mathbf{0} \\
-\widehat{\gamma} & \frac{d}{d S} \mathbf{1}
\end{array}\right]\left[\begin{array}{cc}
\mathbf{c}_{\gamma \gamma} & \mathbf{c}_{\gamma \kappa} \\
\mathbf{c}_{\kappa \gamma} & \mathbf{c}_{\kappa \kappa}
\end{array}\right]\left[\begin{array}{cc}
\frac{d}{d S} \mathbf{1} & \widehat{\gamma} \\
\mathbf{0} & \frac{d}{d S} \mathbf{1}
\end{array}\right]\left\{\begin{array}{c}
\Delta \boldsymbol{x} \\
\Delta \boldsymbol{\omega}
\end{array}\right\} d S
$$

Y en forma compacta

$$
\int_{\Gamma_{0}} \delta \phi^{\top} \overline{\mathbf{b}}^{\top} \overline{\mathbf{d}}^{M} \overline{\mathbf{b}} \Delta \phi d S
$$

con

$$
\overline{\mathbf{d}}^{M}=\left[\begin{array}{ll}
\mathbf{c}_{\gamma \gamma} & \mathbf{c}_{\gamma \kappa} \\
\mathbf{c}_{\kappa \gamma} & \mathbf{c}_{\kappa \kappa}
\end{array}\right]=\mathbf{d}_{11}^{M}
$$

\section{Procediemiento b. Linealización del vector de variaciones y su factor}

El trabajo virtual de las fuerzas internas admite la expresión alternativa (6.1) deducida en la introducción al capítulo, que reproducimos por conveniencia

$$
\delta W_{i n t}=\int_{\Gamma_{0}}\left\{\delta \boldsymbol{x}^{\top} \delta \boldsymbol{\omega}^{\top} \delta \boldsymbol{x}^{\prime \top} \delta \boldsymbol{\omega}^{\prime \top}\right\}\left\{\begin{array}{c}
\mathbf{0} \\
-\widehat{\gamma} \boldsymbol{n} \\
\boldsymbol{n} \\
\boldsymbol{m}
\end{array}\right\} d S
$$

en función del vector de variaciones de la configuración y su derivada, y un vector que depende de los esfuerzos y la deformación generalizada $\gamma$. Su variación, empleando la 
regla de derivación (6.9) en su forma espacial, tiene dos términos

$$
\begin{aligned}
\Delta \delta W_{\text {int }}=\int_{\Gamma_{0}} \Delta\left\{\delta \boldsymbol{x}^{\top} \delta \boldsymbol{\omega}^{\top} \delta \boldsymbol{x}^{\top \top} \delta \boldsymbol{\omega}^{\prime \top}\right\} & \left\{\begin{array}{c}
\mathbf{0} \\
-\widehat{\gamma} \boldsymbol{n} \\
\boldsymbol{n} \\
\boldsymbol{m}
\end{array}\right\} d S \\
& +\int_{\Gamma_{0}}\left\{\delta \boldsymbol{x}^{\top} \delta \boldsymbol{\omega}^{\top} \delta \boldsymbol{x}^{\top \top} \delta \boldsymbol{\omega}^{\top \top}\right\} \Delta\left\{\begin{array}{c}
\boldsymbol{0} \\
-\widehat{\gamma} \boldsymbol{n} \\
\boldsymbol{n} \\
\boldsymbol{m}
\end{array}\right\} d S .
\end{aligned}
$$

El segundo término ha sido analizado en la introducción al capítulo, y como vimos, proporciona la parte constitutiva y geométrica del operador tangente tal y como lo recogen Simó y Vu-Quoc en [62]. La diferencia entre el operador obtenido en la tesis y el de Simó radica pues en el primer término, que no es nulo en virtud de la proposición 6.1.3. Desarrollando su integrando tenemos

$$
-\Delta \delta \boldsymbol{\omega} \cdot \widehat{\gamma} \boldsymbol{n}+\Delta \delta \boldsymbol{x}^{\prime} \cdot \boldsymbol{n}+\Delta \delta \boldsymbol{\omega}^{\prime} \cdot \boldsymbol{m} .
$$

Teniendo en cuenta los resultados obtenidos para las segundas variaciones (6.11)

$$
-(\Delta \widehat{\boldsymbol{\omega}} \delta \boldsymbol{\omega}) \cdot \widehat{\gamma} \boldsymbol{n}+\left(\Delta \widehat{\boldsymbol{\omega}}^{\prime} \delta \boldsymbol{\omega}\right) \cdot \boldsymbol{m}+\left(\Delta \widehat{\boldsymbol{\omega}} \delta \boldsymbol{\omega}^{\prime}\right) \cdot \boldsymbol{m} .
$$

El desarrollo del primer sumando requiere especial atención:

$$
\begin{aligned}
-(\Delta \widehat{\boldsymbol{\omega}} \delta \boldsymbol{\omega}) \cdot \widehat{\gamma} \boldsymbol{n} & =\delta \boldsymbol{\omega}^{\top} \Delta \widehat{\boldsymbol{\omega}}(\boldsymbol{\gamma} \times \boldsymbol{n}) \\
& =\delta \boldsymbol{\omega}^{\top} \Delta \boldsymbol{\omega} \times(\boldsymbol{\gamma} \times \boldsymbol{n}) \\
& =-\delta \boldsymbol{\omega}^{\top}(\widehat{\boldsymbol{\gamma} \times \boldsymbol{n}}) \Delta \boldsymbol{\omega} .
\end{aligned}
$$

Si tenemos en cuenta que $\widehat{\boldsymbol{\gamma} \times \boldsymbol{n}}=\widehat{\gamma} \widehat{\mathbf{n}}-\widehat{\mathbf{n}} \widehat{\gamma}$ (se comprueba fácilmente desarrollando los productos), entonces

$$
-(\Delta \widehat{\boldsymbol{\omega}} \delta \boldsymbol{\omega}) \cdot \widehat{\gamma} \boldsymbol{n}=-\delta \boldsymbol{\omega}^{\top}(\widehat{\gamma} \widehat{\mathbf{n}}-\widehat{\mathbf{n}} \widehat{\gamma}) \Delta \boldsymbol{\omega}
$$

Sustituyendo el primer sumando en la expresión anterior y reordenando el integrando resulta

$$
\delta \boldsymbol{\omega}^{\top}(\widehat{\mathbf{n}} \widehat{\gamma}-\widehat{\gamma} \widehat{\mathbf{n}}) \Delta \boldsymbol{\omega}+\delta \boldsymbol{\omega}^{\top} \widehat{\mathbf{m}} \Delta \boldsymbol{\omega}^{\prime}+\delta \boldsymbol{\omega}^{\prime} \widehat{\mathbf{m}} \Delta \boldsymbol{\omega}
$$

Finalmente el primer sumando de la variación del trabajo virtual de las fuerzas internas se puede expresar así

$$
\int_{\Gamma_{0}}\left\{\delta \boldsymbol{x}^{\top} \delta \boldsymbol{\omega}^{\top} \delta \boldsymbol{x}^{\top \top} \delta \boldsymbol{\omega}^{\prime \top}\right\}\left[\begin{array}{cccc}
\mathbf{0} & \mathbf{0} & \mathbf{0} & \mathbf{0} \\
\mathbf{0} & \widehat{\mathbf{n}} \widehat{\boldsymbol{\gamma}}-\widehat{\boldsymbol{\gamma}} \widehat{\mathbf{n}} & \mathbf{0} & \widehat{\mathbf{m}} \\
\mathbf{0} & \mathbf{0} & \mathbf{0} & \mathbf{0} \\
\mathbf{0} & \widehat{\mathbf{m}} & \mathbf{0} & \mathbf{0}
\end{array}\right]\left\{\begin{array}{c}
\Delta \boldsymbol{x} \\
\Delta \boldsymbol{\omega} \\
\Delta \boldsymbol{x}^{\prime} \\
\Delta \boldsymbol{\omega}^{\prime}
\end{array}\right\} d S .
$$


El núcleo del integrando es una matriz antisimétrica. Además se cumple

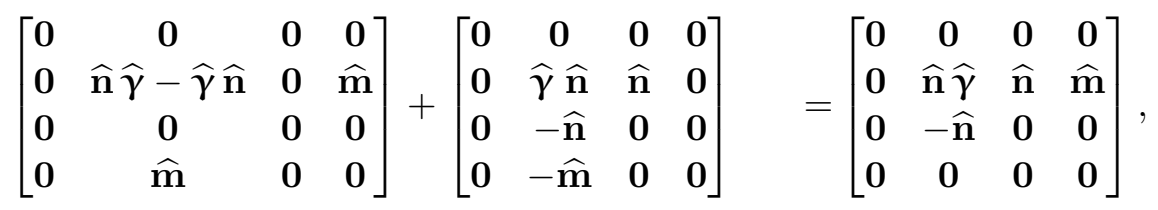

que denotaremos abreviadamente

$$
\left[\begin{array}{ll}
\mathbf{d}_{00}^{G A} & \mathbf{d}_{01}^{G A} \\
\mathbf{d}_{10}^{G A} & \mathbf{d}_{11}^{G A}
\end{array}\right]+\left[\begin{array}{ll}
\mathbf{d}_{00}^{G S} & \mathbf{d}_{01}^{G S} \\
\mathbf{d}_{10}^{G S} & \mathbf{d}_{11}^{G S}
\end{array}\right] \quad=\left[\begin{array}{ll}
\mathbf{d}_{00}^{G} & \mathbf{d}_{01}^{G} \\
\mathbf{d}_{10}^{G} & \mathbf{d}_{11}^{G}
\end{array}\right]
$$

con lo que queda demostrada la relación entre el núcleo de nuestro operador geométrico y el de Simó (que denotamos con el superíndice s), cuya diferencia es precisamente la matriz antisimétrica (abreviada con el superíndice A), así como la equivalencia entre los dos procedimientos presentados para linealizar la ecuación de trabajos virtuales.

\subsubsection{Linealización del trabajo virtual de las fuerzas exteriores}

$\mathrm{Al}$ igual que en el proceso de linealización del trabajo virtual de las fuerzas internas, la linealización consistente de la forma espacial del trabajo virtual de las fuerzas exteriores se puede llevar a cabo utilizando la variación espacial (como en el procedimiento b empleado en la sección anterior). De nuevo aparecen dos sumandos, uno correspondiente a la segunda variación de la configuración, y otro que incluye la variación de las fuerzas exteriores; éste último da lugar a las mismas expresiones que obtuvo Simó, en las que sólo contribuyen las fuerzas perseguidoras (de componentes constantes en el sistema de referencia de la sección).

Supondremos que las fuerzas exteriores dependen de un único parámetro de control $\lambda$ sujeto también a variación. Esta hipótesis permite la solución numérica de las ecuaciones por continuación, cuestión que se aborda en el siguiente capítulo.

El trabajo virtual de las fuerzas exteriores es, según (4.18)

$$
\begin{aligned}
\delta W_{\text {ext }}=\lambda \int_{\Gamma_{0}}\left(\boldsymbol{q}_{n} \cdot \delta \boldsymbol{x}+\boldsymbol{q}_{m} \cdot \delta \boldsymbol{\omega}\right) d S \\
\quad+\lambda\left(\boldsymbol{n}_{1} \cdot \delta \boldsymbol{x}\left(S_{1}\right)+\boldsymbol{m}_{1} \cdot \delta \boldsymbol{\omega}\left(S_{1}\right)\right)+\lambda\left(\boldsymbol{n}_{2} \cdot \delta \boldsymbol{x}\left(S_{2}\right)+\boldsymbol{m}_{2} \cdot \delta \boldsymbol{\omega}\left(S_{2}\right)\right),
\end{aligned}
$$

y se divide en tres partes: (a) el producido por las fuerzas generalizadas sobre la directriz, (b) el trabajo sobre la sección inicial, y (c) el trabajo sobre la sección final. Las fuerzas generalizadas $\boldsymbol{q}_{n}$ y $\boldsymbol{q}_{m}$ pueden no ser conservativas, y en general, en cada instante del proceso de deformación dependerán de la configuración de la pieza, es decir, de $\boldsymbol{x}$ y $\boldsymbol{\Lambda}$. Consideramos en principio la actuación de dos clases de fuerzas: espaciales, cuyas componentes son constantes en el sistema general de referencia, que denotaremos mediante el superíndice $s$, e intrínsecas o perseguidoras, cuyas componentes permanecen constantes en el sistema de referencia de la sección, que denotaremos mediante el superíndice $f$ ya que en la literatura se conocen como follower [53], [62]. 
Por tanto, a partir de (3.50)

$$
\begin{gathered}
\boldsymbol{q}_{n}=\boldsymbol{q}_{n}^{s}+\boldsymbol{q}_{n}^{f}=\boldsymbol{q}_{n}^{s}+\boldsymbol{\Lambda} \boldsymbol{Q}_{n}^{f} \\
\boldsymbol{q}_{m}=\boldsymbol{q}_{m}^{s}+\boldsymbol{q}_{m}^{f}=\boldsymbol{q}_{m}^{s}+\boldsymbol{\Lambda} \boldsymbol{Q}_{m}^{f} .
\end{gathered}
$$

En estas expresiones los vectores $\boldsymbol{q}^{s}$ tienen componentes constantes en el sistema general de referencia, y los vectores $\boldsymbol{q}^{f}$ son de componentes constantes en el sistema actual, por tanto, su forma material $\boldsymbol{Q}^{f}$ también tiene componentes constantes en el sistema global. La misma clasificación establecida para las fuerzas generalizadas se puede considerar para las fuerzas y momentos en extremos de barra

$$
\begin{aligned}
\boldsymbol{n}_{1} & =\boldsymbol{n}_{1}^{s}+\boldsymbol{n}_{1}^{f} \\
\boldsymbol{m}_{1} & =\boldsymbol{m}_{1}^{s}+\boldsymbol{m}_{1}^{f},
\end{aligned}
$$

junto con las expresiones análogas en el extremo $S=S_{2}$. Evidentemente en la situación más general posible, las fuerzas y momentos actuantes no tienen por que ajustarse a estas dos categorías; es posible, por ejemplo, imaginar fuerzas cuya dirección permanece perpendicular a la directriz (otra clase de fuerzas perseguidoras), o bien otras acciones cuya variación siga cierto patrón dependiente de alguna función de la configuración. No obstante, se ha optado por reducir el análisis a los dos tipos mencionados, ya que son suficientemente representativos y muestran con claridad la conclusión del análisis. Una cuestión interesante, que ha sido estudiada en profundidad por Ritto-Corrêa y Camotim [53, [54, es el hecho de que ninguna de las dos clases de momentos considerados tiene carácter conservativo.

La variación (espacial) de las fuerzas espaciales $\boldsymbol{q}^{s}$ es nula, puesto que sus componentes son constantes en el sistema global de referencia

$$
\Delta \boldsymbol{q}^{s}=\mathbf{0}
$$

Por otra parte, la variación de las fuerzas perseguidoras $\boldsymbol{q}^{f}$ se puede calcular teniendo en cuenta que su forma material $\boldsymbol{Q}^{f}$ es de componentes constantes en el sistema global

$$
\begin{aligned}
\Delta \boldsymbol{q}^{f} & =\Delta\left(\boldsymbol{\Lambda} \boldsymbol{Q}^{f}\right)=\Delta \boldsymbol{\Lambda} \boldsymbol{Q}^{f} \\
& =\Delta \boldsymbol{\Lambda} \boldsymbol{\Lambda}^{\top} \boldsymbol{q}^{f}=\Delta \widehat{\boldsymbol{\omega}} \boldsymbol{q}^{f}=-\widehat{\mathbf{q}}^{f} \Delta \boldsymbol{\omega} .
\end{aligned}
$$

Volviendo a la ecuación del trabajo virtual de las fuerzas exteriores, su variación es

$$
\begin{aligned}
\Delta \delta W_{e x t} & =\lambda \Delta \int_{\Gamma_{0}}\left(\boldsymbol{q}_{n} \cdot \delta \boldsymbol{x}+\boldsymbol{q}_{m} \cdot \delta \boldsymbol{\omega}\right) d S \\
& +\lambda \Delta\left(\boldsymbol{n}_{1} \cdot \delta \boldsymbol{x}\left(S_{1}\right)+\boldsymbol{m}_{1} \cdot \delta \boldsymbol{\omega}\left(S_{1}\right)\right)+\lambda \Delta\left(\boldsymbol{n}_{2} \cdot \delta \boldsymbol{x}\left(S_{2}\right)+\boldsymbol{m}_{2} \cdot \delta \boldsymbol{\omega}\left(S_{2}\right)\right) \\
& +\Delta \lambda \int_{\Gamma_{0}}\left(\boldsymbol{q}_{n} \cdot \delta \boldsymbol{x}+\boldsymbol{q}_{m} \cdot \delta \boldsymbol{\omega}\right) d S \\
& +\Delta \lambda\left(\boldsymbol{n}_{1} \cdot \delta \boldsymbol{x}\left(S_{1}\right)+\boldsymbol{m}_{1} \cdot \delta \boldsymbol{\omega}\left(S_{1}\right)\right)+\Delta \lambda\left(\boldsymbol{n}_{2} \cdot \delta \boldsymbol{x}\left(S_{2}\right)+\boldsymbol{m}_{2} \cdot \delta \boldsymbol{\omega}\left(S_{2}\right)\right) .
\end{aligned}
$$

Los tres primeros sumandos corresponden a la variación de las fuerzas exteriores y los tres últimos dependen de la variación del parámetro $\lambda$. A continuación evaluamos: 
1. La variación del trabajo virtual de las fuerzas generalizadas

$$
\begin{aligned}
\Delta \int_{\Gamma_{0}}\left(\boldsymbol{q}_{n} \cdot \delta \boldsymbol{x}+\boldsymbol{q}_{m} \cdot \delta \boldsymbol{\omega}\right) d S & =\int_{\Gamma_{0}}\left(\Delta \delta \boldsymbol{x} \cdot \boldsymbol{q}_{n}+\Delta \delta \boldsymbol{\omega} \cdot \boldsymbol{q}_{m}\right) d S \\
& +\int_{\Gamma_{0}}\left(\delta \boldsymbol{x} \cdot \Delta \boldsymbol{q}_{n}+\delta \boldsymbol{\omega} \cdot \Delta \boldsymbol{q}_{m}\right) d S
\end{aligned}
$$

A su vez, el primer término es

$$
\begin{aligned}
\int_{\Gamma_{0}}\left(\Delta \delta \boldsymbol{x} \cdot \boldsymbol{q}_{n}+\Delta \delta \boldsymbol{\omega} \cdot \boldsymbol{q}_{m}\right) d S & =\int_{\Gamma_{0}}-\delta \boldsymbol{\omega}^{\top} \Delta \widehat{\boldsymbol{\omega}} \boldsymbol{q}_{m} d S \\
& =\int_{\Gamma_{0}} \delta \boldsymbol{\omega}^{\top} \widehat{\mathbf{q}}_{m} \Delta \boldsymbol{\omega} d S,
\end{aligned}
$$

y el segundo

$$
\int_{\Gamma_{0}}\left(\delta \boldsymbol{x} \cdot \Delta \boldsymbol{q}_{n}+\delta \boldsymbol{\omega} \cdot \Delta \boldsymbol{q}_{m}\right) d S=\int_{\Gamma_{0}}-\left(\delta \boldsymbol{x}^{\top} \widehat{\mathbf{q}}_{n}^{f} \Delta \boldsymbol{\omega}+\delta \boldsymbol{\omega}^{\top} \widehat{\mathbf{q}}_{m}^{f} \Delta \boldsymbol{\omega}\right) d S
$$

2. La variación de las fuerzas en los extremos inicial y final ( $a=1$ ó 2 , respectivamente)

$$
\begin{aligned}
\Delta\left(\boldsymbol{n}_{a} \cdot \delta \boldsymbol{x}\left(S_{a}\right)+\boldsymbol{m}_{a} \cdot \delta \boldsymbol{\omega}\left(S_{a}\right)\right) & =\left(\Delta \delta \boldsymbol{x}\left(S_{a}\right) \cdot \boldsymbol{n}_{a}+\Delta \delta \boldsymbol{\omega}\left(S_{a}\right) \cdot \boldsymbol{m}_{a}\right) \\
& +\left(\delta \boldsymbol{x}\left(S_{a}\right) \cdot \Delta \boldsymbol{n}_{a}+\delta \boldsymbol{\omega}\left(S_{a}\right) \cdot \Delta \boldsymbol{m}_{a}\right),
\end{aligned}
$$

cuyo primer término

$$
\begin{aligned}
\Delta \delta \boldsymbol{x}\left(S_{a}\right) \cdot \boldsymbol{n}_{a}+\Delta \delta \boldsymbol{\omega}\left(S_{a}\right) \cdot \boldsymbol{m}_{a} & =-\delta \boldsymbol{\omega}\left(S_{a}\right)^{\top} \Delta \widehat{\boldsymbol{\omega}}\left(S_{a}\right) \boldsymbol{m}_{a} d S \\
& =\delta \boldsymbol{\omega}\left(S_{a}\right)^{\top} \widehat{\mathbf{m}}_{a} \delta \boldsymbol{\omega}\left(S_{a}\right),
\end{aligned}
$$

y segundo término

$$
\delta \boldsymbol{x}\left(S_{a}\right) \cdot \Delta \boldsymbol{n}_{a}+\delta \boldsymbol{\omega}\left(S_{a}\right) \cdot \Delta \boldsymbol{m}_{a}=-\delta \boldsymbol{x}\left(S_{a}\right)^{\top} \widehat{\mathbf{n}}_{a}^{f} \Delta \boldsymbol{\omega}\left(S_{1}\right)-\delta \boldsymbol{\omega}\left(S_{a}\right)^{\top} \widehat{\mathbf{m}}_{a}^{f} \Delta \boldsymbol{\omega}\left(S_{a}\right) .
$$

Por tanto el resultado de linealizar los tres primeros sumandos del trabajo virtual de las fuerzas exteriores se puede dividir en dos partes: la primera está compuesta por términos resultantes de la segunda variación de la configuración,

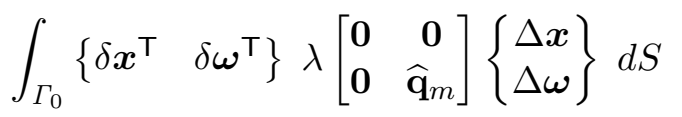

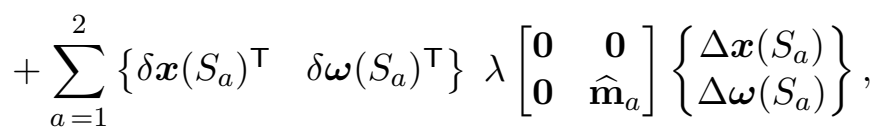

la segunda, por términos consecuencia de la variación de las fuerzas y momentos,

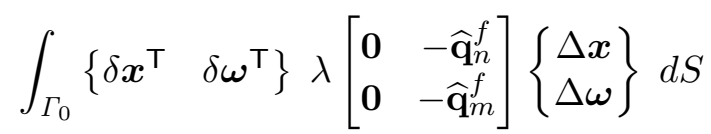

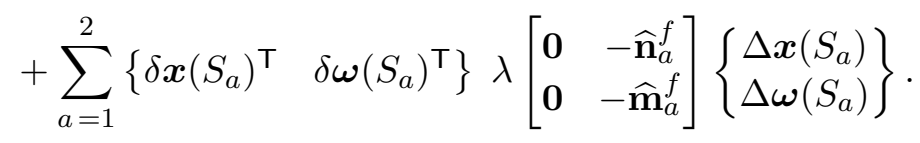


Finalmente podemos abreviar y sumar estas expresiones de la siguiente forma mediante (4.38a):

$$
\begin{array}{r}
\int_{\Gamma_{0}} \delta \phi^{\top} \mathbf{d}^{Q A} \Delta \phi d S+\sum_{a=1}^{2} \delta \phi\left(S_{a}\right)^{\top} \mathbf{f}_{a}^{A} \Delta \phi\left(S_{a}\right) \\
+\int_{\Gamma_{0}} \delta \phi^{\top} \mathbf{d}^{Q S} \Delta \phi d S+\sum_{a=1}^{2} \delta \phi\left(S_{a}\right)^{\top} \mathbf{f}_{a}^{S} \Delta \phi\left(S_{a}\right),
\end{array}
$$

con

$$
\begin{aligned}
\mathbf{d}^{Q A}=\lambda\left[\begin{array}{cc}
\mathbf{0} & \mathbf{0} \\
\mathbf{0} & \widehat{\mathbf{q}}_{m}
\end{array}\right] & \mathbf{f}_{a}^{A}=\lambda\left[\begin{array}{cc}
\mathbf{0} & \mathbf{0} \\
\mathbf{0} & \widehat{\mathbf{m}}_{a}
\end{array}\right], \\
\mathbf{d}^{Q S}=\lambda\left[\begin{array}{cc}
\mathbf{0} & -\widehat{\mathbf{q}}_{n}^{f} \\
\mathbf{0} & -\widehat{\mathbf{q}}_{m}^{f}
\end{array}\right] & \mathbf{f}_{a}^{S}=\lambda\left[\begin{array}{cc}
\mathbf{0} & -\widehat{\mathbf{n}}_{a}^{f} \\
\mathbf{0} & -\widehat{\mathbf{m}}_{a}^{f}
\end{array}\right] .
\end{aligned}
$$

Por lo que respecta a los tres últimos sumandos, admiten la siguiente expresión matricial

$$
\left(\int_{\Gamma_{0}}\left\{\delta \boldsymbol{x}^{\top} \quad \delta \boldsymbol{\omega}^{\top}\right\}\left\{\begin{array}{c}
\boldsymbol{q}_{n} \\
\boldsymbol{q}_{m}
\end{array}\right\} d S+\sum_{a=1}^{2}\left\{\delta \boldsymbol{x}\left(S_{a}\right)^{\top} \delta \boldsymbol{\omega}\left(S_{a}\right)^{\top}\right\}\left\{\begin{array}{c}
\boldsymbol{n}_{a} \\
\boldsymbol{m}_{a}
\end{array}\right\}\right) \Delta \lambda,
$$

o bien en notación abreviada

$$
\left(\int_{\Gamma_{0}} \delta \phi^{\top} \boldsymbol{q} d S+\sum_{a=1}^{2} \delta \phi\left(S_{a}\right)^{\top} \boldsymbol{f}_{a}\right) \Delta \lambda
$$

\subsubsection{Equilibrio tangente}

La expresión del operador tangente resulta de reunir los resultados obtenidos en las secciones anteriores. La ecuación de trabajos virtuales dice que el trabajo virtual de las fuerzas internas es igual al de las fuerzas exteriores

$$
\delta W_{\text {int }}-\delta W_{\text {ext }}=0
$$

para cualquier variación cinemáticamente admisible $\delta \phi$ de la configuración. La linealización consistente de la ecuación de trabajos virtuales lleva a la siguiente expresión variacional

$$
\Delta\left(\delta W_{\text {int }}-\delta W_{\text {ext }}\right)=0,
$$

que debe cumplirse para cualquier variación admisible $\Delta \phi$ de la configuración. Cuando consideramos que las fuerzas exteriores dependen del parámetro $\lambda$ y que este empieza a aumentar desde un valor inicial nulo, podemos imaginar la respuesta de la estructura como una curva $\phi(\lambda)$, función de $\lambda$, que denominamos trayectoria de equilibrio. La condición que garantiza que una cierta configuración $(\phi, \lambda)$ permanece en la trayectoria de equilibrio es, precisamente que se satisfaga la ecuación de trabajos 
virtuales (6.43). En términos incrementales, esto es equivalente a que se cumpla la ecuación (6.44) cuando el parámetro $\lambda$ experimenta un pequeño incremento, es decir

$$
\Delta \delta W_{i n t}=\Delta \delta W_{\text {ext }} .
$$

Diremos que esta expresión define el equilibrio tangente en el modelo RS. A continuación vamos a desarrollarla empleando los resultados obtenidos en las secciones anteriores, primero en su forma espacial, y finalmente en su versión material.

La forma espacial de la variación espacial del trabajo de las fuerzas internas resulta de sumar la contribución de la parte geométrica (6.18) y la constitutiva (6.25). A su vez, la parte geométrica admite la descomposición (6.33b), luego

$$
\begin{aligned}
\Delta \delta W_{i n t} & =\int_{\Gamma_{0}}\left\{\delta \phi^{\top} \quad \delta \phi^{\prime \top}\right\}\left[\begin{array}{ll}
\mathbf{d}_{00}^{G A} & \mathbf{d}_{01}^{G A} \\
\mathbf{d}_{10}^{G A} & \mathbf{d}_{11}^{G A}
\end{array}\right]\left\{\begin{array}{c}
\Delta \phi \\
\Delta \boldsymbol{\phi}^{\prime}
\end{array}\right\} d S \\
& +\int_{\Gamma_{0}}\left\{\delta \boldsymbol{\phi}^{\top} \quad \delta \boldsymbol{\phi}^{\prime \top}\right\}\left(\left[\begin{array}{ll}
\mathbf{d}_{00}^{G S} & \mathbf{d}_{01}^{G S} \\
\mathbf{d}_{10}^{G S} & \mathbf{d}_{11}^{G S}
\end{array}\right]+\left[\begin{array}{ll}
\mathbf{d}_{00}^{M} & \mathbf{d}_{01}^{M} \\
\mathbf{d}_{10}^{M} & \mathbf{d}_{11}^{M}
\end{array}\right]\right)\left\{\begin{array}{c}
\Delta \boldsymbol{\phi} \\
\Delta \boldsymbol{\phi}^{\prime}
\end{array}\right\} d S .
\end{aligned}
$$

La variación de las fuerzas externas se obtiene a partir de las expresiones (6.40) y (6.42), por tanto ${ }^{2}$

$$
\begin{aligned}
\Delta \delta W_{e x t} & =\int_{\Gamma_{0}}\left\{\delta \boldsymbol{\phi}^{\top} \delta \boldsymbol{\phi}^{\prime \top}\right\}\left[\begin{array}{cc}
\mathbf{d}^{Q A} & \mathbf{0} \\
\mathbf{0} & \mathbf{0}
\end{array}\right]\left\{\begin{array}{c}
\Delta \boldsymbol{\phi} \\
\Delta \boldsymbol{\phi}^{\prime}
\end{array}\right\} d S+\sum_{a=1}^{2}\left\{\delta \boldsymbol{\phi}^{\top} \delta \boldsymbol{\phi}^{\prime \top}\right\}\left[\begin{array}{cc}
\mathbf{f}_{a}^{A} & \mathbf{0} \\
\mathbf{0} & \mathbf{0}
\end{array}\right]\left\{\begin{array}{c}
\Delta \boldsymbol{\phi} \\
\Delta \boldsymbol{\phi}^{\prime}
\end{array}\right\} \\
& +\int_{\Gamma_{0}}\left\{\delta \boldsymbol{\phi}^{\top} \delta \boldsymbol{\phi}^{\prime \top}\right\}\left[\begin{array}{cc}
\mathbf{d}^{Q S} & \mathbf{0} \\
\mathbf{0} & \mathbf{0}
\end{array}\right]\left\{\begin{array}{c}
\Delta \boldsymbol{\phi} \\
\Delta \boldsymbol{\phi}^{\prime}
\end{array}\right\} d S+\sum_{a=1}^{2}\left\{\delta \boldsymbol{\phi}^{\top} \delta \boldsymbol{\phi}^{\prime}\right\}\left[\begin{array}{cc}
\mathbf{f}_{a}^{S} & \mathbf{0} \\
\mathbf{0} & \mathbf{0}
\end{array}\right]\left\{\begin{array}{c}
\Delta \boldsymbol{\phi} \\
\Delta \boldsymbol{\phi}^{\prime}
\end{array}\right\} \\
& +\Delta \lambda\left(\int_{\Gamma} \delta \boldsymbol{\phi}^{\top} \boldsymbol{q} d S+\sum_{a=1}^{2} \delta \boldsymbol{\phi}\left(S_{a}\right)^{\top} \boldsymbol{f}_{a}\right) .
\end{aligned}
$$

Igualando las variaciones y reordenando, la expresión del equilibrio tangente queda de la siguiente forma

$$
\begin{aligned}
& \int_{\Gamma_{0}}\left\{\delta \phi^{\top} \delta \phi^{\prime \top}\right\}\left(\left[\begin{array}{cc}
\mathbf{d}_{00}^{G A} & \mathbf{d}_{01}^{G A} \\
\mathbf{d}_{10}^{G A} & \mathbf{d}_{11}^{G A}
\end{array}\right]-\left[\begin{array}{cc}
\mathbf{d}^{Q A} & \mathbf{0} \\
\mathbf{0} & \mathbf{0}
\end{array}\right]\right)\left\{\begin{array}{c}
\Delta \boldsymbol{\phi} \\
\Delta \boldsymbol{\phi}^{\prime}
\end{array}\right\} d S \\
& \left.-\sum_{a=1}^{2}\left\{\delta \phi\left(S_{a}\right)^{\top} \delta \phi\left(S_{a}\right)^{\prime}\right\}^{\top}\right\}\left[\begin{array}{cc}
\mathbf{f}_{a}^{A} & \mathbf{0} \\
\mathbf{0} & \mathbf{0}
\end{array}\right]\left\{\begin{array}{c}
\Delta \phi\left(S_{a}\right) \\
\Delta \phi\left(S_{a}\right)^{\prime}
\end{array}\right\} \\
& +\int_{\Gamma_{0}}\left\{\delta \boldsymbol{\phi}^{\top} \quad \delta \boldsymbol{\phi}^{\prime}{ }^{\top}\right\}\left(\left[\begin{array}{cc}
\mathbf{d}_{00}^{G S} & \mathbf{d}_{01}^{G S} \\
\mathbf{d}_{10}^{G S} & \mathbf{d}_{11}^{G S}
\end{array}\right]+\left[\begin{array}{ll}
\mathbf{d}_{00}^{M} & \mathbf{d}_{01}^{M} \\
\mathbf{d}_{10}^{M} & \mathbf{d}_{11}^{M}
\end{array}\right]-\left[\begin{array}{cc}
\mathbf{d}^{Q S} & \mathbf{0} \\
\mathbf{0} & \mathbf{0}
\end{array}\right]\right)\left\{\begin{array}{c}
\Delta \boldsymbol{\phi} \\
\Delta \boldsymbol{\phi}^{\prime}
\end{array}\right\} d S \\
& -\sum_{a=1}^{2}\left\{\delta \phi\left(S_{a}\right)^{\top} \delta \phi\left(S_{a}\right)^{\prime}{ }^{\top}\right\}\left[\begin{array}{cc}
\mathbf{f}_{a}^{S} & \mathbf{0} \\
\mathbf{0} & \mathbf{0}
\end{array}\right]\left\{\begin{array}{c}
\Delta \phi\left(S_{a}\right) \\
\Delta \phi\left(S_{a}\right)^{\prime}
\end{array}\right\} \\
& =\Delta \lambda\left(\int_{\Gamma_{0}} \delta \boldsymbol{\phi}^{\top} \boldsymbol{q} d S+\sum_{a=1}^{2} \delta \phi\left(S_{a}\right)^{\top} \boldsymbol{f}_{a}\right)
\end{aligned}
$$

\footnotetext{
${ }^{2}$ En esta expresión, por claridad, hemos sustituido $\delta \phi\left(S_{a}\right)$ por $\delta \phi$ en los dos primeros sumatorios.
} 
y es válida para cualquier variación $\delta \phi$ de la configuración. Esta ecuación es la que, tras una adecuada discretización de las variables, va a permitir la formulación de soluciones numéricas del problema.

En la próxima sección analizaremos la primera integral y el primer sumatorio, que como hemos visto, aparecen como consecuencia del sumando que contiene la segunda variación de la configuración. Se ha demostrado que el tercer y el cuarto término coinciden con los obtenidos por Simó y Vu-Quoc [62], y constituyen el operador tangente del modelo de Simó. El integrando se denomina núcleo del operador tangente y tiene un término constitutivo, uno geométrico, y uno debido a las fuerzas exteriores:

$$
\left[\begin{array}{cc}
\mathbf{d}_{00}^{S} & \mathbf{d}_{01}^{S} \\
\mathbf{d}_{10}^{S} & \mathbf{d}_{11}^{S}
\end{array}\right]=\left[\begin{array}{ll}
\mathbf{d}_{00}^{G S} & \mathbf{d}_{01}^{G S} \\
\mathbf{d}_{10}^{G S} & \mathbf{d}_{11}^{G S}
\end{array}\right]+\left[\begin{array}{ll}
\mathbf{d}_{00}^{M} & \mathbf{d}_{01}^{M} \\
\mathbf{d}_{10}^{M} & \mathbf{d}_{11}^{M}
\end{array}\right]-\left[\begin{array}{cc}
\mathbf{d}^{Q S} & \mathbf{0} \\
\mathbf{0} & \mathbf{0}
\end{array}\right]
$$

Por otro lado, el operador tangente deducido en este capítulo está constituido por la suma de todos los términos a la izquierda de la igualdad. Si nos fijamos en la primera integral, podemos denotar así su integrando

$$
\left[\begin{array}{ll}
\mathbf{d}_{00}^{A} & \mathbf{d}_{01}^{A} \\
\mathbf{d}_{10}^{A} & \mathbf{d}_{11}^{A}
\end{array}\right]=\left[\begin{array}{ll}
\mathbf{d}_{00}^{G A} & \mathbf{d}_{01}^{G A} \\
\mathbf{d}_{10}^{G A} & \mathbf{d}_{11}^{G A}
\end{array}\right]-\left[\begin{array}{cc}
\mathbf{d}^{Q A} & \mathbf{0} \\
\mathbf{0} & \mathbf{0}
\end{array}\right]
$$

Por tanto, el núcleo del operador tangente de la tesis (en su forma espacial) resulta

$$
\begin{aligned}
{\left[\begin{array}{ll}
\mathbf{d}_{00} & \mathbf{d}_{01} \\
\mathbf{d}_{10} & \mathbf{d}_{11}
\end{array}\right] } & =\left[\begin{array}{ll}
\mathbf{d}_{00}^{A} & \mathbf{d}_{01}^{A} \\
\mathbf{d}_{10}^{A} & \mathbf{d}_{11}^{A}
\end{array}\right]+\left[\begin{array}{ll}
\mathbf{d}_{00}^{S} & \mathbf{d}_{01}^{S} \\
\mathbf{d}_{10}^{S} & \mathbf{d}_{11}^{S}
\end{array}\right] \\
& =\left[\begin{array}{ll}
\mathbf{d}_{00}^{G} & \mathbf{d}_{01}^{G} \\
\mathbf{d}_{10}^{G} & \mathbf{d}_{11}^{G}
\end{array}\right]+\left[\begin{array}{ll}
\mathbf{d}_{00}^{M} & \mathbf{d}_{01}^{M} \\
\mathbf{d}_{10}^{M} & \mathbf{d}_{11}^{M}
\end{array}\right]-\left[\begin{array}{cc}
\mathbf{d}^{Q} & \mathbf{0} \\
\mathbf{0} & \mathbf{0}
\end{array}\right],
\end{aligned}
$$

donde $\mathbf{d}^{Q}=\mathbf{d}^{Q A}+\mathbf{d}^{Q S}$.

Con esta notación, la ecuación que define el equilibrio tangente es finalmente

$$
\begin{aligned}
& \int_{\Gamma_{0}}\left\{\delta \boldsymbol{\phi}^{\top} \quad \delta \boldsymbol{\phi}^{\prime \top}\right\}\left[\begin{array}{ll}
\mathbf{d}_{00} & \mathbf{d}_{01} \\
\mathbf{d}_{10} & \mathbf{d}_{11}
\end{array}\right]\left\{\begin{array}{c}
\Delta \boldsymbol{\phi} \\
\Delta \boldsymbol{\phi}^{\prime}
\end{array}\right\} d S \\
& -\sum_{a=1}^{2}\left\{\delta \phi\left(S_{a}\right)^{\top} \delta \phi\left(S_{a}\right)^{\prime \top}\right\}\left[\begin{array}{cc}
\mathbf{f}_{a} & \mathbf{0} \\
\mathbf{0} & \mathbf{0}
\end{array}\right]\left\{\begin{array}{c}
\Delta \phi\left(S_{a}\right) \\
\Delta \phi\left(S_{a}\right)^{\prime}
\end{array}\right\} \\
& =\Delta \lambda\left(\int_{\Gamma_{0}} \delta \boldsymbol{\phi}^{\top} \boldsymbol{q} d S+\sum_{a=1}^{2} \delta \phi\left(S_{a}\right)^{\top} \boldsymbol{f}_{a}\right),
\end{aligned}
$$

con $\mathbf{f}_{a}=\mathbf{f}_{a}^{A}+\mathbf{f}_{a}^{S}$. El término a la izquierda de la igualdad es el operador tangente (en la forma espacial), compuesto por el término geométrico, el constitutivo y el de rigidez de las fuerzas generalizadas y las fuerzas en los extremos. Además, el término entre paréntesis es, precisamente, el trabajo virtual de las fuerzas exteriores.

\subsubsection{Análisis de los términos adicionales al operador de Simó}

En esta sección demostramos que los primeros términos de la ecuación de equilibrio tangente, que diferencian nuestro operador del obtenido por Simó, se anulan sobre la trayectoria de equilibrio. 
Proposición 6.2.2. Los términos de la ecuación de equilibrio tangente que derivan de la segunda variación de las variables de la configuración,

$$
\begin{aligned}
& \int_{\Gamma_{0}}\left\{\delta \boldsymbol{\phi}^{\top}\right.\left.\delta \boldsymbol{\phi}^{\prime \top}\right\}\left(\left[\begin{array}{cc}
\mathbf{d}_{00}^{G A} & \mathbf{d}_{01}^{G A} \\
\mathbf{d}_{10}^{G A} & \mathbf{d}_{11}^{G A}
\end{array}\right]-\left[\begin{array}{cc}
\mathbf{d}^{Q A} & \mathbf{0} \\
\mathbf{0} & \mathbf{0}
\end{array}\right]\right)\left\{\begin{array}{c}
\Delta \boldsymbol{\phi} \\
\Delta \boldsymbol{\phi}^{\prime}
\end{array}\right\} d S \\
&-\sum_{a=1}^{2}\left\{\delta \boldsymbol{\phi}\left(S_{a}\right)^{\top} \delta \boldsymbol{\phi}\left(S_{a}\right)^{\prime \top}\right\}\left[\begin{array}{cc}
\mathbf{f}_{a}^{A} & \mathbf{0} \\
\mathbf{0} & \mathbf{0}
\end{array}\right]\left\{\begin{array}{c}
\Delta \boldsymbol{\phi}\left(S_{a}\right) \\
\Delta \boldsymbol{\phi}\left(S_{a}\right)^{\prime}
\end{array}\right\},
\end{aligned}
$$

se anulan sobre la trayectoria de equilibrio.

Demostración. Consideremos en primer lugar el término geométrico antisimétrico y su desarrollo:

$$
\begin{aligned}
& \int_{\Gamma_{0}}\left\{\delta \phi^{\top}\right.\left.\delta \phi^{\prime \top}\right\}\left[\begin{array}{ll}
\mathbf{d}_{00}^{G A} & \mathbf{d}_{01}^{G A} \\
\mathbf{d}_{10}^{G A} & \mathbf{d}_{11}^{G A}
\end{array}\right]\left\{\begin{array}{c}
\Delta \phi \\
\Delta \boldsymbol{\phi}^{\prime}
\end{array}\right\} d S \\
&=\int_{\Gamma_{0}}\left\{\delta \boldsymbol{x}^{\top} \delta \boldsymbol{\omega}^{\top} \delta \boldsymbol{x}^{\prime \top} \delta \boldsymbol{\omega}^{\prime \top}\right\}\left[\begin{array}{cccc}
\mathbf{0} & \mathbf{0} & \mathbf{0} & \mathbf{0} \\
\mathbf{0} & \widehat{\mathbf{n}} \widehat{\boldsymbol{\gamma}}-\widehat{\boldsymbol{\gamma}} \widehat{\mathbf{n}} & \mathbf{0} & \widehat{\mathbf{m}} \\
\mathbf{0} & \mathbf{0} & \mathbf{0} & \mathbf{0} \\
\mathbf{0} & \widehat{\mathbf{m}} & \mathbf{0} & \mathbf{0}
\end{array}\right]\left\{\begin{array}{c}
\Delta \boldsymbol{x} \\
\Delta \boldsymbol{\omega} \\
\Delta \boldsymbol{x}^{\prime} \\
\Delta \boldsymbol{\omega}^{\prime}
\end{array}\right\} d S \\
&=\int_{\Gamma_{0}}\left(\delta \boldsymbol{\omega}^{\top}(\widehat{\mathbf{n}} \widehat{\boldsymbol{\gamma}}-\widehat{\boldsymbol{\gamma}} \widehat{\mathbf{n}}) \Delta \boldsymbol{\omega}+\delta \boldsymbol{\omega}^{\top} \widehat{\mathbf{m}} \Delta \boldsymbol{\omega}^{\prime}+\delta \boldsymbol{\omega}^{\top} \widehat{\mathbf{m}} \Delta \boldsymbol{\omega}\right) d S .
\end{aligned}
$$

Deshaciendo el proceso seguido en la sección anterior y operando, el integrando es

$$
\begin{aligned}
& -(\Delta \widehat{\boldsymbol{\omega}} \delta \boldsymbol{\omega}) \cdot \widehat{\gamma} \boldsymbol{n}+\left(\Delta \widehat{\boldsymbol{\omega}}^{\prime} \delta \boldsymbol{\omega}\right) \cdot \boldsymbol{m}+\left(\Delta \widehat{\boldsymbol{\omega}} \delta \boldsymbol{\omega}^{\prime}\right) \cdot \boldsymbol{m} \\
& =-(\Delta \boldsymbol{\omega} \times \delta \boldsymbol{\omega}) \cdot(\gamma \times \boldsymbol{n})+\left(\Delta \boldsymbol{\omega}^{\prime} \times \delta \boldsymbol{\omega}\right) \cdot \boldsymbol{m}+\left(\Delta \boldsymbol{\omega} \times \delta \boldsymbol{\omega}^{\prime}\right) \cdot \boldsymbol{m} \\
& =-(\Delta \boldsymbol{\omega} \times \delta \boldsymbol{\omega}) \cdot(\gamma \times \boldsymbol{m})+(\Delta \boldsymbol{\omega} \times \delta \boldsymbol{\omega})^{\prime} \cdot \boldsymbol{m} \\
& =-(\Delta \boldsymbol{\omega} \times \delta \boldsymbol{\omega}) \cdot\left(\boldsymbol{m}^{\prime}+\gamma \times \boldsymbol{m}\right)+\frac{d}{d S}[(\Delta \boldsymbol{\omega} \times \delta \boldsymbol{\omega}) \cdot \boldsymbol{m}] .
\end{aligned}
$$

Entonces el término geométrico queda así

$$
-\int_{\Gamma_{0}}(\Delta \boldsymbol{\omega} \times \delta \boldsymbol{\omega}) \cdot\left(\boldsymbol{m}^{\prime}+\gamma \times \boldsymbol{m}\right) d S+[(\Delta \boldsymbol{\omega} \times \delta \boldsymbol{\omega}) \cdot \boldsymbol{m}]_{S=S_{1}}^{S=S_{2}} .
$$

El término debido a las fuerzas generalizadas es

$$
\begin{aligned}
-\int_{\Gamma_{0}} \delta \phi^{\top} \mathbf{d}^{Q A} \Delta \boldsymbol{\phi} d S & =-\int_{\Gamma_{0}} \delta \boldsymbol{\omega}^{\top} \lambda \widehat{\mathbf{q}}_{m} \Delta \boldsymbol{\omega} d S \\
& =\lambda \int_{\Gamma_{0}} \delta \boldsymbol{\omega}^{\top} \Delta \widehat{\boldsymbol{\omega}} \boldsymbol{q}_{m} d S \\
& =-\int_{\Gamma_{0}}(\Delta \boldsymbol{\omega} \times \delta \boldsymbol{\omega}) \cdot \lambda \boldsymbol{q}_{m} d S
\end{aligned}
$$


Por último queda analizar los términos de contorno:

$$
-\sum_{a=1}^{2} \delta \boldsymbol{\omega}\left(S_{a}\right)^{\top} \lambda \widehat{\mathbf{m}}_{a} \Delta \boldsymbol{\omega}\left(S_{a}\right)=-\sum_{a=1}^{2}\left(\Delta \boldsymbol{\omega}\left(S_{a}\right) \times \delta \boldsymbol{\omega}\left(S_{a}\right)\right) \cdot \lambda \widehat{\mathbf{m}}_{a} .
$$

Reuniendo todos estos resultados obtenemos la siguiente expresión

$$
\begin{aligned}
-\int_{\Gamma_{0}}(\Delta \boldsymbol{\omega} \times \delta \boldsymbol{\omega}) \cdot\left(\boldsymbol{m}^{\prime}+\boldsymbol{\gamma} \times \boldsymbol{m}+\lambda \boldsymbol{q}_{m}\right) d S & \\
+\left(\boldsymbol{m}\left(S_{2}\right)-\lambda \boldsymbol{m}_{2}\right) & \cdot\left(\Delta \boldsymbol{\omega}\left(S_{2}\right) \times \delta \boldsymbol{\omega}\left(S_{2}\right)\right) \\
& -\left(\boldsymbol{m}\left(S_{1}\right)+\lambda \boldsymbol{m}_{1}\right) \cdot\left(\Delta \boldsymbol{\omega}\left(S_{1}\right) \times \delta \boldsymbol{\omega}\left(S_{1}\right)\right) .
\end{aligned}
$$

El integrando del primer sumando coincide con la ecuación de equilibrio de momentos a lo largo de la directriz (4.52b). El segundo y el tercer sumandos coinciden precisamente con las ecuaciones de equilibrio de momentos en la sección final (4.54b), e inicial (4.53b). Por lo tanto, sobre la trayectoria de equilibrio los términos correspondientes a la segunda variación de la configuración se anulan.

El resultado de esta proposición tendrá consecuencias en la solución numérica de las ecuaciones, que analizaremos en el próximo capítulo.

Comentario 6.2.3. Sobre las condiciones para la simetría del operador tangente de Simó. Volviendo al núcleo del término geométrico del operador de Simó [62], su fracción antisimétrica es igual a la mitad del núcleo antisimétrico (cambiada de signo) analizado en la proposición precedente:

$$
\begin{aligned}
{\left[\begin{array}{cc}
\mathbf{d}_{00}^{G S} & \mathbf{d}_{01}^{G S} \\
\mathbf{d}_{10}^{G S} & \mathbf{d}_{11}^{G S}
\end{array}\right]^{A} } & =\frac{1}{2}\left(\left[\begin{array}{cc}
\mathbf{d}_{00}^{G S} & \mathbf{d}_{01}^{G S} \\
\mathbf{d}_{10}^{G S} & \mathbf{d}_{11}^{G S}
\end{array}\right]-\left[\begin{array}{cc}
\mathbf{d}_{00}^{G S} & \mathbf{d}_{01}^{G S} \\
\mathbf{d}_{10}^{G S} & \mathbf{d}_{11}^{G S}
\end{array}\right]^{\top}\right) \\
& =\frac{1}{2}\left(\left[\begin{array}{cc}
\mathbf{d}_{00}^{G S} & \mathbf{d}_{01}^{G S} \\
\mathbf{d}_{10}^{G S} & \mathbf{d}_{11}^{G S}
\end{array}\right]-\left[\begin{array}{cc}
\mathbf{d}_{00}^{G} & \mathbf{d}_{01}^{G} \\
\mathbf{d}_{10}^{G} & \mathbf{d}_{11}^{G}
\end{array}\right]\right) \\
& =-\frac{1}{2}\left[\begin{array}{cccc}
\mathbf{0} & \mathbf{0} & \mathbf{0} & \mathbf{0} \\
\mathbf{0} & \widehat{\mathbf{n}} \widehat{\gamma}-\widehat{\gamma} \widehat{\mathbf{n}} & \mathbf{0} & \widehat{\mathbf{m}} \\
\mathbf{0} & \mathbf{0} & \mathbf{0} & \mathbf{0} \\
\mathbf{0} & \widehat{\mathbf{m}} & \mathbf{0} & \mathbf{0}
\end{array}\right]
\end{aligned}
$$

Partiendo de este resultado Simó opera e integra por partes (en la proposición 6.2.2 hemos seguido el mismo procedimiento) obteniendo la siguiente expresión en la que ha sumado y restado el término con $\lambda \boldsymbol{q}_{m}$ :

$$
\begin{aligned}
& \int_{\Gamma_{0}}\left\{\delta \phi^{\top} \delta \phi^{\prime \top}\right\} {\left[\begin{array}{cc}
\mathbf{d}_{00}^{G S} & \mathbf{d}_{01}^{G S} \\
\mathbf{d}_{10}^{G S} & \mathbf{d}_{11}^{G S}
\end{array}\right]^{A}\left\{\begin{array}{c}
\Delta \boldsymbol{\phi} \\
\Delta \boldsymbol{\phi}^{\prime}
\end{array}\right\} d S } \\
&=\frac{1}{2}\left[\int_{\Gamma_{0}}(\Delta \boldsymbol{\omega} \times \delta \boldsymbol{\omega}) \cdot\left(\boldsymbol{m}^{\prime}+\boldsymbol{\gamma} \times \boldsymbol{m}+\lambda \boldsymbol{q}_{m}\right) d S\right. \\
&\left.\quad-\int_{\Gamma_{0}}(\Delta \boldsymbol{\omega} \times \delta \boldsymbol{\omega}) \cdot \lambda \boldsymbol{q}_{m} d S-[(\Delta \boldsymbol{\omega} \times \delta \boldsymbol{\omega}) \cdot \boldsymbol{m}]_{S=S_{1}}^{S=S_{2}}\right] .
\end{aligned}
$$


En el integrando del primer sumando aparece la ecuación de equilibrio de momentos, que se anula sobre la trayectoria de equilibrio. Sobre el segundo término, Simó argumenta que se anula si $\boldsymbol{q}_{m}=\mathbf{0}$, y que esta condición "parece estar en concordancia con el hecho señalado por Ziegler y elaborado por Argyris de que los momentos de eje fijo no son conservativos". Acerca del tercer término indica que se anula para muchos tipos de condición de contorno, pero que "no se anula en el caso de aplicar un momento de eje fijo, pero este tipo de momento no es conservativo", por lo que concluye que (1) "en una configuración de equilibrio la simetría de la rigidez tangente (...) depende del carácter conservativo de las cargas", y (2) "en una configuración no equilibrada el operador tangente es, en general, no simétrico".

Es necesario matizar la primera conclusión de Simó y Vu-Quoc. En primer lugar, es posible imaginar un momento conservativo (que naturalmente no sería de eje fijo) ${ }^{3}$ actuando sobre el extremo libre de una pieza, o también (aunque el origen físico es más difícil de justificar) un momento distribuido conservativo; cualquiera de estas dos acciones supondría la no nulidad del segundo o tercer sumando de la parte antisimétrica del operador geométrico, y por tanto éste no sería simétrico. Por otro lado, para verificar la simetría del operador no sólo hay que analizar el término geométrico, también es necesario tener en cuenta los términos de rigidez de las cargas (tanto las repartidas como las aplicadas en los extremos) que se suman al anterior. En efecto, la parte antisimétrica del núcleo debido a las fuerzas generalizadas es:

$$
\begin{aligned}
{\left[\begin{array}{cc}
\mathbf{d}^{Q S} & \mathbf{0} \\
\mathbf{0} & \mathbf{0}
\end{array}\right]^{A}=} & \frac{1}{2} \lambda\left(\left[\begin{array}{cccc}
\mathbf{0} & -\widehat{\mathbf{q}}_{n}^{f} & \mathbf{0} & \mathbf{0} \\
\mathbf{0} & -\widehat{\mathbf{q}}_{m}^{f} & \mathbf{0} & \mathbf{0} \\
\mathbf{0} & \mathbf{0} & \mathbf{0} & \mathbf{0} \\
\mathbf{0} & \mathbf{0} & \mathbf{0} & \mathbf{0}
\end{array}\right]-\left[\begin{array}{cccc}
\mathbf{0} & \mathbf{0} & \mathbf{0} & \mathbf{0} \\
\widehat{\mathbf{q}}_{n}^{f} & \widehat{\mathbf{q}}_{m}^{f} & \mathbf{0} & \mathbf{0} \\
\mathbf{0} & \mathbf{0} & \mathbf{0} & \mathbf{0} \\
\mathbf{0} & \mathbf{0} & \mathbf{0} & \mathbf{0}
\end{array}\right]\right) \\
& =\frac{1}{2} \lambda\left[\begin{array}{cccc}
\mathbf{0} & -\widehat{\mathbf{q}}_{n}^{f} & \mathbf{0} & \mathbf{0} \\
-\widehat{\mathbf{q}}_{n}^{f} & -2 \widehat{\mathbf{q}}_{m}^{f} & \mathbf{0} & \mathbf{0} \\
\mathbf{0} & \mathbf{0} & \mathbf{0} & \mathbf{0} \\
\mathbf{0} & \mathbf{0} & \mathbf{0} & \mathbf{0}
\end{array}\right],
\end{aligned}
$$

entonces

$$
\begin{aligned}
& -\int_{\Gamma_{0}}\left\{\delta \boldsymbol{\phi}^{\top} \delta \boldsymbol{\phi}^{\prime \top}\right\}\left[\begin{array}{cc}
\mathbf{d}^{Q S} & \mathbf{0} \\
\mathbf{0} & \mathbf{0}
\end{array}\right]^{A}\left\{\begin{array}{c}
\Delta \boldsymbol{\phi} \\
\Delta \boldsymbol{\phi}^{\prime}
\end{array}\right\} d S \\
& =\frac{1}{2} \int_{\Gamma_{0}}\left((\Delta \boldsymbol{x} \times \delta \boldsymbol{\omega}) \cdot \lambda \boldsymbol{q}_{n}^{f}+(\Delta \boldsymbol{\omega} \times \delta \boldsymbol{x}) \cdot \lambda \boldsymbol{q}_{n}^{f}+(\Delta \boldsymbol{\omega} \times \delta \boldsymbol{\omega}) \cdot 2 \lambda \boldsymbol{q}_{m}^{f}\right) d S .
\end{aligned}
$$

La parte antisimétrica del núcleo del operador de las fuerzas en los extremos tiene la

\footnotetext{
${ }^{3}$ Por ejemplo, el denominado momento rotacional por Ritto-Corrêa y Camotim [53].
} 
misma estructura

$$
\begin{aligned}
{\left[\begin{array}{cc}
\mathbf{f}_{a}^{S} & \mathbf{0} \\
\mathbf{0} & \mathbf{0}
\end{array}\right]^{A}=} & \frac{1}{2} \lambda\left(\left[\begin{array}{cccc}
\mathbf{0} & -\widehat{\mathbf{n}}_{a}^{f} & \mathbf{0} & \mathbf{0} \\
\mathbf{0} & -\widehat{\mathbf{m}}_{a}^{f} & \mathbf{0} & \mathbf{0} \\
\mathbf{0} & \mathbf{0} & \mathbf{0} & \mathbf{0} \\
\mathbf{0} & \mathbf{0} & \mathbf{0} & \mathbf{0}
\end{array}\right]-\left[\begin{array}{cccc}
\mathbf{0} & \mathbf{0} & \mathbf{0} & \mathbf{0} \\
\widehat{\mathbf{n}}_{a}^{f} & \widehat{\mathbf{m}}_{a}^{f} & \mathbf{0} & \mathbf{0} \\
\mathbf{0} & \mathbf{0} & \mathbf{0} & \mathbf{0} \\
\mathbf{0} & \mathbf{0} & \mathbf{0} & \mathbf{0}
\end{array}\right]\right) \\
& =\frac{1}{2} \lambda\left[\begin{array}{cccc}
\mathbf{0} & -\widehat{\mathbf{n}}_{a}^{f} & \mathbf{0} & \mathbf{0} \\
-\widehat{\mathbf{n}}_{a}^{f} & -2 \widehat{\mathbf{m}}_{a}^{f} & \mathbf{0} & \mathbf{0} \\
\mathbf{0} & \mathbf{0} & \mathbf{0} & \mathbf{0} \\
\mathbf{0} & \mathbf{0} & \mathbf{0} & \mathbf{0}
\end{array}\right]
\end{aligned}
$$

luego

$$
\begin{aligned}
-\sum_{a=1}^{2}\left\{\delta \phi\left(S_{a}\right)^{\top} \delta \phi\left(S_{a}\right)^{\prime \top}\right\}\left[\begin{array}{rr}
\mathbf{f}_{a}^{S} & \mathbf{0} \\
\mathbf{0} & \mathbf{0}
\end{array}\right]\left\{\begin{array}{l}
\Delta \boldsymbol{\phi}\left(S_{a}\right) \\
\Delta \boldsymbol{\phi}\left(S_{a}\right)^{\prime}
\end{array}\right\} & \\
=\sum_{a=1}^{2} \frac{1}{2}\left((\Delta \boldsymbol{x} \times \delta \boldsymbol{\omega})\left(S_{a}\right) \cdot \lambda \boldsymbol{n}_{a}^{f}+\right. & (\Delta \boldsymbol{\omega} \times \delta \boldsymbol{x})\left(S_{a}\right) \cdot \lambda \boldsymbol{n}_{a}^{f} \\
& \left.+(\Delta \boldsymbol{\omega} \times \delta \boldsymbol{\omega})\left(S_{a}\right) \cdot 2 \lambda \boldsymbol{m}_{a}^{f}\right) .
\end{aligned}
$$

Recordemos que estos últimos términos han sido obtenidas admitiendo que las cargas exteriores se descomponen en fuerzas y momentos de eje fijo, más fuerzas y momentos perseguidores. Por lo tanto, serán nulos si únicamente hay cargas de eje fijo. En cualquier caso, la parte antisimétrica del operador de Simó y Vu-Quoc se obtendrá como suma de (6.51), (6.52) y (6.53). Si hay cargas perseguidoras (que no son conservativas), la expresión final de la parte antisimétrica del operador ya no se anula. Queda abierta la cuestión de si un momento rotacional (conservativo) [53] produce, o no, la anulación de estos términos.

\subsection{Forma material del equilibrio tangente}

\subsubsection{Linealización del trabajo virtual de las fuerzas internas}

La expresión material del trabajo virtual de las fuerzas internas es

$$
\delta W_{i n t}=\int_{\Gamma_{0}}(\boldsymbol{N} \cdot \delta \boldsymbol{\Gamma}+\boldsymbol{M} \cdot \delta \boldsymbol{K}) d S=\int_{\Gamma_{0}} \delta \boldsymbol{E} \cdot \boldsymbol{F} d S .
$$

con las nuevas variables

$$
\begin{aligned}
\delta \boldsymbol{E} & =\left\{\begin{array}{ll}
\delta \boldsymbol{\Gamma} & \delta \boldsymbol{K}
\end{array}\right\}^{\top} \\
\boldsymbol{F} & =\left\{\begin{array}{ll}
\boldsymbol{N} & \boldsymbol{M}
\end{array}\right\}^{\top}
\end{aligned}
$$

que, al igual que en su forma espacial, condensan en símbolos únicos las componentes traslacional y rotacional de la deformación y las componentes de fuerza y de momento 
de los esfuerzos, respectivamente. El operador tangente se obtiene linealizando el trabajo virtual de las fuerzas internas

$$
\Delta \int_{\Gamma_{0}} \delta \boldsymbol{E} \cdot \boldsymbol{F} d S=\int_{\Gamma_{0}} \Delta \delta \boldsymbol{E} \cdot \boldsymbol{F} d S+\int_{\Gamma_{0}} \delta \boldsymbol{E} \cdot \Delta \boldsymbol{F} d S
$$

La linealización de las deformaciones generalizadas da lugar a la parte geométrica de la matriz de rigidez. Teniendo en cuenta la expresión (4.43a) y las definiciones (6.55)

$$
\begin{aligned}
\Delta \delta \boldsymbol{\Gamma} & =\Delta\left(\delta \boldsymbol{\chi}^{\prime}+\boldsymbol{\Gamma} \times \delta \boldsymbol{\Omega}+\boldsymbol{K} \times \delta \boldsymbol{\chi}\right) \\
& =\Delta \delta \boldsymbol{\chi}^{\prime}+\Delta \boldsymbol{\Gamma} \times \delta \boldsymbol{\Omega}+\boldsymbol{\Gamma} \times \Delta \delta \boldsymbol{\Omega} \\
& +\Delta \boldsymbol{K} \times \delta \boldsymbol{\chi}+\boldsymbol{K} \times \Delta \delta \boldsymbol{\chi}
\end{aligned}
$$

Introduciendo en esta expresión la variación de las deformaciones generalizadas (4.43a) y (4.43b), y considerando la forma material de las segundas variaciones de la configuración (6.10)

$$
\begin{aligned}
\Delta \delta \boldsymbol{\Gamma} & =-\Delta \boldsymbol{\Omega}^{\prime} \times \delta \boldsymbol{\chi}-\Delta \boldsymbol{\Omega} \times \delta \boldsymbol{\chi}^{\prime} \\
& +\Delta \boldsymbol{\chi}^{\prime} \times \delta \boldsymbol{\Omega}+(\boldsymbol{\Gamma} \times \Delta \boldsymbol{\Omega}) \times \delta \boldsymbol{\Omega}+(\boldsymbol{K} \times \Delta \boldsymbol{\chi}) \times \delta \boldsymbol{\Omega} \\
& +\Delta \boldsymbol{\Omega}^{\prime} \times \delta \boldsymbol{\chi}+(\boldsymbol{K} \times \Delta \boldsymbol{\Omega}) \times \delta \boldsymbol{\chi}-\boldsymbol{K} \times(\Delta \boldsymbol{\Omega} \times \delta \boldsymbol{\chi}) \\
& =-\Delta \boldsymbol{\Omega} \times \delta \boldsymbol{\chi}^{\prime}+\Delta \boldsymbol{\chi}^{\prime} \times \delta \boldsymbol{\Omega} \\
& +(\boldsymbol{\Gamma} \times \Delta \boldsymbol{\Omega}) \times \delta \boldsymbol{\Omega}+(\boldsymbol{K} \times \Delta \boldsymbol{\chi}) \times \delta \boldsymbol{\Omega} \\
& +(\boldsymbol{K} \times \Delta \boldsymbol{\Omega}) \times \delta \boldsymbol{\chi}-\boldsymbol{K} \times(\Delta \boldsymbol{\Omega} \times \delta \boldsymbol{\chi}) .
\end{aligned}
$$

Entonces

$$
\begin{aligned}
\Delta \delta \boldsymbol{\Gamma} \cdot \boldsymbol{N} & =\delta \boldsymbol{\chi}^{\top}{ }^{\top} \Delta \widehat{\boldsymbol{\Omega}} \boldsymbol{N}-\delta \boldsymbol{\Omega}^{\top} \Delta \widehat{\boldsymbol{\chi}}^{\prime} \boldsymbol{N} \\
& -\delta \boldsymbol{\Omega}^{\top}(\widehat{\boldsymbol{\Gamma \times \Delta} \boldsymbol{\Omega}}) \boldsymbol{N}-\delta \boldsymbol{\Omega}^{\top}(\widehat{\boldsymbol{K} \times \Delta} \boldsymbol{\chi}) \boldsymbol{N} \\
& -\delta \boldsymbol{\chi}^{\top}(\widehat{\boldsymbol{K} \times \Delta} \boldsymbol{\Omega}) \boldsymbol{N}+(\Delta \boldsymbol{\Omega} \times \delta \boldsymbol{\chi})^{\top} \widehat{\mathbf{K}} \boldsymbol{N}
\end{aligned}
$$

El tercer término se puede expresar así

$$
\begin{aligned}
-\delta \boldsymbol{\Omega}^{\top}(\widehat{\boldsymbol{\Gamma} \times \Delta \boldsymbol{\Omega}}) \boldsymbol{N} & =-\delta \boldsymbol{\Omega}^{\top}(\widehat{\boldsymbol{\Gamma}} \Delta \widehat{\boldsymbol{\Omega}}-\Delta \widehat{\boldsymbol{\Omega}} \widehat{\boldsymbol{\Gamma}}) \boldsymbol{N} \\
& =\delta \boldsymbol{\Omega}^{\top} \widehat{\boldsymbol{\Gamma}} \widehat{\mathbf{N}} \Delta \boldsymbol{\Omega}+\delta \boldsymbol{\Omega}^{\top} \Delta \widehat{\boldsymbol{\Omega}}(\boldsymbol{\Gamma} \times \boldsymbol{N}) \\
& =\delta \boldsymbol{\Omega}^{\top} \widehat{\boldsymbol{\Gamma}} \widehat{\mathbf{N}} \Delta \boldsymbol{\Omega}-\delta \boldsymbol{\Omega}^{\top}(\widehat{\boldsymbol{\Gamma}} \widehat{\mathbf{N}}-\widehat{\mathbf{N}} \widehat{\boldsymbol{\Gamma}}) \Delta \boldsymbol{\Omega} \\
& =\delta \boldsymbol{\Omega}^{\top} \widehat{\mathbf{N}} \widehat{\boldsymbol{\Gamma}} \Delta \boldsymbol{\Omega},
\end{aligned}
$$

y los dos siguientes admiten desarrollos análogos. Por último,

$$
\begin{aligned}
(\Delta \boldsymbol{\Omega} \times \delta \boldsymbol{\chi})^{\top} \widehat{\mathbf{K}} \boldsymbol{N} & =(\Delta \boldsymbol{\Omega} \times \delta \boldsymbol{\chi}) \cdot(\boldsymbol{K} \times \boldsymbol{N}) \\
& =-\delta \boldsymbol{\chi}^{\top} \Delta \widehat{\boldsymbol{\Omega}}(\boldsymbol{K} \times \boldsymbol{N}) \\
& =\delta \boldsymbol{\chi}^{\top}(\widehat{\boldsymbol{K} \times \boldsymbol{N}}) \Delta \boldsymbol{\Omega} \\
& =\delta \boldsymbol{\chi}^{\top}(\widehat{\mathbf{K}} \widehat{\mathbf{N}}-\widehat{\mathbf{N}} \widehat{\mathbf{K}}) \Delta \boldsymbol{\Omega} .
\end{aligned}
$$


Operando en función de los resultados anteriores y simplificando los términos que se cancelan, resulta finalmente

$$
\begin{aligned}
\Delta \delta \boldsymbol{\Gamma} \cdot \boldsymbol{N} & =\delta \chi^{\prime \top} \widehat{\mathbf{N}} \Delta \boldsymbol{\Omega}-\delta \boldsymbol{\Omega}^{\top} \widehat{\mathbf{N}} \Delta \chi^{\prime} \\
& +\delta \boldsymbol{\Omega}^{\top} \widehat{\mathbf{N}} \widehat{\boldsymbol{\Gamma}} \Delta \boldsymbol{\Omega}+\delta \boldsymbol{\Omega}^{\top} \widehat{\mathbf{N}} \widehat{\mathbf{K}} \Delta \boldsymbol{\chi}+\delta \chi^{\top} \widehat{\mathbf{K}} \widehat{\mathbf{N}} \Delta \boldsymbol{\Omega} .
\end{aligned}
$$

Por otro lado

$$
\begin{aligned}
\Delta \delta \boldsymbol{K} & =\Delta\left(\delta \boldsymbol{\Omega}^{\prime}+\boldsymbol{K} \times \delta \boldsymbol{\Omega}\right) \\
& =\Delta \delta \boldsymbol{\Omega}^{\prime}+\Delta \boldsymbol{K} \times \delta \boldsymbol{\Omega}+\boldsymbol{K} \times \Delta \delta \boldsymbol{\Omega} .
\end{aligned}
$$

Recordando que $\Delta \delta \boldsymbol{\Omega}=\Delta \delta \boldsymbol{\Omega}^{\prime}=\mathbf{0}$, y empleando la expresión (4.43b)

$$
\Delta \delta \boldsymbol{K}=\Delta \boldsymbol{\Omega}^{\prime} \times \delta \boldsymbol{\Omega}+\left(\boldsymbol{K} \times \Delta \boldsymbol{\Omega}^{\prime}\right) \times \delta \boldsymbol{\Omega},
$$

luego

$$
\begin{aligned}
\Delta \delta \boldsymbol{K} \cdot \boldsymbol{M} & =-\delta \boldsymbol{\Omega}^{\top} \Delta \widehat{\boldsymbol{\Omega}}^{\prime} \boldsymbol{M}-\delta \boldsymbol{\Omega}^{\top}(\widehat{\boldsymbol{K} \times \Delta \boldsymbol{\Omega}}) \boldsymbol{M} \\
& =\delta \boldsymbol{\Omega}^{\top} \widehat{\mathbf{M}} \Delta \boldsymbol{\Omega}^{\prime}+\delta \boldsymbol{\Omega}^{\top} \widehat{\mathbf{M}} \widehat{\mathbf{K}} \Delta \boldsymbol{\Omega} .
\end{aligned}
$$

El integrando del primer sumando de $(6.56)$ se calcula a partir de los resultados anteriores:

$$
\begin{aligned}
\Delta \delta \boldsymbol{\Gamma} \cdot \boldsymbol{N}+\Delta \delta \boldsymbol{K} \cdot \boldsymbol{M} & =\delta \boldsymbol{\chi}^{\prime} \widehat{\mathbf{N}} \Delta \boldsymbol{\Omega}-\delta \boldsymbol{\Omega}^{\top} \widehat{\mathbf{N}} \Delta \boldsymbol{\chi}^{\prime} \\
& +\delta \boldsymbol{\Omega}^{\top} \widehat{\mathbf{N}} \widehat{\boldsymbol{\Gamma}} \Delta \boldsymbol{\Omega}+\delta \boldsymbol{\Omega}^{\top} \widehat{\mathbf{N}} \widehat{\mathbf{K}} \Delta \boldsymbol{\chi}+\delta \chi^{\top} \widehat{\mathbf{K}} \widehat{\mathbf{N}} \Delta \boldsymbol{\Omega} \\
& +\delta \boldsymbol{\Omega}^{\top} \widehat{\mathbf{M}} \Delta \boldsymbol{\Omega}^{\prime}+\delta \boldsymbol{\Omega}^{\top} \widehat{\mathbf{M}} \widehat{\mathbf{K}} \Delta \boldsymbol{\Omega} .
\end{aligned}
$$

Organizando matricialmente este resultado se obtiene el término geométrico del operador tangente en su forma material

$$
\int_{\Gamma_{0}}\left\{\delta \chi^{\top} \delta \boldsymbol{\Omega}^{\top} \delta \chi^{\prime \top} \delta \boldsymbol{\Omega}^{\prime \top}\right\}\left[\begin{array}{cccc}
\mathbf{0} & \widehat{\mathbf{K}} \widehat{\mathbf{N}} & \mathbf{0} & \mathbf{0} \\
\widehat{\mathbf{N}} \widehat{\mathbf{K}} & \widehat{\mathbf{N}} \widehat{\boldsymbol{\Gamma}}+\widehat{\mathbf{M}} \widehat{\mathbf{K}} & \widehat{\mathbf{N}} & \widehat{\mathbf{M}} \\
\mathbf{0} & -\widehat{\mathbf{N}} & \mathbf{0} & \mathbf{0} \\
\mathbf{0} & \mathbf{0} & \mathbf{0} & \mathbf{0}
\end{array}\right]\left\{\begin{array}{c}
\Delta \boldsymbol{\chi} \\
\Delta \boldsymbol{\Omega} \\
\Delta \boldsymbol{\chi}^{\prime} \\
\Delta \boldsymbol{\Omega}^{\prime}
\end{array}\right\} d S
$$

La matriz del integrando es la forma material del núcleo de la parte geométrica del operador.

Si empleamos las variables que condensan las componentes traslacionales y rotacionales de los incrementos de la configuración introducidas en la sección 4.2 -ecuación (4.47a)-

$$
\begin{aligned}
& \delta \Phi=\left\{\begin{array}{ll}
\delta \chi & \delta \boldsymbol{\Omega}
\end{array}\right\}^{\top} \\
& \Delta \boldsymbol{\Phi}=\left\{\begin{array}{ll}
\Delta \chi & \Delta \boldsymbol{\Omega}
\end{array}\right\}^{\top},
\end{aligned}
$$

el término geométrico se puede escribir así

$$
\int_{\Gamma_{0}}\left\{\delta \boldsymbol{\Phi}^{\top} \delta \boldsymbol{\Phi}^{\prime \top}\right\}\left[\begin{array}{ll}
\mathbf{D}_{00}^{G} & \mathbf{D}_{01}^{G} \\
\mathbf{D}_{10}^{G} & \mathbf{D}_{11}^{G}
\end{array}\right]\left\{\begin{array}{c}
\Delta \boldsymbol{\Phi} \\
\Delta \boldsymbol{\Phi}^{\prime}
\end{array}\right\} d S
$$


con el siguiente significado para las submatrices

$$
\begin{aligned}
& \mathbf{D}_{00}^{G}=\left[\begin{array}{cc}
\mathbf{0} & \widehat{\mathbf{K}} \widehat{\mathbf{N}} \\
\widehat{\mathbf{N}} \widehat{\mathbf{K}} & \widehat{\mathbf{N}} \widehat{\mathbf{\Gamma}}+\widehat{\mathbf{M}} \widehat{\mathbf{K}}
\end{array}\right] \\
& \mathbf{D}_{01}^{G}=\left[\begin{array}{ll}
\mathbf{0} & \mathbf{0} \\
\widehat{\mathbf{N}} & \widehat{\mathbf{M}}
\end{array}\right] \\
& \mathbf{D}_{10}^{G}=\left[\begin{array}{cc}
\mathbf{0} & -\widehat{\mathbf{N}} \\
\mathbf{0} & \mathbf{0}
\end{array}\right] \\
& \mathbf{D}_{11}^{G}=\left[\begin{array}{ll}
\mathbf{0} & \mathbf{0} \\
\mathbf{0} & \mathbf{0}
\end{array}\right]
\end{aligned}
$$

El segundo término de (6.56) corresponde a la linealización de los esfuerzos, y da lugar a la parte constitutiva del operador tangente. Empleando la ecuación constitutiva incremental (4.75) el integrando se desarrolla de la siguiente forma

$$
\begin{aligned}
& \delta \boldsymbol{\Gamma} \cdot \Delta \boldsymbol{N}+\delta \boldsymbol{K} \cdot \Delta \boldsymbol{M} \\
& =\left\{\delta \boldsymbol{\Gamma}^{\boldsymbol{\top}} \quad \delta \boldsymbol{K}^{\top}\right\}\left[\begin{array}{ll}
\mathbf{C}_{\Gamma \Gamma} & \mathbf{C}_{\Gamma K} \\
\mathbf{C}_{K \Gamma} & \mathbf{C}_{K K}
\end{array}\right]\left\{\begin{array}{c}
\Delta \boldsymbol{\Gamma} \\
\Delta \boldsymbol{K}
\end{array}\right\} \\
& =\delta \boldsymbol{\Gamma}^{\top} \mathbf{C}_{\Gamma \Gamma} \Delta \boldsymbol{\Gamma}+\delta \boldsymbol{K}^{\top} \mathbf{C}_{K \Gamma} \Delta \boldsymbol{\Gamma}+\delta \boldsymbol{\Gamma}^{\top} \mathbf{C}_{\Gamma K} \Delta \boldsymbol{K}+\delta \boldsymbol{K}^{\top} \mathbf{C}_{K K} \Delta \boldsymbol{K} .
\end{aligned}
$$

Desarrollando la variación y el incremento de las deformaciones generalizadas,

$$
\begin{aligned}
& \delta \boldsymbol{\Gamma}^{\top} \mathbf{C}_{\Gamma \Gamma} \Delta \boldsymbol{\Gamma}=\delta \boldsymbol{\chi}^{\prime \top} \mathbf{C}_{\Gamma \Gamma} \Delta \boldsymbol{\chi}^{\prime}+\delta \boldsymbol{\chi}^{\prime \top} \mathbf{C}_{\Gamma \Gamma} \widehat{\boldsymbol{\Gamma}} \Delta \boldsymbol{\Omega}+\delta \boldsymbol{\chi}^{\prime \top} \mathbf{C}_{\Gamma \Gamma} \widehat{\mathbf{K}} \Delta \boldsymbol{\chi} \\
& -\delta \boldsymbol{\Omega}^{\top} \widehat{\boldsymbol{\Gamma}} \mathbf{C}_{\Gamma \Gamma} \Delta \boldsymbol{\chi}^{\prime}-\delta \boldsymbol{\Omega}^{\top} \widehat{\boldsymbol{\Gamma}} \mathbf{C}_{\Gamma \Gamma} \widehat{\boldsymbol{\Gamma}} \Delta \boldsymbol{\Omega}-\delta \boldsymbol{\Omega}^{\top} \widehat{\boldsymbol{\Gamma}} \mathbf{C}_{\Gamma \Gamma} \widehat{\mathbf{K}} \Delta \boldsymbol{\chi} \\
& -\delta \boldsymbol{\chi}^{\top} \widehat{\mathbf{K}} \mathbf{C}_{\Gamma \Gamma} \Delta \boldsymbol{\chi}^{\prime}-\delta \boldsymbol{\chi}^{\top} \widehat{\mathbf{K}} \mathbf{C}_{\Gamma \Gamma} \widehat{\boldsymbol{\Gamma}} \Delta \boldsymbol{\Omega}-\delta \boldsymbol{\chi}^{\top} \widehat{\mathbf{K}} \mathbf{C}_{\Gamma \Gamma} \widehat{\mathbf{K}} \Delta \boldsymbol{\chi}, \\
& \delta \boldsymbol{K}^{\top} \mathbf{C}_{K \Gamma} \Delta \boldsymbol{\Gamma}=\delta \boldsymbol{\Omega}^{\prime \top} \mathbf{C}_{K \Gamma} \Delta \boldsymbol{\chi}^{\prime}+\delta \boldsymbol{\Omega}^{\prime \top} \mathbf{C}_{K \Gamma} \widehat{\boldsymbol{\Gamma}} \Delta \boldsymbol{\Omega}+\delta \boldsymbol{\Omega}^{\prime \top} \mathbf{C}_{K \Gamma} \widehat{\mathbf{K}} \Delta \boldsymbol{\chi} \\
& -\delta \boldsymbol{\Omega}^{\top} \widehat{\mathbf{K}} \mathbf{C}_{K \Gamma} \Delta \boldsymbol{\chi}^{\prime}-\delta \boldsymbol{\Omega}^{\top} \widehat{\mathbf{K}} \mathbf{C}_{K \Gamma} \widehat{\boldsymbol{\Gamma}} \Delta \boldsymbol{\Omega}-\delta \boldsymbol{\Omega}^{\top} \widehat{\mathbf{K}} \mathbf{C}_{K \Gamma} \widehat{\mathbf{K}} \Delta \boldsymbol{\chi}, \\
& \delta \boldsymbol{\Gamma}^{\top} \mathbf{C}_{\Gamma K} \Delta \boldsymbol{K}=\delta \boldsymbol{\chi}^{\prime \top} \mathbf{C}_{\Gamma K} \Delta \boldsymbol{\Omega}^{\prime}+\delta \boldsymbol{\chi}^{\prime \top} \mathbf{C}_{\Gamma K} \widehat{\mathbf{K}} \Delta \boldsymbol{\Omega} \\
& -\delta \boldsymbol{\Omega}^{\top} \widehat{\boldsymbol{\Gamma}} \mathbf{C}_{\Gamma K} \Delta \boldsymbol{\Omega}^{\prime}-\delta \boldsymbol{\Omega}^{\top} \widehat{\boldsymbol{\Gamma}} \mathbf{C}_{\Gamma K} \widehat{\mathbf{K}} \Delta \boldsymbol{\Omega} \\
& -\delta \chi^{\top} \widehat{\mathbf{K}} \mathbf{C}_{\Gamma K} \Delta \boldsymbol{\Omega}^{\prime}-\delta \chi^{\top} \widehat{\mathbf{K}} \mathbf{C}_{\Gamma K} \widehat{\mathbf{K}} \Delta \boldsymbol{\Omega} \\
& \delta \boldsymbol{K}^{\top} \mathbf{C}_{K K} \Delta \boldsymbol{K}=\delta \boldsymbol{\Omega}^{\prime \top} \mathbf{C}_{K K} \Delta \boldsymbol{\Omega}^{\prime}+\delta \boldsymbol{\Omega}^{\prime \top} \mathbf{C}_{K K} \widehat{\mathbf{K}} \Delta \boldsymbol{\Omega} \\
& -\delta \boldsymbol{\Omega}^{\top} \widehat{\mathbf{K}} \mathbf{C}_{K K} \Delta \boldsymbol{\Omega}^{\prime}-\delta \boldsymbol{\Omega}^{\top} \widehat{\mathbf{K}} \mathbf{C}_{K K} \widehat{\mathbf{K}} \Delta \boldsymbol{\Omega} .
\end{aligned}
$$

El término constitutivo del operador tangente en su forma material se obtiene 
agrupando factores

$$
\begin{aligned}
& \int_{\Gamma_{0}}\left\{\delta \boldsymbol{\chi}^{\top} \delta \boldsymbol{\Omega}^{\top} \delta \boldsymbol{\chi}^{\prime \top} \delta \boldsymbol{\Omega}^{\prime \top}\right\} .
\end{aligned}
$$

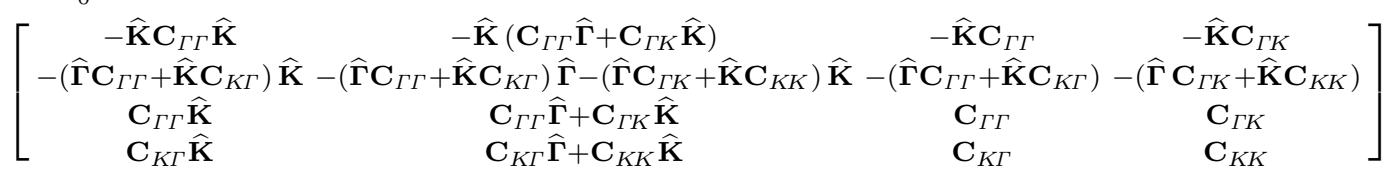

$$
\begin{aligned}
& \left\{\begin{array}{c}
\Delta \chi \\
\Delta \boldsymbol{\Omega} \\
\Delta \chi^{\prime} \\
\Delta \boldsymbol{\Omega}^{\prime}
\end{array}\right\} d S
\end{aligned}
$$

Esta expresión se puede transformar en una mucho más compacta empleando las variables que contienen las componentes translacionales y rotacionales de las variaciones de la configuración

$$
\int_{\Gamma_{0}}\left\{\delta \boldsymbol{\Phi}^{\boldsymbol{\top}} \delta \boldsymbol{\Phi}^{\prime \top}\right\}\left[\begin{array}{ll}
\mathbf{D}_{00}^{M} & \mathbf{D}_{01}^{M} \\
\mathbf{D}_{10}^{M} & \mathbf{D}_{11}^{M}
\end{array}\right]\left\{\begin{array}{c}
\Delta \boldsymbol{\Phi} \\
\Delta \boldsymbol{\Phi}^{\prime}
\end{array}\right\} d S
$$

con

$$
\begin{aligned}
\mathbf{D}_{00}^{M} & =\left[\begin{array}{cc}
-\widehat{\mathbf{K}} & \mathbf{0} \\
-\widehat{\boldsymbol{\Gamma}} & -\widehat{\mathbf{K}}
\end{array}\right]\left[\begin{array}{ll}
\mathbf{C}_{\Gamma \Gamma} & \mathbf{C}_{\Gamma K} \\
\mathbf{C}_{K \Gamma} & \mathbf{C}_{K K}
\end{array}\right]\left[\begin{array}{cc}
\widehat{\mathbf{K}} & \widehat{\boldsymbol{\Gamma}} \\
\mathbf{0} & \widehat{\mathbf{K}}
\end{array}\right] \\
\mathbf{D}_{01}^{M} & =\left[\begin{array}{cc}
-\widehat{\mathbf{K}} & \mathbf{0} \\
-\widehat{\boldsymbol{\Gamma}} & -\widehat{\mathbf{K}}
\end{array}\right]\left[\begin{array}{ll}
\mathbf{C}_{\Gamma \Gamma} & \mathbf{C}_{\Gamma K} \\
\mathbf{C}_{K \Gamma} & \mathbf{C}_{K K}
\end{array}\right] \\
\mathbf{D}_{10}^{M} & =\mathbf{D}_{01}^{M \top} \\
\mathbf{D}_{11}^{M} & =\left[\begin{array}{ll}
\mathbf{C}_{\Gamma \Gamma} & \mathbf{C}_{\Gamma K} \\
\mathbf{C}_{K \Gamma} & \mathbf{C}_{K K}
\end{array}\right] .
\end{aligned}
$$

La simetría de la parte constitutiva del operador se aprecia perfectamente con este tipo de representación.

Aún es posible lograr una expresión alternativa del término constitutivo haciendo uso del operador $\overline{\mathbf{B}}$ definido en (4.46).

$$
\begin{aligned}
& \int_{\Gamma_{0}}\left\{\begin{array}{ll}
\delta \boldsymbol{\chi}^{\top} & \delta \boldsymbol{\Omega}^{\top}
\end{array}\right\}\left[\begin{array}{cc}
\frac{d}{d S} \mathbf{1}-\widehat{\mathbf{K}} & \mathbf{0} \\
-\widehat{\boldsymbol{\Gamma}} & \frac{d}{d S} \mathbf{1}-\widehat{\mathbf{K}}
\end{array}\right]\left[\begin{array}{ll}
\mathbf{C}_{\Gamma \Gamma} & \mathbf{C}_{\Gamma K} \\
\mathbf{C}_{K \Gamma} & \mathbf{C}_{K K}
\end{array}\right] . \\
& \cdot\left[\begin{array}{cc}
\frac{d}{d S} \mathbf{1}+\widehat{\mathbf{K}} & \widehat{\boldsymbol{\Gamma}} \\
\mathbf{0} & \frac{d}{d S} \mathbf{1}+\widehat{\mathbf{K}}
\end{array}\right]\left\{\begin{array}{c}
\Delta \boldsymbol{\chi} \\
\Delta \boldsymbol{\Omega}
\end{array}\right\} d S .
\end{aligned}
$$

$\mathrm{Y}$ en forma compacta

$$
\int_{\Gamma_{0}} \delta \boldsymbol{\Phi}^{\top} \overline{\mathbf{B}}^{\top} \overline{\mathbf{D}}^{M} \overline{\mathbf{B}} \delta \boldsymbol{\Phi} d S
$$

con

$$
\overline{\mathbf{D}}^{M}=\left[\begin{array}{ll}
\mathbf{C}_{\Gamma \Gamma} & \mathbf{C}_{\Gamma K} \\
\mathbf{C}_{K \Gamma} & \mathbf{C}_{K K}
\end{array}\right]=\mathbf{D}_{11}^{M} .
$$


Comentario 6.3.1. El proceso seguido hasta aquí, basado en la linealización directa de los factores del integrando

$$
\delta \boldsymbol{E} \cdot \boldsymbol{F}=\Delta \delta \boldsymbol{E} \cdot \boldsymbol{F}+\delta \boldsymbol{E} \cdot \Delta \boldsymbol{F},
$$

es equivalente al denominado Procedimiento a de la sección anterior

$$
\stackrel{\nabla}{\delta} \boldsymbol{\epsilon} \cdot \boldsymbol{f}=\stackrel{\nabla}{\Delta} \underset{\delta}{\delta} \cdot \boldsymbol{f}+\stackrel{\nabla}{\delta \boldsymbol{\epsilon}} \cdot \stackrel{\nabla}{\Delta} \boldsymbol{f}
$$

ya que cada sumando da como resultado la parte geométrica y la constitutiva, respectivamente. Igual que al operar con la forma espacial, también es posible emplear un procedimiento alternativo basado en expresar el integrando de la expresión del trabajo virtual de las fuerzas internas como producto de un vector de variaciones de la configuración y sus derivadas por otro en el que aparecen los esfuerzos y las deformaciones generalizadas, de este modo:

$$
\boldsymbol{N} \cdot \delta \boldsymbol{\Gamma}+\boldsymbol{M} \cdot \delta \boldsymbol{K}=\left\{\delta \boldsymbol{\chi}^{\top} \delta \boldsymbol{\Omega}^{\top} \delta \chi^{\top} \delta \boldsymbol{\Omega}^{\top}\right\}\left\{\begin{array}{c}
\boldsymbol{N} \times \boldsymbol{K} \\
\boldsymbol{N} \times \boldsymbol{\Gamma}+\boldsymbol{M} \times \boldsymbol{K} \\
\boldsymbol{N} \\
\boldsymbol{M}
\end{array}\right\} .
$$

Linealizando ahora el producto de los dos vectores

$$
\begin{aligned}
\Delta\left\{\delta \chi^{\top} \delta \boldsymbol{\Omega}^{\top} \delta \chi^{\top \top} \delta \boldsymbol{\Omega}^{\prime \top}\right\} & \left\{\begin{array}{c}
\boldsymbol{N} \times \boldsymbol{K} \\
\boldsymbol{N} \times \boldsymbol{\Gamma}+\boldsymbol{M} \times \boldsymbol{K} \\
\boldsymbol{N} \\
\boldsymbol{M}
\end{array}\right\} \\
& +\left\{\delta \chi^{\top} \delta \boldsymbol{\Omega}^{\top} \delta \chi^{\top \top} \delta \boldsymbol{\Omega}^{\prime \top}\right\} \Delta\left\{\begin{array}{c}
\boldsymbol{N} \times \boldsymbol{K} \\
\boldsymbol{N} \times \boldsymbol{M} \times \boldsymbol{K} \times \boldsymbol{K} \\
\boldsymbol{N} \\
\boldsymbol{M}
\end{array}\right\},
\end{aligned}
$$

se obtiene el siguientes resultado: el primer sumando produce el siguiente núcleo

$$
\left[\begin{array}{cccc}
\mathbf{0} & \widehat{\mathbf{K}} \widehat{\mathbf{N}}-\widehat{\mathbf{N}} \widehat{\mathbf{K}} & \mathbf{0} & -\widehat{\mathbf{N}} \\
\mathbf{0} & \mathbf{0} & \mathbf{0} & \mathbf{0} \\
\mathbf{0} & -\widehat{\mathbf{N}} & \mathbf{0} & \mathbf{0} \\
\mathbf{0} & \mathbf{0} & \mathbf{0} & \mathbf{0}
\end{array}\right]
$$

y el segundo da lugar a

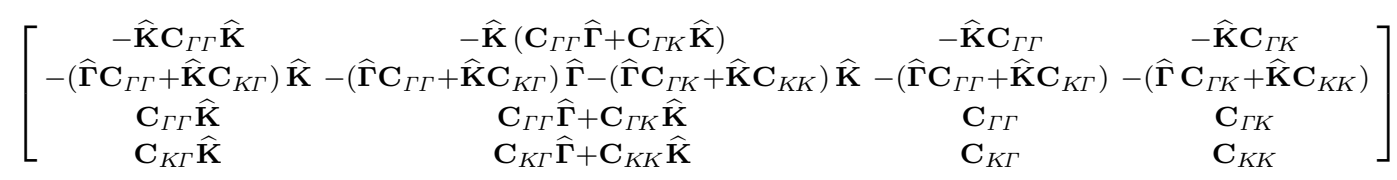

$$
\begin{aligned}
& +\left[\begin{array}{cccc}
0 & \widehat{\mathbf{N}} \widehat{\mathbf{K}} & \mathbf{0} & \widehat{\mathbf{N}} \\
\widehat{\mathbf{N}} \widehat{\mathbf{K}} & \widehat{\mathbf{N}} \widehat{\Gamma}+\widehat{\mathbf{M}} \widehat{\mathbf{K}} & \widehat{\mathbf{N}} & \widehat{\mathbf{M}} \\
\mathbf{0} & \mathbf{0} & \mathbf{0} & \mathbf{0} \\
\mathbf{0} & \mathbf{0} & \mathbf{0} & \mathbf{0}
\end{array}\right]
\end{aligned}
$$


La suma de los términos dependientes de los esfuerzos y las deformaciones generalizadas produce el núcleo geométrico definido en (6.60).

\subsubsection{Linealización del trabajo virtual de las fuerzas exteriores}

En su versión material, el trabajo virtual de las fuerzas exteriores es

$$
\begin{aligned}
\delta W_{\text {ext }}=\lambda & \int_{\Gamma_{0}}\left(\boldsymbol{Q}_{n} \cdot \delta \boldsymbol{\chi}+\boldsymbol{Q}_{m} \cdot \delta \boldsymbol{\Omega}\right) d S \\
& \quad+\lambda\left(\boldsymbol{N}_{1} \cdot \delta \boldsymbol{\chi}\left(S_{1}\right)+\boldsymbol{M}_{1} \cdot \delta \boldsymbol{\Omega}\left(S_{1}\right)\right)+\lambda\left(\boldsymbol{N}_{2} \cdot \delta \boldsymbol{\chi}\left(S_{2}\right)+\boldsymbol{M}_{2} \cdot \delta \boldsymbol{\Omega}\left(S_{2}\right)\right) .
\end{aligned}
$$

Las fuerzas exteriores se dividen también en una parte espacial -superíndice $s$ - y una parte intrínseca (o perseguidora) -superíndice $f-$, de forma que

$$
\begin{gathered}
\boldsymbol{Q}_{n}=\boldsymbol{Q}_{n}^{s}+\boldsymbol{Q}_{n}^{f}=\boldsymbol{\Lambda}^{\top} \boldsymbol{q}_{n}^{s}+\boldsymbol{Q}_{n}^{f} \\
\boldsymbol{Q}_{m}=\boldsymbol{Q}_{m}^{s}+\boldsymbol{Q}_{m}^{f}=\boldsymbol{\Lambda}^{\top} \boldsymbol{q}_{m}^{s}+\boldsymbol{Q}_{m}^{f},
\end{gathered}
$$

donde los vectores $\boldsymbol{q}^{s}$ tienen componentes constantes en el sistema de referencia general, y los vectores $\boldsymbol{Q}^{f}$ son de componentes constantes en el sistema material. La variación de las fuerzas intrínsecas es nula

$$
\Delta \boldsymbol{Q}^{f}=\mathbf{0}
$$

Y operando sobre las fuerzas espaciales

$$
\begin{aligned}
\Delta \boldsymbol{Q}^{s} & =\Delta\left(\boldsymbol{\Lambda}^{\top} \boldsymbol{q}^{s}\right) \\
& =\Delta\left(\boldsymbol{\Lambda}^{\top}\right) \boldsymbol{q}^{s} \\
& =-\boldsymbol{\Lambda}^{\top} \Delta \boldsymbol{\Lambda} \boldsymbol{\Lambda}^{\top} \boldsymbol{q}^{s} \\
& =-\Delta \widehat{\boldsymbol{\Omega}} \boldsymbol{Q}^{s}=\widehat{\mathbf{Q}}^{s} \Delta \boldsymbol{\Omega} .
\end{aligned}
$$

Operando de forma análoga al caso espacial, la variación del trabajo virtual de las fuerzas exteriores está compuesta por la suma de:

1. La variación del trabajo virtual de las fuerzas generalizadas

$$
\begin{aligned}
& \lambda \Delta \int_{\Gamma_{0}}\left(\boldsymbol{Q}_{n} \cdot \delta \boldsymbol{\chi}+\boldsymbol{Q}_{m} \cdot \delta \boldsymbol{\Omega}\right) d S \\
& =\lambda \int_{\Gamma_{0}}\left(\Delta \delta \boldsymbol{\chi} \cdot \boldsymbol{Q}_{n}+\Delta \delta \boldsymbol{\Omega} \cdot \boldsymbol{Q}_{m}\right) d S+\lambda \int_{\Gamma_{0}}\left(\delta \boldsymbol{\chi} \cdot \Delta \boldsymbol{Q}_{n}+\delta \boldsymbol{\Omega} \cdot \Delta \boldsymbol{Q}_{m}\right) d S \\
& =-\lambda \int_{\Gamma_{0}} \delta \boldsymbol{\chi}^{\top} \widehat{\mathbf{Q}}_{n} \Delta \boldsymbol{\Omega} d S+\lambda \int_{\Gamma_{0}}\left(\delta \boldsymbol{\chi}^{\top} \widehat{\mathbf{Q}}_{n}^{s} \Delta \boldsymbol{\Omega}+\delta \boldsymbol{\Omega}^{\top} \widehat{\mathbf{Q}}_{m}^{s} \Delta \boldsymbol{\Omega}\right) d S \\
& =\int_{\Gamma_{0}}\left\{\delta \boldsymbol{\chi}^{\top} \delta \boldsymbol{\Omega}^{\top}\right\} \lambda\left(\left[\begin{array}{cc}
\mathbf{0} & -\widehat{\mathbf{Q}}_{n} \\
\mathbf{0} & \mathbf{0}
\end{array}\right]+\left[\begin{array}{cc}
\mathbf{0} & \widehat{\mathbf{Q}}_{n}^{s} \\
\mathbf{0} & \widehat{\mathbf{Q}}_{m}^{s}
\end{array}\right]\right)\left\{\begin{array}{c}
\Delta \boldsymbol{\chi} \\
\Delta \boldsymbol{\Omega}
\end{array}\right\} d S,
\end{aligned}
$$


que puede abreviarse así empleando las definiciones (6.61)

$$
\int_{\Gamma_{0}} \delta \boldsymbol{\Phi}^{\top} \mathbf{D}^{Q} \Delta \boldsymbol{\Phi} d S
$$

con

$$
\mathbf{D}^{Q}=\lambda\left(\left[\begin{array}{cc}
\mathbf{0} & -\widehat{\mathbf{Q}}_{n} \\
\mathbf{0} & \mathbf{0}
\end{array}\right]+\left[\begin{array}{cc}
\mathbf{0} & \widehat{\mathbf{Q}}_{n}^{s} \\
\mathbf{0} & \widehat{\mathbf{Q}}_{m}^{s}
\end{array}\right]\right)
$$

2. La variación del trabajo virtual de las fuerzas en los extremos, que se obtiene operando del mismo modo

$$
\begin{aligned}
& \lambda \Delta \sum_{a=1}^{2}\left(\boldsymbol{N}_{a} \cdot \delta \chi\left(S_{a}\right)+\boldsymbol{M}_{a} \cdot \delta \boldsymbol{\Omega}\left(S_{a}\right)\right)
\end{aligned}
$$

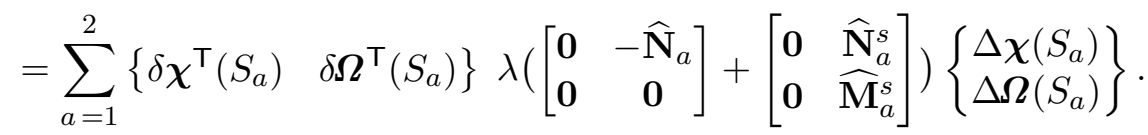

Su expresión abreviada utilizando las definiciones (6.61) es

$$
\sum_{a=1}^{2} \delta \boldsymbol{\Phi}^{\top}\left(S_{a}\right) \mathbf{F}_{a} \Delta \boldsymbol{\Phi}\left(S_{a}\right)
$$

con

$$
\mathbf{F}_{a}=\lambda\left(\left[\begin{array}{cc}
\mathbf{0} & -\widehat{\mathbf{N}}_{a} \\
\mathbf{0} & \mathbf{0}
\end{array}\right]+\left[\begin{array}{cc}
\mathbf{0} & \widehat{\mathbf{N}}_{a}^{s} \\
\mathbf{0} & \widehat{\mathbf{M}}_{a}^{s}
\end{array}\right]\right)
$$

3. Y la variación de $\lambda$. Su expresión vectorial es

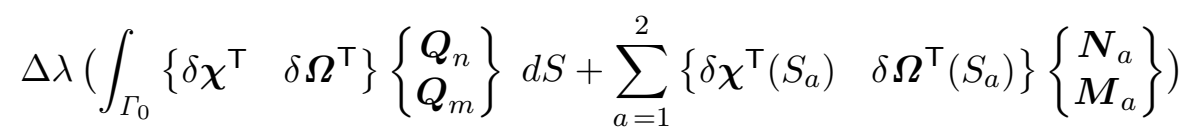

que en forma abreviada queda así

$$
\Delta \lambda\left(\int_{\Gamma_{0}} \delta \boldsymbol{\Phi}^{\top} \boldsymbol{Q} d S+\sum_{a=1}^{2} \delta \boldsymbol{\Phi}\left(S_{a}\right)^{\top} \boldsymbol{F}_{a}\right)
$$

\subsubsection{Ecuación de equilibrio tangente}

En las secciones anteriores se ha obtenido la expresión de la variación del trabajo virtual de las fuerzas internas

$$
\Delta \delta W_{i n t}=\int_{\Gamma_{0}}\left\{\delta \boldsymbol{\Phi}^{\top} \delta \boldsymbol{\Phi}^{\prime} \boldsymbol{\top}\right\}\left(\left[\begin{array}{ll}
\mathbf{D}_{00}^{G} & \mathbf{D}_{01}^{G} \\
\mathbf{D}_{10}^{G} & \mathbf{D}_{11}^{G}
\end{array}\right]+\left[\begin{array}{ll}
\mathbf{D}_{00}^{M} & \mathbf{D}_{01}^{M} \\
\mathbf{D}_{10}^{M} & \mathbf{D}_{11}^{M}
\end{array}\right]\right)\left\{\begin{array}{c}
\Delta \boldsymbol{\Phi} \\
\Delta \boldsymbol{\Phi}^{\prime}
\end{array}\right\} d S
$$


y también de la variación del trabajo virtual de las fuerzas exteriores

$$
\begin{aligned}
\Delta \delta W_{\text {ext }} & =\int_{\Gamma_{0}}\left\{\delta \boldsymbol{\Phi}^{\boldsymbol{\top}} \delta \boldsymbol{\Phi}^{\prime \top}\right\}\left[\begin{array}{cc}
\mathbf{D}^{Q} & \mathbf{0} \\
\mathbf{0} & \mathbf{0}
\end{array}\right]\left\{\begin{array}{c}
\Delta \boldsymbol{\Phi} \\
\Delta \boldsymbol{\Phi}^{\prime}
\end{array}\right\} d S+\sum_{a=1}^{2}\left\{\delta \boldsymbol{\Phi}^{\top} \delta \boldsymbol{\Phi}^{\prime \top}\right\}\left[\begin{array}{cc}
\mathbf{F}_{a} & \mathbf{0} \\
\mathbf{0} & \mathbf{0}
\end{array}\right]\left\{\begin{array}{c}
\Delta \boldsymbol{\Phi} \\
\Delta \boldsymbol{\Phi}^{\prime}
\end{array}\right\} \\
& +\Delta \lambda\left(\int_{\Gamma_{0}} \delta \boldsymbol{\Phi}^{\boldsymbol{\top}} \boldsymbol{Q} d S+\sum_{a=1}^{2} \delta \boldsymbol{\Phi}\left(S_{a}\right)^{\boldsymbol{\top}} \boldsymbol{F}_{a}\right) .
\end{aligned}
$$

La ecuación $\Delta \delta W_{i n t}=\Delta \delta W_{\text {ext }}$ define la trayectoria de equilibrio a medida que varía el parámetro de control $\lambda$. Desarrollándola se obtiene la forma material de la ecuación de equilibrio tangente:

$$
\begin{gathered}
\int_{\Gamma_{0}}\left\{\delta \boldsymbol{\Phi}^{\top} \delta \boldsymbol{\Phi}^{\prime \top}\right\}\left(\left[\begin{array}{ll}
\mathbf{D}_{00}^{G} & \mathbf{D}_{01}^{G} \\
\mathbf{D}_{10}^{G} & \mathbf{D}_{11}^{G}
\end{array}\right]+\left[\begin{array}{ll}
\mathbf{D}_{00}^{M} & \mathbf{D}_{01}^{M} \\
\mathbf{D}_{10}^{M} & \mathbf{D}_{11}^{M}
\end{array}\right]-\left[\begin{array}{cc}
\mathbf{D}^{Q} & \mathbf{0} \\
\mathbf{0} & \mathbf{0}
\end{array}\right]\right)\left\{\begin{array}{c}
\Delta \boldsymbol{\Phi} \\
\Delta \boldsymbol{\Phi}^{\prime}
\end{array}\right\} d S \\
\left.-\sum_{a=1}^{2}\left\{\delta \boldsymbol{\Phi}\left(S_{a}\right)^{\top} \delta \boldsymbol{\Phi}\left(S_{a}\right)^{\prime}\right\}\right\}\left[\begin{array}{cc}
\mathbf{F}_{a} & \mathbf{0} \\
\mathbf{0} & \mathbf{0}
\end{array}\right]\left\{\begin{array}{c}
\Delta \boldsymbol{\Phi}\left(S_{a}\right) \\
\Delta \boldsymbol{\Phi}^{\prime}\left(S_{a}\right)
\end{array}\right\} \\
=\Delta \lambda\left(\int_{\Gamma_{0}} \delta \boldsymbol{\Phi}^{\top} \boldsymbol{Q} d S+\sum_{a=1}^{2} \delta \boldsymbol{\Phi}\left(S_{a}\right)^{\top} \boldsymbol{F}_{a}\right)
\end{gathered}
$$

El término a la izquierda de la igualdad es el operador tangente (en la forma material); está compuesto por el núcleo geométrico, el constitutivo, y el debido a las fuerzas generalizadas, así como por un término consecuencia de las fuerzas y momentos que actúan en los extremos de la pieza.

Comentario 6.3.2. Resulta sencillo comprobar la concordancia entre la forma espacial y material del operador tangente. Para ello basta considerar la relación entre la forma espacial y material de las variaciones de la configuración, y la de sus derivadas

$$
\begin{aligned}
& (\delta \boldsymbol{x})^{\prime}=(\boldsymbol{\Lambda} \delta \boldsymbol{\chi})^{\prime}=\boldsymbol{\Lambda}^{\prime} \delta \boldsymbol{\chi}+\boldsymbol{\Lambda} \delta \boldsymbol{\chi}^{\prime} \\
& (\delta \boldsymbol{\omega})^{\prime}=(\boldsymbol{\Lambda} \delta \boldsymbol{\Omega})^{\prime}=\boldsymbol{\Lambda}^{\prime} \delta \boldsymbol{\Omega}+\boldsymbol{\Lambda} \delta \boldsymbol{\Omega}^{\prime}
\end{aligned}
$$

también válidas en versión incremental. Teniendo en cuanta que $\boldsymbol{\Lambda}^{\top} \boldsymbol{\Lambda}^{\prime}=\widehat{\mathbf{K}}$, la relación entre la forma espacial y material de las variaciones y sus derivadas es

$$
\left\{\begin{array}{l}
\delta \boldsymbol{x} \\
\delta \boldsymbol{\omega} \\
\delta \boldsymbol{x}^{\prime} \\
\delta \boldsymbol{\omega}^{\prime}
\end{array}\right\}=\left[\begin{array}{cccc}
\boldsymbol{\Lambda} & \mathbf{0} & \mathbf{0} & \mathbf{0} \\
\mathbf{0} & \boldsymbol{\Lambda} & \mathbf{0} & \mathbf{0} \\
\boldsymbol{\Lambda} \widehat{\mathbf{K}} & \mathbf{0} & \boldsymbol{\Lambda} & \mathbf{0} \\
\mathbf{0} & \boldsymbol{\Lambda} \widehat{\mathbf{K}} & \mathbf{0} & \boldsymbol{\Lambda}
\end{array}\right]\left\{\begin{array}{l}
\delta \boldsymbol{\chi} \\
\delta \boldsymbol{\Omega} \\
\delta \chi^{\prime} \\
\delta \boldsymbol{\Omega}^{\prime}
\end{array}\right\}
$$

Esta expresión permite cambiar entre la forma espacial y material de los núcleos. El 
núcleo geométrico (6.18), por ejemplo, se transforma así:

$$
\begin{aligned}
& {\left[\begin{array}{cccc}
\boldsymbol{\Lambda}^{\top} & \mathbf{0} & -\widehat{\mathbf{K}} \boldsymbol{\Lambda}^{\top} & \mathbf{0} \\
\mathbf{0} & \boldsymbol{\Lambda}^{\top} & \mathbf{0} & -\widehat{\mathbf{K}} \boldsymbol{\Lambda}^{\top} \\
\mathbf{0} & \mathbf{0} & \boldsymbol{\Lambda}^{\top} & \mathbf{0} \\
\mathbf{0} & \mathbf{0} & \mathbf{0} & \boldsymbol{\Lambda}^{\top}
\end{array}\right]\left[\begin{array}{cccc}
\mathbf{0} & \mathbf{0} & \mathbf{0} & \mathbf{0} \\
\mathbf{0} & \widehat{\mathbf{n}} \widehat{\gamma} & \widehat{\mathbf{n}} & \widehat{\mathbf{m}} \\
\mathbf{0} & -\widehat{\mathbf{n}} & \mathbf{0} & \mathbf{0} \\
\mathbf{0} & \mathbf{0} & \mathbf{0} & \mathbf{0}
\end{array}\right]\left[\begin{array}{cccc}
\boldsymbol{\Lambda} & \mathbf{0} & \mathbf{0} & \mathbf{0} \\
\mathbf{0} & \boldsymbol{\Lambda} & \mathbf{0} & \mathbf{0} \\
\boldsymbol{\Lambda} \widehat{\mathbf{K}} & \mathbf{0} & \boldsymbol{\Lambda} & \mathbf{0} \\
\mathbf{0} & \boldsymbol{\Lambda} \widehat{\mathbf{K}} & \mathbf{0} & \boldsymbol{\Lambda}
\end{array}\right] } \\
&=\left[\begin{array}{cccc}
\mathbf{0} & \widehat{\mathbf{K}} \widehat{\mathbf{N}} & \mathbf{0} & \mathbf{0} \\
\widehat{\mathbf{N}} \widehat{\mathbf{K}} & \widehat{\mathbf{N}} \widehat{\boldsymbol{\Gamma}}+\widehat{\mathbf{M}} \widehat{\mathbf{K}} & \widehat{\mathbf{N}} & \widehat{\mathbf{M}} \\
\mathbf{0} & -\widehat{\mathbf{N}} & \mathbf{0} & \mathbf{0} \\
\mathbf{0} & \mathbf{0} & \mathbf{0} & \mathbf{0}
\end{array}\right]
\end{aligned}
$$

El núcleo constitutivo (6.25) y el de cargas (6.40), se retrotraen a la forma material por el mismo procedimiento. Con ello queda probada la consistencia del procedimiento de linealización propuesto, ya que produce los mismos resultados en la forma material y espacial.

Comentario 6.3.3. Como en el caso espacial, el núcleo geométrico se puede expresar como suma de dos matrices

$$
\begin{aligned}
& {\left[\begin{array}{cccc}
0 & \widehat{\mathbf{K}} \widehat{\mathbf{N}} & \mathbf{0} & 0 \\
\widehat{\mathbf{N}} \widehat{\mathbf{K}} & \widehat{\mathbf{N}} \widehat{\mathbf{\Gamma}}+\widehat{\mathbf{M}} \widehat{\mathbf{K}} & \widehat{\mathbf{N}} & \widehat{\mathbf{M}} \\
\mathbf{0} & -\widehat{\mathbf{N}} & \mathbf{0} & \mathbf{0} \\
\mathbf{0} & \mathbf{0} & \mathbf{0} & \mathbf{0}
\end{array}\right]} \\
& =\left[\begin{array}{lccc}
0 & \widehat{N} \widehat{\Gamma}-\widehat{\Gamma} \widehat{\mathbf{N}}+\widehat{\mathbf{M}} \widehat{\mathbf{K}}-\widehat{\mathbf{K}} \widehat{\mathbf{M}} & \mathbf{0} & \mathbf{0} \\
\mathbf{0} & 0 & 0 & 0 \\
0 & \widehat{M} & 0 & 0
\end{array}\right]+\left[\begin{array}{cccc}
0 & \widehat{\mathbf{K}} \widehat{\mathbf{N}} & 0 & 0 \\
\widehat{\mathbf{N}} \widehat{\mathbf{K}} & \widehat{\Gamma} \widehat{\mathbf{N}}+\widehat{\mathbf{K}} \widehat{\mathbf{M}} & \widehat{\mathbf{N}} & 0 \\
0 & -\widehat{\mathbf{N}} & 0 & 0 \\
0 & -\widehat{\mathbf{M}} & 0 & 0
\end{array}\right] .
\end{aligned}
$$

Con la misma técnica empleada en el comentario anterior se comprueba que cada sumando es la forma material de las matrices de la expresión (espacial) (6.33a). El primer sumando vuelve a ser una matriz antisimétrica y el segundo es la forma material del núcleo geométrico de Simó y Vu-Quoc. Abreviamos esta descomposición de la siguiente forma

$$
\left[\begin{array}{ll}
\mathbf{D}_{00}^{G} & \mathbf{D}_{01}^{G} \\
\mathbf{D}_{10}^{G} & \mathbf{D}_{11}^{G}
\end{array}\right]=\left[\begin{array}{ll}
\mathbf{D}_{00}^{G A} & \mathbf{D}_{01}^{G A} \\
\mathbf{D}_{10}^{G A} & \mathbf{D}_{11}^{G A}
\end{array}\right]+\left[\begin{array}{ll}
\mathbf{D}_{00}^{G S} & \mathbf{D}_{01}^{G S} \\
\mathbf{D}_{10}^{G S} & \mathbf{D}_{11}^{G S}
\end{array}\right]
$$

Por otro lado, si analizamos el núcleo de la rigidez debida a las fuerzas generalizadas $(6.75)$

$$
\mathbf{D}^{Q}=\lambda\left(\left[\begin{array}{cc}
\mathbf{0} & -\widehat{\mathbf{Q}}_{n} \\
\mathbf{0} & \mathbf{0}
\end{array}\right]+\left[\begin{array}{cc}
\mathbf{0} & \widehat{\mathbf{Q}}_{n}^{s} \\
\mathbf{0} & \widehat{\mathbf{Q}}_{m}^{s}
\end{array}\right]\right),
$$

observamos que, teniendo en cuenta que $\widehat{\mathbf{Q}}=\widehat{\mathbf{Q}}^{s}+\widehat{\mathbf{Q}}^{f}$ también puede expresarse como suma de una matriz antisimétrica, más otra que es la versión material de la obtenida por Simó:

$$
\mathbf{D}^{Q}=\lambda\left(\left[\begin{array}{cc}
\mathbf{0} & \mathbf{0} \\
\mathbf{0} & \widehat{\mathbf{Q}}_{m}
\end{array}\right]+\left[\begin{array}{cc}
\mathbf{0} & -\widehat{\mathbf{Q}}_{n}^{f} \\
\mathbf{0} & -\widehat{\mathbf{Q}}_{m}^{f}
\end{array}\right]\right)
$$


es decir

$$
\mathbf{D}^{Q}=\mathbf{D}^{Q A}+\mathbf{D}^{Q S} .
$$

La misma descomposición es aplicable a la rigidez aportada por las fuerzas en los extremos de la pieza

$$
\mathbf{F}_{a}=\lambda\left(\left[\begin{array}{cc}
\mathbf{0} & -\widehat{\mathbf{N}}_{a} \\
\mathbf{0} & \mathbf{0}
\end{array}\right]+\left[\begin{array}{cc}
\mathbf{0} & \widehat{\mathbf{N}}_{a}^{s} \\
\mathbf{0} & \widehat{\mathbf{M}}_{a}^{s}
\end{array}\right]\right)=\lambda\left(\left[\begin{array}{cc}
\mathbf{0} & \mathbf{0} \\
\mathbf{0} & \widehat{\mathbf{M}}_{a}
\end{array}\right]+\left[\begin{array}{cc}
\mathbf{0} & -\widehat{\mathbf{N}}_{a}^{f} \\
\mathbf{0} & -\widehat{\mathbf{M}}_{a}^{f}
\end{array}\right]\right),
$$

o bien, abreviadamente,

$$
\mathbf{F}_{a}^{Q}=\mathbf{F}_{a}^{Q A}+\mathbf{F}_{a}^{Q S}
$$

Con todos estos datos es posible demostrar la contrapartida en forma material de la proposición 6.2.2:

Los términos antisimétricos de la ecuación material de equilibrio tangente (6.79)

$$
\begin{aligned}
& \int_{\Gamma_{0}}\left\{\delta \boldsymbol{\Phi}^{\top} \quad \delta \boldsymbol{\Phi}^{\prime \top}\right\}\left(\left[\begin{array}{ll}
\mathbf{D}_{00}^{G A} & \mathbf{D}_{01}^{G A} \\
\mathbf{D}_{10}^{G A} & \mathbf{D}_{11}^{G A}
\end{array}\right]-\left[\begin{array}{cc}
\mathbf{D}^{Q A} & \mathbf{0} \\
\mathbf{0} & \mathbf{0}
\end{array}\right]\right)\left\{\begin{array}{c}
\Delta \boldsymbol{\Phi} \\
\Delta \boldsymbol{\Phi}^{\prime}
\end{array}\right\} d S \\
& -\sum_{a=1}^{2}\left\{\delta \boldsymbol{\Phi}\left(S_{a}\right)^{\top} \delta \boldsymbol{\Phi}\left(S_{a}\right)^{\prime} \mathrm{\top}\right\}\left[\begin{array}{cc}
\mathbf{F}_{a}^{A} & \mathbf{0} \\
\mathbf{0} & \mathbf{0}
\end{array}\right]\left\{\begin{array}{c}
\Delta \boldsymbol{\Phi}\left(S_{a}\right) \\
\Delta \boldsymbol{\Phi}^{\prime}\left(S_{a}\right)
\end{array}\right\}
\end{aligned}
$$

se anulan sobre la trayectoria de equilibrio.

No desarrollaremos la demostración completa de esta afirmación, pues la técnica es idéntica a la empleada en la proposición mencionada. Basta indicar que el término geométrico antisimétrico se puede desarrollar así:

$$
\begin{aligned}
& \int_{\Gamma_{0}}\left\{\delta \chi^{\top} \delta \boldsymbol{\Omega}^{\top} \delta \chi^{\prime \top} \delta \boldsymbol{\Omega}^{\top \top}\right\}\left[\begin{array}{cccc}
\mathbf{0} & \widehat{\mathbf{N}} \widehat{\boldsymbol{\Gamma}}-\widehat{\boldsymbol{\Gamma}} \widehat{\mathbf{N}}+\widehat{\mathbf{M}} \widehat{\mathbf{K}}-\widehat{\mathbf{K}} \widehat{\mathbf{M}} & \mathbf{0} & \mathbf{0} \\
\mathbf{0} & \mathbf{\mathbf { M }} \\
\mathbf{0} & \widehat{\mathbf{M}} & \mathbf{0} & \mathbf{0} \\
\Delta \boldsymbol{\boldsymbol { N }} & \mathbf{0} & \mathbf{0}
\end{array}\right]\left\{\begin{array}{c}
\Delta \boldsymbol{\chi} \\
\Delta \boldsymbol{\chi}^{\prime} \\
\Delta \boldsymbol{\Omega}^{\prime}
\end{array}\right\} d S \\
& =\int_{\Gamma_{0}}\left(\delta \boldsymbol{\Omega}^{\top}(\widehat{\mathbf{N}} \widehat{\boldsymbol{\Gamma}}-\widehat{\boldsymbol{\Gamma}} \widehat{\mathbf{N}}) \Delta \boldsymbol{\Omega}+\delta \boldsymbol{\Omega}^{\top}(\widehat{\mathbf{M}} \widehat{\mathbf{K}}-\widehat{\mathbf{K}} \widehat{\mathbf{M}}) \Delta \boldsymbol{\Omega}\right. \\
& \left.+\delta \boldsymbol{\Omega}^{\top} \widehat{\mathbf{M}} \Delta \boldsymbol{\Omega}^{\prime}+\delta \boldsymbol{\Omega}^{\prime} \widehat{\top} \widehat{\mathbf{M}} \Delta \boldsymbol{\Omega}\right) d S,
\end{aligned}
$$

y operando resulta

$$
-\int_{\Gamma_{0}}(\Delta \boldsymbol{\Omega} \times \delta \boldsymbol{\Omega}) \cdot\left(\boldsymbol{M}^{\prime}+\boldsymbol{K} \times \boldsymbol{M}+\boldsymbol{\Gamma} \times \boldsymbol{N}\right) d S+[(\Delta \boldsymbol{\Omega} \times \delta \boldsymbol{\Omega}) \cdot \boldsymbol{M}]_{S=S_{1}}^{S=S_{2}} .
$$

El término entre paréntesis en el integrando se puede identificar fácilmente con la forma material de la ecuación de equilibrio de momentos (4.58) (sin el término debido a las fuerzas generalizadas). Para completar la demostración nos remitimos a la proposición 6.2.2. 


\section{Capítulo 7}

\section{Solución numérica basada en la forma espacial de las ecuaciones}

Las expresiones desarrolladas en capítulos anteriores -en concreto, la ecuación de trabajos virtuales, y la expresión del operador tangente- constituyen el punto de partida para la formulación de métodos numéricos de solución de problemas de piezas alargadas ajustados al modelo de Reissner-Simó. La forma en la que se lleve a cabo la discretización del problema conducirá a distintos métodos de solución. En esta tesis hemos optado por formular una solución mediante elementos finitos en desplazamientos, que es la elección natural basada en la ecuación de trabajos virtuales.

A continuación presentamos una solución mediante elementos finitos basada en la forma espacial de las ecuaciones y en la interpolación de rotaciones incrementales (spins). Este camino ha sido seguido por Simó y Vu-Quoc [62], Ibrahimbegović [25], o Kapania y Li [34, entre otros, con distintas variantes relativas al grado de la interpolación o a la posibilidad de considerar geometrías iniciales curvas, por ejemplo. Todos estos autores parten de la forma espacial de las ecuaciones. Es cierto que la forma espacial ofrece, frente a la material, algunas ventajas computacionales, ya que reduce el número de cambios de base necesarios para el ensamblaje; incidiremos sobre esta cuestión en el capítulo siguiente.

El capítulo se estructura de la siguiente forma: en la primera sección se describe la discretización mediante elementos isoparamétricos, basada en la interpolación de desplazamientos y rotaciones incrementales. En segundo lugar se considera la solución de problemas mediante un procedimiento incremental puro; para ello se obtiene la matriz de rigidez tangente a partir de la expresión consistente del operador tangente obtenida en el capítulo anterior. También se llevan a cabo algunas consideraciones sobre la objetividad de la discretización. En la tercera sección se deducen las expresiones necesarias para implementar una solución incremental-iterativa. En la cuarta se resume el flujo del algoritmo de cálculo empleado. Por último se ofrecen los resultados numéricos obtenidos, incluyendo una comparación entre las soluciones proporcionadas por el operador de Simó y las obtenidas con el operador deducido en el capítulo anterior, así como otros resultados correspondientes a diversos ejemplos representativos. 


\subsection{Discretización en elementos finitos}

En esta sección describimos la discretización en elementos finitos rectos, isoparamétricos, con funciones de forma lagrangianas, adecuadas para el modelo propuesto, en el que por la independencia entre las deformaciones traslacionales y las rotacionales únicamente se requiere la continuidad $C^{0}$ de la solución.

\section{Geometría del elemento}

Consideramos elementos rectos de $n$ nodos. El avance sobre la directriz del elemento se describe mediante la coordenada $S \in\left[0, L_{e}\right]$, que tiene dimensiones de longitud. La posición de cada uno de los nodos queda definida por su coordenada $S_{i}$. Para sistematizar la construcción de los elementos se utiliza en los cálculos, como es usual, una coordenada intrínseca $\xi$, de forma que $\xi=-1$ cuando $S=S_{1}$, y $\xi=1$ cuando $S=S_{n}$. A cada nodo $i$ corresponde ahora un valor de $\xi=\xi_{i}$.

La geometría queda descrita por la siguiente ecuación, que representa la transformación que relaciona $S$ y $\xi$

$$
S(\xi)=\left[\begin{array}{lll}
N_{1}(\xi) & \cdots & N_{n}(\xi)
\end{array}\right]\left\{\begin{array}{c}
S_{1} \\
\vdots \\
S_{n}
\end{array}\right\},
$$

donde $N_{i}(\xi)$ son las $n$ funciones de forma basadas en polinomios de Lagrange definidas por la siguiente expresión

$$
N_{i}(\xi)=\prod_{j=1}^{n} \frac{\xi-\xi_{j}}{\xi_{i}-\xi_{j}}, j \neq i
$$

El jacobiano de la transformación (7.1) es

$$
J(\xi)=\frac{d S}{d \xi}=\left[\begin{array}{lll}
N_{1}^{\prime}(\xi) & \cdots & N_{n}^{\prime}(\xi)
\end{array}\right]\left\{\begin{array}{c}
S_{1} \\
\vdots \\
S_{n}
\end{array}\right\}
$$

\section{Interpolación de los incrementos de las variables que describen la} configuración

La interpolación de los incrementos de las variables de la configuración en el elemento se lleva a cabo a partir de los $n$ valores nodales de los mismos, mediante la siguiente fórmula

$$
\left\{\begin{array}{c}
\Delta \boldsymbol{x}(\xi) \\
\Delta \boldsymbol{\omega}(\xi)
\end{array}\right\}=\left[\begin{array}{ccccc}
N_{1}(\xi) \mathbf{1} & \mathbf{0} & \cdots & N_{n}(\xi) \mathbf{1} & \mathbf{0} \\
\mathbf{0} & N_{1}(\xi) \mathbf{1} & \cdots & \mathbf{0} & N_{n}(\xi) \mathbf{1}
\end{array}\right]\left\{\begin{array}{c}
\Delta \boldsymbol{x}_{1} \\
\Delta \boldsymbol{\omega}_{1} \\
\vdots \\
\Delta \boldsymbol{x}_{n} \\
\Delta \boldsymbol{\omega}_{n}
\end{array}\right\}
$$


donde 1 es la identidad de orden 3. Las derivadas de las variables incrementales son

$$
\left\{\begin{array}{l}
d \Delta \boldsymbol{x}(\xi) / d S \\
d \Delta \boldsymbol{\omega}(\xi) / d S
\end{array}\right\}=\frac{1}{J(\xi)}\left[\begin{array}{ccccc}
N_{1}^{\prime}(\xi) \mathbf{1} & \mathbf{0} & \cdots & N_{n}^{\prime}(\xi) \mathbf{1} & \mathbf{0} \\
\mathbf{0} & N_{1}^{\prime}(\xi) \mathbf{1} & \cdots & \mathbf{0} & N_{n}^{\prime}(\xi) \mathbf{1}
\end{array}\right]\left\{\begin{array}{c}
\Delta \boldsymbol{x}_{1} \\
\Delta \boldsymbol{\omega}_{1} \\
\vdots \\
\Delta \boldsymbol{x}_{n} \\
\Delta \boldsymbol{\omega}_{n}
\end{array}\right\}
$$

Agrupando y ordenando estas expresiones resulta

$$
\left\{\begin{array}{c}
\Delta \boldsymbol{x} \\
\Delta \boldsymbol{\omega} \\
d \Delta \boldsymbol{x} / d S \\
d \Delta \boldsymbol{\omega} / d S
\end{array}\right\}=\left[\begin{array}{ccccc}
N_{1} \mathbf{1} & \mathbf{0} & \cdots & N_{n} \mathbf{1} & \mathbf{0} \\
\mathbf{0} & N_{1} \mathbf{1} & \cdots & \mathbf{0} & N_{n} \mathbf{1} \\
\frac{1}{J} N_{1}^{\prime} \mathbf{1} & \mathbf{0} & \cdots & \frac{1}{J} N_{n}^{\prime} \mathbf{1} & \mathbf{0} \\
\mathbf{0} & \frac{1}{J} N_{1}^{\prime} \mathbf{1} & \cdots & \mathbf{0} & \frac{1}{J} N_{n}^{\prime} \mathbf{1}
\end{array}\right]\left\{\begin{array}{c}
\Delta \boldsymbol{x}_{1} \\
\Delta \boldsymbol{\omega}_{1} \\
\vdots \\
\Delta \boldsymbol{x}_{n} \\
\Delta \boldsymbol{\omega}_{n}
\end{array}\right\}
$$

Aún es posible lograr una expresión más compacta empleando la variable $\Delta \phi$ que agrupa incrementos de posición y rotación

$$
\left\{\begin{array}{c}
\Delta \phi \\
\Delta \phi^{\prime}
\end{array}\right\}=\left[\begin{array}{ccc}
N_{1} \mathbf{I} & \cdots & N_{n} \mathbf{I} \\
\frac{1}{J} N_{1}^{\prime} \mathbf{I} & \cdots & \frac{1}{J} N_{n}^{\prime} \mathbf{I}
\end{array}\right]\left\{\begin{array}{c}
\Delta \boldsymbol{\phi}_{1} \\
\cdots \\
\Delta \boldsymbol{\phi}_{n}
\end{array}\right\}
$$

En esta expresión $\mathbf{I}$ es la identidad de orden 6. Emplearemos una versión aun más compacta con la notación que se muestra a continuación

$$
\left\{\begin{array}{c}
\Delta \phi \\
\Delta \phi^{\prime}
\end{array}\right\}=\left[\begin{array}{c}
\mathbb{N} \\
\frac{1}{J} \mathbb{N}^{\prime}
\end{array}\right] \Delta \phi^{e}
$$

en la que $\Delta \phi^{e}$ es el vector que recoge los incrementos nodales de la configuración, y las matrices $\mathbb{N}$ y $\mathbb{N}^{\prime}$ agrupan las funciones de forma y sus derivadas, respectivamente.

\subsection{Solución incremental pura}

El fundamento de la solución incremental pura se encuentra en la ecuación (6.49), expresión del equilibrio tangente que reproducimos por conveniencia

$$
\begin{aligned}
\int_{\Gamma_{0}}\left\{\delta \phi^{\top} \delta \phi^{\prime \top}\right\} & {\left[\begin{array}{ll}
\mathbf{d}_{00} & \mathbf{d}_{01} \\
\mathbf{d}_{10} & \mathbf{d}_{11}
\end{array}\right]\left\{\begin{array}{c}
\Delta \phi \\
\Delta \boldsymbol{\phi}^{\prime}
\end{array}\right\} d S } \\
& -\sum_{a=1}^{2}\left\{\delta \phi\left(S_{a}\right)^{\top} \delta \phi\left(S_{a}\right)^{\prime \top}\right\}\left[\begin{array}{ll}
\mathbf{f}_{a} & \mathbf{0} \\
\mathbf{0} & \mathbf{0}
\end{array}\right]\left\{\begin{array}{c}
\Delta \boldsymbol{\phi}\left(S_{a}\right) \\
\Delta \boldsymbol{\phi}\left(S_{a}\right)^{\prime}
\end{array}\right\} \\
& =\Delta \lambda\left(\int_{\Gamma_{0}} \delta \boldsymbol{\phi}^{\top} \boldsymbol{q} d S+\sum_{a=1}^{2} \delta \phi\left(S_{a}\right)^{\top} \boldsymbol{f}_{a}\right),
\end{aligned}
$$


La discretización del lado izquierdo de la igualdad conduce a la matriz de rigidez tangente de la estructura. Ésta se construye ensamblando (a) la matriz de rigidez tangente de cada uno de los elementos, forma discreta del operador tangente

$$
\int_{\Gamma_{0}}\left\{\delta \phi^{\top} \delta \phi^{\prime \top}\right\}\left[\begin{array}{ll}
\mathbf{d}_{00} & \mathbf{d}_{01} \\
\mathbf{d}_{10} & \mathbf{d}_{11}
\end{array}\right]\left\{\begin{array}{c}
\Delta \phi \\
\Delta \boldsymbol{\phi}^{\prime}
\end{array}\right\} d S
$$

con (b) la contribución de las fuerzas nodales a la rigidez tangente, cuyo valor se obtendría partir de la discretización de esta expresión

$$
-\sum_{a=1}^{2}\left\{\delta \phi\left(S_{a}\right)^{\top} \delta \phi\left(S_{a}\right)^{\prime \top}\right\}\left[\begin{array}{cc}
\mathbf{f}_{a} & \mathbf{0} \\
\mathbf{0} & \mathbf{0}
\end{array}\right]\left\{\begin{array}{c}
\Delta \phi\left(S_{a}\right) \\
\Delta \phi\left(S_{a}\right)^{\prime}
\end{array}\right\} .
$$

Por otra parte, la forma discreta del lado derecho de la igualdad proporciona el vector de fuerzas de la ecuación de equilibrio, multiplicado por el incremento del parámetro de control $\Delta \lambda$. Todos estos términos, cuyas expresiones calculamos a continuación, dan lugar a la ecuación incremental.

\subsubsection{Construcción de la ecuación incremental}

\section{Rigidez tangente del elemento}

La matriz de rigidez tangente de un elemento se puede calcular como forma discreta de la expresión (6.49). El núcleo de la rigidez se descompone, como se vio en el capítulo anterior, en suma de la rigidez geométrica, la constitutiva, y la debida a las fuerzas generalizadas espaciales, esta última cambiada de signo

$$
\left[\begin{array}{ll}
\mathbf{d}_{00} & \mathbf{d}_{01} \\
\mathbf{d}_{10} & \mathbf{d}_{11}
\end{array}\right]=\left[\begin{array}{ll}
\mathbf{d}_{00}^{G} & \mathbf{d}_{01}^{G} \\
\mathbf{d}_{10}^{G} & \mathbf{d}_{11}^{G}
\end{array}\right]+\left[\begin{array}{ll}
\mathbf{d}_{00}^{M} & \mathbf{d}_{01}^{M} \\
\mathbf{d}_{10}^{M} & \mathbf{d}_{11}^{M}
\end{array}\right]-\left[\begin{array}{cc}
\mathbf{d}^{Q} & \mathbf{0} \\
\mathbf{0} & \mathbf{0}
\end{array}\right]
$$

La composición de cada sumando es la siguiente

$$
\begin{aligned}
{\left[\begin{array}{cc}
\mathbf{d}_{00}^{G} & \mathbf{d}_{01}^{G} \\
\mathbf{d}_{10}^{G} & \mathbf{d}_{11}^{G}
\end{array}\right] } & =\left[\begin{array}{cccc}
\mathbf{0} & \mathbf{0} & \mathbf{0} & \mathbf{0} \\
\mathbf{0} & \widehat{\mathbf{n}} \widehat{\gamma} & \widehat{\mathbf{n}} & \widehat{\mathbf{m}} \\
\mathbf{0} & -\widehat{\mathbf{n}} & \mathbf{0} & \mathbf{0} \\
\mathbf{0} & \mathbf{0} & \mathbf{0} & \mathbf{0}
\end{array}\right] \\
{\left[\begin{array}{cc}
\mathbf{d}_{00}^{M} & \mathbf{d}_{01}^{M} \\
\mathbf{d}_{10}^{M} & \mathbf{d}_{11}^{M}
\end{array}\right] } & =\left[\begin{array}{ccccc}
\mathbf{0} & \mathbf{0} & \mathbf{0} & \mathbf{0} \\
\mathbf{0} & -\widehat{\gamma} \mathbf{c}_{\gamma \gamma} \widehat{\gamma} & -\widehat{\gamma} \mathbf{c}_{\gamma \gamma} & -\widehat{\gamma} \mathbf{c}_{\gamma \kappa} \\
\mathbf{0} & \mathbf{c}_{\gamma \gamma} \widehat{\gamma} & \mathbf{c}_{\gamma \gamma} & \mathbf{c}_{\gamma \kappa} \\
\mathbf{0} & \mathbf{c}_{\gamma \kappa} \widehat{\gamma} & \mathbf{c}_{\kappa \gamma} & \mathbf{c}_{\kappa \kappa}
\end{array}\right] \\
{\left[\begin{array}{cc}
\mathbf{d}^{Q} & \mathbf{0} \\
\mathbf{0} & \mathbf{0}
\end{array}\right] } & =\lambda\left[\begin{array}{cccc}
\mathbf{0} & \mathbf{0} & \mathbf{0} & \mathbf{0} \\
\mathbf{0} & \widehat{\mathbf{q}}_{m} & \mathbf{0} & \mathbf{0} \\
\mathbf{0} & \mathbf{0} & \mathbf{0} & \mathbf{0} \\
\mathbf{0} & \mathbf{0} & \mathbf{0} & \mathbf{0}
\end{array}\right]+\lambda\left[\begin{array}{cccc}
\mathbf{0} & -\widehat{\mathbf{q}}_{n}^{f} & \mathbf{0} & \mathbf{0} \\
\mathbf{0} & -\widehat{\mathbf{q}}_{m}^{f} & \mathbf{0} & \mathbf{0} \\
\mathbf{0} & \mathbf{0} & \mathbf{0} & \mathbf{0} \\
\mathbf{0} & \mathbf{0} & \mathbf{0} & \mathbf{0}
\end{array}\right]
\end{aligned}
$$

Introduciendo la discretización (7.6) resulta

$$
\delta \phi^{e \top} \int_{-1}^{1}\left[\begin{array}{ll}
\mathbb{N}^{\top} & \left.\frac{1}{J} \mathbb{N}^{\prime}\right]
\end{array}\right]\left[\begin{array}{ll}
\mathbf{d}_{00} & \mathbf{d}_{01} \\
\mathbf{d}_{10} & \mathbf{d}_{11}
\end{array}\right]\left[\begin{array}{c}
\mathbb{N} \\
\frac{1}{J} \mathbb{N}^{\prime}
\end{array}\right] J d \xi \Delta \boldsymbol{\phi}^{e} .
$$


La integral se evalúa mediante una cuadratura de Gauss de orden $g$. Entonces, la matriz de rigidez tangente del elemento es

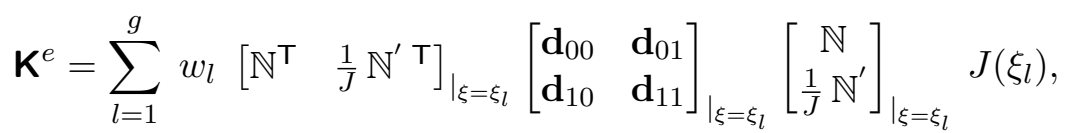

donde las coordenadas $\xi_{l}$ representan los puntos de Gauss y $w_{l}$ los pesos correspondientes de la cuadratura.

\section{Contribución de las fuerzas nodales a la rigidez tangente}

Después del ensamblaje de las matrices de rigidez de los elementos en la matriz de rigidez completa de la estructura es necesario restar de ésta la contribución de las fuerzas espaciales que actúan directamente sobre los nodos de la estructura, de forma que si $\boldsymbol{n}_{i}^{s}$ y $\boldsymbol{m}_{i}^{s}$ son las fuerzas y momentos exteriores de eje fijo sobre el nodo $i$, su contribución a la rigidez general viene dada por la matriz

$$
-\mathbf{f}_{i}=-\lambda\left(\left[\begin{array}{cc}
\mathbf{0} & \mathbf{0} \\
\mathbf{0} & \widehat{\mathbf{m}}_{i}
\end{array}\right]+\left[\begin{array}{cc}
\mathbf{0} & -\widehat{\mathbf{n}}_{i}^{f} \\
\mathbf{0} & -\widehat{\mathbf{m}}_{i}^{f}
\end{array}\right]\right),
$$

que será ensamblada en las posiciones correspondientes a las variables nodales $\Delta \boldsymbol{x}_{i} \mathrm{y}$ $\Delta \boldsymbol{\omega}_{i}$, puesto que interviene en la ecuación del equilibrio tangente mediante el término

$$
\delta \phi_{i}^{\top}\left(-\mathbf{f}_{i}\right) \Delta \phi_{i}
$$

\section{Discretización de las fuerzas totales}

El lado derecho de la ecuación de equilibrio tangente está formado por el producto del incremento del parámetro de control, por las fuerzas totales que actúan sobre la estructura. A su vez, éstas resultan de sumar las fuerzas nodales y las fuerzas generalizadas que actúan sobre la directriz. Denominaremos fuerzas iniciales a la discretización de las fuerzas generalizadas sobre un elemento. Para calcularlas se introduce la discretización en el término

$$
\int_{\Gamma_{0}} \delta \phi^{\top} \boldsymbol{q} d S
$$

con lo que resulta

$$
\delta \phi^{e \top} \int_{-1}^{1} \mathbb{N}^{\top} \boldsymbol{q} d S
$$

La integral, que evaluamos mediante una cuadratura de Gauss de orden $g$, proporciona las fuerzas iniciales

$$
\mathbf{f}^{0 e}=\sum_{l=1}^{g} w_{l} \mathbb{N}^{\top}\left(\xi_{l}\right) \boldsymbol{q}\left(\xi_{l}\right) J_{\left.\right|_{\xi=\xi_{l}}} .
$$

El vector de fuerzas totales se obtiene ensamblando las fuerzas iniciales con las fuerzas que actúan sobre cada nodo

$$
\boldsymbol{f}_{i}=\left\{\begin{array}{ll}
\boldsymbol{n}_{i} & \boldsymbol{m}_{i}
\end{array}\right\}^{\top}
$$

en las posiciones correspondientes a las variables nodales $\Delta \boldsymbol{x}_{i}$ y $\Delta \boldsymbol{\omega}_{i}$. 


\section{Ecuación incremental}

Si denotamos mediante los símbolos $\mathbf{K}$ y $\mathbf{f}$ al resultado de ensamblar la matriz de rigidez tangente y el vector de fuerzas totales, y eliminar las filas (y columnas) correspondientes a las componentes nulas de los incrementos nodales de las variables de la configuración, y mediante el símbolo $\Delta \phi$ al vector que reúne los incrementos nodales incógnita $\Delta \boldsymbol{\Phi}_{i}=\left\{\Delta \boldsymbol{x}_{i} \Delta \boldsymbol{\omega}_{i}\right\}^{\top}$, entonces la ecuación incremental resulta

$$
\mathbf{K} \Delta \boldsymbol{\phi}=\mathbf{f} \Delta \lambda,
$$

que junto con una restricción del tipo

$$
c(\Delta \boldsymbol{\phi}, \Delta \lambda)=0
$$

que define la estrategia de control, proporciona la base para calcular soluciones incrementales puras. En este tipo de soluciones la trayectoria de equilibrio se traza representando el valor del parámetro de control frente al de las variables de la configuración. Todos ellos se calculan paso a paso a partir de los valores al final del paso anterior y de los incrementos calculados. Existen muchas referencias relativas al procedimiento de solución incremental; citeremos únicamente a Crisfield (1991) [14, y a Felippa (2001) [19] como obras en las que se recoge el estado del arte en esta materia. En la figura adjunta se puede observar la interpretación gráfica de las variables que intervienen en el proceso incremental.

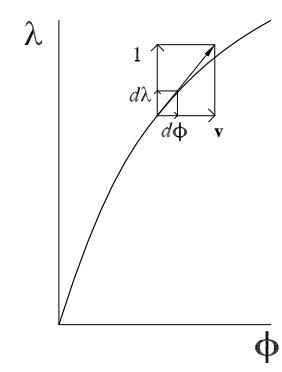

Figura 7.1: Velocidad incremental

Se denomina velocidad incremental a la variable

$$
\mathbf{v}=\mathbf{K}^{-1} \mathbf{f} .
$$

Introduciéndola en la ecuación incremental (7.13) se puede despejar el vector de incrementos de las variables de la configuración

$$
\Delta \boldsymbol{\phi}=\mathbf{v} \Delta \lambda .
$$

Para calcular los incrementos de las variables en el paso $n$ introducimos la magnitud $l_{n}$, que define la amplitud del paso. En adelante denotaremos mediante el subíndice 
(n) los valores de las variables en el paso $n$. Además definimos el número

$$
f_{(n)}=\frac{l_{n}}{\left|\Delta \lambda_{(n)}\right|}
$$

que determina el módulo del vector tangente a la trayectoria de equilibrio. La elección de $f_{n}$ define la estrategia de control. En este trabajo hemos implementado tres estrategias clásicas:

1. Control de carga. Es la estrategia más sencilla, pero no es adecuada si es necesario atravesar puntos críticos de la trayectoria de equilibrio. Se obtiene tomando

$$
f_{(n)}=1,
$$

lo que corresponde a la restricción

$$
c\left(\Delta \boldsymbol{\phi}_{(n)}, \Delta \lambda_{(n)}\right)=\Delta \lambda_{(n)}^{2}-l_{n}^{2}=0 .
$$

El incremento del parámetro de control es

$$
\Delta \lambda_{(n)}= \pm l_{n},
$$

y el signo se toma de forma que el trabajo de las fuerzas sea positivo en el paso.

2. Control de desplazamiento. Resulta apropiado para atravesar puntos críticos pero no puntos de retorno. En este caso se toma

$$
f_{(n)}=\sqrt{\mathbf{v}_{(n)} \cdot \mathbf{v}_{(n)}},
$$

que corresponde a la restricción

$$
c\left(\Delta \boldsymbol{\phi}_{(n)}, \Delta \lambda_{(n)}\right)=\Delta \boldsymbol{\phi}_{(n)} \cdot \Delta \boldsymbol{\Phi}_{(n)}-l_{n}^{2}=0 .
$$

Entonces

$$
\Delta \lambda_{(n)}= \pm \frac{l_{n}}{\sqrt{\mathbf{v}_{(n)} \cdot \mathbf{v}_{(n)}}} .
$$

3. Control de arco. Es más versátil que las estrategias anteriores. Cuando se atraviesa puntos de retorno es necesario emplear un criterio más elaborado que el del trabajo positivo para lograr avanzar por la trayectoria. El valor de las variables es

$$
f_{(n)}=\sqrt{1+\mathbf{v}_{(n)} \cdot \mathbf{v}_{(n)}},
$$

que corresponde a la restricción

$$
c\left(\Delta \boldsymbol{\Phi}_{(n)}, \Delta \lambda_{(n)}\right)=\left\|\left(\Delta \boldsymbol{\Phi}_{(n)}, \Delta \lambda_{(n)}\right)\right\|^{2}-l_{n}^{2}=0 .
$$

Entonces

$$
\Delta \lambda_{(n)}= \pm \frac{l_{n}}{\sqrt{1+\mathbf{v}_{(n)} \cdot \mathbf{v}_{(n)}}} .
$$

En los tres casos los incrementos de las variables de la configuración en el paso $n$ se obtienen mediante la expresión

$$
\Delta \boldsymbol{\phi}_{(n)}=\mathbf{v}_{(n)} \Delta \lambda_{(n)} .
$$




\subsubsection{Actualización de la configuración}

Los procedimientos de actualización que se describe en este sección son válidos tanto en soluciones puramente incrementales como en soluciones iterativas. El punto de partida para la actualización son los valores conocidos al comienzo del paso $n$ (o de la iteración $k$ ) de las distintas variables que definen la solución:

- Los valores nodales de las variables que definen la configuración. $\boldsymbol{x}_{i(n)}$ es el vector que define la posición del nodo $i$, y $\boldsymbol{\Lambda}_{i(n)}$ la matriz ortogonal que representa la rotación de la sección transversal en el nodo.

- Los valores en los puntos de Gauss de cada elemento $e$ de las deformaciones generalizadas. $\boldsymbol{\gamma}_{l(n)}^{e} \mathrm{y} \boldsymbol{\kappa}_{l(n)}^{e}$ definen respectivamente la distorsión y el cambio de orientación de la sección en el punto $\xi_{l}$.

- Los valores en los puntos de Gauss de cada elemento de los esfuerzos (fuerzas internas). $\boldsymbol{n}_{l(n)}^{e}$ y $\boldsymbol{m}_{l(n)}^{e}$ definen respectivamente las componentes con unidades de fuerza y con unidades de momento de los esfuerzos en el punto $\xi_{l}$.

Todas estas variables se expresan en componentes del sistema de referencia general. En este paso, se dispone además de los valores nodales del incremento de posición $\Delta \boldsymbol{x}_{i(n)}$ y de la rotación incremental $\Delta \boldsymbol{\omega}_{i(n)}$, que son las componentes del vector de incrementos $\Delta \boldsymbol{\Phi}_{(n)}$, calculado con alguna de las tres estrategias descritas en el apartado anterior. Las variables $\Delta \boldsymbol{\omega}_{i(n)}$ son vectores de tres componentes, elementos del espacio tangente al grupo de las rotaciones en $\boldsymbol{\Lambda}_{i(n)}$.

\section{Actualización de la posición y de la rotación}

El procedimiento de actualización de las variables de la configuración debe ser consistente con su estructura geométrica. Por una parte, las posiciones nodales se actualizan linealmente

$$
\boldsymbol{x}_{i(n+1)}=\boldsymbol{x}_{i(n)}+\Delta \boldsymbol{x}_{i(n)} .
$$

Sin embargo, la actualización de las rotaciones requiere la evaluación de la composición de la rotación nodal con la rotación incremental. Esta composición es equivalente al cálculo del producto

$$
\boldsymbol{\Lambda}_{i(n+1)}=\exp \left(\Delta \hat{\boldsymbol{\omega}}_{i(n)}\right) \boldsymbol{\Lambda}_{i(n)},
$$

que da como resultado un elemento del grupo ortogonal. A su vez, $\exp \left(\Delta \hat{\boldsymbol{\omega}}_{i(n)}\right)$ se obtiene a partir de la expresión

$$
\exp (\Delta \hat{\boldsymbol{\omega}})=\cos \Delta \omega \mathbf{1}+\frac{\sin \Delta \omega}{\Delta \omega} \Delta \hat{\boldsymbol{\omega}}+\frac{1-\cos \Delta \omega}{\Delta \omega^{2}} \Delta \boldsymbol{\omega} \otimes \Delta \boldsymbol{\omega}
$$

conocida como aplicación exponencial o fórmula de Rodrigues, en la que, por claridad, se ha prescindido de los subíndices. $\Delta \omega$ es el vector rotación nodal incremental, $\Delta \omega$ es su módulo, y $\Delta \hat{\boldsymbol{\omega}}$ es la matriz antisimétrica asociada a $\Delta \boldsymbol{\omega}$. 
La formulación de Simó es independiente de la parametrización escogida para representar las rotaciones de la sección. De hecho, la expresión (7.30) emplea la representación proporcionada por la matriz ortogonal. No obstante, diversos autores (62] 28]) han señalado la conveniencia de emplear cuaterniones unitarios $\left(q_{0}, \mathbf{q}\right)$ como representación de las rotaciones, ya que reducen los requisitos de almacenamiento y facilitan el proceso de actualización de las rotaciones. Este último aspecto ha sido resaltado por Ibrahimbegović y Taylor (2002) [28], que proponen una actualización de las rotaciones en la que es innecesario el cálculo de matrices de rotación, dando lugar al siguiente procedimiento que adoptamos en la tesis (prescindimos, por claridad del subíndice $i$ que denota el nodo):

1. Se parte del valor nodal del cuaternión $\left(q_{0(n)}, \mathbf{q}_{(n)}\right)$ en el paso $n$ (o la iteración $k)$.

2. Se calcula el cuaternión (rotación) correspondiente a la rotación incremental $\Delta \boldsymbol{\omega}_{(n)}$. Este viene dado por la siguiente expresión, equivalente a la fórmula de Rodrigues

$$
\left(q_{0 \omega(n)}, \mathbf{q}_{\omega(n)}\right)=\left(\cos \frac{\Delta \omega_{(n)}}{2}, \frac{\Delta \boldsymbol{\omega}_{(n)}}{\left\|\Delta \boldsymbol{\omega}_{(n)}\right\|} \sin \frac{\Delta \omega_{(n)}}{2}\right)
$$

3. La rotación actualizada viene dada por la expresión

$$
\left(q_{0(n+1)}, \mathbf{q}_{(n+1)}\right)=\left(q_{0 \omega(n)}, \mathbf{q}_{\omega(n)}\right) \star\left(q_{0(n)}, \mathbf{q}_{(n)}\right),
$$

en la que el operador $\star$ denota el producto de cuaterniones que sigue la siguiente regla:

$$
\left(a_{0}, \mathbf{a}\right) \star\left(b_{0}, \mathbf{b}\right)=\left(a_{0} b_{0}-\mathbf{a} \cdot \mathbf{b}, a_{0} \mathbf{b}+b_{0} \mathbf{a}+\mathbf{a} \times \mathbf{b}\right) .
$$

Esta expresión es equivalente a la fórmula de actualización (7.29)

Una vez conocido el valor actual del cuaternión $\left(q_{0(n+1)}, \mathbf{q}_{(n+1)}\right)$ la matriz de rotación correspondiente se puede obtener mediante la expresión

$$
\boldsymbol{\Lambda}_{(n+1)}=\left(q_{0(n+1)}^{2}-1\right) \mathbf{1}+2 q_{0(n+1)} \hat{\mathbf{q}}_{(n+1)}+2 \mathbf{q}_{(n+1)} \otimes \mathbf{q}_{(n+1)} .
$$

\section{Actualización de las deformaciones generalizadas}

\section{Cambio de orientación}

Comenzamos evaluando el cambio de orientación $\widehat{\boldsymbol{\kappa}}$ en el punto de Gauss $\xi_{l}$. Suponemos conocido el valor del cambio de orientación en el paso $n, \widehat{\boldsymbol{\kappa}}_{(n)}$. Su valor en el paso $n+1$ se deduce a partir de la expresión (3.55)

$$
\widehat{\boldsymbol{\kappa}}_{(n+1)}=\boldsymbol{\Lambda}_{(n+1)}^{\prime} \boldsymbol{\Lambda}_{(n+1)}^{\top} .
$$

La rotación actualizada traspuesta se puede expresar como

$$
\boldsymbol{\Lambda}_{(n+1)}^{\top}=\boldsymbol{\Lambda}_{(n)}^{\top} \exp \left(-\Delta \hat{\mathbf{\omega}}_{(n)}\right)
$$


Por otra parte, la derivada de la rotación actualizada se puede calcular en función del valor de la rotación en el paso $n$ de la siguiente forma

$$
\boldsymbol{\Lambda}_{(n+1)}^{\prime}=\frac{d}{d S} \exp \left(\Delta \hat{\boldsymbol{\omega}}_{(n)}\right) \boldsymbol{\Lambda}_{(n)}+\exp \left(\Delta \hat{\boldsymbol{\omega}}_{(n)}\right) \boldsymbol{\Lambda}_{(n)}^{\prime}
$$

Combinando las dos expresiones anteriores

$$
\widehat{\boldsymbol{\kappa}}_{(n+1)}=\frac{d}{d S} \exp \left(\Delta \hat{\boldsymbol{\omega}}_{(n)}\right) \exp \left(-\Delta \hat{\boldsymbol{\omega}}_{(n)}\right)+\exp \left(\Delta \hat{\boldsymbol{\omega}}_{(n)}\right) \boldsymbol{\Lambda}_{(n)}^{\prime} \boldsymbol{\Lambda}_{(n)}^{\top} \exp \left(-\Delta \hat{\boldsymbol{\omega}}_{(n)}\right),
$$

y teniendo en cuenta que $\boldsymbol{\Lambda}_{(n)}^{\prime} \boldsymbol{\Lambda}_{(n)}^{\top}$ es precisamente el cambio de orientación en el paso $n$ resulta

$$
\widehat{\boldsymbol{\kappa}}_{(n+1)}=\frac{d}{d S} \exp \left(\Delta \hat{\boldsymbol{\omega}}_{(n)}\right) \exp \left(-\Delta \hat{\boldsymbol{\omega}}_{(n)}\right)+\exp \left(\Delta \hat{\boldsymbol{\omega}}_{(n)}\right) \widehat{\boldsymbol{\kappa}}_{(n)} \exp \left(-\Delta \hat{\boldsymbol{\omega}}_{(n)}\right) .
$$

Esta es la expresión que proporcionan Simó y Vu-Quoc [62]. Ibrahimbegović (1995) 25] propone una fórmula alternativa, más eficiente desde el punto de vista computacional, que da directamente como resultado el vector que representa el cambio de orientación de la sección

$$
\boldsymbol{\kappa}_{(n+1)}=\mathbf{T}\left(\Delta \boldsymbol{\omega}_{(n)}\right) \Delta \boldsymbol{\omega}_{(n)}^{\prime}+\exp \left(\Delta \hat{\boldsymbol{\omega}}_{(n)}\right) \boldsymbol{\kappa}_{(n)} .
$$

El operador $\mathbf{T}$-Apéndice $\mathrm{B}$, ecuación $(\overline{\mathrm{B} .10})$ - actúa de la siguiente forma sobre $\Delta \omega$

$$
\mathbf{T}(\Delta \boldsymbol{\omega})=\frac{\sin \Delta \omega}{\Delta \omega} \mathbf{1}+\frac{1-\cos \Delta \omega}{\Delta \omega^{2}} \Delta \hat{\boldsymbol{\omega}}+\frac{\Delta \omega-\sin \Delta \omega}{\Delta \omega^{3}} \Delta \boldsymbol{\omega} \otimes \Delta \boldsymbol{\omega},
$$

y la derivada de la rotación incremental en el punto de Gauss se interpola a partir de los valores nodales de ésta

$$
\frac{d}{d S} \Delta \boldsymbol{\omega}\left(\xi_{l}\right)_{(n)}=\frac{1}{J\left(\xi_{l}\right)}\left[\begin{array}{lll}
N_{1}^{\prime}\left(\xi_{l}\right) & \cdots & N_{n}^{\prime}\left(\xi_{l}\right)
\end{array}\right]\left\{\begin{array}{c}
\Delta \boldsymbol{\omega}_{1} \\
\vdots \\
\Delta \boldsymbol{\omega}_{n}
\end{array}\right\}_{(n)} .
$$

\section{Tangente a la directriz}

La deformación generalizada $\gamma$ se calcula en el paso $n+1$ simplemente como

$$
\gamma_{(n+1)}=\boldsymbol{x}_{(n+1)}^{\prime}=\boldsymbol{x}_{(n)}^{\prime}+\Delta \boldsymbol{x}_{(n)}^{\prime}=\gamma_{(n)}+\Delta \boldsymbol{x}_{(n)}^{\prime} \text {. }
$$

Análogamente a la derivada de la rotación incremental, la derivada del cambio de posición de la directriz en los puntos de Gauss se interpola a partir de sus valores nodales

$$
\frac{d}{d S} \Delta \boldsymbol{x}\left(\xi_{l}\right)_{(n)}=\frac{1}{J\left(\xi_{l}\right)}\left[\begin{array}{lll}
N_{1}^{\prime}\left(\xi_{l}\right) & \cdots & N_{n}^{\prime}\left(\xi_{l}\right)
\end{array}\right]\left\{\begin{array}{c}
\Delta \boldsymbol{x}_{1} \\
\vdots \\
\Delta \boldsymbol{x}_{n}
\end{array}\right\}_{(n)} .
$$

Es interesante hacer notar que para actualizar las deformaciones generalizadas no es necesario evaluar las rotaciones en los puntos de Gauss -obsérvese cómo en las expresiones (7.35) y (7.36) sólo interviene $\Delta \boldsymbol{\omega}_{n}$ y su derivada (naturalmente, evaluadas en los puntos de Gauss)-. Sin embargo, como veremos a continuación, sí es necesario disponer de la rotación actualizada en los puntos de Gauss para actualizar la matriz constitutiva. 


\section{Actualización de los esfuerzos}

Los esfuerzos se actualizan directamente a partir del valor de la forma espacial de la matriz constitutiva, y de las deformaciones generalizadas en el paso $n+1$

$$
\begin{gathered}
{\left[\begin{array}{ll}
\mathbf{c}_{\gamma \gamma} & \mathbf{c}_{\gamma \kappa} \\
\mathbf{c}_{\kappa \gamma} & \mathbf{c}_{\kappa \kappa}
\end{array}\right]_{(n+1)}=\left[\begin{array}{cc}
\boldsymbol{\Lambda}_{(n+1)} & \mathbf{0} \\
\mathbf{0} & \boldsymbol{\Lambda}_{(n+1)}
\end{array}\right]\left[\begin{array}{ll}
\mathbf{C}_{\Gamma \Gamma} & \mathbf{C}_{\Gamma K} \\
\mathbf{C}_{K \Gamma} & \mathbf{C}_{K K}
\end{array}\right]\left[\begin{array}{cc}
\boldsymbol{\Lambda}_{(n+1)}^{\top} & \mathbf{0} \\
\mathbf{0} & \boldsymbol{\Lambda}_{(n+1)}^{\top}
\end{array}\right],} \\
\left\{\begin{array}{c}
\boldsymbol{n}_{(n+1)} \\
\boldsymbol{m}_{(n+1)}
\end{array}\right\}=\left[\begin{array}{ll}
\mathbf{c}_{\gamma \gamma} & \mathbf{c}_{\gamma \kappa} \\
\mathbf{c}_{\kappa \gamma} & \mathbf{c}_{\kappa \kappa}
\end{array}\right]_{(n+1)}\left\{\begin{array}{l}
\gamma_{(n+1)}-\gamma_{0} \\
\boldsymbol{\kappa}_{(n+1)}-\boldsymbol{\kappa}_{0}
\end{array}\right\} .
\end{gathered}
$$

donde $\boldsymbol{\Lambda}_{(n+1)}$ hace referencia a la rotación en el punto de interpolación, y por tanto

$$
\boldsymbol{\Lambda}\left(\xi_{l}\right)_{(n+1)}=\exp \left(\Delta \hat{\boldsymbol{\omega}}\left(\xi_{l}\right)_{(n)}\right) \boldsymbol{\Lambda}\left(\xi_{l}\right)_{(n)} .
$$

Esta expresión muestra que la actualización de los esfuerzos requiere almacenar el valor de las rotaciones en los puntos de interpolación.

\subsubsection{Relación entre la interpolación y la objetividad de la solución}

En la sección 3.5.3 demostramos que la forma material de las deformaciones generalizadas del modelo es invariante frente a la superposición de movimientos de sólido rígido. Esta propiedad se denomina objetividad -también frame indifference, en inglés-. Cuando pasamos del modelo a la solución por elementos finitos ya no es evidente que las medidas de la deformación discretas conserven la objetividad.

\section{La objetividad en el sentido de Ibrahimbegović y Taylor}

Ibrahimbegović y Taylor [28, p. 5167] indican que la expresión (7.36) proporciona resultados invariantes frente a rotaciones de sólido rígido en el siguiente sentido: “... la invarianza respecto al sistema de referencia de la discretización de las medidas de la deformación queda asegurada siempre que las rotaciones incrementales correspondan a una rotación de sólido rígido ..."

En efecto, si la diferencia entre el paso $n$ y el $n+1$ es una rotación de sólido rígido $\mathbf{R}$, entonces

$$
\boldsymbol{\Lambda}_{i(n+1)}=\mathbf{Q} \boldsymbol{\Lambda}_{i(n)}, \quad \text { con } \mathbf{Q}=\exp \left(\Delta \hat{\mathbf{\omega}}_{Q}\right)
$$

y en todos los nodos del modelo las rotaciones incrementales son idénticas

$$
\exp \left(\Delta \hat{\boldsymbol{\omega}}_{i(n)}\right)=\exp \left(\Delta \hat{\boldsymbol{\omega}}_{Q}\right)
$$

Por tanto la interpolación de las rotaciones incrementales entre los nodos de un elemento también proporciona el mismo valor

$$
\Delta \boldsymbol{\omega}\left(\xi_{l}\right)_{(n)}=\sum_{i=1}^{n} N_{i}\left(\xi_{l}\right) \Delta \boldsymbol{\omega}_{i(n)}=\Delta \boldsymbol{\omega}_{Q},
$$


entonces

$$
\frac{d}{d S} \Delta \boldsymbol{\omega}\left(\xi_{l}\right)_{(n)}=\frac{d}{d S} \Delta \boldsymbol{\omega}_{Q}=\mathbf{0},
$$

y sustituyendo en la expresión (7.36):

$$
\boldsymbol{\kappa}_{(n+1)}=\boldsymbol{\kappa}_{(n)},
$$

con lo que queda probada la invarianza en el sentido de Ibrahimbegović y Taylor.

\section{La objetividad en el sentido de Crisfield y Jelenić}

En un sentido estricto la invarianza frente a movimientos de sólido rígido de una configuración $(\boldsymbol{x}, \boldsymbol{\Lambda})$ hace referencia a que las deformaciones generalizadas calculadas a partir de ella no varían al calcularlas mediante una nueva configuración $(\underline{\boldsymbol{x}}, \underline{\boldsymbol{\Lambda}})=$ $(\mathbf{Q} \boldsymbol{x}+\boldsymbol{r}, \mathbf{Q} \mathbf{\Lambda})$ consecuencia de la superposición de un movimiento de sólido rígido definido por la traslación $\boldsymbol{r}$ y la rotación $\mathbf{Q}$. Esta propiedad ha sido estudiada en detalle por Crisfield y Jelenić en [16] y [32], que sostienen que una interpolación ${ }^{1}$ del tipo

$$
\Delta \boldsymbol{\omega}^{h}(\xi)=\sum_{i=1}^{n} N_{i}(\xi) \mathbf{1} \Delta \boldsymbol{\omega}_{i}
$$

produce resultados no invariantes, ya que [16, p.1132] "Las rotaciones interpoladas de este modo incluyen en general rotaciones de sólido rígido, de forma que el error introducido por la interpolación hace que las medidas de la deformación resultantes dependan de la rotación de sólido rígido". En [16, Apéndice C] ofrecen una demostración de la no objetividad de la interpolación tradicional mediante un contraejemplo basado en el cálculo de las curvaturas.

Con el fin de lograr una formulación objetiva (invariante) proponen la siguiente interpolación: si se considera un elemento recto de dos nodos $i, j$ de longitud $L_{e}$, en una cierta configuración las rotaciones nodales valdrán $\boldsymbol{\Lambda}_{i}, \boldsymbol{\Lambda}_{j}$ y la rotación en cualquier punto intermedio del elemento se puede obtener mediante la expresión

$$
\boldsymbol{\Lambda}^{h}(S)=\boldsymbol{\Lambda}_{i}\left(\boldsymbol{\Lambda}_{i}^{\top} \boldsymbol{\Lambda}_{j}\right)^{S / L_{e}},
$$

denominada interpolación esférica (SLERP) en el ámbito de los gráficos por ordenador [56, p. 125]. Obsérvese que la rotación entre los nodos $i$ y $j$ en la configuración material está definida por $\exp \left(\widehat{\boldsymbol{\Theta}}_{i j}\right)=\boldsymbol{\Lambda}_{i}^{\top} \boldsymbol{\Lambda}_{j}$.

La objetividad de la interpolación se desprende del siguiente razonamiento

$$
\underline{\boldsymbol{\Lambda}}^{h}=\underline{\boldsymbol{\Lambda}}_{i}\left(\underline{\boldsymbol{\Lambda}}_{i}^{\top} \underline{\boldsymbol{\Lambda}}_{j}\right)^{S / L_{e}}=\mathbf{Q} \boldsymbol{\Lambda}_{i}\left(\boldsymbol{\Lambda}_{i}^{\top} \mathbf{Q}^{\top} \mathbf{Q} \boldsymbol{\Lambda}_{j}\right)^{S / L_{e}}=\mathbf{Q} \boldsymbol{\Lambda}^{h},
$$

que demuestra la invarianza de las rotaciones interpoladas; por tanto en la expresión de las deformaciones generalizadas en su forma material no interviene la rotación de sólido rígido, lo que garantiza su objetividad:

$$
\begin{aligned}
& \underline{\boldsymbol{\Gamma}}^{h}=\underline{\boldsymbol{\Lambda}}^{h \top} \underline{\boldsymbol{x}}^{h^{\prime}}=\boldsymbol{\Lambda}^{h \boldsymbol{\top}} \mathbf{Q}^{\top} \mathbf{Q} \boldsymbol{x}^{h^{\prime}}=\boldsymbol{\Gamma}^{h} \\
& \underline{\widehat{\mathbf{K}}}^{h}=\underline{\boldsymbol{\Lambda}}^{h \top} \underline{\boldsymbol{\Lambda}}^{h^{\prime}}=\boldsymbol{\Lambda}^{h \boldsymbol{\top}} \mathbf{Q}^{\top} \mathbf{Q} \boldsymbol{\Lambda}^{h^{\prime}}=\widehat{\mathbf{K}}^{h} .
\end{aligned}
$$

\footnotetext{
${ }^{1}$ En este apartado denotamos las variables interpoladas mediante el superíndice $h$.
} 


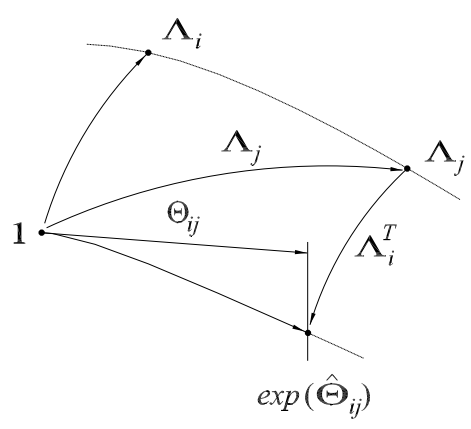

Figura 7.2: Interpolación esférica

Si se analiza la expresión (7.44), el producto $\boldsymbol{\Lambda}_{i}^{\top} \boldsymbol{\Lambda}_{j}$ representa la rotación desde el nodo $i$ al $j$ en la configuración material (Crisfield la denomina local). A partir de esta idea Crisfield y Jelenić generalizan la interpolación a un elemento de $n$ nodos apoyándose en las rotaciones locales (materiales) $\boldsymbol{\Psi}_{i}$ de los nodos referidas a una rotación $\boldsymbol{\Lambda}_{r}$ característica del elemento. El proceso es el siguiente:

1. Se escogen dos nodos $I, J$ del elemento. Se calcula la rotación local entre $I$ y $J$, $\Theta_{I J}$ a partir de la expresión

$$
\exp \widehat{\boldsymbol{\Theta}}_{I J}=\boldsymbol{\Lambda}_{I}^{\top} \boldsymbol{\Lambda}_{J} .
$$

La rotación de referencia se define como rotación "promedio" entre $I$ y $J$

$$
\boldsymbol{\Lambda}_{r}=\boldsymbol{\Lambda}_{I} \exp \left(\frac{1}{2} \widehat{\boldsymbol{\Theta}}_{I J}\right) .
$$

2. Las rotaciones locales $\boldsymbol{\Psi}_{i}$ permiten representar las rotaciones nodales en relación a la rotación de referencia, y se definen a partir de la siguiente expresión

$$
\exp \widehat{\boldsymbol{\Psi}}_{i}=\boldsymbol{\Lambda}_{r}^{\top} \boldsymbol{\Lambda}_{i}
$$

3. La rotación en cada punto se interpola por medio de las rotaciones locales del siguiente modo

$$
\boldsymbol{\Lambda}^{h}(S)=\boldsymbol{\Lambda}_{r} \exp \left(\widehat{\boldsymbol{\Psi}}^{h}(S)\right),
$$

$\operatorname{con} \boldsymbol{\Psi}^{h}(S)=\sum_{i=1}^{n} N_{i}(S) \boldsymbol{\Psi}_{i}$.

A partir de estas definiciones es posible expresar las rotaciones incrementales en función de las rotaciones locales y sus incrementos [16, ec.(3.13)]

$$
\Delta \boldsymbol{\omega}^{h}(S)=\Delta \boldsymbol{\omega}_{r}+\boldsymbol{\Lambda}_{r} \mathbf{T}\left(\boldsymbol{\Psi}^{h}\right) \Delta \boldsymbol{\Psi}^{h} .
$$

Utilizando esta relación, Crisfield y Jelenić consiguen expresar $\Delta \boldsymbol{\omega}^{h}(S)$ como producto de unas funciones de forma generalizadas $I_{i}$ que dependen de $\boldsymbol{\Lambda}_{r}$ y $\boldsymbol{\Psi}_{j}$, por las rotaciones incrementales nodales.

$$
\Delta \boldsymbol{\omega}^{h}(S)=\sum_{i=1}^{n} I_{i}\left(\boldsymbol{\Lambda}_{r}, \boldsymbol{\Psi}_{j}, S\right) \mathbf{1} \Delta \boldsymbol{\omega}_{i}
$$


Con esta nueva interpolación logran formular un elemento finito que conserva la objetividad de las medidas de la deformación.

\section{Una expresión alternativa para el cálculo del cambio de orientación}

Adoptando la fórmula de interpolación (7.44) de Crisfield y Jelenić es posible idear un procedimiento alternativo al propuesto en la sección anterior para el cálculo del cambio de orientación $\boldsymbol{\kappa}$ en los puntos de interpolación -expresiones $(\overline{7.36})$ y $(\overline{7.38})$ - . Consideramos por sencillez un elemento recto de dos nodos $I$ y $J$ con un punto de Gauss en el centro $\left(S / L_{e}=1 / 2\right)$; el proceso sería el siguiente:

1. A partir de las rotaciones incrementales $\Delta \boldsymbol{\omega}_{I(n)}, \Delta \boldsymbol{\omega}_{J(n)}$ en el paso $n$ se calculan las rotaciones nodales $\boldsymbol{\Lambda}_{I(n)}$ y $\boldsymbol{\Lambda}_{J(n)}$ mediante la expresión (7.29) (o su equivalente con cuaterniones).

2. La rotación en el punto de Gauss se calcula mediante la interpolación esférica (7.44). La podemos escoger, además, como rotación de referencia del elemento. Entonces (prescindiendo por claridad del subíndice $n$ ),

$$
\boldsymbol{\Lambda}_{r}=\boldsymbol{\Lambda}_{\xi=0}=\boldsymbol{\Lambda}_{I}\left(\boldsymbol{\Lambda}_{I}^{\top} \boldsymbol{\Lambda}_{J}\right)^{1 / 2}
$$

3. Las rotaciones locales $\boldsymbol{\Psi}_{I}, \boldsymbol{\Psi}_{J}$ se calculan mediante la expresión (7.47).

4. El cambio de orientación (espacial) a lo largo del elemento $\boldsymbol{\kappa}^{h}(S)$ se obtiene directamente a partir de su definición

$$
\begin{aligned}
\boldsymbol{\kappa}^{h}(S) & =\boldsymbol{\Lambda}^{\prime} \boldsymbol{\Lambda}^{\top} \\
& =\boldsymbol{\Lambda}_{r} \frac{d}{d S} \exp \left(\widehat{\boldsymbol{\Psi}}^{h}\right) \exp \left(-\widehat{\boldsymbol{\Psi}}^{h}\right) \boldsymbol{\Lambda}_{r}^{\top} \\
& =\boldsymbol{\Lambda}_{r} \mathbf{T}\left(\boldsymbol{\Psi}^{h}\right) \frac{d}{d S} \boldsymbol{\Psi}^{h} \boldsymbol{\Lambda}_{r}^{\top} .
\end{aligned}
$$

Recordando que $\boldsymbol{\Psi}^{h}=\sum_{i=1}^{2} N_{i} \boldsymbol{\Psi}_{i}$ y teniendo en cuenta que en el punto de interpolación $S=1 / 2(\xi=0)$, y

$$
\begin{array}{ll}
N_{1}(0)=0 & N_{1}^{\prime}(0)=-1 / 2 \\
N_{2}(0)=0 & N_{2}^{\prime}(0)=1 / 2
\end{array}
$$

se tiene

$$
\begin{aligned}
\left.\boldsymbol{\Psi}\right|_{\xi=0} & =\mathbf{0} \\
\left.\frac{d}{d S} \boldsymbol{\Psi}\right|_{\xi=0} & =\left.\frac{2}{L_{e}} \frac{d}{d \xi} \boldsymbol{\Psi}\right|_{\xi=0}=\frac{1}{L_{e}}\left(\boldsymbol{\Psi}_{J}-\boldsymbol{\Psi}_{I}\right) \\
\mathbf{T}\left(\left.\boldsymbol{\Psi}\right|_{\xi=0}\right) & =\mathbf{1}
\end{aligned}
$$

Luego el cambio de orientación en el punto de Gauss es

$$
\left.\boldsymbol{\kappa}\right|_{\xi=0}=\boldsymbol{\Lambda}_{r} \frac{1}{L_{e}}\left(\boldsymbol{\Psi}_{J}-\boldsymbol{\Psi}_{I}\right) \boldsymbol{\Lambda}_{r}^{\top}
$$


Es interesante señalar que la forma material del cambio de orientación en el punto de Gauss calculada a partir de este resultado vale precisamente $\left.\boldsymbol{K}\right|_{\xi=0}=$ $\left(\boldsymbol{\Psi}_{J}-\boldsymbol{\Psi}_{I}\right) / L_{e},[16$, ec. (4.9)].

Empleando esta fórmula de actualización del cambio de orientación se podría construir un elemento cuasi-objetivo, es decir una alternativa intermedia entre el elemento de Simó y Vu-Quoc (objetivo en el sentido de Ibrahimbegović y Taylor), y el elemento estrictamente objetivo de Crisfield y Jelenić, eludiendo la complicación de las funciones de forma generalizadas de este último. No obstante, en el desarrollo numérico llevado a cabo en este capítulo hemos decidido mantener la interpolación original de Simó y Vu-Quoc y la fórmula de actualización de Simó/Ibrahimbegović para disponer de una referencia con resultados comparables a los de la literatura. 


\subsection{Solución incremental-iterativa}

La trayectoria de equilibrio estimada mediante una solución puramente incremental va alejándose de la verdadera trayectoria como consecuencia de la desviación - drift, en inglés- que supone la estimación de cada nuevo punto a partir de la rigidez tangente. Una forma de estimar el error cometido consiste en calcular el residuo, definido como diferencia entre los valores nodales de las fuerzas internas y los valores nodales de las fuerzas exteriores, en cada uno de los pasos de la solución. Los métodos iterativos buscan una corrección de la solución al final de cada paso, que conduzca a la anulación del residuo, de forma que la solución corregida se encuentre de nuevo sobre la trayectoria de equilibrio. La corrección se lleva a cabo aplicando el método de Newton-Raphson al desarrollo en serie del residuo. En esta sección obtendremos las expresiones necesarias para la implementación del método iterativo y justificaremos por qué la matriz tangente apropiada para la iteración, que es la propuesta por Simó [62], no coincide con la deducida para el proceso incremental.

\subsubsection{Expresión del residuo}

Para el cálculo del residuo se parte de la ecuación de trabajos virtuales. La forma espacial del trabajo de las fuerzas internas sobre los incrementos de deformación

$$
\delta W_{\text {int }}=\int_{\Gamma_{0}} \stackrel{\nabla}{\delta} \boldsymbol{\epsilon} \cdot \boldsymbol{f} d S=\int_{\Gamma_{0}}(\stackrel{\nabla}{\delta} \boldsymbol{\gamma} \cdot \boldsymbol{n}+\stackrel{\nabla}{\delta} \boldsymbol{\kappa} \cdot \boldsymbol{m}) d S
$$

se puede expresar, empleando las ecuaciones (4.37),(4.41) y (4.42), como

$$
\begin{aligned}
\delta W_{\text {int }} & =\int_{\Gamma_{0}}\left\{\delta \boldsymbol{x}^{\top} \delta \boldsymbol{\omega}^{\top} \delta \boldsymbol{x}^{\prime \top} \delta \boldsymbol{\omega}^{\prime \top}\right\}\left[\begin{array}{cc}
\mathbf{0} & \mathbf{0} \\
-\widehat{\boldsymbol{\gamma}} & \mathbf{0} \\
\mathbf{1} & \mathbf{0} \\
\mathbf{0} & \mathbf{1}
\end{array}\right]\left\{\begin{array}{c}
\boldsymbol{n} \\
\boldsymbol{m}
\end{array}\right\} d S \\
& =\int_{\Gamma_{0}}\left\{\delta \boldsymbol{\phi}^{\top} \delta \boldsymbol{\phi}^{\top}\right\}\left[\begin{array}{c}
\boldsymbol{\epsilon}^{\top} \\
\mathbf{I}
\end{array}\right] \boldsymbol{f} d S .
\end{aligned}
$$

La contribución de cada elemento finito al incremento de trabajo de las fuerzas internas se puede calcular introduciendo la interpolación (7.6) en la expresión anterior

$$
\delta W_{\text {int }}^{e}=\delta \boldsymbol{\phi}^{e \top} \int_{-1}^{1}\left[\begin{array}{ll}
\mathbb{N}^{\top} & \frac{1}{J} \mathbb{N}^{\top} \top
\end{array}\right]\left[\begin{array}{c}
\mathbf{\epsilon}^{\top} \\
\mathbf{I}
\end{array}\right] \boldsymbol{f} J d \xi=\delta \boldsymbol{\phi}^{e} \cdot \mathbf{p}^{e} .
$$

En esta expresión $\mathbf{p}^{e}$ es la contribución del elemento al vector de fuerzas internas. La integral se evalúa con la misma cuadratura que la empleada en el cálculo de la rigidez tangente y $\mathbf{p}^{e}$ se puede calcular así

$$
\mathbf{p}^{e}=\sum_{l=1}^{g} w_{l}\left[\begin{array}{ll}
\mathbb{N}^{\top} & \frac{1}{J} \mathbb{N}^{\top} \top
\end{array}\right]_{\mid \xi=\xi_{l}}\left[\begin{array}{c}
\mathbf{\epsilon}^{\top} \\
\mathbf{I}
\end{array}\right]_{\left.\right|_{\xi=\xi_{l}}} \boldsymbol{f}\left(\xi_{l}\right) J\left(\xi_{l}\right) .
$$

Ensamblando las fuerzas internas de cada uno de los elementos, y eliminando las filas correspondientes a los grados de libertad coaccionados, se construye el vector de 
fuerzas internas de la estructura p. Entonces, el trabajo virtual de las fuerzas internas es (utilizando la notación de la sección anterior para el vector de variaciones nodales de la configuración)

$$
\delta W_{\text {int }}=\delta \boldsymbol{\phi} \cdot \mathbf{p},
$$

Por lo que respecta a las fuerzas exteriores, el trabajo de las fuerzas generalizadas $\lambda \boldsymbol{q}(S)$ sobre los incrementos de la configuración es

$$
\delta W_{e x t}=\lambda \int_{\Gamma_{0}} \delta \boldsymbol{\phi} \cdot \boldsymbol{q} d S=\lambda \int_{\Gamma_{0}}\left(\delta \boldsymbol{x} \cdot \boldsymbol{q}_{n}+\delta \boldsymbol{\omega} \cdot \boldsymbol{q}_{m}\right) d S,
$$

es decir

$$
\delta W_{\text {ext }}=\lambda \int_{\Gamma_{0}}\left\{\delta \boldsymbol{x}^{\top} \quad \Delta \boldsymbol{\omega}^{\top}\right\}\left\{\begin{array}{c}
\boldsymbol{q}_{n} \\
\boldsymbol{q}_{m}
\end{array}\right\} d S=\lambda \int_{\Gamma_{0}} \delta \boldsymbol{\phi}^{\top} \boldsymbol{q} d S .
$$

Introduciendo en esta expresión la interpolación propuesta se obtiene la contribución de un elemento al trabajo virtual realizado por las fuerzas exteriores

$$
\delta W_{e x t}^{e}=\delta \phi^{e \top} \lambda \int_{-1}^{1} \mathbb{N}^{\top} \boldsymbol{q} J d \xi=\delta \boldsymbol{\phi}^{e} \cdot \lambda \mathbf{f}^{0 e} .
$$

El vector $\mathbf{f}^{0 e}$ es la contribución del elemento al vector de fuerzas exteriores totales y coincide, precisamente, con el vector de fuerzas iniciales introducido en la sección anterior. Resolviendo la integral mediante la cuadratura de Gauss,

$$
\mathbf{f}^{0 e}=\sum_{l=1}^{g} w_{l} \mathbb{N}^{\top}\left(\xi_{l}\right) \boldsymbol{q}\left(\xi_{l}\right) J\left(\xi_{l}\right) .
$$

Los vectores de fuerzas iniciales en cada elemento se ensamblan con las fuerzas exteriores que actúan sobre cada nodo

$$
\lambda \boldsymbol{f}_{i}=\lambda\left\{\boldsymbol{n}_{i} \boldsymbol{m}_{i}\right\}^{\top}
$$

— cuya contribución al trabajo virtual exterior es $\delta \boldsymbol{\phi}_{i} \cdot \lambda \boldsymbol{f}_{i}$ - en las posiciones correspondientes. Después del ensamblaje se eliminan las filas correspondientes a los grados de libertad coaccionados; el resultado es el vector de fuerzas $\lambda \mathbf{f}$. Entonces, el trabajo virtual de las fuerzas exteriores sobre la estructura se puede expresar así

$$
\delta W_{\text {ext }}=\delta \boldsymbol{\phi} \cdot \lambda \mathbf{f},
$$

y la ecuación de trabajos virtuales exige que en el equilibrio, el trabajo virtual de las fuerzas internas sea igual al de las fuerzas exteriores para cualquier variación cinemáticamente admisible de la configuración

$$
\delta \boldsymbol{\phi} \cdot(\mathbf{p}-\lambda \mathbf{f})=0 \quad \forall \delta \boldsymbol{\phi} .
$$

Por tanto, el residuo, que se define como la diferencia entre los vectores de fuerzas nodales internas y externas, debe ser nulo en configuraciones pertenecientes a la trayectoria de equilibrio

$$
\mathbf{r}=\mathbf{p}-\lambda \mathbf{f}=\mathbf{0} .
$$

Conviene recordar que ya hemos eliminado de los vectores $\mathbf{p}$ y $\mathbf{f}$ las posiciones correspondientes a los grados de libertad coaccionados, que multiplican a componentes nulas de la variación de la configuración, y cuya diferencia podría no ser nula. 


\subsubsection{Linealización del residuo}

Supongamos que se lleva a cabo la corrección de la solución definida por la configuración $\boldsymbol{\Phi}_{(n)}, \lambda_{(n)}$ obtenida en el paso $n$ de un proceso incremental; ésta se compone en cada uno de los nodos por el vector posición $\boldsymbol{x}_{i(n)}$ y la rotación $\boldsymbol{\Lambda}_{i(n)}$. Para ello se plantea un proceso iterativo en el que, a partir de la solución inicial, que denotamos $\boldsymbol{\phi}_{(n)}^{(0)}$, se calcula una sucesión de configuraciones $\boldsymbol{\phi}_{(n)}^{(k)}$ con la condición de que al final del proceso, el residuo asociado a la última configuración no supere un cierto valor muy pequeño. El desarrollo en serie del residuo alrededor de la solución $\boldsymbol{\phi}^{(k)}, \lambda^{(k)}$ - prescindimos por claridad del subíndice $(n)$ - se calcula sumando a ésta la derivada direccional (o lo que es lo mismo, la variación espacial) del residuo en la dirección $\Delta \boldsymbol{\phi}$ y en la dirección $\Delta \lambda$

$$
\mathbf{r}(\boldsymbol{\phi}, \lambda)=\mathbf{r}(\boldsymbol{\phi}, \lambda)^{(k)}+\Delta \mathbf{r}(\boldsymbol{\phi}, \lambda)^{(k)}+\frac{\partial \mathbf{r}}{\partial \lambda}(\boldsymbol{\phi}, \lambda)^{(k)} \Delta \lambda
$$

El superíndice hace referencia a que las funciones y derivadas se evalúan en la configuración $\left(\boldsymbol{\phi}^{(k)}, \lambda^{(k)}\right)$. La derivada del residuo en la dirección $\Delta \boldsymbol{\phi}$-compuesta por vectores $\Delta \boldsymbol{x}_{i}, \Delta \boldsymbol{\omega}_{i}$ en cada nodo- se puede expresar de forma simbólica así

$$
\Delta \mathbf{r}(\boldsymbol{\phi}, \lambda)^{(k)}=\left.\frac{d}{d \varepsilon} \mathbf{r}\left(\boldsymbol{x}_{i}^{(k)}+\varepsilon \Delta \boldsymbol{x}_{i}, \exp \left(\varepsilon \Delta \widehat{\boldsymbol{\omega}}_{i}\right) \boldsymbol{\Lambda}_{i}^{(k)}\right)\right|_{\varepsilon=0},
$$

y equivale al cálculo de su variación espacial. Dado que ahora (en el proceso iterativo) la linealización se lleva a cabo después de la discretización, ya no aparecen términos del tipo $\Delta \delta \phi$, que son los que daban lugar a la parte antisimétrica del operador tangente. Por ello, al operar este término se obtiene la matriz de rigidez tangente deducida por Simó y Vu-Quoc. En el desarrollo de la linealización se parte de la expresión de la variación espacial del residuo como diferencia entre la variación del vector de fuerzas internas y el de fuerzas exteriores ${ }^{2}$

$$
\Delta \mathbf{r}=\Delta \mathbf{p}-\lambda \Delta \mathbf{f}
$$

\section{Derivada direccional del vector de fuerzas internas}

Comenzaremos calculando la derivada direccional del vector de fuerzas internas p. Ésta se obtiene ensamblando la variación espacial de los vectores correspondientes a cada uno de los elementos (7.52)

$$
\Delta \mathbf{p}^{e}=\Delta \int_{-1}^{1}\left[\begin{array}{ll}
\mathbb{N}^{\top} & \frac{1}{J} \mathbb{N}^{\prime} \mathrm{T}
\end{array}\right]\left[\begin{array}{c}
\mathbf{\epsilon}^{\top} \\
\mathbf{I}
\end{array}\right] \boldsymbol{f} J d \xi
$$

\footnotetext{
${ }^{2}$ No incluimos en esta expresión el término correspondiente a la variación $\Delta \lambda$, puesto que ya lo hemos tenido en cuenta como último sumando en la ecuación correspondiente a linealización del residuo $(\mathbf{7 . 6 0})$.
} 
que, desarrollando el integrando, también admite esta expresión

$$
\Delta \mathbf{p}^{e}=\Delta \int_{-1}^{1}\left[\begin{array}{cccc}
N_{1} \mathbf{1} & \mathbf{0} & \frac{1}{J} N_{1}^{\prime} \mathbf{1} & \mathbf{0} \\
\mathbf{0} & N_{1} \mathbf{1} & \mathbf{0} & \frac{1}{J} N_{1}^{\prime} \mathbf{1} \\
\vdots & \vdots & \vdots & \vdots \\
N_{n} \mathbf{1} & \mathbf{0} & \frac{1}{J} N_{n}^{\prime} \mathbf{1} & \mathbf{0} \\
\mathbf{0} & N_{n} \mathbf{1} & \mathbf{0} & \frac{1}{J} N_{n}^{\prime} \mathbf{1}
\end{array}\right]\left\{\begin{array}{c}
\mathbf{0} \\
-\widehat{\gamma} \boldsymbol{n} \\
\boldsymbol{n} \\
\boldsymbol{m}
\end{array}\right\} J d \xi
$$

La matriz de funciones de forma no se ve afectada por la variación espacial, entonces

$$
\Delta \mathbf{p}^{e}=\int_{-1}^{1}\left[\begin{array}{cccc}
N_{1} \mathbf{1} & \mathbf{0} & \frac{1}{J} N_{1}^{\prime} \mathbf{1} & \mathbf{0} \\
\mathbf{0} & N_{1} \mathbf{1} & \mathbf{0} & \frac{1}{J} N_{1}^{\prime} \mathbf{1} \\
\vdots & \vdots & \vdots & \vdots \\
N_{n} \mathbf{1} & \mathbf{0} & \frac{1}{J} N_{n}^{\prime} \mathbf{1} & \mathbf{0} \\
\mathbf{0} & N_{n} \mathbf{1} & \mathbf{0} & \frac{1}{J} N_{n}^{\prime} \mathbf{1}
\end{array}\right] \Delta\left\{\begin{array}{c}
\mathbf{0} \\
-\widehat{\gamma} \boldsymbol{n} \\
\boldsymbol{n} \\
\boldsymbol{m}
\end{array}\right\} J d \xi
$$

Ya hemos calculado la variación espacial del vector del integrando en la introducción al capítulo 6. El resultado, que es el obtenido por Simó y Vu-Quoc 62] y Kapania y Li [34], es el siguiente

$$
\begin{array}{r}
\Delta\left\{\begin{array}{c}
\mathbf{0} \\
-\widehat{\gamma} \boldsymbol{n} \\
\boldsymbol{n} \\
\boldsymbol{m}
\end{array}\right\}=\left[\begin{array}{cccc}
\mathbf{0} & \mathbf{0} & \mathbf{0} & \mathbf{0} \\
\mathbf{0} & -\widehat{\gamma} \mathbf{c}_{\gamma \gamma} \widehat{\gamma} & -\widehat{\gamma} \mathbf{c}_{\gamma \gamma} & -\widehat{\gamma} \mathbf{c}_{\gamma \kappa} \\
\mathbf{0} & \mathbf{c}_{\gamma \gamma} \widehat{\gamma} & \mathbf{c}_{\gamma \gamma} & \mathbf{c}_{\gamma \kappa} \\
\mathbf{0} & \mathbf{c}_{\kappa \gamma} \widehat{\gamma} & \mathbf{c}_{\kappa \gamma} & \mathbf{c}_{\kappa \kappa}
\end{array}\right]\left\{\begin{array}{c}
\Delta \boldsymbol{x} \\
\Delta \boldsymbol{\omega} \\
\Delta \boldsymbol{x}^{\prime} \\
\Delta \boldsymbol{\omega}^{\prime}
\end{array}\right\} \\
+\left[\begin{array}{cccc}
\mathbf{0} & \mathbf{0} & \mathbf{0} & \mathbf{0} \\
\mathbf{0} & \widehat{\gamma} \widehat{\mathbf{n}} & \widehat{\mathbf{n}} & \mathbf{0} \\
\mathbf{0} & -\widehat{\mathbf{n}} & \mathbf{0} & \mathbf{0} \\
\mathbf{0} & -\widehat{\mathbf{m}} & \mathbf{0} & \mathbf{0}
\end{array}\right]\left\{\begin{array}{c}
\Delta \boldsymbol{x} \\
\Delta \boldsymbol{\omega} \\
\Delta \boldsymbol{x}^{\prime} \\
\Delta \boldsymbol{\omega}^{\prime}
\end{array}\right\}
\end{array}
$$

y, como se vio, se compone de una parte constitutiva y otra geométrica. Podemos sintetizar estas expresiones utilizando la notación introducida en el capítulo anterior

$$
\begin{aligned}
\Delta \mathbf{p}^{e}=\int_{-1}^{1}\left[\begin{array}{ll}
\mathbb{N}^{\top} & \frac{1}{J} \mathbb{N}^{\prime} \mathrm{T}
\end{array}\right]\left[\begin{array}{ll}
\mathbf{d}_{00}^{M} & \mathbf{d}_{01}^{M} \\
\mathbf{d}_{10}^{M} & \mathbf{d}_{11}^{M}
\end{array}\right] & \left\{\begin{array}{c}
\Delta \phi \\
\Delta \boldsymbol{\phi}^{\prime}
\end{array}\right\} J d \xi \\
& +\int_{-1}^{1}\left[\begin{array}{ll}
\mathbb{N}^{\top} & \frac{1}{J} \mathbb{N}^{\prime} \boldsymbol{\top}
\end{array}\right]\left[\begin{array}{cc}
\mathbf{d}_{00}^{G S} & \mathbf{d}_{01}^{G S} \\
\mathbf{d}_{10}^{G S} & \mathbf{d}_{11}^{G S}
\end{array}\right]\left\{\begin{array}{c}
\Delta \phi \\
\Delta \boldsymbol{\phi}^{\prime}
\end{array}\right\} J d \xi .
\end{aligned}
$$

Si discretizamos las variables de la configuración y sus derivadas resulta

$$
\begin{aligned}
\Delta \mathbf{p}^{e}=\int_{-1}^{1}\left[\begin{array}{ll}
\mathbb{N}^{\top} & \frac{1}{J} \mathbb{N}^{\prime}
\end{array}\right]\left[\begin{array}{ll}
\mathbf{d}_{00}^{M} & \mathbf{d}_{01}^{M} \\
\mathbf{d}_{10}^{M} & \mathbf{d}_{11}^{M}
\end{array}\right]\left[\begin{array}{c}
\mathbb{N} \\
\frac{1}{J} \mathbb{N}^{\prime}
\end{array}\right] J d \xi \Delta \boldsymbol{\phi}^{e} \\
+\int_{-1}^{1}\left[\begin{array}{ll}
\mathbb{N}^{\top} & \frac{1}{J} \mathbb{N}^{\prime} \mathrm{T}
\end{array}\right]\left[\begin{array}{ll}
\mathbf{d}_{00}^{G S} & \mathbf{d}_{01}^{G S} \\
\mathbf{d}_{10}^{G S} & \mathbf{d}_{11}^{G S}
\end{array}\right]\left[\begin{array}{c}
\mathbb{N} \\
\frac{1}{J} \mathbb{N}^{\prime}
\end{array}\right] J d \xi \Delta \boldsymbol{\phi}^{e} .
\end{aligned}
$$


La primera integral es la matriz de rigidez constitutiva y la segunda, la matriz de rigidez geométrica, entonces

$$
\Delta \mathbf{p}^{e}=\left(\mathbf{K}^{M e}+\mathbf{K}^{G S e}\right) \Delta \boldsymbol{\phi}^{e} .
$$

Evaluando las integrales con la cuadratura de Gauss de orden $g$

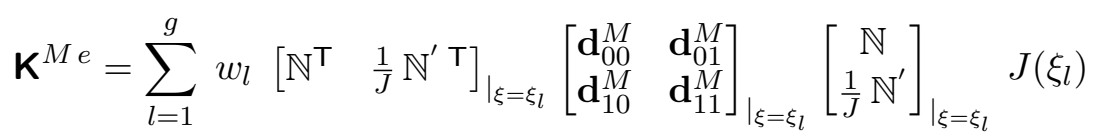

$$
\begin{aligned}
& \mathbf{K}^{G S e}=\sum_{l=1}^{g} w_{l}\left[\begin{array}{ll}
\mathbb{N}^{\top} & \frac{1}{J} \mathbb{N}^{\prime} \mathrm{T}
\end{array}\right]_{\mid \xi=\xi_{l}}\left[\begin{array}{cc}
\mathbf{d}_{00}^{G S} & \mathbf{d}_{01}^{G S} \\
\mathbf{d}_{10}^{G S} & \mathbf{d}_{11}^{G S}
\end{array}\right]_{\left.\right|_{\xi=\xi_{l}}}\left[\begin{array}{c}
\mathbb{N} \\
\frac{1}{J} \mathbb{N}^{\prime}
\end{array}\right]_{\mid \xi=\xi_{l}} J\left(\xi_{l}\right)
\end{aligned}
$$

Ensamblando la contribución de cada uno de los elementos y eliminando las filas y columnas correspondientes a los grados de libertad coaccionados se obtiene la siguiente expresión para el vector variación de las fuerzas internas en la estructura

$$
\Delta \mathbf{p}=\left(\mathbf{K}^{M}+\mathbf{K}^{G S}\right) \Delta \boldsymbol{\phi} .
$$

\section{Derivada direccional del vector de fuerzas exteriores}

El vector de fuerzas exteriores $\mathbf{f}$ se obtiene ensamblando las fuerzas iniciales en cada elemento $\mathbf{f}^{0 e}$ con las fuerzas nodales $\boldsymbol{f}_{i}$. Calculamos en primer lugar la variación espacial de las fuerzas iniciales en un elemento

$$
\Delta \mathbf{f}^{0 e}=\Delta \int_{-1}^{1} \mathbb{N}^{\top} \boldsymbol{q} J d \xi=\int_{-1}^{1} \mathbb{N}^{\top} \Delta \boldsymbol{q} J d \xi
$$

Recordamos que $\boldsymbol{q}=\left\{\begin{array}{ll}\boldsymbol{q}_{n} & \boldsymbol{q}_{m}\end{array}\right\}^{\top}$, y que considerábamos dos tipos de fuerzas: las espaciales, $\boldsymbol{q}^{s}$, de componentes constantes en el sistema de referencia espacial, y las perseguidoras, $\boldsymbol{q}^{f}=\boldsymbol{\Lambda} \boldsymbol{Q}^{f}$, de componentes constantes en el sistema de referencia intrínseco (de la sección). Por tanto (prescindiendo del subíndice)

$$
\Delta \boldsymbol{q}^{s}=\mathbf{0}
$$

y

$$
\begin{aligned}
\Delta \boldsymbol{q}^{f} & =\Delta\left(\boldsymbol{\Lambda} \boldsymbol{Q}^{f}\right)=\Delta \boldsymbol{\Lambda} \boldsymbol{\Lambda}^{\top} \boldsymbol{\Lambda} \boldsymbol{Q}^{f} \\
& =\Delta \widehat{\boldsymbol{\omega}} \boldsymbol{q}^{f}=-\widehat{\mathbf{q}}^{f} \Delta \boldsymbol{\omega} .
\end{aligned}
$$

Por lo tanto

$$
\Delta \mathbf{f}^{0 e}=\int_{-1}^{1}\left[\begin{array}{cc}
N_{1} \mathbf{1} & \mathbf{0} \\
\mathbf{0} & N_{1} \mathbf{1} \\
\vdots & \vdots \\
N_{n} \mathbf{1} & \mathbf{0} \\
\mathbf{0} & N_{n} \mathbf{1}
\end{array}\right]\left[\begin{array}{cc}
\mathbf{0} & -\widehat{\mathbf{q}}_{n}^{f} \\
\mathbf{0} & -\widehat{\mathbf{q}}_{m}^{f}
\end{array}\right]\left\{\begin{array}{c}
\Delta \boldsymbol{x} \\
\Delta \boldsymbol{\omega}
\end{array}\right\} J d \xi
$$


Podemos sintetizar esta expresión para conseguir una disposición de las variables análoga a la de la linealización del vector de fuerzas internas, con objeto de facilitar el ensamblaje

$$
\Delta \mathbf{f}^{0 e}=\int_{-1}^{1}\left[\mathbb{N}^{\top} \quad \frac{1}{J} \mathbb{N}^{\prime}\right]\left[\begin{array}{cc}
\mathbf{d}^{Q S} & \mathbf{0} \\
\mathbf{0} & \mathbf{0}
\end{array}\right]\left\{\begin{array}{c}
\Delta \boldsymbol{\phi} \\
\Delta \boldsymbol{\phi}^{\prime}
\end{array}\right\} J d \xi
$$

con

$$
\mathbf{d}^{Q S}=\left[\begin{array}{ll}
\mathbf{0} & -\widehat{\mathbf{q}}_{n}^{f} \\
\mathbf{0} & -\widehat{\mathbf{q}}_{m}^{f}
\end{array}\right] .
$$

Discretizando los incrementos de la configuración y sus derivadas

$$
\Delta \mathbf{f}^{0 e}=\int_{-1}^{1}\left[\begin{array}{ll}
\mathbb{N}^{\top} & \frac{1}{J} \mathbb{N}^{\prime} \top
\end{array}\right]\left[\begin{array}{cc}
\mathbf{d}^{Q S} & \mathbf{0} \\
\mathbf{0} & \mathbf{0}
\end{array}\right]\left[\begin{array}{c}
\mathbb{N} \\
\frac{1}{J} \mathbb{N}^{\prime}
\end{array}\right] J d \xi \Delta \boldsymbol{\phi}^{e} .
$$

La integral es precisamente la rigidez debida a las fuerzas generalizadas que denotamos $\mathbf{K}^{Q e}$. Por tanto, se puede escribir

$$
\Delta \mathbf{f}^{0 e}=\mathbf{K}^{Q e} \Delta \boldsymbol{\phi}^{e} .
$$

con

$$
\mathbf{K}^{Q e}=\sum_{l=1}^{g} w_{l}\left[\begin{array}{ll}
\mathbb{N}^{\top} & \frac{1}{J}
\end{array} \mathbb{N}^{\prime}\right]_{\left.\right|_{\xi=\xi_{l}}}\left[\begin{array}{cc}
\mathbf{d}^{Q S} & \mathbf{0} \\
\mathbf{0} & \mathbf{0}
\end{array}\right]_{\left.\right|_{\xi=\xi_{l}}}\left[\begin{array}{c}
\mathbb{N} \\
\frac{1}{J} \mathbb{N}^{\prime}
\end{array}\right]_{\left.\right|_{\xi=\xi_{l}}} J\left(\xi_{l}\right)
$$

evaluada mediante una cuadratura de Gauss de orden $g$.

Por otro lado, la variación de las fuerzas nodales $\boldsymbol{f}_{i}=\left\{\begin{array}{ll}\boldsymbol{n}_{i} & \boldsymbol{m}_{i}\end{array}\right\}^{\top}$ también contribuye a la rigidez. Si consideramos que las fuerzas se descomponen en una parte espacial y una perseguidora, entonces su variación espacial sigue las mismas reglas que la de las fuerzas generalizadas

$$
\Delta \boldsymbol{f}_{i}=\Delta\left\{\begin{array}{c}
\boldsymbol{n}_{i} \\
\boldsymbol{m}_{i}
\end{array}\right\}=\left[\begin{array}{cc}
\mathbf{0} & -\widehat{\mathbf{n}}_{i} \\
\mathbf{0} & -\widehat{\mathbf{m}}_{i}
\end{array}\right]\left\{\begin{array}{c}
\Delta \boldsymbol{x}_{i} \\
\Delta \boldsymbol{\omega}_{i}
\end{array}\right\} .
$$

Esta matriz se ensamblará en las posiciones correspondientes a los grados de libertad $\Delta \boldsymbol{x}_{i}$ y $\Delta \boldsymbol{\omega}_{i}$. Ensamblando también la rigidez debida a las fuerzas generalizadas de cada elemento, y eliminando las filas y columnas correspondientes a los grados de libertad coaccionados obtenemos la rigidez tangente debida a las fuerzas exteriores (load stiffness). Entonces

$$
\Delta \mathbf{f}=\mathbf{K}^{Q} \Delta \boldsymbol{\phi}
$$

Finalmente, la derivada direccional del residuo en la dirección $\Delta \boldsymbol{\phi}$ es

$$
\Delta \mathbf{r}=\Delta \mathbf{p}-\lambda \Delta \mathbf{f}=\left(\mathbf{K}^{M}+\mathbf{K}^{S G}-\lambda \mathbf{K}^{Q}\right) \Delta \boldsymbol{\phi}=\mathbf{K}^{S} \Delta \boldsymbol{\phi} .
$$

El superíndice $S$ distingue esta matriz de rigidez tangente, deducida para ser empleada en el proceso iterativo, a partir de la linealización del residuo (después de la discretización), de la matriz tangente $\mathbf{K}$ resultado de linealizar la ecuación de equilibrio tangente (previamente a la discretización), valida para el proceso incremental. La diferencia entre ellas tiene su origen en la parte antisimétrica del operador que ha sido estudiada en el capítulo 6 . 


\subsubsection{Proceso iterativo}

A partir de los resultados anteriores es posible escribir el desarrollo en serie del residuo y de la restricción que define la estrategia de solución entre las iteraciones $(k)$ $\mathrm{y}(k+1)$

$$
\begin{aligned}
\mathbf{r}(\boldsymbol{\phi}, \lambda)^{(k+1)}=\mathbf{r}(\boldsymbol{\phi}, \lambda)^{(k)}+\mathbf{K}^{S}(\boldsymbol{\phi}, \lambda)^{(k)} \Delta \boldsymbol{\phi}^{(k, k+1)} & \\
& +\frac{\partial \mathbf{r}}{\partial \lambda}(\boldsymbol{\phi}, \lambda)^{(k)} \Delta \lambda^{(k, k+1)} \\
c(\Delta \boldsymbol{\phi}, \Delta \lambda)^{(k+1)}=c(\Delta \boldsymbol{\phi}, \Delta \lambda)^{(k)}+\frac{\partial c}{\partial \Delta \boldsymbol{\phi}}(\boldsymbol{\phi}, \lambda)^{(k)} & \Delta \boldsymbol{\phi}^{(k, k+1)} \\
& +\frac{\partial c}{\partial \lambda}(\boldsymbol{\phi}, \lambda)^{(k)} \Delta \lambda^{(k, k+1)} .
\end{aligned}
$$

Si en la iteración $k+1$ se ha retornado a la trayectoria de equilibrio, entonces

$$
\begin{aligned}
\mathbf{r}(\boldsymbol{\phi}, \lambda)^{(k+1)} & =\mathbf{0} \\
c(\Delta \boldsymbol{\phi}, \Delta \lambda)^{(k+1)} & =0 .
\end{aligned}
$$

Entonces los incrementos de las variables de la configuración y del parámetro entre las iteraciones $k$ y $k+1$ se calculan resolviendo el siguiente sistema de ecuaciones (prescindiendo por claridad de los superíndices)

$$
\left[\begin{array}{cc}
\mathbf{K}^{S} & -\mathbf{f} \\
\mathbf{a}^{\mathbf{T}} & g
\end{array}\right]\left\{\begin{array}{c}
\Delta \boldsymbol{\phi} \\
\Delta \lambda
\end{array}\right\}=-\left\{\begin{array}{l}
\mathbf{r} \\
c
\end{array}\right\}
$$

En este sistema todas las componentes de la matriz y del vector se evalúan en la iteración $k$. En el primer bloque de filas aparece $-\mathbf{f}$ que es precisamente $\partial \mathbf{r} / \partial \lambda$. El significado de los elementos de la fila inferior es obvio:

$$
\begin{aligned}
& \mathbf{a}=\frac{\partial c}{\partial \Delta \boldsymbol{\phi}} \\
& g=\frac{\partial c}{\partial \lambda}
\end{aligned}
$$

evaluados en $\left(\boldsymbol{\phi}^{(k)}, \lambda^{(k)}\right)$. Los valores de a y $c$ dependen de la estrategia de control elegida. Veamos las tres posibilidades consideradas:

1. Control de carga. En este caso la restricción es

$$
\Delta \lambda^{(k, k+1)}=0
$$

Entonces

$$
\begin{aligned}
& \mathbf{a}=\mathbf{0} \\
& g=1,
\end{aligned}
$$


y la ecuación se reduce a

$$
\Delta \boldsymbol{\Phi}^{(k, k+1)}=-\left(\mathbf{K}^{S}\right)^{-1} \mathbf{r},
$$

con

$$
\lambda^{(k+1)}=\lambda^{(k)} .
$$

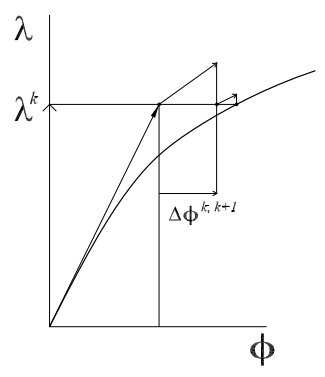

Figura 7.3: Control de carga

2. Control de desplazamientos. La restricción correspondiente al control de desplazamientos es

$$
\Delta \boldsymbol{\Phi}^{(0, k)} \cdot \Delta \boldsymbol{\phi}^{(0, k)}-l_{n}^{2}=0,
$$

para la que

$$
\begin{aligned}
& \mathbf{a}=2 \Delta \boldsymbol{\phi}^{(0, k)} \\
& g=0 .
\end{aligned}
$$

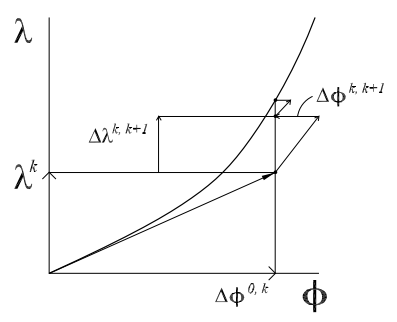

Figura 7.4: Control de desplazamiento 
3. Control de arco. Esta estrategia es algo más complicada. En este caso se puede demostrar que la ecuación de restricción es

$$
\frac{1}{f^{(0)}}\left|\mathbf{v}^{(0)} \cdot \Delta \boldsymbol{\phi}^{(0, k)}+\Delta \lambda^{(0, k)}\right|-l_{n}=0,
$$

con

$$
f^{(0)}=\sqrt{1+\mathbf{v}^{(0)} \cdot \mathbf{v}^{(0)}} .
$$

Entonces

$$
\begin{aligned}
& \mathbf{a}=\frac{1}{f^{(0)}} \mathbf{v}^{(0)} \\
& g=\frac{1}{f^{(0)}}
\end{aligned}
$$

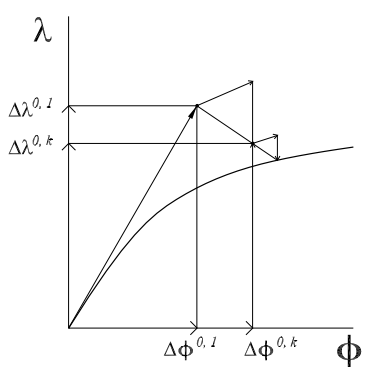

Figura 7.5: Control de arco

Existe un gran número de referencias que contienen detalles del método iterativo, así como de sus variantes y mejoras, por ejemplo [19], que ha sido la seguida en esta tesis. Una vez obtenida la corrección en la iteración $k$ es necesario proceder a la actualización de todas las variables que intervienen en el cálculo siguiendo los procedimientos expuestos en la sección 7.2.2. El proceso se repetirá hasta lograr la convergencia de la solución con alguno de los criterios contenidos en la literatura. En nuestro caso hemos optado por limitar la norma euclídea del residuo así como el cociente entre la norma en el paso de predicción (iteración 0) y la última iteración.

Comentario 7.3.1. Sobre la matriz de rigidez tangente en el proceso incremental - iterativo. En la proposición 6.2.2 del capítulo anterior demostramos que los términos antisimétricos del operador tangente, derivados de la segunda variación de las variables de la configuración, se anulan sobre la trayectoria de equilibrio.

En el proceso incremental - iterativo se parte de la última configuración corregida $\boldsymbol{\Phi}_{(n-1)}$. Ésta se encuentra, prácticamente, sobre la trayectoria de equilibrio, salvo por 
el error admitido en la convergencia. A partir de ella se va a calcular una solución inicial (predicción) $\boldsymbol{\Phi}_{(n)}^{(0)}$ empleando las expresiones 7.15 y 7.27:

$$
\begin{aligned}
\mathbf{v} & =\mathbf{K}\left(\boldsymbol{\phi}_{(n-1)}\right)^{-1} \mathbf{f} \\
\Delta \boldsymbol{\phi}_{(n)}^{(0)} & =\mathbf{v} \Delta \lambda .
\end{aligned}
$$

La rigidez tangente $\mathbf{K}$ se evalúa con las variables correspondientes a la configuración de equilibrio $\boldsymbol{\Phi}_{(n-1)}$. Por la proposición 6.2.2 los términos antisimétricos del operador tangente se cancelan, y por ello $\mathbf{K}=\mathbf{K}^{S}$. Luego para la predicción se puede usar el operador tangente de Simó y Vu-Quoc.

En la fase de corrección de la solución los incrementos de las variables de la configuración se calculan mediante la ecuación (7.75), en la que interviene directamente la matriz tangente de Simó $\mathbf{K}^{S}$. De esta forma queda demostrado que el proceso incremental - iterativo se basa en el operador tangente de Simó, tanto en la predicción como en la corrección; aunque el origen de la matriz tangente es distinto (en la predicción la discretización es posterior a la linealización, y viceversa en la corrección), el resultado final es idéntico en ambos casos.

Por todo lo expuesto, la matriz tangente $\mathbf{K}$ consecuencia del operador deducido en el capítulo anterior únicamente puede conducir a resultados diferentes de los obtenidos por Simó en soluciones puramente incrementales, ya que en ellas se abandona la trayectoria de equilibrio, y los términos antisimétricos derivados de la segunda variación de la configuración dejan de ser nulos. 


\subsection{Flujo del programa}

En esta sección describimos esquemáticamente el algoritmo que se ha diseñado para la solución del problema en forma espacial empleando elementos rectos de dos nodos. Sólo los módulos de programa más relevantes aparecen mencionados de forma explícita.

\subsubsection{Datos iniciales}

\section{Variables nodales}

\begin{tabular}{|l|cc|}
\hline Configuración inicial $^{(1)}$ & $\boldsymbol{x}_{i(0)}$ & $\boldsymbol{\Lambda}_{i(0)}$ \\
Fuerzas espaciales & $\boldsymbol{n}_{i}$ & $\boldsymbol{m}_{i}$ \\
Fuerzas perseguidoras & $\boldsymbol{N}_{i}$ & $\boldsymbol{M}_{i}$ \\
\hline
\end{tabular}

Variables del elemento ${ }^{(2)}$

\begin{tabular}{l|cc|}
\hline Orientación inicial $^{(3)}$ & $\Lambda_{(0)}^{e}$ & \\
Deformaciones iniciales (forma material) $^{(4)}$ & $\boldsymbol{\Gamma}_{(0)}^{e}$ & $\boldsymbol{K}_{(0)}^{e}$ \\
Deformaciones iniciales (forma espacial) $^{(5)}$ & $\gamma_{(0)}^{e}$ & $\boldsymbol{\kappa}_{(0)}^{e}$ \\
\hline
\end{tabular}

1. Las rotaciones nodales iniciales expresadas en el sistema general se toman, por defecto, iguales a la identidad

$$
\boldsymbol{\Lambda}_{i(0)} \equiv\left[\begin{array}{lll}
1 & 0 & 0 \\
0 & 1 & 0 \\
0 & 0 & 1
\end{array}\right]
$$

También se definen inicialmente los grados de libertad coaccionados.

2. Todas las variables relativas al elemento están definidas en el punto de integración (de Gauss), $\xi=0$.

3. Para definir la orientación inicial del elemento se adopta el criterio definido a continuación (suponiendo que el eje $y$ define la dirección vertical). El vector $\boldsymbol{a}_{1}$ es el vector unitario en la dirección determinada por los nodos $i, j$ del elemento.

$$
\boldsymbol{a}_{1}=\frac{\boldsymbol{x}_{j}-\boldsymbol{x}_{i}}{\left\|\boldsymbol{x}_{j}-\boldsymbol{x}_{i}\right\|} .
$$

El vector unitario $\boldsymbol{a}_{3}$ se escoge de forma que sea perpendicular a $\boldsymbol{a}_{1}$ y esté contenido en el plano horizontal, lo que conduce a la siguiente expresión en componentes del sistema general

$$
\boldsymbol{a}_{3} \equiv\left\{\begin{array}{lll}
a_{3}^{1} & 0 & a_{3}^{3}
\end{array}\right\}^{\top},
$$


con

$$
a_{3}^{1}=+\sqrt{\frac{1}{1+\left(a_{1}^{1}\right)^{2} /\left(a_{1}^{3}\right)^{2}}}
$$

y

$$
a_{3}^{3}=-a_{3}^{1} \frac{a_{1}^{1}}{a_{1}^{3}} .
$$

Estas expresiones presentan una singularidad cuando el elemento está orientado en la dirección $y\left(a_{1}^{3}=0\right)$, que se resuelve tomando

$$
\boldsymbol{a}_{3} \equiv\left\{\begin{array}{lll}
0 & 0 & 1
\end{array}\right\}^{\top} .
$$

Por último $\boldsymbol{a}_{2}=\boldsymbol{a}_{3} \times \boldsymbol{a}_{1}$, y la matriz de rotación inicial del elemento se obtiene escribiendo en columnas los vectores anteriores

$$
\boldsymbol{\Lambda}_{0}^{e} \equiv\left[\begin{array}{lll}
\boldsymbol{a}_{1} & \boldsymbol{a}_{2} & \boldsymbol{a}_{3}
\end{array}\right] .
$$

4. Para el elemento de dos nodos (recto) $\boldsymbol{K}_{0}^{e} \equiv\left\{\begin{array}{lll}0 & 0 & 0\end{array}\right\}^{\top}$. Por otro lado $\boldsymbol{\Gamma}_{0}^{e} \equiv$ $\left\{\begin{array}{lll}1 & 0 & 0\end{array}\right\}^{\top}$ define la condición de sección inicialmente normal a la directriz.

5. La forma espacial de las deformaciones iniciales es

$$
\begin{aligned}
\boldsymbol{\gamma}_{0}^{e} & =\boldsymbol{\Lambda}_{0}^{e} \boldsymbol{\Gamma}_{0}^{e} \\
\boldsymbol{\kappa}_{0}^{e} & =\mathbf{0} .
\end{aligned}
$$

\subsubsection{Proceso incremental}

El paso desde el incremento $n$ al $n+1$ constituye el núcleo del proceso incremental puro. Las variables de partida se resumen en la siguiente tabla:

Variables correspondientes al incremento $n$

\begin{tabular}{|l|cc|}
\hline Factor de carga & $\lambda_{(n)}$ & \\
Configuración nodal & $\boldsymbol{x}_{i(n)}$ & $\boldsymbol{\Lambda}_{i(n)}$ \\
Incrementos de la configuración & $\Delta \boldsymbol{x}_{i(n-1, n)}$ & $\Delta \boldsymbol{\omega}_{i(n-1, n)}$ \\
\hline \hline Rotación del elemento & $\boldsymbol{\Lambda}_{(n)}^{e}$ & \\
Deformaciones & $\boldsymbol{\gamma}_{(n)}^{e}$ & $\boldsymbol{\kappa}_{(n)}^{e}$ \\
Esfuerzos & $\boldsymbol{n}_{(n)}^{e}$ & $\boldsymbol{m}_{(n)}^{e}$ \\
\hline \hline Fuerzas nodales espaciales & $\boldsymbol{n}_{i(n)}^{s}$ & $\boldsymbol{m}_{i(n)}^{s}$ \\
Fuerzas nodales perseguidoras & $\boldsymbol{n}_{i(n)}^{f}$ & $\boldsymbol{m}_{i(n)}^{f}$ \\
\hline
\end{tabular}

Las fuerzas perseguidoras se actualizan al principio mediante las expresiones

$$
\begin{gathered}
\boldsymbol{n}_{i(n)}^{f}=\boldsymbol{\Lambda}_{i(n)} \boldsymbol{N}_{i} \\
\boldsymbol{m}_{i(n)}^{f}=\boldsymbol{\Lambda}_{i(n)} \boldsymbol{M}_{i} .
\end{gathered}
$$

El proceso está gobernado por el módulo FEStep cuya estructura, así como la de los módulos incluidos en él, analizamos a continuación. En la columna de la izquierda se hace referencia al módulo ejecutado o a las ecuaciones empleadas. La columna central refleja las variables calculadas, y a la derecha se incluyen aclaraciones. 


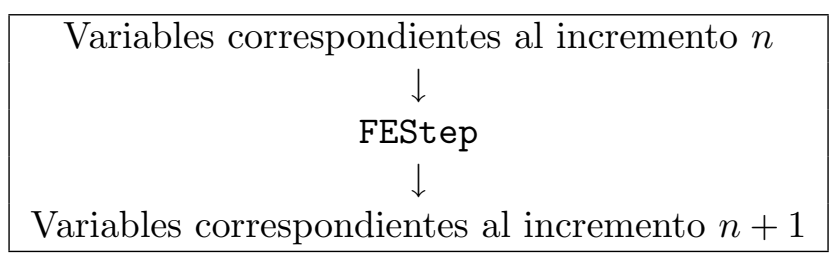

FEStep

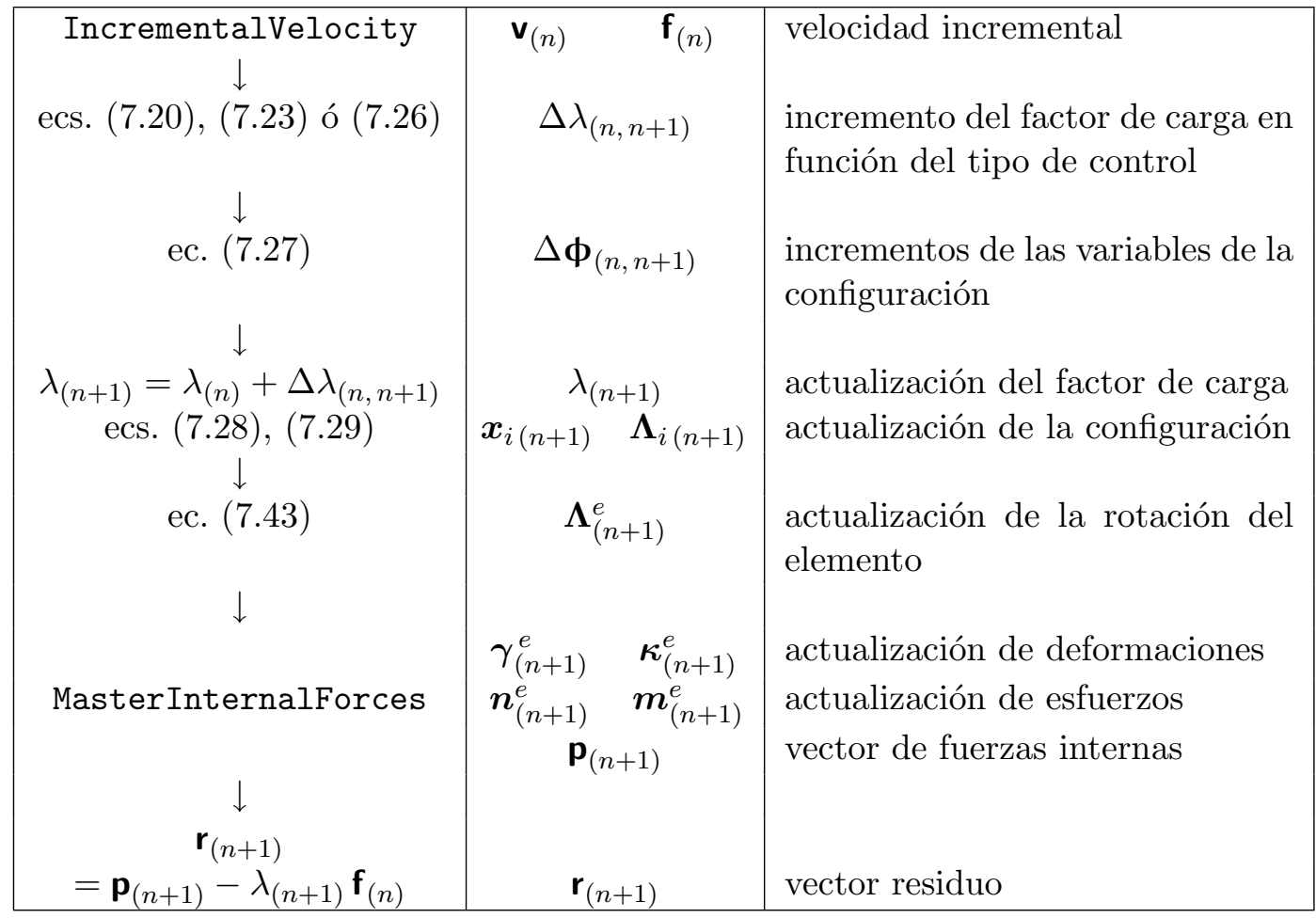

IncrementalVelocity

\begin{tabular}{|c|c|l|}
\hline $\begin{array}{c}\text { MasterTangentStiffness } \\
\downarrow\end{array}$ & $\mathbf{K}_{(n)}$ & rigidez tangente (estructura) \\
ecs. (7.11) y (7.12) & $\mathbf{f}_{(n)}$ & $\begin{array}{l}\text { ensamblaje del vector de fuerzas } \\
\text { exteriores }\end{array}$ \\
$\downarrow$ & & velocidad incremental \\
$\mathbf{v}_{(n)}=\mathbf{K}_{(n)}^{-1} \mathbf{f}_{(n)}$ & $\mathbf{v}_{(n)}$ & \\
\hline
\end{tabular}

MasterTangentStiffness

\begin{tabular}{|c|c|l|}
\hline ec. (7.41) (para el paso $n)$ & {$\left[\begin{array}{ll}\mathbf{c}_{\gamma \gamma} & \mathbf{c}_{\gamma \kappa} \\
\mathbf{c}_{\kappa \gamma} & \mathbf{c}_{\kappa \kappa}\end{array}\right]_{(n)}^{e}$} & matriz constitutiva en $\xi=0$ \\
$\begin{array}{c}\downarrow \\
\text { ecs. (7.8a) } \begin{array}{c}(7.8 \mathrm{~b})(7.8 \mathrm{c}) \\
\mathrm{y}(7.9)\end{array}\end{array}$ & $\mathbf{K}_{(n)}^{e}$ \\
$\begin{array}{c}\downarrow \\
\text { ec. (7.10) }\end{array}$ & $\begin{array}{l}\text { rigidez tangente (elemento) } \\
\mathbf{K}_{(n)}\end{array}$ & $\begin{array}{l}\text { ensamblaje de la rigidez tangente } \\
\text { de la estructura }\end{array}$ \\
\hline
\end{tabular}


MasterInternalForces

\begin{tabular}{|c|c|c|}
\hline $\begin{array}{c}\text { ecs. (7.39) y (7.36) } \\
\downarrow \\
\text { ec. }(\overline{7.41)} \\
\downarrow \\
\text { ec. }(\overline{7.42)} \\
\downarrow \\
\text { ec. }\left(\frac{(7.53)}{}\right. \\
\downarrow \\
\rightarrow\end{array}$ & $\begin{array}{c}\boldsymbol{\gamma}_{(n+1)}^{e} \quad \boldsymbol{\kappa}_{(n+1)}^{e} \\
{\left[\begin{array}{ll}\mathbf{c}_{\gamma \gamma} & \mathbf{c}_{\gamma \kappa} \\
\mathbf{c}_{\kappa \gamma} & \mathbf{c}_{\kappa \kappa}\end{array}\right]_{(n+1)}^{e}} \\
\boldsymbol{n}_{(n+1)}^{e} \\
\boldsymbol{m}_{(n+1)}^{e} \\
\mathbf{p}_{(n+1)}^{e} \\
\mathbf{p}_{(n+1)}\end{array}$ & $\begin{array}{l}\text { matriz constitutiva en } \xi=0 \\
\text { actualización de esfuerzos } \\
\text { fuerzas internas (elemento) } \\
\text { ensamblaje del vector de fuerzas } \\
\text { internas (estructura) }\end{array}$ \\
\hline
\end{tabular}

El proceso incremental se controla fijando de antemano el número de incrementos a considerar, o bien algún valor límite (del factor de carga, o de alguna componente del desplazamiento, por ejemplo). El valor del residuo al final de cada incremento $\left\|\mathbf{r}_{(n+1)}\right\|$ es un indicador de la desviación de la solución incremental de la trayectoria de equilibrio.

\subsubsection{Proceso incremental - iterativo}

El proceso iterativo comienza a partir del resultado del incremento $n$. Empleando las variables asociadas a él (ver cuadro de variables iniciales en el apartado anterior) se lleva a cabo una predicción del estado en el incremento $n+1$, que denominaremos iteración 0 .

\begin{tabular}{c}
\hline Variables correspondientes al paso $n$ \\
$\downarrow$ \\
FEStep \\
$\downarrow$ \\
Resultado de la iteración 0
\end{tabular}

Resultado de la iteración 0

\begin{tabular}{|l|cc|}
\hline Factor de carga & $\lambda^{(0)}$ & \\
Configuración nodal & $\boldsymbol{x}_{i}^{(0)}$ & $\boldsymbol{\Lambda}_{i}^{(0)}$ \\
\hline \hline Rotación del elemento & $\boldsymbol{\Lambda}^{e(0)}$ & \\
Deformaciones & $\boldsymbol{\gamma}^{e(0)}$ & $\boldsymbol{\kappa}^{e(0)}$ \\
Esfuerzos & $\boldsymbol{n}^{e(0)}$ & $\boldsymbol{m}^{e(0)}$ \\
\hline \hline Velocidad incremental & $\mathbf{v}^{(0)}$ & \\
Vector residuo & $\mathbf{r}^{(0)}$ & \\
\hline
\end{tabular}


El proceso de convergencia, que finaliza con la asignación de las variables correspondientes al incremento $n+1$ está controlado por el siguiente diagrama de flujo:

\begin{tabular}{|c|}
\hline Resultado de la iteración 0 \\
Resultado de la iteración $k$ \\
$\downarrow$ \\
NewtonCycle \\
$\downarrow$ \\
Resultado de la iteración $k+1$ \\
$\downarrow$ \\
si $\left\|\mathbf{r}^{(k+1)}\right\| /\left\|\mathbf{r}^{(0)}\right\|<\epsilon_{1}$ \\
o $\left\|\mathbf{r}^{(k+1)}\right\|<\epsilon_{2}$ \\
$\downarrow$ \\
Variables del incremento $n+1 \equiv$ Resultado de la iteración $k+1$ \\
\hline
\end{tabular}

En él se observa que el paso de la iteración $k$ a la $k+1$ está gobernado por el módulo NewtonCycle cuyo funcionamiento se describe a continuación:

NewtonCycle

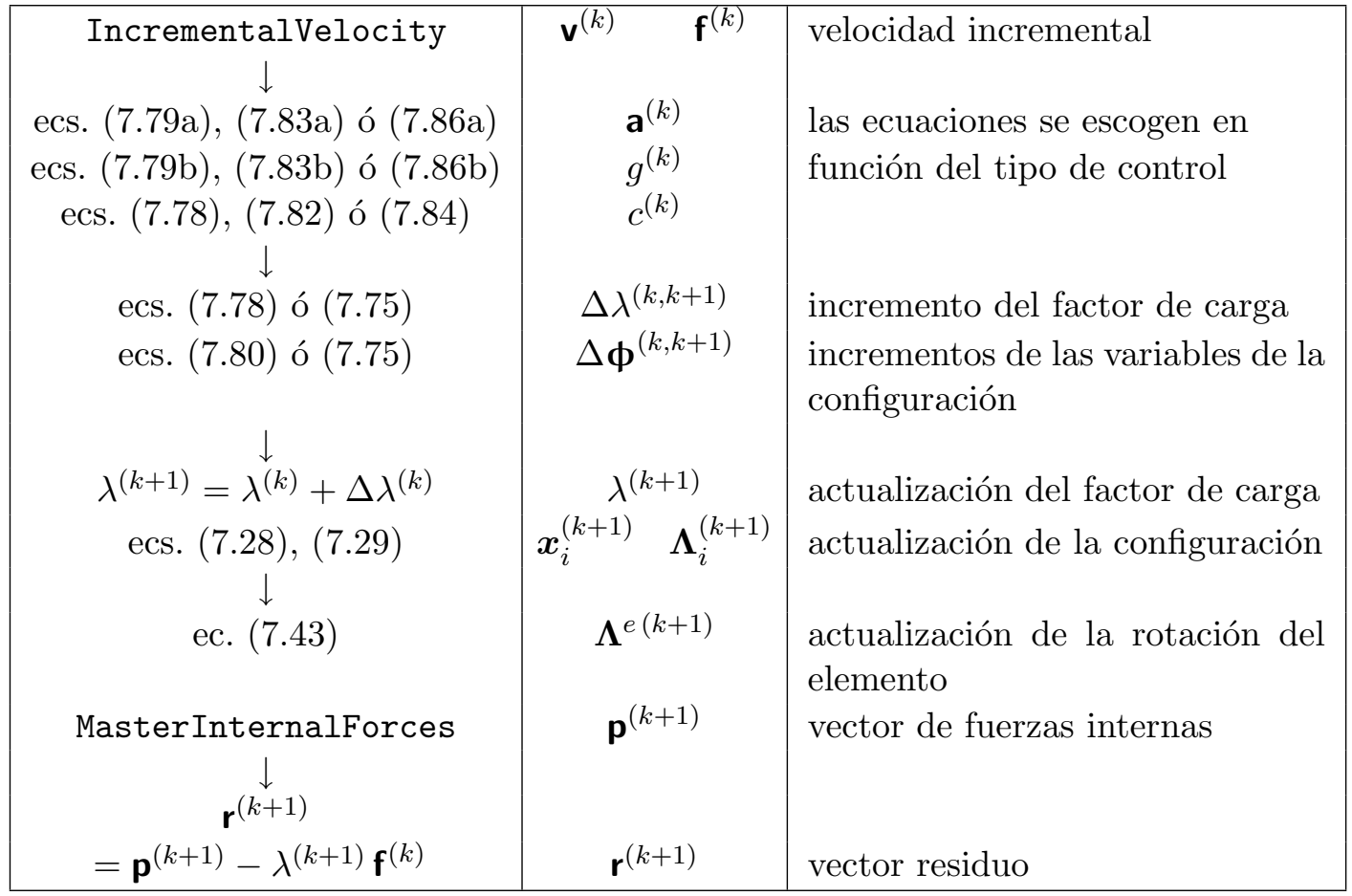




\subsection{Resultados}

Como cierre de este capítulo dedicado a la solución del modelo RS basada en la forma espacial de las ecuaciones, incluimos en esta sección los resultados obtenidos a partir de la implementación en un programa desarrollado en lenguaje MATLAB $\mathbb{R}$ de los procedimientos descritos en las secciones anteriores.

En primer lugar analizamos los resultados de una estructura sencilla para la que se dispone de solución exacta: la ménsula de Simó y Vu-Quoc; este ejemplo, que ha servido como prueba de funcionamiento del programa, da pie a algunos comentarios acerca de los resultados que Simó ofrece en [62]. A continuación nos centramos en el análisis de la precisión alcanzada con soluciones incrementales puras, contrastándolas con soluciones iterativas desde la perspectiva del coste computacional. Por último se ofrecen los resultados correspondientes a distintos ejemplos considerados en la literatura, que ilustran la capacidad del modelo para reproducir comportamientos estructurales complejos.

\subsubsection{Análisis de la solución de Simó y $\mathbf{V u}-$ Quoc al problema de la ménsula}

\section{Solución analítica}

Comenzamos analizando un ejemplo clásico para el que se dispone de solución exacta. Se trata de una ménsula de longitud $L$ cuya directriz se orienta según el eje $x$; el extremo empotrado está en $x=0$. La pieza está sometida en su extremo libre $x=L$ a la acción de un par en la dirección de uno de los ejes principales de inercia de la sección transversal. La ménsula responde en flexión pura, y su deformada es un arco de circunferencia. Suponiendo que el par $M$ se orienta según la dirección del eje cuyo momento de inercia es $I$, la curvatura de la viga es $\kappa=M / E I$. Las expresiones de la solución exacta se toman de la referencia [27] y se reproducen a continuación. La rotación del extremo es

$$
\theta=\frac{M L}{E I}
$$

y las componentes del desplazamiento en el extremo de la pieza

$$
\begin{aligned}
u & =\frac{L}{\theta} \tan \frac{\theta}{2}(1+\cos \theta)-L \\
v & =\frac{L}{\theta} \tan \frac{\theta}{2} \sin \theta .
\end{aligned}
$$

En la figura se representa la trayectoria seguida por el extremo libre para los siguientes datos

$$
L=1 \quad M I=2 \quad M=8 \pi .
$$

Con el valor del par indicado la ménsula se enrolla dos veces sobre si misma. El giro del extremo es $\theta=4 \pi$ y el valor del desplazamiento en el extremo es $u=-L, v=0$, 


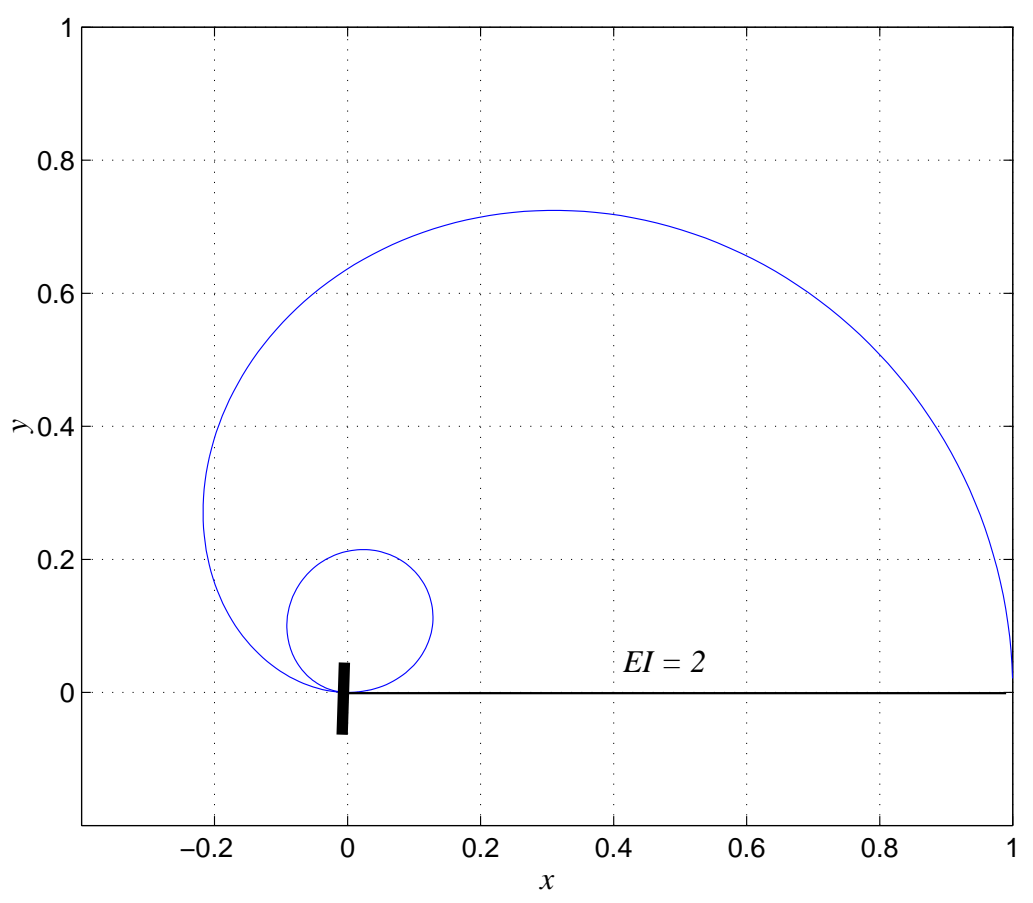

Figura 7.6: Trayectoria del extremo de la ménsula de Simó y Vu-Quoc. Solución exacta

con lo que éste va a parar al origen. El primer paso por el origen $(\theta=2 \pi)$ corresponde a un valor del momento $M=4 \pi$. Es obvio señalar que el resultado no depende de la rigidez a cortante ni a torsion de la pieza.

\section{Crítica de la solución de Simó y Vu-Quoc}

Simo y Vu-Quoc [62] resuelven este problema mediante un proceso iterativo en un único escalón de carga utilizando un modelo con cinco elementos finitos de dos nodos y un punto de integración. En su artículo, los autores únicamente proporcionan los tres datos mencionados antes: la longitud $L=1$, la rigidez a flexión $E I=2$ y el momento en el extremo $M=8 \pi$. Con ellos obtienen resultados espectaculares, pues la convergencia a la solución exacta se logra en un solo escalón de carga y una única iteración. Sin embargo, Ritto-Corrêa y Camotim analizan en [53] un ejemplo análogo y llegan a una conclusión sorprendente, que no se menciona en [62]: la inmejorable velocidad de convergencia de [62] sólo se puede alcanzar si se toma el siguiente conjunto de valores para la rigidez a cortante y axil de la sección

$$
E A=G A_{Q 2}=G A_{Q 3}=2 .
$$

De hecho, el resultado de la solución numérica depende del valor de las características geométricas y mecánicas que no intervienen en la solución analítica, y su influencia es importante. Denominaremos rigidez isótropa a este conjunto de valores 
-puesto que corresponden a una matriz constitutiva $\mathbf{C}_{\Gamma}$ isótropa-, que, por cierto, carecen de sentido físico, ya que $G A_{Q i}$ debe ser necesariamente menor que $E A$.

$\mathrm{Si}$, por el contrario, se adoptan las características mecánicas reales de una sección circular cuya rigidez a flexión $E I$ sea igual a 2, la velocidad de convergencia disminuye de forma apreciable, como comprobaremos a continuación. Estos resultados inducen a pensar que el operador de Simó es sensible a la relación entre el módulo de elasticidad y el módulo de deformación transversal. En un problema tridimensional la relación entre ellos viene dada por un factor que varía entre 2 y 3 , puesto que se cumple

$$
G=\frac{E}{2(1+\nu)},
$$

y $\nu$, el coeficiente de Poisson, debe variar entre 0 y 0,5 . Aunque en el modelo geométricamente exacto $\nu$ no tiene significado físico (ya que se admite que la sección transversal es indeformable), su rango de variación determina los valores de la relación $E / G$ dotados de sentido físico que hemos indicado.

A continuación, vamos reproducir el análisis iterativo en un solo paso de Simó y $\mathrm{Vu}-\mathrm{Quoc}$ empleando su operador tangente (que denominamos SVQ) y comparando resultados para distintos valores de $E / G$. Para ello supondremos en todos los casos que la sección transversal es doblemente simétrica, que los ejes 2 y 3 de la sección se orientan según los ejes principales de inercia, y asumiremos que el valor del área de cortante es el mismo en las dos direcciones principales e igual al área de la sección transversal. Con estas hipótesis consideramos los siguientes conjuntos de datos

1. Rigidez isótropa ${ }^{3}$

$$
\mathbf{C}_{\Gamma}=\left[\begin{array}{lll}
2 & 0 & 0 \\
0 & 2 & 0 \\
0 & 0 & 2
\end{array}\right] \quad \mathbf{C}_{K}=\left[\begin{array}{lll}
2 & 0 & 0 \\
0 & 2 & 0 \\
0 & 0 & 2
\end{array}\right]
$$

2. $E / G=2 \quad(\nu=0)$

$$
\mathbf{C}_{\Gamma}=\left[\begin{array}{ccc}
2 & 0 & 0 \\
0 & 1 & 0 \\
0 & 0 & 1
\end{array}\right] \quad \mathbf{C}_{K}=\left[\begin{array}{lll}
2 & 0 & 0 \\
0 & 2 & 0 \\
0 & 0 & 2
\end{array}\right]
$$

3. $E / G=2,25 \quad(\nu=0,125)$

$$
\mathbf{C}_{\Gamma}=\left[\begin{array}{ccc}
2 & 0 & 0 \\
0 & 8 / 9 & 0 \\
0 & 0 & 8 / 9
\end{array}\right] \quad \mathbf{C}_{K}=\left[\begin{array}{ccc}
16 / 9 & 0 & 0 \\
0 & 2 & 0 \\
0 & 0 & 2
\end{array}\right]
$$

\footnotetext{
${ }^{3}$ Las rigideces a cortante consideradas en este caso, que es el adoptado por Simó en [62] no pueden obtenerse sin violar el rango de variación de $\nu$ físicamente razonable. De hecho, corresponden a $\nu=-0,5$.
} 
4. $E / G=2,5 \quad(\nu=0,25)$

$$
\mathbf{C}_{\Gamma}=\left[\begin{array}{ccc}
2 & 0 & 0 \\
0 & 4 / 5 & 0 \\
0 & 0 & 4 / 5
\end{array}\right] \quad \mathbf{C}_{K}=\left[\begin{array}{ccc}
8 / 5 & 0 & 0 \\
0 & 2 & 0 \\
0 & 0 & 2
\end{array}\right]
$$

5. $E / G=2,75 \quad(\nu=0,375)$

$$
\mathbf{C}_{\Gamma}=\left[\begin{array}{ccc}
2 & 0 & 0 \\
0 & 8 / 11 & 0 \\
0 & 0 & 8 / 11
\end{array}\right] \quad \mathbf{C}_{K}=\left[\begin{array}{ccc}
16 / 11 & 0 & 0 \\
0 & 2 & 0 \\
0 & 0 & 2
\end{array}\right]
$$

6. $E / G=3 \quad(\nu=0,5)$

$$
\mathbf{C}_{\Gamma}=\left[\begin{array}{ccc}
2 & 0 & 0 \\
0 & 2 / 3 & 0 \\
0 & 0 & 2 / 3
\end{array}\right] \quad \mathbf{C}_{K}=\left[\begin{array}{ccc}
4 / 3 & 0 & 0 \\
0 & 2 & 0 \\
0 & 0 & 2
\end{array}\right] .
$$

Como criterio de convergencia limitamos la norma del residuo en fuerzas a $10^{-6}$. Los resultados recogidos en la siguiente tabla muestran cómo la eficacia del proceso de corrección se reduce mucho cuando se toma valores realistas de $E / G$ (pasando de 1 a 5 iteraciones ${ }^{4}$ ). A medida que aumenta $E / G$ disminuye progresivamente la exactitud

\begin{tabular}{|c|c|c|c|c|c|}
\hline$k$ & R. isótropa & $E / G=2$ & $E / G=2,5$ & $E / G=2,75$ & $E / G=3$ \\
\hline 0 & $2,8331 \quad 10^{1}$ & $3,0055 \quad 10^{1}$ & $3,1124 \quad 10^{1}$ & $3,156310^{1}$ & $3,1948 \quad 10^{1}$ \\
\hline 1 & $1,0178 \quad 10^{-13}$ & $6,168910^{0}$ & $6,999510^{0}$ & $8,306310^{0}$ & $1,3812 \quad 10^{1}$ \\
\hline 2 & & $6,562710^{-1}$ & $6,146110^{-1}$ & $8,422010^{-1}$ & $1,478710^{1}$ \\
\hline 3 & & $5,0812 \quad 10^{-2}$ & $5,374410^{-2}$ & $9,5281 \quad 10^{-2}$ & $4,009510^{0}$ \\
\hline 4 & & $2,1865 \quad 10^{-4}$ & $2,5476 \quad 10^{-4}$ & $5,3850 \quad 10^{-4}$ & $1,6262 \quad 10^{0}$ \\
\hline 5 & & $4,2887 \quad 10^{-9}$ & $7,2257 \quad 10^{-9}$ & $3,8674 \quad 10^{-8}$ & $4,9669 \quad 10^{-2}$ \\
\hline 6 & & & & & $1,6373 \quad 10^{-4}$ \\
\hline 7 & & & & & $2,0117 \quad 10^{-9}$ \\
\hline
\end{tabular}
de la solución para un mismo número de iteraciones. Finalmente, cuando $E / G=3$ se requieren 7 iteraciones para la convergencia.

Cuadro 7.1: Proceso de convergencia en el problema de Simó y Vu-Quoc $\left(M_{z}=8 \pi\right)$. Norma del residuo en la iteración $k$ para distintos valores de la relación $E / G$ empleando el operador SVQ

Estos resultados nos han llevado a cuestionar la eficacia del modelo para la resolución de problemas con datos físicamente razonables, no sólo en lo que respecta a los parámetros que describen la respuesta mecánica del material, sino también en cuanto a los datos geométricos de la pieza analizada.

\footnotetext{
${ }^{4}$ Los valores correspondientes a $k=0$ son el resultado de la predicción, por eso se cuenta el número de iteraciones sin tener en cuanta este primer cálculo.
} 
Si escogemos MN y mm como unidades de fuerza y longitud respectivamente, entonces los valores adoptados por Simó y $\mathrm{Vu}$-Quoc para su ménsula corresponden a una estructura con un módulo de elasticidad $E=1 / 2 \pi \mathrm{MN} / \mathrm{mm}^{2}$ (el orden de magnitud corresponde a un material metálico), una longitud $L=1 \mathrm{~mm}$ y una sección transversal circular de $4 \mathrm{~mm}$ de diámetro. Es evidente que una ménsula con estas proporciones es la antítesis de una pieza alargada, y no tiene sentido plantear un problema de grandes rotaciones debidas a la acción de un par en el extremo libre. En vista de ello, parece necesario estudiar la influencia de la esbeltez de la pieza en el resultado del cálculo.

Para ello analizamos cuatro ménsulas cuyo denominador común son las rigideces a axil y flexión $E A=2 \mathrm{MN}, E I=2 \mathrm{MN} \mathrm{mm}^{2}$. El módulo de elasticidad, que vuelve a ser $E=1 / 2 \pi \mathrm{MN} / \mathrm{mm}^{2}$, y el diámetro $(4 \mathrm{~mm})$ se mantienen constantes. Las cuatro estructuras se diferencian en su longitud, que varía entre 1 y $1000 \mathrm{~mm}$. En el cuadro 7.2 se recogen sus características geométricas ${ }^{5}$ y el valor del par aplicado en el extremo libre que enrolla la ménsula una vuelta completa. El caso A corresponde a la ménsula de Simó, la estructura B tiene las proporciones de una viga de gran canto, la C es una pieza esbelta y la D un filamento.

\begin{tabular}{c|rrl} 
estructura & $L(\mathrm{~mm})$ & $\eta$ & $M(\mathrm{~mm} \mathrm{MN})$ \\
\hline & & & \\
$\mathrm{A}$ & 1 & 0,25 & $4 \pi 10^{0}$ \\
$\mathrm{~B}$ & 10 & 2,50 & $4 \pi 10^{-1}$ \\
$\mathrm{C}$ & 100 & 25,00 & $4 \pi 10^{-2}$ \\
$\mathrm{D}$ & 1000 & 250,00 & $4 \pi 10^{-3}$ \\
\hline
\end{tabular}

Cuadro 7.2: Longitud, esbeltez geométrica y par aplicado en el extremo libre, en cuatro ménsulas con rigideces $E A=E I=2$

Resolvemos ahora el problema por un procedimiento incremental-iterativo, empleando el operador de Simó y Vu-Quoc para tres valores representativos de la relación $E / G$. El criterio de convergencia adoptado, como antes, ha sido el de limitar la norma del residuo a $10^{-6}$. También se ha limitado a 25 el número máximo de iteraciones. Si con un número determinado de pasos (escalones carga) no se producía la convergencia, se ha reducido la magnitud del escalón hasta lograrla. En el cuadro 7.3 se recoge el número de pasos y el número de iteraciones por paso necesarios para obtener la solución.

Los resultados muestran cómo se deteriora la convergencia a medida que aumenta la esbeltez de la ménsula. Por ejemplo, la estructura C (esbeltez $\eta=25$ ) con una relación $E / G=2,5$ requiere 5 escalones de carga acumulando un total de 55 iteraciones, lo que supone un volumen de operaciones 10 veces superior al que requiere la ménsula de Simó (estructura A) equivalente. La influencia de la relación $E / G$ es también importante: en los casos B, C y D se observa que cuanto mayor es su valor, menos eficaz resulta la corrección. Es interesante señalar también que al analizar la estructura $\mathrm{D}$ con $E / G=2$ hemos comprobado que se puede alcanzar la convergencia

\footnotetext{
${ }^{5}$ La esbeltez geométrica $\eta$ se define como la relación luz/canto.
} 


\begin{tabular}{|c|c|c|c|c|c|c|c|c|c|c|c|c|}
\hline \multirow[b]{3}{*}{ paso } & \multicolumn{3}{|c|}{$\begin{array}{c}\text { estructura A } \\
\eta=0,25\end{array}$} & \multicolumn{3}{|c|}{$\begin{array}{c}\text { estructura B } \\
\eta=2,5\end{array}$} & \multicolumn{3}{|c|}{$\begin{array}{c}\text { estructura } \mathrm{C} \\
\eta=25\end{array}$} & \multicolumn{3}{|c|}{$\begin{array}{c}\text { estructura D } \\
\quad \eta=250\end{array}$} \\
\hline & & $E / C$ & & & $E / C$ & & & $E / C$ & & & $\overline{E / C}$ & \\
\hline & 2 & 2,5 & 3 & 2 & 2,5 & 3 & 2 & 2,5 & 3 & 2 & 2,5 & 3 \\
\hline 1 & 5 & 5 & 5 & 16 & 8 & 8 & 10 & 11 & 8 & 8 & 9 & 11 \\
\hline 2 & & & & & 8 & 8 & 10 & 11 & 8 & 8 & 9 & 11 \\
\hline 3 & & & & & 8 & 8 & 10 & 11 & 8 & 8 & 9 & 11 \\
\hline 4 & & & & & & & 10 & 11 & 8 & 8 & 9 & 11 \\
\hline 5 & & & & & & & & 11 & 8 & 8 & 9 & 11 \\
\hline 6 & & & & & & & & & 8 & 8 & 9 & 11 \\
\hline 7 & & & & & & & & & 8 & 8 & 9 & 11 \\
\hline 8 & & & & & & & & & 8 & 8 & 9 & 11 \\
\hline total it & 5 & 5 & 5 & 16 & 24 & 24 & 40 & 55 & 64 & 64 & 72 & 88 \\
\hline
\end{tabular}

Cuadro 7.3: Número de iteraciones por paso necesarias para la convergencia en las estructuras A, B, C y D para distintos valores de la relación $E / G$

con menos pasos, aunque con un número total de iteraciones mayor: por ejemplo, es posible lograr la convergencia en 6 escalones con 17 iteraciones cada uno (102 iteraciones en total), o con 7 escalones con 11 iteraciones. Lo mismo sucede para la estructura $\mathrm{C}$ con $E / G=3$, en la que es posible la convergencia con 6 escalones y un total de 78 iteraciones (13 por paso).

En las cuatro alternativas estudiadas antes, el valor numérico de $E A$ es igual al de $E I$. Ahora queremos analizar qué sucede cuando los valores de las rigideces tienen distintos órdenes de magnitud. Para ello consideramos dos variantes de la estructura C $\left(L=100 \mathrm{~mm}, M=4 \pi 10^{-2} \mathrm{~mm} \mathrm{MN}\right)$ cambiando simplemente las unidades en las que se define el problema. Recordamos que el módulo de elasticidad vuelve a ser $E=1 / 2 \pi \mathrm{MN} / \mathrm{mm}^{2}$, y la sección transversal es una circunferencia de $4 \mathrm{~mm}$ de diámetro. La alternativa $\mathrm{C} 2$ emplea $\mathrm{m}$ y $\mathrm{kN}$, unidades habituales en ingeniería civil. Las unidades de las otras dos opciones son, sin embargo, menos usuales. En las opciones $\mathrm{C} 1$ y $\mathrm{C} 2$ las rigideces $E A$ y $E I$ difieren en seis órdenes de magnitud. Los datos se recogen en el cuadro 7.4 .

\begin{tabular}{l|cc|llll} 
alternativa & \multicolumn{2}{|c|}{ unidades } & $L$ & $E A$ & $E I$ & $M$ \\
\hline & & & & & & \\
$\mathrm{C}$ & $\mathrm{mm}$ & $\mathrm{MN}$ & $10^{2}$ & $210^{0}$ & $210^{0}$ & $4 \pi 10^{-2}$ \\
$\mathrm{C} 1$ & $\mathrm{~m}$ & $\mathrm{MN}$ & $10^{-1}$ & $210^{0}$ & $210^{-6}$ & $4 \pi 10^{-5}$ \\
$\mathrm{C} 2$ & $\mathrm{~m}$ & $\mathrm{kN}$ & $10^{-1}$ & $210^{3}$ & $210^{-3}$ & $4 \pi 10^{-2}$ \\
\hline
\end{tabular}

Cuadro 7.4: Longitud, rigidez a axil y flexión, y momento aplicado en el extremo en la estructura $\mathrm{C}$, expresados en distintas unidades

En el cuadro 7.5 se muestran los resultados correspondientes a las tres estructuras. Se puede apreciar que el cambio de unidades no cambia el resultado (evidentemente las diferencias en el exponente se deben a que en la estructura $\mathrm{C}$ se emplea $\mathrm{mm}$ y en la $\mathrm{C} 1$ y la $\mathrm{C} 2 \mathrm{~m}$. El número de iteraciones también es el mismo en los tres casos (40 
para $E / G=2,55$ para $E / G=2,5$ y 64 para $E / G=3$, como ya se vio en el cuadro 7.3).

\begin{tabular}{|c|c|c|c|c|c|c|}
\hline & \multicolumn{2}{|c|}{$E / G=2$} & \multicolumn{2}{|c|}{$E / G=2,5$} & \multicolumn{2}{|c|}{$E / G=3$} \\
\hline & $x$ & $y$ & $x$ & $y$ & $x$ & $y$ \\
\hline $\mathrm{C}$ & $6,01510^{-5}$ & $-7,60810^{-6}$ & $1,69910^{-5}$ & $-2,45910^{-6}$ & $-1,78910^{-7}$ & $2,74210^{-8}$ \\
\hline $\mathrm{C} 1$ & $6,01510^{-8}$ & $-7,60810^{-9}$ & $1,69910^{-8}$ & $-2,45910^{-9}$ & $-1,78910^{-10}$ & $2,74210^{-11}$ \\
\hline $\mathrm{C} 2$ & $6,01510^{-8}$ & $-7,60810^{-9}$ & $1,69910^{-8}$ & $-2,45910^{-9}$ & $-1,78910^{-10}$ & $2,74210^{-11}$ \\
\hline
\end{tabular}

Cuadro 7.5: Posición final (en m) del extremo del voladizo de la estructura C. Comparación entre alternativas $\mathrm{C}, \mathrm{C} 1$ y $\mathrm{C} 2$

\subsubsection{Solución incremental pura de la ménsula de Simó y Vu-Quoc}

Deseamos evaluar a continuación el funcionamiento de la solución incremental pura empleando el operador de Simó. Para ello seguimos tomando la ménsula de Simó sometida a un momento en el extremo como estructura de prueba. Comenzaremos estudiando la estructura A, analizada en el artículo original de Simó y Vu-Quoc [62], y posteriormente analizaremos la que habíamos denominado estructura C, que es una ménsula con una esbeltez $\eta=25$.

\section{Estructura A}

Volvemos a la estructura A, que tiene las siguientes características:

\begin{tabular}{c|cccc} 
estructura & $L$ & $E A$ & $E I$ & $M$ \\
\hline & & & & \\
$\mathrm{A}$ & 1 & 2 & 2 & $4 \pi$ \\
\hline
\end{tabular}

El par de valor $4 \pi$ conduce el extremo libre al origen produciendo una deformada circular. Como tratamos de estudiar la sensibilidad a la relación $E / G$ y al número de escalones de carga (o incrementos) $n$, hemos considerado tres situaciones básicas con

$$
n=50 \quad n=100 \quad n=500,
$$

y para cada una de estas situaciones analizamos la respuesta de cada operador considerando rigidez isótropa o valores de $E / G$ comprendidos entre 2 y 3 . En el cuadro 7.6 se recogen los valores del error relativo en el desplazamiento del extremo del voladizo, y de la norma del residuo en fuerzas definido por la ecuación (7.59) en el último incremento. La medida del error que se ha escogido es la distancia del extremo de la ménsula al origen (posición final exacta) dividida por la longitud de la ménsula, que se toma como longitud de referencia y que en este caso vale 1.

La figura 7.7 muestra la trayectoria del extremo de la ménsula con una relación $E / G=2,5$ para los tres valores de $n$ considerados. Se observa cómo aumenta la precisión del resultado para $n=500$ : el error relativo en la posición del extremo 


\begin{tabular}{l|cc|cc|cc} 
& \multicolumn{2}{|c|}{$n=50$} & \multicolumn{2}{c|}{$n=100$} & \multicolumn{2}{c}{$n=500$} \\
& error & residuo & error & residuo & error & residuo \\
\hline & & & & & & \\
rigidez isótropa & 0,03484 & 0,17696 & 0,01753 & 0,08837 & 0,00352 & 0,01766 \\
$E / G=2$ & 0,04949 & 0,18527 & 0,02545 & 0,09041 & 0,00523 & 0,01774 \\
$E / G=2,5$ & 0,05562 & 0,19008 & 0,02900 & 0,09159 & 0,00606 & 0,01778 \\
$E / G=3$ & 0,06148 & 0,19495 & 0,03240 & 0,09285 & 0,00688 & 0,01782 \\
\hline
\end{tabular}

Cuadro 7.6: Ménsula de Simó y Vu-Quoc (A). Error relativo en el desplazamiento del extremo libre y norma del residuo en la solución incremental pura

se reduce de $5,5 \%$ a $0,6 \%$. En general, los resultados son aceptables incluso para 50 escalones de carga pues el error es siempre inferior al $10 \%$. Como sucedía en la solución corregida, el hecho de considerar la matriz de rigidez isótropa conduce a los mejores resultados, y aunque esta hipótesis carece de sentido físico, también sabemos que en este problema la solución teórica sólo depende de la rigidez a flexión.

Estos resultados corresponden a la estructura A, que es una pieza cuyas dimensiones no tienen proporciones físicamente razonables. Comprobamos a continuación qué sucede en un caso más ajustado a la realidad.

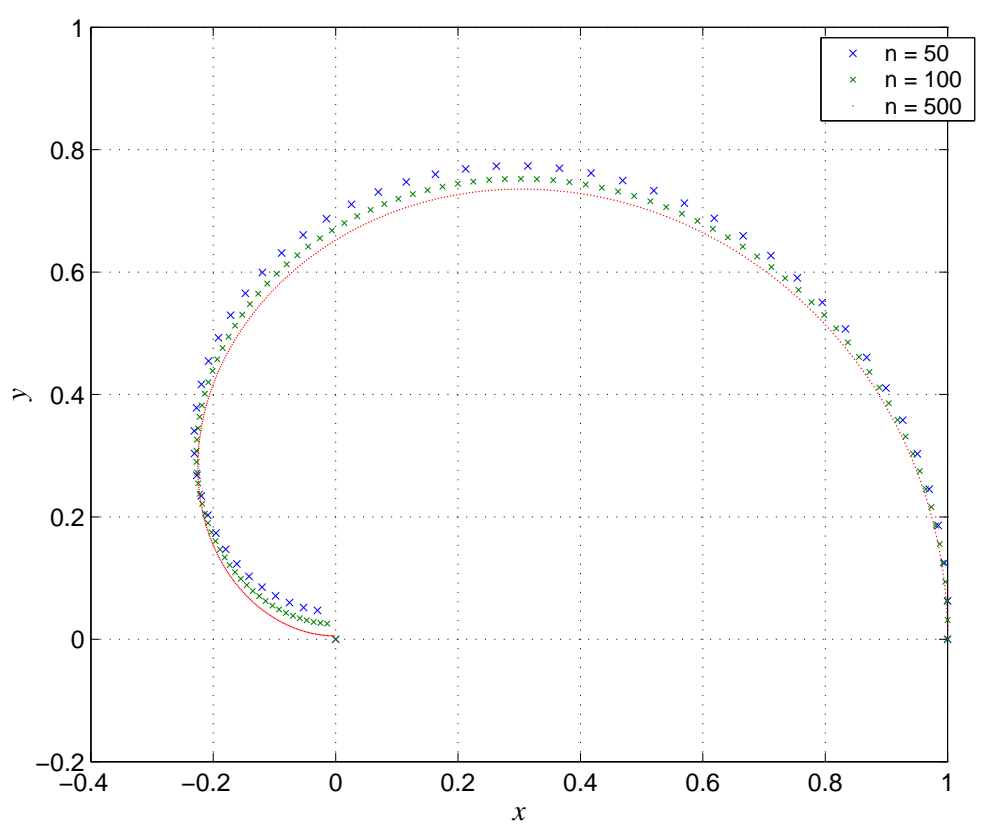

Figura 7.7: Trayectoria del extremo de la estructura A $(E / G=2,5)$. Soluciones incrementales con $n$ variable 


\section{Estructura C2}

Escogemos ahora la estructura $\mathrm{C}$, con proporciones de pieza esbelta, en la variante $\mathrm{C} 2$ (con $\mathrm{m}$ y kN como unidades básicas). Sus características son las siguientes:

\begin{tabular}{c|cccc} 
estructura & $L$ & $E A$ & $E I$ & $M$ \\
\hline $\mathrm{C} 2$ & 0,1 & $210^{3}$ & $210^{-3}$ & $4 \pi 10^{-2}$ \\
\hline
\end{tabular}

El momento aplicado enrolla la ménsula una vuelta completa. Hemos centrado el análisis en el caso $E / G=2,5$. Los resultados, que se reproducen en la figura 7.8 , son extremadamente sensibles al número de pasos en los que se subdivide la carga total: en el intervalo entre 50 y 2500 escalones el resultado obtenido no guarda relación con la respuesta correcta. Es necesario aumentar hasta 5000 la subdivisión para lograr resultados aceptables. La comparación de las soluciones con $n=50,100$ y 500 con las correspondientes a la estructura A (figura 7.7) es elocuente, y muestra cómo en una estructura con proporciones lógicas el proceso incremental no funciona bien.

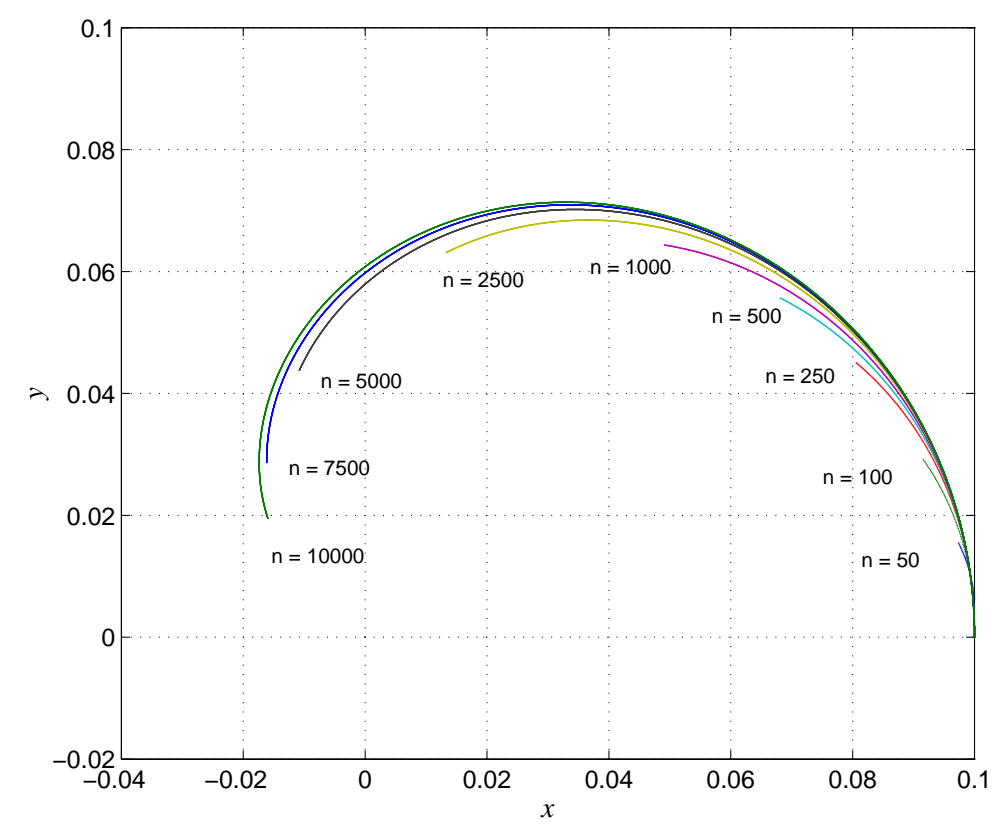

Figura 7.8: Trayectoria del extremo de la estructura $\mathrm{C} 2(E / G=2,5)$. Soluciones incrementales con $n$ variable

El cuadro 7.7 recoge el valor del residuo en fuerzas al final del proceso incremental en cada caso. Tanto en el valor del residuo como en la representación de la trayectoria se observa cómo a partir de 7500 incrementos la mejora del resultado no es relevante. Incluso con 10000 escalones de carga se obtiene una solución mala, con un error relativo en la posición del extremo superior al $25 \%$. 


\begin{tabular}{r|rrrrrrrrr}
$n$ & 50 & 100 & 250 & 500 & 1000 & 2500 & 5000 & 7500 & 10000 \\
\hline $\begin{array}{r}\text { error } \\
\text { residuo }\end{array}$ & 0,986 & 0,961 & 0,923 & 0,880 & 0,809 & 0,645 & 0,451 & 0,331 & 0,252 \\
\hline
\end{tabular}

Cuadro 7.7: Ménsula de Simó y Vu-Quoc (C2). Error relativo en el desplazamiento del extremo libre y norma del residuo en la solución incremental pura

Comentario 7.5.1. La solución puramente incremental del modelo de ReissnerSimó en piezas con proporciones físicamente razonables no produce buenos resultados, ya que el volumen de operaciones a realizar para alcanzar una mínima precisión no guarda proporción alguna con el coste computacional de una solución iterativa, mucho más reducido. Con esta última se alcanza gran precisión con pocas iteraciones: por ejemplo, en la solución de la ménsula de Reissner-Simó sometida a un momento que la enrolla una vuelta se obtiene la solución con un error del orden de $10^{-7}$ en 5 escalones de carga con 11 iteraciones por paso (ver tablas 7.3 y 7.5); sin embargo, con una solución incremental pura, tras 10000 escalones de carga el error relativo en la posición del extremo aún es igual a 0,252. Estos resultados limitan considerablemente el empleo de soluciones incrementales en la práctica.

\subsubsection{Evaluación del efecto de la parte antisimétrica del operador}

En el capítulo 6 demostramos que el operador tangente deducido teniendo en cuenta la segunda variación de las variables de la configuración se puede descomponer en suma del operador obtenido por Simó y Vu-Quoc más un operador antisimétrico que se anula sobre la trayectoria de equilibrio. Por otra parte en el comentario 7.3.1 concluíamos que en una solución incremental-iterativa no tiene sentido utilizar el operador completo, ya que en el paso inicial (predicción) se parte de la trayectoria de equilibrio (sobre la que la parte antisimétrica es nula), y en la fase de corrección se trabaja después de la discretización, linealizando el residuo, es decir, con la matriz tangente de Simó.

Resta analizar qué sucede en una solución incremental pura, que se va alejando de la trayectoria de equilibrio a medida que aumenta el parámetro de control. En este caso es posible que el operador antisimétrico introduzca diferencias respecto del de Simó.

Para evaluar esta situación consideramos dos estructura de prueba cuya geometría se basa en la de la ménsula de Bathe y Bolourchi [8]. La primera se somete a un momento en el extremo, y sus proporciones son equivalentes a la de la estructura A empleada antes (la ménsula original de Simó). La segunda, que tiene una esbeltez análoga a la de la estructura C2 se someterá a la acción de fuerzas puntuales en el extremo. El hecho de analizar cada acción con una estructura diferente está justificado por los resultados obtenidos en el punto anterior: el error de la solución incremental es mucho mayor en una estructura esbelta. El orden de magnitud del error en la primera estructura será, pues, muy inferior al de la segunda, y esta circunstancia permitirá discernir cuál de los dos operadores produce mejores resultados. Además, 
los resultados de la acción del momento sobre la estructura esbelta (la segunda) son proporcionales a los que se obtendrá con la primera, ya que en la estructura sometida únicamente a la acción de momentos en los extremos los desplazamientos obtenidos son escalables $^{6}$. Sin embargo, cuando la estructura se somete a la acción de una fuerza en el extremo ya no existe tal proporcionalidad, puesto que las rigideces correspondientes a axiles y cortantes tienen dimensiones diferentes a las rigideces correspondientes a los momentos. Por ello, en este último caso se ha optado por trabajar con la estructura físicamente realista.

Consideramos ya la primera estructura, que denominamos Estructura 1, y tiene características resistentes similares a la ménsula de Simó. Se trata de un arco de circunferencia de radio $R=1$ y un desarrollo correspondiente a una ángulo de $45^{\circ}$. Uno de sus extremos está empotrado y el otro es libre. La sección transversal es circular con área $A=4 \pi$, los momentos de inercia $I_{y}, I_{z}$ tienen el mismo valor, y módulo de torsión es $J=8 \pi$. La estructura se discretiza mediante 8 elementos rectos de dos nodos con integración reducida, como se muestra en la figura 7.9. El material es elástico y lineal con módulo de elasticidad $E=1 / 2 \pi$ y relación $E / G=2,5$. La

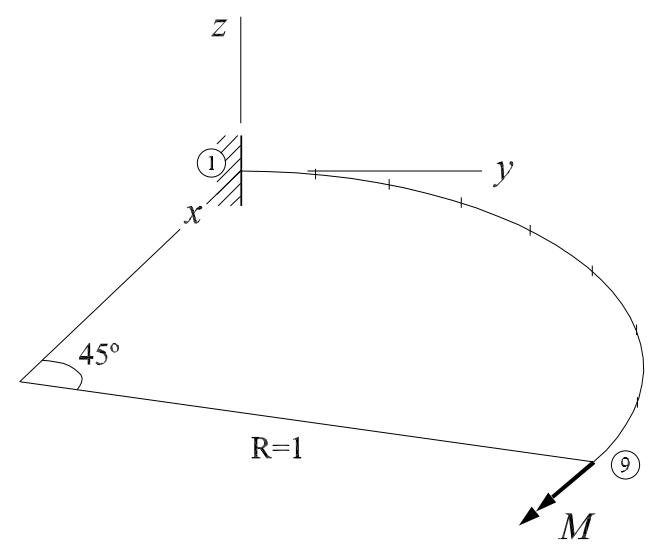

Figura 7.9: Estructura 1

estructura se somete a $(a)$ un momento de eje fijo de módulo $M=4 \pi$ aplicado en la

\footnotetext{
${ }^{6}$ Esto se debe a que en la ecuación constitutiva sólo aparecen términos $\boldsymbol{m}-\boldsymbol{\kappa}$ (no hay esfuerzos $\boldsymbol{n}$ con componentes de fuerza), y en la matriz constitutiva todos los términos tienen las mismas dimensiones. Por ello el valor de $\kappa$ en un punto con la misma posición relativa en ambas estructuras será el mismo, con independencia de la longitud considerada para la pieza. Como $\boldsymbol{n}=0$ las secciones permanecerán perpendiculares a la directriz deformada. Esto implica que la forma de la directriz será la misma en las dos estructuras (serán homotéticas) y las posiciones y los desplazamientos sólo se diferenciarán en el factor de escala. Este hecho, que hemos comprobado numéricamente, permite optimizar el coste computacional en estructuras sometidas únicamente a momentos adoptando longitudes ficticias del mismo orden de magnitud que las características de la sección transversal y corrigiendo el valor de los desplazamientos mediante el factor de escala asociado, y ha sido empleado por Simó y Vu-Quoc para obtener los sorprendentes resultados de su ménsula en 62.
} 
dirección tangente a la ménsula en su extremo, y $(b)$ un momento perseguidor con la misma dirección inicial y la misma magnitud que el momento de eje fijo. Para cada acción analizaremos el error relativo en el desplazamiento de los nodos, tomando como referencia la solución corregida iterativamente, comparando los resultados obtenidos mediante el operador de Simó y el de la tesis. También calcularemos la 1-norma de la matriz tangente de Simó y la de la matriz antisimétrica, que nos orientarán acerca de la importancia relativa entre ellas.

Las soluciones corregidas mediante un proceso incremental - iterativo (empleando el operador de Simó) se recogen en las figuras 7.10 y 7.11

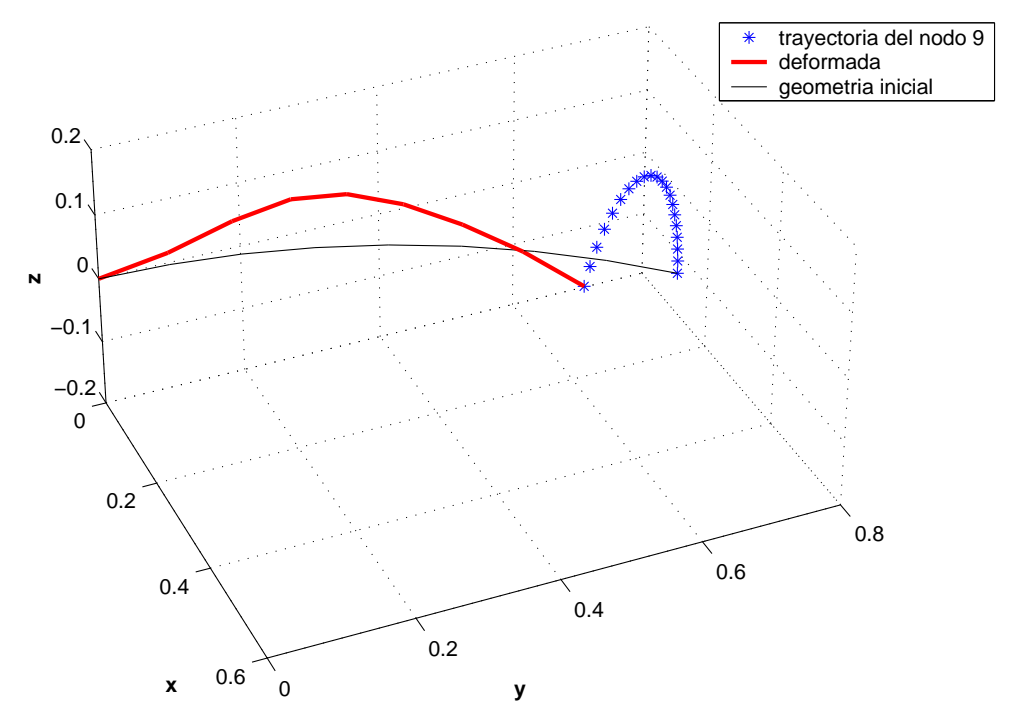

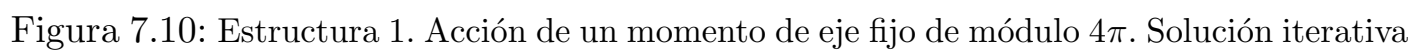

Cuando evaluamos la solución incremental empleando la matriz tangente de la tesis, podemos descomponerla en suma de la matriz de Simó más la antisimétrica y calcular en el último escalón de carga la 1-norma de cada una de ellas ${ }^{7}$; los resultados se muestran en el cuadro 7.8. En él se observa que la norma de la matriz antisimétrica es cuatro órdenes de magnitud inferior a la de la matriz tangente de Simó, lo que indica que la diferencia en el resultado obtenido con la matriz de Simó y la de la tesis va a ser despreciable.

En las figuras 7.12 y 7.13 se ha representado el error relativo en los desplazamientos nodales calculados por el procedimiento incremental puro en 20 escalones de carga, comparando el error obtenido al con el operador de Simó, y el obtenido con la matriz tangente de la tesis. El error relativo en el desplazamiento se calcula como cociente entre el desplazamiento obtenido con el proceso incremental y el calculado mediante la solución incremental - iterativa.

En la estructura cargada con el momento de eje fijo la diferencia entre los dos operadores es prácticamente constante a favor del operador de Simó, para el que se

\footnotetext{
${ }^{7} \mathrm{La}$ 1-norma se define como la suma de los valores absolutos de todos los elementos de la matriz.
} 


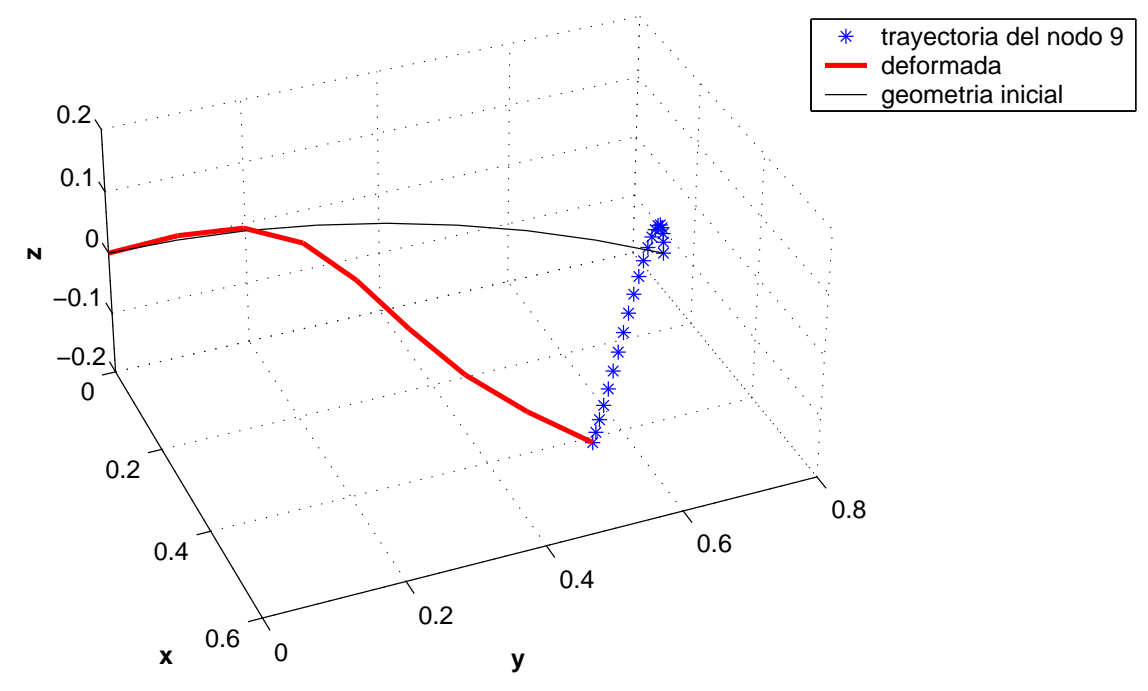

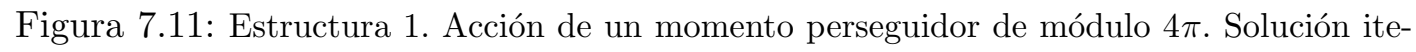
rativa

\begin{tabular}{l|cc} 
& Matriz de Simó & Matriz antisimétrica \\
\hline & 101,00 & 0,05601 \\
momento espacial & 100,11 & 0,08926 \\
momento perseguidor & & \\
\hline
\end{tabular}

Cuadro 7.8: 1-norma de las matrices tangentes para las dos acciones consideradas en la estructura 1

obtiene en todos los nodos un error inferior en $1 \%$ al del operador de la tesis. En la tabla 7.9 se observa también que la norma del residuo tampoco mejora al emplear la matriz obtenida en la tesis.

Cuando actúa el momento perseguidor el error es prácticamente idéntico hasta el nodo 6. A partir de éste y hasta el extremo de la pieza (nodo 9) el operador de la tesis produce un error algo mayor (aproximadamente 1,5\%). El residuo final es muy parecido en ambos casos (ver cuadro 7.10). 


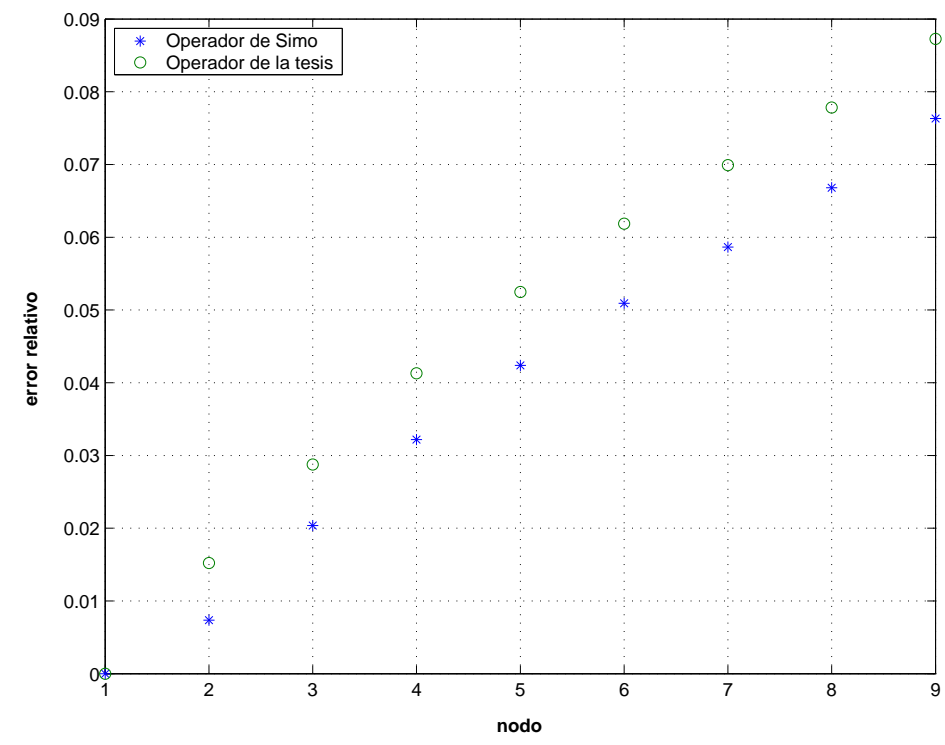

Figura 7.12: Estructura 1 con un momento de eje fijo en el extremo. Error relativo en los desplazamientos nodales en la solución incremental pura en 20 pasos

\begin{tabular}{l|cc} 
& Matriz de Simó & Matriz de la tesis \\
\hline residuo final & 0,12410 & 0,17046 \\
error relativo (nodo 9) & 0,07632 & 0,08726 \\
\hline
\end{tabular}

Cuadro 7.9: Residuo final y error en el extremo en la estructura 1 con un momento de eje fijo 


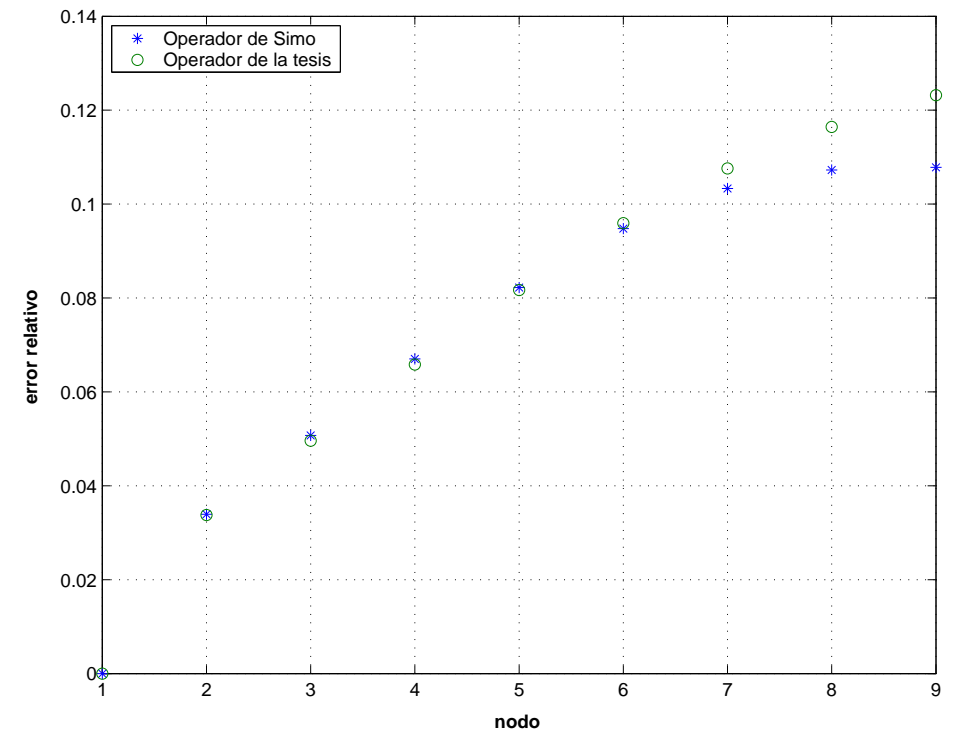

Figura 7.13: Estructura 1 con un momento perseguidor en el extremo. Error relativo en los desplazamientos nodales en la solución incremental pura en 20 pasos

\begin{tabular}{l|cc} 
& Matriz de Simó & Matriz de la tesis \\
\hline & & \\
residuo final & 0,16325 & 0,29698 \\
error relativo (nodo 9) & 0,10781 & 0,12319 \\
\hline
\end{tabular}

Cuadro 7.10: Residuo final y error en el extremo en la estructura 1 con un momento perseguidor 
La Estructura 2 vuelve a ser una ménsula de planta circular que se diferencia de la 1 en la relación entre su longitud y las características resistentes de la sección transversal: en este caso el radio es $R=100$, las características mecánicas de la sección transversal son, de nuevo, $A=I_{y}=I_{z}=4 \pi, J=8 \pi$ y las del material $E=1 / 2 \pi$, y $E / G=2,5$. La discretización es análoga, con 8 elementos de 2 nodos y un punto de integración. En su extremo (nodo 9) se aplica una fuerza de magnitud 1 en dos modalidades: la primera de ellas, de eje fijo, paralela al eje $z$, y la segunda, con la misma dirección inicial, perseguidora. Como en el caso de la estructura 1 se analiza el error relativo en lo desplazamientos nodales para las dos acciones consideradas, comparando los resultados obtenidos con el operador de Simó y con el de la tesis.

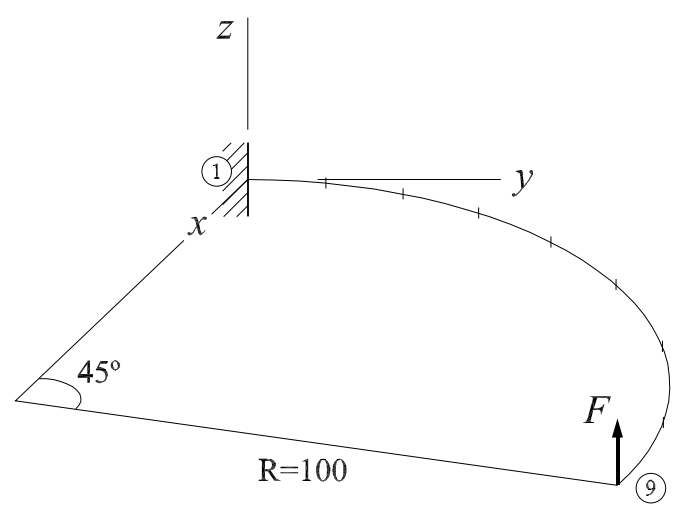

Figura 7.14: Estructura 2

Como punto de comparación para la evaluación de los errores relativos se toman los desplazamientos calculados mediante una solución incremental - iterativa con el operador de Simó en 20 escalones de carga. En las figuras 7.15 y 7.16 se observan los resultados obtenidos para la fuerza de eje fijo y para la perseguidora, respectivamente.

$\mathrm{Al}$ evaluar la solución incremental pura calculamos la 1-norma de la matriz tangente de Simó y de la matriz antisimétrica (cuya suma proporciona la matriz tangente de la tesis). El resultado (tabla 7.11) indica, análogamente a lo obtenido para la estructura 1 sometida a momento, que la norma de la matriz antisimétrica es cuatro órdenes de magnitud inferior a la de la matriz de Simó. 


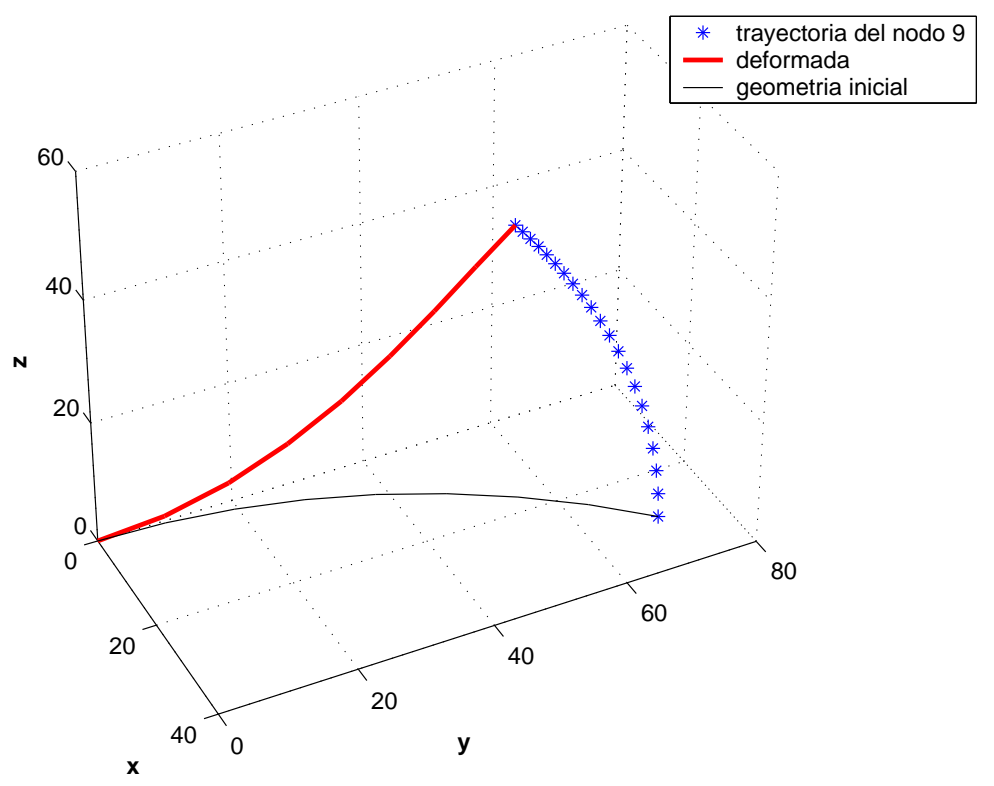

Figura 7.15: Estructura 2. Acción de una fuerza de eje fijo $F_{z}=1$. Solución iterativa

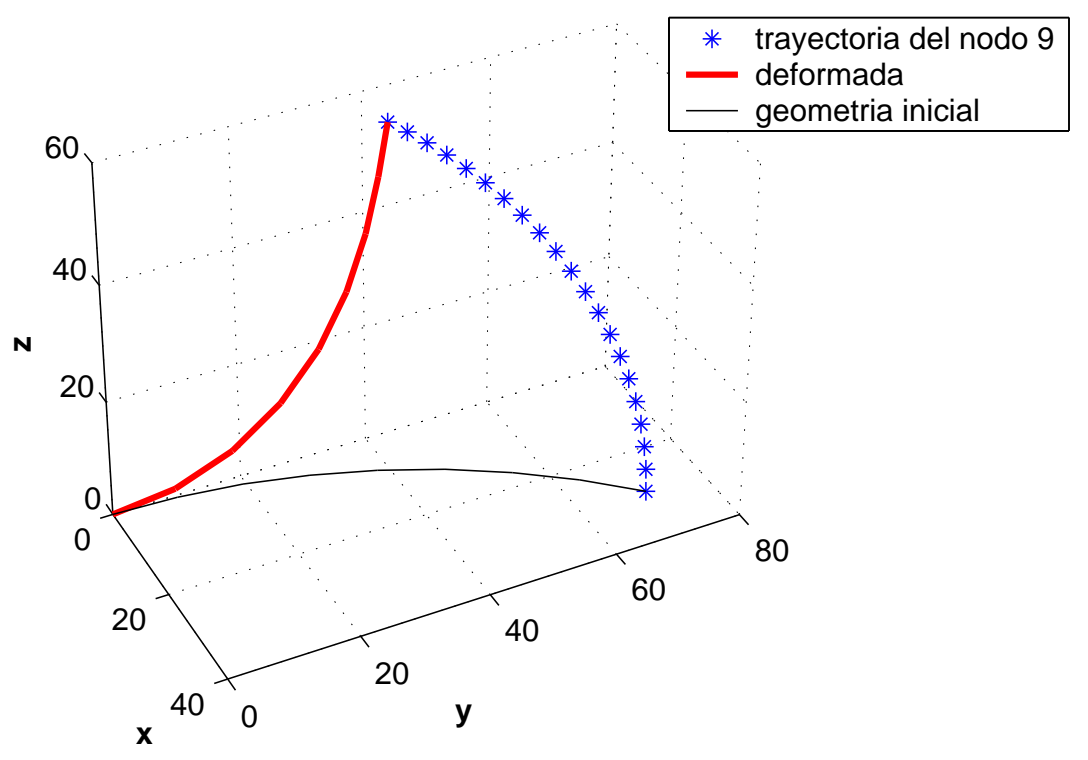

Figura 7.16: Estructura 2. Acción de una fuerza perseguidora de módulo 1. Solución iterativa 


\begin{tabular}{l|cc} 
& Matriz de Simó & Matriz antisimétrica \\
\hline fuerza espacial & 10,936 & $7,92210^{-3}$ \\
fuerza perseguidora & 10,949 & $8,01010^{-3}$ \\
\hline
\end{tabular}

Cuadro 7.11: 1-norma de las matrices tangentes para las dos acciones consideradas en la estructura 2

El error relativo es ahora mucho mayor que en el caso de la estructura 1: varía, para la fuerza perseguidora, entre el $50 \%$ y más del $70 \%$ en los distintos nodos de la estructura (figura 7.17). La precisión de la solución incremental pura es baja, por ello las diferencias entre operadores prácticamente no son perceptibles: en el cuadro 7.12 se observa que la diferencia en el error relativo aparece en el cuarto decimal. La norma del residuo es algo mayor con el operador de la tesis que con el de Simó.

La situación asociada a la acción de la fuerza perseguidora, como se puede ver en la figura 7.18 y en el cuadro 7.13 es análoga.

Comentario 7.5.2. En esta sección hemos probado que la influencia de los términos de la rigidez tangente derivados de la segunda variación de la configuración fuera de la trayectoria de equilibrio (en una solución puramente incremental) es pequeña, y en cualquier caso, su consideración no aporta ventajas en comparación con el uso de la matriz tangente de Simó y Vu-Quoc.

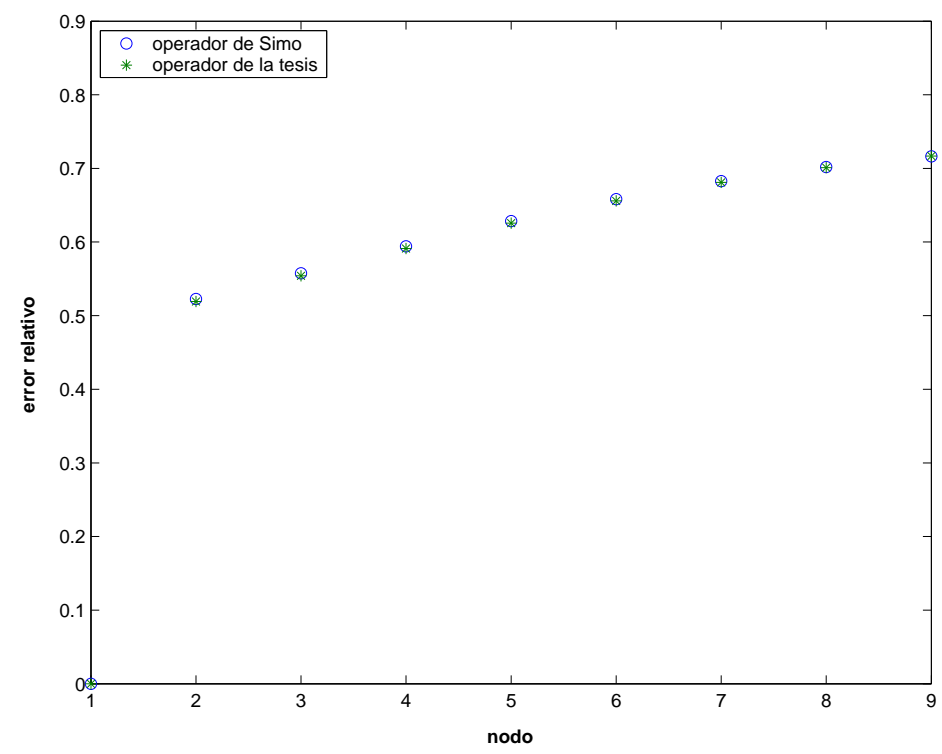

Figura 7.17: Estructura 2 con una fuerza de eje fijo en el extremo. Error relativo en los desplazamientos nodales en la solución incremental pura en 20 pasos 


\begin{tabular}{l|cc} 
& Matriz de Simó & Matriz de la tesis \\
\hline & 0,00994 & 0,01058 \\
residuo final & 0,71643 & 0,71672 \\
\hline
\end{tabular}

Cuadro 7.12: Residuo final y error en el extremo en la estructura 2 con una fuerza de eje fijo

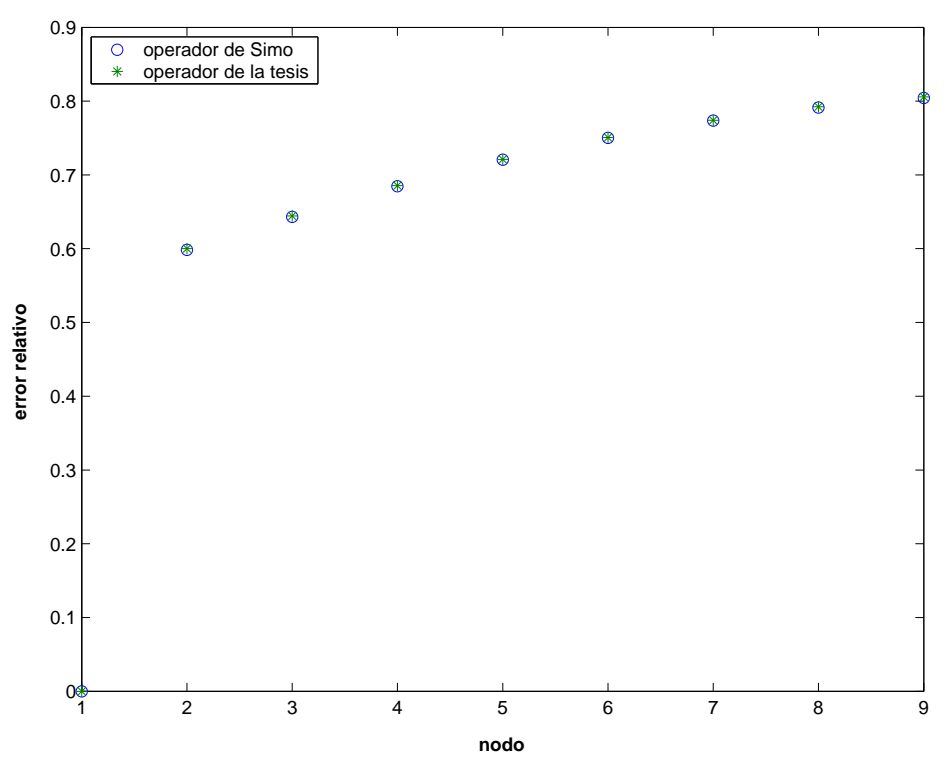

Figura 7.18: Estructura 2 con una fuerza perseguidora en el extremo. Error relativo en los desplazamientos nodales en la solución incremental pura en 20 pasos

\begin{tabular}{l|cc} 
& Matriz de Simó & Matriz de la tesis \\
\hline residuo final & 0,01002 & 0,01067 \\
error relativo (nodo 9) & 0,80426 & 0,80580 \\
\hline
\end{tabular}

Cuadro 7.13: Residuo final y error en el extremo en la estructura 2 con una fuerza perseguidora 


\subsubsection{Ejemplos}

En esta sección estudiamos cuatro ejemplos que muestran las posibilidades y también las dificultades inherentes al modelo de Reissner-Simó. El primero es el problema clásico de la elástica que demuestra la potencia del modelo para resolver un problema de grandes rotaciones con un número limitado de elementos. El siguiente caso, el voladizo curvo de Bathe, sirve como ejemplo de deformación tridimensional de una pieza alargada. A continuación abordamos el problema de la inversión de la geometría de un pórtico biarticulado de dos piezas cuya respuesta es altamente no lineal. Por último analizamos la inversión de la forma de una malla espacial.

\section{La elástica de Euler}

Analizamos el problema en el plano $z=0$ de una pieza simplemente apoyada de longitud $L$ y rigidez a flexión $E I$ sometida en los extremos a una fuerza de compresión en la dirección $x$ que une los apoyos; se trata del problema clásico de estabilidad de una pieza comprimida estudiado por Euler en [18]. Cuando la fuerza alcanza el valor crítico $P_{\text {crit }}=\pi^{2} E I / L^{2}$ se produce una bifurcación de la trayectoria de equilibrio: se puede avanzar por la rama vertical aumentando la compresión en situación de equilibro inestable, o bien por una rama horizontal de equilibrio indiferente en la que los movimientos perpendiculares a la directriz no están determinados.
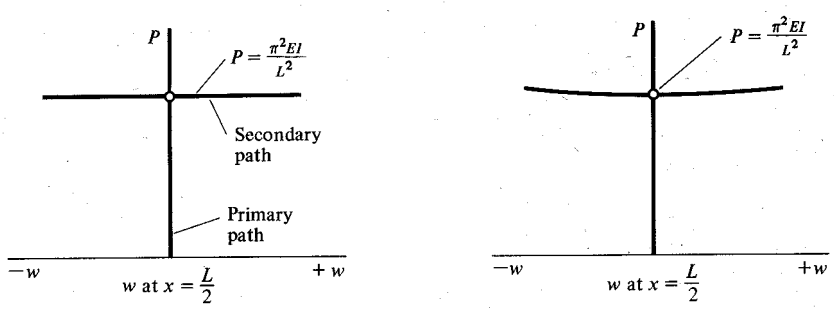

Figura 7.19: Figuras 1.8 y 1.9 de Brush et al.

En el análisis clásico del problema se supone que la pieza es indeformable frente al esfuerzo axil. Además se emplea la medida lagrangiana de la deformación $\epsilon_{x}=$ $\frac{\partial u}{\partial x}+\frac{1}{2}\left(\frac{\partial v}{\partial x}\right)^{2}$ y se asume una relación lineal entre el momento flector y la derivada segunda de la flecha $M=E I v^{\prime \prime}$; esta expresión equivale a aproximar la curvatura por $v^{\prime \prime}$, lo que sólo es válido cuando los desplazamientos son pequeños. Cuando se emplea la expresión exacta de la curvatura en función de las derivadas de la flecha (2.4) la trayectoria de equilibrio en la bifurcación se curva [10, p. 10, 11] -figura 7.19-, y la solución al problema es la elástica de Euler (sección 2.1).

Cuando se aborda la solución por elementos finitos de este problema aparece la dificultad de detectar el punto de bifurcación de la trayectoria de equilibrio. Aunque existen técnicas de detección de este tipo de situación, hemos optado por utilizar una 


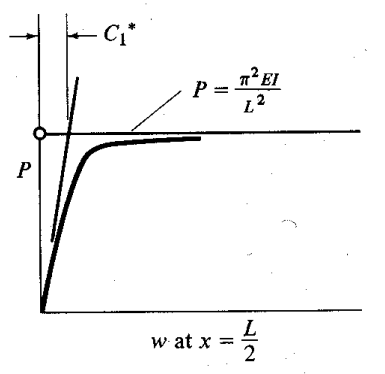

Figura 7.20: Figura 1.10 de Brush et al.

geometría inicial ligeramente perturbada

$$
y_{0}=C \sin \frac{\pi x}{L} \quad C=10^{-3} L,
$$

que elimina la bifurcación correspondiente a la pieza ideal. Brush y Almroth [10, eq. (1.27)] proporcionan la expresión analítica del desplazamiento vertical (calculada mediante la expresión aproximada de la curvatura)

$$
v=\frac{P}{\frac{\pi^{2} E I}{L^{2}}-P} C \sin \frac{\pi x}{L} .
$$

La trayectoria de equilibrio que resulta de esta expresión parte con tangente $P_{\text {crit }} / C$ en el origen y presenta una asíntota horizontal en $P=P_{\text {crit }}$ (figura 7.20).

La viga se orienta en la dirección del eje $x$. Su sección transversal es rectangular, de $0,1 \mathrm{~m}$ de canto y $0,3 \mathrm{~m}$ de anchura. El eje $y$ define la dirección vertical. El apoyo fijo se sitúa en el origen de coordenadas (nodo 1); en el nodo 13 sólo se coacciona el movimiento en la dirección $y$. Las características mecánicas en $\mathrm{m}$ y $\mathrm{kN}$ (admitiendo por sencillez que $A_{Q 2}=A$ ) son:

\begin{tabular}{c|ccccc} 
& $L$ & $A$ & $I_{3}$ & $E$ & $E / G$ \\
\hline pieza comprimida & 10 & 0,03 & $2,5 \cdot 10^{-5}$ & $2,0 \cdot 10^{8}$ & 2,5 \\
\hline
\end{tabular}

La solución se plantea mediante un modelo de 10 elementos rectos (figura 7.21). Para superar la zona de inestabilidad en la trayectoria de equilibrio controlamos el proceso con la estrategia basada en la longitud de arco. En primer lugar vamos a comparar el resultado del modelo con la expresión (7.92) obtenida a partir de la expresión linealizada de la curvatura para una pieza sin rigidez a cortante ni a axil. En la figura 7.22 mostramos las dos trayectorias de equilibrio (a partir de $P=350$ $\mathrm{kN})$, así como la carga crítica de Euler, que en este problema es $P_{\text {crit }}=493,48 \mathrm{kN}$. Las trayectorias se han trazado empleando una longitud de arco $l_{n}=5 \mathrm{y}$ un total de 101 incrementos, lo que ha permitido reflejar correctamente el cambio de pendiente en la trayectoria. Al contrario de lo que sucede con la solución simplificada de Brush, el 


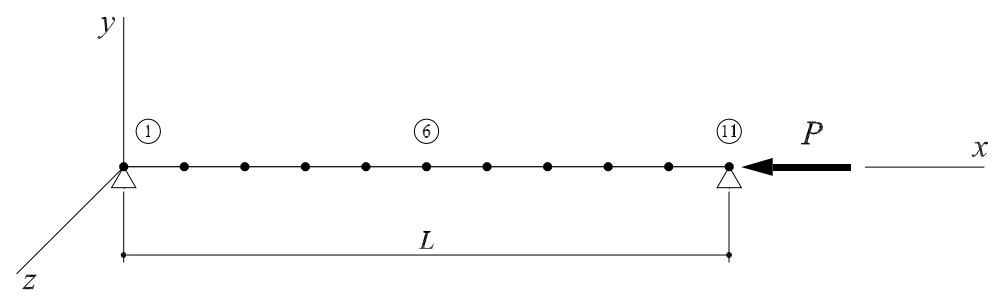

Figura 7.21: Modelo de la viga de Euler

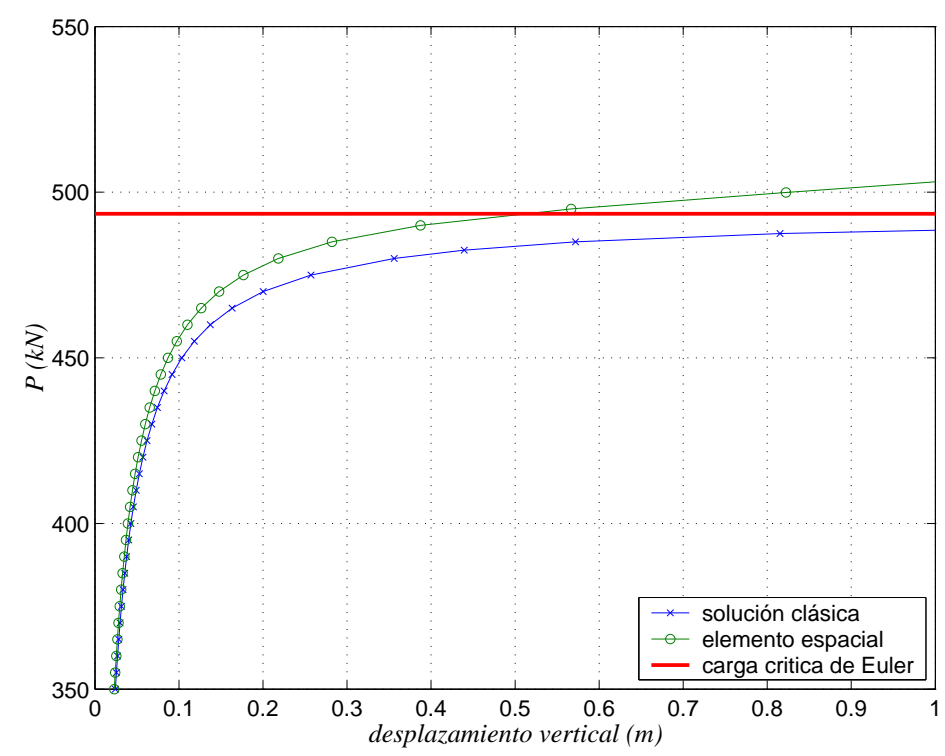

Figura 7.22: Comparación de trayectorias de equilibrio para la pieza comprimida con una perturbación inicial

camino de equilibrio obtenido a partir de la solución con elementos finitos sobrepasa la frontera definida por el valor de la carga crítica de Euler.

Para reproducir las distintas formas de la elástica de Euler necesitamos sobrepasar la frontera de la carga crítica; para ello repetimos el análisis con un valor cinco veces mayor de la longitud de arco $l_{n}=25$ empleando 144 incrementos. Hemos optado por mantener la representación de los elementos como segmentos rectos para recordar que la interpolación de los desplazamientos es lineal, pero que a pesar de ello los resultados son precisos. La trayectoria de equilibrio correspondiente al nodo 6 (centro de la pieza) se puede ver en la figura 7.23. La inestabilidad se produce en el entorno del incremento 20 para $P \simeq 500 \mathrm{kN}$. El incremento 35 proporciona el valor máximo del 
desplazamiento vertical en el nodo 6, asociado a una fuerza de 874, $45 \mathrm{kN}$. Detenemos el proceso en el paso 144 en el que el nodo 11 se sitúa en $x=-5 \mathrm{~m}$ y $P=3599,4$ $\mathrm{kN}$. La corrección en cada incremento es muy efectiva: en la rama inicial ascendente la convergencia se alcanza con 2 iteraciones; para atravesar la barrera de la carga de Euler se requiere puntualmente 11 iteraciones en el paso 20, y a partir de aquí se produce la corrección con un valor estable de 3 iteraciones por paso. En la figura 7.24 recogemos las formas características de la elástica en distintas fases del proceso de deformación, así como la trayectoria del nodo central (6). Los esfuerzos en cada elemento en el último incremento se muestran en la figura 7.25 .

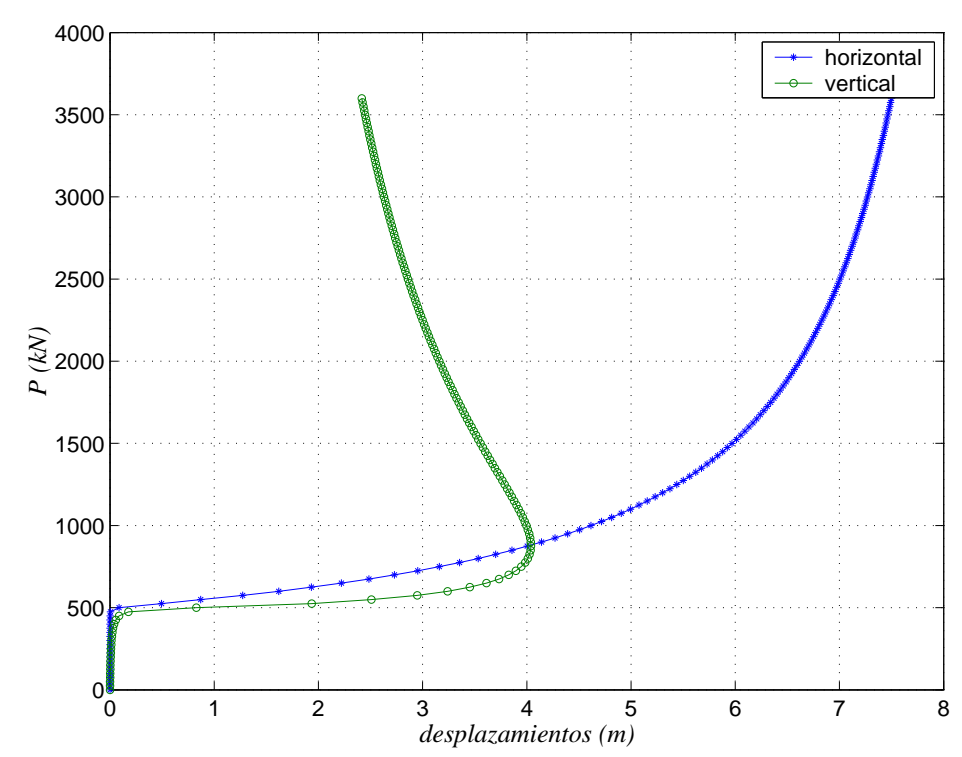

Figura 7.23: Viga de Euler. Trayectoria de equilibrio del nodo 6

En la figura 7.26 se representa el trabajo realizado por la fuerza de compresión en el nodo 11 a lo largo del proceso de deformación — en la integral $u$ representa el desplazamiento horizontal del nodo, y en la gráfica $d=\operatorname{abs}(u)-$

$$
W=\int_{0}^{-15}(-F) d u \simeq-\sum_{n=1}^{144} \frac{1}{2}\left(F_{n+1}+F_{n}\right)\left(u_{n+1}-u_{n}\right)
$$

que se ha evaluado integrando numéricamente la trayectoria de equilibrio del nodo. También se ha incluido en la representación el valor la energía de deformación correspondiente a seis instantes del proceso de carga. Para obtener su valor numérico necesitamos calcular la energía de deformación $U_{e}$ correspondiente a cada elemento:

$$
U=\int_{0}^{10} \mathcal{L}(S) d S \simeq \sum_{e=1}^{10} U_{e}
$$




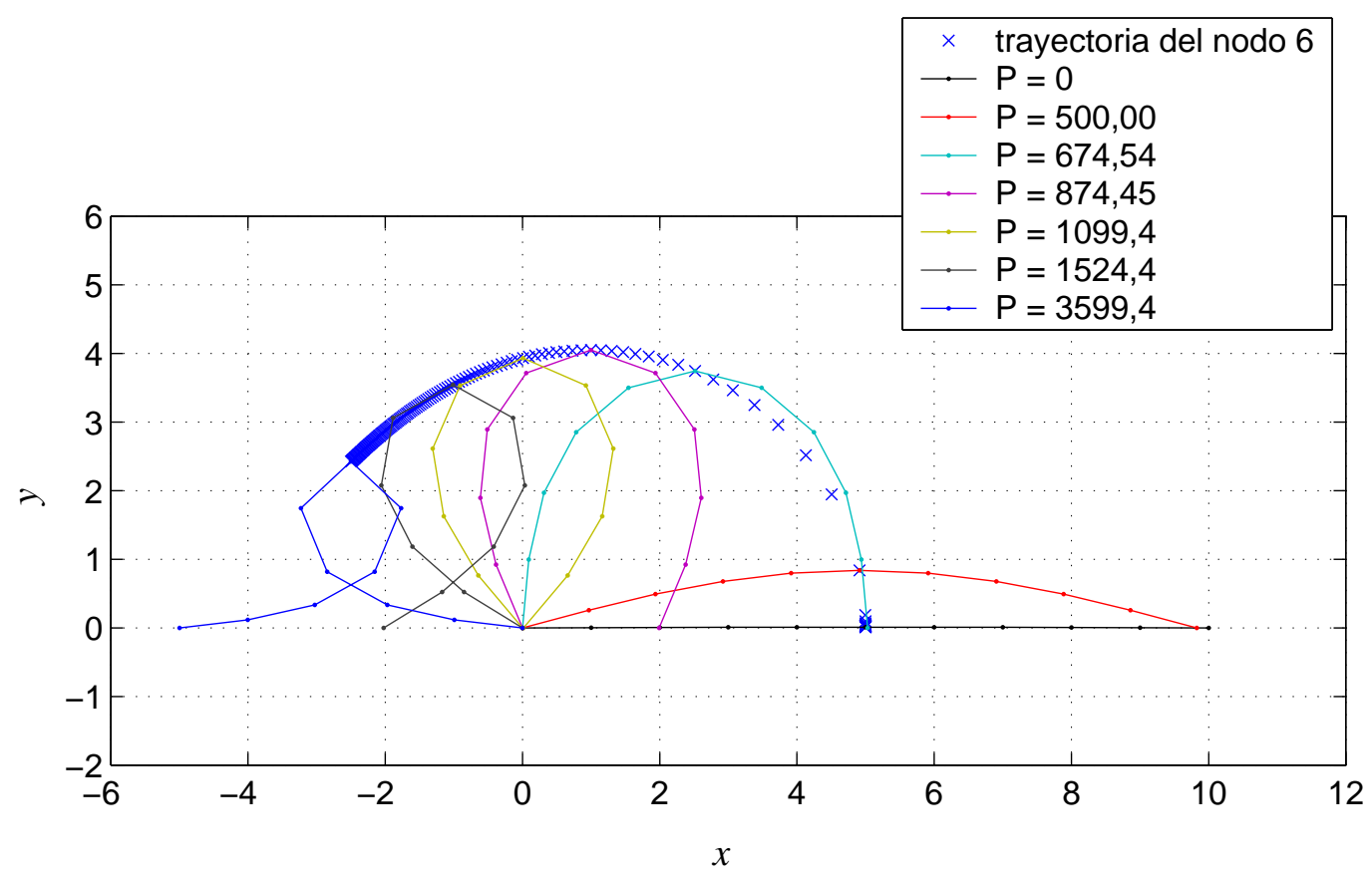

Figura 7.24: Proceso de deformación de la viga de Euler (módulo de la fuerza en kN, coordenadas en $\mathrm{m}$ )
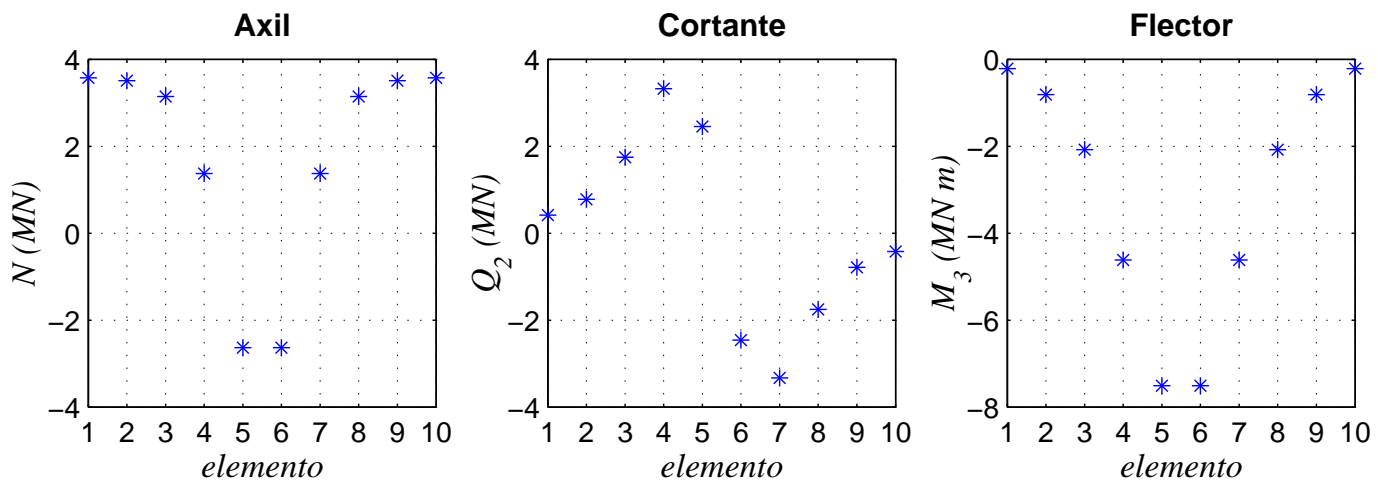

Figura 7.25: Esfuerzos correspondientes a $F=3599,4 k N$

Ésta se calcula teniendo en cuenta que, con la interpolación escogida, el valor numérico de los esfuerzos es constante en el elemento, luego

$$
U_{e}=\left.\int_{-1}^{1} \mathcal{L}(\xi) J d \xi \simeq \frac{1}{2}\left(\frac{N^{2}}{E A}+\frac{\left(Q_{2}\right)^{2}}{G A_{Q 2}}+\frac{\left(M_{3}\right)^{2}}{E I_{3}}\right)\right|_{\xi=0} L_{e},
$$

donde $L_{e}$ es la longitud del elemento ( 1 en todos los elementos de este ejemplo). 


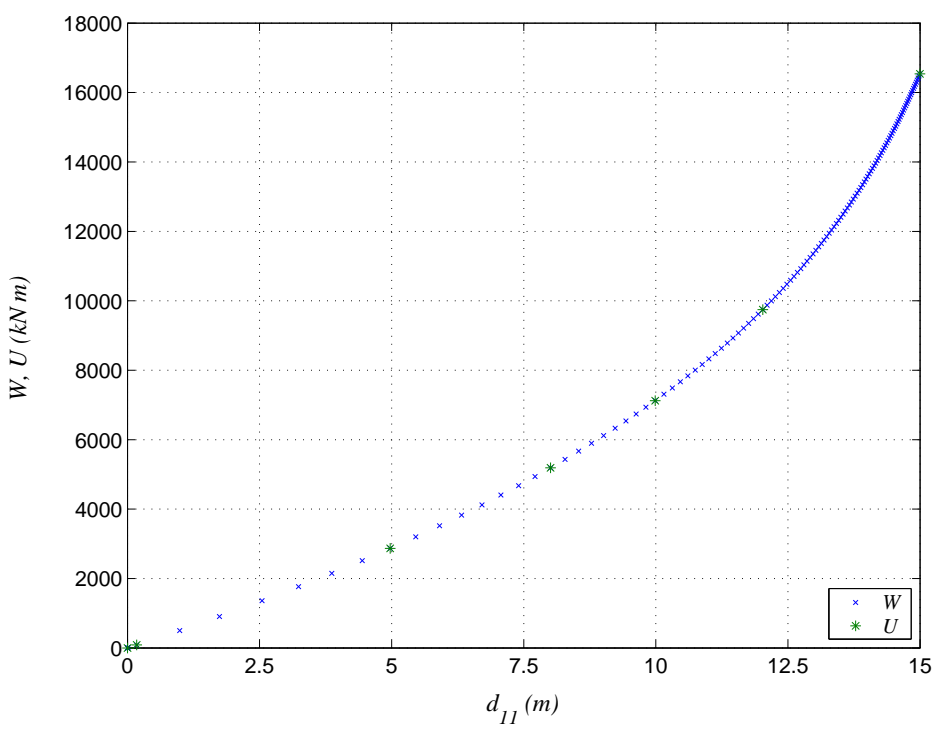

Figura 7.26: Trabajo y energía durante la deformación

En la figura se puede comprobar cómo en los seis escalones de carga considerados la correspondencia entre el valor de la energía de deformación y el trabajo de las fuerzas exteriores es completa. El cuadro 7.14 muestra los valores numéricos que lo corroboran.

\begin{tabular}{ll|rrrrrr}
\multicolumn{2}{c}{ incremento } & \multicolumn{1}{c}{20} & 27 & 35 & 44 & 61 & 144 \\
\hline & & & & & & & \\
$d_{11}$ & $(\mathrm{~m})$ & 0,18 & 4,97 & 8,01 & 9,99 & 12,02 & 15,00 \\
$F$ & $(\mathrm{kN})$ & 500,0 & 674,5 & 874,5 & 1099,4 & 1524,4 & 3599,4 \\
$W$ & $(\mathrm{kNm})$ & 84,6 & 2866,4 & 5189,3 & 7124,7 & 9745,1 & 16538,0 \\
$U$ & $(\mathrm{kNm})$ & 85,7 & 2866,4 & 5188,8 & 7124,0 & 9744,2 & 16537,0 \\
\hline
\end{tabular}

Cuadro 7.14: Trabajo y energía durante la deformación. Comparación de resultados numéricos

Por último deseamos analizar la capacidad de la solución numérica por elementos finitos para conservar los invariantes del modelo $\mathcal{H}, C_{(1)}$ y $C_{(2)}$ ecs. (5.38) y (5.51) presentados en el capítulo 5. En el caso plano, $Q_{3}=M_{1}=M_{2}=0$ y sus expresiones se reducen a:

$$
\begin{aligned}
\mathcal{H} & =\frac{1}{2}\left(\frac{N^{2}}{E A}+\frac{\left(Q_{2}\right)^{2}}{G A_{Q 2}}+\frac{\left(M_{3}\right)^{2}}{E I_{3}}\right)+N \\
C_{(1)} & =N^{2}+\left(Q_{2}\right)^{2} \\
C_{(2)} & =0 .
\end{aligned}
$$

El primer invariante $\mathcal{H}$ es la suma de la densidad de energía complementaria (que en este caso lineal coincide con la densidad de energía de deformación) más la proyección 


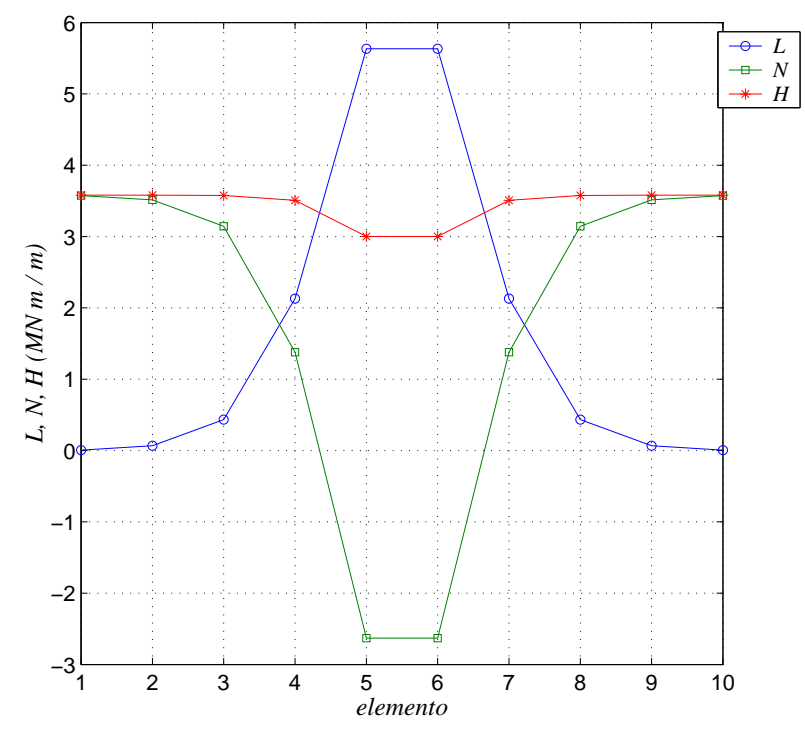

Figura 7.27: Densidad de energía de deformación, esfuerzo axil y valor de $\mathcal{H}$ en cada elemento

del vector de esfuerzos con componentes de fuerza sobre la normal a la sección (que coincide con el esfuerzo axil). En el diagrama 7.27 se puede observar el valor numérico en cada elemento de la densidad de energía, del esfuerzo axil y de $\mathcal{H}$. Resulta obvio que la solución numérica desarrollada no es capaz de conservar el primer invariante, lo que sin embargo no es obstáculo para que la estimación de la energía de deformación, como vimos antes, sea muy precisa.

La situación con el segundo invariante es opuesta: en la figura 7.28 se aprecia la comparación entre la estimación de $\mathcal{H}$ y $C_{(1)}$, y se comprueba que el método numérico sí respeta el valor previsto de $C_{(1)}$. El motivo de este comportamiento estriba en que este último invariante es una forma alternativa de expresar las ecuaciones de equilibrio de fuerzas, y la solución por elementos finitos en desplazamientos, que se basa en la ecuación de trabajos virtuales, también es una expresión del equilibrio de la estructura.

Por último se incluye una representación de las superficies $\mathcal{H}=3599,4 \mathrm{kN} \mathrm{m} / \mathrm{m}$ y $C_{(1)}=3599,4^{2} \mathrm{kN}^{2}$. La intersección de ambas define la órbita en la que se encuentran los puntos $\left(N, Q_{2}, M_{3}\right)$. Los valores numéricos de los esfuerzos, que se encuentran aproximadamente sobre la órbita, se han grafiado en color amarillo. Aunque el dibujo sólo permite apreciar los esfuerzos en los elementos 6 a 10 (el resto queda oculto) se ve cómo la mayor desviación aparece en el punto correspondiente al elemento 6 -máximo flector negativo-, en concordancia con el resultado de la figura 7.27. 


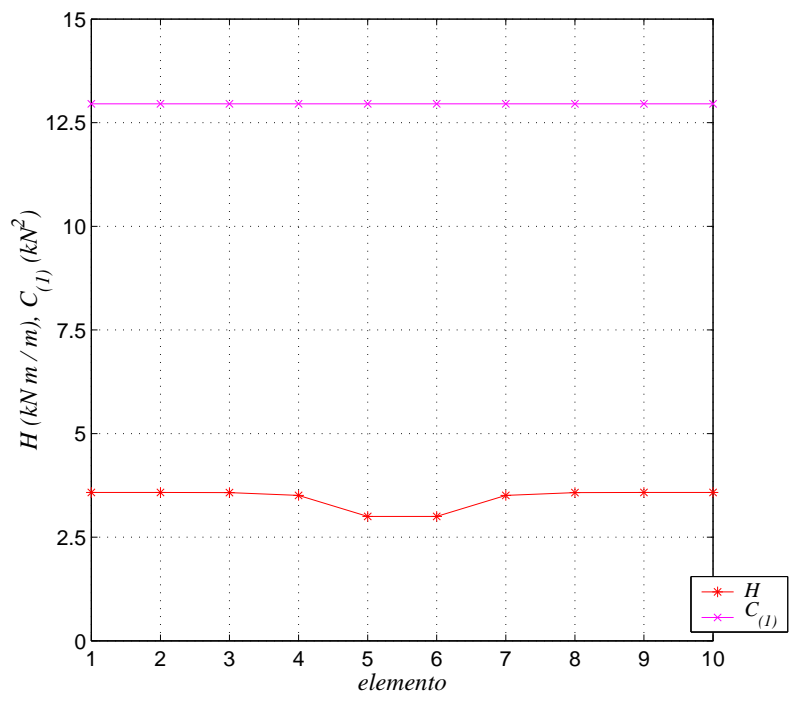

Figura 7.28: Valores del primer $(\mathcal{H})$ y tercer invariante $\left(C_{(1)}\right)$ en cada elemento

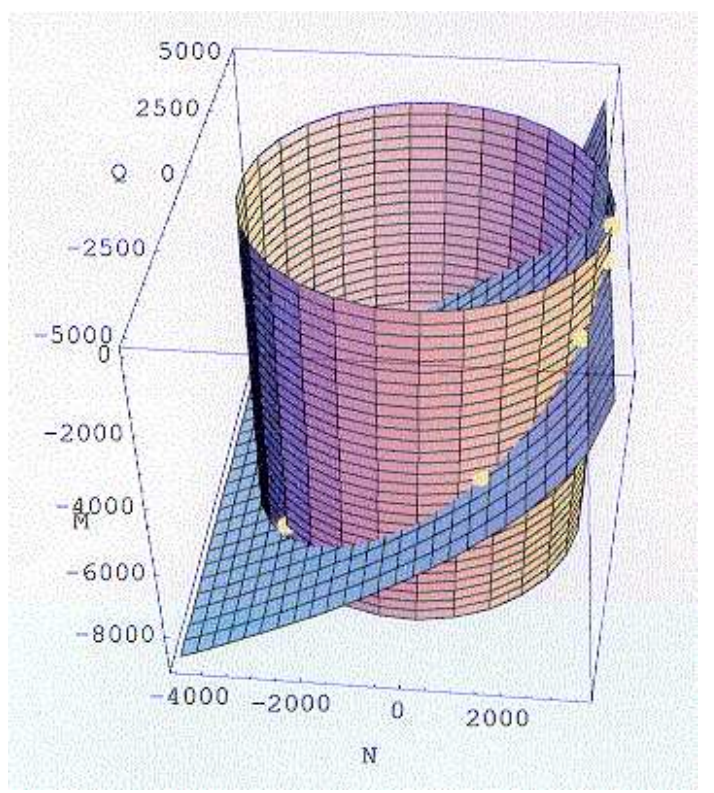

Figura 7.29: Superficies invariantes y esfuerzos 


\section{El voladizo de Bathe y Bolourchi}

Ya habíamos empleado en la sección anterior un modelo de 8 elementos rectos de 2 nodos basado en el problema propuesto por Bathe y Bolourchi [8] de un voladizo de directriz circular de radio $R=100$ in (pulgadas) con un desarrollo de $45^{\circ}$ contenida en el plano $z=0$. Reproducimos de nuevo su geometría en la figura siguiente

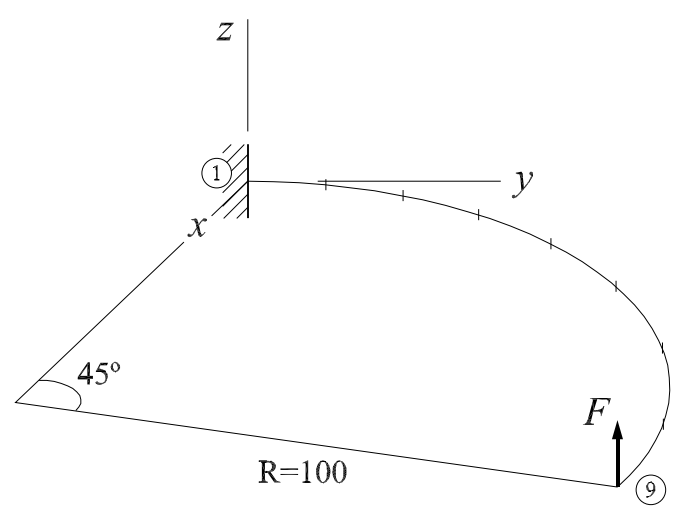

Figura 7.30: Voladizo de Bathe y Bolourchi

La sección transversal es cuadrada de lado 1 in. El área de cortante es $A_{Q 2}=$ $A_{Q 3}=5 / 6$ in $^{2}$ y el módulo de torsión, aplicando la fórmula de Saint-Venant, es

$$
J=\frac{A^{4}}{40\left(I_{y}+I_{z}\right)}=3 / 20 \mathrm{in}^{4},
$$

y con la expresión de Timoshenko/Courbon para secciones rectangulares $J=0,1406$ in $^{4}$.

Aunque ni Bathe ni Simó proporcionan los datos de la sección transversal usados en el cálculo, tras realizar alguna prueba hemos llegado a la conclusión de que $(a)$ emplean como valor del área de cortante el área de la sección transversal (no tienen en cuenta la reducción que se deduce de la teoría de vigas clásica), y (b) para el módulo de torsión usan simplemente el momento polar $I_{y}+I_{z}$. El resumen de los datos de la sección transversal y el material es pues el siguiente (empleando in, lb):

\begin{tabular}{c|cccccccc} 
& $A$ & $A_{Q 2}$ & $A_{Q 3}$ & $J$ & $I_{2}$ & $I_{3}$ & $E$ & $E / G$ \\
\hline voladizo de Bathe & 1 & 1 & 1 & $1 / 6$ & $1 / 12$ & $1 / 12$ & $10^{7}$ & 2 \\
\hline
\end{tabular}

Bathe considera la acción de una fuerza de eje fijo de valor $600 \mathrm{lb}$ actuando en la dirección del eje $z$ y emplea una elemento finito totalmente lagrangiano con la 
cinemática de Timosheko. La solución se alcanza con 60 incrementos. Simó resuelve el problema usando su elemento de tres nodos, con tres escalones de carga (la secuencia es $300-150-150)$ y un máximo de 13 iteraciones. Nuestra solución con el elemento espacial emplea control de carga con tres incrementos iguales y 11, 14 y 7 iteraciones en cada incremento. Los resultados obtenidos se comparan con los de la literatura en el cuadro 7.15, y se puede comprobar que coinciden prácticamente con los de Simó.

\begin{tabular}{c|lll} 
& \multicolumn{1}{|c}{$x$} & \multicolumn{1}{c}{$y$} & \multicolumn{1}{c}{$z$} \\
\hline & & & \\
Bathe & 15,9 & 47,2 & 53,4 \\
Simó & 15,79 & 47,23 & 53,37 \\
elemento espacial & 15,797 & 47,234 & 53,366 \\
\hline
\end{tabular}

Cuadro 7.15: Posición final del extremo del voladizo de Bathe y Bolourchi. Comparación de resultados

Realizamos un segundo experimento, aplicando ahora una fuerza perseguidora en el extremo, orientada inicialmente en la dirección del eje $z$. En este caso Simó proporciona las trayectorias de los desplazamientos, pero no datos numéricos precisos. La tesis de Morán [46] sí proporciona resultados numéricos para un valor de la fuerza de 600 lb que emplearemos como dato de comparación.

\begin{tabular}{c|ccc} 
fuerza perseguidora & $x$ & $y$ & $z$ \\
\hline Morán [46] & $-9,98$ & 25,08 & 59,65
\end{tabular}

Morán, que tampoco detalla los valores empleados para el área de cortante y el módulo de torsión de la sección, obtiene estos resultados por medio de un modelo de rotaciones finitas con una parametrización vectorial, empleando también 8 elementos de dos nodos con interpolación lineal .

Resolvemos el problema con el elemento espacial utilizando las características mecánicas indicadas en la tabla anterior, y empleamos 4 escalones de carga de $150 \mathrm{lb}$ con una secuencia de $9-7-7-9$ iteraciones por incremento. La posición final del extremo del voladizo es

\begin{tabular}{c|ccc} 
fuerza perseguidora & $x$ & $y$ & $z$ \\
\hline elemento espacial (1) & $-4,355$ & 24,143 & 60,516
\end{tabular}

La discrepancia es evidente. Sin embargo, si consideramos el área de cortante $A_{Q 2}=A_{Q 3}=5 / 6$, y el módulo de torsión $J=0,1406$ indicados al principio, la coincidencia de los resultados es mucho mayor, aunque ahora se necesitan 5 incrementos y la secuencia de iteraciones $6-5-6-7-9$.

\begin{tabular}{c|ccc} 
fuerza perseguidora & $x$ & $y$ & $z$ \\
\hline elemento espacial (2) & $-9,953$ & 25,066 & 59,656
\end{tabular}

Por último, analizamos el proceso de deformación del voladizo sometido a una fuerza perseguidora $F_{3}$ (inicialmente paralela al eje $z$ ) actuando en el extremo, mediante una solución incremental-iterativa con control de carga, empleando escalones 
de 50 lb para los que sólo se requieren 3 iteraciones por incremento. Las deformadas correspondientes a valores de la fuerza múltiplos de $500 \mathrm{lb}$ (hasta alcanzar un valor máximo de $3000 \mathrm{lb}$ ), y la trayectoria del extremo (nodo 9) se han representado en la figura 7.31. La trayectoria de equilibrio de éste nodo se recoge en la figura 7.32 .

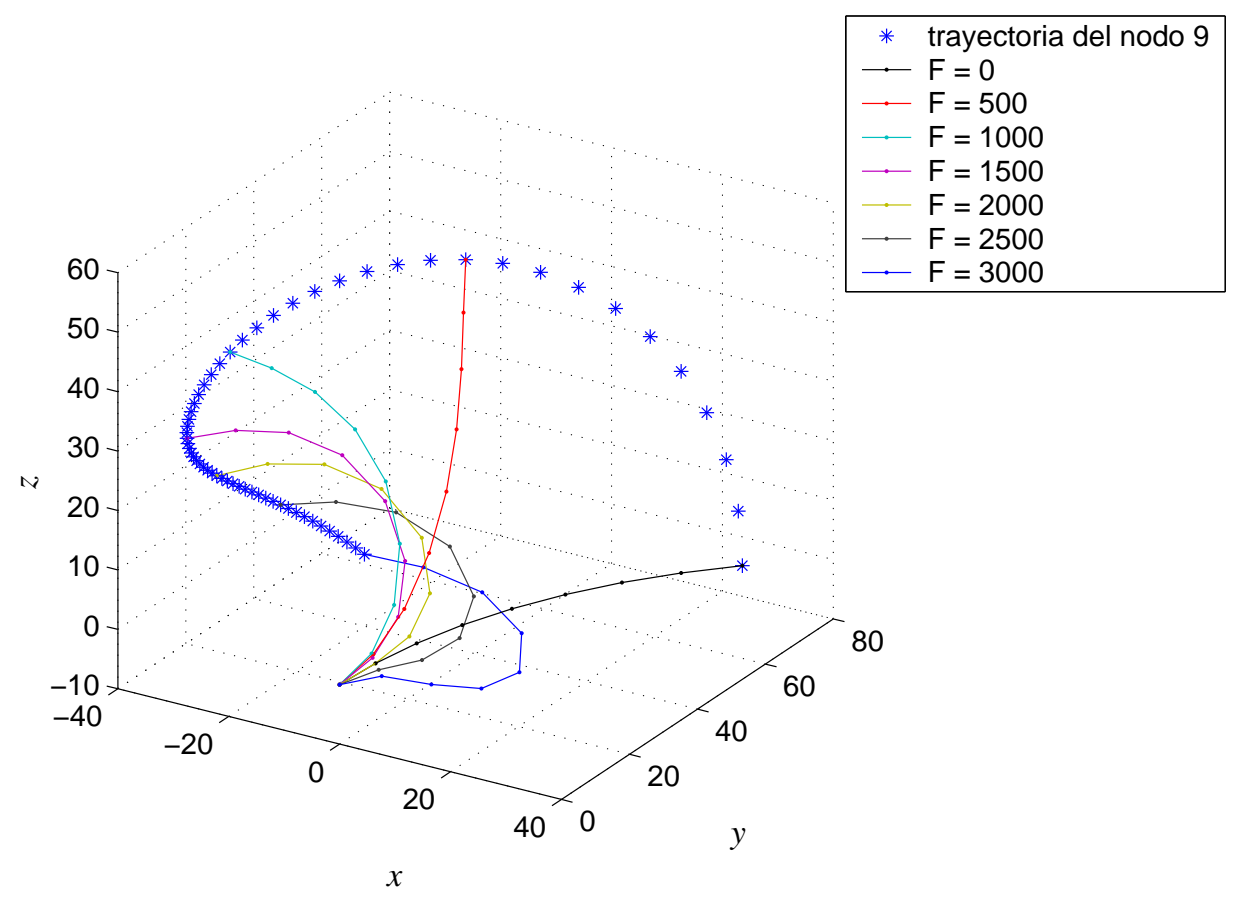

Figura 7.31: Proceso de deformación del voladizo de Bathe. Fuerza perseguidora en el extremo (módulo de la fuerza en lb, coordenadas en in)

También incluimos la representación (fig. 7.33) de las componentes de los esfuerzos en cada elemento correspondientes a $F_{3}=3000 \mathrm{lb}$. Por último, para ésta magnitud de la fuerza exterior, valoramos la conservación del primer invariante $\mathcal{H}$. En la figura 7.34 se aprecia el valor numérico de la densidad de energía de deformación $\mathcal{L}$ (idéntico al de la energía complementaria), el valor del esfuerzo axil $N$, y la suma de ambos $(\mathcal{H})$, cuyo valor fluctúa cerca de 0 . De nuevo se comprueba que la solución numérica por elementos finitos en desplazamientos no conserva exactamente el primer invariante.

El valor teórico de los otros dos invariantes, calculado a partir de las ecuaciones de equilibrio, es $C_{(1)}=9,010^{6}$ y $C_{(2)}=0$. En el cuadro 7.16 recogemos las diferencias entre el valor numérico y el teórico en cada elemento; en ambos casos las diferencias son varios órdenes de magnitud inferiores a los valores teóricos de cada invariante. Nos remitimos a los comentarios que, al respecto de la conservación de los invariantes, formulamos en el ejemplo de la viga de Euler. 


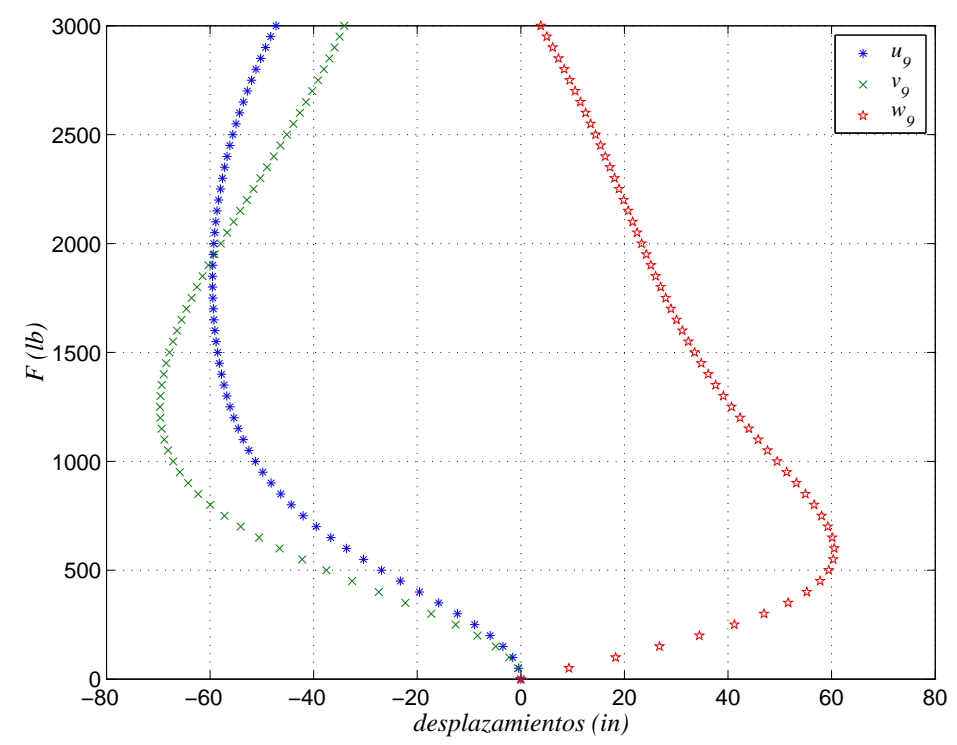

Figura 7.32: Voladizo de Bathe. Trayectoria de equilibrio del nodo 9
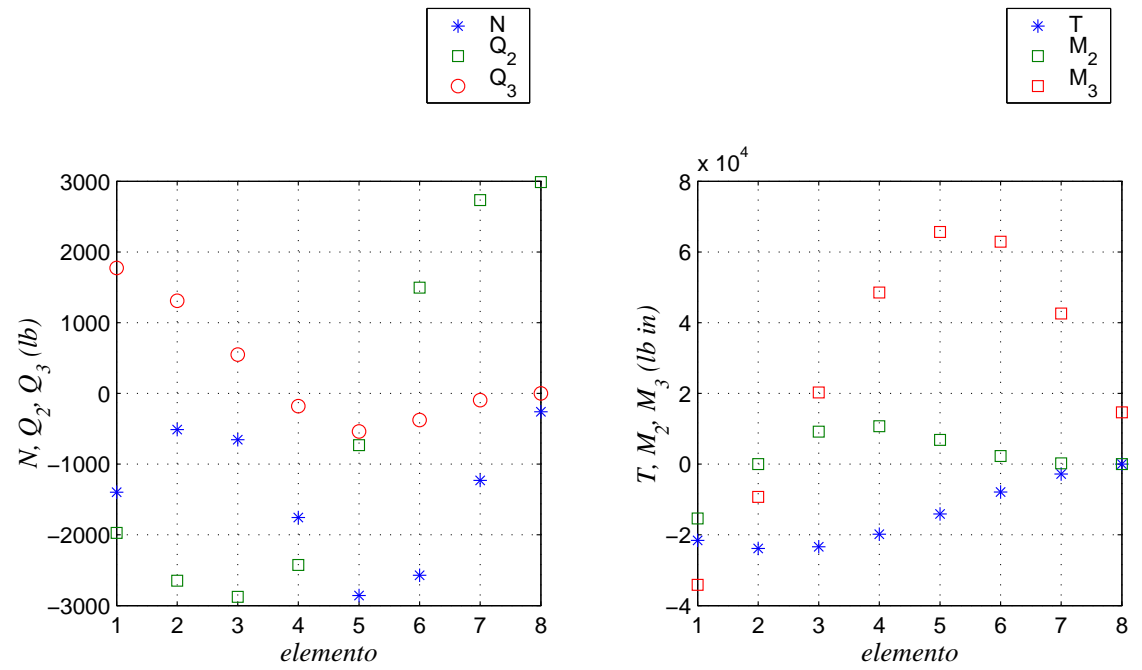

Figura 7.33: Esfuerzos en el voladizo de Bathe. $F=3000 \mathrm{lb}$, perseguidora 


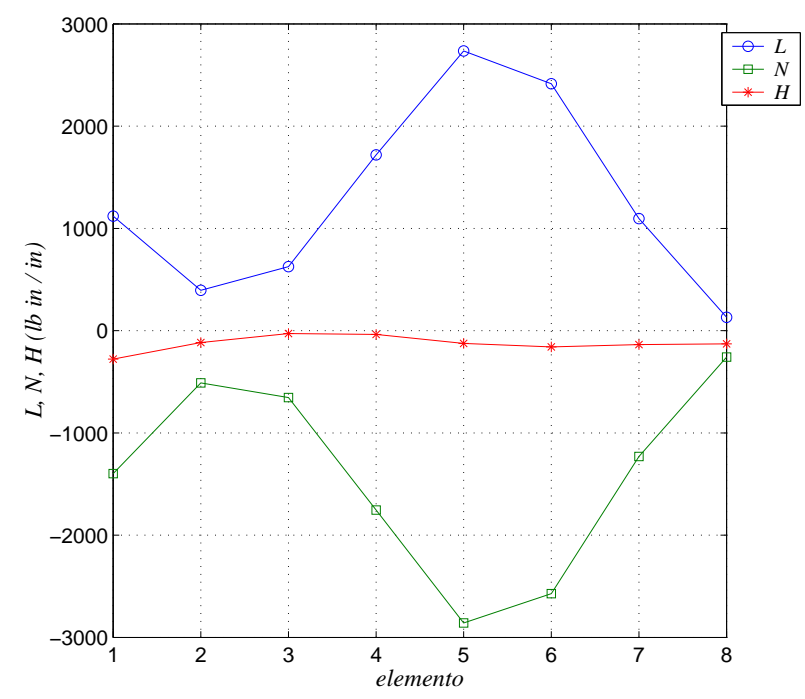

Figura 7.34: Densidad de energía de deformación, esfuerzo axil y valor de $\mathcal{H}$ en cada elemento

\begin{tabular}{l|rr} 
elemento & $C_{(1)}-9,010^{6}$ & $C_{(2)}$ \\
\hline & & \\
1 & $-0,0009$ & $-0,0316$ \\
2 & 0,0021 & $-0,0448$ \\
3 & 0,0027 & $-0,0400$ \\
4 & $-0,0008$ & $-0,0423$ \\
5 & $-0,0036$ & $-0,0659$ \\
6 & $-0,0118$ & $-0,0577$ \\
7 & $-0,0073$ & $-0,0089$ \\
8 & $-0,0042$ & 0,0044 \\
\hline
\end{tabular}

Cuadro 7.16: Diferencias entre $C_{(1)}\left(\mathrm{kN}^{2}\right)$ y $C_{(2)}\left(\mathrm{kN}^{2} \mathrm{~m}\right)$ y sus valores teóricos 


\section{Pórtico de Argyris y Symeonidis}

Argyris y Symeonidis [6] proponen el ejemplo de un pórtico plano formado por un pilar y un dintel rígidamente unidos. La base del pilar y el extremo del dintel se unen al exterior mediante una articulación. El pórtico se somete a la acción de una fuerza vertical excéntrica sobre el dintel. Las características mecánicas de la estructura, en $\mathrm{kN} \mathrm{y} \mathrm{cm}$, son

\begin{tabular}{c|ccccc} 
estructura & $L$ & $A$ & $I_{3}$ & $E$ & $E / G$ \\
\hline pórtico de Argyris & 120 & 6 & 2 & $7,2 \cdot 10^{3}$ & 2,6 \\
\hline
\end{tabular}

La discretización está compuesta por un total de 20 elementos de dos nodos (10 en el pilar y 10 en el dintel). La fuerza se aplica sobre el nodo 13, y se consideran dos situaciones básicas: (a) fuerza de eje fijo actuando en el sentido negativo del eje $y, y(b)$ fuerza perseguidora actuando en el sentido negativo del eje 2 de la sección (inicialmente paralelo al eje $y$ ).

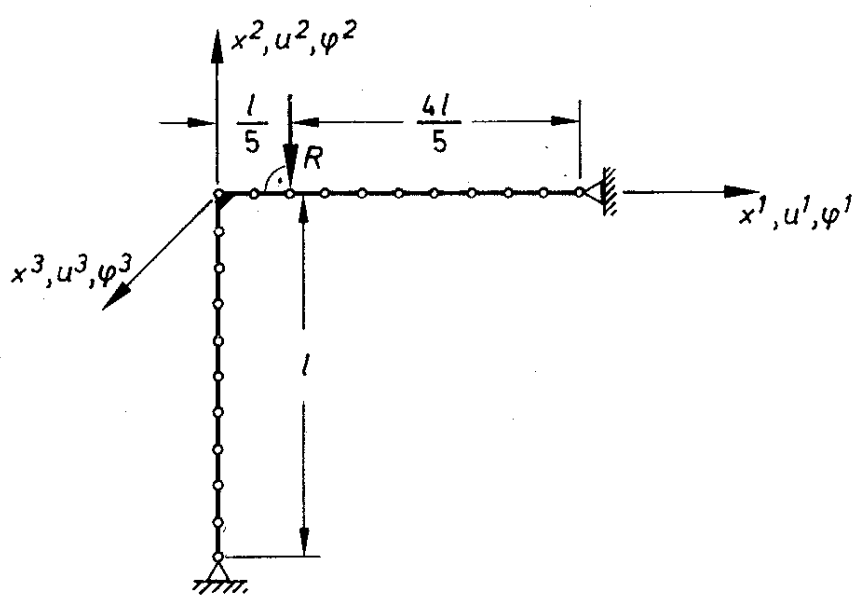

Figura 7.35: Modelo del pórtico de Argyris (figura 6.3.1. de Argyris et al.)

Este problema permite examinar la respuesta del modelo frente al fenómeno denominado snap through o inversión de la forma: la estructura experimenta un cambio brusco de configuración. La trayectoria de equilibrio en un problema de este tipo se caracteriza por la existencia de dos puntos límite, asociados a valores de signo contrario del parámetro de control. Entre ellos se produce la inversión de la forma: a partir del primer punto límite la fuerza disminuye, se anula y cambia de sentido hasta alcanzar un el siguiente punto límite, en el que el parámetro de control presenta un mínimo relativo y el módulo de la fuerza un máximo. Después la fuerza vuelve a disminuir hasta anularse para recuperar su sentido original. Este tipo de comportamiento requiere el empleo de la estrategia de control de la longitud de arco en el avance de la solución. 
El problema de Argyris presenta una dificultad adicional que complica mucho la búsqueda numérica de la solución. En el tramo de la trayectoria situado entre puntos límite van a aparecer, además, dos puntos de retorno (turning points) en los que la tangente a la trayectoria de equilibrio es paralela al eje $\lambda$. Para superar un punto de retorno es necesario aplicar técnicas numéricas específicas. En esta tesis hemos seguido el procedimiento indicado por Felippa [19, sec. 5.3].

En la solución del pórtico sometido a la fuerza espacial se ha escogido un valor de $l_{n}=7,5$ (incremento de la longitud de arco sobre la trayectoria de equilibrio). Con él se obtiene un valor de la fuerza correspondiente al primer punto límite de 18,788 $\mathrm{kN}$ en el incremento 24. En este caso los puntos de retorno se pueden atravesar sin dificultad. El cálculo se ha detenido voluntariamente en la rama de rigidización de la trayectoria en el incremento 92 para un valor de la fuerza de 47,487 kN. En la tabla 7.17 se recogen los datos correspondientes a los puntos singulares.

\begin{tabular}{r|rrrr} 
tipo de singularidad & $n$ & $\lambda$ & $u_{13}$ & $v_{13}$ \\
\hline límite & 24 & 18,788 & 27,58 & $-49,168$ \\
retorno & 42 & 11,631 & 62,88 & $-60,965$ \\
retorno & 56 & $-4,771$ & 81,70 & $-51,044$ \\
límite & 64 & $-9,859$ & 90,80 & $-58,547$ \\
\hline
\end{tabular}

Cuadro 7.17: Puntos singulares de la trayectoria de equilibrio en el pórtico de Argyris sometido a una fuerza de eje fijo

El análisis de la estructura sometida a la acción de la fuerza perseguidora ha resultado más complicado. Finalmente se ha resuelto tomando un incremento de la longitud de $\operatorname{arco} l_{n}=5,5$. En este caso, el pórtico tiene un comportamiento inicial algo más rígido, con el primer punto límite asociado a una fuerza de $36,395 \mathrm{kN}$ obtenida en el incremento 34. El paso del primer punto de retorno no es problemático. Sin embargo, el siguiente se encuentra muy cerca del segundo punto crítico (en el siguiente incremento), y esto complica sobremanera la búsqueda de la solución, que tiene un tramo incompleto. Ha sido necesario probar diferentes valores de $l_{n}$ para evitar el rebote de la solución por la trayectoria original o el estancamiento entre dos puntos fijos del camino de equilibrio. Como en el caso de la fuerza de eje fijo el proceso se ha detenido voluntariamente en el paso 131 con una fuerza de $45,256 \mathrm{kN}$ en el camino de rigidización final de la estructura. Los datos de los puntos singulares se encuentran en el cuadro 7.18 .

\begin{tabular}{r|rrrr} 
tipo de singulridad & $n$ & $\lambda$ & $u_{13}$ & $v_{13}$ \\
\hline límite & 47 & 36,395 & 43,30 & $-69,71$ \\
retorno & 65 & 28,483 & 69,84 & $-77,46$ \\
retorno & 91 & $-16,574$ & 75,81 & $-43,72$ \\
límite & 92 & $-16,645$ & 77,09 & $-44,35$ \\
\hline
\end{tabular}

Cuadro 7.18: Puntos singulares de la trayectoria de equilibrio en el pórtico de Argyris sometido a una fuerza perseguidora 
En la tabla 7.19 se compara los valores de la fuerza correspondiente al primer punto límite con los recogidos en la literatura. Hemos comprobado que los valores de la fuerza en el punto límite son sensibles al valor escogido para $l_{n}$, por eso no resulta sorprendente la discrepancia con [62]. Argyris no llega a superar el primer punto crítico y Simó sí lo hace pero no ofrece datos numéricos para las demás singularidades.

\begin{tabular}{c|ccc} 
& elemento espacial & Simó et al. [62] & Argyris et al. [6] \\
\hline fuerza de eje fijo & 18,788 & & \\
fuerza perseguidora & 36,395 & 18,532 & 18,550 \\
& & 35,447 & $\sim 36$ \\
\hline
\end{tabular}

Cuadro 7.19: Magnitud de la fuerza $(\mathrm{kN})$ correspondiente al primer punto límite en el pórtico de Argyris

Las trayectorias de equilibrio recogidas en las figuras 7.36 y 7.37 corresponden a las componentes $u$ y $v$ del desplazamiento del nodo 13 sobre el que se aplica la fuerza, y concuerdan con las obtenidas por Simó y Vu-Quoc en [62]. El proceso de solución funciona correctamente durante la mayor parte del proceso con un número comprendido entre 2 y 5 iteraciones por paso, para ambos tipos de fuerza. La convergencia empeora algo en las cercanías del segundo punto de retorno, y en el caso de la fuerza perseguidora en la zona del primer cambio de signo del parámetro de control. Simó no menciona las dificultades numéricas reseñadas, ni las técnicas empleadas; probablemente ha modificando el valor de $l_{n}$ a lo largo del proceso, lo cual puede facilitar el paso de las singularidades. Como señala Simó, es curioso observar como las trayectorias correspondientes a cada tipo de fuerza cortan el eje de desplazamientos en el mismo punto. El proceso de deformación, junto con la trayectoria del nodo sobre el que se aplica la fuerza, queda reflejado en los gráficos 7.38 y 7.39 . También conviene llamar la atención sobre la brusquedad del cambio de dirección en la trayectoria del nodo 13, especialmente en el caso de la fuerza perseguidora, así como su coincidencia con la zona de retorno y el segundo punto límite. 


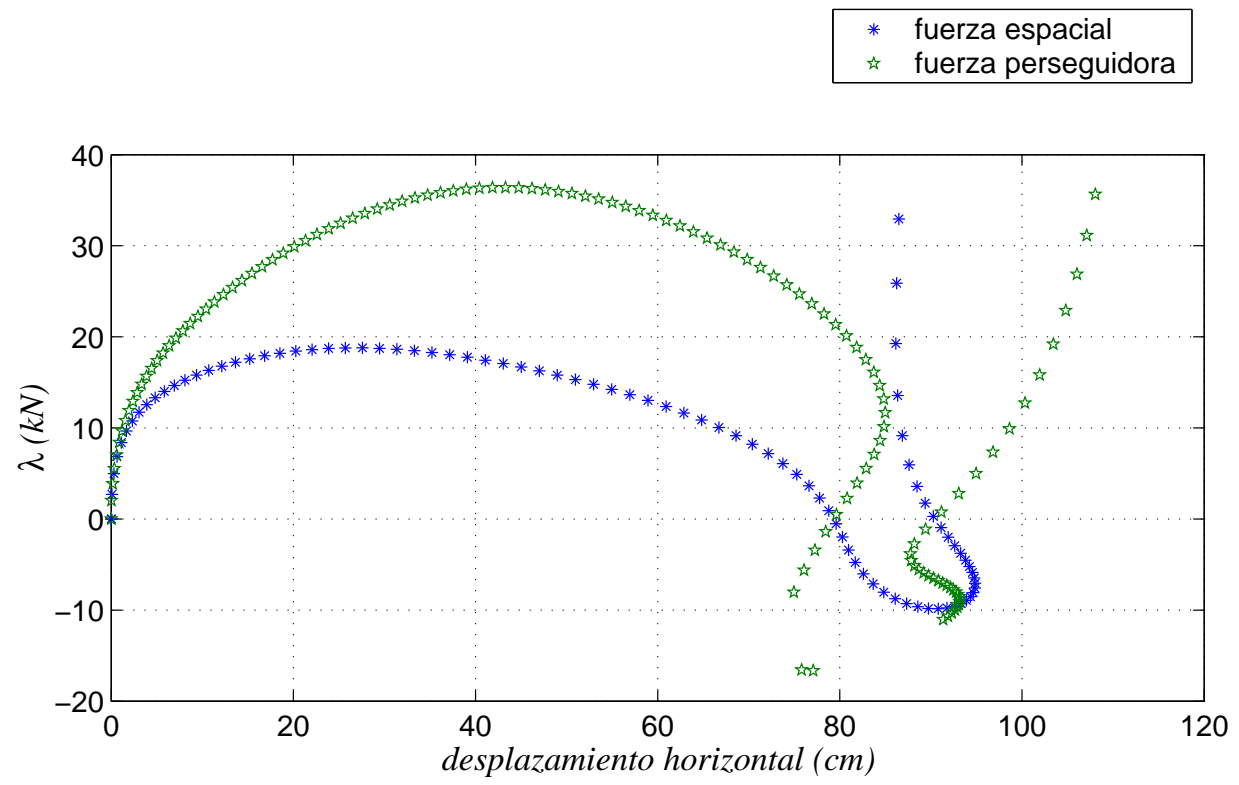

Figura 7.36: Pórtico de Argyris. Trayectorias de equilibrio del desplazamiento horizontal para la fuerza espacial / perseguidora

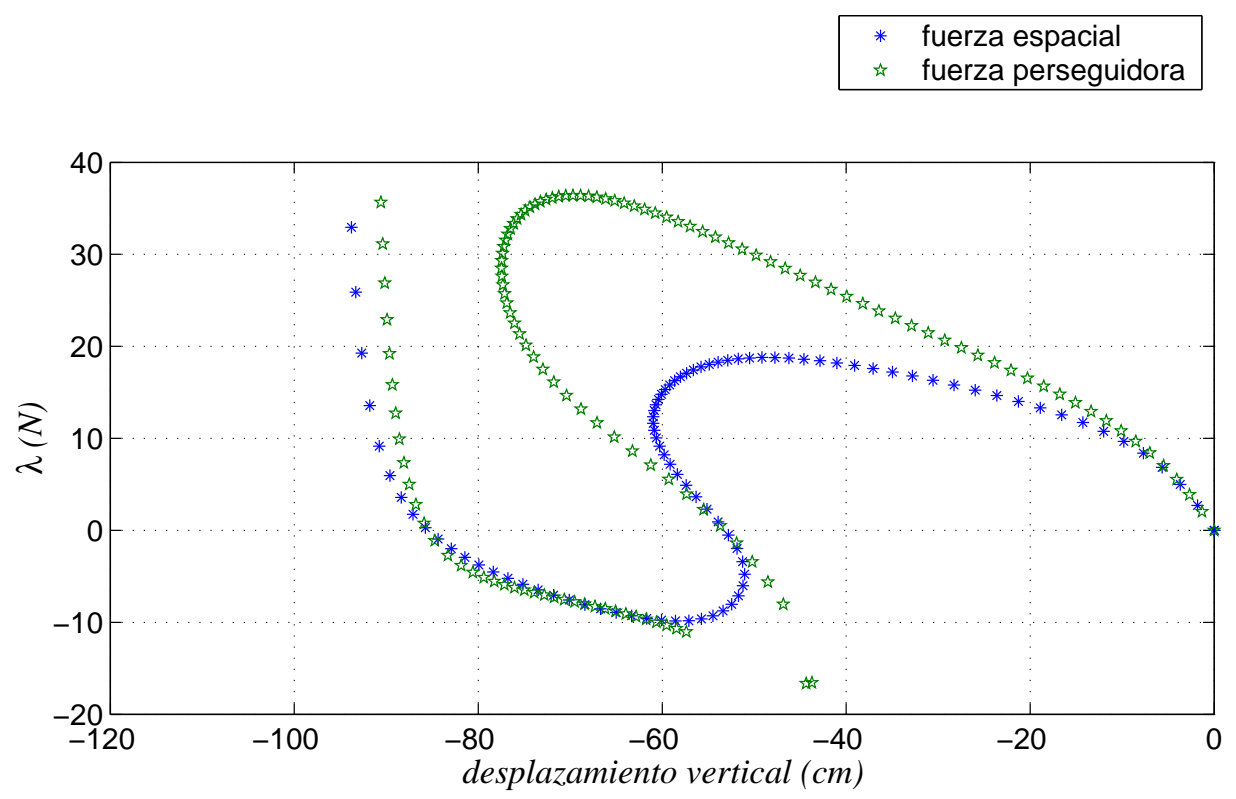

Figura 7.37: Pórtico de Argyris. Trayectorias de equilibrio del desplazamiento vertical para la fuerza espacial / perseguidora 


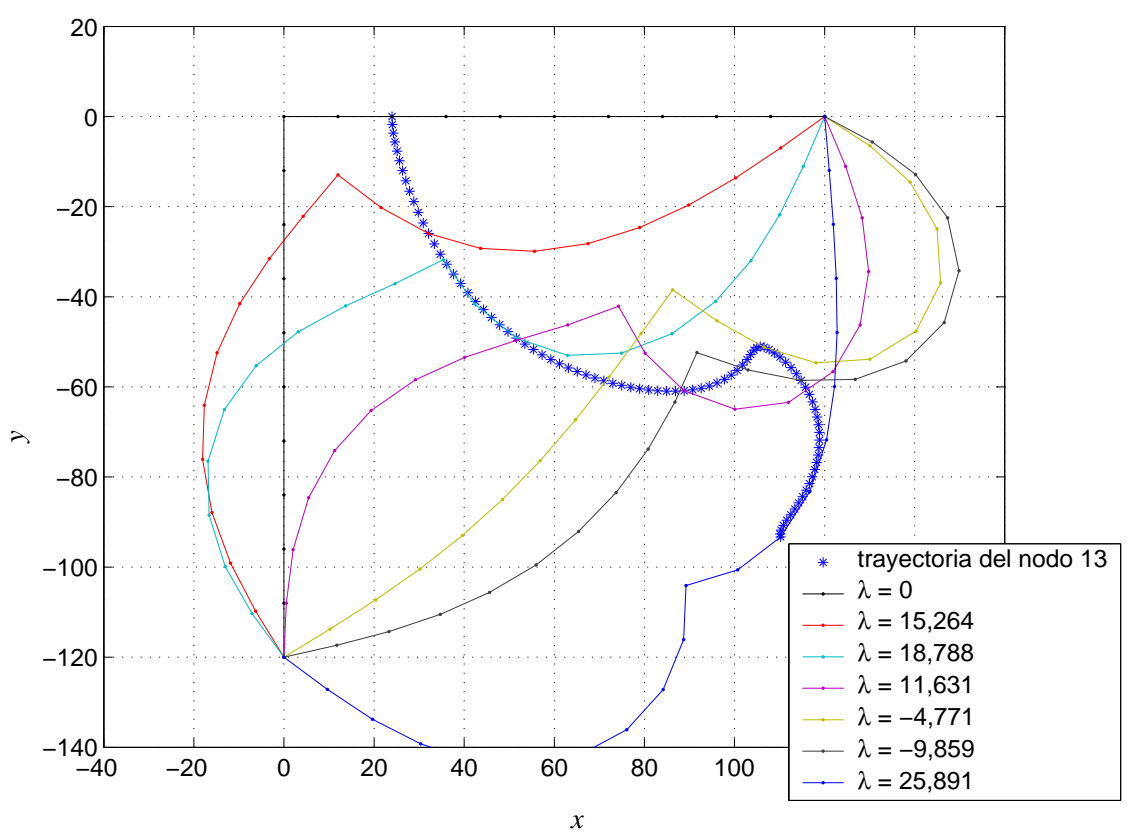

Figura 7.38: Proceso de deformación del pórtico bajo la acción de una fuerza de eje fijo (módulo de la fuerza en $\mathrm{kN}$, coordenadas en $\mathrm{cm}$ )

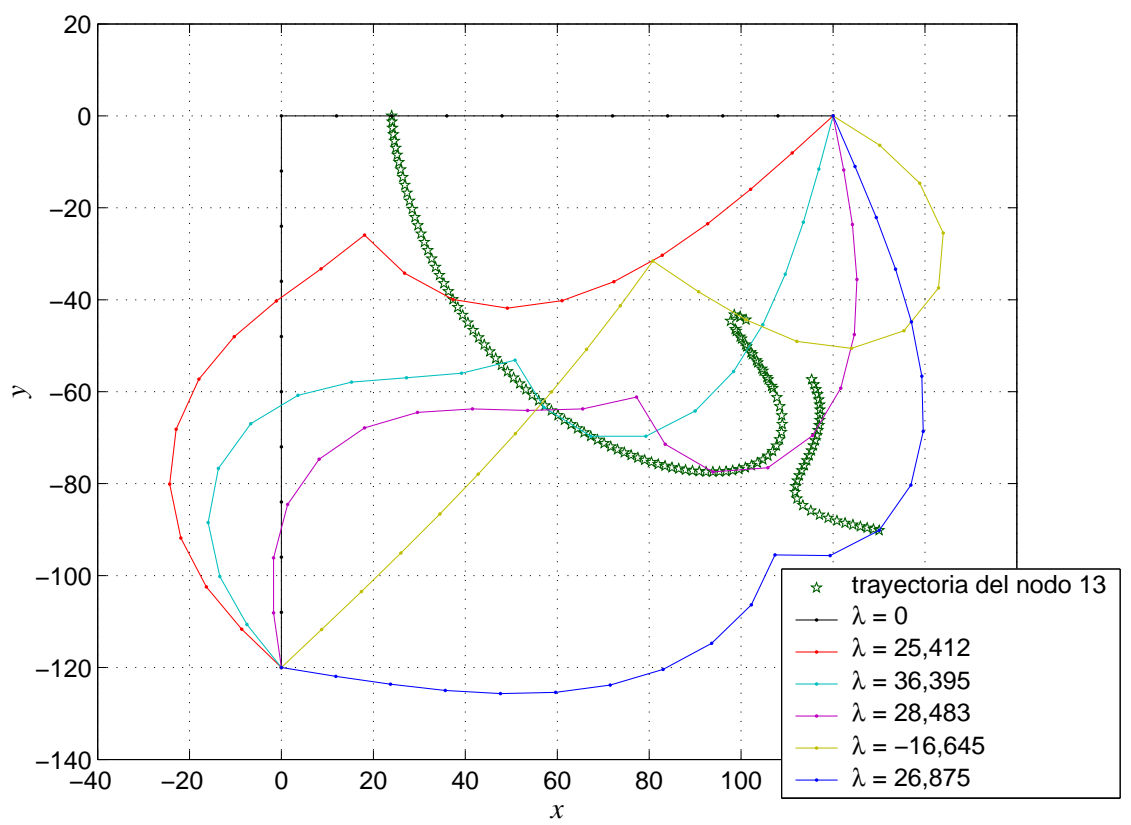

Figura 7.39: Proceso de deformación del pórtico bajo la acción de una fuerza perseguidora (módulo de la fuerza en $\mathrm{kN}$, coordenadas en $\mathrm{cm}$ ) 


\section{Malla geodésica}

Estudiamos, por último, la inversión de la forma (snap through) de una malla geodésica cuyos nodos se inscriben en una esfera de radio $R=25 \mathrm{~m}$. La malla está formada por una capa de 90 barras rígidamente unidas entre si en 37 nodos, y dispone de seis apoyos articulados en el contorno. Todas las barras son de sección transversal tubular hueca, de 0,5 m de diámetro exterior y 0,005 m de espesor. Las características de las secciones y del material se recogen en la tabla siguiente (en $\mathrm{m}$ y GN):

\begin{tabular}{|c|c|c|c|c|c|}
\hline & $A=A_{Q 2}=A_{Q 3}$ & $J$ & $I_{2}=I_{3}$ & $E$ & $E / G$ \\
\hline malla ge & $7,8540 \quad 10^{-4}$ & $4,908710^{-4}$ & $2,4544 \quad 10^{-4}$ & 200 & 2, \\
\hline
\end{tabular}

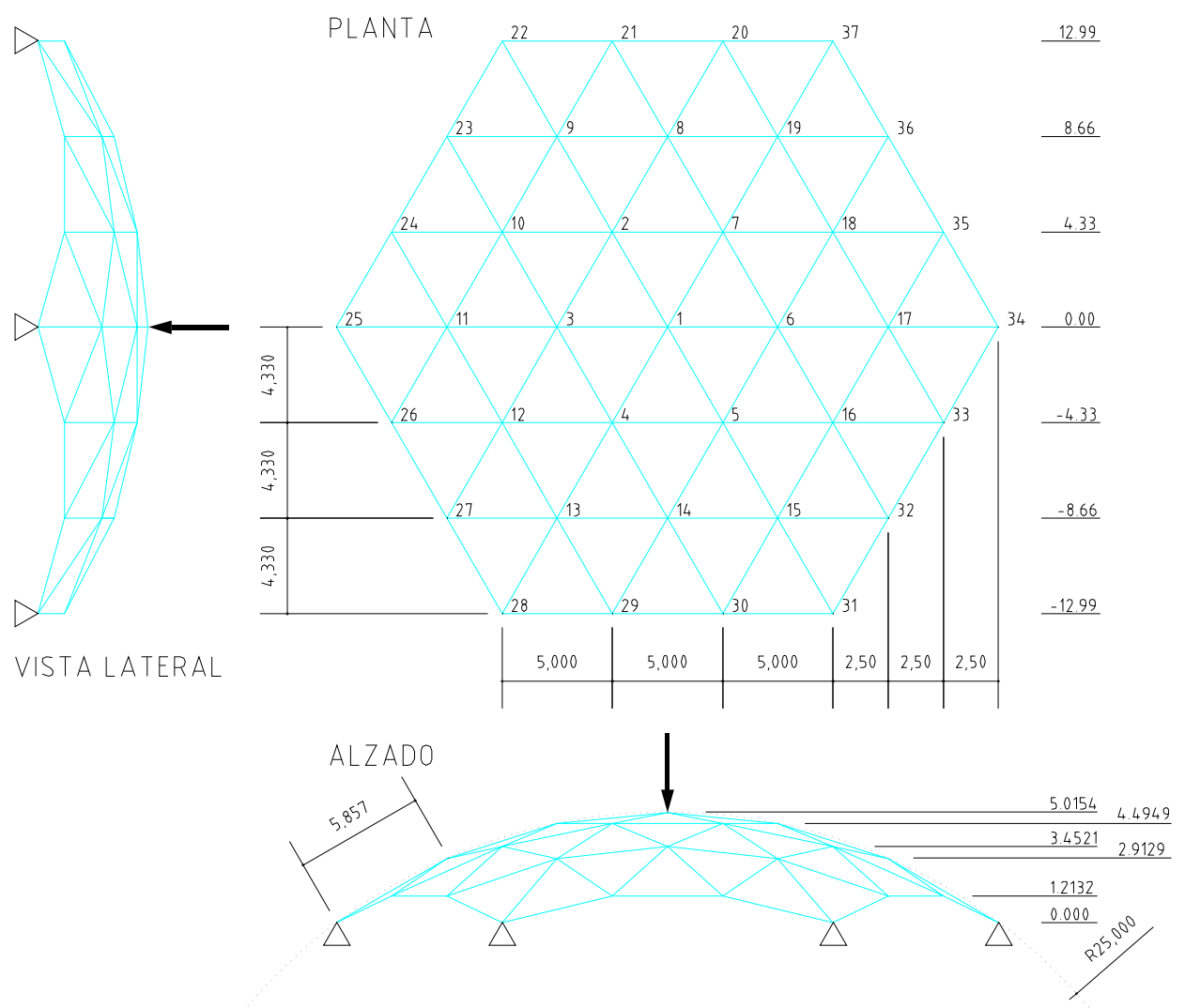

Figura 7.40: Malla geodésica

En este ejemplo nos interesa verificar el funcionamiento de la solución numérica en una estructura reticulada espacial y su capacidad para reproducir su comportamiento empleando el mínimo número de elementos. Por ello se ha generado un modelo en el que cada barra de la estructura se corresponde con un solo elemento de dos nodos. 
Evidentemente, la solución obtenida no será exacta ya que el modelo no podrá reproducir los cambios de curvatura en las barras que aparecerán durante la deformación. No obstante, el comportamiento de esta estructura está muy condicionado por los esfuerzos axiles, para los que la interpolación escogida es suficientemente precisa.

La malla se somete a una fuerza vertical de eje fijo aplicada en la cúspide (nodo 1) que provoca la inestabilidad del conjunto y la inversión de su forma. La trayectoria de equilibrio es muy compleja: reproduce una primera inestabilidad de la zona central de la malla seguida de una recuperación de la forma; en una segunda fase el camino de equilibrio se recorre de forma antisimétrica a la primera. El resultado se refleja en la figura 7.41. Para obtenerlo ha sido necesario emplear dos estrategias de control combinadas - control de desplazamiento $(D C)$ y control de arco $(A C)$ - con distintos valores de $l_{n}$ :

\begin{tabular}{rccccccc}
\hline estrategia & $D C$ & $A C$ & $D C$ & $A C$ & $D C$ & $A C$ & $D C$ \\
hasta el paso & 50 & 75 & 95 & 115 & 135 & 155 & 200 \\
$l_{n}$ & 0.5 & 5 & 0.5 & 5 & 0.5 & 5 & 0.5 \\
\hline
\end{tabular}

Las singularidades de la trayectoria se recogen en el cuadro 7.20. Aunque debería serlo, el camino de equilibrio no es exactamente antisimétrico. Esto se debe a que la discretización escogida no tiene el número de elementos suficiente como para reproducir correctamente el comportamiento local de las barras. Aun así la respuesta global final es muy precisa: en el paso 199 para un valor del parámetro de control próximo a $0(2,571 \mathrm{MN})$ la ordenada del nodo 1 es $z_{1}=-5,046 \mathrm{~m}$, muy parecida -con signo opuesto- a la ordenada inicial $z_{1}=5,015 \mathrm{~m}$. Optamos pues por no refinar el modelo. En la figura 7.42 se muestra la evolución de la energía de deformación. La curva refleja cómo a partir del punto central de la trayectoria la estructura devuelve la energía acumulada hasta que se produce la inversión total ${ }^{8}$ (paso 199). A partir de esa situación la energía vuelve a ser creciente.

\begin{tabular}{r|rrr} 
tipo de singularidad & $n$ & $\lambda(\mathrm{MN})$ & \multicolumn{1}{c}{$z_{1}$} \\
\hline límite & 5 & 36,29 & 3,546 \\
límite & 14 & 29,44 & 1,312 \\
límite & 33 & 50,81 & $-1,914$ \\
retorno & 61 & $-52,08$ & $-3,958$ \\
límite & 79 & $-145,02$ & $-2,325$ \\
retorno & 102 & 30,87 & 0,666 \\
retorno & 109 & 65,82 & 0,596 \\
límite & 124 & 163,33 & 2,499 \\
retorno & 134 & 65,59 & 3,909 \\
límite & 166 & $-45,26$ & 2,000 \\
límite & 185 & $-26,82$ & $-1,269$ \\
límite & 194 & $-36,03$ & $-3,548$ \\
\hline
\end{tabular}

Cuadro 7.20: Puntos singulares de la trayectoria de equilibrio de la malla

\footnotetext{
${ }^{8}$ Teóricamente la figura debería ser simétrica alrededor de $z=0$.
} 


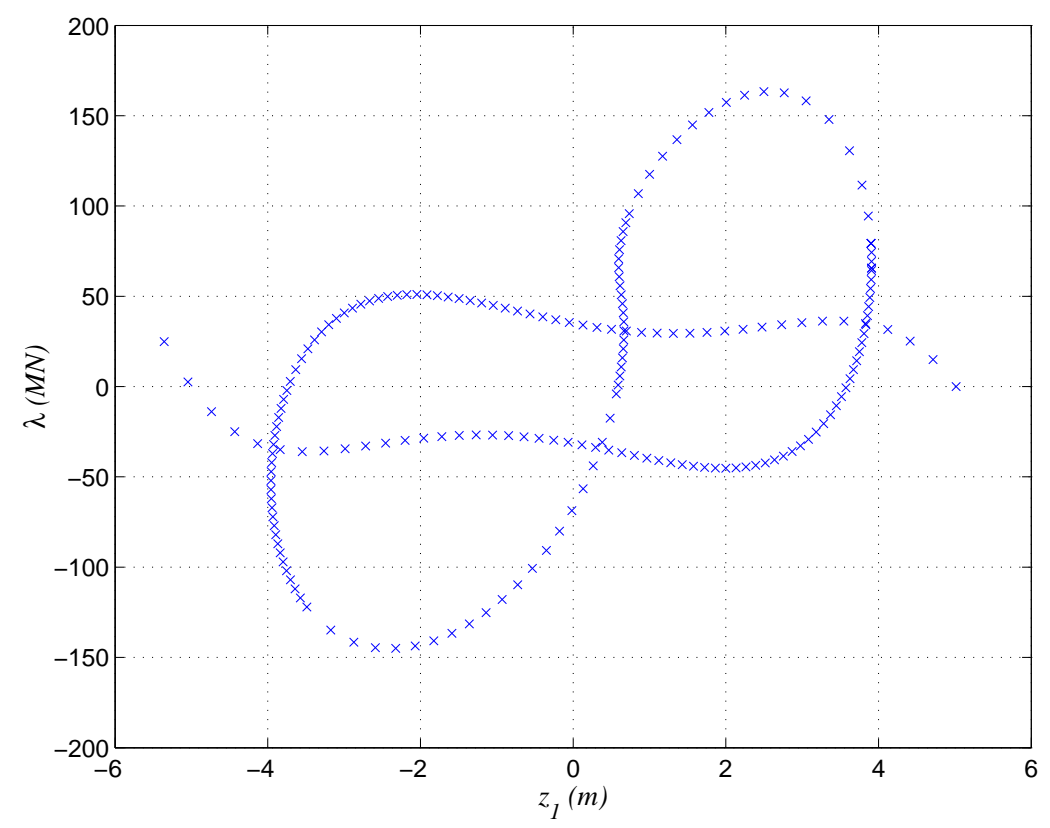

Figura 7.41: Malla geodésica. Trayectoria de equilibrio (desplazamiento vertical de la cúspide)

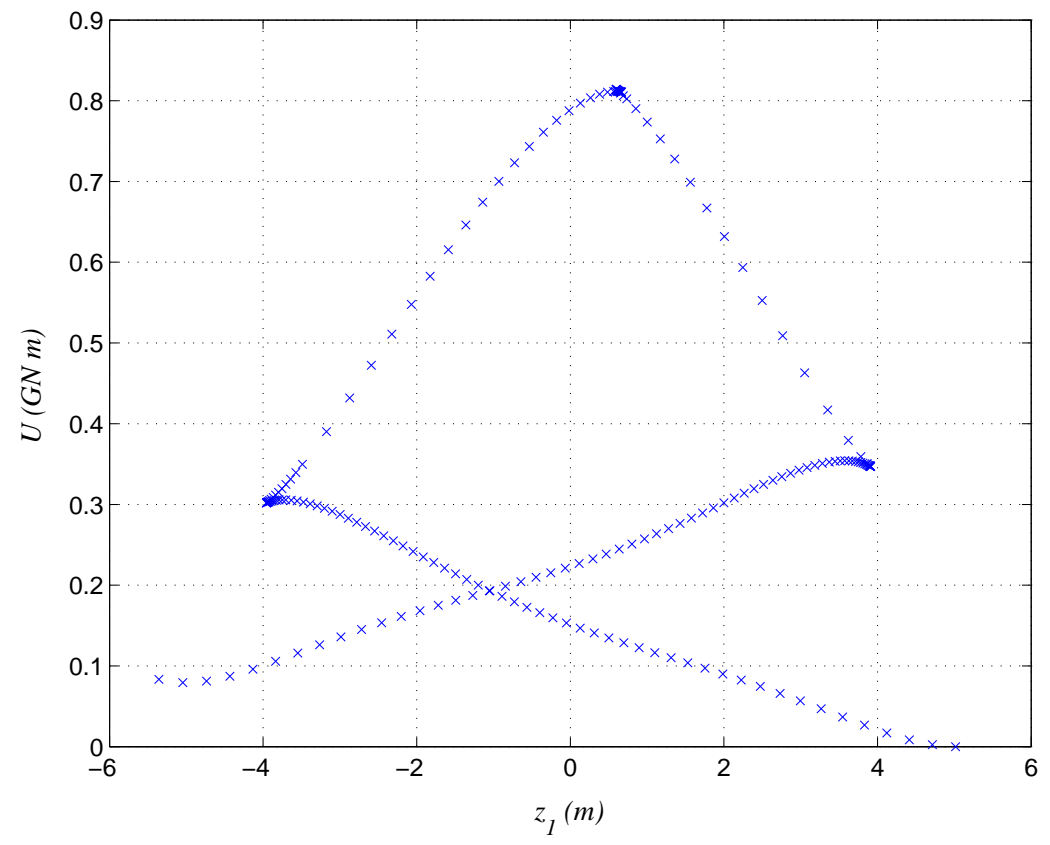

Figura 7.42: Malla geodésica. Energía de deformación 


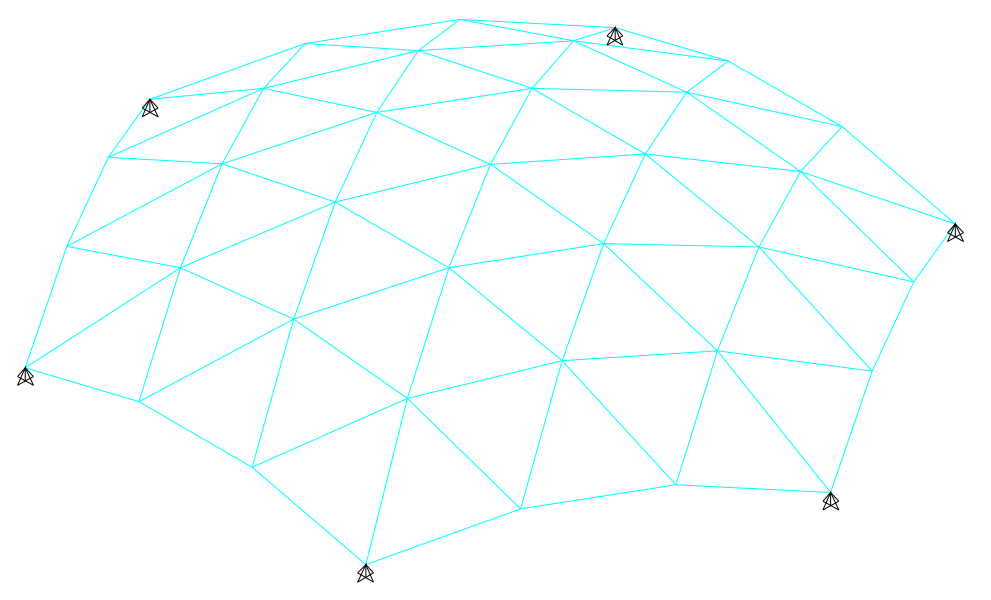

Figura 7.43: Situación inicial

En las siguientes figuras mostramos la secuencia de la deformación en 8 instantes del proceso. El paso 21 muestra la inversión de la forma en la zona central de la malla y es la situación que se produce después de los dos primeros puntos límite. El paso 33 corresponde al tercer punto límite. A partir de aquí el valor de la fuerza decrece hasta anularse y cambiar de sentido. El primer punto de retorno aparece en el paso 61 y corresponde al máximo desplazamiento vertical del nodo central; la fuerza ascendente produce entonces la recuperación de parte de la deformación. El centro de la trayectoria se encuentra aproximadamente en el paso 106: el camino de equilibrio es ahora una imagen antisimétrica del recorrido hasta este punto, ya que la estructura devuelve la energía acumulada trabajando contra la fuerza. El paso 134 es el punto de retorno opuesto al del paso 61 y el paso 178 es la imagen del 21. Finalmente, en el paso 200 ya se ha producido la inversión de la forma y la energía de deformación vuelve a ser creciente. 


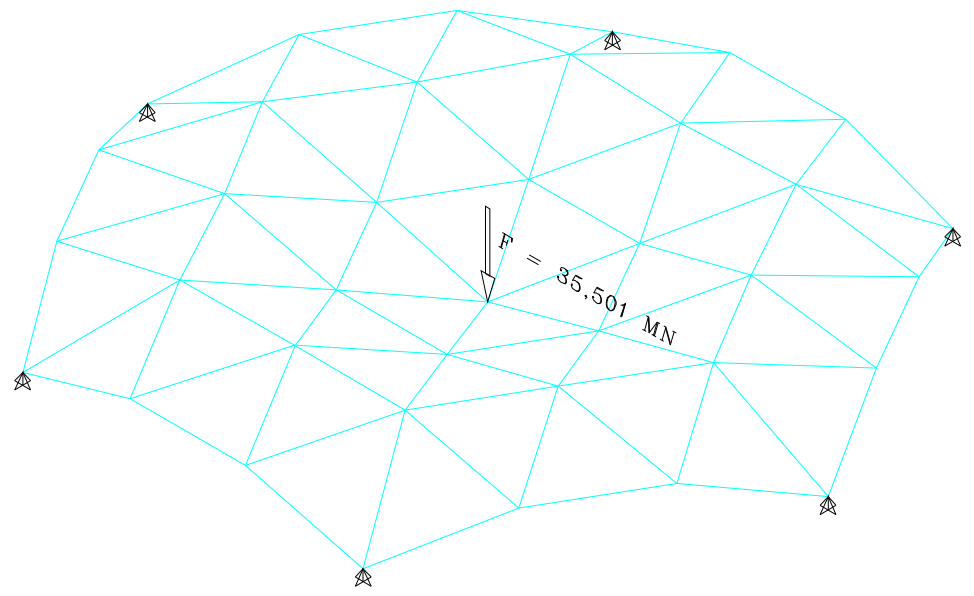

Figura 7.44: Paso 21

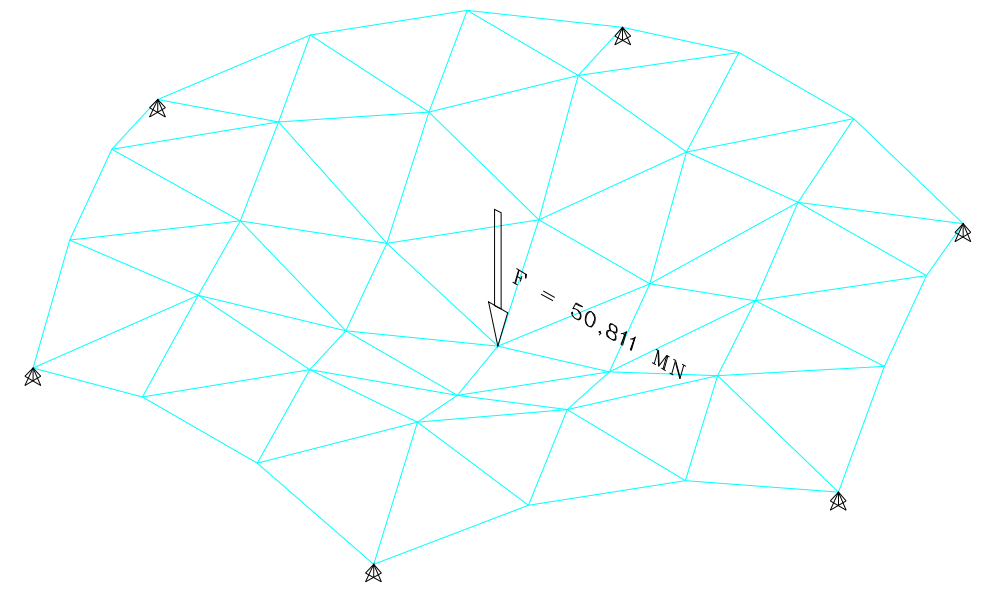

Figura 7.45: Paso 33 


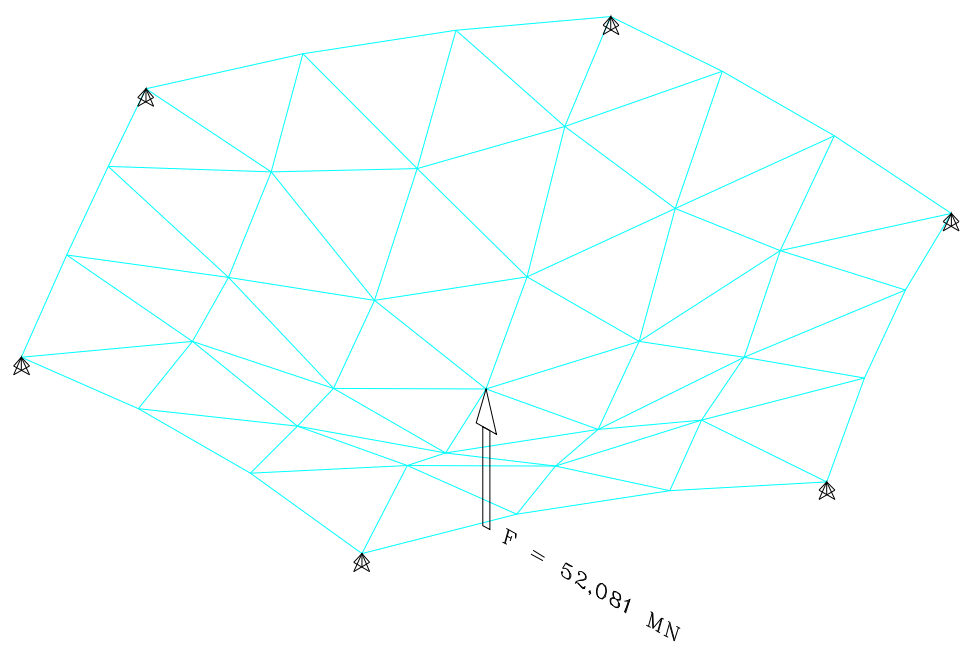

Figura 7.46: Paso 61

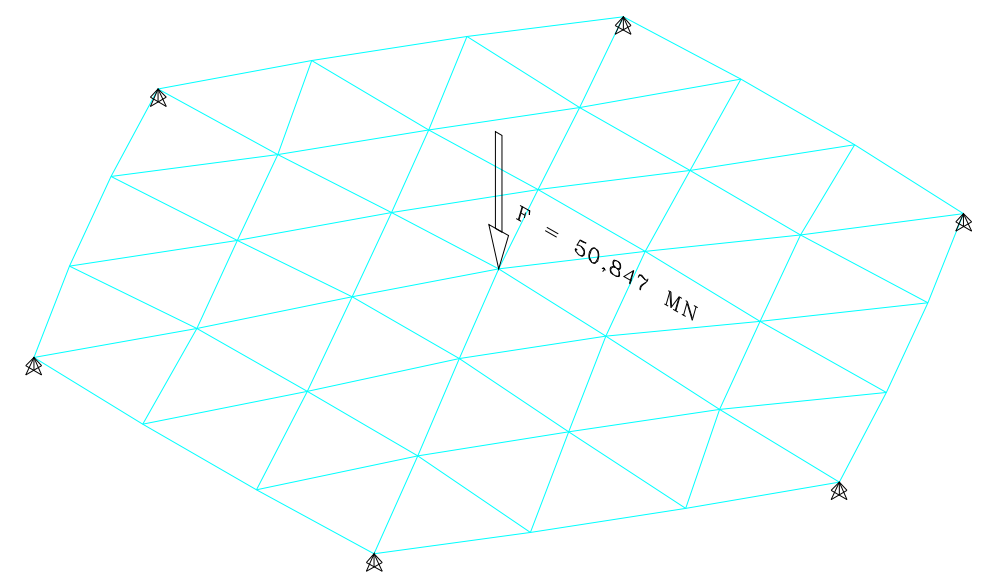

Figura 7.47: Paso 106 


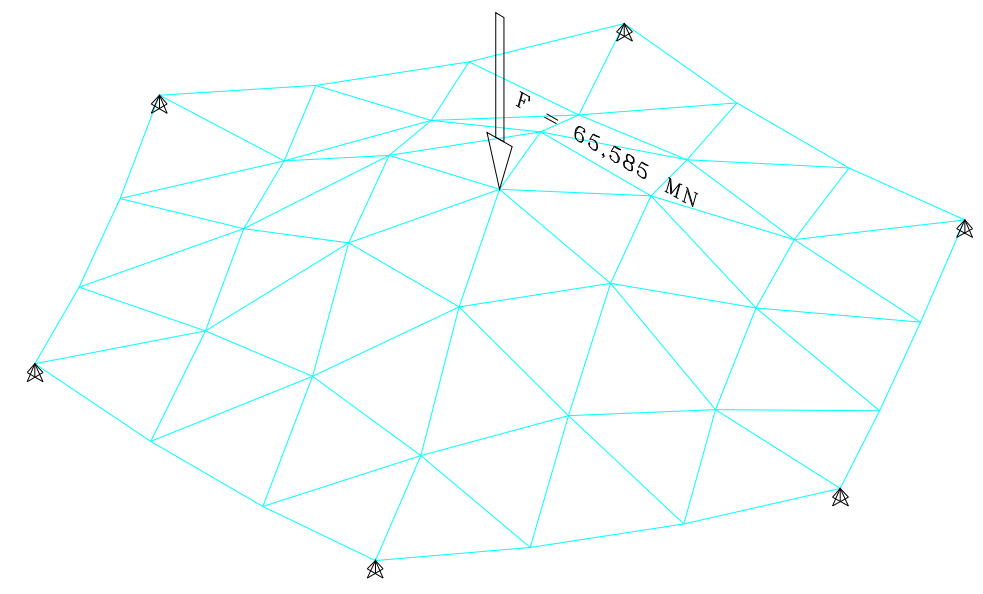

Figura 7.48: Paso 134

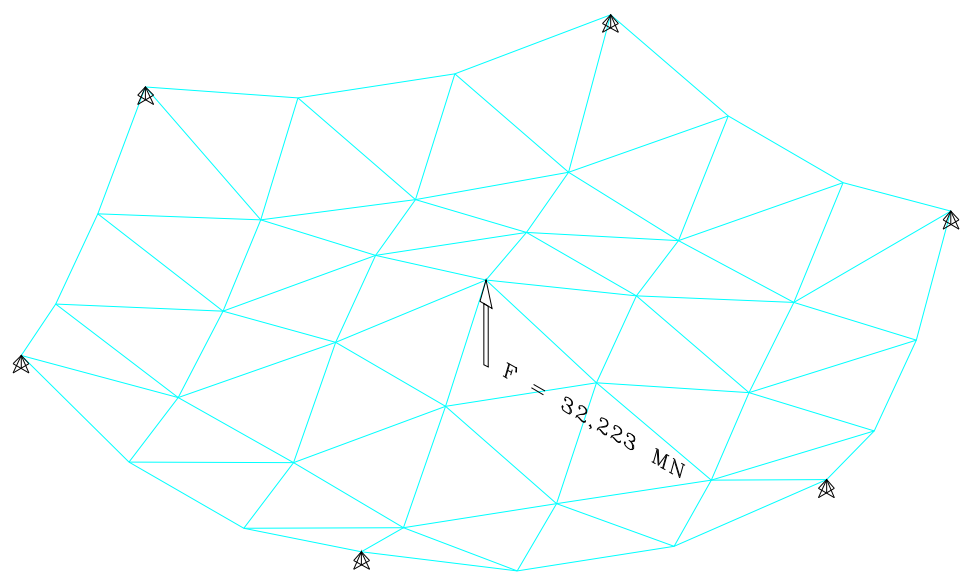

Figura 7.49: Paso 178 


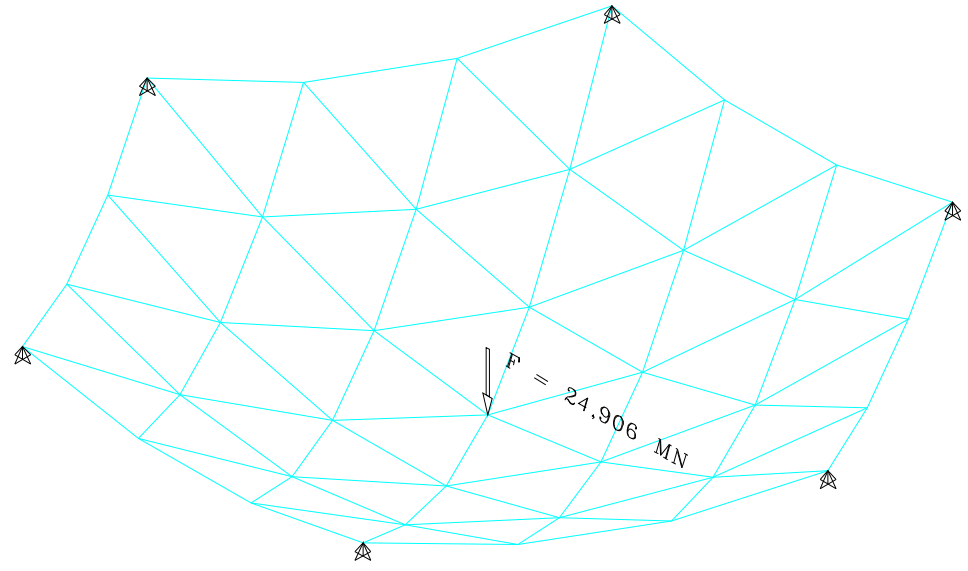

Figura 7.50: Paso 200 



\section{Capítulo 8}

\section{Solución numérica basada en la forma material de las ecuaciones}

En este capítulo presentamos una solución numérica del modelo de ReissnerSimó mediante elementos finitos basada en la forma material de la ecuación de equilibrio tangente. La forma material es, en principio, la vía natural para formular las ecuaciones del problema, ya que las ecuaciones constitutivas tienen en ella su expresión más simple. De hecho, trabajando con la versión material de la ecuación de trabajos virtuales se elude el empleo de la variación corrotacional, aunque esto no evita la necesidad de contar con expresiones específicas para la variación de las deformaciones generalizadas -ecuaciones (4.2.3)-

La idea básica para el desarrollo del nuevo elemento está relacionada en cierto modo con la formulación corrotacional de elementos viga [13]. En ésta, a cada elemento se asocia un sistema de referencia que caracteriza su orientación en la configuración actual. Entonces, el proceso de deformación se descompone en $(a)$ un movimiento global de sólido rígido que define la orientación del sistema de referencia del elemento, y (b) una deformación (pequeña) superpuesta al movimiento de sólido rígido; esto permite emplear las expresiones de la rigidez local de un elemento habituales en teoría lineal.

Cuando trasladamos esta idea al modelo RS es fácil observar que el integrando en el lado izquierdo de la expresión material del equilibrio tangente (6.79) es el núcleo de la rigidez tangente intrínseca o local de la pieza:

$$
\int_{\Gamma_{0}}\left\{\delta \boldsymbol{\Phi}^{\boldsymbol{\top}} \delta \boldsymbol{\Phi}^{\prime \top}\right\}\left[\begin{array}{ll}
\mathbf{D}_{00} & \mathbf{D}_{01} \\
\mathbf{D}_{10} & \mathbf{D}_{11}
\end{array}\right]\left\{\begin{array}{c}
\Delta \boldsymbol{\Phi} \\
\Delta \boldsymbol{\Phi}^{\prime}
\end{array}\right\} d S
$$

Los bloques de la matriz son factores de los incrementos de la configuración en su forma material; las componentes de éstos son la expresión de los vectores incrementales en el sistema de referencia actual de cada sección. La discretización del núcleo conducirá a una expresión material de la rigidez del elemento asociada a desplazamientos nodales y rotaciones incrementales nodales materiales con componentes en el sistema de referencia móvil del nodo. La rigidez obtenida de este modo es, por tanto, una expresión local de la rigidez del elemento. Para el ensamblaje, será necesario 
transformar la matriz a su versión espacial, y para ello se emplearán las matrices de rotación que describen la posición del sistema de referencia móvil de cada nodo del elemento.

En la transformación de la rigidez de un elemento intervienen las rotaciones de todos sus nodos. Esta característica lo diferencia del elemento espacial, ya que como vimos en el capítulo anterior, en éste la transformación opera sobre las variables asociadas al punto de integración -esfuerzos y deformaciones generalizadas, y la matriz constitutiva-, lo que supone cierta economía en el número de operaciones a realizar. El nuevo elemento hereda del modelo RS la ausencia de limitaciones en la magnitud de las deformaciones generalizadas.

El capítulo se estructura del siguiente modo: en la primera sección desarrollamos las expresiones necesarias para la formulación de un nuevo elemento recto de dos nodos a partir de la forma material de las ecuaciones. En la siguiente sección se incluyen los esquemas del algoritmo de cálculo que hemos programado, y en la última analizamos los resultados de diversos ejemplos modelados con el nuevo elemento para valorar su respuesta en comparación con la del elemento espacial.

\subsection{Formulación del elemento}

\subsubsection{Interpolación}

En el elemento material interpolamos los incrementos de la configuración con la expresión análoga a la empleada en el caso espacial

$$
\left\{\begin{array}{c}
\Delta \boldsymbol{\chi}(\xi) \\
\Delta \boldsymbol{\Omega}(\xi)
\end{array}\right\}=\left[\begin{array}{ccccc}
N_{1}(\xi) \mathbf{1} & \mathbf{0} & \cdots & N_{n}(\xi) \mathbf{1} & \mathbf{0} \\
\mathbf{0} & N_{1}(\xi) \mathbf{1} & \cdots & \mathbf{0} & N_{n}(\xi) \mathbf{1}
\end{array}\right]\left\{\begin{array}{c}
\Delta \boldsymbol{\chi}_{1} \\
\Delta \boldsymbol{\Omega}_{1} \\
\vdots \\
\Delta \boldsymbol{\chi}_{n} \\
\Delta \boldsymbol{\Omega}_{n}
\end{array}\right\}
$$

Empleando la notación del capítulo $6, \Delta \boldsymbol{\Phi}=\left\{\begin{array}{ll}\Delta \chi & \Delta \boldsymbol{\Omega}\end{array}\right\}^{\top}$, los incrementos de la configuración y sus derivadas se expresan en función de sus valores nodales mediante la expresión

$$
\left\{\begin{array}{c}
\Delta \boldsymbol{\Phi} \\
\Delta \boldsymbol{\Phi}^{\prime}
\end{array}\right\}=\left[\begin{array}{c}
\mathbb{N} \\
\frac{1}{J} \mathbb{N}^{\prime}
\end{array}\right] \Delta \boldsymbol{\Phi}^{e}
$$

en la que

$$
\mathbb{N}=\left[\begin{array}{lll}
N_{1} \mathbf{I} & \ldots & N_{n} \mathbf{I}
\end{array}\right]
$$

y

$$
\frac{1}{J}\left[\begin{array}{lll}
N_{1}^{\prime} \mathbf{I} & \ldots & N_{n}^{\prime} \mathbf{I}
\end{array}\right]
$$

e I es la identidad de orden 6 . 


\subsubsection{Predicción}

En esta sección nos ocupamos de obtener las expresiones para realizar la predicción de la siguiente solución a partir de la última configuración en la que se ha logrado la convergencia, durante el proceso de solución incremetal - iterativo.

\section{Matriz de rigidez tangente de un elemento}

El cálculo del paso de predicción se realiza a partir de la ecuación de equilibrio tangente, expresada en este caso en forma material (6.79)

$$
\begin{aligned}
\int_{\Gamma_{0}}\left\{\delta \boldsymbol{\Phi}^{\boldsymbol{\top}} \delta \boldsymbol{\Phi}^{\prime \top}\right\} & {\left[\begin{array}{ll}
\mathbf{D}_{00} & \mathbf{D}_{01} \\
\mathbf{D}_{10} & \mathbf{D}_{11}
\end{array}\right]\left\{\begin{array}{c}
\Delta \boldsymbol{\Phi} \\
\Delta \boldsymbol{\Phi}^{\prime}
\end{array}\right\} d S } \\
& -\sum_{a=1}^{2}\left\{\delta \boldsymbol{\Phi}\left(S_{a}\right)^{\boldsymbol{\top}} \delta \boldsymbol{\Phi}\left(S_{a}\right)^{\prime \top}\right\}\left[\begin{array}{cc}
\mathbf{F}_{a} & \mathbf{0} \\
\mathbf{0} & \mathbf{0}
\end{array}\right]\left\{\begin{array}{c}
\Delta \boldsymbol{\Phi}\left(S_{a}\right) \\
\Delta \boldsymbol{\Phi}^{\prime}\left(S_{a}\right)
\end{array}\right\} \\
& =\Delta \lambda\left(\int_{\Gamma_{0}} \delta \boldsymbol{\Phi}^{\boldsymbol{\top}} \boldsymbol{Q} d S+\sum_{a=1}^{2} \delta \boldsymbol{\Phi}\left(S_{a}\right)^{\top} \boldsymbol{F}_{a}\right) .
\end{aligned}
$$

donde el núcleo de la primera integral se descompone en una parte geométrica, una constitutiva y una debida a las fuerzas generalizadas cuyas expresiones se obtuvieron en el capítulo 6

$$
\left[\begin{array}{ll}
\mathbf{D}_{00} & \mathbf{D}_{01} \\
\mathbf{D}_{10} & \mathbf{D}_{11}
\end{array}\right]=\left[\begin{array}{ll}
\mathbf{D}_{00}^{G} & \mathbf{D}_{01}^{G} \\
\mathbf{D}_{10}^{G} & \mathbf{D}_{11}^{G}
\end{array}\right]+\left[\begin{array}{cc}
\mathbf{D}_{00}^{M} & \mathbf{D}_{01}^{M} \\
\mathbf{D}_{10}^{M} & \mathbf{D}_{11}^{M}
\end{array}\right]-\left[\begin{array}{cc}
\mathbf{D}^{Q} & \mathbf{0} \\
\mathbf{0} & \mathbf{0}
\end{array}\right]
$$

con

$$
\begin{aligned}
& {\left[\begin{array}{ll}
\mathbf{D}_{00}^{G} & \mathbf{D}_{01}^{G} \\
\mathbf{D}_{10}^{G} & \mathbf{D}_{11}^{G}
\end{array}\right]=\left[\begin{array}{cccc}
\mathbf{0} & \widehat{\mathbf{K}} \widehat{\mathbf{N}} & \mathbf{0} & \mathbf{0} \\
\widehat{\mathbf{N}} \widehat{\mathbf{K}} & \widehat{\mathbf{N}} \widehat{\mathbf{\Gamma}}+\widehat{\mathbf{M}} \widehat{\mathbf{K}} & \widehat{\mathbf{N}} & \widehat{\mathbf{M}} \\
\mathbf{0} & -\widehat{\mathbf{N}} & \mathbf{0} & \mathbf{0} \\
\mathbf{0} & \mathbf{0} & \mathbf{0} & \mathbf{0}
\end{array}\right]} \\
& \mathbf{D}_{00}^{M}=\left[\begin{array}{cc}
-\widehat{\mathbf{K}} & \mathbf{0} \\
-\widehat{\boldsymbol{\Gamma}} & -\widehat{\mathbf{K}}
\end{array}\right]\left[\begin{array}{ll}
\mathbf{C}_{\Gamma \Gamma} & \mathbf{C}_{\Gamma K} \\
\mathbf{C}_{K \Gamma} & \mathbf{C}_{K K}
\end{array}\right]\left[\begin{array}{cc}
\widehat{\mathbf{K}} & \widehat{\boldsymbol{\Gamma}} \\
\mathbf{0} & \widehat{\mathbf{K}}
\end{array}\right] \\
& \mathbf{D}_{01}^{M}=\left[\begin{array}{cc}
-\widehat{\mathbf{K}} & \mathbf{0} \\
-\widehat{\boldsymbol{\Gamma}} & -\widehat{\mathbf{K}}
\end{array}\right]\left[\begin{array}{ll}
\mathbf{C}_{\Gamma \Gamma} & \mathbf{C}_{\Gamma K} \\
\mathbf{C}_{K \Gamma} & \mathbf{C}_{K K}
\end{array}\right] \\
& \mathbf{D}_{10}^{M}=\mathbf{D}_{01}^{M \top} \\
& \mathbf{D}_{11}^{M}=\left[\begin{array}{ll}
\mathbf{C}_{\Gamma \Gamma} & \mathbf{C}_{\Gamma K} \\
\mathbf{C}_{K \Gamma} & \mathbf{C}_{K K}
\end{array}\right] \\
& {\left[\begin{array}{cc}
\mathbf{D}^{Q} & \mathbf{0} \\
\mathbf{0} & \mathbf{0}
\end{array}\right]=\lambda\left[\begin{array}{cccc}
\mathbf{0} & \mathbf{0} & \mathbf{0} & \mathbf{0} \\
\mathbf{0} & \widehat{\mathbf{Q}}_{m} & \mathbf{0} & \mathbf{0} \\
\mathbf{0} & \mathbf{0} & \mathbf{0} & \mathbf{0} \\
\mathbf{0} & \mathbf{0} & \mathbf{0} & \mathbf{0}
\end{array}\right]+\lambda\left[\begin{array}{cccc}
\mathbf{0} & -\widehat{\mathbf{Q}}_{n}^{f} & \mathbf{0} & \mathbf{0} \\
\mathbf{0} & -\widehat{\mathbf{Q}}_{m}^{f} & \mathbf{0} & \mathbf{0} \\
\mathbf{0} & \mathbf{0} & \mathbf{0} & \mathbf{0} \\
\mathbf{0} & \mathbf{0} & \mathbf{0} & \mathbf{0}
\end{array}\right]}
\end{aligned}
$$


Discretizando el primer sumando podemos representar la contribución de un elemento al operador tangente:

$$
\delta \boldsymbol{\Phi}^{e \boldsymbol{\top}} \int_{-1}^{1}\left[\begin{array}{ll}
\mathbb{N}^{\top} & \frac{1}{J} \mathbb{N}^{\top} \boldsymbol{\top}
\end{array}\right]\left[\begin{array}{ll}
\mathbf{D}_{00} & \mathbf{D}_{01} \\
\mathbf{D}_{10} & \mathbf{D}_{11}
\end{array}\right]\left[\begin{array}{c}
\mathbb{N} \\
\frac{1}{J} \mathbb{N}^{\prime}
\end{array}\right] J d \xi \Delta \boldsymbol{\Phi}^{e} .
$$

La integral se evalúa mediante una cuadratura de Gauss de orden $g$ y da lugar a la matriz de rigidez tangente del elemento

$$
\mathbf{K}_{m a t}^{e}=\sum_{l=1}^{g} w_{l}\left[\begin{array}{ll}
\mathbb{N}^{\top} & \frac{1}{J}
\end{array} \mathbb{N}^{\prime}\right]_{\left.\right|_{\xi=\xi_{l}}}\left[\begin{array}{ll}
\mathbf{D}_{00} & \mathbf{D}_{01} \\
\mathbf{D}_{10} & \mathbf{D}_{11}
\end{array}\right]_{\left.\right|_{\xi=\xi_{l}}}\left[\begin{array}{c}
\mathbb{N} \\
\frac{1}{J} \mathbb{N}^{\prime}
\end{array}\right]_{\mid \xi=\xi_{l}} J\left(\xi_{l}\right),
$$

\section{Transformación de la rigidez material a espacial}

Desarrollamos las expresiones correspondientes al elemento de 2 nodos. Su matriz de rigidez se puede escribir del siguiente modo

$$
\mathbf{K}_{m a t}^{e}=\left[\begin{array}{ll}
\mathbf{K}_{i \text { mat }}^{e} & \mathbf{K}_{i j \text { mat }}^{e} \\
\mathbf{K}_{\text {jimat }}^{e} & \mathbf{K}_{j \text { mat }}^{e}
\end{array}\right]
$$

El cambio desde la forma material a la espacial de las variaciones de la configuración en el nodo $i$ se produce en dos pasos:

1. En primer lugar las variables materiales se expresan en el sistema de referencia inicial por medio de la matriz de rotación inicial $\boldsymbol{\Lambda}_{(0)}^{e}$ (que es la rotación en el punto de Gauss). Como el elemento es recto, la rotación inicial de los nodos es la misma que la del punto de integración, por eso se puede emplear para transformar las variables nodales.

2. A continuación las variables expresadas en el sistema inicial se cambian al sistema general por medio de la rotación $\boldsymbol{\Lambda}_{i}$ del nodo.

$$
\Delta \boldsymbol{\Phi}_{i} \stackrel{\Lambda_{(0)}^{e}}{\longrightarrow} \Delta \phi_{i(0)} \stackrel{\Lambda_{i}}{\longrightarrow} \Delta \phi_{i}
$$

Por lo tanto, la transformación de incrementos de la configuración materiales en espaciales viene gobernada por la siguiente expresión

$$
\left\{\begin{array}{c}
\Delta \boldsymbol{x}_{i} \\
\Delta \boldsymbol{\omega}_{i}
\end{array}\right\}=\left[\begin{array}{cc}
\boldsymbol{\Lambda}_{i} & \mathbf{0} \\
\mathbf{0} & \boldsymbol{\Lambda}_{i}
\end{array}\right]\left[\begin{array}{cc}
\boldsymbol{\Lambda}_{(0)}^{e} & \mathbf{0} \\
\mathbf{0} & \boldsymbol{\Lambda}_{(0)}^{e}
\end{array}\right]\left\{\begin{array}{c}
\Delta \boldsymbol{\chi}_{i} \\
\Delta \boldsymbol{\Omega}_{i}
\end{array}\right\}
$$

Denominando $\mathbf{L}$ a la matriz que contiene las rotaciones

$$
\mathbf{L}_{i}=\left[\begin{array}{cc}
\boldsymbol{\Lambda}_{i} & \mathbf{0} \\
\mathbf{0} & \boldsymbol{\Lambda}_{i}
\end{array}\right]\left[\begin{array}{cc}
\boldsymbol{\Lambda}_{(0)}^{e} & \mathbf{0} \\
\mathbf{0} & \boldsymbol{\Lambda}_{(0)}^{e}
\end{array}\right]=\left[\begin{array}{cc}
\boldsymbol{\Lambda}_{i} \boldsymbol{\Lambda}_{(0)}^{e} & \mathbf{0} \\
\mathbf{0} & \boldsymbol{\Lambda}_{i} \boldsymbol{\Lambda}_{(0)}^{e}
\end{array}\right]
$$

la expresión anterior es

$$
\Delta \phi_{i}=\mathbf{L}_{i} \Delta \boldsymbol{\Phi}_{i}
$$




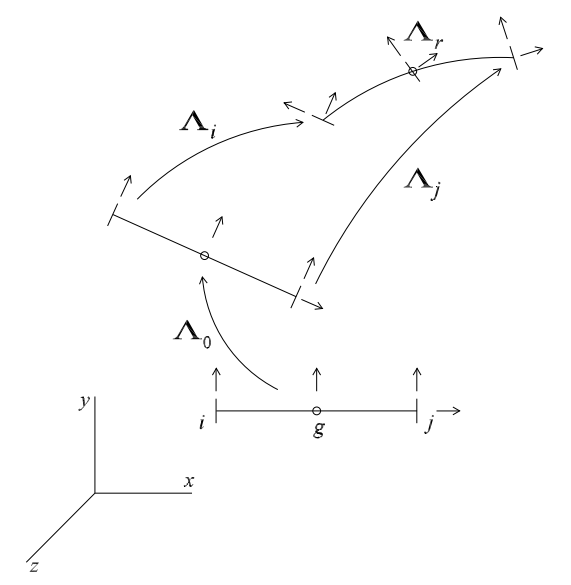

Figura 8.1: Transformaciones en el elemento material

Entonces la forma espacial de la matriz de rigidez del elemento será

$$
\left[\begin{array}{ll}
\mathbf{K}_{i i}^{e} & \mathbf{K}_{i j}^{e} \\
\mathbf{K}_{j i}^{e} & \mathbf{K}_{j j}^{e}
\end{array}\right]=\left[\begin{array}{cc}
\mathbf{L}_{i} & \mathbf{0} \\
\mathbf{0} & \mathbf{L}_{j}
\end{array}\right]\left[\begin{array}{ll}
\mathbf{K}_{i i m a t}^{e} & \mathbf{K}_{i j \text { mat }}^{e} \\
\mathbf{K}_{j i \text { mat }}^{e} & \mathbf{K}_{j j \text { mat }}^{e}
\end{array}\right]\left[\begin{array}{cc}
\mathbf{L}_{i}^{\top} & \mathbf{0} \\
\mathbf{0} & \mathbf{L}_{j}^{\top}
\end{array}\right]
$$

por lo tanto, la contribución de un elemento de dos nodos al operador tangente en forma espacial es

$$
\left\{\begin{array}{ll}
\delta \phi_{i} & \delta \phi_{j}
\end{array}\right\}\left[\begin{array}{ll}
\mathbf{K}_{i i}^{e} & \mathbf{K}_{i j}^{e} \\
\mathbf{K}_{j i}^{e} & \mathbf{K}_{j j}^{e}
\end{array}\right]\left\{\begin{array}{c}
\Delta \phi_{i} \\
\Delta \boldsymbol{\phi}_{j}
\end{array}\right\}
$$

Esta expresión se puede generalizar fácilmente a un elemento con un número arbitrario de nodos.

\section{Ensamblaje de la rigidez completa}

Las matrices de los elementos expresadas ya en forma espacial se pueden ensamblar para construir la matriz de rigidez tangente de la estructura. La contribución a la rigidez tangente de las fuerzas nodales (en forma espacial) se ensambla en las posiciones nodales correspondientes a las variables $\Delta \phi_{i}$ y es, como en el elemento espacial, (7.10)

$$
-\mathbf{f}_{i}=-\lambda\left(\left[\begin{array}{cc}
\mathbf{0} & \mathbf{0} \\
\mathbf{0} & \widehat{\mathbf{m}}_{i}
\end{array}\right]+\left[\begin{array}{cc}
\mathbf{0} & -\widehat{\mathbf{n}}_{i}^{f} \\
\mathbf{0} & -\widehat{\mathbf{m}}_{i}^{f}
\end{array}\right]\right),
$$

Cabe señalar que la rigidez material de las fuerzas nodales deducida en el capítulo 6 -ecuación (6.82)-

$$
-\mathbf{F}_{i}=-\lambda\left(\left[\begin{array}{cc}
\mathbf{0} & \mathbf{0} \\
\mathbf{0} & \widehat{\mathbf{M}}_{i}
\end{array}\right]+\left[\begin{array}{cc}
\mathbf{0} & -\widehat{\mathbf{N}}_{i}^{f} \\
\mathbf{0} & -\widehat{\mathbf{M}}_{i}^{f}
\end{array}\right]\right)
$$

concuerda con la expresión anterior a través de la rotación nodal $\boldsymbol{\Lambda}_{i}$. 


\section{Discretización de las fuerzas totales}

Volviendo a la expresión del equilibrio tangente, el lado derecho de la igualdad contiene, entre paréntesis, la expresión de las fuerzas totales que actúan sobre la estructura: las fuerzas generalizadas sobre la directriz, y las fuerzas sobre los extremos de la pieza. Denominando fuerzas iniciales a la discretización de las fuerzas generalizadas que actúan sobre un elemento, ésta se deducirá de la discretización de la integral

$$
\int_{\Gamma_{0}} \delta \boldsymbol{\Phi}^{\top} \boldsymbol{Q} d S
$$

que proporciona

$$
\delta \boldsymbol{\Phi}^{e \boldsymbol{\top}} \int_{-1}^{1} \mathbb{N}^{\top} \boldsymbol{Q} d S=\delta \boldsymbol{\Phi}^{e} \cdot \mathbf{f}_{m a t}^{0 e}
$$

La integral se resuelve con la cuadratura de Gauss de orden $g$ y proporciona las fuerzas nodales iniciales

$$
\mathbf{f}_{m a t}^{0 e}=\sum_{l=1}^{g} w_{l} \mathbb{N}^{\top}\left(\xi_{l}\right) \boldsymbol{Q}\left(\xi_{l}\right) J_{\left.\right|_{\xi=\xi_{l}}}
$$

En el elemento de dos nodos, el vector de fuerzas se compone de las fuerzas asociadas a los nodos $i$ y $j$

$$
\left\{\begin{array}{c}
\mathbf{f}_{i m a t}^{0 e} \\
\mathbf{f}_{j \text { mat }}^{0} e
\end{array}\right\}
$$

La transformación a la forma material se efectúa a partir de la expresión

$$
\delta \boldsymbol{\Phi}^{e} \cdot \mathbf{f}_{m a t}^{0 e}=\left\{\begin{array}{ll}
\delta \boldsymbol{\Phi}_{i}^{e \boldsymbol{\top}} & \delta \boldsymbol{\Phi}_{j}^{e \boldsymbol{\top}}
\end{array}\right\}\left\{\begin{array}{c}
\mathbf{f}_{i m a t}^{0 e} \\
\mathbf{f}_{j \text { mat }}^{0 e}
\end{array}\right\}=\left\{\begin{array}{ll}
\delta \boldsymbol{\phi}_{i}^{e \top} & \delta \boldsymbol{\phi}_{j}^{e \boldsymbol{\top}}
\end{array}\right\}\left[\begin{array}{cc}
\mathbf{L}_{i} & \mathbf{0} \\
\mathbf{0} & \mathbf{L}_{j}
\end{array}\right]\left\{\begin{array}{c}
\mathbf{f}_{i m a t}^{0 e} \\
\mathbf{f}_{j m a t}^{0 e}
\end{array}\right\} .
$$

Luego la forma espacial del vector de fuerzas iniciales del elemento $e$ es

$$
\left\{\begin{array}{c}
\mathbf{f}_{i}^{0 e} \\
\mathbf{f}_{j}^{0 e}
\end{array}\right\}=\left[\begin{array}{cc}
\mathbf{L}_{i} & \mathbf{0} \\
\mathbf{0} & \mathbf{L}_{j}
\end{array}\right]\left\{\begin{array}{c}
\mathbf{f}_{i m a t}^{0 e} \\
\mathbf{f}_{j \text { mat }}^{0} \text { mat }
\end{array}\right\}
$$

El vector de fuerzas totales se obtiene ensamblando las distintas contribuciones

$$
\left\{\begin{array}{ll}
\mathbf{f}_{i}^{0 e} & \mathbf{f}_{j}^{0 e}
\end{array}\right\}^{\top}
$$

junto con las fuerzas nodales en su forma espacial

$$
\boldsymbol{f}_{i}=\left\{\begin{array}{ll}
\boldsymbol{n}_{i} & \boldsymbol{m}_{i}
\end{array}\right\}^{\top}
$$

en las posiciones nodales correspondientes a $\Delta \phi_{i}$. 


\section{Ecuación incremental}

Empleamos ahora la misma notación que en la solución espacial. $\mathbf{K}$ y $\mathbf{f}$ son resultado de ensamblar la matriz de rigidez tangente y el vector de fuerzas totales, y eliminar las filas (y columnas) correspondientes a las componentes nulas de los incrementos nodales de las variables de la configuración. $\Delta \phi$ reúne los incrementos nodales incógnita $\Delta \boldsymbol{\Phi}_{i}=\left\{\begin{array}{ll}\Delta \boldsymbol{x}_{i} & \Delta \boldsymbol{\omega}_{i}\end{array}\right\}^{\top}$. La ecuación incremental resulta

$$
\mathbf{K} \Delta \boldsymbol{\phi}=\mathbf{f} \Delta \lambda
$$

que junto con una restricción del tipo

$$
c(\Delta \phi, \Delta \lambda)=0
$$

que define la estrategia de control, sirve para calcular el resultado del incremento que denominamos predicción. Las distintas estrategias de control consideradas se han explicado en el capítulo 7 y no añadiremos nada aquí.

\subsubsection{Corrección}

La fase de corrección mediante el proceso iterativo de Newton-Raphson se fundamenta en la linealización del residuo que se calcula a partir de la forma material de la ecuación de trabajos virtuales y proporciona la matriz de rigidez tangente necesaria para el cálculo. Comenzamos calculando el residuo en fuerzas.

\section{Expresión del residuo}

El trabajo virtual de las fuerzas internas viene definido por la ecuación 6.54

$$
\delta W_{i n t}=\int_{\Gamma_{0}} \delta \boldsymbol{E} \cdot \boldsymbol{F} d S=\int_{\Gamma_{0}}(\delta \boldsymbol{\Gamma} \cdot \boldsymbol{N}+\delta \boldsymbol{K} \cdot \boldsymbol{M}) d S,
$$

que, utilizando 6.70, 4.50 y 4.51 también puede expresarse así

$$
\begin{aligned}
\delta W_{\text {int }} & =\int_{\Gamma_{0}}\left\{\delta \boldsymbol{\chi}^{\top} \delta \boldsymbol{\Omega}^{\top} \delta \boldsymbol{\chi}^{\prime \top} \delta \boldsymbol{\Omega}^{\top \top}\right\}\left\{\begin{array}{c}
\boldsymbol{N} \times \boldsymbol{K} \\
\boldsymbol{N} \times \boldsymbol{\Gamma}+\boldsymbol{M} \times \boldsymbol{K} \\
\boldsymbol{N} \\
\boldsymbol{M}
\end{array}\right\} d S \\
& =\int_{\Gamma_{0}}\left\{\delta \boldsymbol{\chi}^{\top} \delta \boldsymbol{\Omega}^{\top} \delta \boldsymbol{\chi}^{\prime \top} \delta \boldsymbol{\Omega}^{\prime \top}\right\}\left[\begin{array}{cc}
-\widehat{\mathbf{K}} & \mathbf{0} \\
-\widehat{\boldsymbol{\Gamma}} & -\widehat{\mathbf{K}} \\
\mathbf{1} & \mathbf{0} \\
\mathbf{0} & \mathbf{1}
\end{array}\right]\left\{\begin{array}{c}
\boldsymbol{N} \\
\boldsymbol{M}
\end{array}\right\} \\
& =\int_{\Gamma_{0}}\left\{\delta \boldsymbol{\Phi}^{\top} \quad \delta \boldsymbol{\Phi}^{\prime \top}\right\}\left[\begin{array}{c}
\mathbf{E}^{\top} \\
\mathbf{I}
\end{array}\right] \boldsymbol{F} d S .
\end{aligned}
$$

La contribución de cada elemento finito al incremento de trabajo de las fuerzas internas se puede calcular introduciendo la interpolación en la expresión anterior

$$
\delta W_{i n t}^{e}=\delta \boldsymbol{\Phi}^{e \boldsymbol{\top}} \int_{-1}^{1}\left[\begin{array}{ll}
\mathbb{N}^{\top} & \frac{1}{J} \mathbb{N}^{\top} \boldsymbol{\top}
\end{array}\right]\left[\begin{array}{c}
\mathbf{E}^{\top} \\
\mathbf{I}
\end{array}\right] \boldsymbol{F} J d \xi=\delta \boldsymbol{\Phi}^{e} \cdot \mathbf{p}_{m a t}^{e},
$$


donde

$$
\mathbf{p}_{\text {mat }}^{e}=\sum_{l=1}^{g} w_{l}\left[\begin{array}{ll}
\mathbb{N}^{\top} & \frac{1}{J} \mathbb{N}^{\prime} \top
\end{array}\right]_{\xi=\xi_{l}}\left[\begin{array}{c}
\mathbf{E}^{\top} \\
\mathbf{I}
\end{array}\right]_{\left.\right|_{\xi=\xi_{l}}} \boldsymbol{F}\left(\xi_{l}\right) J\left(\xi_{l}\right) .
$$

Las fuerzas internas se pueden transformar a su versión espacial empleando las expresiones deducidas en la sección anterior, válida para el elemento de 2 nodos

$$
\left\{\begin{array}{l}
\mathbf{p}_{i}^{0 e} \\
\mathbf{p}_{j}^{0 e}
\end{array}\right\}=\left[\begin{array}{cc}
\mathbf{L}_{i} & \mathbf{0} \\
\mathbf{0} & \mathbf{L}_{j}
\end{array}\right]\left\{\begin{array}{l}
\mathbf{p}_{i e}^{0 e} \\
\mathbf{p}_{j \text { mat }}^{0} e_{\text {mat }}
\end{array}\right\} .
$$

Ensamblándolas se construye el vector de fuerzas internas de la estructura; en éste se procederá a la eliminación de las filas correspondientes a los grados de libertad coaccionados. Finalmente el trabajo virtual de las fuerzas internas en la estructura es

$$
\delta W_{\text {int }}=\delta \boldsymbol{\phi} \cdot \mathbf{p}
$$

Por lo que respecta a las fuerzas exteriores, el trabajo virtual de las fuerzas generalizadas $\lambda \boldsymbol{Q}(S)$ sobre las variaciones de la configuración es

$$
\lambda \int_{\Gamma_{0}}\left(\delta \boldsymbol{\chi} \cdot \boldsymbol{Q}_{n}+\delta \boldsymbol{\Omega} \cdot \boldsymbol{Q}_{m}\right) d S=\lambda \int_{\Gamma_{0}} \delta \boldsymbol{\Phi}^{\top} \boldsymbol{Q} d S .
$$

Introduciendo en esta expresión la interpolación propuesta se obtiene la contribución de un elemento al trabajo virtual realizado por las fuerzas generalizadas

$$
\delta \boldsymbol{\Phi}^{e \top} \lambda \int_{-1}^{1} \mathbb{N}^{\top} \boldsymbol{Q} J d \xi=\delta \boldsymbol{\Phi}^{e} \cdot \lambda \mathbf{f}_{m a t}^{0 e} .
$$

El vector $\mathbf{f}_{\text {mat }}^{0 e}$ coincide con el vector de fuerzas iniciales introducido en la sección anterior. Resolviendo la integral mediante la cuadratura de Gauss,

$$
\mathbf{f}^{0 e}=\sum_{l=1}^{g} w_{l} \mathbb{N}^{\top}\left(\xi_{l}\right) \boldsymbol{Q}\left(\xi_{l}\right) J\left(\xi_{l}\right) .
$$

Su expresión en forma espacial en el caso del elemento de 2 nodos es

$$
\left\{\begin{array}{c}
\mathbf{f}_{i}^{0 e} \\
\mathbf{f}_{j}^{0 e}
\end{array}\right\}=\left[\begin{array}{cc}
\mathbf{L}_{i} & \mathbf{0} \\
\mathbf{0} & \mathbf{L}_{j}
\end{array}\right]\left\{\begin{array}{c}
\mathbf{f}_{i \text { mat }}^{0 e} \\
\mathbf{f}_{j \text { mat }}^{0 e}
\end{array}\right\} .
$$

Las fuerzas exteriores en forma espacial que actúan sobre cada nodo - cuya contribución al trabajo virtual externo es $\delta \boldsymbol{\phi}_{i} \cdot \lambda \boldsymbol{f}_{i}$

$$
\lambda \boldsymbol{f}_{i}=\lambda\left\{\boldsymbol{n}_{i} \boldsymbol{m}_{i}\right\}^{\top},
$$

se ensamblan directamente en las posiciones correspondientes. Después del ensamblaje se eliminan las filas correspondientes a los grados de libertad coaccionados; el resultado es el vector de fuerzas $\lambda \mathbf{f}$. Entonces, el trabajo virtual de las fuerzas exteriores sobre la estructura se puede expresar así

$$
\delta W_{e x t}=\delta \boldsymbol{\phi} \cdot \lambda \mathbf{f}
$$


y la ecuación de trabajos virtuales exige que en el equilibrio, el trabajo virtual de las fuerzas internas sea igual al de las fuerzas exteriores para cualquier variación cinemáticamente admisible de la configuración

$$
\delta \boldsymbol{\phi} \cdot(\mathbf{p}-\lambda \mathbf{f})=0 \quad \forall \delta \boldsymbol{\phi} .
$$

Como en el caso espacial, el residuo, que se define como la diferencia entre los vectores de fuerzas nodales internas y externas,

$$
\mathbf{r}=\mathbf{p}-\lambda \mathbf{f}=\mathbf{0} .
$$

se anula en configuraciones pertenecientes a la trayectoria de equilibrio.

\section{Linealización del residuo}

Evaluamos en primer lugar la derivada direccional del vector de fuerzas internas

$$
\Delta \mathbf{p}_{\text {mat }}^{e}=\Delta \int_{-1}^{1}\left[\begin{array}{ll}
\mathbb{N}^{\top} & \frac{1}{J} \mathbb{N}^{\prime} \mathrm{T}
\end{array}\right]\left[\begin{array}{c}
\mathbf{E}^{\top} \\
\mathbf{I}
\end{array}\right] \boldsymbol{F} J d \xi .
$$

Desarrollando el integrando e introduciendo la variación en la integral (las funciones de forma no están sujetas a variación)

$$
\Delta \mathbf{p}_{\text {mat }}^{e}=\int_{-1}^{1}\left[\begin{array}{cccc}
N_{1} \mathbf{1} & \mathbf{0} & \frac{1}{J} N_{1}^{\prime} \mathbf{1} & \mathbf{0} \\
\mathbf{0} & N_{1} \mathbf{1} & \mathbf{0} & \frac{1}{J} N_{1}^{\prime} \mathbf{1} \\
\vdots & \vdots & \vdots & \vdots \\
N_{n} \mathbf{1} & \mathbf{0} & \frac{1}{J} N_{n}^{\prime} \mathbf{1} & \mathbf{0} \\
\mathbf{0} & N_{n} \mathbf{1} & \mathbf{0} & \frac{1}{J} N_{n}^{\prime} \mathbf{1}
\end{array}\right] \Delta\left\{\begin{array}{c}
\boldsymbol{N} \times \boldsymbol{K} \\
\boldsymbol{N} \times \boldsymbol{\Gamma}+\boldsymbol{M} \times \boldsymbol{K} \\
\boldsymbol{N} \\
\boldsymbol{M}
\end{array}\right\} J d \xi .
$$

La variación espacial del vector del integrando se ha calculado en el comentario 6.3 .1 y su núcleo es suma de dos matrices:

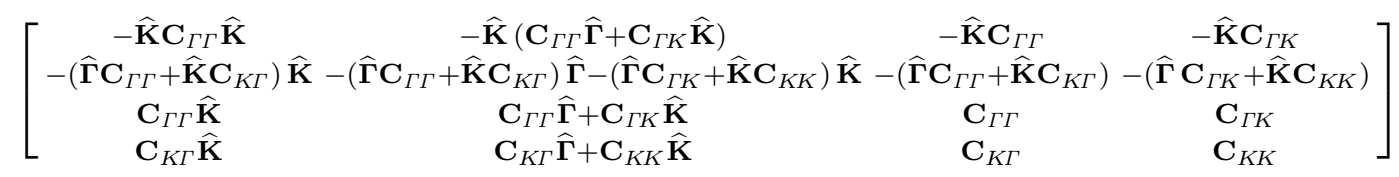

$$
\begin{aligned}
& +\left[\begin{array}{cccc}
0 & \widehat{\mathbf{N}} \widehat{\mathbf{K}} & 0 & \widehat{\mathbf{N}} \\
\widehat{\mathbf{N}} \widehat{\mathbf{K}} & \widehat{\mathbf{N}} \widehat{\Gamma}+\widehat{\mathbf{M}} \widehat{\mathbf{K}} & \widehat{\mathbf{N}} & \widehat{\mathbf{M}} \\
\mathbf{0} & \mathbf{0} & \mathbf{0} & \mathbf{0} \\
\mathbf{0} & \mathbf{0} & \mathbf{0} & \mathbf{0}
\end{array}\right] .
\end{aligned}
$$

La primera matriz es el núcleo constitutivo en su forma material. La segunda matriz, que constituye la parte geométrica, no coincide con el núcleo geométrico empleado en la predicción.

Empleamos la siguiente notación para resumir las expresiones anteriores

$$
\begin{aligned}
\Delta \mathbf{p}_{\text {mat }}^{e}=\int_{-1}^{1}\left[\begin{array}{ll}
\mathbb{N}^{\top} & \frac{1}{J} \mathbb{N}^{\prime} \top
\end{array}\right]\left[\begin{array}{ll}
\mathbf{D}_{00}^{M} & \mathbf{D}_{01}^{M} \\
\mathbf{D}_{10}^{M} & \mathbf{D}_{11}^{M}
\end{array}\right]\left\{\begin{array}{c}
\Delta \boldsymbol{\Phi} \\
\Delta \boldsymbol{\Phi}^{\prime}
\end{array}\right\} J d \xi \\
\\
+\int_{-1}^{1}\left[\begin{array}{ll}
\mathbb{N}^{\top} & \frac{1}{J} \mathbb{N}^{\prime} \mathrm{T}
\end{array}\right]\left[\begin{array}{cc}
\widetilde{\mathbf{D}}_{00}^{G} & \widetilde{\mathbf{D}}_{01}^{G} \\
\widetilde{\mathbf{D}}_{10}^{G} & \widetilde{\mathbf{D}}_{11}^{G}
\end{array}\right]\left\{\begin{array}{c}
\Delta \boldsymbol{\Phi} \\
\Delta \boldsymbol{\Phi}^{\prime}
\end{array}\right\} J d \xi .
\end{aligned}
$$


La tilde en el término geométrico se introduce para diferenciarlo del término geométrico deducido para la predicción. Discretizando las variables de la configuración y sus derivadas resulta

$$
\begin{aligned}
& \Delta \mathbf{p}_{\text {mat }}^{e}=\int_{-1}^{1}\left[\begin{array}{ll}
\mathbb{N}^{\top} & \frac{1}{J} \mathbb{N}^{\prime} \top
\end{array}\right]\left[\begin{array}{ll}
\mathbf{D}_{00}^{M} & \mathbf{D}_{01}^{M} \\
\mathbf{D}_{10}^{M} & \mathbf{D}_{11}^{M}
\end{array}\right]\left[\begin{array}{c}
\mathbb{N} \\
\frac{1}{J} \mathbb{N}^{\prime}
\end{array}\right] J d \xi \Delta \boldsymbol{\Phi}^{e} \\
& +\int_{-1}^{1}\left[\begin{array}{ll}
\mathbb{N}^{\top} & \frac{1}{J} \mathbb{N}^{\prime} \top
\end{array}\right]\left[\begin{array}{ll}
\widetilde{\mathbf{D}}_{00}^{G} & \widetilde{\mathbf{D}}_{01}^{G} \\
\widetilde{\mathbf{D}}_{10}^{G} & \widetilde{\mathbf{D}}_{11}^{G}
\end{array}\right]\left[\begin{array}{c}
\mathbb{N} \\
\frac{1}{J} \mathbb{N}^{\prime}
\end{array}\right] J d \xi \Delta \boldsymbol{\Phi}^{e} .
\end{aligned}
$$

La primera integral es la matriz de rigidez constitutiva y la segunda, la matriz de rigidez geométrica. Entonces podemos escribir

$$
\Delta \mathbf{p}_{m a t}^{e}=\left(\mathbf{K}_{m a t}^{M e}+\widetilde{\mathbf{K}}_{m a t}^{G e}\right) \Delta \boldsymbol{\Phi}^{e},
$$

con

$$
\begin{aligned}
& \mathbf{K}_{m a t}^{M e}=\sum_{l=1}^{g} w_{l}\left[\begin{array}{ll}
\mathbb{N}^{\top} & \frac{1}{J} \mathbb{N}^{\prime} \mathrm{T}
\end{array}\right]_{\left.\right|_{\xi=\xi_{l}}}\left[\begin{array}{ll}
\mathbf{D}_{00}^{M} & \mathbf{D}_{01}^{M} \\
\mathbf{D}_{10}^{M} & \mathbf{D}_{11}^{M}
\end{array}\right]_{\left.\right|_{\xi=\xi_{l}}}\left[\begin{array}{c}
\mathbb{N} \\
\frac{1}{J}
\end{array} \mathbb{N}^{\prime}\right]_{\left.\right|_{\xi=\xi_{l}}} J\left(\xi_{l}\right) \\
& \widetilde{\mathbf{K}}_{m a t}^{G e}=\sum_{l=1}^{g} w_{l}\left[\begin{array}{ll}
\mathbb{N}^{\top} & \frac{1}{J}
\end{array} \mathbb{N}^{\prime}\right]_{\left.\right|_{\xi=\xi_{l}}}\left[\begin{array}{cc}
\widetilde{\mathbf{D}}_{00}^{G} & \widetilde{\mathbf{D}}_{01}^{G} \\
\widetilde{\mathbf{D}}_{10}^{G} & \widetilde{\mathbf{D}}_{11}^{G}
\end{array}\right]_{\left.\right|_{\xi=\xi_{l}}}\left[\begin{array}{c}
\mathbb{N} \\
\frac{1}{J} \mathbb{N}^{\prime}
\end{array}\right]_{\mid \xi=\xi_{l}} J\left(\xi_{l}\right)
\end{aligned}
$$

A continuación calcularemos la derivada direccional de las fuerzas generalizadas.

$$
\Delta \mathbf{f}_{m a t}^{0 e}=\int_{-1}^{1} \mathbb{N}^{\top} \Delta \boldsymbol{Q} J d \xi
$$

y recordando que, según 6.72 y 6.73

$$
\begin{aligned}
& \Delta \boldsymbol{Q}^{f}=\mathbf{0} \\
& \Delta \boldsymbol{Q}^{s}=\widehat{\mathbf{Q}}^{s} \Delta \boldsymbol{\Omega},
\end{aligned}
$$

la expresión anterior queda

$$
\Delta \mathbf{f}_{m a t}^{0 e}=\int_{-1}^{1}\left[\mathbb{N}^{\top} \quad \frac{1}{J} \mathbb{N}^{\prime}\right]\left[\begin{array}{cc}
\widetilde{\mathbf{D}}^{Q} & \mathbf{0} \\
\mathbf{0} & \mathbf{0}
\end{array}\right]\left\{\begin{array}{c}
\Delta \boldsymbol{\Phi} \\
\Delta \boldsymbol{\Phi}^{\prime}
\end{array}\right\} J d \xi
$$

con la matriz del núcleo

$$
\widetilde{\mathbf{D}}^{Q}=\left[\begin{array}{ll}
\mathbf{0} & \widehat{\mathbf{Q}}_{n}^{s} \\
\mathbf{0} & \widehat{\mathbf{Q}}_{m}^{s}
\end{array}\right],
$$

que también distinguimos con una tilde para diferenciarla de la empleada en la predicción. Completando la discretización

$$
\Delta \mathbf{f}_{m a t}^{0 e}=\int_{-1}^{1}\left[\begin{array}{ll}
\mathbb{N}^{\top} & \frac{1}{J} \mathbb{N}^{\prime}
\end{array}\right]\left[\begin{array}{cc}
\widetilde{\mathbf{D}}^{Q} & \mathbf{0} \\
\mathbf{0} & \mathbf{0}
\end{array}\right]\left[\begin{array}{c}
\mathbb{N} \\
\frac{1}{J} \mathbb{N}^{\prime}
\end{array}\right] J d \xi \Delta \boldsymbol{\Phi}^{e} .
$$


La integral es precisamente la contribución a la rigidez del elemento debida a las fuerzas generalizadas (en forma material) que denotamos $\widetilde{\mathbf{K}}_{m a t}^{Q e}$. Por tanto, se puede escribir

$$
\Delta \mathbf{f}_{m a t}^{0 e}=\widetilde{\mathbf{K}}_{m a t}^{Q e} \Delta \boldsymbol{\Phi}^{e} .
$$

con

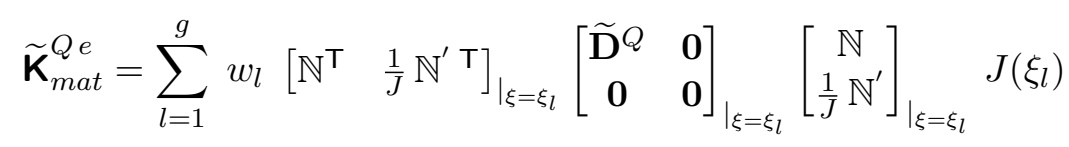

evaluada mediante una cuadratura de Gauss de orden $g$.

Con todos estos resultados concluimos que la forma material de la rigidez tangente del elemento para la corrección es

$$
\widetilde{\mathbf{K}}_{m a t}^{e}=\mathbf{K}_{m a t}^{M}+\widetilde{\mathbf{K}}_{m a t}^{G e}-\lambda \widetilde{\mathbf{K}}_{m a t}^{Q e}
$$

La transformación a ejes globales en el elemento de dos nodos sigue la misma regla que se usó en la matriz de rigidez para la predicción:

$$
\left[\begin{array}{cc}
\widetilde{\mathbf{K}}_{i i}^{e} & \widetilde{\mathbf{K}}_{i j}^{e} \\
\widetilde{\mathbf{K}}_{j i}^{e} & \widetilde{\mathbf{K}}_{j j}^{e}
\end{array}\right]=\left[\begin{array}{cc}
\mathbf{L}_{i} & \mathbf{0} \\
\mathbf{0} & \mathbf{L}_{j}
\end{array}\right]\left[\begin{array}{ll}
\widetilde{\mathbf{K}}_{i i m a t}^{e} & \widetilde{\mathbf{K}}_{i j m a t}^{e} \\
\widetilde{\mathbf{K}}_{j i m a t}^{e} & \widetilde{\mathbf{K}}_{j j m a t}^{e}
\end{array}\right]\left[\begin{array}{cc}
\mathbf{L}_{i}^{\top} & \mathbf{0} \\
\mathbf{0} & \mathbf{L}_{j}^{\top}
\end{array}\right]
$$

Resta linealizar la contribución de las fuerzas nodales al residuo

$$
-\lambda \boldsymbol{F}_{i}=-\lambda\left\{\begin{array}{ll}
\boldsymbol{N}_{i} & \boldsymbol{M}_{i}
\end{array}\right\}^{\top}
$$

Teniendo en cuenta las propiedades de la variación sobre la forma material de las fuerzas espaciales y perseguidoras $6.72,6.73$ resulta

$$
-\lambda \Delta \boldsymbol{F}_{i}=-\lambda\left[\begin{array}{ll}
\mathbf{0} & \widehat{\mathbf{N}}_{i}^{s} \\
\mathbf{0} & \widehat{\mathbf{M}}_{i}^{s}
\end{array}\right]\left\{\begin{array}{l}
\delta \boldsymbol{\chi}^{\top} \\
\delta \boldsymbol{\Omega}^{\top}
\end{array}\right\} .
$$

La forma espacial de la matriz que aparece en esta expresión, que será la empleada en el ensamblaje, es

$$
-\lambda\left[\begin{array}{cc}
\mathbf{0} & \widehat{\mathbf{n}}_{i}^{s} \\
\mathbf{0} & \widehat{\mathbf{m}}_{i}^{s}
\end{array}\right] .
$$

Finalmente, la matriz de rigidez completa para la corrección resulta de ensamblar la contribución de cada uno de los elementos en forma espacial con la rigidez de las fuerzas nodales (8.40), y eliminar las filas y columnas correspondientes a los grados de libertad coaccionados. Entonces

$$
\Delta \mathbf{p}=\widetilde{\mathrm{K}} \Delta \boldsymbol{\phi}
$$

El proceso iterativo que permite la anulación del residuo, así como las estrategias de control han sido tratados con detalle en la sección 7.3 .3 del capítulo anterior. 


\subsubsection{Actualización de la configuración}

\section{Actualización de la posición y la rotación}

Empleamos en este caso el mismo procedimiento definido en la solución espacial (7.28), (7.29), basado en la actualización aditiva de las posiciones nodales

$$
\boldsymbol{x}_{i(n+1)}=\boldsymbol{x}_{i(n)}+\Delta \boldsymbol{x}_{i(n)},
$$

y la actualización multiplicativa de las rotaciones

$$
\boldsymbol{\Lambda}_{i(n+1)}=\exp \left(\Delta \hat{\boldsymbol{\omega}}_{i(n)}\right) \boldsymbol{\Lambda}_{i(n)} .
$$

Para la implementación numérica se emplean cuaterniones unitarios, según la propuesta de Ibrahimbegović y Taylor [28] concretada en las ecuaciones (7.31), (7.32), (7.33), (7.34).

\section{Cálculo de las deformaciones generalizadas}

En este caso vamos a emplear la interpolación esférica propuesta por Crisfield y Jelenić [16] comentada en 7.2.3. Este procedimiento permite calcular las deformaciones directamente a partir de las variables de la configuración, sin necesidad de almacenar el valor de las mismas en el incremento o iteración anterior. Las medidas de la deformación obtenidas son, además, objetivas en el sentido estricto definido en la sección 3.5.3. El desarrollo de este punto se circunscribe al elemento de 2 nodos.

Las rotaciones totales se expresan como producto de la rotación inicial y la deformacional (sección 3.3 )

$$
\boldsymbol{\Lambda}(S)=\boldsymbol{\Lambda}_{d}(S) \boldsymbol{\Lambda}_{0}(S) .
$$

En el elemento de dos nodos la rotación inicial $\boldsymbol{\Lambda}_{0}(S)$ es constante y se denota $\boldsymbol{\Lambda}_{(0)}^{e}$. Por otro lado definimos las rotaciones nodales $\boldsymbol{\Lambda}_{i}$ y $\boldsymbol{\Lambda}_{j}$ de forma que representen el cambio desde la configuración inicial a la configuración actual en los nodos. Por tanto, se trata de rotaciones deformacionales $\boldsymbol{\Lambda}_{\mathrm{d}}$. Su discretización se basa en la interpolación esférica (7.44): como rotación deformacional de referencia para el elemento escogemos la correspondiente al punto de Gauss. Entonces (prescindiendo por claridad del subíndice $n$ ),

$$
\boldsymbol{\Lambda}_{r}=\left.\boldsymbol{\Lambda}_{d}\right|_{\xi=0}=\boldsymbol{\Lambda}_{i}\left(\boldsymbol{\Lambda}_{i}^{\top} \boldsymbol{\Lambda}_{j}\right)^{1 / 2},
$$

A partir de la rotación de referencia es posible definir las rotaciones locales de los nodos

$$
\begin{aligned}
\exp \widehat{\boldsymbol{\Psi}}_{i} & =\boldsymbol{\Lambda}_{r}^{\top} \boldsymbol{\Lambda}_{i} \\
\exp \widehat{\boldsymbol{\Psi}}_{j} & =\boldsymbol{\Lambda}_{r}^{\top} \boldsymbol{\Lambda}_{j}
\end{aligned}
$$

Con ellas se construye la interpolación

$$
\boldsymbol{\Lambda}_{d}^{h}(S)=\boldsymbol{\Lambda}_{r} \exp \left(\widehat{\boldsymbol{\Psi}}^{h}(S)\right),
$$




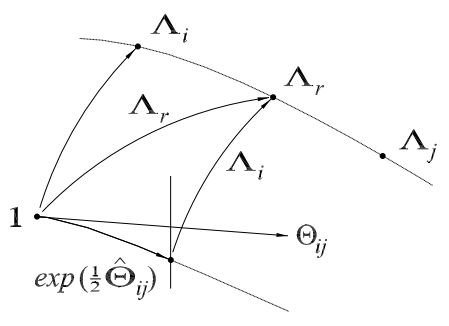

Figura 8.2: Rotación de referencia

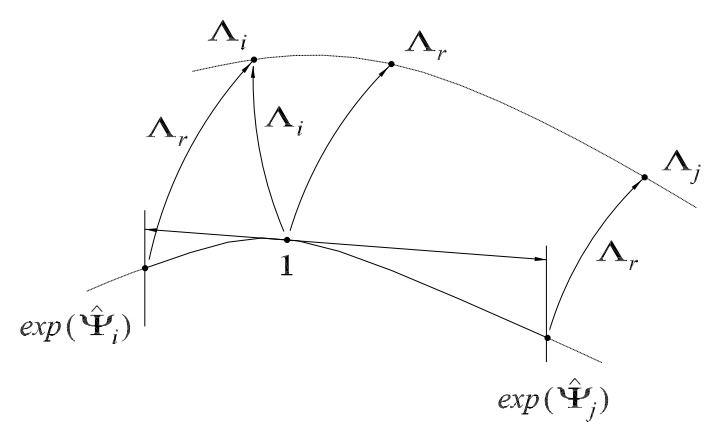

Figura 8.3: Rotaciones locales

$\operatorname{con} \boldsymbol{\Psi}^{h}=\sum_{a=i, j} N_{a} \boldsymbol{\Psi}_{a}$, luego

$$
\boldsymbol{\Lambda}^{h}(S)=\boldsymbol{\Lambda}_{d}^{h}(S) \boldsymbol{\Lambda}_{(0)}^{e}=\boldsymbol{\Lambda}_{r} \exp \left(\widehat{\mathbf{\Psi}}^{h}(S)\right) \boldsymbol{\Lambda}_{(0)}^{e}
$$

De (3.72)

$$
\begin{gathered}
\widehat{\mathbf{K}}^{h}(S)=\boldsymbol{\Lambda}^{\top} \boldsymbol{\Lambda}^{\prime}=\boldsymbol{\Lambda}_{(0)}^{\top e} \exp \left(-\widehat{\boldsymbol{\Psi}}^{h}\right) \frac{d}{d S} \exp \left(\widehat{\boldsymbol{\Psi}}^{h}\right) \boldsymbol{\Lambda}_{(0)}^{e}, \\
\boldsymbol{K}^{h}(S)=\boldsymbol{\Lambda}_{(0)}^{\top e} \mathbf{T}\left(\boldsymbol{\Psi}^{h}\right)^{\top} \frac{d}{d S} \boldsymbol{\Psi}^{h} .
\end{gathered}
$$

Teniendo en cuenta que en el punto de interpolación $S=1 / 2(\xi=0)$, y

$$
\begin{array}{ll}
N_{i}(0)=0 & N_{i}^{\prime}(0)=-1 / 2 \\
N_{j}(0)=0 & N_{j}^{\prime}(0)=1 / 2
\end{array}
$$

se tiene

$$
\begin{aligned}
\left.\boldsymbol{\Psi}\right|_{\xi=0} & =\mathbf{0} \\
\left.\frac{d}{d S} \boldsymbol{\Psi}\right|_{\xi=0} & =\left.\frac{2}{L_{e}} \frac{d}{d \xi} \boldsymbol{\Psi}\right|_{\xi=0}=\frac{1}{L_{e}}\left(\boldsymbol{\Psi}_{j}-\boldsymbol{\Psi}_{i}\right) \\
\mathbf{T}\left(\left.\boldsymbol{\Psi}\right|_{\xi=0}\right) & =\mathbf{1}
\end{aligned}
$$


Luego el cambio de orientación en el punto de Gauss es

$$
\left.\boldsymbol{K}\right|_{\xi=0}=\boldsymbol{\Lambda}_{(0)}^{\top e} \frac{1}{L_{e}}\left(\boldsymbol{\Psi}_{j}-\boldsymbol{\Psi}_{i}\right)
$$

De $(3.72)$

como

$$
\boldsymbol{\Gamma}^{h}(S)=\boldsymbol{\Lambda}^{\top} \boldsymbol{x}^{\prime}=\boldsymbol{\Lambda}_{(0)}^{\top e} \exp \left(-\widehat{\boldsymbol{\Psi}}^{h}\right) \boldsymbol{\Lambda}_{r}^{\top} \frac{d}{d S} \boldsymbol{x}^{h}
$$

$$
\begin{aligned}
\boldsymbol{\Psi}^{h} & =\sum_{a=i, j} N_{a} \boldsymbol{\Psi}_{a} \\
\boldsymbol{x}^{h} & =\sum_{a=i, j} N_{a} \boldsymbol{x}_{a},
\end{aligned}
$$

y las funciones de forma son lineales

$$
\begin{aligned}
\left.\boldsymbol{\Psi}\right|_{\xi=0} & =\mathbf{0} \\
\exp \left(-\left.\widehat{\boldsymbol{\Psi}}\right|_{\xi=0}\right) & =\mathbf{1} \\
\left.\frac{d}{d S} \boldsymbol{x}\right|_{\xi=0} & =\left.\frac{2}{L_{e}} \frac{d}{d \xi} \boldsymbol{x}\right|_{\xi=0}=\frac{1}{L_{e}}\left(\boldsymbol{x}_{j}-\boldsymbol{x}_{i}\right)
\end{aligned}
$$

luego

$$
\left.\boldsymbol{\Gamma}\right|_{\xi=0}=\boldsymbol{\Lambda}_{(0)}^{\top e} \boldsymbol{\Lambda}_{r}^{\top} \frac{1}{L_{e}}\left(\boldsymbol{x}_{j}-\boldsymbol{x}_{i}\right) .
$$

Las expresiones (8.48) y (8.49) son las medidas discretas de la deformación en el punto de integración del elemento de dos nodos.

\section{Cálculo de los esfuerzos}

En el caso del material lineal los esfuerzos en el punto de integración se pueden calcular directamente a partir del valor de las deformaciones generalizadas calculado.

$$
\left\{\begin{array}{l}
\left.\boldsymbol{N}\right|_{\xi=0} \\
\left.\boldsymbol{M}\right|_{\xi=0}
\end{array}\right\}=\left[\begin{array}{ll}
\mathbf{C}_{\Gamma \Gamma} & \mathbf{C}_{\Gamma K} \\
\mathbf{C}_{K \Gamma} & \mathbf{C}_{K K}
\end{array}\right]\left\{\begin{array}{c}
\left.\boldsymbol{\Gamma}\right|_{\xi=0}-\boldsymbol{\Gamma}_{0} \\
\left.\boldsymbol{K}\right|_{\xi=0}-\boldsymbol{K}_{0}
\end{array}\right\}
$$




\subsection{Flujo del programa}

En esta sección describimos esquemáticamente el algoritmo que se ha diseñado para la solución del problema en forma material empleando elementos rectos de dos nodos. Sólo los módulos de programa más relevantes aparecen mencionados de forma explícita.

\subsubsection{Datos iniciales}

Variables nodales

\begin{tabular}{|l|cc|}
\hline Configuración inicial $^{(1)}$ & $\boldsymbol{x}_{i(0)}$ & $\boldsymbol{\Lambda}_{i(0)}$ \\
Fuerzas nodales espaciales & $\boldsymbol{n}_{i}$ & $\boldsymbol{m}_{i}$ \\
Fuerzas nodales perseguidoras & $\boldsymbol{N}_{i}$ & $\boldsymbol{M}_{i}$ \\
\hline
\end{tabular}

Variables del elemento ${ }^{(2)}$

\begin{tabular}{|l|ll|}
\hline Orientación inicial $^{(3)}$ & $\boldsymbol{\Lambda}_{(0)}^{e}$ & \\
Deformaciones iniciales (forma material) $^{(4)}$ & $\Gamma_{(0)}^{e}$ & $\boldsymbol{K}_{(0)}^{e}$
\end{tabular}

1. Las rotaciones nodales iniciales expresadas en el sistema general se toman, por defecto, iguales a la identidad

$$
\boldsymbol{\Lambda}_{i(0)} \equiv\left[\begin{array}{lll}
1 & 0 & 0 \\
0 & 1 & 0 \\
0 & 0 & 1
\end{array}\right]
$$

Para nuestro elemento material es importante remarcar cuál es su sentido físico: las rotaciones nodales representan el cambio desde la configuración inicial a la configuración actual. Por tanto constituyen la parte de la rotación total que produce deformaciones $\boldsymbol{\Lambda}_{\mathrm{d}}$, que había sido definida en la sección 3.3 .

2. Todas las variables relativas al elemento están definidas en el punto de integración (de Gauss), $\xi=0$.

3. Para definir la orientación inicial del elemento se adopta el criterio definido a continuación (suponiendo que el eje $y$ define la dirección vertical). El vector $\boldsymbol{a}_{1}$ es el vector unitario en la dirección determinada por los nodos $i, j$ del elemento.

$$
\boldsymbol{a}_{1}=\frac{\boldsymbol{x}_{j}-\boldsymbol{x}_{i}}{\left\|\boldsymbol{x}_{j}-\boldsymbol{x}_{i}\right\|}
$$

El vector unitario $\boldsymbol{a}_{3}$ se escoge de forma que sea perpendicular a $\boldsymbol{a}_{1}$ y esté contenido en el plano horizontal, lo que conduce a la siguiente expresión en componentes del sistema general

$$
\boldsymbol{a}_{3} \equiv\left\{\begin{array}{lll}
a_{3}^{1} & 0 & a_{3}^{3}
\end{array}\right\}^{\top},
$$


con

$$
a_{3}^{1}=+\sqrt{\frac{1}{1+\left(a_{1}^{1}\right)^{2} /\left(a_{1}^{3}\right)^{2}}}
$$

y

$$
a_{3}^{3}=-a_{3}^{1} \frac{a_{1}^{1}}{a_{1}^{3}} .
$$

Estas expresiones presentan una singularidad cuando el elemento está orientado en la dirección $y\left(a_{1}^{3}=0\right)$, que se resuelve tomando

$$
\boldsymbol{a}_{3} \equiv\left\{\begin{array}{lll}
0 & 0 & 1
\end{array}\right\}^{\top} .
$$

Por último $\boldsymbol{a}_{2}=\boldsymbol{a}_{3} \times \boldsymbol{a}_{1}$, y la matriz de rotación inicial del elemento se obtiene escribiendo en columnas los vectores anteriores

$$
\boldsymbol{\Lambda}_{(0)}^{e} \equiv\left[\begin{array}{lll}
\boldsymbol{a}_{1} & \boldsymbol{a}_{2} & \boldsymbol{a}_{3}
\end{array}\right]
$$

4. Para el elemento de dos nodos (recto) $\boldsymbol{K}_{(0)}^{e} \equiv\left\{\begin{array}{lll}0 & 0 & 0\end{array}\right\}^{\top}$. Por otro lado $\boldsymbol{\Gamma}_{(0)}^{e} \equiv$ $\left\{\begin{array}{lll}1 & 0 & 0\end{array}\right\}^{\top}$ define la condición de sección inicialmente normal a la directriz.

\subsubsection{Predicción}

El proceso iterativo comienza a partir del resultado del incremento $n$. Empleando las variables asociadas a él (ver cuadro de variables iniciales en el apartado anterior) se lleva a cabo una predicción del estado en el incremento $n+1$, que denominaremos iteración 0. Las variables de partida se resumen en la siguiente tabla:

\begin{tabular}{|c|}
\hline Variables correspondientes al paso $n$ \\
$\downarrow$ \\
FEStep \\
$\downarrow$ \\
Resultado de la iteración 0 \\
\hline
\end{tabular}

Variables correspondientes al incremento $n$

\begin{tabular}{|l|cc|}
\hline Factor de carga & $\lambda_{(n)}$ & \\
Configuración nodal & $\boldsymbol{x}_{i(n)}$ & $\boldsymbol{\Lambda}_{i(n)}$ \\
Incrementos de la configuración & $\Delta \boldsymbol{x}_{i(n-1, n)}$ & $\Delta \boldsymbol{\omega}_{i(n-1, n)}$ \\
\hline \hline Rotación del elemento & $\boldsymbol{\Lambda}_{(n)}^{e}$ & \\
Deformaciones & $\boldsymbol{\Gamma}_{(n)}^{e}$ & $\boldsymbol{K}_{(n)}^{e}$ \\
Esfuerzos & $\boldsymbol{N}_{(n)}^{e)}$ & $\boldsymbol{M}_{(n)}^{e}$ \\
\hline \hline Fuerzas nodales espaciales & $\boldsymbol{n}_{i(n)}^{s}$ & $\boldsymbol{m}_{i(n)}^{s}$ \\
Fuerzas nodales perseguidoras & $\boldsymbol{n}_{i(n)}^{f}$ & $\boldsymbol{m}_{i(n)}^{f}$ \\
\hline
\end{tabular}


Las fuerzas perseguidoras se actualizan al principio mediante las expresiones

$$
\begin{gathered}
\boldsymbol{n}_{i(n)}^{f}=\boldsymbol{\Lambda}_{i(n)} \boldsymbol{N}_{i} \\
\boldsymbol{m}_{i(n)}^{f}=\boldsymbol{\Lambda}_{i(n)} \boldsymbol{M}_{i} .
\end{gathered}
$$

El proceso está gobernado por el módulo FEStep cuya estructura, así como la de los módulos incluidos en él, analizamos a continuación. En la columna de la izquierda se hace referencia al módulo ejecutado o a las ecuaciones empleadas. La columna central refleja las variables calculadas, y a la derecha se incluyen aclaraciones. Prescindimos, por claridad del subíndice $(n)$ en las variables que resulten de la predicción.

\begin{tabular}{|c|c|c|}
\hline 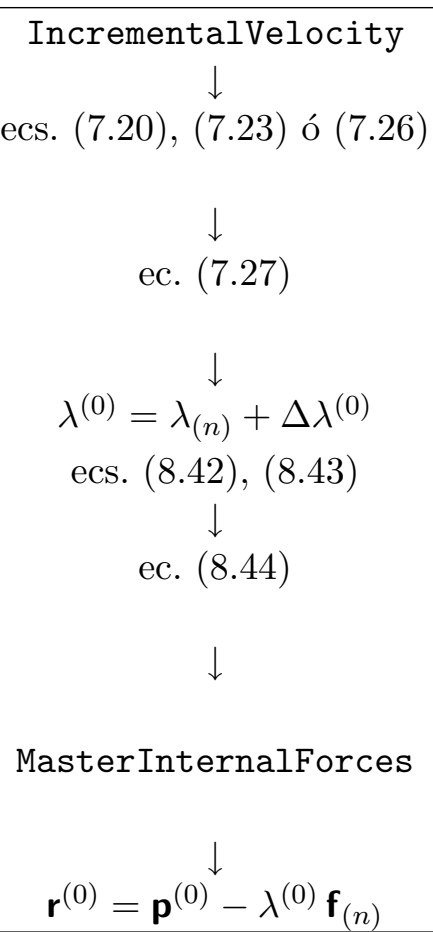 & $\begin{array}{c}\boldsymbol{x}_{i}^{(0)}{ }^{\lambda^{(0)}} \boldsymbol{\Lambda}_{i}^{(0)} \\
\boldsymbol{\Lambda}^{e(0)} \\
\boldsymbol{\Gamma}^{e(0)} \quad \boldsymbol{K}^{e(0)} \\
\boldsymbol{N}^{e(0)} \quad \boldsymbol{M}^{e(0)} \\
\mathbf{p}^{(0)} \\
\mathbf{r}^{(0)}\end{array}$ & $\begin{array}{l}\text { incremento del factor de carga en } \\
\text { función del tipo de control } \\
\text { incrementos de las variables de la } \\
\text { configuración } \\
\text { actualización del factor de carga } \\
\text { actualización de la configuración } \\
\text { actualización de la rotación del } \\
\text { elemento } \\
\text { actualización de deformaciones } \\
\text { actualización de esfuerzos } \\
\text { vector de fuerzas internas } \\
\text { vector residuo }\end{array}$ \\
\hline
\end{tabular}

\section{FEStep}


IncrementalVelocity

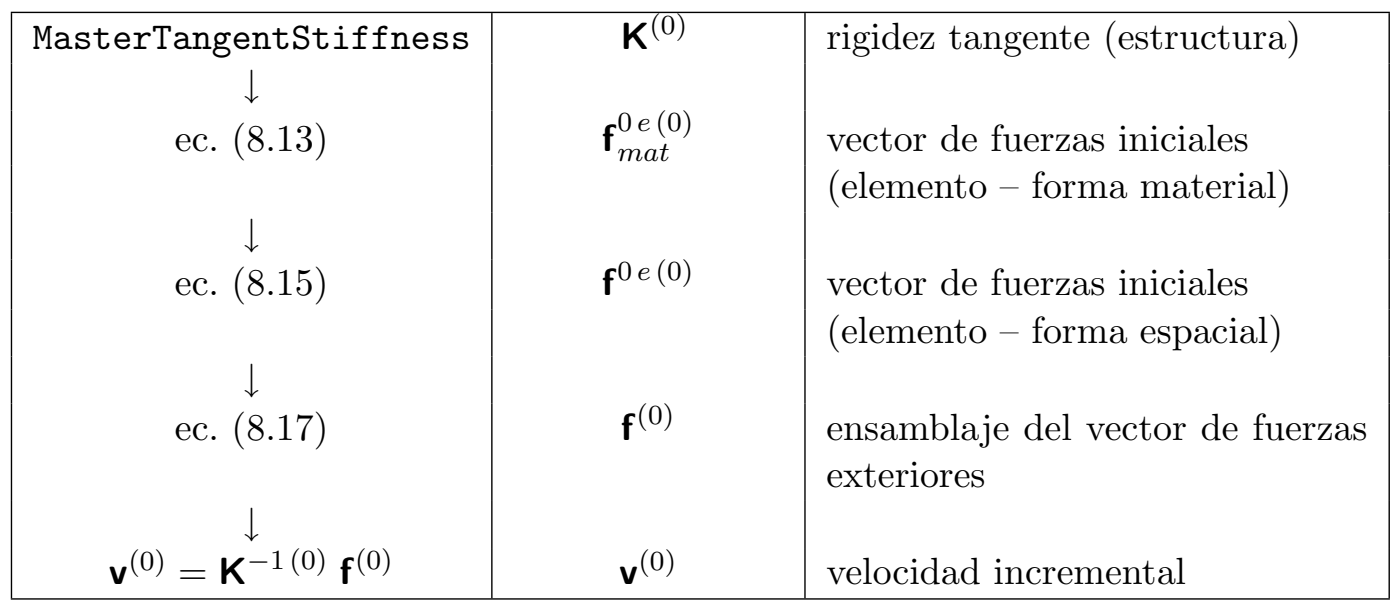

MasterTangentStiffness

\begin{tabular}{|c|l|l|}
\hline ecs. (8.3a) $-(8.3 \mathrm{f})$ & $\mathbf{K}_{\text {mat }}^{e(0)}$ & $\begin{array}{l}\text { rigidez tangente } \\
\text { (elemento }- \text { forma material })\end{array}$ \\
$\begin{array}{c}\downarrow \\
\text { ec. }(8.9)\end{array}$ & $\mathbf{K}^{e(0)}$ & $\begin{array}{l}\text { rigidez tangente } \\
(\text { elemento }- \text { forma espacial }) \\
\downarrow\end{array}$ \\
ec. (8.11) & $\mathbf{K}^{(0)}$ & $\begin{array}{l}\text { ensamblaje de la rigidez tangente } \\
\text { de la estructura }\end{array}$ \\
\hline
\end{tabular}

MasterInternalForces

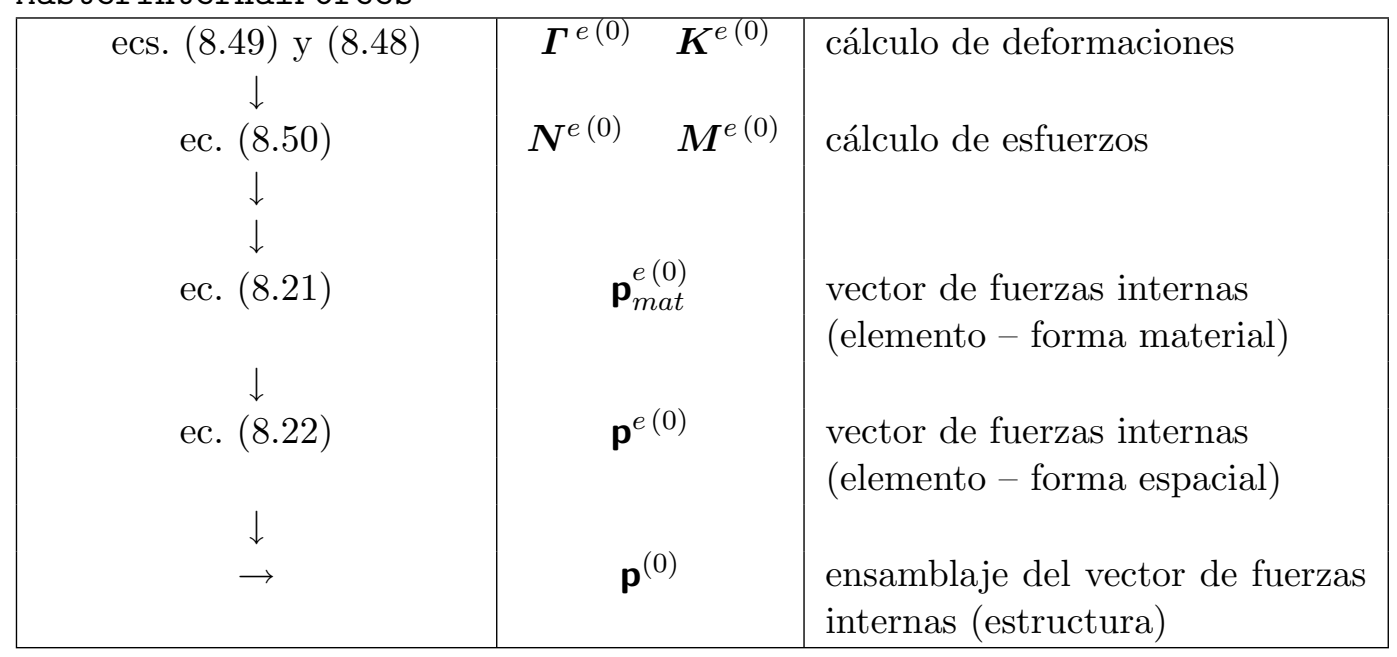




\subsubsection{Corrección}

El proceso iterativo comienza a partir del resultado del incremento $n$. Empleando las variables asociadas a él (ver cuadro de variables iniciales en el apartado anterior) se lleva a cabo una predicción del estado en el incremento $n+1$, que denominaremos iteración 0 .

\section{Resultado de la iteración 0}

\begin{tabular}{|l|cc|}
\hline Factor de carga & $\lambda^{(0)}$ & \\
Configuración nodal & $\boldsymbol{x}_{i}^{(0)}$ & $\boldsymbol{\Lambda}_{i}^{(0)}$ \\
\hline \hline Rotación del elemento & $\boldsymbol{\Lambda}^{e(0)}$ & \\
Deformaciones & $\boldsymbol{\Gamma}^{(0)}$ & $\boldsymbol{K}^{(0)}$ \\
Esfuerzos & $\boldsymbol{N}^{e(0)}$ & $\boldsymbol{M}^{e(0)}$ \\
\hline \hline Velocidad incremental & $\mathbf{v}^{(0)}$ & \\
Vector residuo & $\mathbf{r}^{(0)}$ & \\
\hline
\end{tabular}

El proceso de convergencia, que finaliza con la asignación de las variables correspondientes al incremento $n+1$ está controlado por el siguiente diagrama de flujo:

Resultado de la iteración 0
Resultado de la iteración $k$
$\downarrow$
NewtonCycle
$\downarrow$
Resultado de la iteración $k+1$
$\downarrow$
si $\left\|\mathbf{r}^{(k+1)}\right\| /\left\|\mathbf{r}^{(0)}\right\|<\epsilon_{1}$
o $\left\|\mathbf{r}^{(k+1)}\right\|<\epsilon_{2}$
$\downarrow$
Variables del incremento $n+1 \equiv$ Resultado de la iteración $k+1$

En él se observa que el paso de la iteración $k$ a la $k+1$ está gobernado por el módulo NewtonCycle cuyo funcionamiento se describe a continuación: 
NewtonCycle

\begin{tabular}{|c|c|c|}
\hline 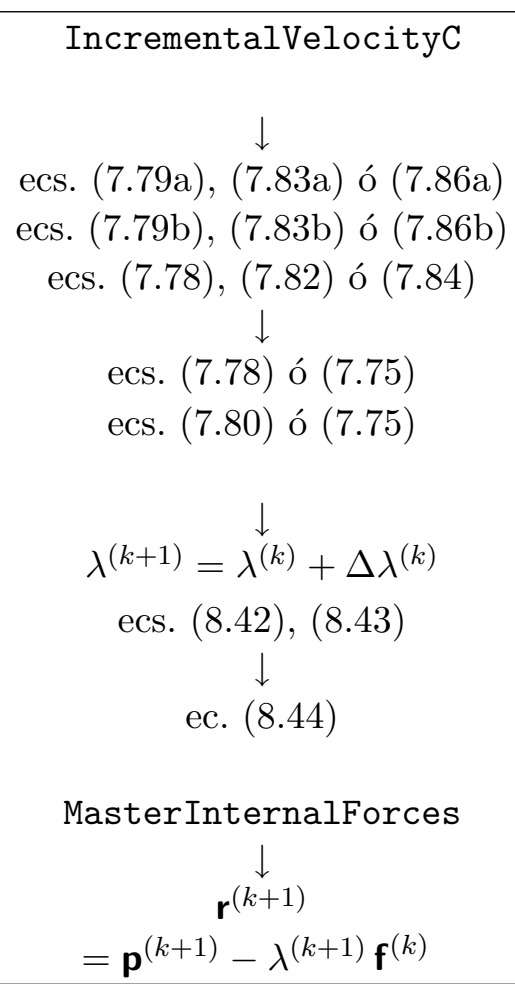 & $\begin{array}{c}\boldsymbol{x}_{i}^{\lambda^{(k+1)}} \boldsymbol{\Lambda}_{i}^{(k+1)} \\
\boldsymbol{\Lambda}^{e(k+1)} \\
\mathbf{p}^{(k+1)}\end{array}$ & $\begin{array}{l}\text { incremento del factor de carga } \\
\text { incrementos de las variables de la } \\
\text { configuración } \\
\text { actualización del factor de carga } \\
\text { actualización de la configuración } \\
\text { actualización de la rotación del } \\
\text { elemento } \\
\text { vector de fuerzas internas }\end{array}$ \\
\hline
\end{tabular}

IncrementalVelocityC

\begin{tabular}{|c|c|l|}
\hline $\begin{array}{c}\text { MasterTangentStiffnessC } \\
\downarrow\end{array}$ & $\widetilde{\mathbf{K}}^{(k)}$ & $\begin{array}{l}\text { rigidez tangente para la } \\
\text { corrección (estructura) }\end{array}$ \\
$\begin{array}{c}\downarrow \\
\text { ec. (8.13) }\end{array}$ & $\mathbf{f}_{\text {mat }}^{0 e(k)}$ & $\begin{array}{l}\text { vector de fuerzas iniciales } \\
(\text { elemento }- \text { forma material) }\end{array}$ \\
$\begin{array}{c}\downarrow \\
\text { ec. (8.15) }\end{array}$ & $\mathbf{f}^{0 e(k)}$ & $\begin{array}{l}\text { vector de fuerzas iniciales } \\
\text { (elemento }- \text { forma espacial) }\end{array}$ \\
$\downarrow$ & $\mathbf{f}^{(k)}$ & $\begin{array}{l}\text { ensamblaje del vector de fuerzas } \\
\text { exteriores }\end{array}$ \\
$\mathbf{v}^{(k)}=\mathbf{K}^{-1(k)} \mathbf{f}^{(k)}$ & $\mathbf{v}^{(k)}$ & velocidad incremental \\
\hline
\end{tabular}


MasterTangentStiffnessC

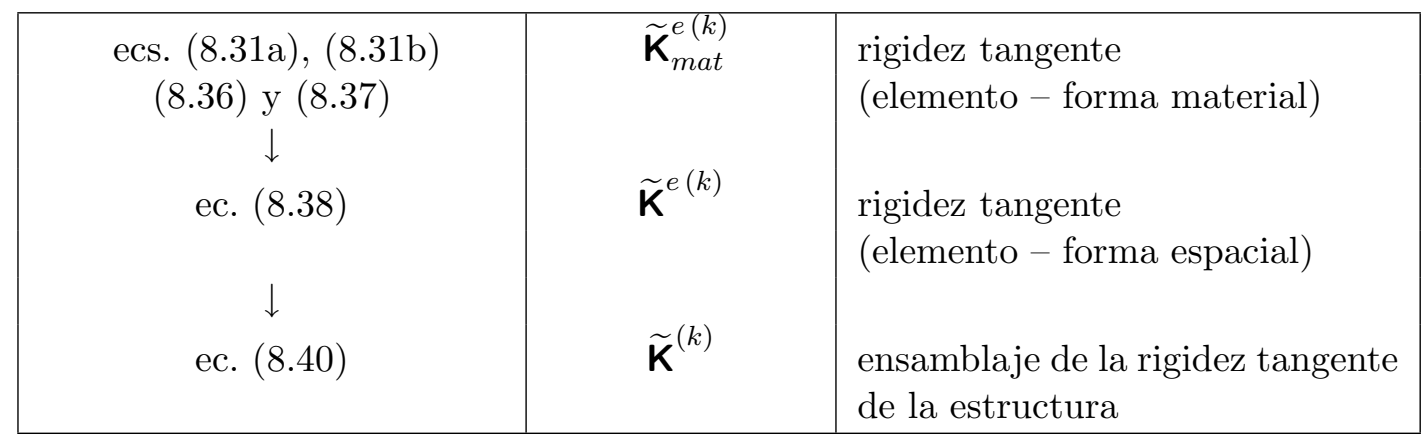




\subsection{Resultados}

\section{Estructura A}

Probamos en primer lugar el comportamiento del nuevo elemento en la solución de la ménsula de Simó y Vu-Quoc (estructura A) con el momento que la enrolla una vuelta. Recordamos que sus características son

\begin{tabular}{c|ccc} 
estructura & $L$ & $E A$ & $E I$ \\
\hline $\mathrm{A}$ & 1 & 2 & 2 \\
\hline
\end{tabular}

Comparamos el elemento material con el espacial sobre la base de una solución incremental pura en 50 escalones de carga. En la figura 8.4 hemos reproducido sobre los ejes $x$ e $y$ la trayectoria de su extremo en ambos casos, así como la solución exacta cuya expresión analítica es (7.88).

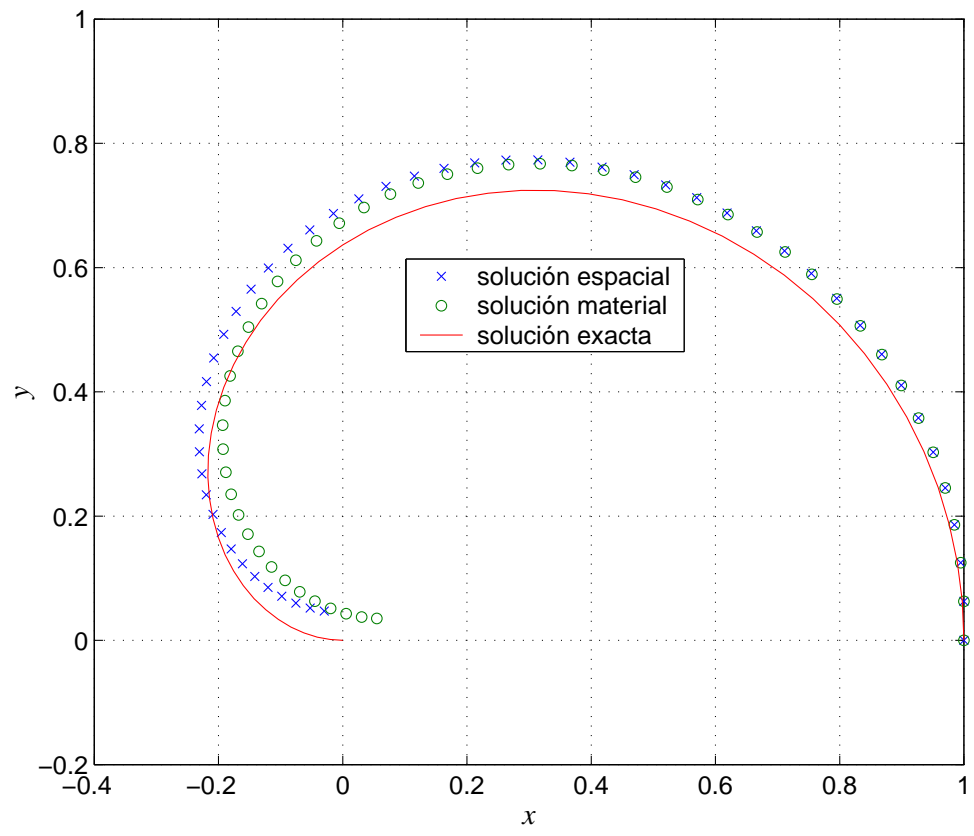

Figura 8.4: Trayectoria del extremo de la estructura A $(E / G=2,5)$. Solución incremental pura en 50 incrementos. Comparación de la forma espacial y material

El comportamiento parece análogo en las dos soluciones, y para obtener una información más precisa de su calidad hemos obtenido una medida del error en cada uno de los pasos del cálculo incremental. Éste se define simplemente como la distancia entre la posición exacta del extremo, y la calculada numéricamente, normalizada dividiendo por una longitud de referencia (que en este caso es la longitud de la ménsula, igual a la unidad). El resultado se presenta en la figura 8.5. 


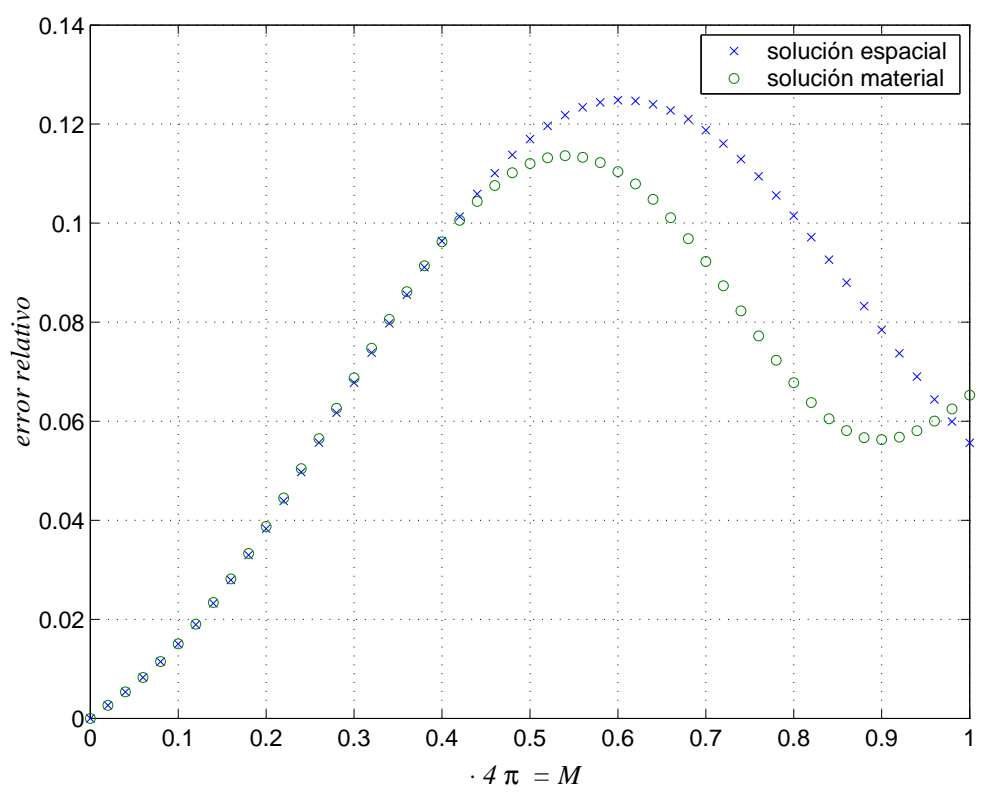

Figura 8.5: Error relativo en la posición del extremo de la estructura A $(E / G=2,5)$. Solución incremental pura en 50 incrementos. Comparación de la forma espacial y material

Se puede observar que el error es prácticamente idéntico hasta un $40 \%$ del momento de enrrollamiento $4 \pi$. A partir de este valor el error de la solución material es menor que el de la solución espacial. La primera alcanza un error máximo de 0,115 cuando el momento es igual al $0,54 \cdot 4 \pi$. Entre este valor y $0,90 \cdot 4 \pi$ el error es decreciente; con valores mayores del momento vuelve a aumentar. El error de la solución espacial tiene un máximo de 0,125\% para un $60 \%$ del momento de referencia. Luego disminuye hasta el final. Con el momento $4 \pi$ el error de la solución espacial es 0,056, inferior a 0,065 correspondiente a la solución material.

En el siguiente experimento numérico estudiamos el comportamiento del proceso de solución incremental - iterativo. En este caso aplicamos al voladizo de Simó y $\mathrm{Vu}$-Quoc un momento $M=2 \pi$ en el extremo que produce una deformación que lo convierte en una semicircunferencia. Consideramos discretizaciones con un número creciente de elementos. En la figura 8.6 hemos representado el valor de la coordenada $y$ del extremo para cada discretización, frente al valor exacto, que es igual a 0,63662 . A partir de 4 elementos la distancia entre la solución numérica y la exacta es inferior a 0,02 y se reduce asintóticamente cuando la discretización es más fina.

Dado que en este problema no hay deformación de extensión ni de corte en las soluciones corregidas (como sucede en la solución exacta), su geometría es, en cada caso, una poligonal con un número de lados igual al de elementos; sus extremos se encuentran sobre el eje $y$, y la longitud de los lados es igual a la longitud inicial de los elementos (figura 8.7); por ello la exactitud de la aproximación mediante elementos finitos queda únicamente determinada por el número de elementos escogido para la 
discretización.

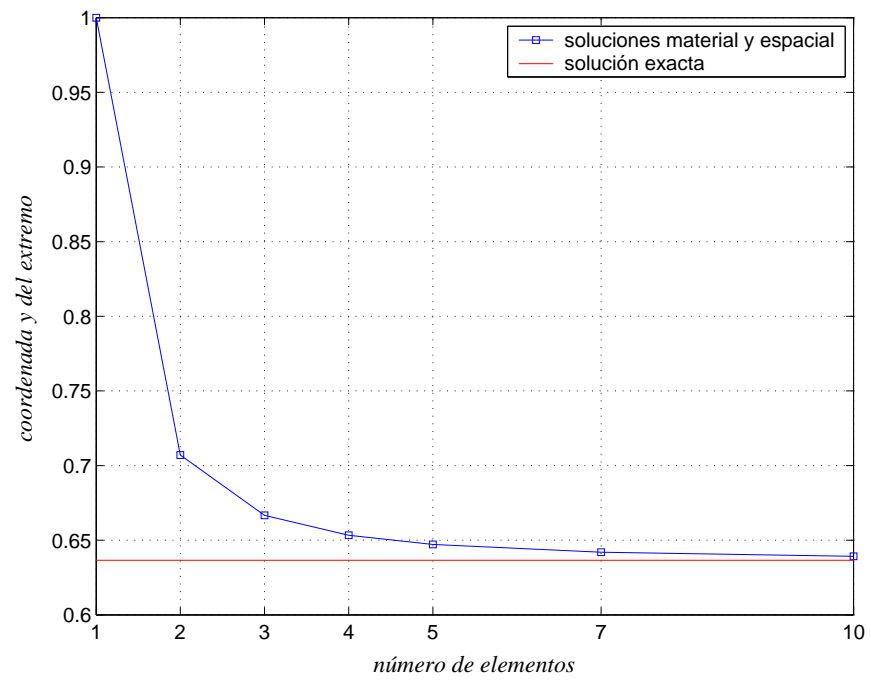

Figura 8.6: Estructura A $(E / G=2,5)$ con un momento $2 \pi$ aplicado en el extremo. Aproximación a la solución exacta en función del número de elementos

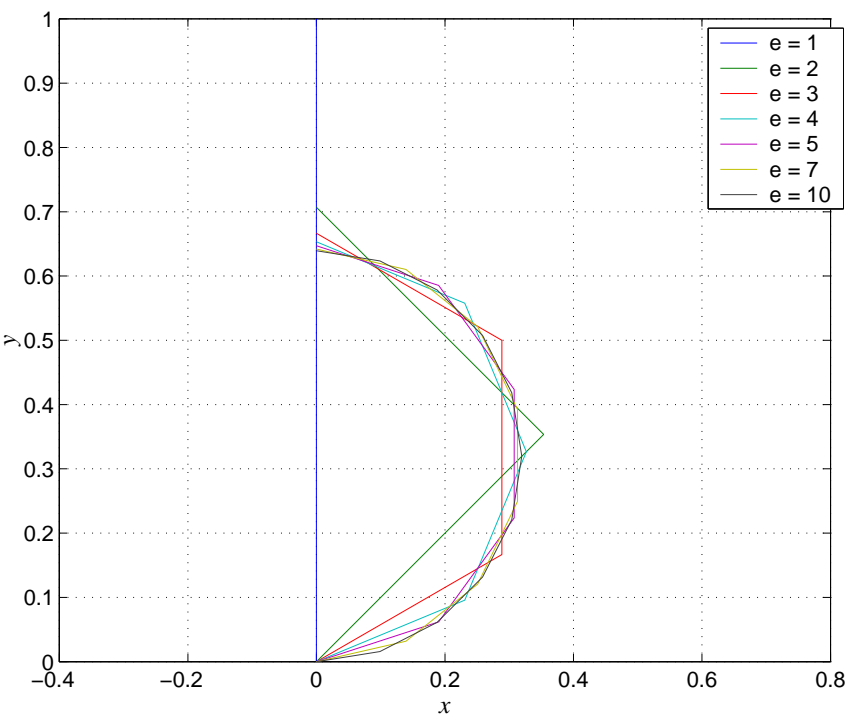

Figura 8.7: Estructura A $(E / G=2,5)$ con un momento $2 \pi$ aplicado en el extremo. Geometría de la solución en función del número de elementos 
El conjunto de soluciones obtenidas es idéntico tanto para el elemento basado en la forma material como en la espacial (las coordenadas y las rotaciones nodales son iguales); sin embargo, la velocidad de convergencia hacia la solución corregida es totalmente diferente. En la figura 8.8 se representa el número de iteraciones en función del número de elementos empleados en la discretización para los dos tipos de solución. La solución espacial proporciona una respuesta muy uniforme que tiende a estabilizarse en 5 iteraciones (la discretización con 2 elementos sólo requiere 3). Por otra parte, la solución espacial muestra un comportamiento asintótico que comienza con 72 iteraciones para la discretización con 1 elemento y termina con 7 iteraciones necesarias en el modelo con 10 elementos, y cierta tendencia a seguir disminuyendo.

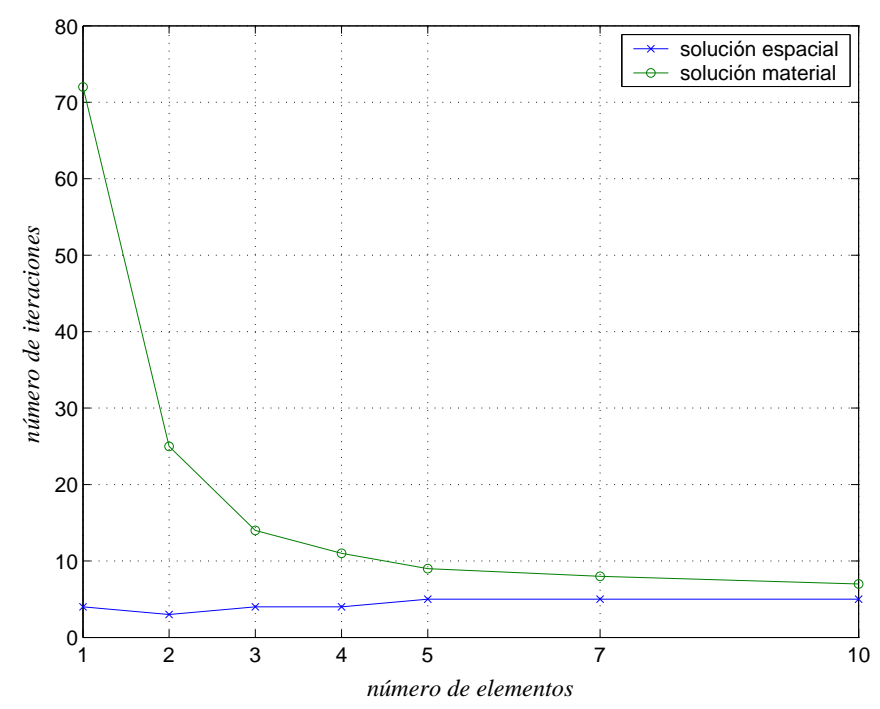

Figura 8.8: Estructura A $(E / G=2,5)$ con un momento $2 \pi$ aplicado en el extremo. Número de iteraciones para la convergencia en función del número de elementos

De este resultado es posible avanzar que, para una misma discretización, el elemento basado en la forma material no podrá competir contra el elemento espacial, como consecuencia de su baja velocidad de convergencia. Será necesario emplear discretizaciones considerablemente más finas para obtener resultados equiparables.

\section{Estructura C2}

La estructura C2, cuyas características recordamos a continuación, es una ménsula

\begin{tabular}{c|cccc} 
estructura & $L$ & $E A$ & $E I$ & $E / G$ \\
\hline $\mathrm{C} 2$ & 0,1 & 2000 & 0,002 & 2,5 \\
\hline
\end{tabular}

esbelta $(\eta=25)$ con una longitud de $0,1 \mathrm{~m}$, una sección transversal circular de $0,002 \mathrm{~m}$ de radio, formada por un material con un módulo de elasticidad $E=1 / 2 \pi$. 
$10^{9} \mathrm{kN} / \mathrm{m}^{2}$. En este caso, para enrollarla media vuelta es necesario aplicar un par $M=2 \pi 10^{-2} \mathrm{~m} \mathrm{kN}$.

La solución incremental - iterativa basada en la forma espacial se obtiene con 4 escalones de carga y 9 iteraciones por escalón. La coordenada y del extremo es $y=0,064721 \mathrm{~m}$ (que es el valor escalado correspondiente a la solución con 5 elementos de la estructura A. Sin embargo la solución de ese problema con el elemento basado en la forma material es muy problemática. Con la misma discretización no se logra la convergencia con 4 escalones de carga. Aumentando el número de escalones se observa que al principio del proceso, en los primeros escalones, la velocidad de convergencia es buena, pero se deteriora rápidamente: con 100 escalones de carga se requiere más de 75 iteraciones hacia el final del proceso.

Refinando la discretización se logra mejorar el comportamiento de la solución material. Con 10 elementos y 50 pasos el número de iteraciones por paso crece desde 3 hasta 26 en el último, y el movimiento final del extremo es el esperado $y=0,063925$. Finalmente hemos probado una discretización con 20 elementos y también 50 escalones de carga, para la que el número de iteraciones aumenta desde 3 hasta 7 , que ya es un número aceptable. Los resultados se muestran en la figura 8.9. El aumento del número de iteraciones crece asintóticamente en el entorno del paso número 30.

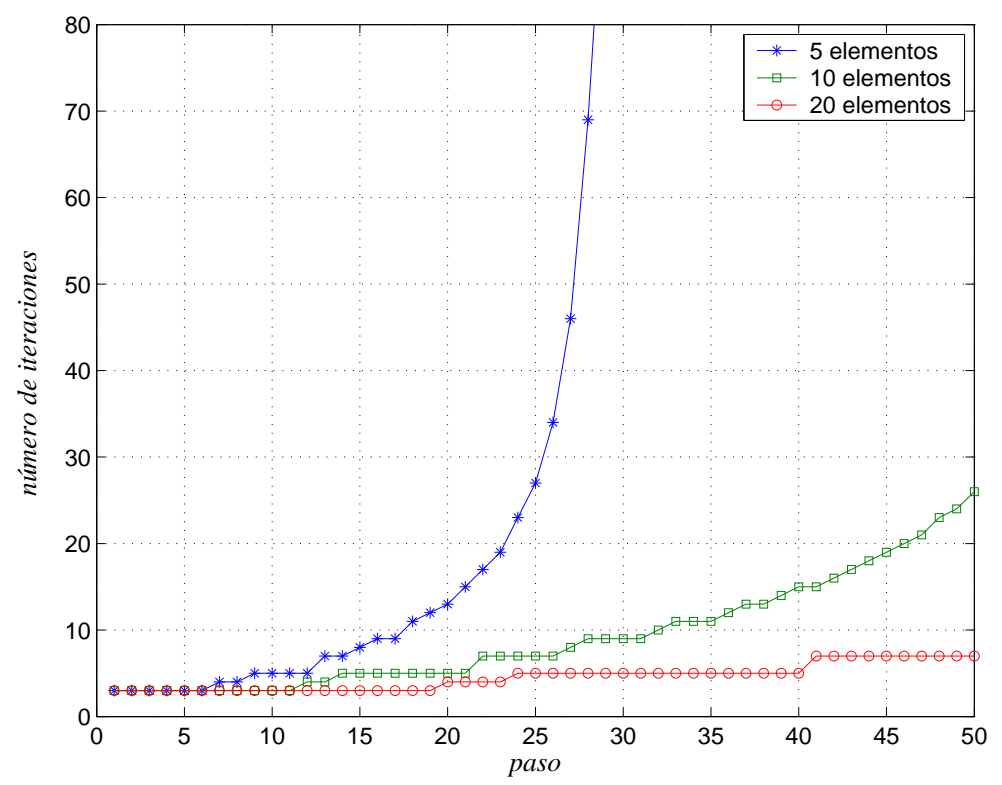

Figura 8.9: Estructura C2 con $M=2 \pi 10^{-2}$. Solución material. Número de iteraciones por paso necesarias para la convergencia

Incluso con discretizaciones mucho más finas, la respuesta del elemento material es menos efectiva que la del espacial debido a su baja velocidad de convergencia. Los resultados numéricos para los desplazamientos y esfuerzos son, sin embargo, idénticos en ambos casos una vez alcanzada la convergencia. 


\section{Ménsula curva}

Volvemos a continuación a la estructura 2 que se empleó en la sección 7.5.3. De nuevo se trata de una estructura esbelta que someteremos a la acción de una fuerza en el extremo libre para analizar el funcionamiento del elemento en un problema con deformaciones de corte y extensión. La longitud de la directriz es de $100 \mathrm{~mm}$, la sección transversal es circular de $2 \mathrm{~mm}$ de radio y el módulo de elasticidad es $1 / 2 \pi$ MN.

Estudiamos la respuesta numérica en dos situaciones :(a) cuando se aplica una fuerza de $0,5 \mathrm{kN}$ de eje fijo, orientada en la dirección del eje $z, \mathrm{y}(b)$ al aplicar una fuerza perseguidora de la misma magnitud orientada en la dirección del eje 3 de la sección transversal (inicialmente paralelo al eje $z$ ). La posición del extremo final obtenida mediante un proceso incremental - iterativo en 20 escalones de carga se recogen en las tablas 8.1 y 8.2 , En ellas se observa que los resultados obtenidos con los elementos espacial y material son prácticamente idénticos (iguales en el caso de la fuerza de eje fijo). La velocidad de convergencia del elemento material es, como cabía esperar, algo menor: necesita entre 3 y 6 iteraciones por paso en el problema con la fuerza fija, y entre 3 y 9 iteraciones en el de la fuerza perseguidora, frente a las 2 o 3 iteraciones requeridas por la solución espacial para ambos tipos de solicitación.

En la figura 8.12 hemos representado la distancia entre las posiciones nodales obtenidas con cada elemento, para mostrar que los resultados obtenidos son prácticamente idénticos. Con ello podemos validar el funcionamiento del elemento basado en la forma material también para solicitaciones que producen extensión y corte.

\begin{tabular}{c|ccc} 
& $x$ & $y$ & $z$ \\
\hline & & & \\
solución espacial & 24,877 & 63,218 & 32,435 \\
solución material & 24,877 & 63,218 & 32,435 \\
\hline
\end{tabular}

Cuadro 8.1: Ménsula curva sometida a una fuerza de eje fijo $F_{z}=0,5 \cdot 10^{-3} \mathrm{MN}$. Posición final del extremo

\begin{tabular}{l|ccc} 
& $x$ & $y$ & $z$ \\
\hline & & & \\
solución espacial & 21,219 & 61,955 & 36,293 \\
solución material & 21,218 & 61,955 & 36,294 \\
\hline
\end{tabular}

Cuadro 8.2: Ménsula curva sometida a una fuerza perseguidora $F_{3}=0,5 \cdot 10^{-3} \mathrm{MN}$. Posición final del extremo

Finalmente hemos comparado los esfuerzos obtenidos con el elemento espacial y material, comprobando las dos soluciones proporcionan los mismos resultados. En las figuras 8.10 y 8.11 representamos los esfuerzos en cada uno de los elementos de la estructura obtenidos para las dos acciones consideradas (espacial y perseguidora) al final del proceso incremental - iterativo. 

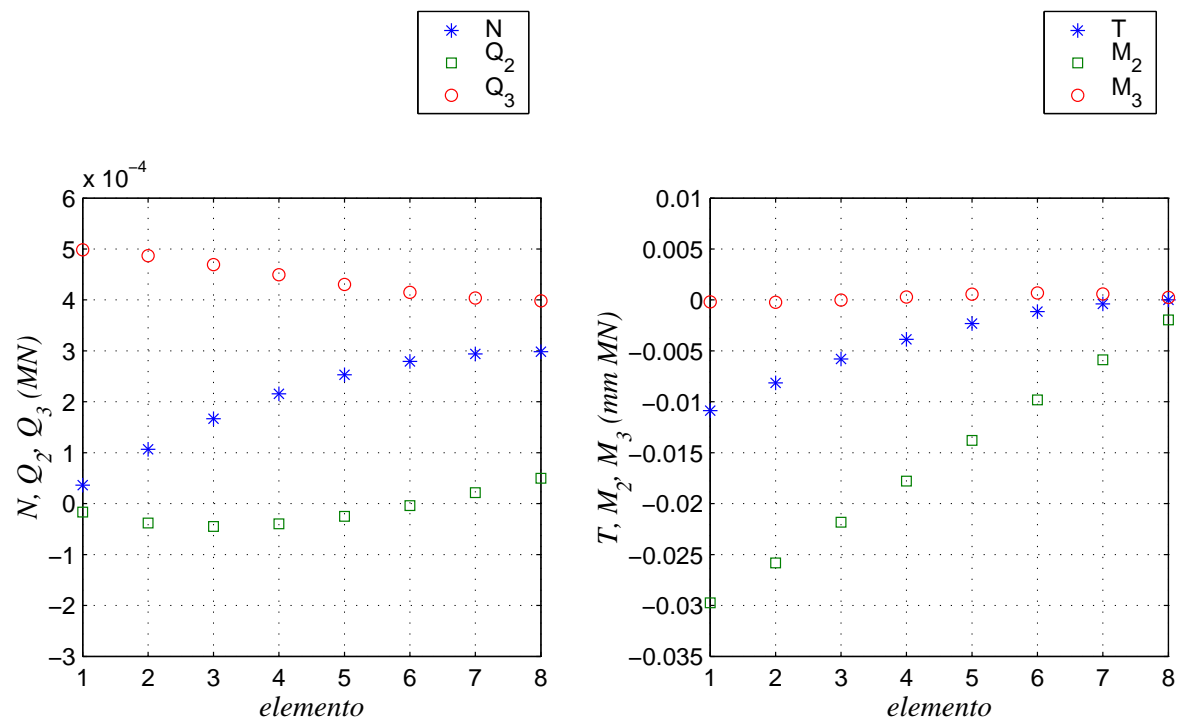

Figura 8.10: Esfuerzos en la ménsula curva sometida a una fuerza de eje fijo
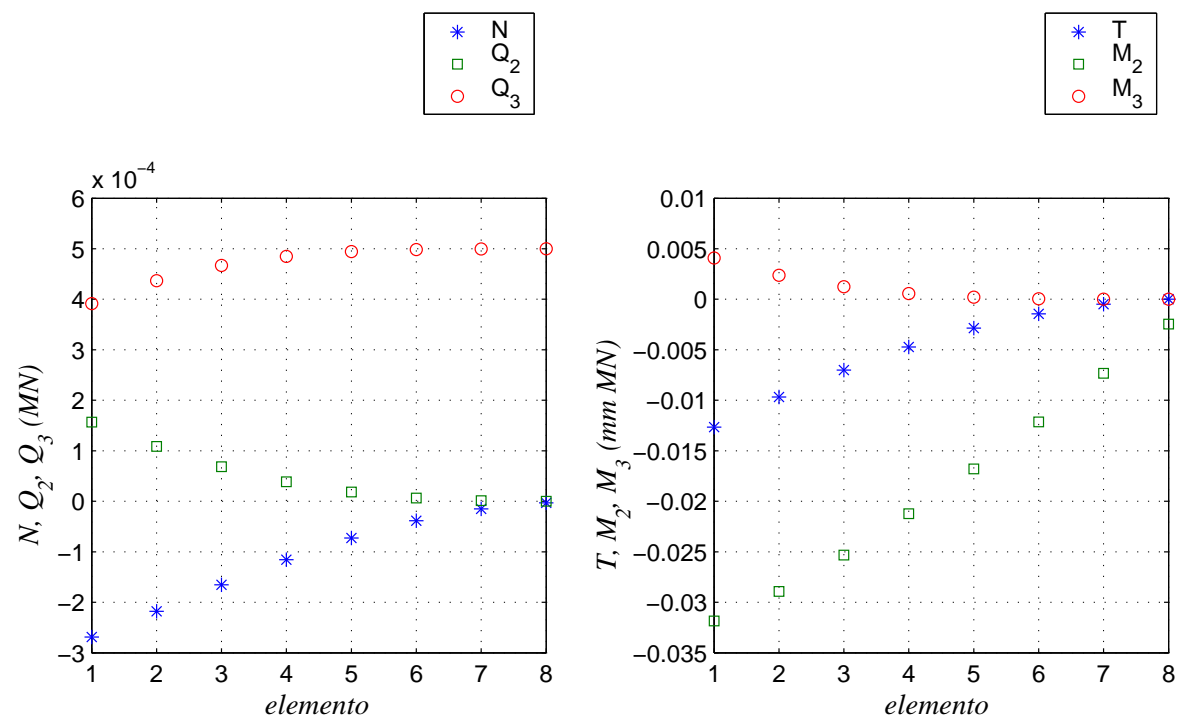

Figura 8.11: Esfuerzos en la ménsula curva sometida a una fuerza perseguidora 


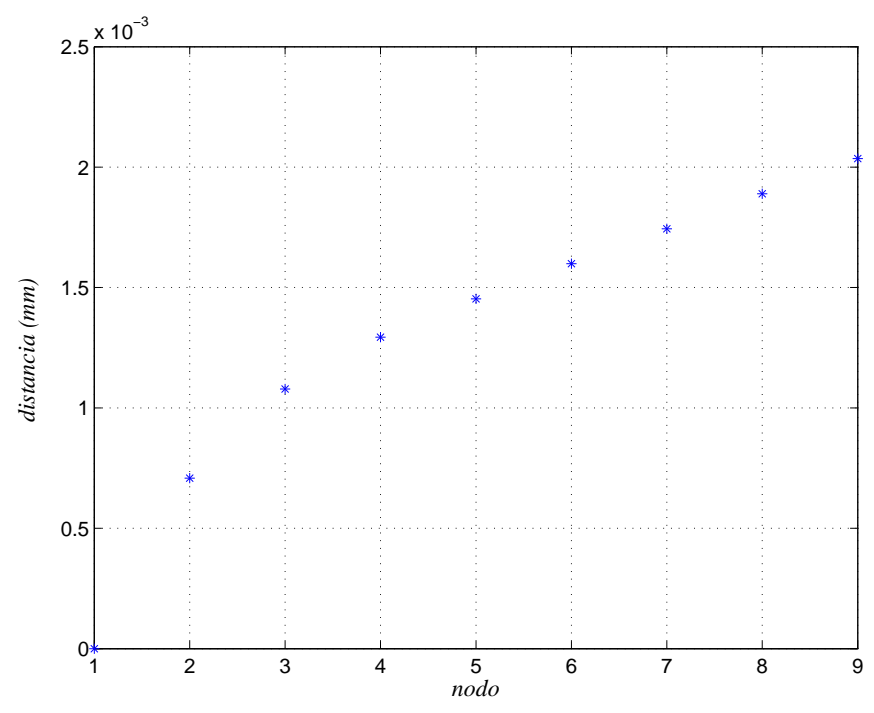

Figura 8.12: Distancia entre las posiciones nodales obtenidas con las soluciones espacial y material en el problema de la ménsula curva sometida a una fuerza perseguidora en el extremo

\section{Consideraciones finales}

En esta sección hemos llevado a cabo algunos experimentos numéricos para valorar el comportamiento del elemento finito basado en la forma material de las ecuaciones. Las expresiones desarrolladas en la primera parte del capítulo muestran que la transformación de la matriz del elemento de dos nodos de su forma material a la espacial requiere el cálculo de un cambio de ejes de cada uno de los dieciséis bloques que la componen ${ }^{1}$. Esta operación tiene un coste computacional más elevado que en el caso del elemento espacial, ya que en este último únicamente se necesita transformar los cuatro bloques diagonales de la matriz constitutiva (sólo tres si es simétrica, pues el cuarto se obtiene trasponiendo). Como contrapartida, los esfuerzos y las deformaciones generalizadas que intervienen en el cálculo son los correspondientes a la forma material, y se pueden interpretar directamente sin necesidad de cambiar el sistema de referencia.

En cuanto a la respuesta numérica, ya hemos indicado que aunque los resultados obtenidos al final del proceso de corrección son los mismos que se obtiene con el elemento basado en la forma espacial, la velocidad de convergencia es mucho menor. En procesos incrementales - iterativos, el número de iteraciones hasta la convergencia, que en los primeros pasos puede ser pequeño, crece rápidamente a medida que aumenta el número de incrementos. En general, en comparación con el elemento espacial es necesario emplear discretizaciones más finas y un número mayor de escalones de carga.

\footnotetext{
${ }^{1}$ Cuatro bloques de filas y columnas, correspondientes a los vectores de desplazamientos incrementales y rotaciones incrementales de cada nodo.
} 



\section{Capítulo 9}

\section{Conclusiones}

\subsection{Resumen del trabajo realizado}

Esta tesis doctoral tiene por objeto analizar el fundamento y explorar las posibilidades del modelo geométricamente exacto de piezas alargadas hiperelásticas en el marco de los problemas de equilibrio estático. El modelo admite grandes desplazamientos de la directriz, considera rotaciones arbitrariamente grandes de la sección transversal, y permite deformaciones de extensión y corte sin restringir su magnitud.

En la primera parte del trabajo se ha desarrollado la cinemática del modelo, obteniendo la expresión de las deformaciones generalizadas; también se ha establecido la relación entre variables materiales y espaciales a través de la transformación definida por la rotación de cada sección. A continuación se ha formulado la ecuación de trabajos virtuales del modelo a partir de la ecuación correspondiente al sólido tridimensional -lo que ha permitido introducir los esfuerzos como variables estáticas- y se han deducido las ecuaciones de campo -en su versión material y espacial-. Finalmente se ha establecido la equivalencia entre la definición estática y la definición constitutiva de los esfuerzos. El análisis del problema desde el punto de vista variacional ha puesto de manifiesto las conexiones formales entre las ecuaciones de la estática de las piezas alargadas y las ecuaciones de la dinámica de sólidos rígidos, y ha conducido a actualizar y generalizar la analogía cinética de Kirchhoff.

Como paso previo al desarrollo de soluciones numéricas se ha deducido la expresión espacial del operador tangente a partir de la linealización consistente de la ecuación de trabajos virtuales. Cuando este proceso se lleva a cabo antes de la discretización da lugar a la aparición de términos adicionales en el operador que no habían sido considerados hasta el momento. También se ha obtenido la forma material del operador y se ha demostrado su relación con la forma espacial.

La parte final de la tesis se centra en la solución numérica del modelo. En una primera etapa se ha desarrollado un elemento finito basado en la forma espacial del operador, que es esencialmente el propuesto por Simó y Vu-Quoc con algunas modificaciones introducidas por Ibrahimbegović y Taylor. El análisis de diversos ejemplos muestra la potencia del modelo, pero también pone de manifiesto algunos problemas numéricos. Por último se ha desarrollado un nuevo elemento finito empleando el ope- 
rador tangente en forma material, que incorpora la interpolación esférica propuesta por Crisfield y Jelenić.

\subsection{Conclusiones}

En relación con la formulación teórica del modelo:

1. Existe una relación explícita, determinada por la hipótesis cinemática, entre las deformaciones generalizadas del modelo de Reissner-Simó y los vectores que componen el gradiente de la deformación del sólido tridimensional. Del mismo modo, la introducción de la hipótesis cinemática permite justificar la definición de las variaciones de la configuración $\delta \boldsymbol{x}, \delta \boldsymbol{\omega}$. La última es el spin o rotación virtual y tiene un papel relevante en la solución numérica.

2. La ecuación de trabajos virtuales del modelo se puede deducir de la ecuación de trabajos virtuales correspondiente al sólido a partir de las relaciones anteriores, e introduciendo unas variables estáticas -los esfuerzos- definidas como resultantes (integrales) de los vectores tensión sobre las secciones transversales. La estructura del integrando de estas resultantes vuelve a estar determinada por la hipótesis cinemática. Con ello se asegura una definición consistente de los esfuerzos.

3. Para deducir las ecuaciones de equilibrio del modelo integrando por partes la ecuación de trabajos virtuales es necesario obtener las expresiones de la variación corrotacional de las deformaciones generalizadas en función de las variaciones de la configuración. Las primeras se pueden deducir a partir de la definición de variación corrotacional.

4. Admitiendo la existencia de una función de densidad de la energía de deformación en el sólido se demuestra que la densidad de energía de deformación del modelo admite una expresión invariante en función de las deformaciones generalizadas en forma material. Partiendo de esta expresión se puede demostrar que la definición estática de los esfuerzos (introducida al formular el principio de trabajos virtuales) coincide con su definición energética como derivadas de la energía de deformación respecto de las deformaciones generalizadas. Esta conclusión es fundamental para el desarrollo de la analogía dinámica.

\section{Relativas a la analogía dinámica:}

1. La analogía cinética de Kirchhoff - entre el problema estático de flexión (sin extension ni corte) de una pieza alargada, y el problema del movimiento de una peonza de Lagrange- se puede generalizar en el marco de la teoría de Reissner-Simó, estableciéndola entre el problema general de deformación de la pieza alargada y una categoría de problemas dinámicos gobernados por las ecuaciones denominadas de Euler-Poincaré. 
2. Como consecuencia de la analogía es posible concluir que en un problema homogéneo, es decir, en una pieza hiperelástica sometida únicamente a fuerzas en equilibrio en sus extremos, existen tres funciones de los esfuerzos que son invariantes a lo largo de la directriz:

$$
\begin{aligned}
\mathcal{H} & =\frac{1}{2}\left(\frac{N^{2}}{E A}+\frac{\left(Q_{2}\right)^{2}}{G A_{Q 2}}+\frac{\left(Q_{3}\right)^{2}}{G A_{Q 3}}+\frac{\left(M_{1}\right)^{2}}{G J}+\frac{\left(M_{2}\right)^{2}}{E I_{2}}+\frac{\left(M_{3}\right)^{2}}{E I_{3}}\right)+N \\
C_{(1)} & =N^{2}+\left(Q_{2}\right)^{2}+\left(Q_{3}\right)^{2} \\
C_{(2)} & =N M_{1}+Q_{2} M_{2}+Q_{3} M_{3} .
\end{aligned}
$$

$\mathrm{Su}$ interpretación física es la siguiente: la primera es la densidad de energía complementaria más el esfuerzo axil en cada sección transversal, la segunda es equivalente a la ecuación de equilibrio de fuerzas de la parte de la pieza situada entre la sección transversal y uno de los extremos, y la tercera equivale a la ecuación de equilibrio de momentos.

\section{Respecto a la estructura del operador tangente:}

1. Cuando se lleva a cabo la linealización de la ecuación de trabajos virtuales previamente a la discretización (es decir, cuando se calcula su segunda variación) es necesario tener en cuenta que la segunda variación afecta también a las variaciones de la configuración. Si el espacio de las configuraciones es lineal la segunda variación de la configuración es nula, sin embargo, en nuestro caso el carácter no lineal del grupo de las rotaciones implica la no nulidad de dos de ellas. Al introducir las expresiones de la segunda variación en el cálculo aparecen nuevos términos en el operador tangente, adicionales a los obtenidos por Simó y Vu-Quoc. El integrando o núcleo de estos términos está compuesto por matrices antisimétricas, y se puede demostrar, integrando por partes, que los nuevos términos se anulan en una configuración de equilibrio. La demostración ha permitido contemplar los razonamientos de Simó relativos a la simetría del operador desde otro prisma. En nuestra opinión no es correcta la afirmación de Simó que indica que el operador tangente es simétrico cuando las fuerzas exteriores son conservativas, ya que el problema de la ménsula con un momento conservativo (dependiente de la rotación) aplicado en el extremo produciría un operador no simétrico.

2. La formulación de la solución numérica iterativa del modelo geométricamente exacto se basa en la linealización del residuo. Como éste se define después de la discretización, en el resultado de la linealización ya no aparecen las segundas variaciones de la configuración. Por tanto se puede concluir que en una solución numérica incremental-iterativa los términos adicionales del operador tangente no tienen ninguna influencia: en el paso de predicción se parte de la trayectoria de equilibrio y los términos se anulan, y durante la corrección no forman parte de la matriz tangente. Por eso podemos asegurar que los nuevos términos del operador sólo serán relevantes en soluciones puramente incrementales. La influencia en la solución incremental se ha valorado mediante varios experimentos 
numéricos. Podemos afirmar que ésta es pequeña, y que la consideración de los nuevos términos en la solución numérica no aporta ventajas en comparación con el uso de la matriz tangente de Simó y Vu-Quoc.

3. La teoría se completa con las expresiones correspondientes al operador tangente en forma material deducidas a partir de la expresión material de la ecuación de trabajos virtuales, y cuya concordancia con la forma espacial a través de la rotación de las secciones es fácilmente demostrable.

En lo tocante a la solución numérica del modelo:

1. El elemento finito espacial propuesto por Simó para el análisis de piezas alargadas posee un fundamento físico riguroso proporcionado por la teoría de piezas alargadas de Reissner-Simó. El empleo de las rotaciones incrementales spins como variables incrementales $(a)$ es la elección natural consistente con la teoría, (b) independiza la formulación de la parametrización escogida para la representación de las rotaciones, y $(c)$ conduce a expresiones sencillas de la rigidez tangente, en comparación con las obtenidas en otras formulaciones que usan parámetros globales de la rotación como variables incrementales. La actualización de las rotaciones es multiplicativa: la rotación correspondiente al paso o incremento anterior se multiplica por la izquierda por la exponencial del spin.

2. Los modelos numéricos basados en el elemento de Simó proporcionan resultados muy precisos incluso cuando se emplea un número reducido de elementos, y la interpolación es lineal. La velocidad de convergencia es muy elevada; en muchos de los casos analizados ha mostrado ser superior a la cuadrática. El elemento permite simular procesos de deformación altamente no lineales atravesando todo tipo de singularidades en la trayectoria de equilibrio (puntos críticos y puntos de retorno). Sin embargo también presenta algunas particularidades que deben ser mencionadas: el funcionamiento del elemento empeora a medida que aumenta la esbeltez de la pieza alargada. Esto es consecuencia del mal condicionamiento de la rigidez tangente, ya que el orden de magnitud las rigideces a extensión y corte es diferente del de las debidas a flexión y torsión.

3. El problema del mal condicionamiento en piezas esbeltas se pone especialmente de manifiesto en análisis puramente incrementales. Los experimentos numéricos realizados en modelos sencillos con pocos elementos han proporcionado resultados completamente alejados de los obtenidos con análisis iterativos. Incluso empleando un número muy elevado de incrementos se sigue obteniendo precisiones muy bajas en los resultados.

4. Es posible construir elementos basados en la forma material del operador tangente que guardan ciertas similitudes conceptuales con los elementos finitos corrotacionales. La idea fundamental se concreta en dos pasos $(a)$ la evaluación de la rigidez local de un elemento a partir de la forma material del operador tangente, y $(b)$ la transformación de la rigidez local(material) a una rigidez global (espacial) por medio de las rotaciones nodales del elemento. Una de las 
particularidades del elemento material es que la matriz tangente en la predicción es distinta de la necesaria para la corrección; la primera se obtiene a partir del operador tangente deducido antes de la discretización, y la segunda de la linealización del residuo después de la discretización. En el elemento material hemos optado por emplear la interpolación esférica propuesta por Crisfield y Jelenić que permite calcular la rotación en los puntos de integración, así como las deformaciones generalizadas, directamente a partir de las rotaciones nodales. Con ello se evita el uso de la fórmula de actualización de $\boldsymbol{\kappa}$ propuesta por Simó, que tiene un mayor coste computacional.

5. Los modelos basados en el nuevo elemento material proporcionan la misma precisión en los resultados que los construidos con elementos de Simó, pero su velocidad de convergencia es muy inferior, lo que obliga a emplear un número muy superior de elementos para reducir a límites razonables el número de iteraciones por paso. Otra desventaja del elemento material es su mayor coste computacional en comparación con el elemento espacial, ya que el número de rotaciones (cambios de base) necesarias previas al ensamblaje es mayor: en el elemento de dos nodos es necesario operar sobre los dieciséis bloques de la rigidez tangente (asimétrica), mientras en el elemento espacial basta con transformar los dos bloques diagonales y el bloque cruzado de la matriz constitutiva (asimétrica). A cambio, el modelo maneja esfuerzos materiales que tienen una interpretación física directa y no requieren ser transformados.

\subsection{Aportaciones originales}

Destacamos a continuación las aportaciones originales realizadas en la tesis.

Relativas al desarrollo del modelo geométricamente exacto:

1. El planteamiento global sistemático seguido en el desarrollo del modelo (capítulos 3 y 4) a partir de las expresiones correspondientes al sólido hiperelástico, obteniendo todas las ecuaciones en forma material y espacial, es una aportación nueva, aunque está basada en la idea original de Simó [58] y los desarrollos de Cardona y Geradin [11] que fundamentan el paso del 3D al modelo en una descomposición apropiada del gradiente de la deformación $\mathbf{F}^{*}$. La mayor parte de las referencias obtienen la ecuación de trabajos virtuales como forma débil las ecuaciones de equilibrio [52], 62], [27], [16]. Únicamente Cardona y Geradin proponen su construcción desde la ecuación correspondiente al sólido.

2. La expresión de la forma material de la variación de las deformaciones generalizadas (4.43) (necesaria para obtener las ecuaciones de equilibrio a partir de la expresión de los trabajos virtuales), y el procedimiento para su cálculo (proposición 4.2.3) aún no han sido publicados.

3. También es nueva la demostración de la dependencia de la densidad de energía de deformación del modelo únicamente de las deformaciones generalizadas mate- 
riales (proposición 4.4.1), así como de su objetividad (proposición 4.4.2), cuando se admite que el material es hiperelástico.

4. Por último señalaremos la originalidad de la demostración de la equivalencia entre la definición estática y la definición constitutiva de los esfuerzos del modelo (proposición 4.4 .3 y desarrollo previo).

En relación con el estudio de la analogía:

1. Es original la generalización de la analogía cinética de Kirchhoff, que se hace patente al demostrar que las ecuaciones tienen la estructura de Euler-Poincaré cuando se expresan en función de las deformaciones generalizadas (proposición 5.1.1), y de Lie-Poisson cuando se expresan en función de los esfuerzos (proposición 5.2.1).

2. La identificación de los tres invariantes del problema homogéneo de la pieza alargada -la función de Hamilton (5.38), y las funciones de Casimir (5.46) - es novedosa (aunque los dos últimos ya han sido referidos por [44] su deducción a partir de las funciones de Casimir es original)

3. También es original la definición del corchete de la pieza alargada o corchete de Reissner-Simó (5.41) como caso particular del corchete de Lie-Poisson. Esta definición ha permitido escribir las ecuaciones del modelo en forma compacta empleando el formalismo de Hamilton (5.42).

En cuanto al cálculo del operador tangente:

1. Es novedoso el cálculo y las expresiones de la segunda variación de las variables de la configuración en la forma material (proposición 6.1.2) y espacial (proposición 6.1.3).

2. La demostración de que la consideración de las segundas variaciones conduce a la aparición de nuevos términos en operador tangente (6.33a) y la prueba de que éstos se anulan en una configuración de equilibrio (comentario 6.2.3) es original.

3. El desarrollo y las expresiones de la forma material del operador tangente sección 6.3 y ecuación (6.79) - aún no han sido publicadas.

Relativas a la solución numérica del modelo

1. El análisis de la respuesta del elemento de Simó en piezas con esbeltez creciente, que pone de manifiesto las dificultades numéricas debidas al mal condicionamiento de la matriz es original (sección 7.5.1).

2. También es novedoso el análisis de soluciones incrementales puras empleando el elemento de Simó (sección 7.5.2).

3. Por último, todo el desarrollo del elemento basado en la forma material de las ecuaciones (capítulo 8) es una aportación original. 


\subsection{Líneas de investigación propuestas}

En lo tocante a la formulación de elementos finitos basados en el modelo geométricamente exacto, han sido numerosos los artículos que proponen variantes o modificaciones del elemento de Simó. Las dificultades numéricas típicas de los elementos de barra, como el bloqueo de extensión y corte, aparecen sobre todo al formular elementos curvos, y hay avances interesantes en esa dirección. En otro sentido existe cierta inquietud por la formulación de elementos que garanticen la objetividad de las medidas de la deformación y la independencia de la solución del camino recorrido; también ha habido aportaciones importantes en esa campo. Una línea de trabajo interesante puede encaminarse hacia la aplicación de técnicas para corregir el mal condicionamiento de la rigidez tangente al elemento de Simó. Esto redundaría en un funcionamiento aún más potente del elemento y en la posibilidad de utilizar soluciones incrementales puras en aquellos problemas que lo requieran (como ciertos problemas de plasticidad).

La extensión de la formulación a problemas dinámicos es un aspecto tratado por Simó en la vertiente teórica desde el principio. Son ya numerosas las publicaciones en las que se presentan soluciones numéricas a problemas dinámicos en el marco de la teoría geométricamente exacta. En este ámbito se puede volver a retomar la idea de emplear técnicas modernas de integración geométrica en variedades, aplicándolas esta vez a problemas de dinámica de piezas alargadas.

La consideración de la no linealidad/plasticidad en la respuesta del material es un aspecto que ha sido menos tratado en relación con el modelo de Reissner-Simó y que tiene un campo de aplicación claro, puesto que frecuentemente las piezas flexibles exhiben comportamientos plásticos.

En todo caso, tenemos el convencimiento de que las líneas de investigación más fructíferas en el ámbito de los modelos mecánicos de piezas alargadas muy flexibles serán aquellas que busquen la solución de problemas reales de sistemas estructurales y mecanismos formados por este tipo de sólidos; en ese sentido el modelo geométricamente exacto ofrece la base conceptual adecuada. El reto consistirá en desarrollar modelos numéricos que sean capaces de considerar de forma conjunta el comportamiento dinámico, la no linealidad del material, y la interacción de las piezas con contornos o fluidos exteriores al sistema. 



\section{Apéndice A}

\section{Algunas propiedades de la variación corrotacional}

Recordamos que la variación corrotacional se definió de la siguiente forma

$$
\stackrel{\nabla}{\Delta} \boldsymbol{w}=\Lambda \Delta\left(\boldsymbol{\Lambda}^{\top} \boldsymbol{w}\right)=\boldsymbol{\Lambda} \Delta \boldsymbol{W}
$$

Esta expresión muestra su relación con la variación respecto de los ejes fijos (variación espacial), de objetos materiales. Consideramos las siguientes propiedades:

1. Propiedad de Jacobi en relación con el producto escalar de objetos espaciales (mencionada antes)

$$
\begin{aligned}
\Delta(\boldsymbol{a} \cdot \boldsymbol{b})=\Delta\left(\boldsymbol{a}^{\top} \boldsymbol{\Lambda} \boldsymbol{\Lambda}^{\top} \boldsymbol{b}\right) & =\Delta\left(\boldsymbol{a}^{\top} \boldsymbol{\Lambda}\right) \boldsymbol{\Lambda}^{\top} \boldsymbol{b}+\boldsymbol{a}^{\top} \boldsymbol{\Lambda} \Delta\left(\boldsymbol{\Lambda}^{\top} \boldsymbol{b}\right) \\
& =\stackrel{\nabla}{ } \boldsymbol{a} \cdot \boldsymbol{b}+\boldsymbol{a} \cdot \stackrel{\nabla}{\Delta} \boldsymbol{b} .
\end{aligned}
$$

2. Propiedad de Jacobi en relación con el producto tensor-vector

$$
\begin{aligned}
\stackrel{\nabla}{\Delta}(\boldsymbol{\sigma} \boldsymbol{b})=\boldsymbol{\Lambda} \Delta\left(\boldsymbol{\Lambda}^{\top} \boldsymbol{\sigma} \boldsymbol{\Lambda} \boldsymbol{\Lambda}^{\top} \boldsymbol{b}\right) & =\boldsymbol{\Lambda} \Delta\left(\boldsymbol{\Lambda}^{\top} \boldsymbol{\sigma} \boldsymbol{\Lambda}\right) \boldsymbol{\Lambda}^{\top} \boldsymbol{b}+\boldsymbol{\Lambda} \boldsymbol{\Lambda}^{\top} \boldsymbol{\sigma} \boldsymbol{\Lambda} \Delta\left(\boldsymbol{\Lambda}^{\top} \boldsymbol{b}\right) \\
& =\stackrel{\nabla}{\Delta} \cdot \boldsymbol{b}+\boldsymbol{\sigma} \cdot \stackrel{\nabla}{\Delta} \boldsymbol{b} .
\end{aligned}
$$

3. Propiedad de Jacobi en relación con el producto vectorial. Se demuestra particularizando la propiedad anterior al caso de un tensor antisimétrico, y teniendo en cuenta que el incremento espacial de un tensor antisimétrico también lo es

$$
\stackrel{\nabla}{\Delta}(\boldsymbol{a} \times \boldsymbol{b})=\stackrel{\nabla}{\Delta} \boldsymbol{a} \times \boldsymbol{b}+\boldsymbol{a} \times \stackrel{\nabla}{\Delta} \boldsymbol{b}
$$


4. Variación corrotacional de la derivada de un vector

$$
\begin{aligned}
& \stackrel{\nabla}{\Delta}\left(\boldsymbol{a}^{\prime}\right)=\boldsymbol{\Lambda} \Delta\left(\boldsymbol{\Lambda}^{\top} \boldsymbol{a}^{\prime}\right)=-\boldsymbol{\Lambda} \boldsymbol{\Lambda}^{\top} \Delta \boldsymbol{\Lambda} \boldsymbol{\Lambda}^{\top} \boldsymbol{a}^{\prime}+\boldsymbol{\Lambda} \boldsymbol{\Lambda}^{\top} \Delta\left(\boldsymbol{a}^{\prime}\right) \\
& =-\Delta \boldsymbol{\Lambda} \boldsymbol{\Lambda}^{\top} \boldsymbol{a}^{\prime}+\frac{d}{d S} \Delta(\boldsymbol{\Lambda} \boldsymbol{A}) \\
& =-\Delta \widehat{\boldsymbol{\omega}} \boldsymbol{a}^{\prime}+\frac{d}{d S}(\Delta \boldsymbol{\Lambda} \boldsymbol{A}+\boldsymbol{\Lambda} \Delta \boldsymbol{A}) \\
& =-\Delta \widehat{\boldsymbol{\omega}} \boldsymbol{a}^{\prime}+\frac{d}{d S}\left(\Delta \boldsymbol{\Lambda} \boldsymbol{\Lambda}^{\top} \boldsymbol{a}+\stackrel{\nabla}{\Delta} \boldsymbol{a}\right) \\
& =(\Delta \vec{a})^{\prime}+\Delta \widehat{\boldsymbol{\omega}}^{\prime} \boldsymbol{a} \text {. }
\end{aligned}
$$




\section{Apéndice B}

\section{Cálculo del operador $\mathrm{T}$}

Si $\boldsymbol{\theta}$ es la parametrización vectorial total del grupo $\mathrm{SO}(3)$ de las rotaciones, entonces cualquier elemento del grupo se puede expresar así

$$
\boldsymbol{\Lambda}=\exp \widehat{\boldsymbol{\theta}}
$$

Consideremos ahora una perturbación $\boldsymbol{\Lambda}_{\varepsilon}$ alrededor de $\boldsymbol{\Lambda}$

$$
\boldsymbol{\Lambda}_{\varepsilon}=\exp (\widehat{\boldsymbol{\theta}}+\varepsilon \delta \widehat{\boldsymbol{\theta}}) .
$$

La misma perturbación se puede expresar en función de la parametrización local $\delta \widehat{\boldsymbol{\omega}}$, constituida por los elementos del espacio tangente a $\mathrm{SO}(3)$ en $\boldsymbol{\Lambda}$

$$
\boldsymbol{\Lambda}_{\varepsilon}=\exp (\varepsilon \delta \widehat{\boldsymbol{\omega}}) \boldsymbol{\Lambda} .
$$

Igualando ambas expresiones resulta

$$
\exp (\varepsilon \delta \widehat{\boldsymbol{\omega}})=\exp (\widehat{\boldsymbol{\theta}}+\varepsilon \delta \widehat{\boldsymbol{\theta}}) \exp (-\widehat{\boldsymbol{\theta}}) .
$$

Si derivamos el lado izquierdo de la igualdad

$$
\left.\frac{d}{d \varepsilon} \exp (\varepsilon \delta \widehat{\boldsymbol{\omega}})\right|_{\varepsilon=0}=\delta \widehat{\boldsymbol{\omega}} .
$$

Entonces

$$
\delta \widehat{\boldsymbol{\omega}}=\frac{d}{d \varepsilon}[\exp (\widehat{\boldsymbol{\theta}}+\varepsilon \delta \widehat{\boldsymbol{\theta}}) \exp (-\widehat{\boldsymbol{\theta}})]_{\varepsilon=0} .
$$

Esta expresión muestra la dependencia entre los parámetros locales $\delta \widehat{\boldsymbol{\omega}}$ y la variación de los parámetros totales $\delta \boldsymbol{\theta}$. A continuación vamos a operar la derivada en el lado derecho de la igualdad para obtener una expresión compacta del lado derecho de la igualdad.

$$
\begin{aligned}
& \frac{d}{d \varepsilon}[\exp (\widehat{\boldsymbol{\theta}}+\varepsilon \delta \widehat{\boldsymbol{\theta}}) \exp (-\widehat{\boldsymbol{\theta}})]_{\varepsilon=0} \\
&=\frac{d}{d \varepsilon}\left[\mathbf{1}+\frac{\operatorname{sen}(\|\boldsymbol{\theta}+\varepsilon \delta \boldsymbol{\theta}\|)}{\|\boldsymbol{\theta}+\varepsilon \delta \boldsymbol{\theta}\|}(\widehat{\boldsymbol{\theta}}+\varepsilon \delta \widehat{\boldsymbol{\theta}})+\frac{1-\cos (\|\boldsymbol{\theta}+\varepsilon \delta \boldsymbol{\theta}\|)}{\|\boldsymbol{\theta}+\varepsilon \delta \boldsymbol{\theta}\|^{2}}(\widehat{\boldsymbol{\theta}}+\varepsilon \delta \widehat{\boldsymbol{\theta}})^{2}\right]_{\varepsilon=0} \\
& \cdot\left[\mathbf{1}+\frac{\sin \theta}{\theta} \widehat{\boldsymbol{\theta}}+\frac{1-\cos \theta}{\theta^{2}} \widehat{\boldsymbol{\theta}}^{2}\right] .
\end{aligned}
$$


Derivamos ahora los términos en el corchete. La derivada del tensor 1 es nula; la derivada del segundo término (con la notación $\delta \theta=\|\delta \boldsymbol{\theta}\|$ y $\theta=\|\boldsymbol{\theta}\|$ ) es:

$$
\begin{aligned}
\frac{d}{d \varepsilon} & {\left[\frac{\operatorname{sen}(\|\boldsymbol{\theta}+\varepsilon \delta \boldsymbol{\theta}\|)}{\|\boldsymbol{\theta}+\varepsilon \delta \boldsymbol{\theta}\|}(\widehat{\boldsymbol{\theta}}+\varepsilon \delta \widehat{\boldsymbol{\theta}})\right]_{\varepsilon=0} } \\
& =\left\{\left[\frac{\cos (\|\boldsymbol{\theta}+\varepsilon \delta \boldsymbol{\theta}\|) \delta \theta\|\boldsymbol{\theta}+\varepsilon \delta \boldsymbol{\theta}\|-\operatorname{sen}(\|\boldsymbol{\theta}+\varepsilon \delta \boldsymbol{\theta}\|) \delta \theta}{\|\boldsymbol{\theta}+\varepsilon \delta \boldsymbol{\theta}\|^{2}}\right](\widehat{\boldsymbol{\theta}}+\varepsilon \delta \widehat{\boldsymbol{\theta}})\right. \\
& \left.+\left[\frac{\operatorname{sen}(\|\boldsymbol{\theta}+\varepsilon \delta \boldsymbol{\theta}\|)}{\|\boldsymbol{\theta}+\varepsilon \delta \boldsymbol{\theta}\|}\right] \delta \widehat{\boldsymbol{\theta}}\right\}_{\varepsilon=0} \\
& =\frac{\theta \cos \theta-\operatorname{sen} \theta}{\theta^{2}} \delta \theta \widehat{\boldsymbol{\theta}}+\frac{\operatorname{sen} \theta}{\theta} \delta \widehat{\boldsymbol{\theta}} \\
& =\frac{\cos \theta}{\theta^{2}}(\boldsymbol{\theta} \cdot \delta \boldsymbol{\theta}) \widehat{\boldsymbol{\theta}}-\frac{\operatorname{sen} \theta}{\theta^{3}}(\boldsymbol{\theta} \cdot \delta \boldsymbol{\theta}) \widehat{\boldsymbol{\theta}}+\frac{\operatorname{sen} \theta}{\theta} \delta \widehat{\boldsymbol{\theta}} .
\end{aligned}
$$

En este último paso se ha empleado la siguiente relación

$$
\delta \theta=\frac{\boldsymbol{\theta} \cdot \delta \boldsymbol{\theta}}{\theta}
$$

deducida teniendo en cuenta que

$$
\frac{d}{d \varepsilon}[(\boldsymbol{\theta}+\varepsilon \delta \boldsymbol{\theta}) \cdot(\boldsymbol{\theta}+\varepsilon \delta \boldsymbol{\theta})]_{\varepsilon=0}=2[(\boldsymbol{\theta}+\varepsilon \delta \boldsymbol{\theta}) \cdot \delta \boldsymbol{\theta}]_{\varepsilon=0}=2 \boldsymbol{\theta} \cdot \delta \boldsymbol{\theta}
$$

y

$$
\frac{d}{d \varepsilon}[(\boldsymbol{\theta}+\varepsilon \delta \boldsymbol{\theta}) \cdot(\boldsymbol{\theta}+\varepsilon \delta \boldsymbol{\theta})]_{\varepsilon=0}=\frac{d}{d \varepsilon}\left[\|\boldsymbol{\theta}+\varepsilon \delta \boldsymbol{\theta}\|^{2}\right]_{\varepsilon=0}=2[\|\boldsymbol{\theta}+\varepsilon \delta \boldsymbol{\theta}\| \delta \theta]_{\varepsilon=0}=2 \theta \delta \theta
$$

Falta calcular la derivada del tercer término:

$$
\begin{aligned}
& \frac{d}{d \varepsilon}\left[\frac{1-\cos (\|\boldsymbol{\theta}+\varepsilon \delta \boldsymbol{\theta}\|)}{\|\boldsymbol{\theta}+\varepsilon \delta \boldsymbol{\theta}\|^{2}}(\widehat{\boldsymbol{\theta}}+\varepsilon \delta \widehat{\boldsymbol{\theta}})^{2}\right]_{\varepsilon=0} \\
& =\left\{\left[\frac{\operatorname{sen}(\|\boldsymbol{\theta}+\varepsilon \delta \boldsymbol{\theta}\|) \delta \theta\|\boldsymbol{\theta}+\varepsilon \delta \boldsymbol{\theta}\|^{2}-(1-\cos (\|\boldsymbol{\theta}+\varepsilon \delta \boldsymbol{\theta}\|))^{2} 2\|\boldsymbol{\theta}+\varepsilon \delta \boldsymbol{\theta}\| \delta \theta}{\|\boldsymbol{\theta}+\varepsilon \delta \boldsymbol{\theta}\|^{4}}\right](\widehat{\boldsymbol{\theta}}+\varepsilon \delta \widehat{\boldsymbol{\theta}})^{2}\right. \\
& \left.+\left[\frac{1-\cos (\|\boldsymbol{\theta}+\varepsilon \delta \boldsymbol{\theta}\|)}{\|\boldsymbol{\theta}+\varepsilon \delta \boldsymbol{\theta}\|^{2}}\right][\delta \widehat{\boldsymbol{\theta}}(\widehat{\boldsymbol{\theta}}+\varepsilon \delta \widehat{\boldsymbol{\theta}})+(\widehat{\boldsymbol{\theta}}+\varepsilon \delta \widehat{\boldsymbol{\theta}}) \delta \widehat{\boldsymbol{\theta}}]\right\}_{\varepsilon=0} \\
& =\frac{\operatorname{sen} \theta}{\theta^{3}}(\boldsymbol{\theta} \cdot \delta \boldsymbol{\theta}) \widehat{\boldsymbol{\theta}}^{2}-2 \frac{1-\cos \theta}{\theta^{4}}(\boldsymbol{\theta} \cdot \delta \boldsymbol{\theta}) \widehat{\boldsymbol{\theta}}^{2}+\frac{1-\cos \theta}{\theta^{2}}(\delta \widehat{\boldsymbol{\theta}} \widehat{\boldsymbol{\theta}}-\widehat{\boldsymbol{\theta}} \delta \widehat{\boldsymbol{\theta}}) .
\end{aligned}
$$

Entonces, podemos expresar la ecuación (B.1) así

$$
\begin{aligned}
\delta \widehat{\boldsymbol{\omega}}=[ & \left(\frac{\cos \theta}{\theta^{2}}-\frac{\operatorname{sen} \theta}{\theta^{3}}\right)(\boldsymbol{\theta} \cdot \delta \boldsymbol{\theta}) \widehat{\boldsymbol{\theta}}+\left(\frac{\operatorname{sen} \theta}{\theta^{3}}-2 \frac{1-\cos \theta}{\theta^{4}}\right)(\boldsymbol{\theta} \cdot \delta \boldsymbol{\theta}) \widehat{\boldsymbol{\theta}}^{2} \\
& \left.+\frac{\operatorname{sen} \theta}{\theta} \delta \widehat{\boldsymbol{\theta}}+\frac{1-\cos \theta}{\theta^{2}}(\delta \widehat{\boldsymbol{\theta}} \widehat{\boldsymbol{\theta}}-\widehat{\boldsymbol{\theta}} \delta \widehat{\boldsymbol{\theta}})\right]\left[\mathbf{1}+\frac{\sin \theta}{\theta} \widehat{\boldsymbol{\theta}}+\frac{1-\cos \theta}{\theta^{2}} \widehat{\boldsymbol{\theta}}^{2}\right]
\end{aligned}
$$


Teniendo en cuenta que $\widehat{\boldsymbol{\theta}}^{3}=-\theta^{2} \widehat{\boldsymbol{\theta}}$, operando y simplificando se obtiene

$$
\begin{aligned}
\delta \widehat{\boldsymbol{\omega}}=\left(\frac{\cos \theta}{\theta^{2}}-\right. & \left.\frac{\operatorname{sen} \theta}{\theta^{3}}+\frac{\operatorname{sen}^{2} \theta}{\theta^{2}}-\frac{\operatorname{sen} \theta(1-\cos \theta)}{\theta^{3}}-\frac{\cos \theta(1-\cos \theta)}{\theta^{2}}\right)(\boldsymbol{\theta} \cdot \delta \boldsymbol{\theta}) \widehat{\boldsymbol{\theta}} \\
+ & \left.-2 \frac{1-\cos \theta}{\theta^{4}}+\frac{\operatorname{sen}^{2} \theta}{\theta^{4}}+2 \frac{(1-\cos \theta)^{2}}{\theta^{4}}\right)(\boldsymbol{\theta} \cdot \delta \boldsymbol{\theta}) \widehat{\boldsymbol{\theta}}^{2} \\
+\frac{\operatorname{sen} \theta}{\theta} \delta \widehat{\boldsymbol{\theta}}- & \frac{1-\cos \theta}{\theta^{2}} \delta \widehat{\boldsymbol{\theta}} \widehat{\boldsymbol{\theta}}+\frac{1-\cos \theta}{\theta^{2}} \widehat{\boldsymbol{\theta}} \delta \widehat{\boldsymbol{\theta}} \\
& -\frac{\operatorname{sen} \theta(1-\cos \theta)}{\theta^{3}} \widehat{\boldsymbol{\theta}} \delta \widehat{\boldsymbol{\theta}} \widehat{\boldsymbol{\theta}}+\frac{(1-\cos \theta)^{2}}{\theta^{4}} \widehat{\boldsymbol{\theta}} \delta \widehat{\boldsymbol{\theta}} \widehat{\boldsymbol{\theta}}^{2} .
\end{aligned}
$$

Esta expresión se puede simplificar más considerando las siguientes igualdades (cuya verificación es inmediata)

$$
\widehat{\boldsymbol{\theta}} \delta \widehat{\boldsymbol{\theta}} \widehat{\boldsymbol{\theta}}=-(\boldsymbol{\theta} \cdot \delta \boldsymbol{\theta}) \widehat{\boldsymbol{\theta}} \quad \widehat{\boldsymbol{\theta}} \delta \widehat{\boldsymbol{\theta}} \widehat{\boldsymbol{\theta}}^{2}=-(\boldsymbol{\theta} \cdot \delta \boldsymbol{\theta}) \widehat{\boldsymbol{\theta}}^{2}
$$

de modo que

$$
\delta \widehat{\boldsymbol{\omega}}=\frac{\operatorname{sen} \theta}{\theta} \delta \widehat{\boldsymbol{\theta}}+\frac{1-\cos \theta}{\theta^{2}}(\widehat{\boldsymbol{\theta}} \delta \widehat{\boldsymbol{\theta}}-\delta \widehat{\boldsymbol{\theta}} \widehat{\boldsymbol{\theta}})+\frac{\theta-\operatorname{sen} \theta}{\theta^{3}}(\boldsymbol{\theta} \cdot \delta \boldsymbol{\theta}) \widehat{\boldsymbol{\theta}} .
$$

A partir de aquí, interesa factorizar esta expresión en $\delta \boldsymbol{\theta}$. Para ello tenderemos en cuenta la siguiente igualdad:

$$
\widehat{\boldsymbol{\theta}} \delta \widehat{\boldsymbol{\theta}}-\delta \widehat{\boldsymbol{\theta}} \widehat{\boldsymbol{\theta}}=\widehat{\boldsymbol{\theta} \times \delta \boldsymbol{\theta}}=\widehat{\widehat{\boldsymbol{\theta}} \delta \boldsymbol{\theta}},
$$

Por otro lado

$$
\widehat{\boldsymbol{\theta}} \widehat{\boldsymbol{\theta}} \delta \boldsymbol{\theta}=-\theta^{2} \delta \boldsymbol{\theta}+(\boldsymbol{\theta} \cdot \delta \boldsymbol{\theta}) \cdot \boldsymbol{\theta}
$$

luego

$$
(\boldsymbol{\theta} \cdot \delta \boldsymbol{\theta}) \cdot \boldsymbol{\theta}=\widehat{\boldsymbol{\theta}} \widehat{\boldsymbol{\theta}} \delta \boldsymbol{\theta}+\theta^{2} \delta \boldsymbol{\theta}
$$

y la expresión matricial equivalente es

$$
(\boldsymbol{\theta} \cdot \delta \boldsymbol{\theta}) \widehat{\boldsymbol{\theta}}=\widehat{\widehat{\boldsymbol{\theta}} \widehat{\boldsymbol{\theta}} \delta \boldsymbol{\theta}}+\theta^{2} \delta \widehat{\boldsymbol{\theta}} .
$$

Introduciendo (B.6) y (B.7) en (B.5) se obtiene

$$
\delta \widehat{\boldsymbol{\omega}}=\left(\frac{\operatorname{sen} \theta}{\theta}+\frac{\theta-\operatorname{sen} \theta}{\theta}\right) \delta \widehat{\boldsymbol{\theta}}+\frac{1-\cos \theta}{\theta^{2}} \widehat{\widehat{\boldsymbol{\theta}} \delta \boldsymbol{\theta}}+\frac{\theta-\operatorname{sen} \theta}{\theta^{3}} \widehat{\widehat{\boldsymbol{\theta}} \widehat{\boldsymbol{\theta}} \delta \boldsymbol{\theta}},
$$

y la expresión vectorial equivalente es

$$
\delta \boldsymbol{\omega}=\left(\mathbf{1}+\frac{1-\cos \theta}{\theta^{2}} \widehat{\boldsymbol{\theta}}+\frac{\theta-\operatorname{sen} \theta}{\theta^{3}} \widehat{\boldsymbol{\theta}}^{2}\right) \delta \boldsymbol{\theta}=\mathbf{T}(\boldsymbol{\theta}) \delta \boldsymbol{\theta} .
$$

Por lo tanto la expresión del operador es

$$
\mathbf{T}(\boldsymbol{\theta})=\mathbf{1}+\frac{1-\cos \theta}{\theta^{2}} \widehat{\boldsymbol{\theta}}+\frac{\theta-\operatorname{sen} \theta}{\theta^{3}} \widehat{\boldsymbol{\theta}}^{2}
$$

o bien

$$
\mathbf{T}(\boldsymbol{\theta})=\frac{\operatorname{sen} \theta}{\theta} \mathbf{1}+\frac{1-\cos \theta}{\theta^{2}} \widehat{\boldsymbol{\theta}}+\frac{\theta-\operatorname{sen} \theta}{\theta^{3}} \boldsymbol{\theta} \otimes \boldsymbol{\theta} .
$$





\section{Bibliografía}

[1] S.S. Antman, The Theory of Rods, Handbuch der Physik, vol. VI a/2, SpringerVerlag, Berlin, 1972.

[2] K Kirchhoff's problem for nonlinearly elastic rods, Quarterly of Applied Mathematics 32 (1974), no. 3, 221-240.

[3] - Ordinary differential equations of non-linear elasticity I: Foundations of the theories of non-linearly elastic rods and shells, Arch. Rational Mech. Anal. 61 (1976), no. 4, 307-351.

[4] J.H. Argyris, An excursion into large rotations, Computer Methods in Applied Mechanics and Engineering 32 (1982), 85-155.

[5] J.H. Argyris, P.C. Dunne, and D.W. Scharpf, On large displacement-small strain analysis of structures with rotational degrees of freedom, Computer Methods in Applied Mechanics and Engineering 14 (1978), 401-451.

[6] J.H. Argyris and S. Symeonidis, Nonlinear finite element analysis of elastic systems under nonconservative loading - natural formulation. Part I. Quasistatic problems, Computer Methods in Applied Mechanics and Engineering 26 (1981), $75-123$.

[7] V.I. Arnold, Mathematical Methods of Classical Mechanics, 2nd ed., Graduate Texts in Mathematics, vol. 60, Springer, New York, 1989, traducción del original en ruso publicado en 1974 por Nauka (Moscú).

[8] K.J. Bathe and S. Bolourchi, Large displacement analysis of three dimensional beam structures, International Journal for Numerical Methods in Engineering 14 (1979), 961-986.

[9] P. Betsch, A. Menzel, and E. Stein, On the parametrization of finite rotations in computational mechanics. A classification of concepts with application to smooth shells, Computer Methods in Applied Mechanics and Engineering 155 (1998), 273-305.

[10] D.O. Brush and B.O. Almroth, Buckling of Bars, Plates and Shells, McGrawHill, 1975. 
[11] A. Cardona and M. Geradin, A beam finite element non-linear theory with finite rotations, International Journal for Numerical Methods in Engineering 26 (1988), 2403-2438.

[12] E. Cosserat and F. Cosserat, Theorie des Corps Deformables, A. Hermann et Fils, Paris, 1909.

[13] M.A. Crisfield, A consistent co-rotational formulation for non-linear, threedimensional beam elements, Computer Methods in Applied Mechanics and Engineering 81 (1990), 131-150.

[14] _ Non-linear Finite Element Analysis of Solids and Structures, vol. 1: Essentials, John Wiley \& Sons, 1991.

[15] _ Non-linear Finite Element Analysis of Solids and Structures, vol. 2: Advanced Topics, John Wiley \& Sons, 1997.

[16] M.A. Crisfield and G. Jelenić, Objectivity of strain measures in the geometrically exact three-dimensional beam theory and its finite-element implementation, Proc. R. Soc. Lond. A 455 (1999), 1125-1147.

[17] A. Dou, Leonhard Euler. Método de Máximos y Mínimos, Publicacions de la Universitat Autonoma de Barcelona, Edicions de la Universitat Politecnica de Catalunya, Barcelona, 1993.

[18] L. Euler, Methodus Inveniendi Lineas Curvas Maximi Minimivi Proprietate Gaudentes, Marcum-Michaelem Bousquet \& Socios, Lausanne \& Geneve, 1744, Reproducción facsímil del original, editada por el Servei de Publicacions de la Universitat Autònoma de Barcelona y Edicions de la Universitat Politècnica de Catalunya, Barcelona 1993.

[19] C.A. Felippa, Nonlinear Finite Element Methods, http://caswww.colorado.edu/courses.d/NFEM.d/Home.html, 2001, notes for a graduate course offered at the University of Colorado (Boulder).

[20] H. Goldstein, Classical Mechanics, second ed., Addison-Wesley, 1980.

[21] A.E. Green, P.M. Naghdi, and M.L. Wenner, On the theory of rods. I. Derivations from the three-dimensional equations, Proc. Royal Soc. Lond. A 337 (1974), 451483.

[22] _ On the theory of rods. II. Developments by the direct approach, Proc. Royal Soc. Lond. A 337 (1974), 485-507.

[23] E. Hairer, C. Lubich, and G. Wanner, Geometric Numerical Integration. Structure-Preserving Algorithms for Ordinary Differential Equations, Springer Series in Computational Mathematics, vol. 31, Springer, Berlin, 2002. 
[24] D.D. Holm, J.E. Marsden, and T.S. Ratiu, The Euler-Poincaré equations and semidirect products with applications to continuum theories, Adv. in Math. 137 (1998), 1-81.

[25] A. Ibrahimbegović, On finite element implementation of geometrically nonlinear Reissner's beam theory: three-dimensional curved beam elements, Computer Methods in Applied Mechanics and Engineering 122 (1995), 11-26.

[26] - On the choice of finite rotation parameters, Computer Methods in Applied Mechanics and Engineering 149 (1997), 49-71.

[27] A. Ibrahimbegović, F. Frey, and I. Kozar, Computational aspects of vector-like parametrization of three-dimensional finite rotations, International Journal for Numerical Methods in Engineering 38 (1995), 3653-3673.

[28] A. Ibrahimbegović and R.L. Taylor, On the role of frame-invariance in structural mechanics models at finite rotations, Computer Methods in Applied Mechanics and Engineering 191 (2002), 5159-5176.

[29] A. Iserles, Solving linear ordinary differential equations by exponentials of iterated commutators, Numerische Mathematik 45 (1984), 183-199.

[30] A. Iserles and S.P. Nørsett, On the solution of linear differential equations in Lie groups, R. Soc. Lond. Philos. Trans. Ser. A Math. Phys. Eng. Sci. 357 (1999), 983-1019.

[31] L. Vu-Quoc J.C. Simo, A geometrically-exact rod model incorporating shear and torsion-warping deformation, International Journal of Solids and Structures $\mathbf{2 7}$ (1991), no. 3, 371-393.

[32] G. Jelenić and M.A. Crisfield, Geometrically exact 3D beam theory: implementation of a strain-invariant finite element for statics and dynamics, Computer Methods in Applied Mechanics and Engineering 171 (1999), 141-171.

[33] G. Jelenić and M. Saje, A kinematically exact space finite strain beam model - finite element formulation by generalizad virtual work principle, Computer Methods in Applied Mechanics and Engineering 120 (1995), 131-161.

[34] R.K. Kapania and J. Li, A formulation and implementation of geometrically exact curved beam elements incorporating finite strains and finite rotations, Computational Mechanics 30 (2003), 44-459.

[35] G. Kirchhoff, Über das Gleichgewicht und die Bewegung eines unendlich dünnen elastischen Stabes, Journal für reine und angewandte Mathematik (Crelle) 56 (1859), 285-313.

[36] J.L. Lagrange, Mécanique Analytique, Chez la Veuve Desaint, 1788.

[37] C. Lanczos, The Variational Principles of Mechanics, Dover, New York, 1986, reedición del original publicado en 1949 por University of Toronto Press. 
[38] C. Lázaro, Formulación hamiltoniana de problemas de equilibrio en vigas, arcos, placas y láminas, trabajo de investigación presentado en el Departamento de Mecánica de Medios Continuos y Teoría de Estructuras de la Universidad Politécnica de Valencia, 2002.

[39] _ Técnicas numéricas de linealización a trozos vs. expansiones de Magnus y Fer en la solución de problemas elásticos lineales 1D. Aplicación a la viga de canto variable, trabajo de investigación presentado en el Departamento de Mecánica de Medios Continuos y Teoría de Estructuras de la Universidad Politécnica de Valencia, 2002.

[40] A.E.H. Love, A Treatise on the Mathematical Theory of Elasticity, Dover, New York, 1944, reimpresión del original publicado en 1927 por Cambridge University Press.

[41] J.E. Marsden and T.J.R. Hughes, Mathematical Foundations of Elasticity, Dover, New York, 1994, revisión de la edición original publicada por Prentice-Hall, Inc. en 1983.

[42] J.E. Marsden, S. Pekarsky, and S. Shkoller, Discrete Euler-Poincaré and LiePoisson equations, Nonlinearity 12 (1999), 1647-1662.

[43] J.E. Marsden and T.S. Ratiu, Einführung in die Mechanik und Symmetrie, Springer, Berlin, 2001, traducido del original en inglés "Introduction to Mechanics and Symmetry" por Stefan Hackmann und Ulrich Krähmer.

[44] A. Mielke and P. Holmes, Spatially complex equilibria of buckled rods, Arch. Rational Mech. Anal. 101 (1988), no. 4, 319-348.

[45] S. Monleón, Análisis de Vigas, Arcos, Placas y Láminas: Una Presentación Unificada, Editorial de la Universidad Politécnica de Valencia, Valencia, 1999.

[46] A. Morán, Formulaciones tangente y secante en análisis no lineal de vigas Cosserat, tesis doctoral, E.T.S. Ingenieros de Caminos, Canales y Puertos, Universidad Politècnica de Catalunya, 2005.

[47] P.M. Naghdi, Finite deformations of elastic rods and shells, Proceedings of the IUTAM Symposium on Finite Elasticity, Lehigh University, August 10-15, 1980 (The Hague/Boston/London) (D.E. Carlson and R.T. Shield, eds.), Martinus Nijhoff Publishers, 1981, pp. 47-103.

[48] P.K.V.V. Nukala and D.W. White, A mixed finite element for three-dimensional nonlinear analysis of steel frames, Computer Methods in Applied Mechanics and Engineering 193 (2004), 2507-2545.

[49] P. J. Olver, Applications of Lie Groups to Differential Equations, Graduate Texts in Mathematics, vol. 107, Springer, New York, 1986.

[50] H. Poincaré, Sur une forme nouvelle des équations de la mécanique, C. R. Acad. Sci. 132 (1901), 369-371. 
[51] E. Reissner, On one-dimensional large-displacement finite-strain beam theory, Studies in Applied Mathematics 52 (1973), no. 2, 87-95.

[52] _ On finite deformations of space-curved beams, Journal of Applied Mathematics and Physics (ZAMP) 32 (1981), 734-744.

[53] M. Ritto-Corrêa and D. Camotim, On the differentiation of the Rodrigues formula and its significance fot the vector-like parametrization of Reissner-Simo beam theory, International Journal for Numerical Methods in Engineering 55 (2002), $1005-1032$.

[54]__ Work-conjugacy between rotation-dependent moments and finite rotations, International Journal of Solids and Structures 40 (2003), no. 11, 28512873 .

[55] O. Rodrigues, Des lois géométriques qui régissent les déplacements d'un système solide dans l'espace et de la variation des coordinnées provenant de ces déplacements considéres indépendment des causes qui peuvent les produire, J. Math Pures Appl. 5 (1840), 380-440.

[56] I. Romero, The interpolation of rotations and its application to finite element models of geometrically exact rods, Computational Mechanics 34 (2004), 121133.

[57] I. Romero and F. Armero, An objective finite element approximation of the kinematics of geometrically exact rods and its use in the formulation of an energymomentum conserving scheme in dynamics, International Journal for Numerical Methods in Engineering 54 (2002), 1683-1716.

[58] J. C. Simo, A finite strain beam formulation. The three-dimensional dynamic problem. Part I, Computer Methods in Applied Mechanics and Engineering 49 (1985), 55-70, North-Holland.

[59] J.C. Simo, The (symmetric) Hessian for geometrically nonlinear models in solid mechanics: Intrinsic definition and geometric interpretation, Computer Methods in Applied Mechanics and Engineering 96 (1992), 189-200.

[60] J.C. Simo and D.D. Fox, On a stress resultant geometrically exact shell model. Part I: Formulation and optimal parametrization, Computer Methods in Applied Mechanics and Engineering 72 (1989), 267-304.

[61] J.C. Simó, N. Tarnow, and M. Doblaré, Non-linear dynamics of threedimensional rods: Exact energy and momentum conserving algorithms, International Journal for Numerical Methods in Engineering 38 (1995), 1431-1473.

[62] J.C. Simo and L. Vu-Quoc, A three-dimensional finite-strain rod model. Part II: Computational aspects, Computer Methods in Applied Mechanics and Engineering 58 (1986), 79-116, North-Holland. 
[63] R.A. Spurrier, Comment on "Singularity-free extraction of a quaternion from a direction-cosine matrix", J. Spacecraft 15 (1978), no. 4, 255.

[64] J. Stuelpnagel, On the parametrization of the three-dimensional rotation group, SIAM Review 6 (1964), no. 4, 422-430.

[65] J.M. Wendlandt and J.E. Marsden, Mechanical integrators derived from a discrete variational principle, Physica D 106 (1997), 223-246.

[66] D. Zupan and M. Saje, Finite-element formulation of geometrically exact threedimensional beam theories based on interpolation of strain measures, Computer Methods in Applied Mechanics and Engineering 192 (2003), 5209-5248.

[67] _ Rotational invariants in finite element formulation of three-dimensional beam theories, Computers and Structures 82 (2004), 2027-2040. 